\title{
BRINE SAMPLING AND EVALUATION PROGRAM \\ 1992-1993 REPORT AND SUMMARY OF \\ BSEP DATA SINCE 1982
}

\section{DOE-WIPP 94-011}

April 1995

\author{
AUTHORS \\ D. E. Deal-IT Corporation \\ R. J. Abitz-IT Corporation \\ D. S. Belski-Westinghouse Electric Corporation \\ J. B. Case-IT Corporation \\ M. E. Crawley-IT Corporation \\ C. A. Givens-IT Corporation \\ P. P. James Lipponer-IT Corporation \\ D. J. Milligan-IT Corporation \\ J. Myers-IT Corporation \\ D. W. Powers-Consulting Geologist \\ M. A. Valdivia-IT Corporation
}

Any comments or questions regarding this report should

be directed to the

U.S. Department of Energy

WIPP Project Office

P. O. Box 3090

Carlsbad, New Mexico 88221

\author{
or to the \\ Manager \\ Engineering Department \\ Westinghouse Electric Corporation \\ Waste Isolation Division \\ P.O. Box 2078 \\ Carlsbad, New Mexico 88221
}

This report was prepared for the U.S. Department of Energy by the Engineering Department of the Management and Operating Contractor, Waste Isolation Pilot Plant, under Contract No. DE-AC04-86AL31950. 


\section{DISCLAIMER}

Portions of this document may be illegible in electronic image products. Images are produced from the best available original document. 


\title{
BRINE SAMPLING AND EVALUATION PROGRAM 1992-1993 REPORT AND SUMMARY OF \\ BSEP DATA SINCE 1982 \\ DOE-WIPP 94-011.
}

April 1995

\author{
AUTHORS \\ D. E. Deal-IT Corporation \\ R. J. Abitz-IT Corporation \\ D. S: Belski-Westinghouse Electric Corporation \\ J. B. Case-IT Corporation \\ M. E. Crawley-IT Corporation \\ C. A. Givens-IT Corporation \\ P. P. James Lipponer-IT Corporation \\ D. J. Milligan-IT Corporation \\ J. Myers-IT Corporation \\ D. W. Powers-Consulting Geologist \\ M. A. Valdivia-IT Corporation
}

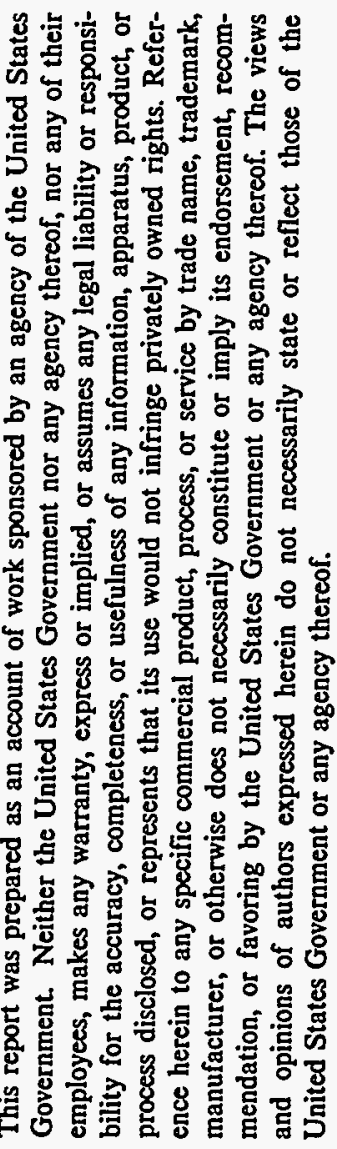

Any comments or questions regarding this report should

be directed to the

U.S. Department of Energy

WIPP Project Office

P. O. Box 3090

Carlsbad, New Mexico 88221

\author{
or to the \\ Manager \\ Engineering Department \\ Westinghouse Electric Corporation \\ Waste Isolation Division \\ P.O. Box 2078 \\ Carlsbad, New Mexico 88221
}

This report was prepared for the U.S. Department of Energy by the Engineering Department of the Management and Operating Contractor, Waste Isolation Pilot Plant, under Contract No. DE-AC04-86AL31950. 
This document is issued by Westinghouse Electric Corporation, Waste Isolation Division, as the Management and Operating Contractor for the U. S. Department of Energy, Waste Isolation Pilot Plant, Carlsbad, New Mexico 88221.

\section{DOE CONTRACT NUMBER: DE-ACO4-86AL31950}

This document has been submitted as required to:

Office of Scientific and Technical Information

PO Box 62

Oak Ridge, TN 37831

(615) $576-8401$

Additional information about this document may be obtained by calling 1-800-336-9477. Copies may be obtained by contacting the National Technical Information Service, US Department of Commerce, 5285 Port Royal Roacl, Springfield, VA 22161. 


\section{Acknowledgments}

Dr. Dwight Deal provides overall direction to the Brine Sampling and Evaluation Program (BSEP) at the Waste Isolation Pilot Plant (WIPP) located in Carlsbad, New Mexico.

Dr. Rich Abitz coordinates the geochemical analyses.

Mr. Dave Belski is responsible for the routine collection of brine from the drill holes in the repository, sample measurement and processing, and data analysis.

Mr. Darin Milligan prepared the statistical analysis of the geochemical data (Chapter 3.0).

Mr. Mark Crawley prepared the 1993 file report of the hydrologic testing of the fractured part of the disturbed rock zone beneath the WIPP excavations, which is summarized in Chapter 4.0 and Appendix E.

Dr. Dennis Powers contributed the observations in the air intake shaft (Appendix C).

Ms. Pamela James-Lipponer was responsible for entry, analysis, and quality assurance of the brine inflow data and prepared Appendices A, B, and D.

Mr. Craig Givens edited and condensed Appendices $C$ and E, prepared the final graphs in Appendix B, and wrote Chapter 4.0.

Dr. John Case performed the numerical modeling of brine seepage from clay compaction presented in Appendix F.

Mr. Miguel Valdivia provided extensive support to both the statistical analysis and the numerical modeling. He prepared the final version of Appendix F.

Dr. Jonathan Myers provided input to the discussion of brine geochemistry and on the importance of the BSEP to the assessment of long-term facility performance. 


\section{THIS PAGE INTENTIONALLY LEFT BLANK}




\section{Table of Contents}

Acknowledgments $\ldots \ldots \ldots \ldots \ldots \ldots \ldots \ldots \ldots \ldots \ldots \ldots \ldots \ldots \ldots$

List of Tables $\ldots \ldots \ldots \ldots \ldots \ldots \ldots \ldots \ldots \ldots \ldots \ldots \ldots \ldots \ldots \ldots$

List of Figures $\ldots \ldots \ldots \ldots \ldots \ldots \ldots \ldots \ldots \ldots \ldots \ldots \ldots \ldots \ldots$

List of Abbreviations/Acronyms $\ldots \ldots \ldots \ldots \ldots \ldots \ldots \ldots \ldots \ldots \ldots \ldots$

Executive Summary $\ldots \ldots \ldots \ldots \ldots \ldots \ldots \ldots \ldots \ldots \ldots \ldots \ldots$

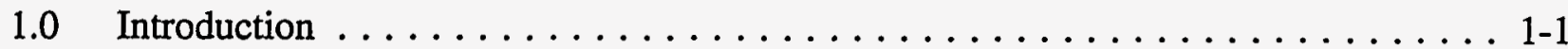

2.0 Monitoring of Brine Inflow Parameters $\ldots \ldots \ldots \ldots \ldots \ldots \ldots \ldots \ldots \ldots$

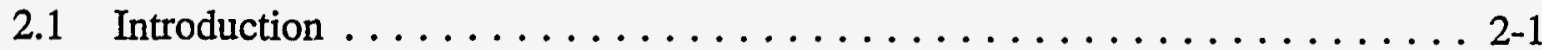

2.2 Damp or Wet Areas on Drift Floors ................. 2-1

2.3 Downholes and Brine Beneath the Floor . . . . . . . . . . . . 2-1

2.3.1 Downholes ......................... 2-1

2.3.2 Shaft Sumps ........................ 2-6

2.4 Upholes and Brine Above the Roof ................. 2-6

2.5 Subhorizontal Holes $\ldots \ldots \ldots \ldots \ldots \ldots \ldots \ldots \ldots \ldots \ldots \ldots$

2.6 Air Intake Shaft . . . . . . . . . . . . . . . . . . . . 2-9

2.7 Discussion of Data Acquisition and Analysis . . . . . . . . . . . . 2-10

3.0 Statistical Analysis of the BSEP Brines . . . . . . . . . . . . . . 3-1

3.1 Introduction $\ldots \ldots \ldots \ldots \ldots \ldots \ldots \ldots \ldots \ldots \ldots \ldots \ldots \ldots \ldots \ldots$

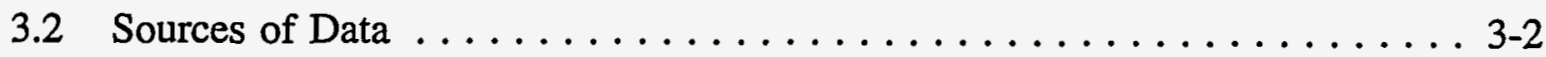

3.3 Temporal Trends . . . . . . . . . . . . . . . . . . . 3-2

3.4 Duplicate Analysis . . . . . . . . . . . . . . . . . . . . 3-4

3.5 Determination of Statistical Distributions . . . . . . . . . . . . . . 3-4

3.6 Handling of Values . . . . . . . . . . . . . . . . . . 3-7

3.7 Rejection of Outliers . . . . . . . . . . . . . . . . 3-7

3.8 Average Brine Chemistry . . . . . . . . . . . . . . . . 3-9

3.9 Composition of Non-Salado Brine from the WIPP Underground . . . . . 3-19

3.10 Conclusions . . . . . . . . . . . . . . . . . . . . . 3-19

4.0 Hydrologic Testing of the Fractured Part of the Disturbed Rock Zone Beneath

the WIPP Excavations . . . . . . . . . . . . . . . . . . . . 4-1

5.0 Numerical Modeling of Brine Seepage as a Result of Clay Compaction . . . . . . 5-1

5.1 Introduction $\ldots \ldots \ldots \ldots \ldots \ldots \ldots \ldots \ldots \ldots \ldots \ldots \ldots \ldots \ldots$

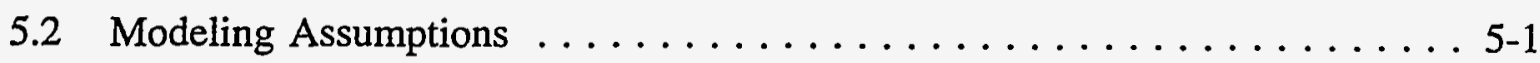

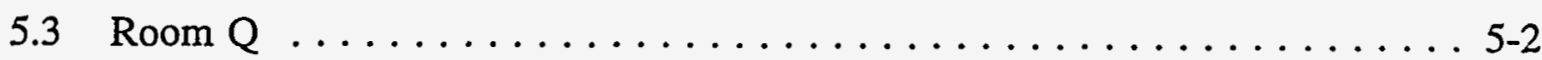




\section{Table of Contents (Continued)}

5.4 Standard WIPP Waste Storage Room $\ldots \ldots \ldots \ldots \ldots \ldots \ldots \ldots .5-2$

5.5 Axial Consolidation Around a Borehole $\ldots \ldots \ldots \ldots \ldots \ldots \ldots \ldots .5-3$

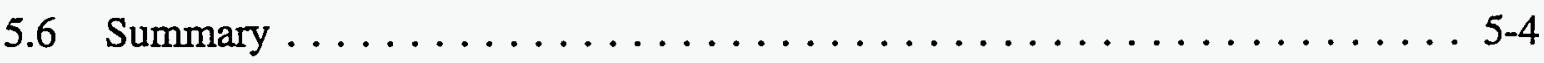

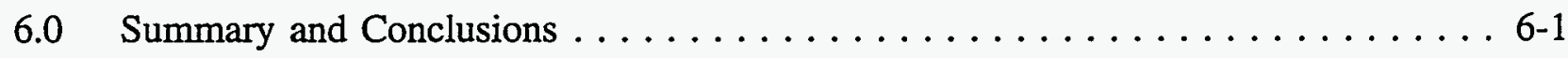

7.0 References ............................. 7-1

Appendix A-Brine Accumulation

Appendix B-Graphs of Brine Accumulation Data

Appendix C-AIS Observations

Appendix D-1993 Analytical Results

Appendix E-Hydrologic Testing

Appendix F-Numerical Modeling of Brine Seepage from Clay Compaction 


\section{List of Tables}

Table

Title

1-1 Points to be Considered When Evaluating BSEP Data

2-1 Brine Accumulation Summary

3-1 BSEP Drillholes Sampled for Brine between 1987 and 1993

3-2 Simple Statistics for BSEP Analyses

3-3 Average Composition of Salado Formation Brine

3-4 Composition of Salado and Nonsalado Brines

5-1 Seepage Rate in Drillholes Penetrating Clay B 


\section{List of Figures}

Figure

1-1 WIPP Location in Southeastern New Mexico

1-2 Surface and Underground Layout of the WIPP Facility

1-3 Generalized Stratigraphic Cross Section of the WIPP Site

2-1 Map of the WIPP Underground Workings Showing BSEP Observation Locations as of December 31, 1993

2-2 Correlation of the Stratigraphy to the Downholes in the Northern Part of the Facility

2-3 Correlation of the Stratigraphy to the Upholes in the Northern Part of the Facility

3-1 Uphole $\mathrm{A} 1 \mathrm{X} 02$ Concentration vs. Time for $\mathrm{B}, \mathrm{Br}, \mathrm{Mg}$, and $\mathrm{K}$

3-2 Means Plots for BSEP Drill Holes for $\mathrm{B}, \mathrm{Br}, \mathrm{K}$, and $\mathrm{Mg}$ 


\section{List of Abbreviations/Acronyms}

AIS

Al

ALK

ANOVA

As

ASTM

B

$\mathrm{Ba}$

$\mathrm{Br}$

BSEP

$\mathrm{Ca}$

$\mathrm{Cl}$

$\mathrm{cm}$

$\mathrm{cm} / \mathrm{s}$

Cs

DOE

DRZ

EPA

$\mathrm{F}$

$\mathrm{Fe}$

$\mathrm{ft}$

I

$\mathrm{K}$

$\mathrm{Kg}$

L

$\mathrm{m}$

$M$

$\mathrm{MB}$

$\mathrm{Mg}$

$\mathrm{mg} / \mathrm{kg}$

$\mathrm{mL}$

$\mathrm{mm}$

$\mathrm{Mn}$

$\mathrm{MPa}$

$\mathrm{N}$

$\mathrm{Na}$

ND

$\mathrm{NH}_{4}$

$\mathrm{NO}_{3}$

$\mathrm{P}$

$\mathrm{Pa}$
Air Intake Shaft

aluminum

alkalinity

Analysis of Variance

arsenic

American Society for Testing and Materials

boron

barium

bromine

Brine Sampling and Evaluation Program

calcium

chlorine

centimeter(s)

centimeter/second

cesium

U.S. Department of Energy

disturbed rock zone

U.S. Environmental Protection Agency

florine

iron

foot/feet

iodine

potassium

kilogram(s)

liter(s)

meter(s)

Mean

Marker Bed

magnesium

milligrams per kilogram

milliliter(s)

millimeter(s)

manganese

megapascal(s)

number of samples

sodium

Not detected

Ammonium

Nitrate

phosphorous

pascal(s) 


\section{List of Abbreviations/Acronyms (Continued)}

$\begin{array}{ll}\mathrm{PA} & \text { Performance Assessment } \\ \mathrm{Rb} & \text { ribodium } \\ \mathrm{S} & \text { standard deviation } \\ \mathrm{SO}_{4} & \text { sulfate } \\ \mathrm{SG} & \text { specific gravity } \\ \mathrm{Si} & \text { silicon } \\ \mathrm{SNL} / \mathrm{NM} & \text { Sandia National Laboratories/New Mexico } \\ \mathrm{SPDV} & \text { Site and Preliminary Design Validation } \\ \mathrm{Sr} & \text { strontium } \\ \mathrm{TDS} & \text { total dissolved solids } \\ \mathrm{TIC} & \text { total inorganic carbon } \\ \mathrm{TOC} & \text { total organic carbon } \\ \mathrm{TRU} & \text { transuranic } \\ \text { WIPP } & \text { Waste Isolation Pilot Plant }\end{array}$




\section{Executive Summary}

The data in this report are the result of activities associated with the Brine Sampling and Evaluation Program (BSEP) at the Waste Isolation Pilot Plan (WIPP) during 1992 and 1993. This report is the last one that is currently scheduled in the sequence of reports of new data, and therefore, also includes summary comments referencing important data obtained by BSEP since 1983. These BSEP activities document and investigate the origins, hydraulic characteristics, extent, and composition of brine occurrences in the Permian Salado Formation and seepage of that brine into the excavations at the WIPP. A project concern is that enough brine might be present after sealing and closure to generate large quantities of hydrogen gas by corroding the metal in the waste drums and waste inventory.

When excavations began at the WIPP in 1982, small brine seepages (weeps) were observed on the walls. Brine studies began as part of the Site Validation Program and were formalized as the BSEP in 1985. During eleven years of observations (1982-1993), evidence has mounted that the amount of brine seeping into the WIPP excavations is limited, local, and only a small fraction of that required to produce a maximum amount of hydrogen gas. The data collected through 1991 are discussed in detail and are summarized by Deal and others (1993). This report describes progress made during the calendar years 1992 and 1993 and focuses on four major areas: (1) monitoring of brine inflow, e.g., measuring brines recovered from holes drilled downward from the underground drifts (downholes), upward from the underground drifts (upholes), and from subhorizontal holes from the underground drifts; (2) observations of weeps in the Air Intake Shaft (AIS); (3) further characterization of brine geochemistry; and (4) additional characterization of the hydrologic conditions in the fractured zone beneath the excavations.

Damp or Wet Areas on Drift Floors. Seepage into the one persistently wet area on the floor of the WIPP excavations in Room G (GSEEP), continued to decline in 1992 and 1993, reaching a low value of 0.03 liter $(\mathrm{L})$ per day by December see Comment Section 2.2. GSEEP had, for all practical purposes, dried up by December 31, 1993. No evidence was found of brine flowing upward out of fractures beneath the drift floors in the northern part of the workings. Observations of drillholes penetrating anhydrite Marker Bed (MB) 139 in Room G and in Site and Preliminary Design Validation (SPDV) Room 4 showed that the anhydrite is fractured; however, no brine was seeping out of either the anhydrite or the fractures, providing evidence for no significant flow of brine into the excavations from MB 139. If far-field flow exists through $M B 139$, moisture or evidence of moisture should be observed at these locations. No evidence of moisture was found. (In the context of brine flow toward the WIPP excavations, far-field flow refers to flow far enough beyond the disturbed rock zone [DRZ] where the salt does not deform in response to the presence of the WIPP excavations.)

Seepage into Drillholes. Seepage into selected vertical downholes in the repository floor were monitored. Four of the ten downholes monitored in 1993 showed fairly steady seepage rates ranging from 0.008 to $0.1 \mathrm{~L}$ per day. Six downholes showed decreasing seepage trends. In those downholes where MB 139 could be observed, seepage was not found to be entering 
the hole from MB 139. Rather, seepage was observed to be from deeper horizons, which will not be intersected by waste storage rooms or be subjected to the fracturing expected to occur around waste storage rooms.

None of the monitored upholes continue to produce brine. Eleven subhorizontal observation holes (drilled at a slight downward angle) continue to be monitored. Only those four that intersect the orange band (Map Unit 1) continue to have measurable seepage, which was 0.005 to $0.01 \mathrm{~L}$ per day.

Seepage into Shaft Sumps. Observations in the shaft sumps show that open fractures in MB 139 remain dry. The shaft sumps are, in effect, long-term far-field flow experiments.

Seepage into the Air Intake Shaft. The AIS was inspected for evidence of brine inflow and only one small moist area was observed. It occurs at the base of MB 103, in the upper part of the Salado Formation not far below the Rustler-Salado contact. Salt encrustations (evidence of past brine seepage) occur more commonly below 1,500-ft depth, are clearly stratigraphically controlled, and are associated with clay interbeds and argillaceous halite. The AIS is, in effect, a long-term far-field flow experiment. The anhydrite exposures are typically dry and free of salt encrustations, indicating that no significant amount of brine flows through them to the shaft.

Geochemistry. The general trends of the 1992-1993 geochemistry data are similar to those discussed by Deal and others (1991b, Chapter 3, Table 3-5 and 3-3). Long-term trends of strontium values have been decreasing for samples collected from drillhole DHP402A. A high strontium signature is characteristic of brine that originated as water from the Rustler Formation and was spread for dust control. The lowering of strontium values is consistent with the hypothesis that there is less dilution from construction water derived from the Rustler Formation occurring at the location of DHP402A in Panel 1.

Data from eleven drillholes that have not been contaminated with construction brine form two very similar brine populations. One population are those holes that contain brine derived from the repository horizon and the other, holes that contain brine only from horizons below the floor of the repository. Brines associated with the repository horizon are slightly higher in $\mathrm{Mg}$ and $\mathrm{Br}$ and lower in $\mathrm{K}$ and $\mathrm{B}$ than brines from stratigraphic horizons (probably clay $\mathrm{B}$ ) below the excavations. An average composition for the repository horizon brines that might come into contact with the waste after sealing and closure is presented in Table 3-3.

Hydraulic Tests in the Fractured Part of the DRZ. Fractures are common in the WIPP underground, and fracture systems locally connect brine-filled drillholes at some drift intersections. Fracturing creates pathways that can greatly alter apparent flow rates to individual downholes.

Hydrologic tests were performed in the fractured part of the DRZ at the following three locations: 
- Intersection of $S 90$ and W620 near the AIS

- Intersection of W170 and S400 at the Underground Core Storage Area

- Intersection of E0 and N620.

The test results confirmed that the width of an excavation influences the development of integrated fractures and showed that integrated fracture systems were only found beneath intersections. This supports the concept of limited, bounded, fractured fluid reservoirs. Since brine stands at different levels in closely spaced drillholes in the floor and is not seeping out of fractures observed in the Salt Shaft and Waste Shaft sumps, it can be concluded that largescale hydrologically interconnected fracture systems do not exist below the WIPP underground excavation.

Numerical Modeling of Brine Seepage as a Result of Clay Compaction. It has previously been pointed out (Deal and others, 1993, Sections 4 and 5; Deal and Bills, 1994) that there appears to be enough moisture present in the clays within the Salado Formation to account for all the brine that is observed to seep into the WIPP excavations. The excavation of WIPP rooms result in stress redistribution around those openings that can cause the consolidation of thin clays within the stratigraphic sequence. Additionally, the excavations (including drillholes) provide a sink at atmospheric pressure allowing brine to flow from the consolidating clays.

A series of order-of-magnitude seepage calculations (Appendix F) were made in order to numerically model clay consolidation and estimate the resultant brine seepage into the WIPP.

These order-of-magnitude seepage calculations compare well with the observed seepage into Room Q. Calculated seepage rate after 1,600 days is on the order of $0.3 \mathrm{~L} /$ day, where the actual observed rate is $0.17 \mathrm{~L} / \mathrm{day}$. In this case the model is for flow towards the room along a thin clay seam. Extending this model to a waste storage room predicts that the total seepage into the room will be on the order of $9,000 \mathrm{~L}$, far short of the $220,000 \mathrm{~L}$ necessary to react anoxically with all the susceptible metal placed in the room (Deal and others, 1991b, Section 4.6). Furthermore, seepage into the room will cease after about 100 years.

The case for seepage into a downhole drilled into the strata below a WIPP excavation behaves differently, as flow is radially toward the drillhole rather than parallel to the wall of a room. In this case, some seepage continues for a long time, perhaps a thousand years or more. It is clear that seepage into drillholes is strikingly different from seepage into a repository excavation. Deal and others (1994, Section 2.7.2) pointed out that seepage into drillholes probably should not be used to predict long term seepage into a WIPP waste storage room. The calculation provides additional support for this caution. 


\section{THIS PAGE INTENTIONALLY LEFT BLANK}




\subsection{Introduction}

The Waste Isolation Pilot Plant (WIPP), a U.S. Department of Energy (DOE) research facility, was established to demonstrate the safe disposal of defense-generated transuranic (TRU) waste in the United States. The WIPP facility is 26 miles (42 kilometers) east of Carlsbad, New Mexico (Figure 1-1). The surface and underground layout of the facility is presented in Figure 1-2. The repository is approximately 2,150 feet (ft) (655 meters [m]) below the surface in the Salado Formation. The Salado Formation and underlying Castile Formation make up an evaporite sequence over 3,300 ft (1,000 m) thick (Figure 1-3). An extensive program of site characterization was initiated in 1976 (Powers and others, 1978; Bechtel, 1983) and continued through 1991 (Deal and others, 1993). The hydrogeological activities of the Brine Sampling and Evaluation Program (BSEP) are part of a continuing effort to refine the understanding of the repository geology. The data in this report update the previous studies summarized in Deal and others (1993) and in Deal and Bills (1994).

Brine studies began in 1982 as part of the Site Validation Program (Black and others, 1983) and were formalized in 1985 by Morse and Hassinger (1985). The focus of the BSEP is the origin, hydraulic characteristics, extent, and chemical composition of brine in the Salado Formation at the repository horizon and seepage of brine into the excavations at the WIPP. Although the repository is dry, brine weeps from exposed surfaces, accumulates in drillholes and sumps, and forms encrustations on the walls as the brine evaporates over time. The chemistry of the brine may affect chemical reactions in the buried waste, and the volume of brine and the hydrologic system that drives the brine seepage need to be known in order to assess the long-term performance of the repository after closure.

Brine inflow systems have been discussed in previous BSEP reports. There are three different conceptual models that have been considered:

- Far-field Flow Model: flow from the far-field, either by radial flow to the excavation or laterally through stratigraphically-constrained flow.

- Redistribution Model: movement of interstitial brine within the disturbed rock zone (DRZ) toward the excavations by excavation-induced stress redistribution. This does not consider displacement of brine from inside the clays, only redistribution of brine already in available pore spaces in the salt, polyhalite, and anhydrite units. 


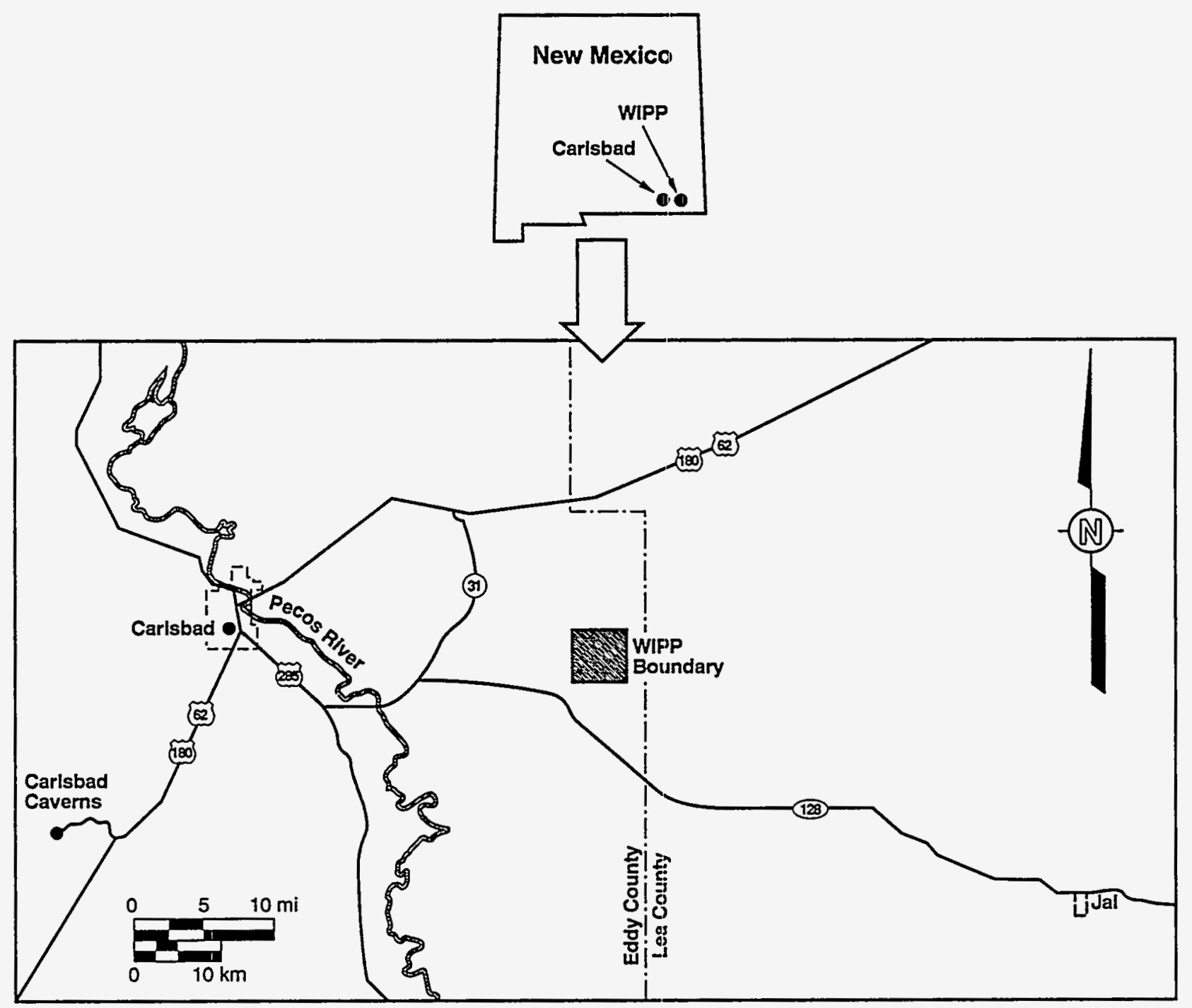

Figure 1-1

WIPP Location in Southeastern New Mexico 


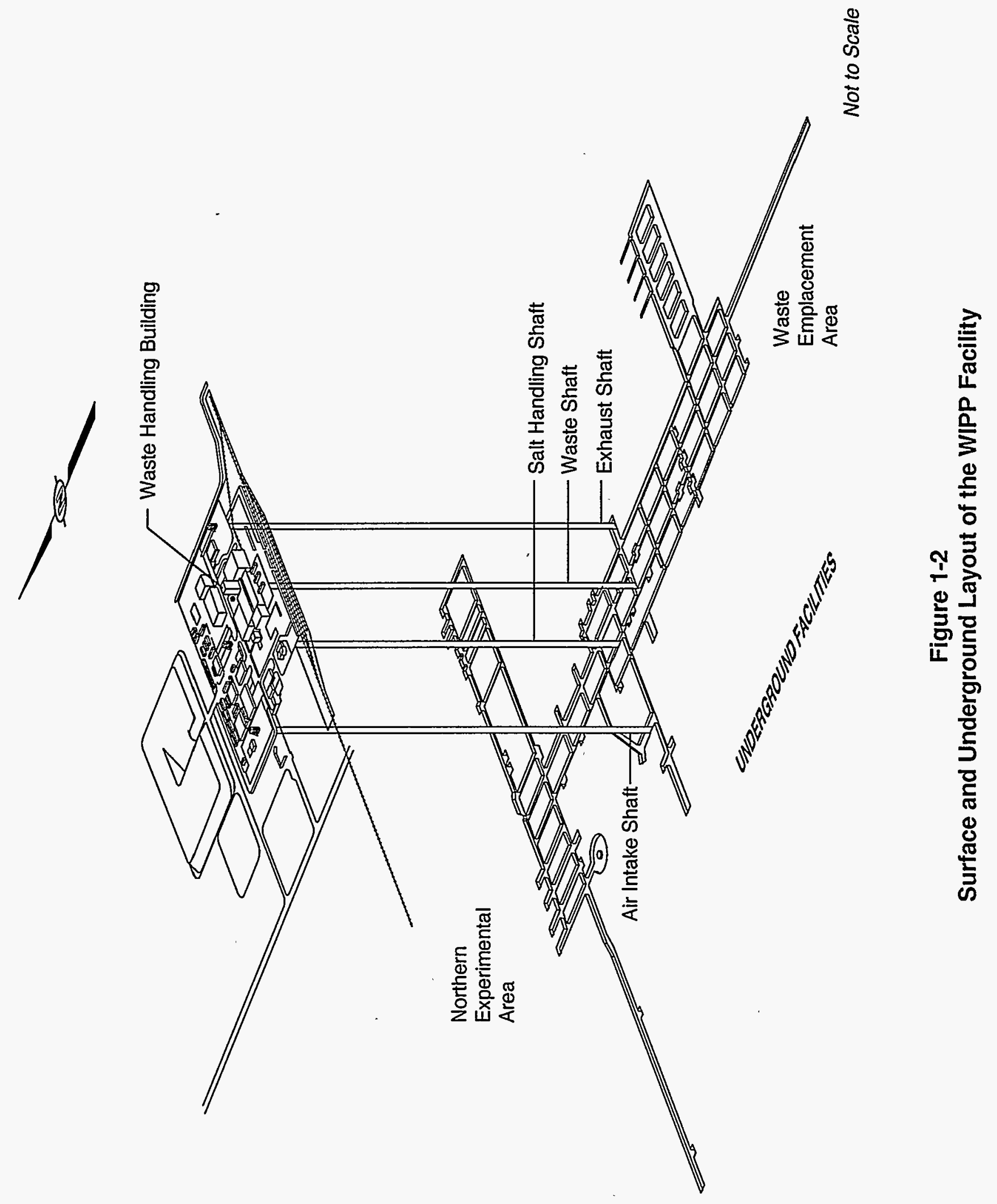




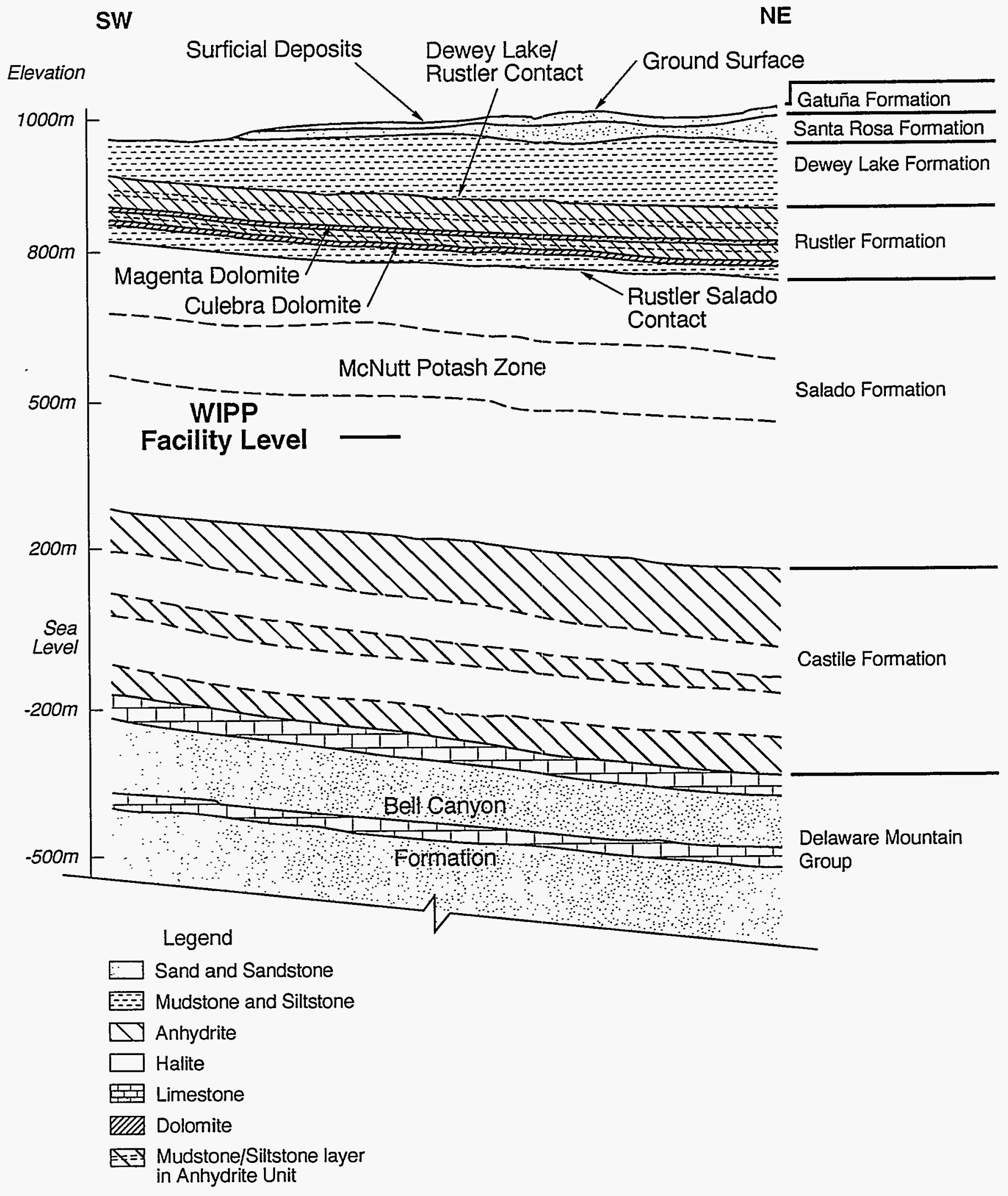

Figure 1-3

Generalized Stratigraphic Cross Section of the WIPP Site 
- Clay Compaction Model: brine squeezed from clays within a short distance (a few $m$ ) of the excavations.

Additional effects, such as gas exsolution, development of enhanced porosity and permeability within the DRZ, and preferential flow along bedding planes, may modify brine inflow. However, it is fundamentally important to distinguish between far-field sources and local, relatively limited redistribution of brine in the immediate vicinity of the WIPP excavations. In both cases, the driving mechanism is the pressure gradient caused by the excavation of the underground openings. Flow pathways are through permeable interbeds, along stratigraphic discontinuities, or through fractures.

The relative importance of these systems needs to be determined. For example, if there is sufficient far-field flow into the repository, enough brine may come into the excavations to completely corrode the metal in the waste and the waste drums. In that case, the potential for hydrogen generation due to the corrosion would be limited by the total metal inventory. If brine seepage is a purely local phenomenon that occurs as a results of redistribution of brine in the immediate vicinity of the excavations, there would be insufficient brine available to cause much corrosion after closure. In the latter case, gas generation would be limited by brine availability and would not be a problem. Evidence suggests that brine is derived from clay within a few meters of the excavations, and will not result in the production of large quantities of hydrogen gas by anoxic corrosion.

The predicted consequences of human-intrusion events, the fate of the waste-generated gases, and the migration of the hazardous constituents during undisturbed performance are all sensitive to brine inflow assumptions. If the far-field model is valid, a human-intrusion event (i.e., drilling into the sealed repository at a future date) will lower fluid pressure in the waste storage rooms, create pressure gradients toward the rooms, and reinstate far-field flow. This will lead to a greater release of radionuclides from the repository, because the inflowing brine would infiltrate through the waste and flow up the drillhole. Alternatively, if a near-field model is valid, the only brine available for transport of radionuclides is the volume of brine that is trapped in the room at the time of sealing.

Collection techniques and certain general observations should be kept in mind when evaluating the BSEP data. These are listed in Table 1-1. Care should also be exercised when 


\section{Table 1-1 \\ Points to be Considered When Evaluating BSEP Data}

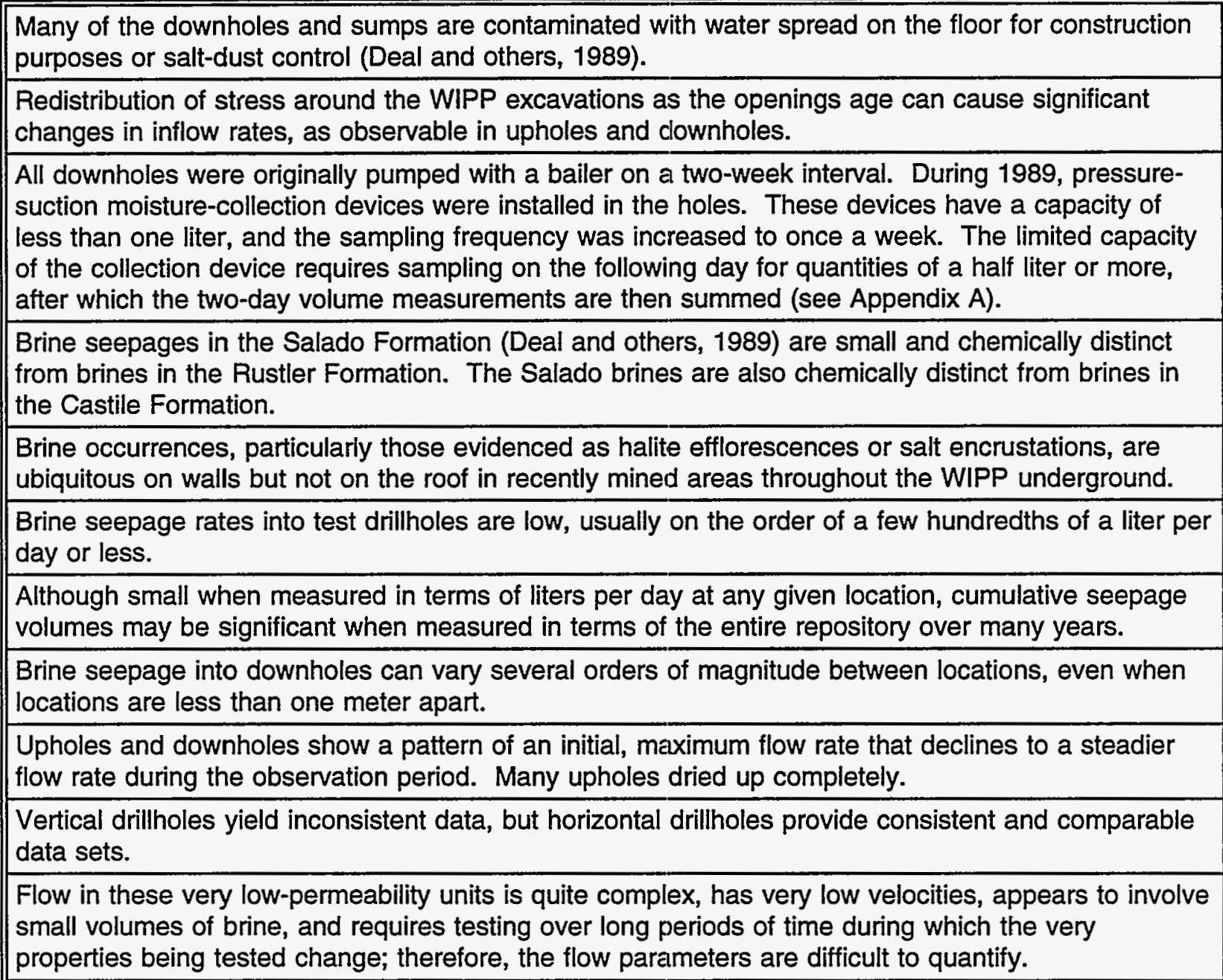


interpreting the various diagrams of drillhole lengths and stratigraphic thicknesses. Although the strata at the WIPP are quite uniform in both composition and thickness, some variation occurs.

Activities in 1992 and 1993 provided additional information on the brine seepage in the repository (Chapter 2.0), geochemical properties of the brine (Chapter 3.0 ), and additional hydrologic testing (Chapter 4.0). This report supplements the summary of data through 1991 reported and discussed by Deal and others (1993).

Appendix A provides detailed information of the brine seepage into drillholes monitored for this program. The information includes the name of the drillhole; the date and time of brine collection or sampling; the volume (in liters) removed; the number of days since January 1 , 1985 (an arbitrary reference date); the cumulative volume (L) collected; the inflow rates in $\mathrm{L}$ per day, and specific remarks. Appendix B contains graphs of the data from Appendix A, presented as an 11-point moving average of the data. This averaging reduces variation introduced by collection techniques and presents a more realistic picture of the real variations in brine seepage rates than would be presented by plots of raw data. Appendix $\mathrm{C}$ documents brine weeps observed in the AIS. Appendix D shows the results of the chemical analyses, including ion concentrations in milligrams per liter $(\mathrm{mg} / \mathrm{L}), \mathrm{pH}$, specific conductivity, and alkalinity. Appendix $\mathrm{E}$ documents additional hydrologic testing of the fractured zone beneath the floor of the WIPP excavations. 


\subsection{Monitoring of Brine Inflow Parameters}

\subsection{Introduction}

Brine seepage observations have been made at underground locations at the WIPP from 1982 to December 31, 1993. Information regarding the inflow of brine was derived from observations and mapping of moist areas and measurements of brine seeping into downholes, upholes, and subhorizontal holes drilled from the underground excavation. The locations of the 1992-1993 BSEP observation holes, along with other reference locations, are shown in Figure 2-1. Descriptions and the underground locations of these boreholes are listed in Table A-1 of Appendix A. Part II of Appendix A lists the quantity of brine removed, calculated inflow rates in liters per day, and cumulative volume in liters for all of the boreholes monitored in 1992 and 1993.

\subsection{Damp or Wet Areas on Drift Floors}

A brine seep, GSEEP, on the floor of Room G, at approximately N1100-W1140, has been the only persistently moist area in the WIPP excavations. Inflow data for GSEEP are contained in Appendix A, with a smoothed, moving-average graph of the data presented in Appendix B. A description of the location and a discussion of the brine chemistry and seepage history through December 1990 are contained in Deal and others (1991b, Section 2.5), who conclude that the brine from GSEEP has a component from brine spread in the G Access drift for saltdust control. Note that although no construction water was spread at the location of GSEEP in Room G, water was spread in the G Access Drift which is topographically higher (uphill) of GSEEP. The seepage rate reached a maximum of $0.75 \mathrm{~L}$ per day in April 1989 but declined to $0.03 \mathrm{~L}$ per day by December 1993. GSEEP had, for all practical purposes, dried up by December 31, 1993. A total of 1,099 $\mathrm{L}$ have been collected, and a thick salt encrustation on the floor indicates that more has evaporated into the air circulated through the WIPP workings for ventilation.

\subsection{Downholes and Brine Beneath the Floor}

\subsubsection{Downholes}

Downholes are drilled vertically downward into the repository floor. Deal and Case (1987, Table 3-1) discussed brine inflow in 13 downholes, with observations beginning in late 1984 and early 1985. A detailed discussion of sampling, data scattering, and inflow rates through 


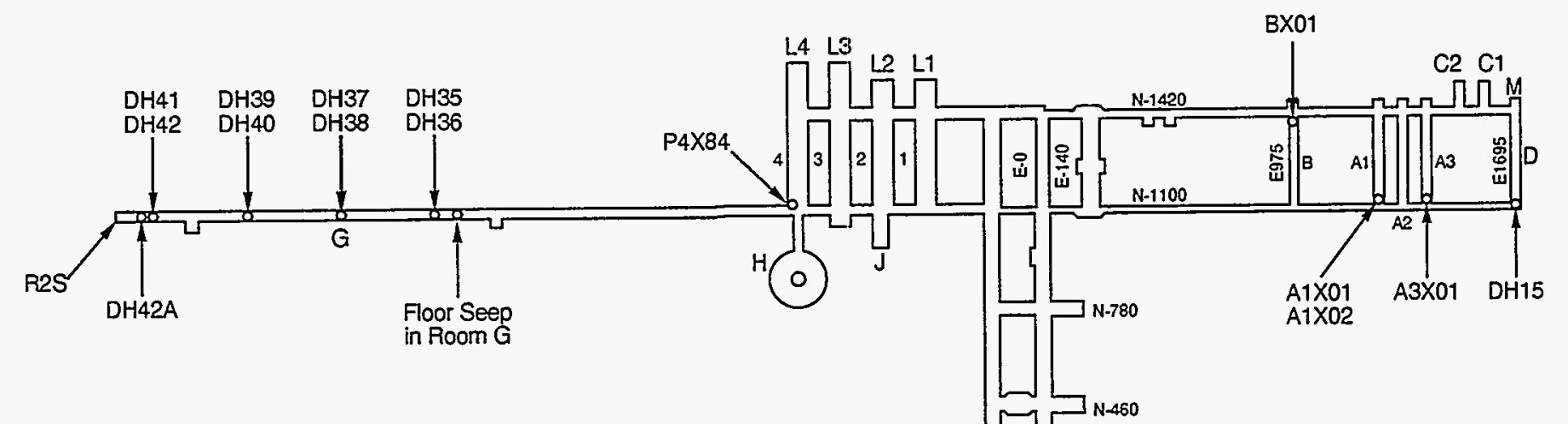

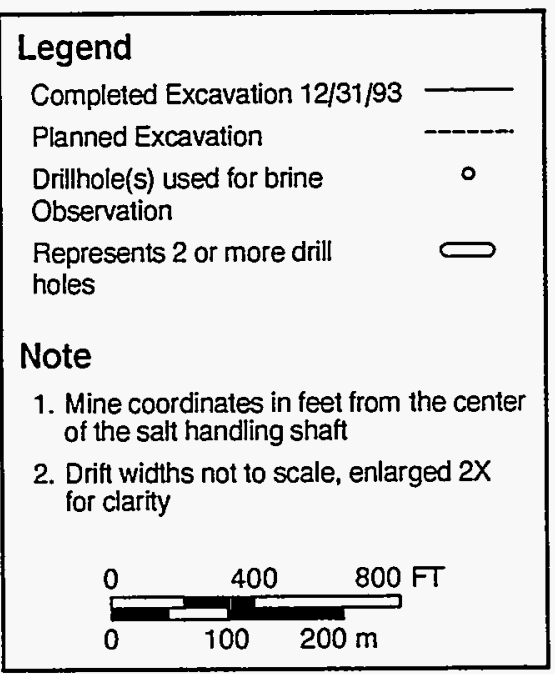

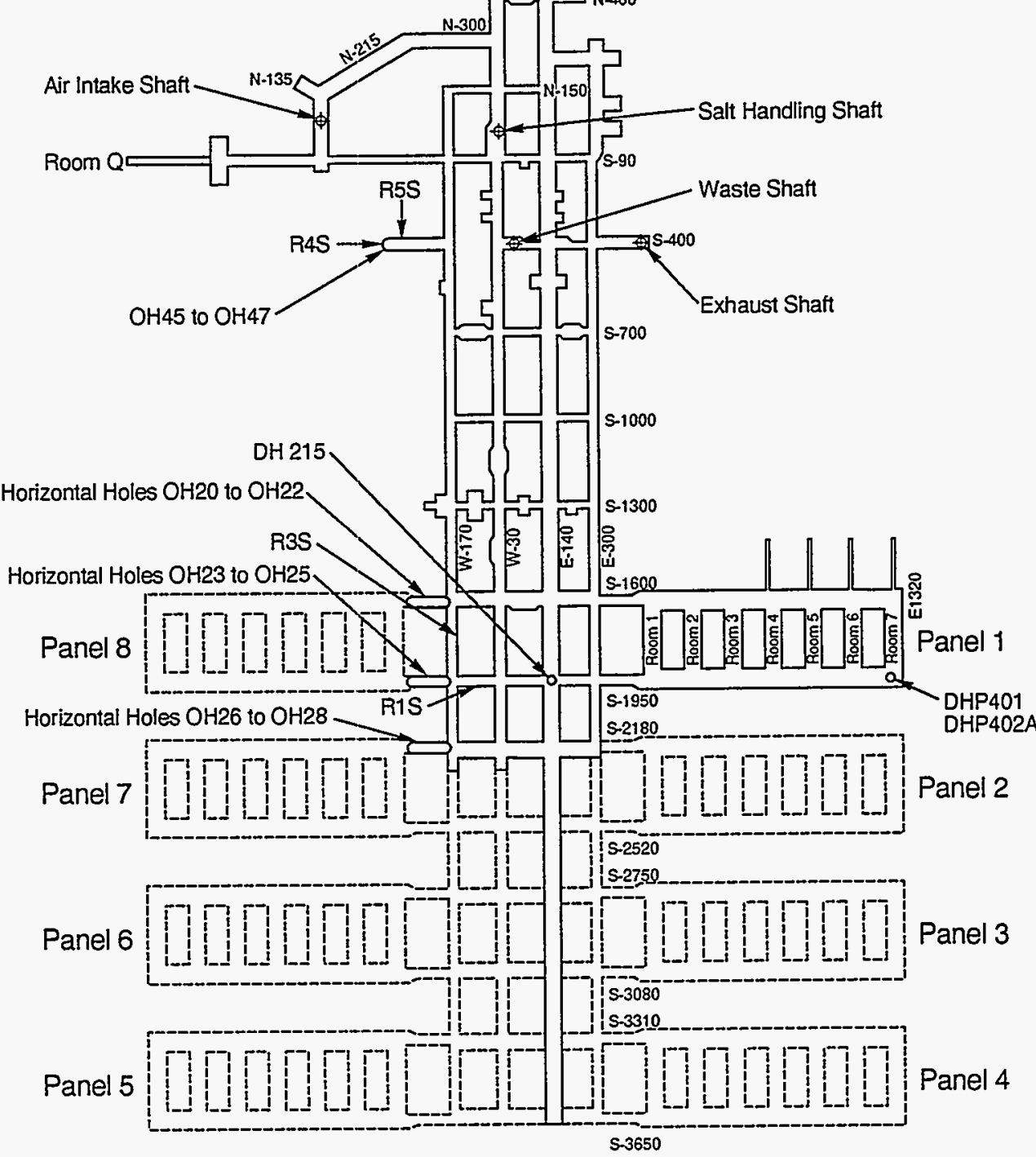

Figure 2-1

Map of WIPP Underground Workings Showing BSEP

Observation Locations as of December 31, 1993 
the end of 1990 is presented in Deal and others (1991b). Five of the ten downholes monitored in 1993 showed steady inflow (A3X01, BX01, DH36, DH38, and DH40). Downholes DH42 and DH42A showed a decrease in inflow rate as did DHP402A in Panel 1 and OH46 neither of which were included in the original 13 downholes drilled in 1984. Five of the original 13 holes (A1X01, IG201, IG202, L1X00, and NG252) could no longer be observed. Table 2-1 summarizes the data obtained from the downholes, with additional information presented in Appendix A.

Contamination with non-Salado water used for construction purposes has been confirmed in most downholes by the chemical composition of the brine, which clearly indicates the mixing of waters with discrete and different chemical signatures (Deal and others, 1989; 1991b; 1993). In some cases, inflow rates vary directly with known water-spreading practices. The first seven downholes in Table 2-1 are located in the northern part of the repository (Figure 2-1), where water was not spread during construction; therefore, the brine collected from these downholes was derived from within the Salado Formation. Brine chemistries from these holes differ from chemical signatures associated with construction brines.

These seven downholes have a similar nine-year seepage pattern (Appendix B), although these holes penetrate different stratigraphic horizons (Figure 2-2) and the seepage rates vary more than two orders of magnitude. These holes were drilled into relatively undisturbed salt shortly after excavation and then monitored. The following observations, first made in 1986 (Deal and Case, 1987), have been confirmed:

- After drilling a hole, a few days elapse where little or no brine seeps into the hole.

- After the initial no-flow or low-flow period, brine seepage quickly reaches a maximum and then begins to decline.

- Seepage rates decrease over a period of several months to steadier, long-term trends.

Five of the downholes demonstrated a steady flow (within the scatter of the data points) through 1993, though slightly decreased from past years, and four showed a decrease in inflow. 
Table 2-1

\section{Brine Accumulation Summary}

\begin{tabular}{|c|c|c|c|c|c|c|c|c|}
\hline \multicolumn{9}{|c|}{ Downholes } \\
\hline Hole & Location & $\begin{array}{l}\text { Date Area } \\
\text { Excavated }\end{array}$ & $\begin{array}{l}\text { Date Hole } \\
\text { Drilled }\end{array}$ & $\begin{array}{l}\text { Date First } \\
\text { Observed }\end{array}$ & $\begin{array}{l}\text { Approx. } \\
\text { Maximum } \\
\text { Inflow } \\
(\text { L/Day) }\end{array}$ & $\begin{array}{l}\text { Approx. } \\
\text { Inflow } \\
12 / 93 \\
\text { (LDay) }\end{array}$ & $\begin{array}{l}\text { Inflow } \\
\text { Trend } \\
12 / 93^{b}\end{array}$ & $\begin{array}{c}\text { Approx. Total } \\
\text { Vol. } \\
\text { Removed by } \\
12 / 93 \text { (L) }\end{array}$ \\
\hline$A 3 \times 01$ & Room A3 & $11 / 84$ & $1 / 85$ & $2 / 85$ & 0.03 & 0.02 & $S$ & 65 \\
\hline BX01 & Room $B$ & $6 / 84$ & $1 / 85$ & $1 / 85$ & 0.06 & 0.03 & $\mathbf{S}$ & 118 \\
\hline DH36 & Room G & $12 / 84$ & $1 / 85$ & $1 / 85$ & 0.28 & 0.1 & $\mathbf{S}$ & 482 \\
\hline DH38 & Room G & $12 / 84$ & $1 / 85$ & $1 / 85$ & 0.18 & 0.03 & $\mathbf{S}$ & 135 \\
\hline $\mathrm{DH} 40$ & Room G & $12 / 84$ & $1 / 85$ & $1 / 85$ & 0.04 & 0.008 & $\mathbf{S}$ & 22 \\
\hline $\mathrm{DH} 42$ & Room $G$ & $12 / 84$ & $1 / 85$ & $1 / 85$ & 0.05 & 0.01 & $D$ & 68 \\
\hline $\mathrm{DH} 42 \mathrm{~A}$ & Room G & $12 / 84$ & $1 / 85$ & $1 / 85$ & 0.2 & 0.02 & $D$ & 245 \\
\hline DHP402A & S1950/E1330 & $10 / 86$ & $12 / 86$ & $12 / 86$ & 4.0 & 0.02 & $D$ & 644 \\
\hline $\mathrm{OH} 46$ & S390/W320 & $5 / 89$ & $6 / 89$ & $7 / 89$ & 0.04 & 0.005 & $D$ & 21 \\
\hline \multicolumn{9}{|c|}{ Upholes } \\
\hline Hole & Location & $\begin{array}{l}\text { Date Area } \\
\text { Excavated }\end{array}$ & $\begin{array}{c}\text { Date Hole } \\
\text { Drilled }\end{array}$ & $\begin{array}{l}\text { Date First } \\
\text { Observed }\end{array}$ & $\begin{array}{l}\text { Approx. } \\
\text { Maximum } \\
\text { Inflow } \\
\text { (L/Day) }^{a}\end{array}$ & $\begin{array}{c}\text { Approx. } \\
\text { Inflow } \\
12 / 93 \\
\text { (L/Day) }\end{array}$ & $\begin{array}{l}\text { Inflow } \\
\text { Trend } \\
12 / 93^{b}\end{array}$ & $\begin{array}{c}\text { Approx. Total } \\
\text { Vol. } \\
\text { Removed by } \\
12 / 93 \text { (L) }\end{array}$ \\
\hline A1X02 & Room A1 & $10 / 84$ & $3 / 85$ & $3 / 85$ & 0.09 & 0 & DRY & 83 \\
\hline $\mathrm{DH} 15$ & N1104/E1688 & $3 / 84$ & $3 / 84$ & $5 / 86$ & 0.01 & 0 & DRY & 4 \\
\hline DH35 & Room G & $12 / 84$ & $1 / 85$ & $2 / 85$ & 0.02 & 0 & DRY & 4 \\
\hline $\mathrm{DH} 37$ & Room G & $12 / 84$ & $1 / 85$ & $2 / 85$ & 0.01 & 0 & DRY & 1 \\
\hline DH39 & Room G & $12 / 84$ & $1 / 85$ & $2 / 85$ & Trace & 0 & DRY & 0 \\
\hline $\mathrm{DH} 41$ & Room G & $12 / 84$ & $1 / 85$ & $2 / 85$ & Trace & 0 & DRY & 0 \\
\hline $\mathrm{DH} 215$ & S1960/E153 & $1 / 83$ & $2 / 83$ & $4 / 84$ & 0.09 & 0 & DRY & 18 \\
\hline DHP401 & S1950/E1330 & $10 / 86$ & $1 / 87$ & $3 / 87$ & 0.008 & 0 & DRY & 2 \\
\hline $\mathrm{OH} 47$ & S390NW320 & $5 / 89$ & $7 / 89$ & $8 / 89$ & 0.030 & 0 & DRY & 4 \\
\hline \multicolumn{9}{|c|}{ Subhorizontal Holes } \\
\hline Hole & Location & $\begin{array}{l}\text { Date Area } \\
\text { Excavated }\end{array}$ & $\begin{array}{l}\text { Date Hole } \\
\text { Drilled }\end{array}$ & $\begin{array}{l}\text { Date First } \\
\text { Observed }\end{array}$ & $\begin{array}{l}\text { Approx. } \\
\text { Maximum } \\
\text { Inflow } \\
\text { (L/Day) }^{\text {a }}\end{array}$ & $\begin{array}{c}\text { Approx. } \\
\text { Inflow } \\
12 / 93 \\
\text { (L/Day) }\end{array}$ & $\begin{array}{l}\text { Inflow } \\
\text { Trend } \\
12 / 93^{b}\end{array}$ & $\begin{array}{c}\text { Approx. Total } \\
\text { Vol. } \\
\text { Removed by } \\
12 / 93 \text { (L) }\end{array}$ \\
\hline $\mathrm{OH} 2 \mathrm{O}$ & S1600/W170 & $9 / 85$ & $3 / 89$ & $3 / 89$ & 0.02 & 0.005 & $s$ & 16 \\
\hline $\mathrm{OH} 21$ & $S 1600 / W 170$ & $9 / 85$ & $12 / 88$ & $2 / 89$ & 0 & 0 & DRY & 0 \\
\hline $\mathrm{OH} 22$ & $S 1600 / W 170$ & $9 / 85$ & $12 / 88$ & $2 / 89$ & 0.006 & 0 & DRY & 1 \\
\hline $\mathrm{OH} 23$ & $\mathrm{~S} 1950 / \mathrm{W} 170$ & $12 / 85$ & $2 / 89$ & $2 / 89$ & 0.06 & 0.01 & $D$ & 28 \\
\hline $\mathrm{OH} 24$ & S1950/W170 & $12 / 85$ & $3 / 89$ & $3 / 89$ & 0.002 & 0 & DAY & 1 \\
\hline $\mathrm{OH} 25$ & S1950/W170 & $12 / 85$ & $3 / 89$ & $3 / 89$ & 0.001 & 0 & DRY & 0.1 \\
\hline $\mathrm{OH} 26$ & S2150/W170 & $8 / 86$ & $3 / 89$ & $3 / 89$ & 0.04 & 0.01 & $D$ & 27 \\
\hline $\mathrm{OH} 28$ & S2150/W170 & $8 / 86$ & $4 / 89$ & $4 / 89$ & 0.008 & 0 & DRY & 2 \\
\hline $\mathrm{OH} 45$ & S390NW325 & $5 / 89$ & $6 / 89$ & $6 / 89$ & 0.03 & 0.003 & $S$ & $\overline{7}$ \\
\hline
\end{tabular}

aLiters (L) per day.

'Trend derived from data presented in Appendices A and B-Dry; Decreasing (D); Steady (S). 


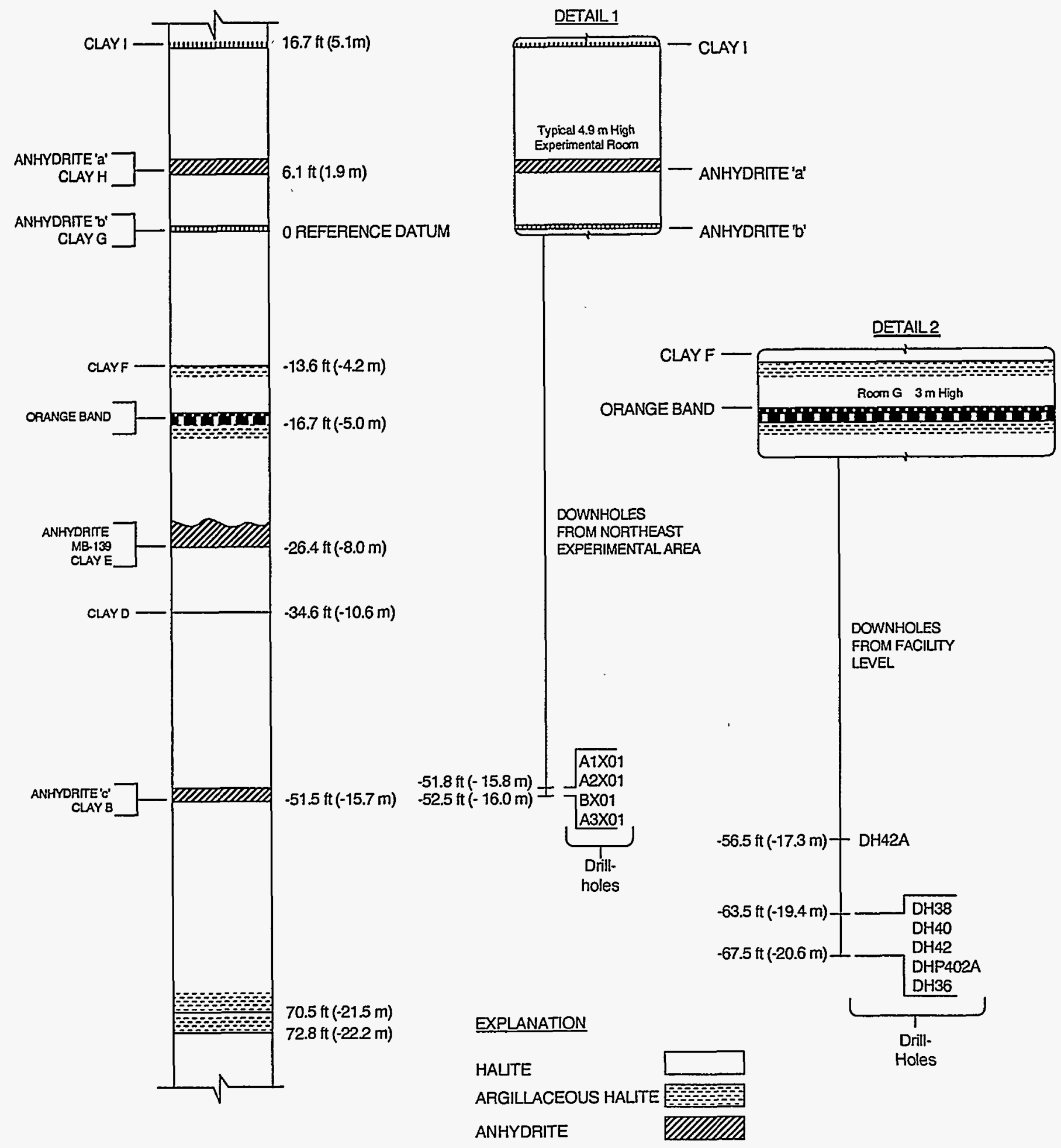

NOTE: Distances above and below anhydrite "b" (clay G) vary from place to place in the WIPP excavations due to natural changes in stratigraphic thickness. This figure represents thicknesses in the northem part of the facility. Distances from clay $E$ down are from Room $G$ and from the orange band up are from Room $A 1$.

Figure 2-2

Correlation of the Stratigraphy to the Downholes in the Northern Part of the Facility 


\subsubsection{Shaft Sumps}

Deal and others (1991b, Section 2.7.1) discuss observations made in the Salt Shaft and Waste Shaft sumps, where MB 139 and open fractures can be seen. The sumps were inspected again in 1991 (Deal and others, 1993) and again in 1993. The fractures and MB 139 were found to be dry and did not contain large quantities of salt encrustations. The shaft sumps are, in effect, long-term far-field brine inflow experiments. If significant amounts of brine were flowing toward the repository through $M B 139$, brine should be found in the shaft sumps. The fact that brine is not observed seeping from MB 139 in the shafts is evidence that significant far-field flow does not exist.

\subsection{Upholes and Brine Above the Roof}

Upholes are drilled vertically upward into the repository roof. Upholes characteristically produce less brine for shorter periods of time than downholes. Part of this can be attributed to greater evaporation caused by less effective sealing of upholès (Deal and Case, 1987) and loss of moisture by dispersion from the hole collar into the salt. Loss of moisture by evaporation is evident from salt-crust buildup in and around most of the upholes. Chemical data (Chapter 3.0 of this report; Deal and others, 1989, 1991a, and 1991b; Abitz and others, 1990) confirm compositional differences between brine samples from upholes and downholes, which can be explained by the partial evaporation of a brine with typical downhole composition to produce the uphole brine. Although the stratigraphy exposed in the upholes (Figure 2-3) is slightly different from the stratigraphy exposed in the downholes, it is unclear whether this contributes significantly to the differences in either brine quantity or chemistry (Deal and others, 1989).

Summary data for selected upholes are presented in Table 2-1. None of the nine upholes listed in 1985 continue to produce brine (upholes A2X02, A3X02, and BX02 are no longer monitored). As discussed in Deal and others (1991b), A1X02 is longer than any of the other upholes $(59 \mathrm{ft}[18 \mathrm{~m}])$ and intersects an additional anhydrite unit not penetrated by any other uphole. No associated clay was observed in the core, but clay commonly occurs below anhydrite stringers and may be discontinuous at this horizon. Additional data are presented in Appendix A. During 1992 and 1993, inflow data for A1X02 continues to be sporadic. The hole is in Room A1, which is inaccessible. A1X02 has not been monitored since August 19, 1993. 


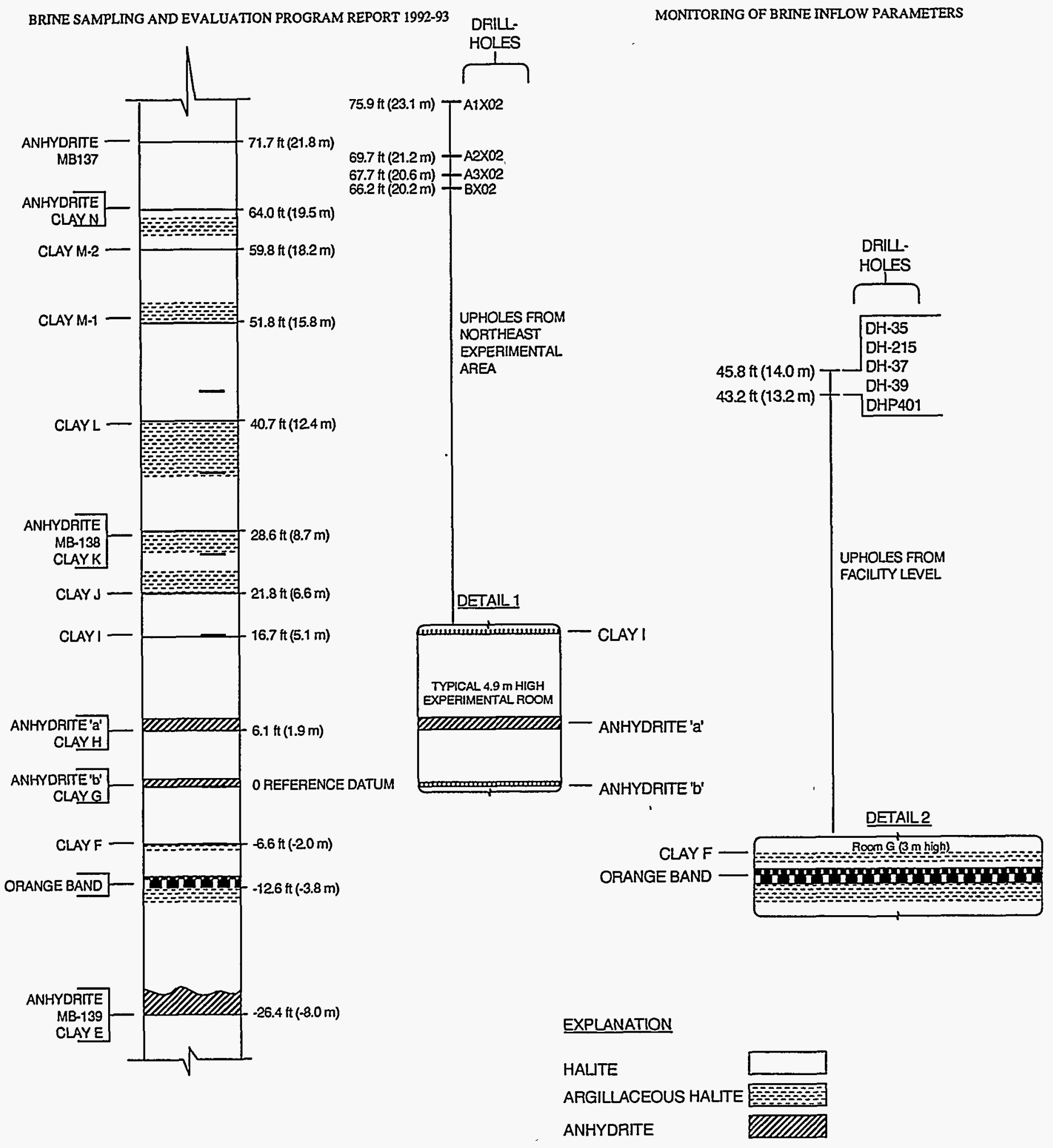

NOTE: Distances above and below anhydrite "b" (clay G) vary from place to place in the WIPP excavations due to natural changes in stratigraphic thickness. This figure represents thicknesses in the northem part of the facility. Distances below the zero datum (clay $\mathrm{G}$ ) are from Room $\mathrm{G}$, distances above clay $\mathrm{G}$ are from Room $\mathrm{A} 1$.

Figure 2-3

Correlation of the Stratigraphy to the Upholes in the Northern Part of the Facility 
Drillholes in the roof that intersect overlying clay layers (clays $\mathrm{J}$ and $\mathrm{K}$ and argillaceous halite between the two clays), including those for the placement of rock bolts, commonly drip brine for a period of several months, often forming halite stalactites. Seepage is particularly notable when the drifts are allowed to age for several years, allowing bed separations to form prior to drilling.

The undisturbed roof of the workings at the WIPP rarely shows evidence of brine seeps or weeps (Deal and others, 1987, Section 2.2). Drill holes provide a route for brine to move across effectively impermeable clear halite beds, and seepage from drillholes in the roof is a common occurrence at the WIPP. Typically upholes start to show evidence of brine seepage a month or so after drilling, exhibit their most active seepage for the following year or so, and then gradually dry up. Rooms $\mathrm{C} 1$ and $\mathrm{C} 2$ show this very typical behavior (Deal and others, 1991b, Section 2.8.1).

\subsection{Subhorizontal Holes}

Subhorizontal brine sampling holes are drilled at a slight downward angle. During 1989, 11 subhorizontal holes were drilled to investigate brine seepage from the WIPP facility stratigraphic horizon. The holes were oriented slightly downward from the opening to accumulate brine at the end of the hole where it could be collected and measured without loss to fractures near the surface of excavations. Ten of the eleven holes were drilled westward from the W170 drift at the location of future entries to Panels 7 and 8 at S1600, S1950, and S2180 (Figure 2-1). These portions of the W170 were excavated in September 1985 at S1600, in December 1985 at S1950, and in August 1986 at S2180 and are considered to have a mature DRZ around them. Three of the holes (OH20, OH23, and $\mathrm{OH} 26$ ), which are $150 \mathrm{ft}$ (46 m) long and 3 in. (7.6 centimeters [cm]) in diameter, started in the clayey halite (Map Unit 4) above the orange band (Map Unit 1) and are deflected slightly downward (Deal and others, 1993, Figures 2-18, 2-19, and 2-20), so that they end in the clear halite (Map Unit 0) below the orange band. The $150-\mathrm{ft}(46-\mathrm{m})$ holes reached the orange band about $50 \mathrm{ft}(15 \mathrm{~m})$ into the holes. Hole OH27A was started at the initial location for $\mathrm{OH} 27$ but was terminated at a depth of $4 \mathrm{ft}(1.2 \mathrm{~m})$ because of drilling problems. The six remaining $50-\mathrm{ft}(15-\mathrm{m})$ holes were drilled either above or below the orange band. One 50-ft (15-m) hole (OH45), which cuts the same stratigraphic interval as the three $150-\mathrm{ft}(46-\mathrm{m})$ holes, was drilled in a newer excavation in May 1989 at S400. 
Several of these holes have produced measurable quantities of brine (Table 2-1, Appendix A). The 150-ft (46-m) holes provide the most uniform and comparable set of measurements yet obtained in the BSEP and have all produced several orders of magnitude more brine than the $50-\mathrm{ft}(15-\mathrm{m})$ holes. The longer holes are still producing, while the shorter holes are essentially dry (i.e., they have not produced enough brine to be measured by the equipment and techniques used), with the exception of OH45. OH45 is a 50-ft (15-m) hole that cuts the same stratigraphic interval as the $150-\mathrm{ft}(46-\mathrm{m})$ longer holes but that was drilled in a more recently mined area at $\mathrm{S} 400$, over $1,000 \mathrm{ft} \mathrm{(300} \mathrm{m)} \mathrm{north} \mathrm{of} \mathrm{OH} 20, \mathrm{OH} 23$, and $\mathrm{OH} 26$. Lateral variation may play a minor role in the difference in brine seepage. This is considered to be unlikely, as Deal and others (1989) found no significant lateral variation in moisture content for any of the stratigraphic units exposed in the excavations.

Two explanations have been offered for the brine seepage observations (Deal and others, 1991b, Section 2.9): (1) The longer holes are tapping an area that is not dewatered, because they extend past the relatively old W170 drift DRZ. As a result, they may only tap about $100 \mathrm{ft}(30 \mathrm{~m})$ of undisturbed salt (in this case, the one $50-\mathrm{ft}(15-\mathrm{m})$ hole would still produce brine, because it was drilled from a young excavation where a significant DRZ had not yet developed), and (2) Brine flows preferentially from the clay units, so the clay at the top and bottom of the orange band may be the only significant source of brine. Therefore, only the four holes (OH20, $\mathrm{OH} 23, \mathrm{OH} 26$, and $\mathrm{OH} 45)$ that cut the orange band accumulate brine. Evidence presented in this report suggests that the second explanation is the more likely one.

\subsection{Air Intake Shaft}

The Air Intake Shaft (AIS) was inspected for evidence of brine inflow. The entire length of the shaft was viewed from the man cage, and photographs were taken of various intervals. Evidence of weep was noted, mainly in the form of salt encrustations. Appendix $C$ provides details of the AIS inspection and includes photographs of some of the weep surfaces.

Salt encrustations, or weeps, are more common at depths below 1,500 $\mathrm{ft}$, about the midpoint of the Salado Formation exposed within the AIS. Many of the weeps are stratigraphically controlled by bedding plains, as indicated by encrustations at single horizons. Most of the zones of weeping are associated with argillaceous halite; however, some weeps occur at the claystones underlying sulfate marker beds. There are few weeps within the purer halite beds deposited subaqueously, and only one wet surface (MB 103) was observed. 
The anhydrite surfaces are typically dry and free of salt encrustations, indicating that no significant amount of brine flows through them to the shaft.

\subsection{Discussion of Data Acquisition and Analysis}

Several different sampling techniques have been used in an attempt to uniformly collect the very small amounts of brine that seep into the hole between sampling rounds; each technique has unique problems. The change in sampling methods and difficulties in sampling techniques was discussed in detail by Deal and others (1991b) and is sometimes reflected as apparent variations in seepage rates (Appendix B).

To compensate for sampling-induced apparent variations in seepage rates, the graphs of the seepage data presented in Appendix $B$ have been smoothed using an 11-point moving average (the average of the data point and the five points on each side of the data point). At the beginning and end of each curve, the trend is distorted by the smoothing function, because the eleven point moving average reduces to a 9,7,5,3 average and actual data point on both ends of the curve for a more accurate graphical representation of the seepage trends. There are slight differences between the curves presented in this report and in previous BSEP reports, because a different software package was used to create the plots. 


\subsection{Statistical Analysis of the BSEP Brines}

\subsection{Introduction}

A major objective of the BSEP has been to characterize the composition of brine that seeps into the WIPP excavations from the Salado Formation. Statistical analysis of BSEP geochemical data has been used to approximate the chemistry of typical Salado Formation brine that may come into contact with waste after closure of the WIPP repository. The analysis of BSEP brine compositions contained here updates previously analysis (Deal and others 1989, 1991a, 1991b, and 1993).

The geochemistry of brines recovered from the WIPP repository horizon have been the subject of numerous studies (Stein and Krumhansl, 1986; Krumhansl and Stockman, 1987; Stein and Krumhansl, 1988; Deal and others 1989; Abitz and others, 1990; Krumhansl and others, 1991; Deal and others, 1991b; Deal and others, 1993). Both the major and traceelement compositions of the WIPP brines suggest that the brine originated from evaporating seawater, as substantiated by the high magnesium, potassium, and bromine content of the brines, which differs from the composition of a brine formed by dissolving the Salado evaporites in infiltrating groundwater (Deal and others, 1991b). The brine chemistry indicates that seawater has precipitated carbonate minerals, anhydrite, and halite and has been further modified by diagenetic reactions with gypsum, magnesite, polyhalite, and clay minerals. The major-element compositions of brines recovered from BSEP holes are distinct from fluid inclusion in WIPP halite (Stein and Krumhansl, 1988), implying that the brine recovered from the drillholes is mostly intergranular fluid, rather than fluid released by migration of fluid inclusions to grain boundaries in response to stress relief.

During 1992, 40 brine samples were recovered from 18 drillholes in the Salado Formation at the repository horizon. These brine samples were analyzed for up to 27 chemical parameters by Rust Geotech (formerly UNC Geotech of Grand Junction, Colorado). Brine chemistry data for all samples collected from 1987 to 1992 are tabulated in Appendix D.

The statistical analysis of BSEP brine compositions includes a measure of the central tendency of each measured parameter for each drillhole. In order to calculate a central tendency, such as a mean or a median, the following issues were considered: 
- Evaluation of data sources

- Analysis of data for the presence of temporal trends

- Handling of duplicate analysis

- Determination of the type of statistical distributions

- Handling of values less than the detection limit of the laboratory equipment

- Rejection of outliers.

The statistical analysis also includes the calculation of an average brine chemistry for the repository horizon. This average brine chemistry was determined by grouping data together from drillholes that sample brine from below and within the repository horizon. Data were tested using an analysis-of-variance calculation to determine if it is statistically valid to group the analyses from different drillholes together.

\subsection{Sources of Data}

BSEP brine samples have been collected over five years from several drillholes at various locations in the underground. Many of the drillholes discussed in previous BSEP reports are no longer producing brine, and some new holes were added to sampling locations. Only drillholes that produced a significant volume of brine since sampling began in 1987 are considered in these calculations. Additionally, some BSEP drillholes have been contaminated by water spread for dust control and floor consolidation. This report only discusses data from those drillholes that were not considered to have been contaminated with waters used for dust control (spread waters), drilling fluids, or synthetic brine used in Room J. These drillholes, sampled in 1992, are located in areas where contaminating brines have not been spread (Rooms A1, A2, A3, B, and G) or in subhorizontal holes located where water spread on floors could not enter them (Table 3-1).

Only geochemical data from Rust Geotech were used in the statistical analysis. Previous sampling rounds were analyzed by both Rust Geotech and IT analytical laboratories.

Comparisons of geochemical data analyzed by these two laboratories are misleading, because differences in laboratory technique produce slightly different values for parameters analyzed (Deal and others, 1991b).

\subsection{Temporal Trends}

In order to perform a statistical analysis of the brine compositions, it was necessary to first determine if brine chemistry changes as a function of time. Changes in brine chemistry with 
Table 3-1

BSEP Drillholes Sampled for Brine between 1987 and 1993

\begin{tabular}{|c|c|c|c|}
\hline Downholes & "Suspect ${ }^{\mathrm{a}}$ Downholes & Upholes & Subhorizontal Holes \\
\hline$\overline{A 1 \times 01^{*}}$ & DH28 & A1X02 & $\overline{\mathrm{OH} 2 \mathrm{O}}$ \\
\hline $\mathrm{A} 2 \times 01^{*}$ & $\mathrm{DH} 30$ & $\mathrm{OH} 47$ & $\mathrm{OH} 23^{*}$ \\
\hline A3X01* & $\mathrm{DH} 32$ & & $\mathrm{OH}_{26}^{*}$ \\
\hline $\mathrm{BXO1^{* }}$ & $\mathrm{DH} 34$ & & $\mathrm{OH} 45^{\star}$ \\
\hline $\mathrm{DH} 36^{*}$ & DHP402A & & \\
\hline $\mathrm{DH} 38^{\star}$ & G090 & & \\
\hline $\mathrm{DH} 40$ & GSEEP & & \\
\hline $\mathrm{DH} 42^{*}$ & H09O & & \\
\hline$\overline{D H} 42 A^{*}$ & L1X00 & & \\
\hline NG252 & $\mathrm{OH} 62$ & & \\
\hline \multirow[t]{3}{*}{$\mathrm{OH} 46$} & $\mathrm{OH} 63$ & & \\
\hline & $\mathrm{OH} 66$ & & \\
\hline & $\mathrm{OH} 67$ & סמ & \\
\hline
\end{tabular}

asuspect holes may be contaminated with water spread on drift floor for construction purposes. *Drillholes used for statistical analysis.

time may indicate that physical processes such as evaporation or mixing are occurring. Brine chemistry affected by these processes may not be reflective of in situ conditions.

Chemical parameters that are nonsolubility-constrained (i.e., not controlled by precipitation of evaporite minerals) will behave similarly when evaporation occurs and will become concentrated in the brine. Likewise, mixing of brine with spread waters will also change the concentration of the nonsolubility constrained parameters with time. These include boron, bromide, magnesium, and potassium. Parameters that are controlled by solubility and precipitate with evaporite minerals included sodium, chloride, calcium, and sulfate.

Temporal trends were analyzed by plotting the concentration data against the sampling date for the downholes, upholes, and subhorizontal holes. No temporal trends were evident for nonsolubility-constrained parameters from the downholes and the subhorizontal holes. Thus, brine from downholes and subhorizontal holes have not been evaporated or mixed with other 
waters. However, Figure 3-1 shows that uphole A1X02 is affected by evaporation. Magnesium, boron, and bromine display similar changes in concentration with time. Concentrations for these elements in uphole A2X01 all increase and decrease in the same samples. Concentrations of potassium, however, are not similar to magnesium, boron, and bromine in the latest sampling rounds. This suggests that perhaps some potassium is substituting into halite, which is precipitating from the brine during evaporation. Because magnesium, boron, and bromine have similar changes in concentrations with time and because the ratio of these parameters with each other is constant with time, brine from uphole A1X02 has undergone various amounts of evaporation between sampling events. It has been previously suspected that partial evaporation has altered the concentrations in the upholes (Deal and others, 1991b).

\subsection{Duplicate Analysis}

In order to measure the concentration of dissolved constituents in brine samples from the repository horizon, it was necessary for the analytical laboratories to dilute the samples. Because dilution factors were high for the BSEP brines, measurement errors sometimes occurred, particularly in earlier sampling rounds. Consequently, duplicate analyses were performed on the brine samples. Duplicate analyses were used to identify analytical errors and to indicate how precisely the concentrations can be measured.

For the purposes of the statistical analysis, the concentration values for duplicate analyses were averaged. If one of the duplicate samples was obviously erroneous (i.e., an obvious data outlier), then only the single best value of the duplicates was included. Additionally, if one of the duplicates had a value below detection limits and the other duplicate had a detectable concentration, then only the detected value was chosen for statistics.

\subsection{Determination of Statistical Distributions}

The first step in data analysis is to determine the distribution of each data set. In this case, a data set would consist of all data collected for a particular parameter in a particular drillhole. The specific statistical procedure used to analyze the data and the methods used to identify outliers are dependent on the assumed distributions of the data sets. If a data set was determined to be normally distributed, then a mean and a standard deviation were calculated. If a data set was not normal, then nonparametric techniques were used. For the purposes of this report, the term "nonparametric techniques" refer to statistical procedures that do not 


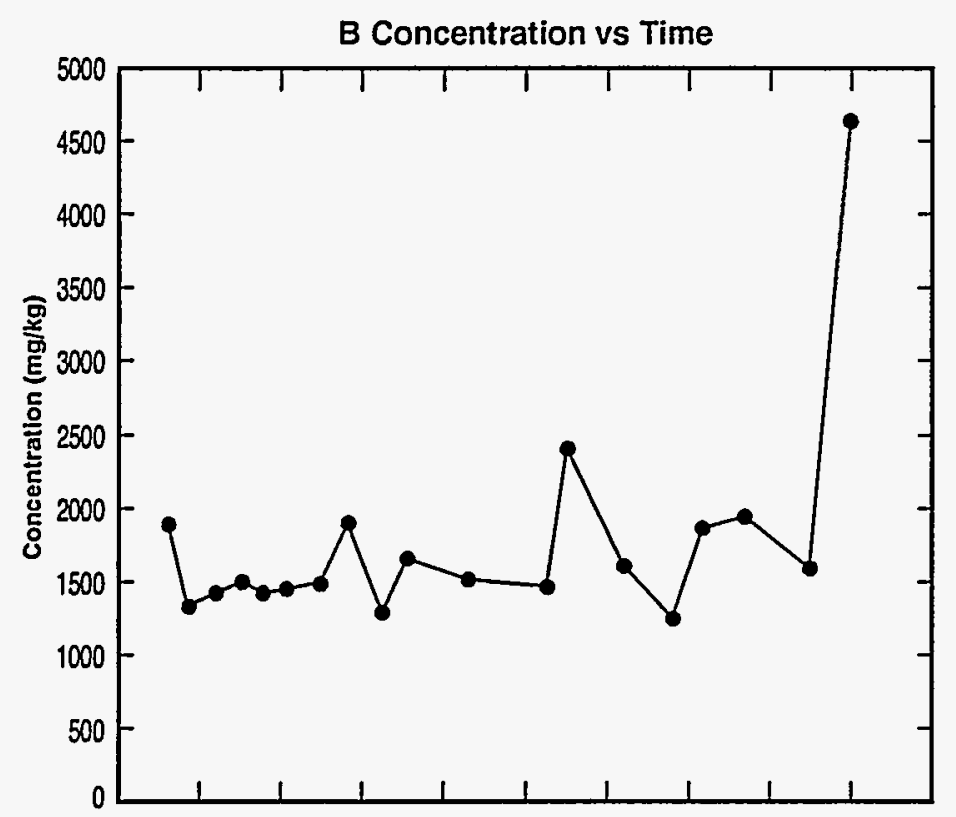

8/1/87 2/27/88 9/14/88 4/2899 10/19/89 57/90 11/23/90 6/11/91 12/28/91 7/15/92 1/31/93

Date

Mg Concentration vs Time

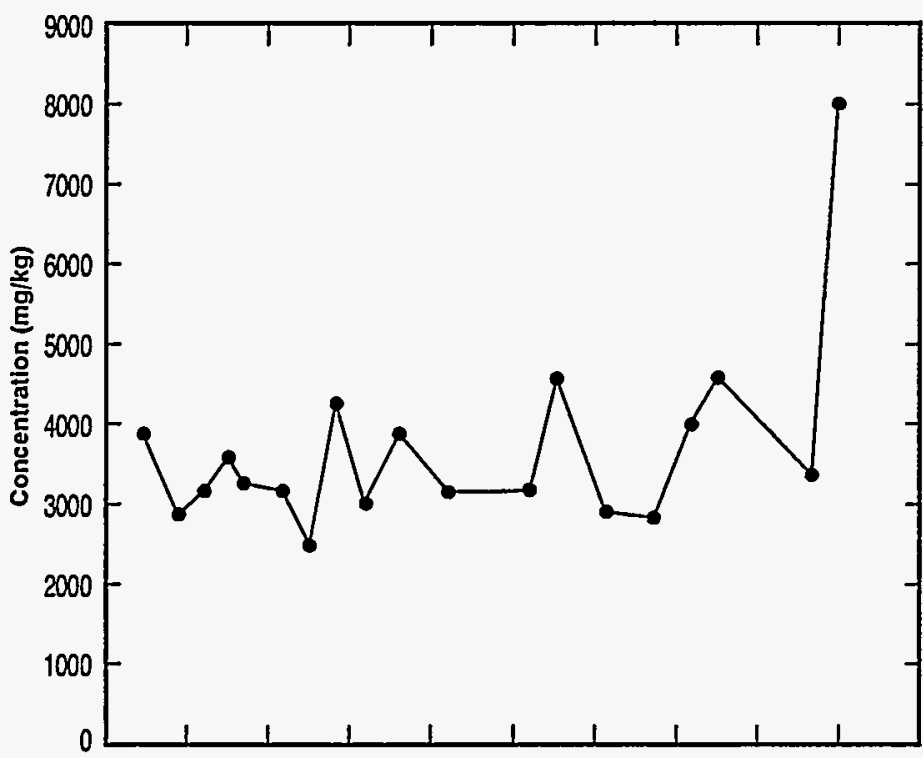

8/11/87 2/27/88 $9 / 14 / 88$ 4/2/89 10/19/89 57/90 11/23/90 6/11/91 12/28/91 7/15/92 1/31/83

Date

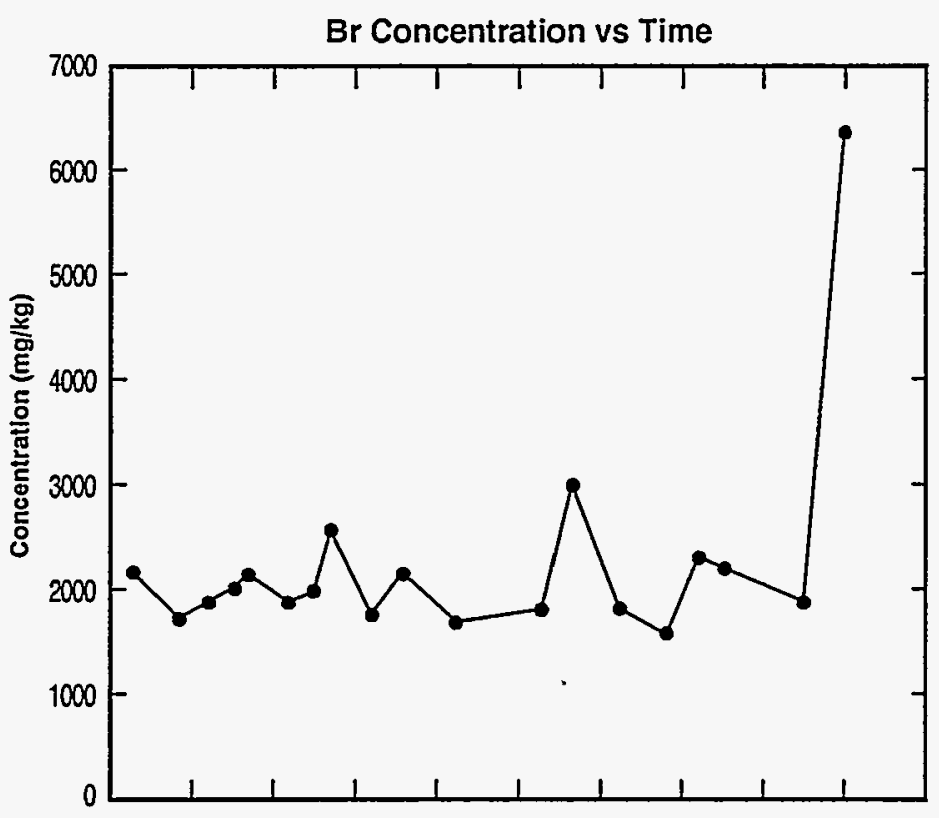

$8 / 11 / 87$ 2/27/88 9/14/88 4/2/89 10/19/89 5/7/90 1//23/90 6/11/91 12/28/91 7/15/92 1/31/98

Date

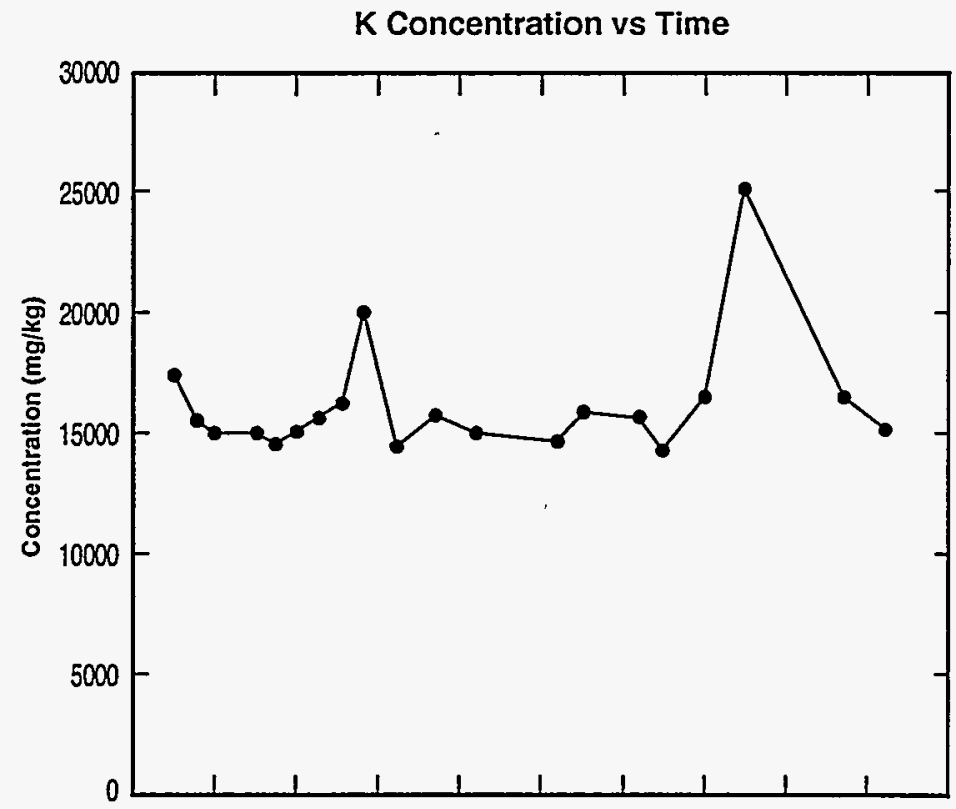

8/11/87 2/27/88 9/14/88 4/2/89 10/19/89 5/7/90 11/23/906/11/91 12/28917/15/92 1/31/93

Date

Figure 3-1

Uphole A1X02 Concentration vs. Time for $\mathrm{B}, \mathrm{Br}, \mathrm{Mg}$, and $\mathrm{K}$ 
require the data to fit any particular distribution. Only a median was reported for a nonparametric data set.

For each drillhole, 27 parameters were analyzed. Thus, there are 27 data sets for each drillhole, and there are 11 drillholes that were considered in this statistical analysis (Table 3-1) for a total of 297 data sets. Because there are so many data sets, it was impractical to test each one for normality. Consequently, only the nonsolubility-constrained parameters (boron, bromine, magnesium, and potassium) were tested for normality.

A Kolmogorov-Smirnov statistical test (Kennedy and Neville, 1986) for the 95 percent confidence level was applied to the data from each drillhole (Table 3-1) for each nonsolubility-constrained parameter to test for normality. This statistical test determines how well a set of observations fit a theoretical normal distribution by calculating the maximum distance between the cumulative distribution functions of the sample and the theoretical normal distribution. If the distance is too large, the hypothesis that the theoretical distribution fits the observed distribution is rejected. In all cases, the geochemical data collected from 1987 to 1993 for each nonsolubility-constrained parameter in each drillhole were normally distributed. After it was determined that the data from nonsolubility-constrained parameters were normally distributed, it was assumed that data sets for other parameters were also normally distributed.

Because each data set was not rigorously tested to determine if it was normally distributed, the coefficient of variation was also calculated for each data set assumed to be normal. The coefficient of variation (V) is defined by Kennedy and Neville (1986) as:

$$
V=S / X * 100
$$

where

$$
\begin{aligned}
& \mathrm{S}=\text { Population standard deviation } \\
& \mathrm{X}=\text { Population mean }
\end{aligned}
$$

The coefficient of variation expresses the dispersion of samples on a percentage basis. If the coefficient of variation is larger than approximately 10 percent, the assumption of normality for that particular data set is questionable. Thus, data sets with a coefficient of variation larger than 10 percent were tested for normality using the Kolmogorov-Smirnov test described above. If a data set had a large coefficient of variation and did not pass the Kolmogorov- 
Smirnov test at the 95 percent confidence level, the distribution was assumed to be nonparametric, and only a median was reported.

\subsection{Handling of Values}

A certain proportion of the values presented in this report were reported as being below the detection limits of the analytical equipment. The U.S. Environmental Protection Agency (EPA) guidance (EPA, 1989 [EPA/530-SW-89-026]) for dealing with such values was used for this report. If the data set was normal and if less than 15 percent of the values were below detection limits, the nondetected values were replaced with a value equal to one half of the detection limit, and a mean and a standard deviation were calculated. This approach should not have introduced a large bias, because the proportion of nondetected values was low, and the difference between the detection limit and zero is small using modern analytical methods.

If the percentage of nondetected values were greater than 15 percent of the data set, those values were replaced with one half of the detection limit, and a median was calculated. The percentage of those values below the detection limit was also reported. Some of the data sets contain older data points that have considerably higher detection limits than more recent data. In fact, the detection limits for some older below-detection-limit data points are higher than the median of the population. These "high nondetect" data points were deleted from the data sets because they did not add any additional information and because including them with an arbitrarily assigned value of one half the detection limit would have added a bias to the calculated median.

\subsection{Rejection of Outliers}

Outliers are data points whose values are anomalously high or low in relation to the rest of the data set. The following are possible reasons for outliers:

- Improper sampling, analytical error, or laboratory contamination

- Errors in transcription of data values, decimal points, or units

- The presence of foreign substances or contamination in the sample

- A true natural value that is unusually high.

Each data set that was assumed to be normally distributed was screened for outliers using the EPA-recommended technique (EPA, 1989 [EPA/530-SW-89-026]), which is based on American Society for Testing and Materials (ASTM) Procedure E178-80. This procedure determines if there is statistical evidence that an observation which appears extreme does not 
fit the distribution of the rest of the data. The procedure calculates the statistic $T_{n}$, which is defined as:

$$
T_{n}=\left(X_{n}-X\right) / S
$$

where

$$
\begin{aligned}
& X_{n}=\text { Observation } \\
& X=\text { Population mean } \\
& S=\text { Population standard deviation. }
\end{aligned}
$$

The calculated $T_{n}$ value is then compared to a table of one-sided critical values for the appropriate significance level (upper 5 percent) and sample size (a suitable table is provided in EPA, 1989 [EPA/530-SW-89-026]). The $\mathrm{T}_{\mathrm{n}}$ statistic differs from the standard " $\mathrm{t}$ " critical value distribution in that the $T_{n}$ statistic is calculated from the entire population, including the suspected outliers. The standard " $t$ " critical values are used to determine if a new sample value (not yet included in the population statistics) is an outlier.

If the $T_{n}$ value for the suspect data is greater than the critical value from the table, then there is evidence that the value is a statistical outlier. Because of symmetry considerations, the above equation can be applied to a suspected minimum outlier value by taking the absolute value of $T_{n}$ equation and comparing it with the tabulated values. Both minimum and maximum suspected outliers can be screened from the data sets.

The specific procedure used in this investigation for the identification of outliers is as follows:

- Normal data sets. Calculate a mean and standard deviation. Calculate a $T_{n}$ statistic and compare to the table. If outliers are confirmed, delete them from the data set and recalculate the mean standard deviation.

- Nonparametric data sets. The screening using the $T_{n}$ statistic is not applied. The $T_{n}$ procedure described above is based on an assumption of a normal distribution in which one can calculate the probability of a given value being a member of a population. Nonparametric data sets are not predictable in this sense.

For all data sets that were assumed to be normal, outliers (if present) were removed from the data sets, and the average and the standard deviation for each parameter were calculated. If a data set was nonparametric, the median, the number of nondetects, and the percentage of 
nondetects was determined. Values of the mean or median and standard deviation for each drillhole are given in Table 3-2.

\subsection{Average Brine Chemistry}

An average brine chemistry was determined by grouping data together from drillholes used to sample brine from the repository horizon. To check the validity of grouping these drillholes together, a one-way analysis of variance (ANOVA) calculation was performed.

Drillholes were separated into two different groupings, based upon whether or not they sampled stratigraphy within the repository horizon. One group consisted of drillholes DH36, $\mathrm{DH} 38, \mathrm{DH} 42$, and DH42A. These drillholes are used to sample brine encountered only in stratigraphy beneath the repository horizon. The second group consisted of drillholes A1X01, A2X01, A3X01, BX01, OH23, OH26, and $\mathrm{OH} 45$. These drillholes are used to sample brine encountered in stratigraphy within and below the repository horizon and are the most representative of overall repository brine chemistry. Figure 2-3 shows the stratigraphic locations of the down holes. The subhorizontal holes start just above the orange band (Figure 2-2, Detail 2) and end below it, just above the floor of the drift. The subhorizontal holes are primarily to sample brine from the clays above and below the orange band.

A one-way ANOVA was performed for each of the nonsolubility-limited parameters (boron, bromine, potassium, and magnesium) to determine if the data for a particular parameter from the drillholes in their respective groupings were part of the same statistical population. ANOVA is a general method in which the total statistical variation in a set of data is considered in order to simultaneously test the differences between subpopulation means at a certain confidence level to determine if the subpopulations can be grouped. In this case, the subpopulation means consisted of a given parameter from each evaluated drillhole (listed in Table 3-2). The ANOVA calculation was performed for the 95 percent confidence level.

ANOVA calculations performed on the combined data from drillholes A1X01, A2X01, $\mathrm{A} 3 \mathrm{X} 01, \mathrm{BX} 01, \mathrm{OH} 23, \mathrm{OH} 26$, and $\mathrm{OH} 45$ showed that analyses for boron, bromine, and potassium are members of the same population (i.e., they have significance at the 95 percent confidence level). Magnesium analyses for these drillholes did not have significance at the 95 percent confidence level. It is unclear why magnesium failed the ANOVA test for 
Table 3-?

Simple Statistics for ESEP Analyses

(in $\mathrm{mg} / \mathrm{L}$ )

\begin{tabular}{|c|c|c|c|c|c|c|c|c|c|c|c|c|c|}
\hline \multicolumn{7}{|c|}{ Downhole A1X01 } & \multicolumn{7}{|c|}{ Downhole A2X01 } \\
\hline & $\mathbf{N}$ & $x$ & $s$ & Median & No. ND & $\%$ ND & & $N$ & $x$ & $\mathbf{s}$ & Median & No. ND & $\%$ ND \\
\hline$S G$ & 14 & 1.23 & 0.01 & & & & SG & 13 & 1.23 & 0.01 & & & \\
\hline TDS & $13^{*}$ & 376000 & 10000 & & & & TDS & $12^{*}$ & 400,000 & 8000 & & & \\
\hline $\mathrm{pH}$ & 14 & 6.1 & & & & & $\mathrm{pH}$ & $12^{*}$ & 6.1 & & & & \\
\hline ALK & 14 & 980 & 33 & & & & ALK & 13 & 989 & 88 & & & \\
\hline TIC & $13^{*}$ & 5.6 & 4.5 & & & & TIC & 13 & 26.4 & 26.7 & & & \\
\hline TOC & 12 & 22 & 20 & & & & TOC & 10 & 53 & 49 & & & \\
\hline $\mathrm{Br}-$ & 14 & 1500 & 60 & & & & $\mathrm{Br}-$ & $12^{*}$ & 1510 & 40 & & & \\
\hline $\mathrm{Cl}$ & 14 & 193000 & 2000 & & & & $\mathrm{Cl}-$ & $12^{*}$ & 200,000 & 2000 & & & \\
\hline F- & 14 & 6 & 1 & & & & F- & 13 & 7 & 1 & & & \\
\hline 1- & 14 & 14.6 & 2.6 & & & & 1- & 13 & 13.5 & 1.0 & & & \\
\hline $\mathrm{NH}_{4}^{+}$ & 14 & 150 & 13 & & & & $\mathrm{NH}_{4}^{+}$ & $12^{*}$ & 148 & 10 & & & \\
\hline $\mathrm{NO}_{3}^{-}$ & 10 & & & 0.8 & 3 & 30 & $\mathrm{NO}_{3}^{-}$ & 9 & & & 0.8 & 3 & 33 \\
\hline$P$ & 8 & & & $<0.1$ & 7 & 88 & $P$ & 7 & & & $<0.1$ & 6 & 86 \\
\hline $\mathrm{SO}_{4}^{-2}$ & 14 & 17500 & 600 & & & & $\mathrm{SO}_{4}^{-2}$ & 13 & 17300 & 1000 & & & \\
\hline Al & $13^{*}$ & 0.18 & 0.16 & & & & $\mathrm{Al}$ & 12 & & & 0.13 & 2 & 17 \\
\hline As & $13^{*}$ & 0.003 & 0.004 & & & & As & 13 & & & $<0.001$ & 8 & 62 \\
\hline B & 14 & 1460 & 110 & & & & $B$ & 13 & 1430 & 100 & & & \\
\hline $\mathrm{Ba}$ & $13^{*}$ & 0.03 & 0.02 & & & & $\mathrm{Ba}$ & 13 & 0.07 & 0.04 & & & \\
\hline $\mathrm{Ca}$ & $13^{*}$ & 265 & 32 & & & & $\mathrm{Ca}$ & 13 & 290 & 49 & & & \\
\hline Cs & 9 & 0.36 & 0.04 & & & & Cs & 7 & 0.37 & 0.04 & & & \\
\hline $\mathrm{Fe}$ & 14 & & & $<0.5$ & 8 & 57 & $\mathrm{Fe}$ & 13 & 17.1 & 16.3 & & & \\
\hline$K$ & 14 & 15900 & 800 & & & & $k$ & $12^{*}$ & 16100 & 500 & & & \\
\hline $\mathrm{Mg}$ & 14 & 23300 & 1000 & & & & $\mathrm{Mg}$ & 13 & 23100 & 1700 & & & \\
\hline $\mathrm{Mn}$ & 14 & 1.6 & 0.2 & & & & $M n$ & 13 & 1.8 & 0.1 & & & \\
\hline $\mathrm{Na}$ & 14 & 79000 & 2000 & & & & $\mathrm{Na}$ & $12^{*}$ & 78700 & 2300 & & & \\
\hline $\mathrm{Rb}$ & 5 & 16.5 & 1.2 & & & & $\mathrm{Rb}$ & 3 & 16.1 & 1.5 & & & \\
\hline $\mathrm{Si}$ & $13^{*}$ & 1.4 & 0.4 & & & & $\mathrm{Si}$ & 13 & 1.5 & 1.2 & & & \\
\hline $\mathrm{Sr}$ & $13^{*}$ & 1.7 & 0.1 & & & & $\mathrm{Sr}$ & $12^{*}$ & 1.0 & 0.2 & & & \\
\hline
\end{tabular}

"Outlier values omitted in statistical calculations.

$\mathrm{N}=$ Number of samples.

$X=$ Mean.

$\mathrm{S}=$ Standard deviation.

ND $=$ Not detected. 
Table 3-2 (Continued)

Simple Statistics for BSEP Analyses

(in $\mathrm{mg} / \mathrm{L}$ )

\begin{tabular}{|c|c|c|c|c|c|c|c|c|c|c|c|c|c|}
\hline \multicolumn{7}{|c|}{ Downhole A3X01 } & \multicolumn{7}{|c|}{ Downhole BX01 } \\
\hline & $N$ & $x$ & $\mathbf{S}$ & Median & No. ND & $\% N D$ & & $N$ & $x$ & $S$ & Median & No. ND & $\%$ ND \\
\hline SG & 18 & 1.22 & 0.01 & & & & SG & 17 & 1.22 & 0.01 & & & 1 \\
\hline TDS & $17^{*}$ & 374000 & 14000 & & & & TDS & $16^{*}$ & 400,000 & 12000 & & & \\
\hline $\mathrm{pH}$ & $17^{*}$ & 6.1 & & & & & $\mathrm{pH}$ & 17 & 6 & & & & \\
\hline ALK & 18 & 980 & 41 & & & & ALK & 17 & 873 & 31 & & & \\
\hline TIC & 18 & & & 4.8 & 1 & 6 & TIC & $16^{*}$ & 7.1 & 7.2 & & & \\
\hline TOC & $14^{*}$ & 29 & 17 & & & & TOC & 14 & 27 & 19 & & & \\
\hline $\mathrm{Br}-$ & 18 & 1490 & 70 & & & & $\mathrm{Br}-$ & 17 & 1470 & 60 & & & \\
\hline Cl- & 18 & 192000 & 5000 & & & & $\mathrm{Cl}-$ & 17 & 200,000 & 4000 & & & \\
\hline F. & 18 & 7 & 1 & & & & F. & $16^{*}$ & 7 & 1 & & & \\
\hline I- & $17^{*}$ & 14.2 & 2.8 & & & & 1- & 17 & 14.0 & 1.6 & & & \\
\hline $\mathrm{NH}_{4}^{+}$ & 18 & 150 & 17 & & & & $\mathrm{NH}_{4}^{+}$ & 17 & 150 & 15 & & & \\
\hline $\mathrm{NO}_{3}^{-}$ & 14 & & & 0.7 & 4 & 29 & $\mathrm{NO}_{3}^{-}$ & 15 & & & 0.7 & 5 & 33 \\
\hline$P$ & 14 & & & $<0.1$ & 13 & 93 & $P$ & 11 & & & $<0.1$ & 11 & 100 \\
\hline $\mathrm{SO}_{4}^{-2}$ & 18 & 16900 & 900 & & & & $\mathrm{SO}_{4}^{-2}$ & 17 & 17100 & 700 & & & \\
\hline Al & 18 & & & 0.08 & 8 & 44 & Al & 17 & & & 0.08 & 6 & 35 \\
\hline As & 18 & & & 0.002 & 4 & 22 & As & $16^{*}$ & 0.002 & 0.001 & & & \\
\hline B & 18 & 1490 & 120 & & & & B & 17 & 1470 & 100 & & & \\
\hline $\mathrm{Ba}$ & 18 & 0.05 & 0.02 & & & & $\mathrm{Ba}$ & 17 & 0.04 & 0.02 & & & \\
\hline $\mathrm{Ca}$ & 18 & 273 & 32 & & & & $\mathrm{Ca}$ & 17 & 270 & 23 & & & \\
\hline Cs & 13 & 0.36 & 0.03 & & & & Cs & 12 & 0.34 & 0.04 & & & \\
\hline $\mathrm{Fe}$ & 18 & & & $<0.5$ & 10 & 56 & $\mathrm{Fe}$ & 17 & & & 0.7 & 8 & 47 \\
\hline$K$ & 18 & 15700 & 800 & & & & $K$ & 17 & 16100 & 800 & & & \\
\hline $\mathrm{Mg}$ & 18 & 23200 & 1300 & & & & $\mathrm{Mg}$ & 17 & 22500 & 1100 & & & \\
\hline$M n$ & 18 & 1.5 & 0.1 & & & & Mn & $16^{*}$ & 1.3 & 0.2 & & & \\
\hline $\mathrm{Na}$ & $17^{*}$ & 78300 & 2000 & & & & $\mathrm{Na}$ & 17 & 79800 & 1700 & & & \\
\hline $\mathrm{Rb}$ & $8^{*}$ & 15.9 & 0.6 & & & & $R b$ & 8 & 15.8 & 1.0 & & & \\
\hline Si & $17^{\star}$ & 1.7 & 0.3 & & & & $\mathrm{Si}$ & $16^{\star}$ & 1.6 & 0.8 & & & \\
\hline $\mathrm{Sr}$ & $17^{*}$ & 1.9 & 0.2 & & & & $\mathrm{Sr}$ & 17 & 2.0 & 0.2 & & & \\
\hline
\end{tabular}

-Outlier values omitted in statistical calculations.

$\mathrm{N}=$ Number of samples.

$X=$ Mean.

$S=$ Standard deviation.

ND $=$ Not detected. 
Table 3-2 (Cortinued)

Simple Statistics for ESEP Analyses

(in $\mathrm{mg} / \mathrm{L}$ )

\begin{tabular}{|c|c|c|c|c|c|c|c|c|c|c|c|c|c|}
\hline \multicolumn{7}{|c|}{ OH23-horizontal hole } & \multicolumn{7}{|c|}{ OH26-horizontal hole } \\
\hline & $N$ & $x$ & S & Median & No. ND & $\%$ ND & & $\mathbf{N}$ & $x$ & S & Median & No. ND & $\%$ ND \\
\hline SG & 15 & 1.22 & 0.01 & & & & $S G$ & 12 & 1.22 & 0.01 & & & \\
\hline TDS & $14^{*}$ & 373000 & 1500 & & & & TDS & 12 & 400,000 & 15000 & & & \\
\hline $\mathrm{pH}$ & 15 & 6 & & & & & $\mathrm{pH}$ & 12 & 6 & & & & \\
\hline ALK & 15 & 716 & 87 & & & & ALK & $11^{*}$ & 731 & 38 & & & \\
\hline TIC & 15 & 4.0 & 1.3 & & & & TIC & $11^{*}$ & 3.8 & 0.7 & & & \\
\hline TOC & 15 & 97 & 78 & & & & TOC & 12 & 70 & 25 & & & \\
\hline Br- & 15 & 1520 & 60 & & & & $\mathrm{Br}-$ & 12 & 1490 & 30 & & & \\
\hline $\mathrm{Cl}-$ & 15 & 193000 & 3000 & & & & $\mathrm{Cl}-$ & 12 & 200,000 & 3000 & & & \\
\hline F- & 15 & 4 & 1 & & & & F- & 12 & 4 & 1 & & & \\
\hline 1. & $14^{*}$ & 16.3 & 5.0 & & & & $1-$ & $11^{*}$ & 16.0 & 3.0 & & & \\
\hline $\mathrm{NH}_{4}^{+}$ & 15 & 147 & 14 & & & & $\mathrm{NH}_{4}^{+}$ & 12 & 144 & 18 & & & \\
\hline $\mathrm{NO}_{3}{ }^{-}$ & 15 & & & 1 & 3 & 20 & $\mathrm{NO}_{3}{ }^{\circ}$ & 12 & 0.9 & 0.3 & & & \\
\hline$P$ & 15 & & & $<0.1$ & 9 & 60 & $P$ & 12 & & & 0.1 & 3 & 25 \\
\hline $\mathrm{SO}_{4}^{-2}$ & 15 & 16800 & 900 & & & & $\mathrm{SO}_{4}^{-2}$ & 12 & 16500 & 800 & & & \\
\hline Al & 15 & 0.13 & 0.08 & & & & Al & 12 & & & 0.15 & 2 & 17 \\
\hline As & $14^{*}$ & & & 0.002 & 0 & 0 & As & 12 & & & 0.001 & 5 & 42 \\
\hline$B$ & $14^{*}$ & 1450 & 60 & & & & $B$ & 12 & 1400 & 110 & & & \\
\hline $\mathrm{Ba}$ & $14^{*}$ & 0.06 & 0.02 & & & & $8 a$ & 12 & 0.07 & 0.03 & & & \\
\hline $\mathrm{Ca}$ & 15 & 303 & 36 & & & & $\mathrm{Ca}$ & 12 & 295 & 32 & & & \\
\hline Cs & $14^{*}$ & 0.29 & 0.03 & & & & Cs & 12 & 0.29 & 0.03 & & & \\
\hline $\mathrm{Fe}$ & 15 & & & $<0.5$ & 15 & 100 & $\mathrm{Fe}$ & 12 & & & $<0.5$ & 12 & 100 \\
\hline $\mathrm{K}$ & 15 & 15900 & 900 & & & & $K$ & 12 & 15300 & 600 & & & \\
\hline $\mathrm{Mg}$ & 15 & 22700 & 1500 & & & & $\mathrm{Mg}$ & 12 & 22100 & 1100 & & & \\
\hline $\mathrm{Mn}$ & 15 & 2.0 & 0.4 & & & & $\mathrm{Mn}$ & 12 & 1.6 & 0.1 & & & \\
\hline $\mathrm{Na}$ & 15 & 79400 & 1900 & & & & $\mathrm{Na}$ & 12 & 79200 & 2700 & & & \\
\hline $\mathrm{Rb}$ & 9 & 15.6 & 1.1 & & & & $R b$ & $8^{*}$ & 15.2 & 0.7 & & & \\
\hline Si & 15 & 1.9 & 0.7 & & & & $\mathrm{Si}$ & $11^{*}$ & 1.2 & 0.4 & & & \\
\hline $\mathrm{Sr}$ & 15 & 1.1 & 0.3 & & & & $\mathrm{Sr}$ & $11^{*}$ & 1.0 & 0.2 & & & \\
\hline
\end{tabular}

*Outlier values omitted in statistical calculations.

$\mathrm{N}=$ Number of samples.

$X=$ Mean.

$S=$ Standard deviation.

$\mathrm{ND}=$ Not detected. 
Table 3-2 (Continued)

Simple Statistics for BSEP Analyses

(in $\mathrm{mg} / \mathrm{L}$ )

\begin{tabular}{|c|c|c|c|c|c|c|c|c|c|c|c|c|c|}
\hline \multicolumn{7}{|c|}{ OH45-horizontal hole } & \multicolumn{7}{|c|}{ Downhole DH36 } \\
\hline & $\mathbf{N}$ & $\mathrm{x}$ & $\mathbf{S}$ & Median & No. ND & $\%$ ND & & $N$ & $\mathrm{x}$ & $S$ & Median & No. ND & $\% N D$ \\
\hline SG & $6^{*}$ & 1.22 & 0.01 & & & & SG & 20 & 1.22 & 0.01 & & & \\
\hline TDS & $6^{*}$ & 372000 & 14000 & & & & TDS & 20 & 400,000 & 10000 & & & \\
\hline $\mathrm{pH}$ & 7 & 6.2 & & & & & $\mathrm{pH}$ & $19^{*}$ & 6.1 & & & & \\
\hline ALK & $6^{*}$ & 856 & 50 & & & & ALK & $19^{*}$ & 843 & 17 & & & \\
\hline TIC & 7 & 5.5 & 2.4 & & & & TIC & 18 & & & 5.2 & 0 & 0 \\
\hline TOC & 7 & 91 & 28 & & & & TOC & 15 & 23 & 17 & & & \\
\hline $\mathrm{Br}-$ & $6^{*}$ & 1550 & 60 & & & & $\mathrm{Br}-$ & $19^{*}$ & 1430 & 70 & & & \\
\hline $\mathrm{Cl}-$ & $6^{*}$ & 193000 & 5000 & & & & $\mathrm{Cl}-$ & 20 & 200,000 & 3000 & & & \\
\hline F. & 7 & 5 & 1 & & & & F- & 20 & 5 & 1 & & & \\
\hline 1- & 7 & 16.2 & 3.9 & & & & $1-$ & $17^{*}$ & 15.4 & 1.8 & & & \\
\hline $\mathrm{NH}_{4}{ }^{+}$ & 7 & 145 & 23 & & & & $\mathrm{NH}_{4}^{+}$ & $17^{\star}$ & 164 & 17 & & & \\
\hline $\mathrm{NO}_{3}^{-}$ & 7 & 1.0 & 0.3 & & & & $\mathrm{NO}_{3}^{-}$ & 16 & & & 1.0 & 6 & 38 \\
\hline$P$ & 7 & & & 0.1 & 2 & 29 & $P$ & 11 & & & $<0.1$ & 10 & 91 \\
\hline $\mathrm{SO}_{4}^{-2}$ & $6^{*}$ & 16400 & 600 & & & & $\mathrm{SO}_{4}^{-2}$ & 20 & 16300 & 600 & & & \\
\hline Al & 7 & & & 0.06 & 3 & 43 & Al & 20 & & & 0.19 & 5 & 25 \\
\hline As & 7 & 0.002 & 0.001 & & & & As & 20 & 0.010 & 0.004 & & & \\
\hline$B$ & 7 & 1350 & 230 & & & & $B$ & 18 & 1520 & 110 & & & \\
\hline $\mathrm{Ba}$ & 7 & 0.08 & 0.03 & & & & $\mathrm{Ba}$ & 19 & 0.04 & 0.03 & & & \\
\hline $\mathrm{Ca}$ & 7 & 289 & 62 & & & & $\mathrm{Ca}$ & 20 & 322 & 23 & & & \\
\hline Cs & 7 & 0.25 & 0.03 & & & & Cs & 12 & 0.27 & 0.03 & & & \\
\hline $\mathrm{Fe}$ & 7 & & & $<0.5$ & 7 & 100 & $\mathrm{Fe}$ & 20 & & & $<0.5$ & 14 & 70 \\
\hline K & $6^{*}$ & 16100 & 1000 & & & & $K$ & 20 & 17900 & 800 & & & \\
\hline $\mathrm{Mg}$ & $6^{*}$ & 21100 & 900 & & & & $\mathrm{Mg}$ & 20 & 18600 & 900 & & & \\
\hline Mn & $6^{*}$ & 1.5 & 0.1 & & & & $\mathrm{Mn}$ & 20 & 1.0 & 0.1 & & & \\
\hline $\mathrm{Na}$ & $6^{*}$ & 78900 & 2400 & & & & $\mathrm{Na}$ & 20 & 85900 & 2000 & & & \\
\hline $\mathrm{Rb}$ & $4^{*}$ & 15.3 & 0.3 & & & & $\mathrm{Rb}$ & 8 & 14.8 & 0.6 & & & \\
\hline Si & 6 & 1.3 & 0.4 & & & & $\mathrm{Si}$ & 20 & 2.6 & 1.0 & & & \\
\hline Sr & 7 & 2.5 & 0.6 & & & & $\mathrm{Sr}$ & 20 & 1.3 & 0.1 & & & \\
\hline
\end{tabular}

- Outlier values omitted in statistical calculations.

$\mathrm{N}=$ Number of samples.

$\mathrm{X}=$ Mean.

$\mathrm{S}=$ Standard deviation

ND $=$ Not detected. 
Table 3-2 (Continued)

Simple Statistics for BSEP Analyses

(in $\mathrm{mg} / \mathrm{L}$ )

\begin{tabular}{|c|c|c|c|c|c|c|c|c|c|c|c|c|c|}
\hline \multicolumn{7}{|c|}{ Downhole DH38 } & \multicolumn{7}{|c|}{ Downhole DH42 } \\
\hline & $\mathbf{N}$ & $x$ & $S$ & Median & No. ND & $\%$ ND & & $\mathbf{N}$ & $x$ & $\mathrm{~s}$ & Median & No. ND & $\%$ ND \\
\hline$S G$ & 20 & 1.22 & 0.01 & & & & SG & 16 & 1.23 & 0.01 & & & \\
\hline TDS & 20 & 371000 & 9000 & & & & TDS & $15^{*}$ & 400,000 & 5000 & & & \\
\hline $\mathrm{pH}$ & $19^{*}$ & 6.2 & & & & & $\mathrm{pH}$ & $15^{*}$ & 6.3 & & & & \\
\hline ALK & 19 & 939 & 68 & & & & ALK & $15^{*}$ & 927 & 33 & & & \\
\hline TIC & 19 & & & 6.1 & 0 & 0 & TIC & 16 & & & 6.1 & 0 & 0 \\
\hline TOC & 16 & 29 & 21 & & & & TOC & 13 & 36 & 17 & & & \\
\hline $\mathrm{Br}-$ & $19^{*}$ & 1410 & 60 & & & & $\mathrm{Br}-$ & 16 & 1410 & 60 & & & \\
\hline $\mathrm{Cl}-$ & 20 & 193000 & 4000 & & & & $\mathrm{Cl}-$ & 16 & 200,000 & 4000 & & & \\
\hline F- & $19^{*}$ & 5 & 1 & & & & F- & 16 & 4 & 1 & & & \\
\hline I- & $18^{*}$ & 16.3 & 2.3 & & & & 1- & $15^{\star}$ & 16.0 & 1.8 & & & \\
\hline $\mathrm{NH}_{4}^{+}$ & 19 & 165 & 12 & & & & $\mathrm{NH}_{4}^{+}$ & 16 & 169 & 16 & & & \\
\hline $\mathrm{NO}_{3}^{-}$ & 16 & & & 0.7 & 3 & 19 & $\mathrm{NO}_{3}^{-}$ & 14 & & & 1.0 & 2 & 14 \\
\hline$P$ & 13 & & & $<0.1$ & 9 & 69 & $P$ & 9 & & & $<0.1$ & 5 & 56 \\
\hline $\mathrm{SO}_{4}^{-2}$ & $19^{*}$ & 15800 & 600 & & & & $\mathrm{SO}_{4}^{-2}$ & 16 & 15800 & 800 & & & \\
\hline Al & 20 & & & 0.20 & 7 & 35 & Al & 16 & & & 0.1 & 6 & 38 \\
\hline As & 20 & & & 0.004 & 4 & 20 & As & 16 & 0.005 & 0.002 & & & \\
\hline B & 19 & 1510 & 90 & & & & $B$ & 16 & 1490 & 100 & & & \\
\hline $\mathrm{Ba}$ & $19^{*}$ & 0.03 & 0.01 & & & & $\mathrm{Ba}$ & $15^{*}$ & 0.04 & 0.02 & & & \\
\hline $\mathrm{Ca}$ & 20 & 317 & 24 & & & & $\mathrm{Ca}$ & $15^{*}$ & 319 & 25 & & & \\
\hline Cs & 13 & 0.26 & 0.03 & & & & Cs & $9^{*}$ & 0.26 & 0.02 & & & \\
\hline $\mathrm{Fe}$ & 20 & & & $<0.5$ & 17 & 85 & $\mathrm{Fe}$ & 16 & & & $<0.5$ & 9 & 56 \\
\hline$K$ & 20 & 18000 & 700 & & & & $K$ & 16 & 17800 & 900 & & & \\
\hline $\mathrm{Mg}$ & $19^{*}$ & 18200 & 800 & & & & $\mathrm{Mg}$ & $15^{*}$ & 17800 & 400 & & & \\
\hline $\mathrm{Mn}$ & 20 & 1.0 & 0.1 & & & & Mn & $15^{*}$ & 1.1 & 0.1 & & & \\
\hline $\mathrm{Na}$ & 20 & 85700 & 2000 & & & & $\mathrm{Na}$ & 16 & 86400 & 1500 & & & \\
\hline$R b$ & 9 & 14.4 & 0.7 & & & & $\mathrm{Rb}$ & $7^{*}$ & 14.0 & 0.4 & & & \\
\hline $\mathrm{Si}$ & 20 & 2.4 & 0.8 & & & & $\mathrm{Si}$ & $15^{*}$ & 2.6 & 1.3 & & & \\
\hline $\mathrm{Sr}$ & $18^{*}$ & 0.8 & 0.1 & & & & Sr & $15^{*}$ & 0.9 & 0.2 & & & \\
\hline
\end{tabular}

"Outlier values omitted in statistical calculations.

$\mathrm{N}=$ Number of samples.

$X=$ Mean.

$S=$ Standard deviation.

ND $=$ Not detected. 
Table 3-2 (Continued)

Simple Statistics for BSEP Analyses (in $\mathrm{mg} / \mathrm{L}$ )

\begin{tabular}{|c|c|c|c|c|c|c|}
\hline \multicolumn{7}{|c|}{ Downhole DH42A } \\
\hline & $\mathbf{N}$ & $x$ & $S$ & Median & No.ND & \%ND \\
\hline SG & 20 & 1.23 & 0.01 & & & \\
\hline TDS & 20 & 372000 & 9000 & & & \\
\hline $\mathrm{pH}$ & $19^{*}$ & 6.2 & & & & \\
\hline ALK & 19 & 882 & 39 & & & \\
\hline TIC & $17^{*}$ & 5.0 & 1.2 & & & \\
\hline TOC & 15 & & & 20 & 3 & 20 \\
\hline $\mathrm{Br}-$ & $19^{*}$ & 1400 & 50 & & & \\
\hline $\mathrm{Cl}-$ & 20 & 194000 & 3000 & & & \\
\hline F- & 20 & 4 & 1 & & & \\
\hline 1- & 18 & 16.3 & 3.8 & & & \\
\hline $\mathrm{NH}_{4}^{+}$ & $17^{*}$ & 174 & 17 & & & \\
\hline $\mathrm{NO}_{3} \cdot$ & 16 & & & 1.0 & 5 & 31 \\
\hline$P$ & 11 & & & $<0.1$ & 9 & 82 \\
\hline $\mathrm{SO}_{4}^{-2}$ & $19^{*}$ & 15700 & 600 & & & \\
\hline $\mathrm{Al}$ & 20 & & & 0.12 & 7 & 35 \\
\hline As & 20 & & & 0.004 & 3 & 15 \\
\hline B & 18 & 1480 & 110 & & & \\
\hline $\mathrm{Ba}$ & 19 & 0.03 & 0.02 & & & \\
\hline $\mathrm{Ca}$ & 20 & 322 & 27 & & & \\
\hline Cs & $11^{*}$ & 0.24 & 0.03 & & & \\
\hline $\mathrm{Fe}$ & 20 & & & $<0.5$ & 15 & 75 \\
\hline$K$ & 20 & 18200 & 800 & & & \\
\hline $\mathrm{Mg}$ & 20 & 17700 & 900 & & & \\
\hline $\mathrm{Mn}$ & 20 & 1.0 & 0.1 & & & \\
\hline $\mathrm{Na}$ & 20 & 87100 & 2000 & & & \\
\hline $\mathrm{Rb}$ & 8 & 14.1 & 0.1 & & & \\
\hline Si & 20 & 2.5 & 0.7 & & & \\
\hline $\mathrm{Sr}$ & 20 & 0.8 & 0.1 & & & \\
\hline
\end{tabular}

"Outtier values omitted in statistical calculations.

$\mathrm{N}=$ Number of samples.

$X=$ Mean.

$S=$ Standard deviation

ND $=$ Not detected. 
these drillholes since the accuracy and precision for the magnesium measurements are similar to the other nonsolubility-constrained parameters.

ANOVA calculations were also performed on the data from drillholes $\mathrm{DH} 36, \mathrm{DH} 38, \mathrm{DH} 42$, and DH42A that sampled the lower stratigraphic units below the repository horizon. ANOVA calculations indicated that geochemical analyses for boron, bromine, and potassium may be grouped together for these drillholes. Again, magnesium analyses for these drillholes did not have significance at the 95 percent confidence level. In addition to the ANOVA calculation, means plots were also produced. Means plots were created using a Tukey's honest significant differences method at a 95 percent confidence level. Means plots showed the mean of each data set as well as the upper and lower 95th confidence interval of each individual population. Means plots for the nonsolubility-limited parameters indicated the two distinct groupings of drillholes mentioned above (Figure 3-2). Means plots for bromine, potassium, and magnesium show the greatest differences between the two groups of drillholes (Figure 3-2).

Because data from drillholes A1X01, A2X01, A3X01, BX01, OH23, OH26, and OH45 for the nonsolubility-limited parameters (boron, bromine, and potassium) comprise a statistically significant population, it is reasonable to assume that data for other parameters in these drillholes can also form a statistically significant population. As mentioned previously, data from drillholes A1X01, A2X01, A3X01, BX01, OH23, OH26, and OH45 are most representative of the repository brine chemistry because these drillholes sample brine encountered in the stratigraphy within and below the repository horizon. Since data from these drillholes can be grouped together, a measure of the central tendency for each parameter can be calculated; however, it was necessary to determine which type of data distribution each parameter possesses. The data was normally distributed for the nonsolubility-limited parameters. This was achieved by again applying the Kolmogorov-Smirnov test for normality to the combined data from each of the drillholes mentioned above. It was then assumed that other parameters were also normally distributed, as long as the data distributions for each drillhole were also normal. With this assumption, a mean and a standard deviation were calculated for each parameter. If the data distributions for individual drillholes were nonparametric, then only a median was calculated. The average representative brine chemistry is given in Table $3-3$. 

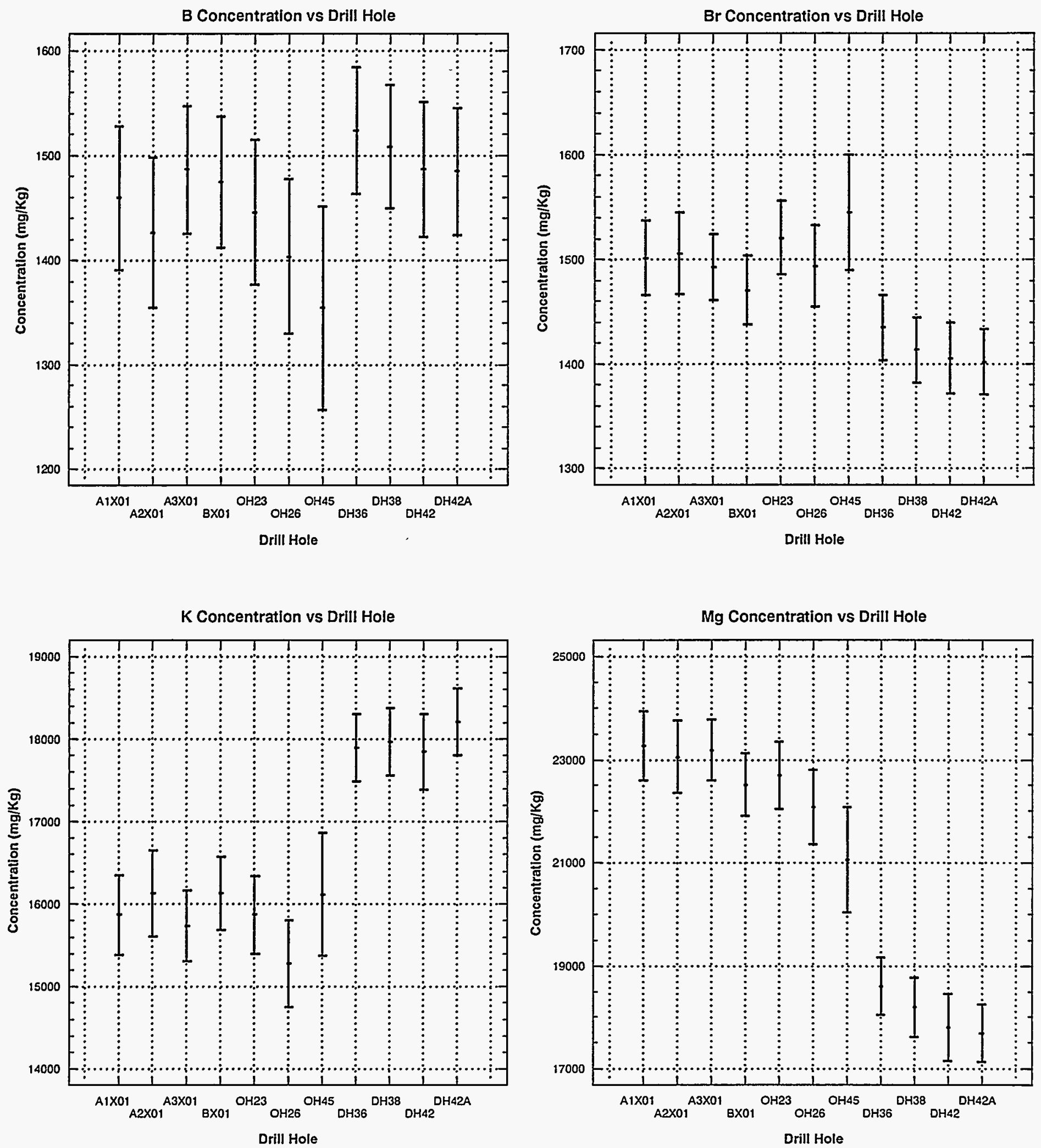

Figure 3-2

Means Plots for BSEP Drill Holes for $\mathrm{B}, \mathrm{Br}, \mathrm{K}$, and $\mathrm{Mg}$ 
Table 3-3

Average Composition of Salado Formation Brine

\begin{tabular}{|l|c|c|c|c|c|c||}
\hline & $\mathrm{N}$ & $\mathrm{X}$ & $\mathrm{S}$ & Median & No. ND & $\%$ ND \\
\hline \hline $\mathrm{SG}$ & 95 & 1.22 & 0.01 & & & \\
\hline $\mathrm{TDS}$ & 90 & 374000 & 13000 & & & \\
\hline $\mathrm{pH}$ & 94 & 6.1 & & & & \\
\hline $\mathrm{ALK}$ & 94 & 883 & 123 & & & \\
\hline $\mathrm{TIC}$ & 96 & & & 4.6 & 3 & 3 \\
\hline $\mathrm{TOC}$ & 84 & 54 & 50 & & & \\
\hline $\mathrm{Br}-$ & 94 & 1500 & 60 & & & \\
\hline $\mathrm{Cl}-$ & 94 & 193000 & 4000 & & & \\
\hline $\mathrm{F}-$ & 95 & 6 & 1 & & & \\
\hline $\mathrm{I}-$ & 93 & 14.8 & 3.1 & & & \\
\hline $\mathrm{NH}{ }^{+}$ & 95 & 148 & 16 & & & \\
\hline $\mathrm{NO}{ }_{3}^{-}$ & 82 & & & 0.8 & 19 & 23 \\
\hline $\mathrm{P}$ & 74 & & & $<0.1$ & 51 & 69 \\
\hline $\mathrm{SO}{ }_{4}^{-2}$ & 95 & 17000 & 900 & & & \\
\hline $\mathrm{Al}$ & 95 & & & 0.10 & 21 & 22 \\
\hline $\mathrm{As}$ & 96 & & & 0.002 & 19 & 20 \\
\hline $\mathrm{B}$ & 95 & 1450 & 120 & & & \\
\hline $\mathrm{Ba}$ & 94 & 0.05 & 0.03 & & & \\
\hline $\mathrm{Ca}$ & 95 & 282 & 38 & & & \\
\hline $\mathrm{Cs}$ & 74 & 0.32 & 0.05 & & & \\
\hline $\mathrm{Fe}$ & 96 & & & $<0.5$ & 61 & 64 \\
\hline $\mathrm{K}$ & 94 & 15900 & 800 & & & \\
\hline $\mathrm{Mg}$ & 95 & 22700 & 1400 & & & \\
\hline $\mathrm{Mn}$ & 94 & 1.6 & 0.3 & & & \\
\hline $\mathrm{Na}$ & 93 & 79100 & 2100 & & & \\
\hline $\mathrm{Rb}$ & 45 & 15.7 & 1.0 & & & \\
\hline $\mathrm{Si}$ & 91 & 1.6 & 0.7 & & & \\
\hline $\mathrm{Sr}$ & 92 & 1.6 & 0.6 & & & \\
\hline \hline & & & & & & \\
\hline
\end{tabular}

$\mathrm{N}=$ Number of samples.

$M=$ Mean

$\mathrm{S}=$ Standard deviation.

$\mathrm{ND}=$ Not detected. 


\subsection{Composition of Non-Salado Brine from the WIPP Underground}

The BSEP Brine Chemistry database also contains data on some non-Salado brines that have been encountered in the WIPP excavations. The most important of these are water from the Culebra Dolomite that has been piped to the repository horizon through temporary drains in the shafts, the brine in the AIS sump, and artificial brine produced commercially (B\&E Artificial Brine).

Previously unreported data are included in Part III of Appendix D and summarized in Table 3-4.

The sample for the Culebra brine was collected on March 3, 1990, at the discharge of the AIS drain on the north side of the AIS station. The Sr concentration was found to be $11 \mathrm{mg} / \mathrm{l}$ in this sample, which is considered a low value for Culebra water. The data collected for the Culebra in the vicinity of the WIPP site by the Water Quality Sampling Program show that there is quite a variation in the Sr values. Data from an individual location (well H-03b3) range between 12 and $30 \mathrm{mg} / \mathrm{l}$ as sampling is repeated (DOE/WIPP 92-007, 1992). Underground brine samples with a relatively high (more than $3 \mathrm{mg} / \mathrm{l}$ ) suggest the possibility of contamination by Culebra water or of partial evaporation of the sample prior to laboratory analysis.

The water from the Culebra is collected in a sump at the AIS, where it dissolved additional salt from the Salado. The AIS sump brine was often used for construction purposes and has been the main source of underground brine contamination. The salinity of the water in the sump varies considerable from time to time, ranging from nearly unaltered Culebra water to a saturated brine. As a result, it is not appropriate to average the analyses for the sump brines, but rather to show great variations in the chemistry as is done in Table 3-4. The only way to determine a mixing curve for any given sample that is suspected of being contaminated would be to have performed an analyses of the actual batch of brine that was spread to cause that contamination. Such analyses was performed in August 1988 and reported in Deal and others (1989), Section 3.1.1.3.

\subsection{Conclusions}

Temporal trends for geochemical data collected as part of the BSEP were determined for uphole A1X02. Simultaneous changes in nonsolubility-constrained parameters indicated that evaporation had occurred in this drillhole. No temporal trends were evident in other 
Table 3-4

Composition of Salado and Nonsalado Brines

\begin{tabular}{|c|c|c|c|c|}
\hline & $\begin{array}{l}\text { WIPP } \\
\text { Repository } \\
\text { Horizon }\end{array}$ & $\begin{array}{c}\text { Culebra } \\
\text { from AIS } \\
\text { Drain }\end{array}$ & Culebra from AIS Sump & $\begin{array}{c}\text { B \& E } \\
\text { Artificial } \\
\text { Brine }\end{array}$ \\
\hline$S G$ & 1.22 & 1.04 & $1.06-1.21$ & 1.22 \\
\hline TDS & 374,000 & 48,000 & $96,000-333,000$ & 324,000 \\
\hline $\mathrm{pH}$ & 6.1 & 8.1 & $7.1-8.2$ & 6.6 \\
\hline ALK & 883 & 113 & $116-177$ & 191 \\
\hline TIC & 4.6 & 73 & $78-113$ & 190 \\
\hline TOC & 54 & 10 & $5-91$ & 31 \\
\hline $\mathrm{Br}-$ & 1,500 & 28 & $35-90$ & 23 \\
\hline $\mathrm{Cl}-$ & 193,000 & 20,600 & $50,700-190,000$ & 187,000 \\
\hline F- & 6 & 1 & $<1-4$ & 3 \\
\hline I- & 14.8 & 0.2 & $<0.1-0.2$ & 1.5 \\
\hline $\mathrm{NH}_{4}^{+}$ & 148 & 0.34 & $0.33-4.5$ & 0.31 \\
\hline $\mathrm{NO}_{3}^{-}$ & 0.8 & 0.1 & $<1-10$ & 4 \\
\hline$P$ & $<0.1$ & $<0.1$ & $<1$ & $<0.3$ \\
\hline $\mathrm{SO}_{4}^{-2}$ & 17,000 & 8,200 & $6,2,00-11,000$ & 3,600 \\
\hline $\mathrm{Al}$ & 0.10 & $<0.05$ & $0.17-2.4$ & $<0.05$ \\
\hline As & 0.002 & $<0.001$ & 0.002 & 0.003 \\
\hline$B$ & 1,450 & 35 & $12-31$ & 2 \\
\hline $\mathrm{Ba}$ & 0.05 & 0.03 & $0.07-2.4$ & 0.1 \\
\hline $\mathrm{Ca}$ & 282 & 822 & $669-989$ & 1,520 \\
\hline $\mathrm{Cs}$ & 0.32 & $\mathrm{NA}$ & $<0.01-0.02$ & 0.02 \\
\hline $\mathrm{Fe}$ & $<0.5$ & 0.5 & $0.1-1.4$ & $<0.5$ \\
\hline K & 15,900 & 376 & $496 \cdot 3,210$ & 11 \\
\hline $\mathrm{Mg}$ & 22,700 & 568 & $629-1,630$ & 43 \\
\hline $\mathrm{Mn}$ & 1.6 & $<0.5$ & $\leq 0.1-0.4$ & $<0.1$ \\
\hline $\mathrm{Na}$ & 79,100 & 15,800 & $32,600-121,000$ & 120,000 \\
\hline $\mathrm{Rb}$ & 15.7 & $\mathrm{NA}$ & $\mathrm{NA}$ & NA \\
\hline $\mathrm{Si}$ & 1.6 & 3.8 & $3.3-8.6$ & 50 \\
\hline $\mathrm{Sr}$ & 1.6 & 11 & $14-33$ & 24 \\
\hline
\end{tabular}


drillholes. Data distributions were assumed for each parameter in each drillhole, duplicate analyses were averaged, outliers were removed, and simple statistics were calculated for each drillhole (Table 3-2).

Data from different drillholes were then grouped together. One group consisted of drillholes A1X01, A2X01, A3X01, BX01, OH23, OH26, and OH45. These drillholes are used to sample brine from stratigraphy located within and below the WIPP repository. A second group consisted of data from drillholes DH36, DH38, DH42, and DH42A. These drillholes are used to sample brine from stratigraphy located beneath the repository. An ANOVA calculation indicated two separate populations for the nonsolubility-limited geochemical parameters. Because brine recovered from drillholes A1X01, A2X01, A3X01, BX01, OH23, $\mathrm{OH} 26$, and $\mathrm{OH} 45$ are more representative of the repository horizon conditions, an average geochemical composition for brine from these drillholes was calculated (Table 3-3). This brine composition was the average representative brine composition for the repository horizon. 


\section{THIS PAGE INTENTIONALLY LEFT BLANK}




\subsection{Hydrologic Testing of the Fractured Part of the Disturbed Rock Zone Beneath the WIPP Excavations}

The main objective of the Hydrologic Testing of the Fractured Part of the Disturbed Rock Zone Beneath the WIPP Excavations Program is to characterize the fracture system beneath the floor of the repository. The data resulting from this program will be used by Waste Isolation Division personnel to develop operational plans for predicting brine and gas movement through the fracture system. Additionally, the data obtained may be useful in refining the design of seals to be used within the repository and in assessing the long-term behavior of flow through the fractured zone.

As salt creeps into the WIPP underground excavations, macrofractures develop in the DRZ beneath the excavations (Bechtel, 1986; also, see review by Deal and Roggenthen, 1991). The fractures tend to concentrate in, but are not limited to, MB 139, which is about $1 \mathrm{~m}$ thick, lying 1 to $2 \mathrm{~m}$ below the floor of most of the WIPP excavations. The developing fracture systems may provide pathways for rapid movement of brine and gas (Deal and Case, 1987; Deal and others, 1989; Deal and others, 1991b) and are considered to be one of the most likely pathways for migration of constituents away from the waste storage panels. The hydrologic characteristics of the fractured zone must be understood to predict and, if necessary, modify the movement of fluids and constituents within MB 139 if a release occurred during operation of the facility.

In 1989, a hydraulic test of short duration was conducted in the DRZ beneath the floor of the intersection of the S90 and W620 drifts (Deal and others, 1991b). The results indicated that drawdown-type pump testing in the underlying fracture system could be performed successfully and could yield useful hydrologic data about the DRZ. After evaluating the results from the preliminary testing effort, a more comprehensive field testing program was developed, and hydraulic testing was implemented at two additional underground test sites.

This section summarizes the results of short-duration hydraulic tests conducted at the two additional sites. The original file report (Crawley and others, 1992) without the test appendices, is edited and presented as Appendix E.

The hydrologic testing areas were selected to evaluate various room and drift dimensions, excavation ages, areas where water was introduced for construction purposes, and areas 
isolated from construction fluids. Three sites were selected for drilling and testing as part of this program because of their age, their physical characteristics, their relationship to other excavations, the existence of fractures, and exposure to long periods of water spread for construction purposes.

- Test Site No. 1 is at the intersection of the S90 and W620 drifts near the AIS. This site consists of 20 test holes drilled at the intersection and along the length of the S90 drift (Appendix E, Figure E-2-2). This test site was not accessible during this field investigation period, but was described in detail by Deal and others (1991a, Section 4).

- Test Site No. 2 is located in the E0 drift in the general area of N620. The site includes nine test holes drilled along the E0 drift (Appendix E, Figure E-2-2).

- Test Site No. 3 is located in the W170 drift immediately in front of the underground core storage room at $\$ 400$. This site consists of 11 test holes drilled along the W170 drift and into the core storage room (Appendix E, Figure E-2-2).

Test results indicate that the significant fracture systems that yield water to test holes are restricted to $\mathrm{MB} 139$. For the two sites tested during this reporting period, there appears to be separate, saturated, unconnected fracture systems of fairly low transmissivity. At the E0 test site, fracture systems that are connected are confined to the immediate intersection of the drift and alcove. For the W170 site, the intersection did not contain significant connected fractures. Based on the observed drawdown response to pumping, the area within the core storage room appeared to be underlain by a somewhat more connected fracture system. This condition could be influenced by the width of the individual excavations. The W170 drift, though much older, has a relatively narrow opening in comparison to the core storage room. These data indicate that excavation dimensions may have a more important role than age in fracture development.

The post-test fluid-level recovery observed at the test sites suggests that the fracture systems beneath these areas are limited, and the available fluid reservoirs are small. Although longterm fluid-level monitoring was not conducted as part of this field program, the data gathered indicate that pumping at these sites was dewatering the fracture systems.

The results of the pumping tests support the concept of limited, bounded fractured fluid reservoir that was developed during the 1989 testing program (Deal and others, 1991a). Data 
analysis from the EO test site showed clear changes in the slope of the plotted drawdown curves for some test holes, indicating the presence of nearby no-flow or low-permeability boundaries. Testing at the W170 site did not produce adequate data for aquifer test analysis.

The Jacob and Theis methods (Lohman, 1972) were used to determine transmissivity and storage coefficients for the first test at the EO site. The calculated transmissivities for all holes were 0.7 to $9.9 \mathrm{ft}^{2} /$ day. Storage coefficients ranged from 0.00038 to 0.0034 , indicating that the fracture system at the EO site is partially confined.

Additional test sites should be developed to better define the nature of fracturing in areas other than the intersections of drifts and rooms. The E0 test site could be expanded to both the north and south of the present site to allow comparative testing. If the test site was expanded, the results of pump testing away from the drift and alcove intersection could be compared to the results produced by this study, and the effects of excavation geometry could be quantified. Additional testing should be conducted at the lowest possible flow rates for the longest time achievable, and fluid-level recovery should be monitored long-term. 


\section{THIS PAGE INTENTIONALLY LEFT BLANK}




\subsection{Numerical Modeling of Brine Seepage as a Result of Clay Compaction}

\subsection{Introduction}

There appears to be enough moisture present in the clays within the Salado Formation to account for all the brine that is observed to seep into the WIPP excavations (Deal and others, 1993, Section 5; Deal and Bills, 1994). The excavation of WIPP rooms result in stress redistribution around those openings that can cause the consolidation of thin clays within the stratigraphic sequence. Additionally, the excavations (including drillholes) provide a sink at atmospheric pressure allowing brine to flow from the consolidating clays.

A series of order-of-magnitude calculations were made for this report (Appendix F) in order to numerically model clay consolidation and estimate the resultant brine seepage into the repository horizon.

\subsection{Modeling Assumptions}

The modeling assumptions are as follows:

- Stress redistribution results in a localized increase in stress that is far more significant in generating excess pore pressure than in near ground surface consolidation. The stress redistribution deforms the clay plastically generating an excess pore pressure of several megapascals (MPa) within the DRZ.

- Transient flow to the excavation or boundary dissipates the excess pore pressure within the clay layer.

- The rate of flow depends on the consolidation properties of the clay (hydraulic conductivity, compressibility, and porosity), the cross sectional area of the clay seams intercepting the excavation, and the extent of the DRZ.

- The tributary method predicts the resulting increase in total stress of $3 \mathrm{MPa}$. Consider that after 1,000 days (Deal and others, 1989, Section 5), the stress abutment zone extends out about 5 excavation diameters. The average diameter for the room is about $3 \mathrm{~m}$.

- The compressibility of the clay is $10^{-7} \mathrm{~Pa}^{-1}$ corresponding to a clay of medium compressibility. The hydraulic conductivity of the clay is $10^{-8} \mathrm{~cm} / \mathrm{s}$. Under a change in effective stress of $3 \mathrm{MPa}$ after consolidation is complete, the change in porosity is 30 percent. 


\subsection{Room $Q$}

For the case of Room $\mathrm{Q}$, the room has a radius of $1.5 \mathrm{~m}$ and a length of $100 \mathrm{~m}$. Two thin clay seams occur, above and below the orange band. Both are about $3.5 \mathrm{~mm}$ thick (Deal and others, 1993, Table 4-3) and are modeled as a single clay $7 \mathrm{~mm}$ thick, centered in the room. In this case flow occurs linearly along the clay seams toward the room. From previous modeling analyses (Deal and others, 1989, Section 5), the stress abutment zone around Room $\mathrm{Q}$ will affect the clay seams out to a distance of about $9 \mathrm{~m}$. No brine was collected from Room $Q$ for the first 800 days (Howarth and others, 1994, Section 4.2.2.3). For this calculation, brine inflow was assumed to have begun as soon as Room $\mathrm{Q}$ was excavated, but because no records of brine volume were made for the first 800 days, the first 800 days of predicted seepage were subtracted from these calculations so that the plot (Appendix $F$, Fig. F-2-2) shows calculated inflow from 800 days to 25 years after excavation. The cumulative inflow 1,600 days after excavation was calculated to be about $300 \mathrm{~L}$, slightly more than the $200 \mathrm{~L}$ that was observed (Howarth and others, 1994, Fig. 2). Calculated inflow rates after 1600 days are on the order of $0.3 \mathrm{~L} /$ day (Appendix F, Fig. F-2-2), close to the observed value of $0.17 \mathrm{~L}$ /day (Howarth and others, 1994, Fig. 3). The calculation shows that seepage ceases after about 25 years (Appendix F, Fig. F-2-2).

\subsection{Standard WIPP Waste Storage Room}

In order to estimate the amount of brine that might come in contact with waste stored at the WIPP after sealing and closure, a similar calculation was made for a standard waste storage room. A waste storage room was approximated as a circular opening $3.6 \mathrm{~m}$ in radius and $91.4 \mathrm{~m}$ long with an abutment zone extending $20 \mathrm{~m}$ into the salt from the wall of the room. Three clay layers are observed in the walls of the rooms, the two clays associated with the orange band that are exposed in Room $Q$ (each about $3.5 \mathrm{~mm}$ thick), and clay $F$, which is about $10 \mathrm{~mm}$ thick (Deal and others, 1993, Table 4-3). For this calculation, the three clays were combined as a single clay $17 \mathrm{~mm}$ thick occurring at the mid-point of the room. This model predicts rapid initial inflow of about $2 \mathrm{~L}$ /day rapidly dropping to less than $0.5 \mathrm{~L} /$ day after about 10 years (Appendix F, Fig. F-3-2). This calculation shows that the pore pressure is completely depleted after about 100 years (Appendix F, Fig. F-3-2) and inflow then ceases. The total inflow would be about $9,000 \mathrm{~L}$, much of which would be evaporated during excavation and emplacement of waste into the air circulated for ventilation. 


\subsection{Axial Consolidation Around a Borehole}

Brine seepage occurs into drillholes drilled vertically downward from WIPP excavations. This calculation was performed to estimate inflow into $15 \mathrm{~m}$-deep downholes drilled from Room G. The vertical drillhole has a radius of $8.9 \mathrm{~cm}$ and intersects the clay B layer about $10 \mathrm{~m}$ below the floor of the room. Clay B is about $1 \mathrm{~cm}$ thick (Deal and others, 1991b, Section 2.7.3.2). Stress redistribution around Room $G$ will result in compaction of clay B for a distance of about $20 \mathrm{~m}$ from the borehole. Brine flow is radially to the borehole along the thin clay seam. As a result, complete compaction will take a fairly long time, over 1,000 years, and would ultimately yield about $340 \mathrm{~L}$ of brine. Over a period of 60 to 100 years, approximately 100 to $150 \mathrm{~L}$ of brine will seep into the borehole (Appendix F, Fig. F-4-2). After about 10 years, inflow rate is calculated to be about $.006 \mathrm{~L} / \mathrm{day}$, an order of magnitude lower than the observed inflow below Room G (Table 5-1). The only other

\section{Table 5-1}

Seepage Rate in Drillholes Penetrating Clay B

\begin{tabular}{|c|c|c||}
\hline Drillhole & Location & $\begin{array}{c}\text { Seepage Rate } \\
\text { (L/day) }\end{array}$ \\
\hline \hline $\mathrm{DH} 36$ & Room G & 0.1 \\
\hline $\mathrm{DH} 38$ & Room G & 0.03 \\
\hline $\mathrm{DH} 40$ & Room G & 0.008 \\
\hline $\mathrm{DH} 42$ & Room G & 0.01 \\
\hline $\mathrm{DH} 42 \mathrm{~A}$ & Room G & 0.02 \\
\hline $\mathrm{OH} 46$ & S390/W320 & 0.005 \\
\hline
\end{tabular}

drillhole that penetrates the same stratigraphy and is probably not contaminated with construction brines is $\mathrm{OH} 46$, which is drilled from the underground core storage area. Consolidation response should be about the same for OH46 as for the holes in Room G.

All of the drillholes listed in Table 5-1 also intersect clay E and clay D, which are potential sources for additional brine. Clay $\mathrm{D}$ is thin and discontinuous and was not considered in the above calculation. The intersection with clay $\mathrm{E}$ can be observed from the drillhole collars and is not providing brine to the downholes in Room G. 


\subsection{Summary}

These order-of-magnitude seepage calculations compare well with the observed seepage into Room Q. Calculated seepage rate after 1,600 days is on the order of $0.3 \mathrm{~L} / \mathrm{day}$, where the actual observed rate is $0.17 \mathrm{~L} /$ day. In this case the model is for flow towards the room along a thin clay seam. Extending this model to a waste storage room predicts that the total seepage into the room will be on the order of 9,000 liters, far short of the $220,000 \mathrm{~L}$ necessary to react anoxically with all the susceptible metal placed in the room (Deal and others, 1991b, Section 4.6). Furthermore, seepage into the room will cease after about 100 years.

The case for seepage into a downhole drilled into the strata below an excavation behaves differently, as flow is radially toward the drillhole. In this case, some seepage continues for a long time, perhaps a thousand years or more. It is clear that seepage into drillholes is strikingly different from seepage into a repository excavation. Deal and others (1994, Section 2.7.2) pointed out that seepage into drillholes probably should not be used to predict longterm seepage into a WIPP waste storage room. This calculation provides additional support for this caution. 


\subsection{Summary and Conclusions}

During eleven years of observations (1982 to 1993) the amount of brine seeping into the WIPP excavations is local, limited, and finite. Even a small amount of brine may produce hydrogen gas by anoxic corrosion of the metal in the CH-TRU waste drums and waste inventory. However, the amount of brine that will be available will be only a small percentage of that necessary to corrode all of the metal. The data through 1990 are discussed in detail by Deal and others (1991b). It was concluded that it will take on the order of $220,000 \mathrm{~L}$ of brine to corrode all the susceptible metal (iron and aluminum) and that there is probably less than 10 percent available $(20,000 \mathrm{~L})$, unless it can be proven that far-field flow does occur at the WIPP. Far-field flow is theoretically unlikely or impossible (Deal and Roggenthen, 1991), and evidence so far confirms that significant seepage of brine ceases about three years after the excavation of an opening, although small seeps can continue for a longer period of time (Deal and others, 1993, Section 5; Deal and Bills, 1994). Calculations presented in Chapter 5 of this report indicate that less than $9,000 \mathrm{~L}$ will be available from clay consolidation.

Data gathered in 1992 and 1993 additionally support those conclusions. Continued observations of downholes and Salt Shaft and Waste Shaft sumps where fractured MB 139 can be observed confirm that the exposed surfaces are still dry and show very little evidence of moisture. Inspection of the AIS showed that there was little evidence of moisture or past seepage. Salt encrustations are more common below a depth of $1,500 \mathrm{ft}$, are clearly stratigraphically controlled, and are associated with clay interbeds and argillaceous halite. Anhydrite exposures are typically dry and free of salt encrustations, indicating that no significant amount of brine flows through them to the shaft.

Both the shaft sumps and the AIS are, in effect, long-term far-field flow experiments. There is no evidence confirming that enough flow exists to supply the needed volume of brine for complete anoxic corrosion of the susceptible metal waste and waste containers that will be emplaced at the WIPP.

Hydrologic testing was performed during this reporting period at two additional areas in order to obtain data on the hydrologic properties of the fractured part of the DRZ that has formed beneath the WIPP excavations. The test results confirmed that the width of an excavation 
influences the development of integrated fractures and showed that, in the tested areas in the EO drift and near the AIS, integrated fracture systems only exist beneath intersections. This supports the concept of limited, bounded, fractured fluid reservoirs. Additional evidence that extensive, large-scale hydrologically interconnected fracture system apparently do not exist under much of the WIPP excavation is supplied by the fact that brine stands at different levels in closely spaced drillholes in the floor and that brine is not seeping out of fractures observed in the Salt Shaft and Waste Shaft sumps.

Long-term observations of the salt encrustations (Deal and others, 1993, Section 2.2) confirm and semiquantify that the brine weeps cease about three years (1,000 days) after excavation. Calculations estimate total seepage into a full-sized waste storage room from wall weeps between 43 and $604 \mathrm{~L}$, with an average of less than $300 \mathrm{~L}$ (Deal and others, 1993; Table 2-4 and Figure 2-14), much less than 1 percent of the $220,000 \mathrm{~L}$ of brine needed to corrode all the susceptible metal in the CH-TRU waste and waste storage drums.

Previous efforts to calculate the amount of moisture that might be released to the repository by clay consolidation (Deal and others, 1993, Section 4) to a full-sized waste storage room was on the order of $400 \mathrm{~L}$ of brine. In order to provide a somewhat more rigorous estimate, numerical calculations were performed for this report in order to provide order-of-magnitude estimates of brine seepage that might result from clay compaction. The calculations compare well with the observed seepage into Room Q. Calculated seepage rate after 1,600 days is on the order of $0.3 \mathrm{~L} /$ day, where the actual observed rate is $0.17 \mathrm{~L} /$ day. In this case the model is for flow towards the room along a thin clay seam. Extending this model to a waste storage room predicts that the total seepage into the room will be on the order of $9,000 \mathrm{~L}$, much of which will evaporate during operations. Furthermore, seepage into the room will cease after about 100 years.

The case for seepage into a downhole drilled into the strata below a WIPP excavation behaves differently, as flow is radially toward the drillhole. In this case, some seepage continues for a long time, perhaps a thousand years or more. It is clear that seepage into drillholes is strikingly different from seepage into a repository excavation. Deal and others (1994, Section 2.7.2) pointed out that seepage into drillholes probably should not be used to predict long term seepage into a WIPP waste storage room. This calculation provides additional support for this caution. 
Although there is no observed evidence from the WIPP excavations that brine will seep into the workings from the underlying anhydrite MB 139 (Deal and Bills, 1994), Deal and others (1994) calculated that even if far-field flow occurred in the anhydrite, only about $6,000 \mathrm{~L}$ could flow into a WIPP storage room over a 200-year period of time. They point out that due to evaporation during the period of time the excavations are open for waste storage, and because creep closure will repressurize the room even in the absence of gas generation, a more realistic figure may be on the order of $1,700 \mathrm{~L}$.

All of these estimates and calculations are far short of the $220,000 \mathrm{~L}$ required to corrode all of the metal and cause maximum gas generation by anoxic corrosion. 


\section{THIS PAGE INTENTIONALLY LEFT BLANK}




\subsection{References}

Abitz, R. J., J. Myers, P. E. Drez, and D. E. Deal, 1990, "Geochemistry of Salado Formation Brines Recovered from the Waste Isolation Pilot Plant (WIPP) Repository," Proceedings of Waste Management '90, Waste Processing, Transportation, Storage and Disposal, Technical Programs and Public Education, R. G. Post, ed., Tucson, Arizona, Vol. 2, pp. 881-891.

American Society for Testing and Materials (ASTM), 1980, "Recommended Practice for Dealing with Outlying Observations," Procedure E178-80, American Society for Testing and Materials, Philadelphia, Pennsylvania.

ASTM, see American Society for Testing and Materials.

Bechtel National, Inc. (Bechtel), 1986, "Waste Isolation Pilot Plant Design Validation Final Report," DOE-WIPP 86-010, prepared for the U.S. Department of Energy by Bechtel National, Inc., San Francisco, California.

Bechtel National, Inc. (Bechtel), 1983, "Waste Isolation Pilot Plant Preliminary Design Validation Report," prepared for the U.S. Department of Energy by Bechtel National, Inc., San Francisco, California.

Black, S. R., R. S. Newton, and D. K. Shukla, eds., 1983, "Results of Site Validation Experiments, Waste Isolation Pilot Plant," DOE-TME-3177, TSC-D'Appolonia Consulting Engineers, Albuquerque, New Mexico.

Crawley, M. E., T. W. Cooper, R. G. Richardson, 1992, "Hydrologic Testing of the Fractured Part of the Disturbed Rock Zone Beneath the WIPP Excavations," file report prepared for the U.S. Department of Energy by IT Corporation and Westinghouse Electric Corporation, Carlsbad, New Mexico.

Deal, D. E., and J. B. Case, 1987, "Brine Sampling and Evaluation Program, Phase I Report," DOE-WIPP 87-008, prepared for the U.S. Department of Energy by IT Corporation and Westinghouse Electric Corporation, Carlsbad, New Mexico, 163 pp.

Deal, D. E., and R. A. Bills, 1994, "Conclusions After Eleven Years of Studying Brine at the Waste Isolation Pilot Plant," Waste Management '94, Tucson, Arizona, March 2, 1994, IT Corporation, Albuquerque, New Mexico, and U.S. Department of Energy, Carlsbad, New Mexico.

Deal, D. E., and R. M. Roggenthen, 1991, "Evolution of Hydrologic Systems and Brine Geochemistry in a Deforming Salt Medium: Data from WIPP Brine Seeps," Waste Management '91, Waste Processing, Transportation, Storage and Disposal, Technical Programs and Public Education, R. G. Post, ed., Vol. 2, pp. 507-516. 
Deal, D. E., R. H. Holt, J. M. Melvin, and S. M. Djordevic, 1994, "Calculation of Brine Seepage from Anhydrite Marker Bed 139 into a Waste Storage Room at the Waste Isolation Pilot Plant," DOE-WIPP 94-007, Westinghouse Electric Corporation, Carlsbad, New Mexico.

Deal, D. E., R. J. Abitz, D. S. Belski, J. B. Case, M. E. Crawley, R. M. Deshler, P. E. Drez, C. A. Givens, R. B King, B. A. Lauctes, J. Myers, S. Niou, J. M. Pietz, W. M. Roggenthen, J. R. Tyburski, and M. G. Wallace, 1989, "Brine Sampling and Evaluation Program Report, 1988," DOE-WIPP 89-015, prepared for the U.S. Department of Energy by IT Corporation and Westinghouse Electric Corporation, Carlsbad, New Mexico.

Deal, D. E., R. J. Abitz, D. S. Belski, J. B. Clark, M. E. Crawley, and M. L. Martin, 1991a, "Brine Sampling and Evaluation Program Report, 1989," DOE-WIPP 91-009, prepared for U.S. Department of Energy by IT Corporation and Westinghouse Electric Corporation, Carlsbad, New Mexico.

Deal, D. E., R. J. Abitz, J. Myers, D. S. Belski, M. L. Martin, D. J. Milligan, R. W. Sobocinski, and P. P. James Lipponer, 1993, "Brine Sampling and Evaluation Program Report 1991," DOE-WIPP 93-026, prepared for U.S. Department of Energy by IT Corporation and Westinghouse Electric Corporation, Carlsbad, New Mexico.

Deal, D. E., R. J. Abitz, J. Myers, J. B. Case, D. S. Belski, M. L. Martin, W. M. Roggenthen, 1991b, "Brine Sampling and Evaluation Program Report, 1990," DOE-WIPP 91-036, prepared for U.S. Department of Energy by IT Corporation and Westinghouse Electric Corporation, Carlsbad, New Mexico.

EPA, see U.S. Environmental Protection Agency.

Howarth, S., K. Larson, T. Christian-Frear, R. Beauheim, D. Borns, D. Deal, A. L. Jensen, K. Pickens, R. Roberts, M. Tierney, P. Vaughn, and S. Webb, 1994, "Salado Formation Fluid Flow and Transport Containment Group-White Paper for Systems Prioritization and Technical Baseline, Rev. 1," prepared by Sandia National Laboratories/New Mexico for the U.S. Department of Energy, Carlsbad, New Mexico.

Kennedy and Neville, 1986, (ref) (II 3.5)

Krumhansl, J. L., and H. W. Stockman, 1987, Memorandum to M. A. Molecke, Sandia National Laboratories, New Mexico ,"Test Progress Report-Room J."

Krumhansl, J. L., K. M. Kimball, and C. L. Stein, 1991, "Intergranular Fluid Compositions from the Waste Isolation Pilot Plant (WIPP), Southeastern New Mexico," SAND90-0584, Sandia National Laboratories, New Mexico.

Lohman, S. W., 1972, "Ground-Water Hydraulics," U.S. Geological Survey Professional Paper 708, U.S. Government Printing Office, 70 pp. 
Morse, J. G., and B. W. Hassinger, April, 1985, "Brine Testing Program Plan: Waste Isolation Pilot Plant (WIPP) Project, Carlsbad, New Mexico, Revision 2," WD:85:01214, internal document transmitted as a letter from W. R. Cooper to R. H. Neil, Waste Isolation Pilot Plant, AEH 85:086.

Powers, D. W., S. J. Lambert, S. E. Shaffer, L. R. Hill, and W. D. Weart, eds., 1978, "Geological Characterization Report, Waste Isolation Pilot Plant (WIPP) Site, Southeastern New Mexico," SAND78-1596, Vols. I and II, Sandia National Laboratories, Albuquerque, New Mexico.

SNL/NM, see Sandia National Laboratories/New Mexico.

Stein, C. L., and J. L. Krumhansl, 1988, "A Model for the Evolution of Brines in Salt from the Lower Salado Formation, Southeastern New Mexico," Geochimica et Cosmochimica Acta, Vol. 52, pp. 1037-1046.

Stein, C. L., and J. L. Krumhansl, 1986, "Chemistry of Brines in Salt From the Waste Isolation Pilot Plant (WIPP), Southeastern New Mexico: A Preliminary Investigation," SAND85-0897, Sandia National Laboratories, New Mexico.

U.S. Environmental Protection Agency (EPA), 1989, (supply elements) I3.6 530-SW-89-026.

DOE/WIPP 92-007, 1992, Waste Isolation Pilot Plant Site Environmental Report for Calendar Year 1991, prepared for the U. S. Department of Energy by Westinghouse Electric Corporation and IT Corporation, Carlsbad, New Mexico. 


\section{THIS PAGE INTENTIONALLY LEFT BLANK}




\section{APPENDIX A \\ BRINE ACCUMULATION}

PART I-LIST OF UNDERGROUND LOCATIONS WHERE BRINE OCCURRENCES WERE OBSERVED AND MONITORED

PART II—BRINE ACCUMULATION DATA TABLES 


\section{THIS PAGE INTENTIONALLY LEFT BLANK}




\section{APPENDIX A BRINE ACCUMULATION}

PART I-LIST OF UNDERGROUND LOCATIONS WHERE BRINE
OCCURRENCES WERE OBSERVED AND MONITORED 


\section{THIS PAGE INTENTIONALLY LEFT BLANK}


Table A-1

List of Underground Locations Where Brine Occurrences

Were Observed and Monitored Through December, 1993

\section{As Part of the Brine Sampling and Evaluation Program at WIPP}

\begin{tabular}{|c|c|c|c|c|c|c|c|c|c|c|c|}
\hline $\begin{array}{l}\text { Hole } \\
\text { Number }\end{array}$ & $\begin{array}{l}\text { Room } \\
\text { or } \\
\text { Location }\end{array}$ & $\begin{array}{c}\text { Survey } \\
\text { Accuracy } \\
\text { S=Surveyed } \\
A=\text { Approximate }\end{array}$ & $\begin{array}{l}\text { North-South } \\
\text { Coordinates* }\end{array}$ & $\begin{array}{l}\text { East-West } \\
\text { Coordinates* }\end{array}$ & $\begin{array}{l}\text { Elevation } \\
\mathrm{m}\end{array}$ & $\begin{array}{l}\text { Dia. } \\
\text { cm }\end{array}$ & $\begin{array}{l}\text { Length } \\
\text { m }\end{array}$ & $\begin{array}{l}\text { Direction } \\
\begin{array}{l}\text { U=Up } \\
D=\text { Down } \\
H=\text { Horiz. }\end{array}\end{array}$ & $\begin{array}{l}\text { Angle } \\
\text { in } \\
\text { Degrees }\end{array}$ & References** & Remarks \\
\hline A1X01 & A1 & S & N1147.02 & E1254.40 & 400.28 & 10 & 15.2 & D & 90 & $B, D, E$ & Monitored as part of the BSEP from $3 / 85$ to $2 / 91$. \\
\hline $\mathrm{A} 1 \times 02$ & Al & $\mathrm{s}$ & N1146.88 & E1254.24 & 405.78 & 10 & 18 & $\mathrm{U}$ & 90 & $B, D, E$ & $\begin{array}{l}\text { Monitored as part of the BSEP since it was drilled in } \\
3 / 85, \text { to } 8 / 93 \text { when collecting device malfunctioned } \\
\text { and became erratic. }\end{array}$ \\
\hline A2X01 & A2 & s & N1393.72 & E1338.88 & 399.65 & 10 & 15.3 & D & 90 & $B, D, E$ & Monitored as part of the BSEP from 2/85 to $10 / 90$. \\
\hline $\mathrm{A} 2 \times 02$ & A2 & S & N1393.65 & E1338.89 & 405.03 & 10 & 16.1 & U & 90 & $B, D, E$ & Monitored as part of the BSEP from $2 / 85$ to $9 / 89$. \\
\hline $\mathrm{A} 3 \times 01$ & A3 & $s$ & N1137.94 & E1406.84 & 399.22 & 10 & 15.4 & D & 90 & $B, D, E$ & $\begin{array}{l}\text { Monitored as part of the BSEP from when it was } \\
\text { drilled in } 1 / 85 \text { to } 6 / 93 \text {. Drillers did not report any } \\
\text { moisture while drilling. Hole started producing brine } \\
\text { a few weeks later. }\end{array}$ \\
\hline $\mathrm{A} 3 \times 02$ & $A 3$ & $\mathrm{~s}$ & $N 1138.00$ & E1406.89 & 404.75 & 10 & 15.5 & $U$ & 90 & $B, D, E$ & $\begin{array}{l}\text { Monitored from } 1 / 85 \text { to } 9 / 89 \text {. Drillers did not } \\
\text { encounter moisture while drilling. Hole started } \\
\text { producing brine a few weeks later. }\end{array}$ \\
\hline BTPA1 & $S 1620 / W 170$ & A & $\mathrm{S} 1638$ & $W 162$ & 384 & 7.6 & 1.6 & D & 90 & B & $\begin{array}{l}\text { Open from } 0 \text { to } 1.6 \mathrm{~m} \text {. Drilled for the BSEP study } \\
7 / 86 \text { and monitored until } 12 / 02 / 88 \text {. }\end{array}$ \\
\hline BTPA2 & $S 1620 W 170$ & A & $S 1638$ & W166 & 384 & 7.6 & 2.8 & $D$ & 90 & B & $\begin{array}{l}\text { Cased from } 0 \text { to } 1.6 \mathrm{~m} \text {. Open from } 1.6 \text { to } 2.8 \mathrm{~m} \text {. } \\
\text { Drilled for the BSEP study } 7 / 86 \text { and monitored until } \\
12 / 02 / 88 \text {. }\end{array}$ \\
\hline ВТРАЗ & $S 1620 \mathrm{~W} 170$ & $A$ & S1638 & $W 170$ & 384 & 7.6 & 4.1 & $D$ & 90 & B & $\begin{array}{l}\text { Cased from } 0 \text { to } 3.1 \mathrm{~m} \text {. Open from } 3.1 \text { to } 4.1 \mathrm{~m} \text {. } \\
\text { Drilled for the BSEP study } 7 / 86 \text { and monitored until } \\
12 / 02 / 88 \text {. }\end{array}$ \\
\hline BTPA4 & $S 1620 W 170$ & $A$ & S1638 & $W 166$ & 388 & 7.6 & 1.4 & $U$ & 90 & B & $\begin{array}{l}\text { Open from } 0 \text { to } 1.4 \mathrm{~m} \text {. Drilled for the BSEP study } \\
7 / 86 \text { and monitored until } 9 / 27 / 88 \text {. Dry. }\end{array}$ \\
\hline BTPA5 & $S 1620 W 170$ & $A$ & S1638 & W170 & 388 & 7.6 & 1.6 & U & 90 & $\mathrm{~B}$ & $\begin{array}{l}\text { Open from } 0 \text { to } 1.6 \mathrm{~m} \text {. Drilled for the BSEP study } \\
7 / 86 \text { and monitored until } 9 / 27 / 88 \text {. Dry. }\end{array}$ \\
\hline
\end{tabular}

"The repository is referenced in feet; therefore, the North-South and East-West coordinates are presented in feet.

**For references, see footnote at end of table. 
Table A-1 (Continued)

List of Underground Locations Where Brine Occurrences

Were Observed and Monitored Through December, 1993

As Part of the Brine Sampling and Evaluation Program at WIPP

\begin{tabular}{|c|c|c|c|c|c|c|c|c|c|c|c|}
\hline $\begin{array}{c}\text { Hole } \\
\text { Number }\end{array}$ & $\begin{array}{l}\text { Room } \\
\text { or } \\
\text { Location }\end{array}$ & $\begin{array}{c}\text { Survey } \\
\text { Accuracy } \\
\text { S=Surveyed } \\
\text { A=Approximate }\end{array}$ & $\begin{array}{l}\text { North-South } \\
\text { Coordinates" }\end{array}$ & $\begin{array}{c}\text { East-West } \\
\text { Coordinates* }\end{array}$ & $\begin{array}{c}\text { Elevation } \\
\mathrm{m}\end{array}$ & $\begin{array}{l}\text { Dia. } \\
\mathrm{cm}\end{array}$ & $\begin{array}{c}\text { Length } \\
\text { m }\end{array}$ & $\begin{array}{l}\text { Direction } \\
\text { U=Up } \\
\text { D=Down } \\
H=\text { Horiz. }\end{array}$ & $\begin{array}{l}\text { Angle } \\
\text { in } \\
\text { Degrees }\end{array}$ & References"** & Remarks \\
\hline BTPB1 & $S 1620 / W 170$ & $A$ & S1636 & W162 & 384 & 7.6 & 1.6 & $D$ & 90 & B & $\begin{array}{l}\text { Open from } 0 \text { to } 1.6 \mathrm{~m} \text {. Drilled for the BSEP study } \\
7 / 86 \text { and monitored until } 9 / 27 / 88 \text {. }\end{array}$ \\
\hline BTPB2 & $S 1620 N 170$ & A & S1636 & W166 & 384 & 7.6 & 2.9 & D & 90 & B & $\begin{array}{l}\text { Cased } 0 \text { to } 1.8 \mathrm{~m} \text {. Open from } 1.8 \text { to } 2.9 \mathrm{~m} \text {. Drilled } \\
\text { for the BSEP study } 7 / 86 \text { and monitored until } 9 / 27 / 88 \text {. }\end{array}$ \\
\hline ВTPB3 & $S 1620 / W 170$ & A & $\mathrm{S} 1636$ & W170 & 384 & 7.6 & 4.1 & D & 90 & B & $\begin{array}{l}\text { Cased } 0 \text { to } 3.1 \mathrm{~m} \text {. Open from } 3.0 \text { to } 4.1 \mathrm{~m} \text {. Drilled } \\
\text { for the BSEP study } 7 / 86 \text { and monitored until } 9 / 27 / 88 \text {. }\end{array}$ \\
\hline BTPB4 & S1620N170 & A & $S 1636$ & W166 & 388 & 7.6 & 3.0 & $U$ & 90 & B & $\begin{array}{l}\text { Cased } 0 \text { to } 2.1 \mathrm{~m} \text {. Open from } 2.1 \text { to } 3.0 \mathrm{~m} \text {. Drilled } \\
\text { for the BSEP study } 7 / 86 \text { and monitored until } 9 / 27 / 88 \text {. }\end{array}$ \\
\hline BTPB5 & $\mathrm{S} 1620 \mathrm{~W} 170$ & A & S1636 & W170 & 388 & 7.6 & 3.1 & $U$ & 90 & B & $\begin{array}{l}\text { Cased } 0 \text { to } 1.9 \mathrm{~m} \text {. Open from } 1.9 \text { to } 3.1 \mathrm{~m} \text {. Drilled } \\
\text { for the BSEP study } 7 / 86 \text { and monitored until } 9 / 27 / 88 \text {. }\end{array}$ \\
\hline BTPC1 & $S 1620 M 170$ & A & $\mathrm{S} 1634$ & W162 & 384 & 7.6 & 1.5 & D & 90 & B & $\begin{array}{l}\text { Open from } 0 \text { to } 1.5 \mathrm{~m} \text {. Drilled for the BSEP study } \\
7 / 86 \text { and monitored until } 9 / 27 / 88 \text {. }\end{array}$ \\
\hline BTPC2 & $S 1620 N 170$ & A & S1634 & W166 & 384 & 7.6 & 3.0 & D & 90 & B & $\begin{array}{l}\text { Cased from } 0 \text { to } 1.7 \mathrm{~m} \text {. Open from } 1.8 \text { to } 3.0 \mathrm{~m} \text {. } \\
\text { Drilled for the BSEP study } 8 / 86 \text { and monitored until } \\
9 / 27 / 88 \text {. }\end{array}$ \\
\hline BTPC3 & $S 1620 / W 170$ & A & S1634 & W170 & 384 & 7.6 & 4.4 & $\mathrm{D}$ & 90 & B & $\begin{array}{l}\text { Cased from } 0 \text { to } 3.0 \mathrm{~m} \text {. Open from } 3.0 \text { to } 4.4 \mathrm{~m} \text {. } \\
\text { Drilled for the BSEP study } 8 / 86 \text { and monitored until } \\
9 / 27 / 88 \text {. }\end{array}$ \\
\hline BTPC4 & $S 1620 / W 170$ & A & S1634 & W166 & 388 & 7.6 & 5.4 & $\mathbf{U}$ & 90 & B & $\begin{array}{l}\text { Cased from } 0 \text { to } 4.2 \mathrm{~m} \text {. Open from } 4.2 \text { to } 5.4 \mathrm{~m} \text {. } \\
\text { Drilled for the BSEP study } 7 / 86 \text { and monitored until } \\
\text { 9/27/88. }\end{array}$ \\
\hline BTPC5 & S1620/W170 & A & 51634 & W170 & 388 & 7.6 & 5.5 & $U$ & 90 & B & $\begin{array}{l}\text { Cased from } 0 \text { to } 4.3 \mathrm{~m} \text {. Open from } 4.3 \text { to } 5.5 \mathrm{~m} \text {. } \\
\text { Drilled for the BSEP study } 7 / 86 \text { and monitored until } \\
9 / 27 / 88 \text {. Dry. }\end{array}$ \\
\hline
\end{tabular}

-The repository is referenced in feet; therefore, the North-South and East-West coordinates are presented in feet.

"For references, see footnote at end of table. 
Table A-1 (Continued)

List of Underground Locations Where Brine Occurrences

Were Observed and Monitored Through December, 1993

As Part of the Brine Sampling and Evaluation Program at WIPP

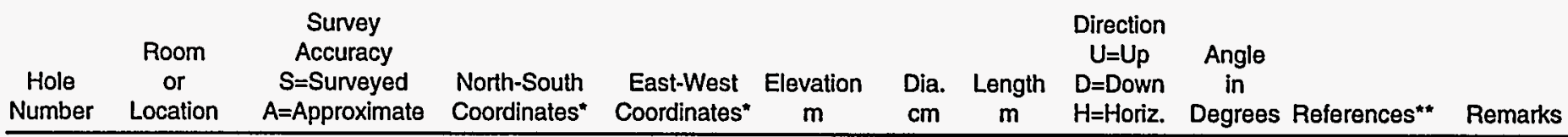

\begin{tabular}{|c|c|c|c|c|c|c|c|c|c|c|c|}
\hline BTR1 & S1950/E100 & A & S1942 & E98 & 387 & 8.3 & 0.3 & $H$ & 5 & B & $\begin{array}{l}\text { Hole slightly declined below horizontal. Collar above } \\
\text { upper clay seam, about } 0.3 \mathrm{~m} \text { below back. Drilled } \\
6 / 86 \text { and monitored until } 9 / 27 / 88 \text {. Dry. }\end{array}$ \\
\hline BTR2 & S1950/E100 & A & S1942 & $\mathrm{E} 100$ & 387 & 8.3 & 1.0 & $\mathrm{H}$ & 5 & B & $\begin{array}{l}\text { Hole slightly declined below horizontal. Collar above } \\
\text { upper clay seam, about } 0.3 \mathrm{~m} \text { below back. Drilled } \\
6 / 86 \text { and monitored until } 12 / 02 / 88 \text {. }\end{array}$ \\
\hline ВТАЗ & S1950/E100 & A & S1942 & E101 & 387 & 8.3 & 1.0 & $\mathrm{H}$ & 5 & B & $\begin{array}{l}\text { Hole slightly declined below horizontal. Collar above } \\
\text { upper clay seam, about } 0.3 \mathrm{~m} \text { below back. Drilled } \\
6 / 86 \text { and monitored until } 12 / 02 / 88 \text {. }\end{array}$ \\
\hline BTA4 & S1950/E100 & A & S1942 & E98 & 386 & 8.3 & 0.3 & $H$ & 5 & B & $\begin{array}{l}\text { Hole slightly declined below horizontal. Collar in } \\
\text { halite about } 1.1 \mathrm{~m} \text { below back. Drilled } 6 / 86 \text { and } \\
\text { monitored until } 12 / 02 / 88 \text {. }\end{array}$ \\
\hline BTR5 & S1950/E100 & A & $\mathrm{S} 1942$ & E100 & 386 & 8.3 & 0.9 & $H$ & 5 & B & $\begin{array}{l}\text { Hole slightly declined below horizontal. Collar in } \\
\text { halite about } 1.1 \mathrm{~m} \text { below back. Drilled } 6 / 86 \text { and } \\
\text { monitored until } 12 / 02 / 88 \text {. }\end{array}$ \\
\hline BTR6 & S1950/E100 & A & S1942 & E101 & 386 & 8.3 & 0.9 & $H$ & 5 & B & $\begin{array}{l}\text { Hole slightly declined below horizontal. Collar in } \\
\text { halite about } 1.1 \mathrm{~m} \text { below back. Drilled } 6 / 86 \text { and } \\
\text { monitored until } 12 / 02 / 88 \text {. }\end{array}$ \\
\hline BTR7 & S1950/E100 & A & S1942 & E98 & 386 & 8.3 & 0.3 & $\mathrm{H}$ & 5 & B & $\begin{array}{l}\text { Hole slightly declined below horizontal. Collar just } \\
\text { above orange band. Drilled } 6 / 86 \text { and monitored until } \\
12 / 02 / 88 \text {. Dry. }\end{array}$ \\
\hline BTR8 & S1950/E100 & A & 51942 & E100 & 386 & 8.3 & 0.9 & $H$ & 5 & B & $\begin{array}{l}\text { Hole slightly declined below horizontal. Collar just } \\
\text { above orange band. Drilled } 6 / 86 \text { and monitored until } \\
12 / 02 / 88 \text {. }\end{array}$ \\
\hline BTR9 & S1950/E100 & A & S1942 & E101 & 386 & 8.3 & 0.9 & $\mathrm{H}$ & 5 & B & $\begin{array}{l}\text { Hole slightly declined below horizontal. Collar just } \\
\text { above orange band. Drilled } 6 / 86 \text { and monitored until } \\
12 / 02 / 88 \text {. }\end{array}$ \\
\hline
\end{tabular}

"The repository is referenced in feet; therefore, the North-South and East-West coordinates are presented in feet.

"For references, see footnote at end of table. 
Table A-1 (Continued)

List of Underground Locations Where Brine Occurrences

Were Observed and Monitored Through December, 1993

\section{As Part of the Brine Sampling and Evaluation Program at WIPP}

\begin{tabular}{|c|c|c|c|c|c|c|c|c|c|c|c|}
\hline $\begin{array}{l}\text { Hole } \\
\text { Number }\end{array}$ & $\begin{array}{c}\text { Room } \\
\text { or } \\
\text { Location }\end{array}$ & $\begin{array}{c}\text { Survey } \\
\text { Accuracy } \\
\text { S=Surveyed } \\
A=\text { Approximate }\end{array}$ & $\begin{array}{l}\text { North-South } \\
\text { Coordinates }\end{array}$ & $\begin{array}{l}\text { East-West } \\
\text { Coordinates }\end{array}$ & $\begin{array}{l}\text { Elevation } \\
\mathrm{m}\end{array}$ & $\begin{array}{l}\text { Dia. } \\
\mathrm{cm}\end{array}$ & $\begin{array}{c}\text { Length } \\
\mathrm{m}\end{array}$ & $\begin{array}{l}\text { Direction } \\
U=\text { Up } \\
D=\text { Down } \\
H=\text { Horiz. }\end{array}$ & $\begin{array}{l}\text { Angle } \\
\text { in } \\
\text { Degrees }\end{array}$ & References** & Remarks \\
\hline BTR10 & S1950/E100 & A & S1942 & E98 & 385 & 8.3 & 0.4 & $H$ & 5 & B & $\begin{array}{l}\text { Hole slightly declined below horizontal. Collar about } \\
0.8 \mathrm{~m} \text { above floor. Drilled } 6 / 86 \text { and monitored until } \\
12 / 02 / 88 \text {. Dry. }\end{array}$ \\
\hline BTR11 & S1950/E100 & A & S1942 & E100 & 385 & 8.3 & 0.9 & $\mathrm{H}$ & 5 & B & $\begin{array}{l}\text { Hole slightly declined below horizontal. Collar about } \\
0.8 \mathrm{~m} \text { above floor. Drilled } 6 / 86 \text { and monitored until } \\
12 / 02 / 88 \text {. }\end{array}$ \\
\hline BTR12 & S1950/E100 & A & $\mathrm{S} 1942$ & E101 & 385 & 8.3 & 0.9 & $H$ & 5 & B & $\begin{array}{l}\text { Hole slightly declined below horizontal. Collar about } \\
0.8 \mathrm{~m} \text { above floor. Drilled } 6 / 86 \text { and monitored until } \\
12 / 02 / 88 \text {. }\end{array}$ \\
\hline$B \times 01$ & B & $\mathbf{S}$ & $N 1384.68$ & E982.33 & 401.56 & 10 & 15.3 & D & 90 & $B, E$ & $\begin{array}{l}\text { Monitored as part of the BSEP from when it was } \\
\text { drilled in } 1 / 85 \text { to } 4 / 93 \text {. Core moist from } 10.6 \text { to } 11.1 \\
\mathrm{~m} \text { in coarsely crystalline clear halite. MB139 at } 7.1 \text { to } \\
7.9 \mathrm{~m} \text {. }\end{array}$ \\
\hline $\mathrm{BX02}$ & $B$ & $S$ & N1384.44 & E982.87 & 407.05 & 10 & 15.0 & $\mathrm{U}$ & 90 & $B, E$ & Monitored as part of the BSEP from $1 / 85$ to $12 / 89$. \\
\hline $\mathrm{DH} 15$ & N1140/E1689 & A & N1140 & E1688.5 & 402 & 7.6 & 15.5 & $\mathbf{U}$ & 90 & B & $\begin{array}{l}\text { Moisture noticed at collar in } 4 / 86 \text {. Collecting device } \\
\text { installed } 5 / 86 \text { and monitored as part of the BSEP } \\
\text { since then. At present no brine is collected because } \\
\text { of insufficient inflow. }\end{array}$ \\
\hline DH35 & $\mathbf{G}$ & A & N1102 & W1882 & 395 & 8.9 & 15.8 & U & 90 & $A 3, B$ & $\begin{array}{l}\text { Monitored as part of the BSEP since } 2 / 85 \text {. At present } \\
\text { no brine is collected because of insufficient inflow. }\end{array}$ \\
\hline DH36 & G & A & N1102 & W1882 & 392 & 8.9 & 15.7 & D & 90 & A3, B & Monitored as part of the BSEP since $1 / 85$. \\
\hline $\mathrm{DH} 37$ & G & A & N1101 & W2182 & 396 & 8.9 & 15.7 & U & 90 & $A 3, B$ & $\begin{array}{l}\text { Monitored as part of the BSEP since } 1 / 85 \text {. At the } \\
\text { present no brine is collected because of insufficient } \\
\text { inflow. }\end{array}$ \\
\hline DH38 & $\mathbf{G}$ & A & N1101 & W2182 & 392 & 8.9 & 14.5 & $\mathbf{D}$ & 90 & $A 3, B$ & Monitored as part of the BSEP since $1 / 85$. \\
\hline
\end{tabular}

"The repository is referenced in feet; therefore, the North-South and East-West coordinates are presented in feet.

**For references, see footnote at end of table. 
Table A-1 (Continued)

\section{List of Underground Locations Where Brine Occurrences \\ Were Observed and Monitored Through December, 1993 \\ As Part of the Brine Sampling and Evaluation Program at WIPP}

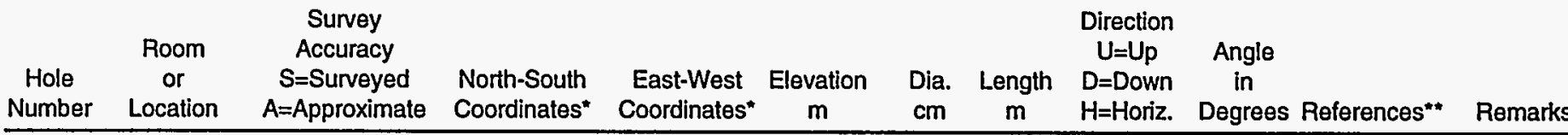

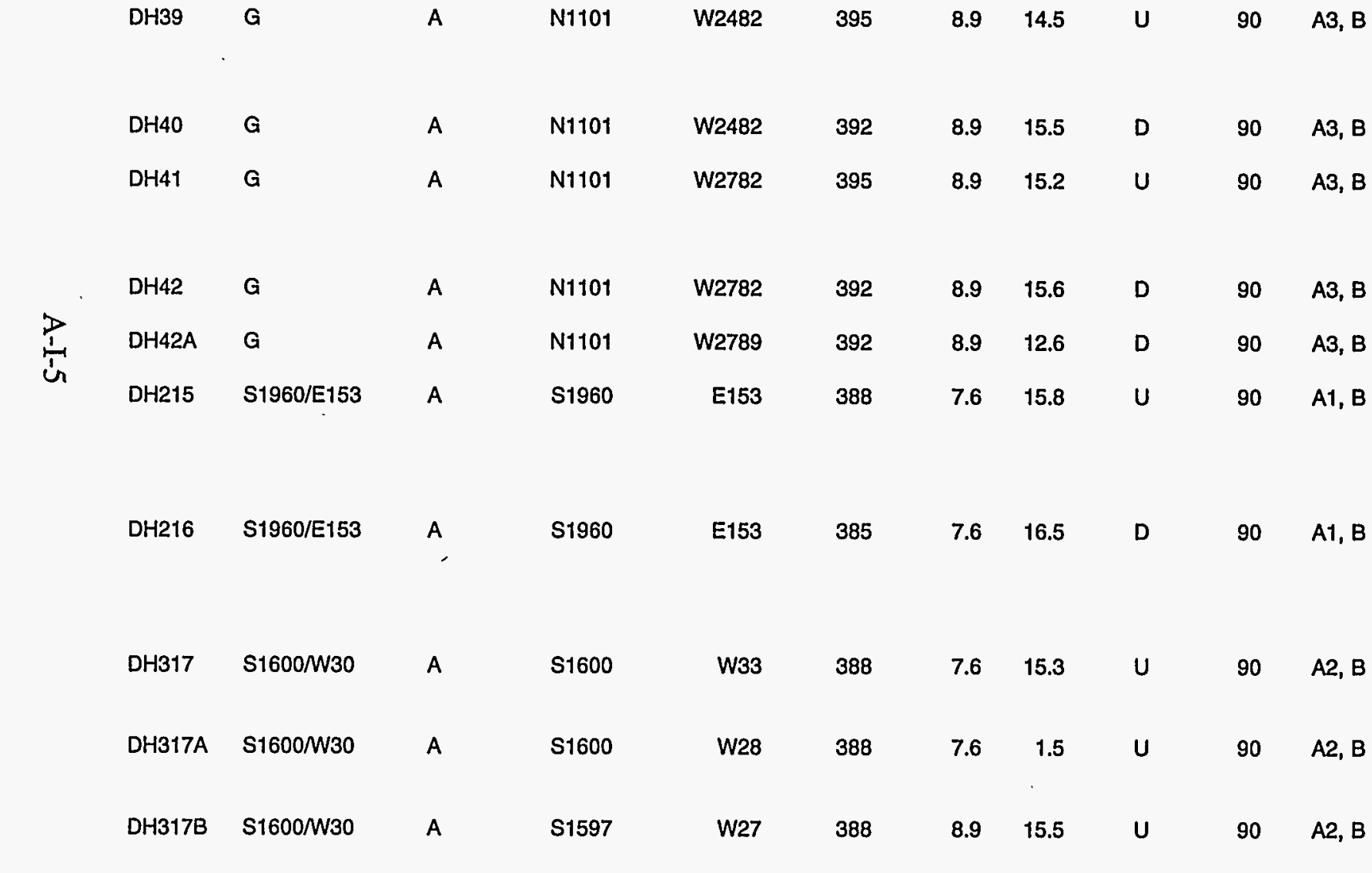

Monitored as part of the BTP since 2/85. At the present no brine is collected because of insufficient inflow.

Monitored as part of the BSEP since 1/85.

Monitored as part of the BSEP since 2/85. At the present no brine is collected because of insufficient inflow.

Monitored as part of the BSEP since 2/85.

Monitored as part of the BSEP since 2/85.

Gas releases had been observed in this hole. Monitored as part of the BSEP from $1 / 85$ to $11 / 90$. At the present no brine is collected due to insufficient inflow.

Gas releases had been observed in this hole. Monitored as part of the BSEP from 1/85 to 6/85 when collar was destroyed and hole plugged by mining.

Stalactite growth monitored as part of the BSEP from $5 / 85$ to $2 / 86$.

Stalactite growth monitored as part of the BSEP from $5 / 85$ to $2 / 86$.

Gas pocket at $14.0 \mathrm{~m}$. Brine seeped from hole after drill rods were broken at end of run at depth of $5 \mathrm{~m}$. Probable source was anhydrite "a". Stalactite growth monitored as part of the BSEP from $5 / 85$ to $2 / 86$. 
Table A-1 (Continued)

List of Underground Locations Where Brine Occurrences

Were Observed and Monitored Through December, 1993

\title{
As Part of the Brine Sampling and Evaluation Program at WIPP
}

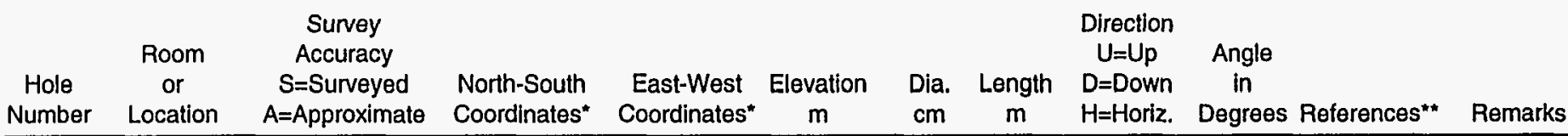

DHP401 S1950/E1330 A

S1950

E1330

387

10

15.1

U

DHP402A S1950/E1330

$\$ 1950$

$E 1330$

383

10

15.2

D

EES12B

N1430/E0140

A

N1430

E140

398

$4.7 \quad 3$

D

90

K

$\frac{5}{1}$

EES21B S0700/E0066

A

$\$ 700$

E66

381

$\begin{array}{ll}4.7 & 2.7\end{array}$

N1095

W1837

391

B

GSEEP
A

(n)

$S \quad N$

1 G202

1

\author{
S
}

N1264.79

W246.11

395.17

7.3

14.7

D

K

JV8

S

N1067

W374

393

91

2.5

D

90

90

90
A3, B, H, J Monitored as part of the BSEP from 11/84 to $9 / 87$ when shear closure pinched hole shut so that sampler would not go to bottom.

$A 3, B, H, J$ Monitored as part of the BSEP from $11 / 84$ to $7 / 87$ when shear closure pinched hole shut so that sampler would not go to bottom. Last BSEP brine data collected in $3 / 87$.

Drilled 8/08/85; drillers reported water at $2.4 \mathrm{~m}$. Not monitored after initial observation. 
Table A-1 (Continued)

List of Underground Locations Where Brine Occurrences

Were Observed and Monitored Through December, 1993

As Part of the Brine Sampling and Evaluation Program at WIPP

\begin{tabular}{|c|c|c|c|c|c|c|c|c|c|c|c|}
\hline $\begin{array}{c}\text { Hole } \\
\text { Number }\end{array}$ & $\begin{array}{c}\text { Room } \\
\text { or } \\
\text { Location }\end{array}$ & $\begin{array}{c}\text { Survey } \\
\text { Accuracy } \\
\text { S=Surveyed } \\
\text { A=Approximate }\end{array}$ & $\begin{array}{l}\text { North-South } \\
\text { Coordinates" }\end{array}$ & $\begin{array}{c}\text { East-West } \\
\text { Coordinates" }\end{array}$ & $\begin{array}{c}\text { Elevation } \\
\mathbf{m}\end{array}$ & $\begin{array}{l}\text { Dia. } \\
\mathrm{cm}\end{array}$ & $\begin{array}{l}\text { Length } \\
\mathrm{m}\end{array}$ & $\begin{array}{l}\text { Direction } \\
U=U p \\
D=\text { Down } \\
H=\text { Horiz. }\end{array}$ & $\begin{array}{c}\text { Angle } \\
\text { in } \\
\text { Degrees }\end{array}$ & References** & Remarks \\
\hline JV9 & $J$ & $s$ & N1067 & W378 & 393.3 & 91 & 2.5 & D & 90 & D, G & $\begin{array}{l}\text { Brine in bottom of pilot hole on } 8 / 20 / 85 \text {. Not } \\
\text { monitored after initial observation. }\end{array}$ \\
\hline L1S25 & $\mathrm{L} 1$ & A & N1524 & W218 & 400 & 10 & 3.6 & D & 90 & $B, H$ & Monitored as part of the BSEP from $8 / 85$ to $6 / 89$. \\
\hline L1S26 & L1 & A & N1524 & W220 & 400 & 10 & 3.6 & D & 90 & B, $\mathrm{H}$ & Monitored as part of the BSEP from $8 / 85$ to $6 / 89$. \\
\hline L1S27 & L1 & A & N1524 & W222 & 400 & 10 & 3.6 & D & 90 & B, H & Monitored as part of the BSEP from $8 / 85$ to $6 / 89$. \\
\hline L1S28 & L1 & A & N1524 & W224 & 400 & 10 & 3.7 & D & 90 & $B, H$ & Monitored as part of the BSEP from $8 / 85$ to $6 / 89$. \\
\hline L1S29 & L1 & A & N1524 & W226 & 400 & 10 & 3.7 & D & 90 & B, H & Monitored as part of the BSEP from $8 / 85$ to $6 / 89$. \\
\hline L1S30 & L1 & A & N1524 & W228 & 400 & 10 & 3.7 & D & 90 & $B, H$ & Monitored as part of the BSEP from $8 / 85$ to $6 / 89$. \\
\hline L1S31 & L1 & A & N1524 & W235 & 400 & 10 & 3.6 & D & 90 & B, H & Monitored as part of the BSEP from $8 / 85$ to $6 / 89$. \\
\hline L1S32 & L1 & A & N1524 & W237 & 400 & 10 & 3.6 & D & 90 & B, H & Monitored as part of the BSEP from $8 / 85$ to $6 / 89$. \\
\hline L1S33 & L1 & A & N1524 & W239 & 400 & 10 & 3.6 & D & 90 & $B_{1} H$ & Monitored as part of the BSEP from $8 / 85$ to $6 / 89$. \\
\hline L1S34 & L1 & A & N1524 & W241 & 400 & 10 & 3.7 & $\mathrm{D}$ & 90 & $B, H$ & Monitored as part of the BSEP from $8 / 85$ to $6 / 89$. \\
\hline L1S35 & L1 & A & N1524 & W243 & 400 & 10 & 3.8 & D & 90 & B, H & Monitored as part of the BSEP from $8 / 85$ to $6 / 89$. \\
\hline L1S36 & L1 & A & N1524 & W245 & 400 & 10 & 3.7 & D & 90 & $B, H$ & Monitored as part of the BSEP from $8 / 85$ to $6 / 89$. \\
\hline L1X00 & L1 & A & N1538.5 & W225 & 400 & 10 & 3.8 & D & 90 & B, H & $\begin{array}{l}\text { Drillers found water in hole at } 3 \mathrm{~m}, 5 / 13 / 84 \text {. } \\
\text { Monitored as part of the BSEP from } 10 / 84 \text { to } 4 / 89 \text {. }\end{array}$ \\
\hline $\mathrm{L} 2 \mathrm{CO}$ & L2 & A & N1510 & W365 & 400 & 41 & 3.7 & D & 90 & B, H & $\begin{array}{l}\text { Drilled } 4 / 85 \text { overcoring and destroying L2C25. Brine } \\
\text { and gas enters hole quickly through open fractures. } \\
\text { Monitored intermittently as part of the BSEP from } \\
12 / 85 \text { through } 12 / 86 \text {. }\end{array}$ \\
\hline
\end{tabular}

"The repository is referenced in feet; therefore, the North-South and East-West coordinates are presented in feet.

"*For references, see footnote at end of table. 
Table A-1 (Continued)

List of Underground Locations Where Brine Occurrences

Were Observed and Monitored Through December, 1993

As Part of the Brine Sampling and Evaluation Program at WIPP

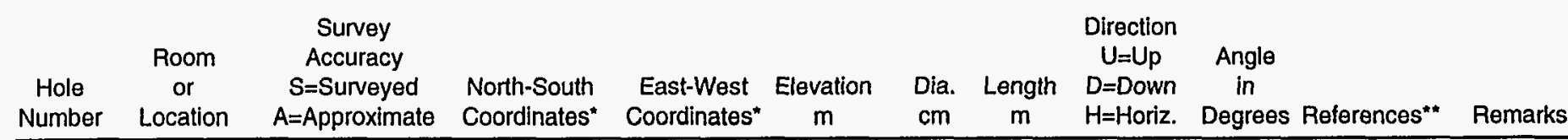

\begin{tabular}{llllllllllll}
\hline L2C25 & L1 & A & N1510 & W365 & 400 & 12.7 & 3.5 & D & 90 & B,
\end{tabular}

$\mathrm{H} \quad \mathrm{L} 2 \mathrm{C} 25$ is a $12.7 \mathrm{~cm}$ overcore of a previously grouted SNL/NM test hole. The overcore was drilled $3 / 85$ and air and brine was blown through fractures into hole L2C29, $1.2 \mathrm{~m}$ to the north. In $4 / 85$, a $40 \mathrm{~cm}$ overcore was made destroying this hole. The larger hole is designated L2C03.

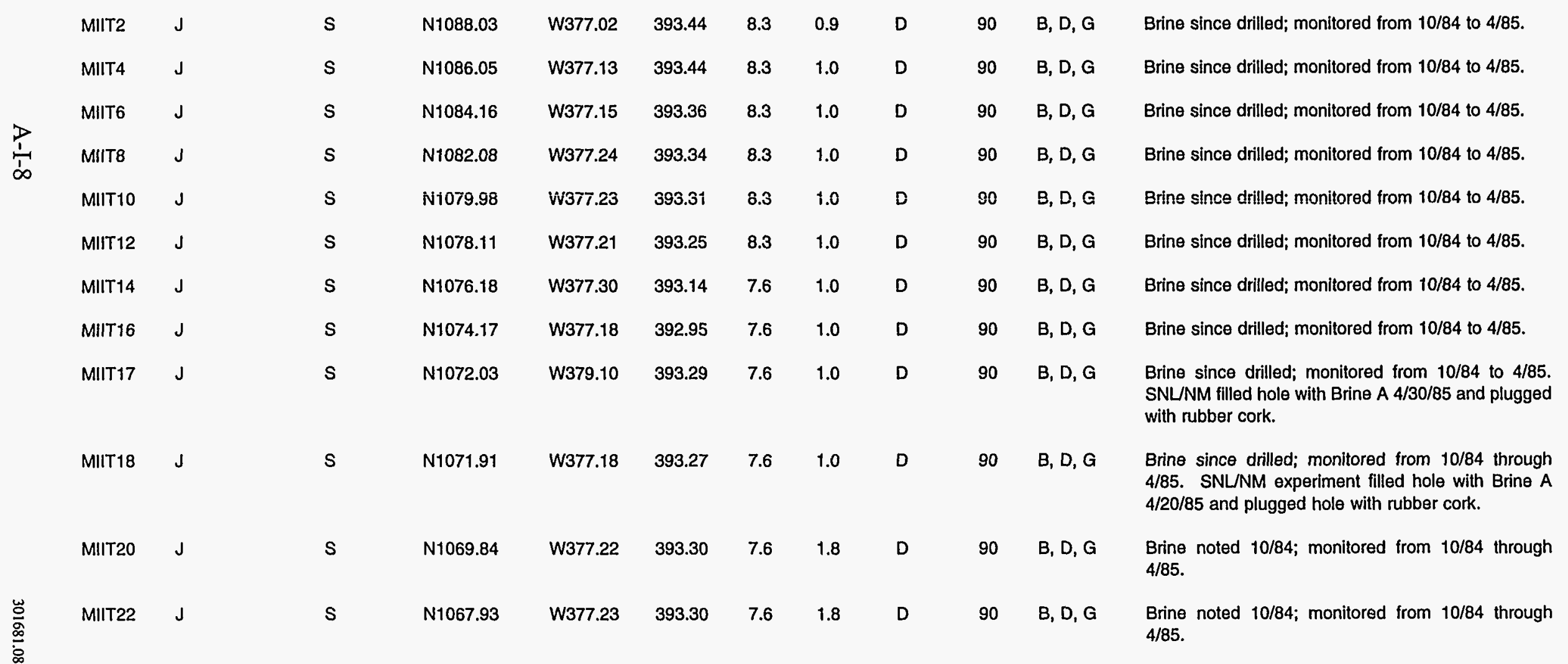


Table A-1 (Continued)

\section{List of Underground Locations Where Brine Occurrences \\ Were Observed and Monitored Through December, 1993 \\ As Part of the Brine Sampling and Evaluation Program at WIPP}

\begin{tabular}{|c|c|c|c|c|c|c|c|c|c|c|c|}
\hline $\begin{array}{c}\text { Hole } \\
\text { Number }\end{array}$ & $\begin{array}{l}\text { Room } \\
\text { or } \\
\text { Location }\end{array}$ & $\begin{array}{c}\text { Survey } \\
\text { Accuracy } \\
S=\text { Surveyed } \\
A=\text { Approximate }\end{array}$ & $\begin{array}{l}\text { North-South } \\
\text { Coordinates* }\end{array}$ & $\begin{array}{c}\text { East-West } \\
\text { Coordinates* }\end{array}$ & $\begin{array}{c}\text { Elevation } \\
\mathrm{m}\end{array}$ & $\begin{array}{l}\text { Dia. } \\
\mathrm{cm}\end{array}$ & $\begin{array}{c}\text { Length } \\
\text { m }\end{array}$ & $\begin{array}{l}\text { Direction } \\
\begin{array}{l}U=U p \\
D=\text { Down } \\
H=\text { Horiz. }\end{array}\end{array}$ & $\begin{array}{l}\text { Angle } \\
\text { in } \\
\text { Degrees }\end{array}$ & References*" & Remarks \\
\hline MIIT24 & $\mathrm{J}$ & s & N1065.79 & W377.21 & 393.42 & 7.6 & 1.8 & D & 90 & B, D, G & $\begin{array}{l}\text { Brine noted } 10 / 84 \text {; monitored } 10 / 84 \text { through } 4 / 85 \text {, } \\
\text { SNL/NM experiment added Brine A to hole } 4 / 30 / 85 \\
\text { and plugged with rubber cork. }\end{array}$ \\
\hline MITP & J & A & N1067 & W378 & 393 & 3.8 & 2.7 & D & 90 & $B, F$ & $\begin{array}{l}\text { Brine since drilled; pilot hole for } 0.9-\text { m-diameter hole } \\
\text { that was never completed. Monitored from } 4 / 02 / 85 \\
\text { through } 4 / 23 / 85 \text {. }\end{array}$ \\
\hline NG252 & 2 & S & N1275.86 & W381.05 & 394.68 & 3.8 & 2.3 & D & 90 & $A 3, B, H, J$ & $\begin{array}{l}\text { Monitored as part of the BSEP from } 11 / 84 \text { to } 4 / 89 \text {. } \\
\text { This hole constantly produced gas. First time noticed } \\
\text { was before } 10 / 84 \text {. Room closed } 6 / 89 \text {. }\end{array}$ \\
\hline $\mathrm{OH} 2 \mathrm{O}$ & $S 1600 / W 170$ & $\mathbf{S}$ & $\mathrm{S} 1610.36$ & W177.16 & 386.22 & 8.9 & 47.2 & $H$ & $0-3$ & L & $\begin{array}{l}\text { Collared about } 0.3 \mathrm{~m} \text { above the orange band, } \\
\text { bottoms in Map Unit } 0 \text { below the orange band. } \\
\text { Monitored as part of the BSEP since it was drilled } \\
3 / 89 \text {. }\end{array}$ \\
\hline $\mathrm{OH} 21$ & $\mathrm{~S} 1600 / \mathrm{W} 170$ & s & S1605.36 & W177.16 & 385.50 & 8.9 & 16.2 & $H$ & $0-3$ & $\mathbf{L}$ & $\begin{array}{l}\text { Collared about } 0.3 \mathrm{~m} \text { below the orange band. } \\
\text { Monitored for the BSEP since it was drilled } 12 / 88 \text {. }\end{array}$ \\
\hline $\mathrm{OH} 22$ & $S 1600 / W 170$ & s & S1615.36 & W177.16 & 386.65 & 8.9 & 15.1 & $H$ & $0-3$ & L & $\begin{array}{l}\text { Collared about } 0.6 \mathrm{~m} \text { above the orange band. } \\
\text { Monitored for the BSEP since it was drilled } 12 / 8 B \text {. }\end{array}$ \\
\hline $\mathrm{OH} 23$ & S1950/W170 & S & S1950.41 & W178.86 & 384.94 & 8.9 & 46.0 & $\mathrm{H}$ & $0-3$ & L & $\begin{array}{l}\text { Collared about } 0.3 \mathrm{~m} \text { above the orange band, } \\
\text { bottoms in Map Unit } 0 \text { below the orange band. } \\
\text { Monitored for the BSEP since it was drilled } 2 / 89 \text {. }\end{array}$ \\
\hline $\mathrm{OH} 24$ & $\mathrm{~S} 1950 \mathrm{~W} 170$ & $\mathbf{S}$ & S1945.41 & W178.86 & 384.11 & 8.9 & 15.2 & $\mathrm{H}$ & $0-3$ & $\mathbf{L}$ & $\begin{array}{l}\text { Collared about } 0.3 \mathrm{~m} \text { below the orange band. } \\
\text { Monitored for the BSEP from } 3 / 89 \text { to } 8 / 90 \text {. }\end{array}$ \\
\hline $\mathrm{OH} 25$ & S1950W170 & S & S1955.41 & W178.86 & 385.27 & 8.9 & 15.2 & $H$ & $0-3$ & L & $\begin{array}{l}\text { Collared about } 0.6 \mathrm{~m} \text { above the orange band. } \\
\text { Monitored for the BSEP from } 3 / 89 \text { to } 8 / 90 \text {. }\end{array}$ \\
\hline $\mathrm{OH} 26$ & S2180 W170 & S & S2183.01 & W177.14 & 384.70 & 8.9 & 45.7 & $\mathrm{H}$ & $0-3$ & $L$ & $\begin{array}{l}\text { Collared about } 0.3 \mathrm{~m} \text { above the orange band, } \\
\text { bottoms in Map Unit } 0 \text { below the orange band. } \\
\text { Monitored for the BSEP since it was drilled } 3 / 89 \text {. }\end{array}$ \\
\hline
\end{tabular}

The repository is referenced in feet; therefore, the North-South and East-West coordinates are presented in feet.

*For references, see footnote at end of table. 
Table A-1 (Continued)

List of Underground Locations Where Brine Occurrences

Were Observed and Monitored Through December, 1993

As Part of the Brine Sampling and Evaluation Program at WIPP

\begin{tabular}{|c|c|c|c|c|c|c|c|c|c|c|c|}
\hline $\begin{array}{l}\text { Hole } \\
\text { Number }\end{array}$ & $\begin{array}{l}\text { Room } \\
\text { or } \\
\text { Location }\end{array}$ & $\begin{array}{c}\text { Survey } \\
\text { Accuracy } \\
\text { S=Surveyed } \\
A=\text { Approximate }\end{array}$ & $\begin{array}{l}\text { North-South } \\
\text { Coordinates" }\end{array}$ & $\begin{array}{l}\text { East-West } \\
\text { Coordinates* }\end{array}$ & $\begin{array}{l}\text { Elevation } \\
\mathrm{m}\end{array}$ & $\begin{array}{l}\text { Dia. } \\
\text { cm }\end{array}$ & $\begin{array}{l}\text { Length } \\
\mathrm{m}\end{array}$ & $\begin{array}{l}\text { Direction } \\
U=\text { Up } \\
D=\text { Down } \\
H=\text { Horiz. }\end{array}$ & $\begin{array}{l}\text { Angle } \\
\text { in } \\
\text { Degrees }\end{array}$ & References** & Remarks \\
\hline $\mathrm{OH} 27$ & $S 2180 / W 170$ & $S$ & S2178.01 & $W 177.14$ & 385 & 8.9 & 15.1 & $H$ & 0.3 & L. & $\begin{array}{l}\text { Collared about } 0.6 \mathrm{~m} \text { above the orange band. } \\
\text { Monitored for the BSEP since it was drilled } 4 / 89 \text { to } \\
10 / 91 \text {. Hole dry. }\end{array}$ \\
\hline $\mathrm{OH} 27 \mathrm{~A}$ & S2180/W170 & $\mathrm{s}$ & S2177.01 & W177.14 & 385 & 8.9 & 1.2 & $H$ & $0-3$ & $L$ & $\begin{array}{l}\text { Short offset hole to OH27. Collared about } 0.6 \mathrm{~m} \\
\text { above the orange band. Monitored for the BSEP } \\
\text { since it was drilled } 4 / 89 \text { to } 12 / 89 \text {. Hole dry. }\end{array}$ \\
\hline $\mathrm{OH} 28$ & S2180/W170 & $S$ & S2188.01 & W177.14 & 383.78 & 8.9 & 15.1 & $\mathrm{H}$ & $0-3$ & $\mathbf{L}$ & $\begin{array}{l}\text { Collared about } 0.3 \mathrm{~m} \text { below the orange band. } \\
\text { Monitored for the BSEP since it was drilled } 4 / 89 \text {. }\end{array}$ \\
\hline $\mathrm{OH} 35$ & AIS/S90 & $\mathrm{s}$ & $S 100.73$ & W628.97 & 383.45 & 8.9 & 3.1 & $D$ & 90 & $M$ & $\begin{array}{l}\text { Drilled for hydrologic testing of fractures beneath the } \\
\text { floor. Not a part of routine BSEP sampling. }\end{array}$ \\
\hline $\mathrm{OH} 36$ & AIS/S90 & $\mathrm{s}$ & S96.71 & W623.11 & 383.39 & 8.9 & 3.1 & D & 90 & $M$ & $\begin{array}{l}\text { Drilled for hydrologic testing of fractures beneath the } \\
\text { floor. Not a part of routine BSEP sampling. }\end{array}$ \\
\hline $\mathrm{OH} 37$ & AIS/S90 & $S$ & S97.66 & W609.39 & 383.35 & 8.9 & 3.1 & D & 90 & $M$ & $\begin{array}{l}\text { Drilled for hydrologic testing of fractures beneath the } \\
\text { floor. Not a part of routine BSEP sampling. }\end{array}$ \\
\hline $\mathrm{OH} 38$ & AIS/S90 & $S$ & S97.35 & W595.62 & 383.36 & 8.9 & 3.1 & D & 90 & $M$ & $\begin{array}{l}\text { Drilled for Marker Bed } 139 \text { hydrologic testing. Not a } \\
\text { part of routine BSEP sampling. }\end{array}$ \\
\hline OH39 & AIS/S90 & A & S97 & W540 & 383 & 8.9 & 3 & $\mathrm{D}$ & 90 & $M$ & $\begin{array}{l}\text { Drilled for hydrologic testing of fractures beneath the } \\
\text { floor. Not a part of routine BSEP sampling. }\end{array}$ \\
\hline $\mathrm{OH} 40$ & AIS/S90 & $S$ & S96.91 & W485.10 & 383.02 & 8.9 & 3 & D & 90 & $M$ & $\begin{array}{l}\text { Drilled for hydrologic testing of fractures beneath the } \\
\text { floor. Not a part of routine BSEP sampling. }\end{array}$ \\
\hline $\mathrm{OH} 41$ & AIS/S90 & $S$ & $S 110.52$ & W622.79 & 383.44 & 8.9 & 3.5 & $\mathrm{D}$ & 90 & $M$ & $\begin{array}{l}\text { Drilled for hydrologic testing of fractures beneath the } \\
\text { floor. Not a part of routine BSEP sampling. }\end{array}$ \\
\hline $\mathrm{OH} 42$ & AIS/S90 & $S$ & $\mathrm{~S} 43.44$ & W622.54 & 383.62 & 8.9 & 3.2 & D & 90 & $M$ & $\begin{array}{l}\text { Drilled for hydrologic testing of fractures beneath the } \\
\text { floor. Not a part of routine BSEP sampling. }\end{array}$ \\
\hline
\end{tabular}

"The repository is referenced in feet; therefore, the North-South and East-West coordinates are presented in feet.

*For references, see footnote at end of table. 
Table A-1 (Continued)

List of Underground Locations Where Brine Occurrences

Were Observed and Monitored Through December, 1993

As Part of the Brine Sampling and Evaluation Program at WIPP

\begin{tabular}{|c|c|c|c|c|c|c|c|c|c|c|c|}
\hline $\begin{array}{l}\text { Hole } \\
\text { Number }\end{array}$ & $\begin{array}{l}\text { Room } \\
\text { or } \\
\text { Location }\end{array}$ & $\begin{array}{c}\text { Survey } \\
\text { Accuracy } \\
\text { S=Surveyed } \\
A=\text { Approximate }\end{array}$ & $\begin{array}{l}\text { North-South } \\
\text { Coordinates* }\end{array}$ & $\begin{array}{l}\text { East-West } \\
\text { Coordinates* }\end{array}$ & $\begin{array}{l}\text { Elevation } \\
\mathrm{m}\end{array}$ & $\begin{array}{l}\text { Dia. } \\
\text { cm }\end{array}$ & $\begin{array}{l}\text { Length } \\
\mathrm{m}\end{array}$ & $\begin{array}{c}\text { Direction } \\
\text { U=Up } \\
D=\text { Down } \\
H=\text { Horiz. }\end{array}$ & $\begin{array}{l}\text { Angle } \\
\text { in } \\
\text { Degrees }\end{array}$ & References** & Remarks \\
\hline $\mathrm{OH} 43$ & AIS/S90 & $\mathbf{S}$ & S124.01 & W622.52 & 383.45 & 8.9 & 3.7 & D & 90 & $M$ & $\begin{array}{l}\text { Drilled for hydrologic testing of fractures beneath the } \\
\text { floor. Not a part of routine BSEP sampling. }\end{array}$ \\
\hline $\mathrm{OH} 44$ & AIS/S90 & $\mathbf{S}$ & $S 134.53$ & W622.31 & 383.46 & 8.9 & 3.4 & D & 90 & $M$ & $\begin{array}{l}\text { Drilled for hydrologic testing of fractures beneath the } \\
\text { floor. Not a part of routine BSEP sampling. }\end{array}$ \\
\hline $\mathrm{OH} 45$ & Core Library & S & S391.51 & W326.35 & 384.15 & 8.9 & 14.9 & $\mathrm{H}$ & $0-3$ & $L$ & Monitored for the BSEP since it was drilled 6/89. \\
\hline $\mathrm{OH} 46$ & Core Library & S & S391.51 & W319.01 & 381.65 & 8.9 & 15.3 & D & 90 & $L$ & Monitored for the BSEP since it was drilled 6/89. \\
\hline $\mathrm{OH} 47$ & Core Library & S & $\$ 391.51$ & W319.01 & 385.90 & 8.9 & 15.2 & U & 90 & $\mathbf{L}$ & $\begin{array}{l}\text { Monitored for the BSEP since it was drilled } 7 / 89 \text {. Hole } \\
\text { dry. }\end{array}$ \\
\hline P4X84 & SPDV Room & A & N1138 & W0644 & 394 & 91.4 & 4.8 & D & 90 & B & $\begin{array}{l}\text { Large diameter downhole in south end of Room } 4 \\
\text { often shown to visitors. MB } 139 \text { and fractures } \\
\text { beneath the floor are well exposed, both of which are } \\
\text { dry. This is good evidence that no far-field flow } \\
\text { exists. }\end{array}$ \\
\hline PR2 & S1600/E140 & A & $\$ 1600$ & $\mathrm{E} 140$ & 388 & 5 & 6.1 & U & 90 & $\mathrm{~B}, \mathrm{C}$ & $\begin{array}{l}\text { Stalactite growth monitored as part of the BSEP from } \\
5 / 85 \text { to } 2 / 86 \text {. }\end{array}$ \\
\hline PR3 & S1282/E140 & A & S2182 & $\mathrm{E} 140$ & 385 & 5 & 6.1 & U & 90 & $\mathrm{~B}, \mathrm{C}$ & $\begin{array}{l}\text { Stalactite growth monitored as part of the BSEP from } \\
5 / 85 \text { to } 2 / 86 \text {. }\end{array}$ \\
\hline PR4 & S2748/E140 & A & S2748 & E140 & 381 & 5 & 6.1 & $\mathrm{u}$ & 90 & $B, C$ & $\begin{array}{l}\text { Stalactite growth monitored as part of the BSEP from } \\
5 / 85 \text { to } 2 / 86 \text {. }\end{array}$ \\
\hline WWC1 & Room C1 & A. & N1420 & E1572 & 398.96 & 91 & 4.9 & $H$ & 0 & B & $\begin{array}{l}\text { Large horizontal hole on south rib of } \mathrm{N} 1420 \text { drift, } \\
\text { across from Room } \mathrm{C} 1 \text {. Photographically monitored } \\
\text { for salt buildup. }\end{array}$ \\
\hline
\end{tabular}

"The repository is referenced in feet; therefore, the North-South and East-West coordinates are presented in feet.

**For references, see footnote at end of table. 
Table A-1 (Concluded)

\section{List of Underground Locations Where Brine Occurrences}

Were Observed and Monitored Through December, 1993

As Part of the Brine Sampling And Evaluation Program at WIPP

Footnote

A1 TSC-D'Appolonia, 1983 (WIPP-DOE-163)

A2 Bechtel, 1984 (WIPP-DOE-202)

A3 Bechtel, 1985 (WIPP-DOE-213)

B Brine Sampling and Evaluation Program File

C Records of Special Drill Holes, September 12, 1983: BSEP Files

D As-Built Survey Calculation Sheets: BSEP Files

E Field Notes, J. Gallerani, Bechtel: BSEP Files

F Field Notes, D. Deal, IT Corporation: BSEP Files

G Room J Brine Survey: BSEP Files

H Room L1 and L2 Field Notes: BSEP Files

J Geotechnical Instrumentation List, November 2, 1983: BSEP files

Excavation Effects Drilling Program, Data Transmittal August 12, 1986: Excavation Effects Files: WIPP Geotechnical Engineering Files Drilling Record Log: BSEP Files

Survey Data Sheet: WIPP Geotechnical Engineering Files 
APPENDIX A

BRINE ACCUMULATION

PART II-BRINE ACCUMULATION DATA TABLES 


\section{THIS PAGE INTENTIONALLY LEFT BLANK}


TABLE A-2

BRINE ACCUMULATION DATA TABLE

Data through December 31, 1993

$\begin{array}{llclc} & \text { DAYS } & \text { DAYS } & & \text { CUAULATIVE } \\ \text { LITERS } & \text { SIMCE } & \text { USED FOR } & \text { LITERS } & \text { LITERS } \\ \text { REMOVED } & 1 / 1 / 85 & \text { CALCULATION } & \text { PER DAY } & \text { COLLECTED }\end{array}$

LOCATION DATE TIME REMOVED 1/1/85 CALCULATION PER DAY COLLECTED REMARKS

\begin{tabular}{|c|c|c|c|c|c|c|c|c|}
\hline$A 1 \times 02$ & $10 / 10 / 84$ & $00: 00$ & NA & 0.000 & 0.000 & 0.000 & 0.00 & Room Al completed. \\
\hline A1X02 & 03/07/85 & $09: 30$ & HA & 65.396 & 0.000 & 0.000 & 0.00 & $\begin{array}{l}\text { Uphole drilled } 2 / 27 / 85 \text { to } 3 / 07 / 85 \text {. Hit } \\
\text { brine at } 12 \mathrm{ft} \text {. on } 2 / 27 / 85 \text {. }\end{array}$ \\
\hline A1X02 & $03 / 12 / 85$ & $12: 00$ & HA & 70.500 & 0.000 & 0.000 & 0.00 & $\begin{array}{l}\text { Trace brine, deepened hole to clay seam. } \\
\text { Moisture on back } 1 \mathrm{ft} \text { radius. }\end{array}$ \\
\hline A1X02 & $03 / 20 / 85$ & 13:00 & HA & 78.542 & 0.000 & 0.000 & 0.00 & Trace brine, drip missing funnel. \\
\hline A1X02 & $03 / 26 / 85$ & $11: 25$ & HA & 84.476 & 0.000 & 0.000 & 0.00 & $\begin{array}{l}\text { Repositioned funnel, collected one cup of } \\
\text { salt crystals with trace of brine. }\end{array}$ \\
\hline A1X02 & 04/02/85 & $12: 15$ & 00.21 & 91.510 & 174.510 & 0.001 & 0.21 & Some drips missing funnel. \\
\hline A1X02 & $04 / 10 / 85$ & $12: 20$ & 00.22 & 99.514 & 8.004 & 0.027 & 0.43 & Collecting container had leak. \\
\hline A1X02 & $04 / 17 / 85$ & $11: 30$ & 00.12 & 106.479 & 6.965 & 0.017 & 0.55 & Some drips missing funnel. \\
\hline A1X02 & $04 / 23 / 85$ & $10: 50$ & 00.12 & 112.451 & 5.972 & 0.020 & 0.67 & Some drips missing funnel. \\
\hline A1X02 & $04 / 30 / 85$ & 13:16 & 00.12 & 119.553 & 7.102 & 0.017 & 0.79 & Some drips missing funnel. \\
\hline A1X02 & 05/07/85 & $09: 05$ & 00.16 & 126.378 & 6.825 & 0.023 & 0.95 & \\
\hline A1X02 & $05 / 14 / 85$ & $10: 04$ & 00.19 & 133.419 & 7.041 & 0.027 & 1.14 & \\
\hline A1X02 & $05 / 21 / 85$ & $11: 35$ & 00.13 & 140.483 & 7.064 & 0.018 & 1.27 & Some drips missing funnel. \\
\hline A1X02 & 05/29/85 & $10: 00$ & 00.21 & 148.417 & 7.934 & 0.026 & 1.48 & \\
\hline A1X02 & 06/04/85 & $10: 25$ & 00.17 & 154.434 & 6.017 & 0.028 & 1.65 & \\
\hline A $1 \times 02$ & $06 / 11 / 85$ & $09: 40$ & 00.05 & 169.403 & 6.969 & 0.007 & 1.70 & \\
\hline$A 1 \times 02$ & $06 / 18 / 85$ & $09: 30$ & 00.08 & 168.396 & 6.993 & 0.011 & 1.78 & $\begin{array}{l}\text { Some drips missing funnel, big stalactite } \\
\text { formed. }\end{array}$ \\
\hline A1X02 & $06 / 25 / 85$ & $09: 45$ & 00.16 & 175.406 & 7.010 & 0.023 & 1.94 & \\
\hline $\mathrm{A} 1 \times 02$ & $07 / 02 / 85$ & $11: 00$ & 00.10 & 182.458 & 7.052 & 0.014 & 2.04 & \\
\hline A1X02 & $07 / 09 / 85$ & $09: 58$ & 00.15 & 189.415 & 6.957 & 0.022 & 2.19 & . \\
\hline $\mathrm{A} 1 \times 02$ & $07 / 16 / 85$ & $10: 53$ & 00.24 & 196.453 & 7.038 & 0.034 & 2.43 & \\
\hline $\mathrm{A} 1 \times 02$ & $07 / 24 / 85$ & $09: 49$ & 00.24 & 204.409 & 7.956 & 0.030 & 2.67 & \\
\hline A1X02 & $07 / 30 / 85$ & $09: 30$ & 00.15 & 210.396 & 5.987 & 0.025 & 2.82 & \\
\hline A1X02 & 08/06/85 & $09: 35$ & 00.14 & 217.399 & 7.003 & 0.020 & 2.96 & \\
\hline A1X02 & $08 / 14 / 85$ & $09: 26$ & 00.05 & 225.393 & 7.994 & 0.006 & 3.01 & \\
\hline$A 1 \times 02$ & $08 / 20 / 85$ & $10: 13$ & 00.09 & 231.426 & 6.033 & 0.015 & 3.10 & \\
\hline A1X02 & $08 / 28 / 85$ & $09: 08$ & 00.06 & 239.381 & 7.955 & 0.008 & 3.16 & \\
\hline A1X02 & $09 / 04 / 85$ & $09: 44$ & 00.07 & 246.406 & 7.025 & 0.010 & 3.23 & \\
\hline A1X02 & $09 / 10 / 85$ & $09: 24$ & 00.12 & 252.392 & 5.986 & 0.020 & 3.35 & \\
\hline A1X02 & $09 / 17 / 85$ & $09: 08$ & 00.13 & 259.381 & 6.989 & 0.019 & 3.48 & Some drips missing funnel. \\
\hline A1X02 & $09 / 24 / 85$ & 09:07 & 00.17 & 266.380 & 6.999 & 0.024 & 3.65 & \\
\hline A1X02 & 10/01/85 & $09: 21$ & 00.14 & 273.390 & 7.010 & 0.020 & 3.79 & $:$ \\
\hline A1X02 & $10 / 08 / 85$ & $12: 19$ & 00.16 & 280.513 & .7 .123 & 0.022 & 3.95 & Room A1 heaters turned on $10 / 02 / 85$. \\
\hline A1X02 & $10 / 15 / 85$ & $09: 41$ & 00.12 & 287.403 & 6.890 & 0.017 & 4.07 & \\
\hline A1X02 & $10 / 23 / 85$ & $09: 43$ & 00.19 & 295.405 & 8.002 & 0.024 & 4.26 & \\
\hline A1X02 & $10 / 29 / 85$ & $11: 02$ & 00.12 & 301.460 & 6.055 & 0.020 & 4.38 & \\
\hline A1X02 & $11 / 05 / 85$ & $08: 46$ & 00.12 & 308.365 & 6.905 & 0.017 & 4.50 & \\
\hline A1X02 & $11 / 13 / 85$ & $09: 16$ & 00.13 & 316.386 & 8.021 & 0.016 & 4.63 & Some drips missing funnel. \\
\hline A1X02 & $11 / 21 / 85$ & $10: 45$ & 00.13 & 324.448 & 8.062 & 0.016 & 4.76 & Some drips missing funnel. \\
\hline A1X02 & $12 / 04 / 85$ & $14: 07$ & 00.14 & 337.588 & 13.140 & 0.011 & 4.90 & Sample for chemical analysis, \#12. \\
\hline A1X02 & $12 / 10 / 85$ & $10: 31$ & 00.08 & 343.438 & 5.850 & 0.014 & 4.98 & \\
\hline A1X02 & $12 / 17 / 85$ & $13: 56$ & 00.03 & 350.581 & 7.143 & 0.004 & 5.01 & \\
\hline A1X02 & $01 / 03 / 86$ & $09: 40$ & 00.01 & 367.403 & 16.822 & 0.001 & 5.02 & Some drips missing funnel. \\
\hline
\end{tabular}


TABLE A-2 (Continued)

BRINE ACCUMULATION DATA TABLE

Data through December 31, 1993

\begin{tabular}{lllllll} 
& \multicolumn{2}{c}{ DAYS } & DAYS & \multicolumn{2}{c}{ CUAULATIVE } \\
LOCATIOH & LATE & LITERS & SINCE & USED FOR & LITERS & LITERS \\
\hline
\end{tabular}

\begin{tabular}{|c|c|c|c|c|c|c|c|c|}
\hline A1X02 & $01 / 23 / 86$ & $10: 10$ & 00.06 & 387.424 & 20.021 & 0.003 & 5.08 & New, larger funnel since $01 / 17$. \\
\hline A1X02 & $01 / 31 / 86$ & $11: 05$ & 00.23 & 395.462 & 8.038 & 0.029 & 5.31 & \\
\hline A $1 \times 02$ & $02 / 12 / 86$ & $10: 10$ & 00.22 & 407.424 & 11.962 & 0.018 & 5.53 & \\
\hline A $9 \times 02$ & $02 / 19 / 86$ & $10: 50$ & 00.07 & 414.451 & 7.027 & 0.010 & 5.60 & \\
\hline A1X02 & $02 / 28 / 86$ & $14: 00$ & 00.02 & 423.583 & 9.132 & 0.002 & 5.62 & \\
\hline A1X02 & $03 / 13 / 86$ & $09: 30$ & 00.05 & 436.396 & 12.813 & 0.004 & 5.67 & \\
\hline A $1 \times 02$ & $03 / 26 / 86$ & $09: 20$ & 00.05 & 449.389 & 12.993 & 0.004 & 5.72 & \\
\hline A1X02 & $04 / 02 / 86$ & $09: 00$ & 00.08 & 456.375 & 6.986 & 0.011 & 5.80 & \\
\hline A1X02 & $04 / 16 / 86$ & $11: 30$ & 00.10 & 470.479 & 14.104 & 0.007 & 5.90 & Sample for chemical analysis, $\# 2$. \\
\hline A1X02 & $04 / 24 / 86$ & $09: 35$ & 00.05 & 478.399 & 7.920 & 0.006 & 5.95 & Sample for chemistry. \\
\hline$A 1 \times 02$ & $04 / 30 / 86$ & $10: 10$ & 00.07 & 484.424 & 6.025 & 0.012 & 6.02 & Sample for chemistry. \\
\hline$A 1 \times 02$ & $05 / 06 / 86$ & $09: 40$ & 00.16 & 490.403 & 5.979 & 0.027 & 6.18 & \\
\hline A1X02 & $05 / 13 / 86$ & $09: 25$ & 00.02 & 497.392 & 6.989 & 0.003 & 6.20 & Sample for chemistry. \\
\hline A1X02 & $05 / 20 / 86$ & $10: 16$ & 00.04 & 504.428 & 7.036 & 0.006 & 6.24 & \\
\hline A1X02 & $05 / 27 / 86$ & $15: 05$ & 00.15 & 511.628 & 7.200 & 0.021 & 6.39 & \\
\hline A1X02 & $06 / 03 / 86$ & $09: 28$ & 00.13 & 518.394 & 6.766 & 0.019 & 6.52 & \\
\hline A1X02 & $06 / 10 / 86$ & $10: 50$ & 00.10 & 525.451 & 7.057 & 0.014 & 6.62 & \\
\hline A1X02 & $06 / 17 / 86$ & $09: 59$ & 00.12 & 532.416 & 6.965 & 0.017 & 6.74 & \\
\hline A $1 \times 02$ & $06 / 24 / 86$ & $10: 10$ & 00.25 & 539.424 & 7.008 & 0.036 & 6.99 & \\
\hline A1X02 & $07 / 01 / 86$ & $12: 44$ & 00.23 & 546.531 & 7.107 & 0.032 & 7.22 & \\
\hline A $1 \times 02$ & $07 / 08 / 86$ & $10: 05$ & 00.11 & 553.420 & 6.889 & 0.016 & 7.33 & \\
\hline$A 1 \times 02$ & $07 / 16 / 86$ & $09: 54$ & 00.25 & 561.413 & 7.993 & 0.031 & 7.58 & \\
\hline A1X02 & $07 / 22 / 86$ & $09: 26$ & 00.16 & 567.393 & 5.980 & 0.027 & 7.74 & \\
\hline$A 1 \times 02$ & $07 / 29 / 86$ & $10: 05$ & 00.26 & 574.420 & 7.027 & 0.037 & 8.00 & \\
\hline A1X02 & $08 / 05 / 86$ & $10: 19$ & 00.22 & 581.430 & 7.010 & 0.031 & 8.22 & \\
\hline A1X02 & $08 / 12 / 86$ & $09: 58$ & 00.28 & 588.415 & 6.985 & 0.040 & 8.50 & \\
\hline A1X02 & $08 / 19 / 86$ & $10: 38$ & 00.26 & 595.443 & 7.028 & 0.037 & 8.76 & \\
\hline$A 1 \times 02$ & $08 / 26 / 86$ & $10: 07$ & 00.24 & 602.422 & 6.979 & 0.034 & 9.00 & Sample \#6. \\
\hline$A 1 \times 02$ & $09 / 04 / 86$ & $10: 01$ & 00.35 & 611.417 & 8.995 & 0.039 & 9.35 & \\
\hline A1X02 & $09 / 09 / 86$ & $10: 25$ & 00.17 & 616.434 & 5.017 & 0.034 & 9.52 & \\
\hline A $1 \times 02$ & $09 / 16 / 86$ & $09: 35$ & 00.27 & 623.399 & 6.965 & 0.039 & 9.79 & \\
\hline$A 1 \times 02$ & $09 / 23 / 86$ & $09: 39$ & 00.26 & 630.402 & 7.003 & 0.037 & 10.05 & \\
\hline A1X02 & $10 / 01 / 86$ & $11: 39$ & 00.24 & 638.485 & 8.083 & 0.030 & 10.29 & \\
\hline$A 1 \times 02$ & $10 / 08 / 86$ & $10: 32$ & 00.17 & 645.439 & 6.954 & 0.024 & 10.46 & \\
\hline A1X02 & $10 / 14 / 86$ & $10: 53$ & 00.13 & 651.453 & 6.014 & 0.022 & 10.59 & \\
\hline A $9 \times 02$ & $11 / 05 / 86$ & $10: 30$ & 0.30 & 673.438 & 21.985 & 0.014 & 10.89 & \\
\hline A1X02 & $11 / 20 / 86$ & $11: 43$ & 00.11 & 688.488 & 15.050 & 0.007 & 11.00 & \\
\hline A $1 \times 02$ & $12 / 31 / 86$ & $12: 10$ & 00.14 & 729.507 & 41.019 & 0.003 & 11.14 & $\begin{array}{l}\text { Low readings from } 11 / 20 / 86 \text { to } 6 / 20 / 87 \text { may } \\
\text { be due to blockage in collecting system. }\end{array}$ \\
\hline A $1 \times 02$ & $02 / 03 / 87$ & $12: 16$ & NA & 763.511 & 0.000 & 0.000 & 11.14 & \\
\hline A $1 \times 02$ & $03 / 06 / 87$ & $11: 55$ & 0.05 & 794.497 & 64.990 & 0.001 & 11.19 & \\
\hline A $1 \times 02$ & $03 / 30 / 87$ & $11: 55$ & 0.01 & 818.497 & 24.000 & 0.000 & 11.20 & Tubing plugged, unable to open. \\
\hline A1X02 & 05/07/87 & $10: 45$ & 0.01 & 856.448 & 37.951 & 0.000 & 11.21 & Tubing plugged, unable to open. \\
\hline$A 1 \times 02$ & $07 / 28 / 87$ & $12: 00$ & 1.58 & 910.500 & 54.052 & 0.029 & 12.79 & $\begin{array}{l}\text { Removed metal funnel, which was plugged. } \\
\text { Most of the brine collected was in the } \\
\text { funnel. Installed a large plastic funnel. } \\
\text { Collected for chemistry, sample } \# 148 \text {. }\end{array}$ \\
\hline
\end{tabular}


TABLE A-2 (Continued)

\section{BRINE ACCUMULATION DATA TABLE}

Data through December 31, 1993

\begin{tabular}{|c|c|c|c|c|c|c|c|}
\hline & & & & DAYS & DAYS & & CUAULATIVE \\
\hline 104 & DATE & TIME & LITERS & SINCE & USED FOR & LITERS & LITERS \\
\hline
\end{tabular}

\begin{tabular}{|c|c|c|c|c|c|c|c|c|}
\hline A1X02 & $09 / 01 / 87$ & $11: 55$ & 0.94 & 973.497 & 35.007 & 0.027 & 14.58 & Collected for chemistry, sample \#159 A\&B. \\
\hline A1X02 & $10 / 20 / 87$ & $10: 59$ & 1.84 & 1022.458 & 48.961 & 0.038 & 16.42 & \\
\hline A1X02 & $11 / 19 / 87$ & $10: 30$ & 1.09 & 1052.438 & 29.980 & 0.036 & 17.51 & Collected for chemistry, sample 226. \\
\hline A1X02 & $01 / 04 / 88$ & 11:05 & 3.73 & 1098.462 & 46.024 & 0.081 & 21.24 & \\
\hline A1X02 & $02 / 08 / 88$ & $13: 17$ & 1.65 & 1133.553 & 35.091 & 0.047 & 22.89 & $\begin{array}{l}\text { Collected for chemistry, sample } \# 299, \$ 300 \text {, } \\
\# 301 \text { \& } \$ 302 \text {. }\end{array}$ \\
\hline A1X02 & $03 / 30 / 88$ & $12: 20$ & 4.86 & 1184.514 & 50.961 & 0.095 & 27.75 & $\begin{array}{l}\text { Collected for chemistry, sample } \$ 343 \text { - } \\
\$ 352 \text {. }\end{array}$ \\
\hline A1X02 & $06 / 14 / 88$ & $09: 00$ & 5.15 & 1260.375 & 75.861 & 0.068 & 32.90 & $\begin{array}{l}\text { Collected for chemistry, sample } \# 02 \text { - } \\
\# 406 \text {. Removed to provide room for further } \\
\text { collection. }\end{array}$ \\
\hline A1X02 & $07 / 12 / 88$ & $09: 30$ & 1.11 & 1288.396 & 28.021 & 0.040 & 34.01 & $\begin{array}{l}\text { Collected for chemistry, sample \#458 \& } \\
\# 459 \text {. }\end{array}$ \\
\hline A1X02 & $09 / 15 / 88$ & $11: 00$ & 0.18 & 1353.458 & 0.000 & 0.000 & 34.19 & $\begin{array}{l}\text { Not fully evacuated. Do not use for } \\
\text { calculation. }\end{array}$ \\
\hline A1X02 & $09 / 27 / 88$ & $08: 30$ & 3.00 & 1365.354 & 76.958 & 0.041 & 37.19 & $\begin{array}{l}\text { Collected for chemistry, sample } \# 514 \text { - } \\
\# 519 . \text { Used } 3.18 \text { liters for calculation } \\
(0.18 \text { on } 9 / 15+3.00 \text { on } 9 / 27) \text {. }\end{array}$ \\
\hline A1X02 & $12 / 13 / 88$ & $09: 30$ & 2.50 & 1442.396 & 77.042 & .0 .032 & 39.69 & $\begin{array}{l}\text { Collected for chemistry, sample } \# 597 \text { - } \\
\# 601 \text {. }\end{array}$ \\
\hline A1X02 & $03 / 14 / 89$ & $09: 30$ & 2.96 & 1533.396 & 91.000 & 0.033 & 42.65 & \\
\hline A1X02 & 04/06/89 & $11: 55$ & NA & 1556.497 & 0.000 & 0.000 & 42.65 & Room locked. \\
\hline A1X02 & $04 / 20 / 89$ & $10: 00$ & NA & 1570.417 & 0.000 & 0.000 & 42.65 & Room locked. \\
\hline A1X02 & $05 / 17 / 89$ & $12: 05$ & 4.47 & 1597.503 & 64.107 & 0.070 & 47.12 & $\begin{array}{l}\text { Sample saved for chemistry, sample } \# 750 \text { - } \\
751 \text { A \& B. }\end{array}$ \\
\hline A1X02 & $07 / 11 / 89$ & $10: 05$ & 2.32 & 1652.420 & 54.917 & 0.042 & 49.44 & \\
\hline A1X02 & $09 / 12 / 89$ & $11: 35$ & 2.77 & 1715.483 & 63.063 & 0.044 & 52.21 & Sample saved for chemistry. \\
\hline A1X02 & $10 / 10 / 89$ & $09: 25$ & 1.57 & 1743.392 & 27.909 & 0.056 & 53.78 & Sample saved for chemistry, sample $\# 847$. \\
\hline A1X02 & $10 / 10 / 89$ & $10: 00$ & NA & 1743.417 & 0.000 & 0.000 & 53.78 & $\begin{array}{l}\text { Repositioned collecting tube from funnel. } \\
\text { Collection point for brine located outside } \\
\text { room. }\end{array}$ \\
\hline A1X02 & $10 / 20 / 89$ & $10: 44$ & NA & 1753.447 & 0.000 & 0.000 & 53.78 & No sample. \\
\hline A1X02 & $11 / 10 / 89$ & $10: 08$ & 1.90 & 1774.422 & 31.030 & 0.061 & 55.68 & $\begin{array}{l}\text { Sample saved for chemistry, sample } \\
\# 862-1,2,3,4 \text {. }\end{array}$ \\
\hline A1X02 & $11 / 29 / 89$ & $12: 10$ & 0.53 & 1793.507 & 19.085 & 0.028 & 56.21 & Sample saved for chemistry, sample $\# 873$. \\
\hline A1X02 & $12 / 12 / 89$ & $09: 20$ & 0.05 & 1806.389 & 12.882 & 0.004 & 56.26 & Sample saved for chemistry, sample $\# 884$. \\
\hline A1X02 & $01 / 04 / 90$ & $10: 50$ & 0.22 & 1829.451 & 23.062 & 0.010 & 56.48 & $\begin{array}{l}\text { Hose broken, some brine leaked to floor. } \\
\text { fixed hose, funnel full of brine. }\end{array}$ \\
\hline A $1 \times 02$ & 01/17/90 & $11: 35$ & 1.20 & 1842.483 & 13.032 & 0.092 & 57.68 & \\
\hline A1X02 & $01 / 31 / 90$ & $10: 27$ & 0.53 & 1856.435 & 13.952 & 0.038 & 58.21 & \\
\hline A1X02 & $02 / 13 / 90$ & $09: 53$ & 0.29 & 1869.412 & 12.977 & 0.022 & 58.50 & \\
\hline A1X02 & $02 / 27 / 90$ & $12: 17$ & 0.45 & 1883.512 & 14.100 & 0.032 & 58.95 & \\
\hline A1X02 & $03 / 05 / 90$ & $11: 11$ & 0.58 & 1889.466 & 5.954 & 0.097 & 59.53 & \\
\hline A1X02 & $03 / 21 / 90$ & $11: 26$ & 0.18 & 1905.476 & 16.010 & 0.011 & 59.71 & \\
\hline$A 1 \times 02$ & $04 / 06 / 90$ & $10: 40$ & 0.34 & 1921.444 & 15.968 & 0.021 & 60.05 & \\
\hline A1X02 & $04 / 17 / 90$ & $11: 53$ & 0.17 & 1932.495 & 11.051 & 0.015 & 60.22 & \\
\hline A $1 \times 02$ & $04 / 24 / 90$ & $10: 40$ & 0.01 & 1939.444 & 6.949 & 0.001 & 60.23 & \\
\hline
\end{tabular}


TABLE A-2 (Continued) BRINE ACCUMULATION DATA TABLE

Data through December 31, 1993

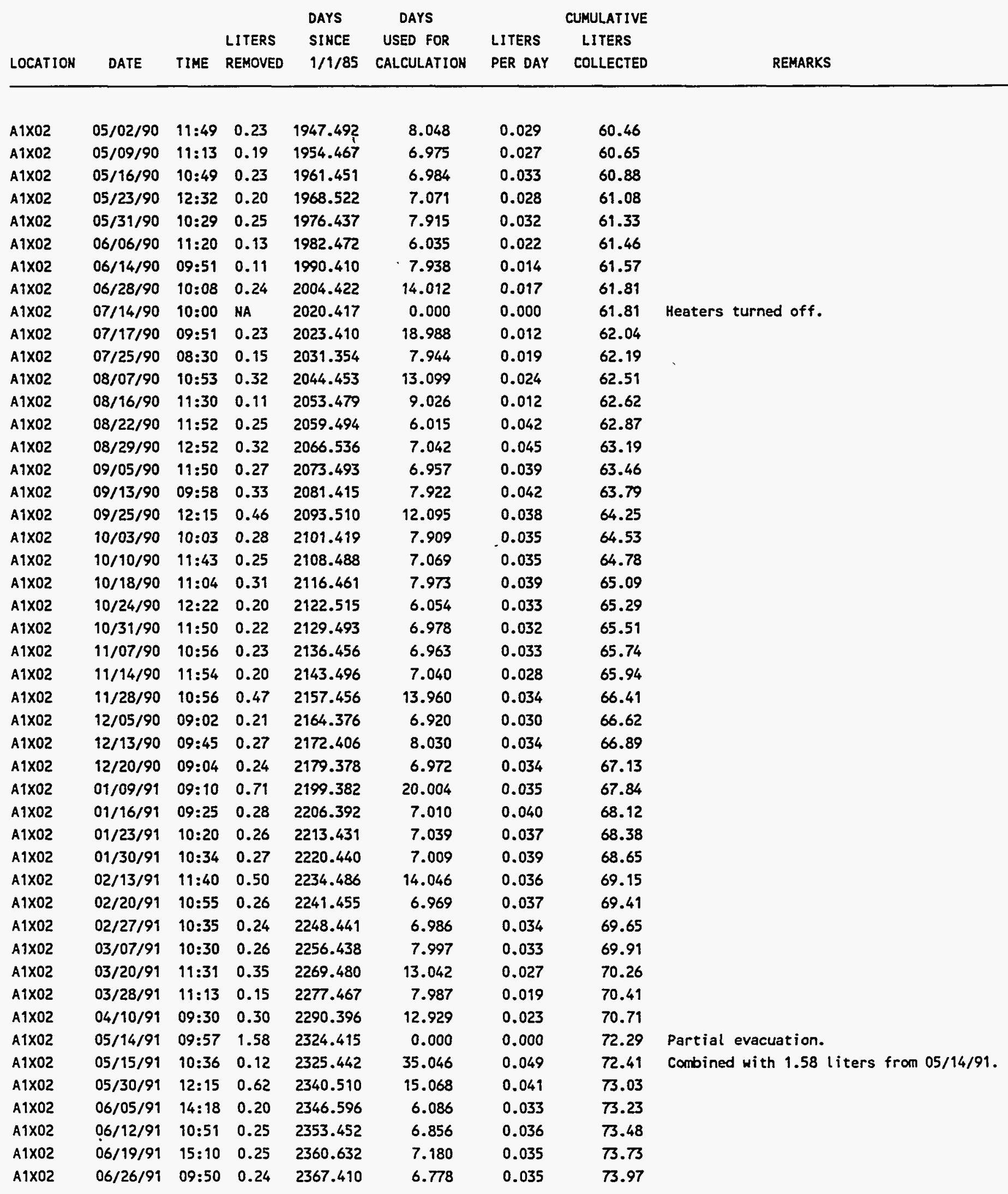


TABLE A-2 (Continued) BRINE ACCUMULATION DATA TABLE

Data through December 31, 1993

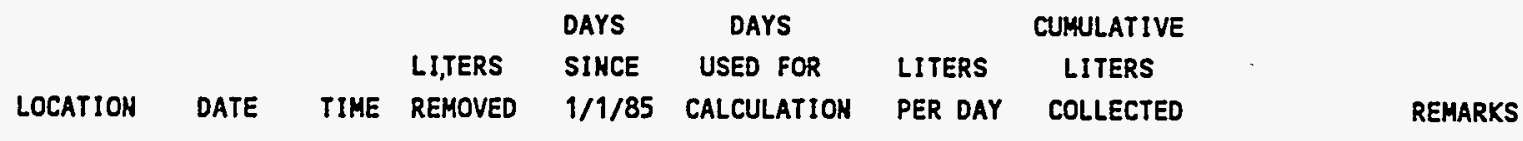

\begin{tabular}{|c|c|c|c|c|c|c|c|c|}
\hline A1X02 & $07 / 11 / 91$ & 11:01 & 0.65 & 2382.459 & 15.049 & 0.043 & 74.62 & \\
\hline$A 1 \times 02$ & $07 / 17 / 91$ & $10: 15$ & 0.26 & 2388.427 & 5.968 & 0.044 & 74.88 & \\
\hline A1X02 & $07 / 30 / 91$ & $09: 55$ & 0.49 & 2401.413 & 12.986 & 0.038 & 75.37 & \\
\hline A1X02 & $08 / 08 / 91$ & $08: 35$ & 0.32 & 2410.358 & 8.945 & 0.036 & 75.69 & \\
\hline$A 1 \times 02$ & $08 / 14 / 91$ & $09: 32$ & 0.15 & 2416.397 & 6.039 & 0.025 & 75.84 & \\
\hline A1X02 & $08 / 21 / 91$ & $09: 40$ & 0.39 & 2423.403 & 7.006 & 0.056 & 76.23 & \\
\hline A1X02 & $08 / 28 / 91$ & $09: 06$ & 0.29 & 2430.379 & 6.976 & 0.042 & 76.52 & \\
\hline A1X02 & $09 / 04 / 91$ & $10: 15$ & 0.16 & 2437.427 & 7.048 & 0.023 & 76.68 & - \\
\hline A1X02 & $09 / 11 / 91$ & $11: 15$ & 0.43 & 2444.469 & 7.042 & 0.061 & 77.11 & \\
\hline A1X02 & $10 / 02 / 91$ & $10: 30$ & 1.15 & 2465.438 & 20.969 & 0.055 & 78.26 & \\
\hline A1X02 & $10 / 16 / 91$ & $10: 35$ & 0.73 & 2479.441 & 14.003 & 0.052 & 78.99 & \\
\hline A1X02 & $10 / 31 / 91$ & $10: 28$ & 0.68 & 2494.436 & 14.995 & 0.045 & 79.67 & \\
\hline A1X02 & $11 / 06 / 91$ & $11: 40$ & 0.12 & 2500.486 & 6.050 & 0.020 & 79.79 & \\
\hline A1X02 & $11 / 13 / 91$ & $10: 10$ & 0.11 & 2507.424 & 6.938 & 0.016 & 79.90 & \\
\hline A1X02 & $11 / 20 / 91$ & $09: 45$ & 0.04 & 25.14 .406 & 6.982 & 0.006 & 79.94 & \\
\hline A1X02 & $11 / 27 / 91$ & $08: 55$ & 0.02 & 2521.372 & 6.966 & 0.003 & 79.96 & \\
\hline A1X02 & $12 / 04 / 91$ & $10: 25$ & 0.05 & 2528.434 & 7.062 & 0.007 & 80.01 & \\
\hline A1X02 & $12 / 11 / 91$ & $10: 20$ & 0.05 & 2535.431 & 6.997 & 0.007 & 80.06 & \\
\hline A1X02 & $12 / 18 / 91$ & $10: 20$ & 0.02 & 2542.431 & 7.000 & .0 .003 & 80.08 & \\
\hline A1X02 & $01 / 08 / 92$ & 09:09 & 0.04 & 2563.381 & 20.950 & 0.002 & 80.12 & \\
\hline A1X02 & 02/12/92 & $09: 20$ & 0.15 & 2598.389 & 35.008 & 0.004 & 80.27 & \\
\hline A1X02 & $02 / 26 / 92$ & $08: 55$ & 0.06 & 2612.372 & 13.983 & 0.004 & 80.33 & \\
\hline A1X02 & $04 / 22 / 92$ & $09: 57$ & 0.05 & 2668.415 & 56.043 & 0.001 & 80.38 & \\
\hline A1X02 & 05/06/92 & $10: 15$ & & 2682.427 & 14.012 & 0.000 & 80.38 & \\
\hline A1X02 & $05 / 21 / 92$ & $11: 06$ & 0.0 & 2697.463 & 15.036 & 0.000 & 80.38 & Bucket is empty. \\
\hline A $1 \times 02$ & $07 / 29 / 92$ & $10: 20$ & 0.22 & 2766.431 & 68.968 & 0.003 & 80.60 & \\
\hline A1X02 & $08 / 18 / 92$ & $09: 33$ & 0.52 & 2786.398 & 19.967 & 0.026 & 81.12 & $\begin{array}{l}\text { Hole has returned to normal, restriction } \\
\text { seems to have corrected itself. }\end{array}$ \\
\hline A1X02 & $09 / 02 / 92$ & $09: 35$ & 0.49 & 2801.399 & 15.001 & 0.033 & 81.61 & \\
\hline A1X02 & $09 / 09 / 92$ & $09: 32$ & 0.25 & 2808.397 & 6.998 & 0.036 & 81.86 & \\
\hline A1X02 & $09 / 17 / 92$ & $10: 00$ & 0.22 & 2816.417 & 8.020 & 0.027 & 82.08 & \\
\hline A1X02 & 09/23/92 & $09: 40$ & 0.01 & 2822.403 & 5.986 & 0.002 & 82.09 & \\
\hline A1X02 & $09 / 30 / 92$ & $10: 25$ & 0.01 & 2829.434 & 7.031 & 0.001 & 82.10 & \\
\hline A1X02 & $10 / 12 / 92$ & $13: 00$ & 0.05 & 2841.542 & 12.108 & 0.004 & 82.15 & \\
\hline A1X02 & 10/21/92 & $12: 35$ & Trace & 2850.524 & 0.000 & 0.000 & 82.15 & Did not remove trace. \\
\hline A1X02 & $10 / 28 / 92$ & $08: 40$ & Trace & 2857.361 & 0.000 & 0.000 & 82.15 & Did not remove trace. \\
\hline A1X02 & $11 / 25 / 92$ & $10: 05$ & Trace & 2885.420 & 0.000 & 0.000 & 82.15 & Did not remove trace. \\
\hline A $1 \times 02$ & $01 / 07 / 93$ & $09: 25$ & 0.12 & 2928.392 & 86.850 & 0.001 & 82.27 & \\
\hline A1X02 & $02 / 11 / 93$ & $09: 20$ & 0.00 & 2963.389 & 34.997 & 0.000 & 82.27 & Bucket empty. \\
\hline A1X02 & $04 / 28 / 93$ & $10: 45$ & 0.00 & 3039.448 & 76.059 & 0.000 & 82.27 & \\
\hline$A 1 \times 02$ & $06 / 16 / 93$ & $09: 35$ & 1.02 & 3088.399 & 48.951 & 0.021 & 83.29 & \\
\hline A $\times 02$ & $08 / 19 / 93$ & $13: 30$ & & 3152.563 & 0.000 & 0.000 & 83.29 & 0.50 liters of urine. Last time sampl \\
\hline
\end{tabular}

\begin{tabular}{|c|c|c|c|c|c|c|c|c|}
\hline$A 3 \times 01$ & $11 / 06 / 84$ & $00: 00$ & NA & 0.000 & 0.000 & 0.000 & 0.00 & Room A3 completed. \\
\hline A3X01 & $01 / 14 / 85$ & $00: 00$ & NA & 13.000 & 0.000 & 0.000 & 0.00 & Downhole drilled $12 / 20 / 85$ to $1 / 14 / 85$. \\
\hline A3X01 & $02 / 05 / 85$ & $11: 10$ & NA & 35.465 & 0.000 & 0.000 & 0.00 & Moist muck at the botton. \\
\hline
\end{tabular}


TABLE A-2 (Continued)

BRINE ACCUMULATION DATA TABLE

Data through December 31, 1993

\begin{tabular}{|c|c|c|c|c|c|c|c|c|}
\hline LOCATION & DATE & TIME & $\begin{array}{l}\text { LITERS } \\
\text { REMOVED }\end{array}$ & $\begin{array}{l}\text { DAYS } \\
\text { SINCE } \\
1 / 1 / 85\end{array}$ & $\begin{array}{l}\text { DAYS } \\
\text { USED FOR } \\
\text { CALCULATION }\end{array}$ & $\begin{array}{l}\text { LITERS } \\
\text { PER DAT }\end{array}$ & $\begin{array}{l}\text { CUMULATIVE } \\
\text { LITERS } \\
\text { COLLECTED }\end{array}$ & REMARKS \\
\hline$A 3 \times 01$ & $02 / 19 / 85$ & $13: 40$ & 00.30 & 49.569 & 14.104 & 0.021 & 0.30 & Some oil. First time collected. \\
\hline$A 3 \times 01$ & $02 / 26 / 85$ & $13: 20$ & 00.23 & 56.556 & 6.987 & 0.033 & 0.53 & Brine and oil. \\
\hline$A 3 \times 01$ & $03 / 07 / 85$ & $09: 45$ & 00.26 & 65.406 & 8.850 & 0.029 & 0.79 & \\
\hline$A 3 \times 01$ & $03 / 12 / 85$ & $11: 45$ & 00.17 & 70.490 & 5.084 & 0.033 & 0.96 & \\
\hline$A 3 \times 01$ & $03 / 20 / 85$ & $13: 14$ & 00.19 & 78.551 & 8.061 & 0.024 & 1.15 & $\begin{array}{l}\text { Valved leaked, some brine drained back down } \\
\text { hole. }\end{array}$ \\
\hline$A 3 \times 01$ & $03 / 26 / 85$ & $11: 12$ & 00.22 & 84.467 & 5.916 & 0.037 & 9.37 & \\
\hline$A 3 \times 01$ & $04 / 02 / 85$ & $12: 00$ & 00.21 & 91.500 & 7.033 & 0.030 & 1.58 & - \\
\hline$A 3 \times 01$ & $04 / 10 / 85$ & $12: 00$ & 00.23 & 99.500 & 8.000 & 0.029 & 1.81 & \\
\hline$A 3 \times 01$ & $04 / 17 / 85$ & $11: 20$ & 00.20 & 106.472 & 6.972 & 0.029 & 2.01 & \\
\hline A3X01 & $04 / 23 / 85$ & $10: 41$ & 00.16 & 112.445 & 5.973 & 0.027 & 2.17 & \\
\hline$A 3 \times 01$ & $04 / 30 / 85$ & $13: 35$ & 00.20 & 119.566 & 7.121 & 0.028 & 2.37 & \\
\hline A3X01 & $05 / 07 / 85$ & $08: 55$ & 00.20 & 126.372 & 6.806 & 0.029 & 2.57 & \\
\hline$A 3 \times 01$ & $05 / 14 / 85$ & $09: 56$ & 00.17 & 133.414 & 7.042 & 0.024 & 2.74 & \\
\hline$A 3 \times 01$ & $05 / 21 / 85$ & $12: 00$ & 00.20 & 140.500 & 7.086 & 0.028 & 2.94 & \\
\hline A3X01 & $05 / 29 / 85$ & $09: 25$ & 00.21 & 148.392 & 7.892 & 0.027 & 3.15 & \\
\hline$A 3 \times 01$ & $06 / 04 / 85$ & $09: 55$ & 00.16 & 154.413 & 6.021 & 0.027 & 3.31 & \\
\hline$A 3 \times 01$ & $06 / 11 / 85$ & $09: 25$ & 00.18 & 161.392 & 6.979 & 0.026 & 3.49 & \\
\hline$A 3 \times 01$ & $06 / 18 / 85$ & $09: 27$ & 00.18 & 168.394 & 7.002 & 0.026 & 3.67 & \\
\hline$A 3 \times 01$ & $06 / 25 / 85$ & $09: 30$ & 00.19 & 175.396 & 7.002 & 0.027 & 3.86 & \\
\hline$A 3 \times 01$ & $07 / 02 / 85$ & $11: 00$ & 00.19 & 182.458 & 7.062 & 0.027 & 4.05 & \\
\hline$A 3 \times 01$ & $07 / 09 / 85$ & $09: 50$ & 00.17 & 189.410 & 6.952 & 0.024 & 4.22 & \\
\hline$A 3 \times 01$ & $07 / 16 / 85$ & $10: 50$ & 00.18 & 196.451 & 7.041 & 0.026 & 4.40 & Brine effervesces. \\
\hline$A 3 \times 01$ & $07 / 24 / 85$ & $09: 47$ & 00.21 & 204.408 & 7.957 & 0.026 & 4.61 & \\
\hline$A 3 \times 01$ & $07 / 30 / 85$ & $09: 30$ & 00.15 & 210.396 & 5.988 & 0.025 & 4.76 & \\
\hline$A 3 \times 01$ & $08 / 06 / 85$ & $09: 30$ & 00.17 & 217.396 & 7.000 & 0.024 & 4.93 & \\
\hline$A 3 \times 01$ & $08 / 14 / 85$ & $09: 21$ & 00.20 & 225.390 & 7.994 & 0.025 & 5.13 & \\
\hline$A 3 \times 01$ & $08 / 20 / 85$ & $10: 08$ & 00.16 & 231.422 & 6.032 & 0.027 & 5.29 & \\
\hline A3X01 & $08 / 28 / 85$ & $09: 05$ & 00.21 & 239.378 & 7.956 & 0.026 & 5.50 & \\
\hline$A 3 \times 01$ & $09 / 04 / 85$ & $09: 29$ & 00.17 & 246.395 & 7.017 & 0.024 & 5.67 & \\
\hline$A 3 \times 01$ & $09 / 10 / 85$ & $09: 20$ & 00.15 & 252.389 & 5.994 & 0.025 & 5.82 & \\
\hline$A 3 \times 01$ & $09 / 17 / 85$ & $09: 06$ & 00.16 & 259.379 & 6.990 & 0.023 & 5.98 & \\
\hline$A 3 \times 01$ & $09 / 24 / 85$ & $09: 03$ & 00.17 & 266.377 & 6.998 & 0.024 & 6.15 & \\
\hline$A 3 \times 01$ & $10 / 01 / 85$ & $09: 18$ & 00.18 & 273.388 & 7.011 & 0.026 & 6.33 & \\
\hline$A 3 \times 01$ & $10 / 08 / 85$ & $12: 35$ & 00.18 & 280.524 & 7.136 & 0.025 & 6.51 & Room A3 heaters turned on $10 / 02 / 85$ \\
\hline A3X01 & $10 / 15 / 85$ & $09: 35$ & 00.16 & 287.399 & 6.875 & 0.023 & 6.67 & \\
\hline$A 3 \times 01$ & $10 / 23 / 85$ & $09: 40$ & 00.19 & 295.403 & 8.004 & 0.024 & 6.86 & \\
\hline A3X01 & $10 / 29 / 85$ & $11: 11$ & 00.14 & 301.466 & 6.063 & 0.023 & 7.00 & \\
\hline$A 3 \times 01$ & $11 / 05 / 85$ & $08: 42$ & 00.16 & 308.363 & 6.897 & 0.023 & 7.16 & \\
\hline$A 3 \times 01$ & $11 / 13 / 85$ & $09: 30$ & 00.19 & 316.396 & 8.033 & 0.024 & 7.35 & \\
\hline A3X01 & $11 / 21 / 85$ & $10: 30$ & 00.19 & 324.438 & 8.042 & 0.024 & 7.54 & \\
\hline A3X01 & $11 / 26 / 85$ & $09: 55$ & 00.10 & 329.413 & 4.975 & 0.020 & 7.64 & \\
\hline$A 3 \times 01$ & $12 / 04 / 85$ & $14: 03$ & 00.18 & 337.585 & 8.172 & 0.022 & 7.82 & Sample for chemical analysis, \#10. \\
\hline$A 3 \times 01$ & $12 / 10 / 85$ & $10: 46$ & 00.14 & 343.449 & 5.864 & 0.024 & 7.96 & \\
\hline$A 3 \times 01$ & $12 / 17 / 85$ & $13: 55$ & 00.14 & 350.580 & 7.131 & 0.020 & 8.10 & \\
\hline$A 3 \times 01$ & $01 / 03 / 86$ & $10: 00$ & 00.39 & 367.417 & 16.837 & 0.023 & 8.49 & \\
\hline$A 3 \times 01$ & $01 / 08 / 86$ & $10: 10$ & 00.11 & 372.424 & 5.007 & 0.022 & 8.60 & \\
\hline
\end{tabular}


TABLE A-2 (Continued) BRINE ACCUMULATION DATA TABLE

Data through December 31, 1993

\begin{tabular}{|c|c|c|c|c|c|c|c|c|}
\hline & & & & DAYS & DAYS & & CUMULATIVE & \\
\hline & & & LITERS & SINCE & USED FOR & LITERS & LITERS & \\
\hline LOCATION & DATE & TIME & REMOVED & $1 / 1 / 85$ & CALCULATION & PER DAY & COLLECTED & REMARKS \\
\hline
\end{tabular}

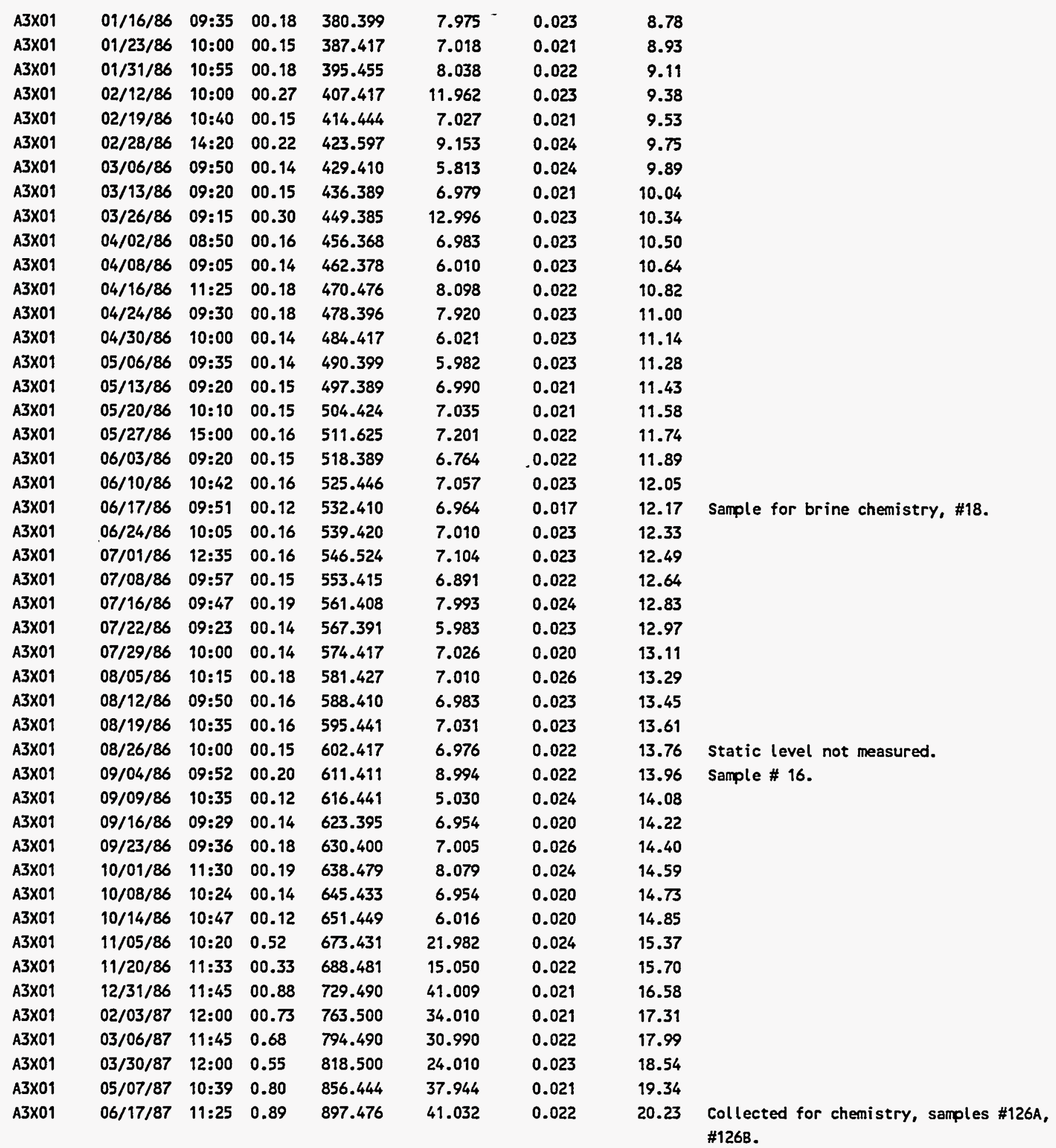


TABLE A-2 (Continued)

\section{BRINE ACCUMULATION DATA TABLE}

Data through December 31, 1993

\begin{tabular}{|c|c|c|c|c|c|c|c|c|}
\hline LOCATION & DATE & TIME & $\begin{array}{l}\text { LITERS } \\
\text { REMOVED }\end{array}$ & $\begin{array}{l}\text { DAYS } \\
\text { SINCE } \\
1 / 1 / 85\end{array}$ & $\begin{array}{l}\text { DAYS } \\
\text { USED FOR } \\
\text { CALCULATION }\end{array}$ & $\begin{array}{l}\text { LITERS } \\
\text { PER DAY }\end{array}$ & $\begin{array}{l}\text { CUMULATIVE } \\
\text { LITERS } \\
\text { COLLECTED }\end{array}$ & REMARKS \\
\hline A3X01 & $07 / 28 / 87$ & $12: 02$ & 0.92 & 938.501 & 41.025 & 0.022 & 21.15 & \\
\hline$A 3 \times 01$ & $09 / 01 / 87$ & $91: 45$ & 0.77 & 973.490 & 34.989 & 0.022 & 21.92 & Collected for chemistry, sample \#172 A\&B. \\
\hline A3X01 & $10 / 20 / 87$ & $10: 55$ & 1.10 & 1022.455 & 48.965 & 0.022 & 23.02 & \\
\hline A3X01 & $11 / 19 / 87$ & $10: 20$ & 0.66 & 1052.431 & 29.976 & 0.022 & 23.68 & Collected for chemistry, sample $\# 220$. \\
\hline A3X01 & $01 / 04 / 88$ & $11: 00$ & 1.01 & 1098.458 & 46.027 & 0.022 & 24.69 & \\
\hline A3X01 & $02 / 08 / 88$ & $13: 30$ & 0.67 & 1133.563 & 35.105 & 0.019 & 25.36 & $\begin{array}{l}\text { Collected for chemistry, sample } \# 297 \text { \& } \\
\# 298 \text {. }\end{array}$ \\
\hline$A 3 \times 01$ & $03 / 30 / 88$ & $12: 10$ & 1.02 & 1984.507 & 50.944 & 0.020 & 26.38 & $\begin{array}{l}\text { Collected for chemistry, sample } \$ 387 \text { \& } \\
\$ 388 \text {. }\end{array}$ \\
\hline$A 3 \times 01$ & $05 / 12 / 88$ & $10: 20$ & 0.88 & 1227.431 & 42.924 & 0.021 & 27.26 & Sampled for SHL/HH PA. \\
\hline$A 3 \times 01$ & $07 / 12 / 88$ & $09: 40$ & 1.28 & 1288.403 & 60.972 & 0.021 & 28.54 & $\begin{array}{l}\text { Collected for chemistry, sample } \# 456 \text { \& } \\
\# 457 \text {. }\end{array}$ \\
\hline$A 3 \times 01$ & $09 / 27 / 88$ & $08: 20$ & & 1365.347 & 0.000 & 0.000 & 28.54 & Cannot be sampled. Room has bad back. \\
\hline$A 3 \times 01$ & $12 / 13 / 88$ & $09: 25$ & 3.35 & 1442.392 & 153.989 & 0.022 & 31.89 & $\begin{array}{l}\text { Collected for chemistry, sample } \# 591 \text { - } \\
\# 596 \text {. }\end{array}$ \\
\hline A3X01 & $03 / 14 / 89$ & $09: 15$ & 1.90 & 1533.385 & 90.993 & 0.021 & 33.79 & Collected for chemistry, sample $\# 656$ - 659. \\
\hline$A 3 \times 01$ & $04 / 06 / 89$ & $12: 04$ & HA & 1556.503 & 0.000 & 0.000 & 33.79 & Room locked. \\
\hline A3X01 & $04 / 20 / 89$ & $10: 00$ & MA & 1570.417 & 0.000 & 0.000 & 33.79 & Room locked. \\
\hline$A 3 \times 01$ & $05 / 17 / 89$ & $11: 45$ & 1.42 & 1597.490 & 64.105 & 0.022 & 35.21 & Collected for chemistry, sample \#758 A \& B. \\
\hline$A 3 \times 01$ & $07 / 11 / 89$ & $09: 55$ & 0.93 & 1652.413 & 54.923 & 0.017 & 36.14 & \\
\hline A3X01 & $09 / 12 / 89$ & $11: 26$ & 1.51 & 1715.476 & 63.063 & 0.024 & 37.65 & Sample saved for chemistry. \\
\hline A3X01 & $10 / 10 / 89$ & $09: 43$ & NA & 1743.405 & 0.000 & 0.000 & 37.65 & $\begin{array}{l}\text { Installed collection device. Collection } \\
\text { point for brine located outside room. }\end{array}$ \\
\hline$A 3 \times 01$ & $10 / 20 / 89$ & $10: 39$ & 0.36 & 1753.444 & 37.968 & 0.009 & 38.01 & Collected for chemistry, sample $\$ 850$. \\
\hline A3X01 & $11 / 10 / 89$ & $09: 40$ & 0.50 & 1774.403 & 20.959 & 0.024 & 38.51 & Collected for chemistry, sample $\# 860-1$. \\
\hline A3X01 & $11 / 29 / 89$ & $11: 56$ & 0.63 & 1793.497 & 19.094 & 0.033 & 39.14 & Collected for chemistry, sample $\# 871$. \\
\hline$A 3 \times 01$ & $12 / 12 / 89$ & $09: 00$ & 0.43 & 1806.375 & 12.878 & 0.033 & 39.57 & Collected for chemistry, sample \#882. \\
\hline A3X01 & $01 / 04 / 90$ & $10: 00$ & 0.50 & 1829.417 & 23.042 & 0.022 & 40.07 & \\
\hline A3X01 & $01 / 17 / 90$ & $11: 24$ & 0.25 & 1842.475 & 13.058 & 0.019 & 40.32 & \\
\hline A3X01 & $01 / 31 / 90$ & $09: 40$ & 0.24 & 1856.403 & 13.928 & 0.017 & 40.56 & \\
\hline$A 3 \times 01$ & $02 / 13 / 90$ & $09: 21$ & 0.31 & 1869.390 & 12.987 & 0.024 & 40.87 & \\
\hline$A 3 \times 01$ & $02 / 27 / 90$ & $11: 43$ & 0.32 & 1883.488 & 14.098 & 0.023 & 41.19 & \\
\hline A3X01 & $03 / 05 / 90$ & $10: 45$ & 0.30 & 1889.448 & 5.960 & 0.050 & 41.49 & \\
\hline$A 3 \times 01$ & $03 / 21 / 90$ & $11: 15$ & 0.15 & 1905.469 & 16.021 & 0.005 & 41.64 & Brine probably left in hole. \\
\hline$A 3 \times 01$ & $04 / 06 / 90$ & $10: 29$ & 0.35 & 1921.437 & 15.968 & 0.022 & 41.99 & \\
\hline$A 3 \times 01$ & $04 / 17 / 90$ & $11: 13$ & 0.13 & 1932.467 & 11.030 & 0.012 & 42.12 & \\
\hline$A 3 \times 01$ & $04 / 24 / 90$ & $10: 26$ & 0.02 & 1939.435 & 0.000 & 0.000 & 42.14 & \\
\hline$A 3 \times 01$ & $04 / 25 / 90$ & $09: 35$ & 0.15 & 1940.399 & 7.932 & 0.021 & 42.29 & $\begin{array}{l}\text { Reinstalled sampler. Combined with } 0.02 \\
\text { liters from } 04 / 24 / 90 \text {. Used } 0.17 \text { liters for } \\
\text { calculation. }\end{array}$ \\
\hline A3X01 & $05 / 02 / 90$ & $11: 20$ & 0 & 1947.472 & 0.000 & 0.000 & 42.29 & Could not sample. \\
\hline$A 3 \times 01$ & $05 / 16 / 90$ & $10: 26$ & NA & 1961.435 & 0.000 & 0.000 & 42.29 & Sampler malfunction. \\
\hline$A 3 \times 01$ & $05 / 23 / 90$ & $12: 35$ & 0.08 & 1968.524 & 28.125 & 0.003 & 42.37 & \\
\hline A3X01 & $05 / 31 / 90$ & $10: 51$ & 0.14 & 1976.452 & 7.928 & 0.018 & 42.51 & \\
\hline$A 3 \times 01$ & $06 / 01 / 90$ & $10: 25$ & HA & 1977.434 & 0.000 & 0.000 & 42.51 & Replaced sampler. \\
\hline$A 3 \times 01$ & $06 / 06 / 90$ & $11: 06$ & 0.49 & 1982.463 & 6.011 & $0.08 ?$ & 43.00 & \\
\hline$A 3 \times 01$ & $06 / 14 / 90$ & $08: 38$ & 0.17 & 1990.360 & 7.897 & 0.022 & 43.17 & \\
\hline
\end{tabular}




\section{TABLE A-2 (Continued) BRINE ACCUMULATION DATA TABLE}

Data through December 31, 1993

\begin{tabular}{|c|c|c|c|c|c|c|c|c|}
\hline LOCATION & DATE & TIME & $\begin{array}{l}\text { LITERS } \\
\text { REMOVED }\end{array}$ & $\begin{array}{l}\text { DAYS } \\
\text { SINCE } \\
1 / 1 / 85\end{array}$ & $\begin{array}{l}\text { DAYS } \\
\text { USED FOR } \\
\text { CALCULATION }\end{array}$ & $\begin{array}{l}\text { LITERS } \\
\text { PER DAY }\end{array}$ & $\begin{array}{l}\text { CUHULATIVE } \\
\text { LITERS } \\
\text { COLLECTED }\end{array}$ & REMARKS \\
\hline$A 3 \times 01$ & $07 / 17 / 90$ & $10: 18$ & 0.60 & 2023.429 & 0.000 & 0.000 & 43.77 & \\
\hline$A 3 \times 01$ & $07 / 18 / 90$ & $10: 11$ & 0.09 & 2024.424 & 34.064 & 0.020 & 43.86 & $\begin{array}{l}\text { Combined with } 0.60 \text { liters from } 07 / 17 / 90 \text {. } \\
\text { Used } 0.69 \text { liters for calculation. }\end{array}$ \\
\hline$A 3 \times 01$ & $08 / 07 / 90$ & $11: 21$ & 0.24 & 2044.473 & 20.049 & 0.047 & 44.80 & $\begin{array}{l}\text { Combined with } 0.7 \text { liters from } 07 / 25 / 90 \text {. } \\
\text { Used } 0.94 \text { liters for calculation. }\end{array}$ \\
\hline$A 3 \times 01$ & $08 / 16 / 90$ & $11: 11$ & 0.27 & 2053.466 & 8.993 & 0.030 & 45.07 & \\
\hline$A 3 \times 01$ & $08 / 22 / 90$ & $11: 42$ & 0.15 & 2059.488 & 6.022 & 0.025 & 45.22 & \\
\hline A3X01 & $08 / 23 / 90$ & $10: 00$ & HA & 2060.417 & 0.000 & 0.000 & 45.22 & Heaters turned off. \\
\hline$A 3 \times 01$ & $09 / 25 / 90$ & $12: 34$ & 0.25 & 2093.524 & 12.110 & 0.021 & 45.96 & \\
\hline$A 3 \times 01$ & $09 / 26 / 90$ & $11: 09$ & 0.02 & 2094.465 & 0.941 & 0.021 & 45.98 & \\
\hline$A 3 \times 01$ & $10 / 03 / 90$ & $09: 50$ & 0.16 & 2101.410 & 6.945 & 0.023 & 46.14 & \\
\hline$A 3 \times 01$ & $10 / 10 / 90$ & $11: 40$ & 0.15 & 2108.486 & 7.076 & 0.021 & 46.29 & \\
\hline$A 3 \times 01$ & $10 / 18 / 90$ & $10: 53$ & 0.16 & 2116.453 & 7.967 & 0.020 & 46.45 & \\
\hline$A 3 \times 01$ & $10 / 24 / 90$ & $12: 08$ & 0.14 & 2122.506 & 6.053 & 0.023 & 46.59 & \\
\hline$A 3 \times 01$ & $10 / 31 / 90$ & $11: 35$ & 0.16 & 2129.483 & 6.977 & 0.023 & 46.75 & \\
\hline$A 3 \times 01$ & $11 / 07 / 90$ & $10: 52$ & 0.15 & 2136.453 & 6.970 & 0.022 & 46.90 & \\
\hline$A 3 \times 01$ & $11 / 14 / 90$ & $11: 50$ & 0.15 & 2143.493 & 7.040 & 0.021 & 47.05 & \\
\hline$A 3 \times 01$ & $11 / 28 / 90$ & $10: 51$ & 0.30 & 2157.452 & 13.959 & 0.021 & 47.35 & \\
\hline$A 3 \times 01$ & $12 / 05 / 90$ & $08: 55$ & 0.15 & 2164.372 & 6.920 & 0.022 & 47.50 & \\
\hline$A 3 \times 01$ & $02 / 20 / 91$ & $10: 45$ & 0.16 & 2241.448 & 6.974 & 0.023 & 49.23 & \\
\hline$A 3 \times 01$ & $02 / 27 / 91$ & $10: 20$ & 0.14 & 2248.431 & 6.983 & 0.020 & 49.37 & \\
\hline$A 3 \times 01$ & $03 / 07 / 91$ & $10: 15$ & 0.26 & 2256.427 & 7.996 & 0.033 & 49.63 & \\
\hline$A 3 \times 01$ & $03 / 20 / 91$ & $11: 21$ & 0.28 & 2269.473 & 13.046 & 0.021 & 49.91 & \\
\hline$A 3 \times 01$ & $03 / 28 / 91$ & $11: 07$ & 0.18 & 2277.463 & 7.990 & 0.023 & 50.09 & \\
\hline$A 3 \times 01$ & $04 / 10 / 91$ & $09: 19$ & 0.26 & 2290.388 & 12.925 & 0.020 & 50.35 & \\
\hline$A 3 \times 01$ & $05 / 14 / 91$ & $09: 50$ & 0.34 & 2324.410 & 0.000 & 0.000 & 50.69 & Partial evacuation. \\
\hline A3X01 & $05 / 15 / 91$ & $10: 20$ & 0.30 & 2325.431 & 35.043 & 0.018 & 50.99 & Combined with 0.34 liters from $05 / 14 / 91$. \\
\hline A3X01 & $05 / 30 / 91$ & $11: 45$ & 0.31 & 2340.490 & 15.059 & 0.021 & 51.30 & \\
\hline A3X01 & 06/05/91 & $14: 22$ & 0.16 & 2346.599 & 6.109 & 0.026 & 51.46 & \\
\hline A3X01 & $06 / 12 / 91$ & $10: 50$ & 0.15 & 2353.451 & 6.852 & 0.022 & 51.61 & \\
\hline$A 3 \times 01$ & $06 / 19 / 91$ & $15: 12$ & 0.15 & 2360.633 & 7.182 & 0.021 & 51.76 & \\
\hline$A 3 \times 01$ & $06 / 26 / 91$ & $09: 45$ & 0.14 & 2367.406 & 6.773 & 0.021 & 51.90 & \\
\hline$A 3 \times 01$ & $07 / 11 / 91$ & $11: 16$ & 0.30 & 2382.469 & 15.063 & 0.020 & 52.20 & \\
\hline$A 3 \times 01$ & $07 / 17 / 91$ & $10: 10$ & 0.15 & 2388.424 & 5.955 & 0.025 & 52.35 & \\
\hline$A 3 \times 01$ & $07 / 30 / 91$ & $09: 50$ & 0.40 & 2401.410 & 12.986 & 0.031 & 52.75 & \\
\hline$A 3 \times 01$ & $08 / 08 / 91$ & $08: 30$ & 0.24 & 2410.354 & 8.944 & 0.027 & 52.99 & \\
\hline
\end{tabular}


TABLE A-2 (Continued)

\section{BRINE ACCUMULATION DATA TABLE}

Data through December 31, 1993

\begin{tabular}{|c|c|c|c|c|c|c|c|c|}
\hline LOCATION & DATE & TIME & $\begin{array}{l}\text { LITERS } \\
\text { REMOVED }\end{array}$ & $\begin{array}{l}\text { DAYS } \\
\text { SINCE } \\
1 / 1 / 85\end{array}$ & $\begin{array}{l}\text { DAYS } \\
\text { USED FOR } \\
\text { CALCULATION }\end{array}$ & $\begin{array}{l}\text { LITERS } \\
\text { PER DAY }\end{array}$ & $\begin{array}{l}\text { CUMULATIVE } \\
\text { LITERS } \\
\text { COLLECTEO }\end{array}$ & REMARKS \\
\hline$A 3 \times 01$ & $08 / 14 / 91$ & $09: 25$ & 0.13 & 2416.392 & 6.038 & 0.022 & 53.12 & \\
\hline$A 3 \times 01$ & $08 / 21 / 91$ & $09: 22$ & 0.15 & 2423.390 & 6.998 & 0.021 & 53.27 & \\
\hline$A 3 \times 01$ & $08 / 28 / 91$ & $08: 53$ & 0.13 & 2430.370 & 6.980 & 0.019 & 53.40 & \\
\hline$A 3 \times 01$ & $09 / 04 / 91$ & $10: 14$ & 0.22 & 2437.426 & 7.056 & 0.031 & 53.62 & \\
\hline$A 3 \times 01$ & $09 / 11 / 91$ & $11: 10$ & 0.17 & 2444.465 & 7.039 & 0.024 & 53.79 & \\
\hline$A 3 \times 01$ & $10 / 02 / 91$ & $10: 25$ & 0.13 & 2465.434 & 20.969 & 0.006 & 53.92 & \\
\hline$A 3 \times 01$ & $10 / 16 / 91$ & $10: 25$ & 0.48 & 2479.434 & 14.000 & 0.034 & 54.40 & \\
\hline$A 3 \times 01$ & $10 / 31 / 91$ & $10: 40$ & 0.01 & 2494.444 & 0.000 & 0.000 & 54.41 & $\begin{array}{l}\text { Some brine may have been left in hole. } \\
\text { Lost vacuum prior to sampling. }\end{array}$ \\
\hline$A 3 \times 01$ & $11 / 06 / 91$ & $11: 35$ & 0.06 & 2500.483 & 0.000 & 0.000 & 54.47 & $\begin{array}{l}\text { Some brine may have been left in hole. } \\
\text { Line clogged. }\end{array}$ \\
\hline$A 3 \times 01$ & $11 / 20 / 91$ & $9: 30$ & 0.53 & 2514.375 & 34.941 & 0.017 & 55.00 & $\begin{array}{l}\text { Combined with } 0.01 \text { liters from } 10 / 31 / 91 \text { and } \\
0.06 \text { liters from } 11 / 06 / 91 \text {. }\end{array}$ \\
\hline$A 3 \times 01$ & $11 / 27 / 91$ & $09: 04$ & 0.21 & 2521.378 & 7.003 & 0.030 & 55.21 & \\
\hline$A 3 \times 01$ & $12 / 04 / 91$ & $10: 20$ & 0.17 & 2528.431 & 7.053 & 0.024 & 55.38 & \\
\hline$A 3 \times 01$ & $12 / 11 / 91$ & $10: 15$ & 0.13 & 2535.427 & 6.996 & 0.019 & 55.51 & \\
\hline$A 3 \times 01$ & $12 / 18 / 91$ & $10: 15$ & 0.17 & 2542.427 & 7.000 & 0.024 & 55.68 & \\
\hline$A 3 \times 01$ & $12 / 23 / 91$ & $09: 10$ & 0.14 & 2547.382 & 4.955 & 0.028 & 55.82 & \\
\hline$A 3 \times 01$ & $01 / 08 / 92$ & $09: 05$ & 0.15 & 2563.378 & 15.996 & .0 .009 & 55.97 & \\
\hline$A 3 \times 01$ & $01 / 15 / 92$ & $09: 21$ & 0.35 & 2570.390 & 7.012 & 0.050 & 56.32 & \\
\hline$A 3 \times 01$ & $01 / 22 / 92$ & $09: 45$ & 0.15 & 2577.406 & 7.016 & 0.021 & 56.47 & \\
\hline$A 3 \times 01$ & $01 / 29 / 92$ & $10: 26$ & 0.14 & 2584.435 & 7.029 & 0.020 & 56.61 & \\
\hline$A 3 \times 01$ & $02 / 12 / 92$ & $09: 15$ & 0.38 & 2598.385 & 13.950 & 0.027 & 56.99 & \\
\hline$A 3 \times 01$ & $02 / 19 / 92$ & $10: 00$ & 0.14 & 2605.417 & 7.032 & 0.020 & 57.13 & \\
\hline$A 3 \times 01$ & $02 / 26 / 92$ & $08: 45$ & 0.15 & 2612.365 & 6.948 & 0.022 & 57.28 & \\
\hline$A 3 \times 01$ & $03 / 11 / 92$ & $08: 55$ & 0.28 & 2626.372 & 14.007 & 0.020 & 57.56 & \\
\hline$A 3 \times 01$ & $03 / 18 / 92$ & $08: 40$ & 0.16 & 2633.361 & 6.989 & 0.023 & 57.72 & \\
\hline$A 3 \times 01$ & $03 / 25 / 92$ & $09: 55$ & 0.14 & 2640.413 & 7.052 & 0.020 & 57.86 & \\
\hline A3X01 & $04 / 01 / 92$ & $09: 50$ & 0.15 & 2647.410 & 6.997 & 0.021 & 58.01 & \\
\hline$A 3 \times 01$ & $04 / 07 / 92$ & $09: 55$ & 0.12 & 2653.413 & 6.003 & 0.020 & 58.13 & \\
\hline$A 3 \times 01$ & $04 / 15 / 92$ & $08: 35$ & 0.16 & 2661.358 & 7.945 & 0.020 & 58.29 & \\
\hline A3X01 & $04 / 22 / 92$ & $09: 55$ & 0.15 & 2668.413 & 7.055 & 0.021 & 58.44 & \\
\hline$A 3 \times 01$ & $05 / 06 / 92$ & $10: 29$ & 0.27 & 2682.437 & 14.024 & 0.019 & 58.71 & \\
\hline$A 3 \times 01$ & $05 / 13 / 92$ & $13: 15$ & 0.17 & 2689.552 & 7.115 & 0.024 & 58.88 & \\
\hline$A 3 \times 01$ & $05 / 21 / 92$ & $10: 57$ & 0.15 & 2697.456 & 7.904 & 0.019 & 59.03 & \\
\hline$A 3 \times 01$ & $05 / 27 / 92$ & $09: 40$ & 0.12 & 2703.403 & 5.947 & 0.020 & 59.15 & \\
\hline$A 3 \times 01$ & $06 / 09 / 92$ & $09: 50$ & 0.25 & 2716.410 & 13.007 & 0.019 & 59.40 & \\
\hline$A 3 \times 01$ & $06 / 18 / 92$ & $08: 45$ & 0.19 & 2725.365 & 8.955 & 0.021 & 59.59 & \\
\hline$A 3 \times 01$ & $06 / 25 / 92$ & $10: 15$ & 0.14 & 2732.427 & 7.062 & 0.020 & 59.73 & \\
\hline$A 3 \times 01$ & $07 / 01 / 92$ & $09: 04$ & 0.12 & 2738.378 & 5.951 & 0.020 & 59.85 & \\
\hline$A 3 \times 01$ & $07 / 08 / 92$ & $09: 50$ & 0.14 & 2745.410 & 7.032 & 0.020 & 59.99 & \\
\hline$A 3 \times 01$ & $07 / 15 / 92$ & $09: 01$ & 0.15 & 2752.376 & 6.966 & 0.022 & 60.14 & \\
\hline$A 3 \times 01$ & $07 / 22 / 92$ & $10: 15$ & 0.14 & 2759.427 & 7.051 & 0.020 & 60.28 & \\
\hline$A 3 \times 01$ & $07 / 29 / 92$ & $10: 15$ & 0.14 & 2766.427 & 7.000 & 0.020 & 60.42 & \\
\hline$A 3 \times 01$ & $08 / 04 / 92$ & $09: 11$ & 0.14 & 2772.383 & 5.956 & 0.026 & 60.56 & \\
\hline$A 3 \times 01$ & $08 / 18 / 92$ & $09: 30$ & 0.25 & 2786.396 & 14.013 & 0.018 & 60.81 & \\
\hline$A 3 \times 01$ & $09 / 02 / 92$ & $09: 30$ & 0.30 & 2801.396 & 15.000 & 0.020 & 61.11 & \\
\hline
\end{tabular}


TABLE A-2 (Continued)

BRINE ACCUMULATION DATA TABLE

Data through December 31, 1993

\begin{tabular}{|c|c|c|c|c|c|c|c|}
\hline & - & & & DAYS & DAYS & & CUMULATIVE \\
\hline & & & & SINCE & USED FOR & LITERS & LITERS \\
\hline
\end{tabular}

\begin{tabular}{|c|c|c|c|c|c|c|c|c|}
\hline A3X01 & $09 / 09 / 92$ & $09: 30$ & 0.15 & 2808.396 & 7.000 & 0.021 & 61.26 & \\
\hline$A 3 \times 01$ & $09 / 17 / 92$ & $09: 55$ & 0.15 & 2816.413 & 8.017 & $0.019^{\circ}$ & 61.41 & \\
\hline$A 3 \times 01$ & $09 / 23 / 92$ & $09: 45$ & 0.14 & 2822.406 & 5.993 & 0.023 & 61.55 & \\
\hline$A 3 \times 01$ & $09 / 30 / 92$ & $10: 20$ & 0.13 & 2829.431 & 7.025 & 0.019 & 69.68 & \\
\hline A3X01 & $10 / 12 / 92$ & $12: 59$ & 0.25 & 2841.541 & 12.110 & 0.021 & 61.93 & \\
\hline$A 3 \times 01$ & $10 / 21 / 92$ & $12: 31$ & 0.16 & 2850.522 & 8.981 & 0.018 & 62.09 & \\
\hline$A 3 \times 01$ & $10 / 28 / 92$ & $08: 45$ & 0.14 & 2857.365 & 6.843 & 0.020 & 62.23 & \\
\hline$A 3 \times 01$ & $11 / 11 / 92$ & $10: 45$ & 0.26 & 2871.448 & 14.083 & 0.018 & 62.49 & \\
\hline$A 3 \times 01$ & $11 / 18 / 92$ & $12: 50$ & 0.15 & 2878.535 & 7.087 & 0.021 & 62.64 & \\
\hline A3X01 & $11 / 25 / 92$ & $10: 00$ & 0.05 & 2885.417 & 6.882 & 0.007 & 62.69 & \\
\hline A3X01 & $12 / 09 / 92$ & $13: 20$ & 0.36 & 2899.556 & 14.139 & 0.025 & 63.05 & \\
\hline$A 3 \times 01$ & $12 / 16 / 92$ & $09: 45$ & 0.14 & 2906.406 & 6.850 & 0.020 & 63.19 & \\
\hline A3X01 & $01 / 07 / 93$ & $09: 20$ & 0.37 & 2928.389 & 21.983 & 0.017 & 63.56 & \\
\hline$A 3 \times 01$ & $01 / 13 / 93$ & $09: 20$ & 0.15 & 2934.389 & 6.000 & 0.025 & 63.71 & \\
\hline$A 3 \times 01$ & $01 / 28 / 93$ & $09: 46$ & 0.29 & 2949.407 & 15.018 & 0.019 & 64.00 & \\
\hline A3X01 & $02 / 11 / 93$ & $09: 20$ & 0.00 & 2963.389 & 0.000 & 0.000 & 64.00 & Ho suction. \\
\hline$A 3 \times 01$ & $02 / 26 / 93$ & & 0.00 & 2978.000 & 0.000 & 0.000 & 64.00 & Ho vacuum. \\
\hline A3X01 & $03 / 19 / 93$ & $10: 35$ & 0.15 & 2999.441 & 0.000 & 0.000 & 64.15 & Partial evacuation. \\
\hline A3X01 & $03 / 25 / 93$ & $09: 30$ & 0.13 & 3005.396 & 55.989 & .0 .005 & 64.28 & Combine with 0.15 liters from 03-19-93. \\
\hline A3X01 & $03 / 31 / 93$ & $12: 10$ & 0.14 & 3011.507 & 6.111 & 0.023 & 64.42 & \\
\hline A3X01 & $06 / 16 / 93$ & $09: 45$ & 0.34 & 3088.406 & 76.899 & 0.004 & 64.76 & Last time sampled. \\
\hline $8 \times 01$ & $06 / 02 / 84$ & $00: 00$ & ' $\mathrm{HA}$ & 0.000 & 0.000 & 0.000 & 0.00 & Room B completed. \\
\hline BX01 & $01 / 27 / 85$ & $00: 00$ & NA & 26.000 & 0.000 & 0.000 & 0.00 & $\begin{array}{l}\text { Downhole drilled } 1 / 24 / 85 \text { to } 1 / 27 / 85 \text {. Wet } \\
\text { core and brine encountered } 1 / 26 / 85 \text { at } 35 \text { to } \\
36.5 \text { feet. }\end{array}$ \\
\hline BX01 & $02 / 05 / 85$ & $11: 00$ & 00.39 & 35.458 & 248.458 & 0.002 & 0.39 & First time collected. \\
\hline BX01 & $02 / 11 / 85$ & $12: 00$ & 00.72 & 41.500 & 6.042 & 0.119 & 1.11 & \\
\hline BX01 & $02 / 19 / 85$ & $13: 00$ & 00.70 & 49.542 & 8.042 & 0.087 & 1.81 & \\
\hline BX01 & $02 / 26 / 85$ & $12: 45$ & 00.61 & 56.531 & 6.989 & 0.087 & 2.42 & \\
\hline BX01 & $03 / 07 / 85$ & $09: 15$ & 00.70 & 65.385 & 8.854 & 0.079 & 3.12 & \\
\hline BX01 & $03 / 12 / 85$ & $11: 45$ & 00.41 & 70.490 & 5.105 & 0.080 & 3.53 & \\
\hline $8 \times 01$ & $03 / 20 / 85$ & $12: 50$ & 00.61 & 78.535 & 8.045 & 0.076 & 4.14 & \\
\hline 8X01 & $03 / 26 / 85$ & $10: 45$ & 00.45 & 84.448 & 5.913 & 0.076 & 4.59 & \\
\hline BX01 & $04 / 02 / 85$ & $11: 44$ & 00.51 & 91.489 & 7.041 & 0.072 & 5.10 & - \\
\hline BX01 & $04 / 10 / 85$ & $11: 38$ & 00.55 & 99.485 & 7.996 & 0.069 & 5.65 & \\
\hline BX01 & $04 / 17 / 85$ & $11: 00$ & 00.45 & 106.458 & 6.973 & 0.065 & 6.10 & \\
\hline BX01 & $04 / 23 / 85$ & $10: 05$ & 00.38 & 112.420 & 5.962 & 0.064 & 6.48 & Room B heaters turned on $4 / 23 / 85$. \\
\hline BX01 & $05 / 01 / 85$ & $11: 40$ & 00.46 & 120.486 & 8.066 & 0.057 & 6.94 & \\
\hline BX01 & $06 / 04 / 85$ & $09: 30$ & 02.00 & 154.396 & 33.910 & 0.059 & 8.94 & First check in several weeks. \\
\hline BX01 & $07 / 16 / 85$ & $10: 15$ & 02.34 & 196.427 & 42.031 & 0.056 & 11.28 & Brine effervesces. \\
\hline BX01 & $08 / 26 / 85$ & $13: 56$ & 02.38 & 237.581 & 41.154 & 0.058 & 13.66 & $\begin{array}{l}\text { Room temperature } 98 \text { degrees } F \text { at collar, } \\
103 \mathrm{~F} \text { in center of room. }\end{array}$ \\
\hline BX01 & $10 / 08 / 85$ & $12: 00$ & 02.27 & 280.500 & 42.919 & 0.053 & 15.93 & \\
\hline BX01 & $11 / 21 / 85$ & $10: 05$ & 02.42 & 324.420 & 43.920 & 0.055 & 18.35 & \\
\hline $8 \times 01$ & $12 / 04 / 85$ & $13: 35$ & 00.69 & 337.566 & 13.146 & 0.052 & 19.04 & Collected for chemistry, sample \#8. \\
\hline
\end{tabular}


TABLE A-2 (Continued)

\section{BRINE ACCUMULATION DATA TABLE}

Data through December 31, 1993

\begin{tabular}{|c|c|c|c|c|c|c|c|c|}
\hline LOCATIOH & DATE & TIME & $\begin{array}{l}\text { LITERS } \\
\text { REMOVED }\end{array}$ & $\begin{array}{l}\text { DAYS } \\
\text { SINCE } \\
1 / 1 / 85\end{array}$ & $\begin{array}{l}\text { DAYS } \\
\text { USED FOR } \\
\text { CALCULATION }\end{array}$ & $\begin{array}{l}\text { LITERS } \\
\text { PER DAY }\end{array}$ & $\begin{array}{l}\text { CUMULATIVE } \\
\text { LITERS } \\
\text { COLLECTED }\end{array}$ & REMARKS \\
\hline BX01 & $01 / 31 / 86$ & $10: 25$ & 02.95 & 395.434 & 57.868 & 0.051 & 21.99 & \\
\hline BX01 & $02 / 12 / 86$ & $09: 30$ & 00.80 & 407.396 & 11.962 & 0.067 & 22.79 & \\
\hline BX01 & $04 / 16 / 86$ & $11: 00$ & 03.45 & 470.458 & 63.062 & 0.055 & 26.24 & \\
\hline BX01 & $04 / 30 / 86$ & $09: 45$ & 00.73 & 484.406 & 13.948 & 0.052 & 26.97 & \\
\hline BXO1 & $05 / 06 / 86$ & $09: 18$ & 00.30 & 490.388 & 5.982 & 0.050 & 27.27 & \\
\hline $8 \times 01$ & $06 / 10 / 86$ & $10: 20$ & 01.85 & 525.431 & 35.043 & 0.053 & 29.12 & Collected for chemistry, sample \#12. \\
\hline BX01 & $08 / 19 / 86$ & $10: 50$ & 03.21 & 595.451 & 70.020 & 0.046 & 32.33 & \\
\hline BX01 & $09 / 09 / 86$ & $11: 00$ & 01.30 & 616.458 & 21.007 & 0.062 & 33.63 & \\
\hline BX01 & $10 / 01 / 86$ & $11: 08$ & 01.16 & 638.464 & 22.006 & 0.053 & 34.79 & \\
\hline BX01 & $11 / 05 / 86$ & $10: 00$ & NA & 673.417 & 0.000 & 0.000 & 34.79 & Not collected. \\
\hline $8 \times 01$ & $11 / 20 / 86$ & $10: 39$ & 02.40 & 688.444 & 49.980 & 0.048 & 37.19 & \\
\hline BX01 & $12 / 30 / 86$ & $14: 10$ & 01.75 & 728.590 & 40.146 & 0.044 & 38.94 & \\
\hline BX01 & $02 / 03 / 87$ & $11: 00$ & 01.67 & 763.458 & 34.868 & 0.048 & 40.61 & \\
\hline $8 \times 01$ & $03 / 06 / 87$ & $11: 50$ & NA & 794.493 & 0.000 & 0.000 & 40.61 & Room closed, bad back, not sampled. \\
\hline BX01 & $10 / 20 / 87$ & & & 1022.000 & 0.000 & 0.000 & 40.61 & $\begin{array}{l}\text { Room closed, could not sample. Ho } \\
\text { calculation. }\end{array}$ \\
\hline BX01 & $11 / 16 / 87$ & $11: 10$ & 12.86 & 1049.465 & 286.007 & 0.045 & 53.47 & $\begin{array}{l}\text { Collected for chemistry, sample \#198A, } \\
\# 201 A, \# 204 A, \# 207 A, \# 210 A \text { \#198B, \#201B, } \\
\# 204 B \text {, \#207B, \& \#210B. }\end{array}$ \\
\hline $8 \times 01$ & $01 / 04 / 88$ & & & 1098.000 & 0.000 & 0.000 & 53.47 & Could not sample. Room elosed. \\
\hline $8 \times 01$ & $02 / 08 / 88$ & $12: 35$ & 3.71 & 1133.524 & 84.059 & 0.044 & 57.18 & $\begin{array}{l}\text { Collected for chemistry, sample \#287, \#288, } \\
\# 289, \# 290, \# 291, \# 292 \# 293 \text { \& \#294. }\end{array}$ \\
\hline $8 \times 01$ & $03 / 29 / 88$ & $12: 00$ & 2.30 & 1183.500 & 49.976 & 0.046 & 59.48 & $\begin{array}{l}\text { Collected for chemistry, sample } \# 379 \text { - } \\
\# 383 \text {. }\end{array}$ \\
\hline $8 \times 01$ & $05 / 12 / 88$ & $10: 44$ & 1.67 & 1227.447 & 43.947 & 0.038 & 61.15 & Sampled for SHL PA. \\
\hline $8 \times 01$ & $07 / 12 / 88$ & $09: 50$ & 2.23 & 1288.410 & 60.963 & 0.037 & 63.38 & $\begin{array}{l}\text { Collected for chemistry, sample \#449. } \\
\# 452 \text {. }\end{array}$ \\
\hline BX01 & $09 / 27 / 88$ & $08: 00$ & 2.61 & 1365.333 & 76.923 & 0.034 & 65.99 & $\begin{array}{l}\text { Collected for chemistry, sample } \$ 504 \text { - } \\
\# 509 \text {. }\end{array}$ \\
\hline BX01 & $12 / 13 / 88$ & $09: 00$ & 0 & 1442.375 & 0.000 & 0.000 & 65.99 & Could not sample. Roon locked. \\
\hline BX01 & $01 / 30 / 89$ & NA & NA & 1490.000 & 0.000 & 0.000 & 65.99 & $\begin{array}{l}\text { Heaters in Room } 8 \text { turned off at } 14: 20 \text { on } \\
1 / 30 / 89 \text {. }\end{array}$ \\
\hline $8 \times 01$ & $03 / 14 / 89$ & $08: 40$ & 6.17 & 1533.361 & 168.028 & 0.037 & 72.16 & Collected for chemistry, sample \#646 - 651 . \\
\hline $8 \times 01$ & $04 / 06 / 89$ & $11: 53$ & NA & 1556.495 & 0.000 & 0.000 & 72.16 & Room locked. \\
\hline BX01 & $04 / 20 / 89$ & $10: 00$ & MA & 1570.417 & 0.000 & 0.000 & 72.16 & Room locked. \\
\hline $8 \times 01$ & $05 / 17 / 89$ & $11: 00$ & 2.90 & 1597.458 & 64.097 & $0.04 \%$ & 75.06 & $\begin{array}{l}\text { Sample saved for chemistry, sample \#759 - } \\
761 \text { A \& B. }\end{array}$ \\
\hline $8 \times 01$ & $07 / 11 / 89$ & $09: 30$ & 1.77 & 1652.396 & 54.938 & 0.032 & 76.83 & \\
\hline$B \times 01$ & $09 / 12 / 89$ & $10: 50$ & 1.90 & 1715.451 & 63.055 & 0.030 & 78.73 & $\begin{array}{l}\text { Increased buildup of salt crust on cap. Ho } \\
\text { indication of leakage into hole, walls dry. }\end{array}$ \\
\hline BX01 & $10 / 11 / 89$ & $10: 30$ & HA & 1744.438 & 0.000 & 0.000 & 78.73 & $\begin{array}{l}\text { Installed collection device. Collection } \\
\text { point for brine located outside heated } \\
\text { room. }\end{array}$ \\
\hline BX01 & $10 / 20 / 89$ & $10: 30$ & 0.61 & 1753.438 & 37.987 & 0.016 & 79.34 & Sample saved for chemistry, sample \#848. \\
\hline BX01 & $11 / 10 / 89$ & $08: 50$ & 0.65 & 1774.368 & 20.930 & 0.031 & 79.99 & $\begin{array}{l}\text { Sample saved for chemistry, sample } \\
\# 858-1,2 \text {. }\end{array}$ \\
\hline BX01 & $11 / 29 / 89$ & $10: 50$ & 0.66 & 1793.451 & 19.083 & 0.035 & 80.65 & Sample saved for chemistry, sample $\# 869$. \\
\hline
\end{tabular}


TABLE A-2 (Continued)

BRINE ACCUMULATION DATA TABLE

Data through December 31, 1993

\begin{tabular}{|c|c|c|c|c|c|c|c|c|}
\hline & & & & DAYS & DAYS & & CUMULATIVE & \\
\hline & & & LITERS & SINCE & USED FOR & LITERS & LITERS & \\
\hline LOCATION & DATE & TIME & REMOVED & $1 / 1 / 85$ & CALCULATIOH & PER DAY & COLLECTED & REMARKS \\
\hline
\end{tabular}

\begin{tabular}{|c|c|c|c|c|c|c|c|c|}
\hline BX01 & $12 / 12 / 89$ & $08: 49$ & 0.63 & 1806.367 & 12.916 & 0.049 & 81.28 & Sample saved for chemistry, sample $\$ 880$. \\
\hline BX01 & $01 / 04 / 90$ & 09:03 & 0.14 & 1829.377 & 23.010 & 0.006 & 81.42 & \\
\hline $8 \times 01$ & $01 / 17 / 90$ & $10: 10$ & 0.17 & 1842.424 & 13.047 & 0.013 & 81.59 & \\
\hline$B \times 01$ & $01 / 31 / 90$ & $08: 57$ & 0.20 & 1856.373 & 13.949 & 0.014 & 81.79 & \\
\hline BX01 & $02 / 13 / 90$ & $10: 23$ & 0.41 & 1869.433 & 13.060 & 0.031 & 82.20 & \\
\hline BX01 & $02 / 27 / 90$ & $11: 12$ & 0.61 & 1883.467 & 14.034 & 0.043 & 82.81 & \\
\hline $8 \times 01$ & $03 / 05 / 90$ & $10: 24$ & 0.35 & 1889.433 & 5.966 & 0.059 & 83.16 & \\
\hline$B \times 01$ & $03 / 21 / 90$ & $10: 59$ & 0.58 & 1905.458 & 16.025 & 0.036 & 83.74 & \\
\hline BX01 & $04 / 04 / 90$ & $10: 26$ & 0.60 & 1919.435 & 13.977 & 0.043 & 84.34 & \\
\hline $8 \times 01$ & $04 / 17 / 90$ & $10: 47$ & 0.71 & 1932.449 & 0.000 & 0.000 & 85.05 & \\
\hline BX01 & $04 / 24 / 90$ & $09: 45$ & 0.63 & 1939.406 & 0.000 & 0.000 & 85.68 & \\
\hline BX01 & $04 / 25 / 90$ & 09:00 & 0.76 & 1940.375 & 20.940 & 0.100 & 86.44 & $\begin{array}{l}\text { Combined with } 0.71 \text { liters from } 04 / 17 / 90 \text { and } \\
0.63 \text { liters from } 04 / 24 / 90 \text {. Used } 2.1 \text { liters } \\
\text { for calculation. }\end{array}$ \\
\hline BX01 & $05 / 02 / 90$ & $10: 59$ & 0.67 & 1947.458 & 7.083 & 0.095 & 87.11 & \\
\hline $8 \times 01$ & $05 / 09 / 90$ & $10: 39$ & 0.19 & 1954.444 & 6.986 & 0.027 & 87.30 & \\
\hline BX01 & $05 / 16 / 90$ & $09: 56$ & 0.20 & 1961.414 & 6.970 & 0.029 & 87.50 & \\
\hline $8 \times 01$ & $05 / 23 / 90$ & $12: 55$ & 0.03 & 1968.538 & 7.124 & 0.004 & 87.53 & \\
\hline $8 \times 01$ & $05 / 31 / 90$ & $11: 11$ & 0.13 & 1976.466 & 7.928 & .0 .016 & 87.66 & \\
\hline $8 \times 01$ & $06 / 01 / 90$ & $10: 15$ & NA & 1977.427 & 0.000 & 0.000 & 87.66 & Replaced sampler. \\
\hline $8 \times 01$ & $06 / 06 / 90$ & $10: 53$ & 0.41 & 1982.453 & 5.987 & 0.068 & 88.07 & \\
\hline $8 \times 01$ & $06 / 14 / 90$ & $09: 14$ & 0.28 & 1990.385 & 7.932 & 0.035 & 88.35 & . \\
\hline $8 \times 01$ & $06 / 20 / 90$ & $08: 42$ & 0.05 & 1996.363 & 5.978 & 0.008 & 88.40 & \\
\hline$B \times 01$ & $06 / 28 / 90$ & $09: 35$ & 0.40 & 2004.399 & 8.036 & 0.050 & 88.80 & \\
\hline $8 \times 01$ & $07 / 17 / 90$ & $10: 20$ & 0.12 & 2023.431 & 0.000 & 0.000 & 88.92 & Partial evacuation. \\
\hline $\mathrm{B} \times 01$ & $07 / 18 / 90$ & $09: 54$ & 0.47 & 2024.413 & 20.014 & 0.029 & 89.39 & $\begin{array}{l}\text { Combined with } 0.12 \text { liters from } 07 / 17 / 90 \text {. } \\
\text { Used } 0.59 \text { liters for calculation. }\end{array}$ \\
\hline BX01 & $07 / 25 / 90$ & $08: 10$ & 0.38 & 2031.340 & 6.927 & 0.055 & 89.77 & \\
\hline $8 \times 01$ & $08 / 07 / 90$ & $11: 40$ & 0.40 & 2044.486 & 13.146 & 0.030 & 90.17 & \\
\hline BX01 & $08 / 16 / 90$ & $10: 52$ & 0.31 & 2053.453 & 8.967 & 0.035 & 90.48 & \\
\hline BX01 & $08 / 22 / 90$ & $11: 40$ & 0.21 & 2059.486 & 6.033 & 0.035 & 90.69 & \\
\hline $\mathrm{B} \times 01$ & $08 / 29 / 90$ & $12: 27$ & 0.09 & 2066.519 & 7.033 & 0.013 & 90.78 & \\
\hline BX01 & $09 / 05 / 90$ & $11: 10$ & 0.12 & 2073.465 & 6.946 & 0.017 & 90.90 & \\
\hline BX01 & $09 / 13 / 90$ & $09: 27$ & 0.30 & 2081.394 & 7.929 & 0.038 & 91.20 & \\
\hline BX01 & $09 / 25 / 90$ & $12: 51$ & 0.48 & 2093.535 & 0.000 & 0.000 & 91.68 & Brine probably left in hole. \\
\hline BX01 & $09 / 26 / 90$ & $11: 18$ & 0.02 & 2094.471 & 13.077 & 0.038 & 91.70 & $\begin{array}{l}\text { Combined with } 0.48 \text { liters from } 09 / 25 / 90 \text {. } \\
\text { Used } 0.50 \text { liters for calculation. }\end{array}$ \\
\hline BX01 & $10 / 03 / 90$ & $09: 25$ & 0.21 & 2101.392 & 6.921 & 0.030 & 91.91 & \\
\hline BX01 & $10 / 10 / 90$ & $11: 10$ & 0.23 & 2108.465 & 7.073 & 0.033 & 92.14 & \\
\hline BX01 & $10 / 18 / 90$ & $10: 46$ & 0.23 & 2116.449 & 7.984 & 0.029 & 92.37 & \\
\hline BX01 & $10 / 24 / 90$ & $12: 02$ & 0.20 & 2122.501 & 6.052 & 0.033 & 92.57 & \\
\hline BX01 & $10 / 31 / 90$ & $11: 26$ & 0.22 & 2129.476 & 6.975 & 0.032 & 92.79 & \\
\hline $8 \times 01$ & $11 / 07 / 90$ & $10: 49$ & 0.15 & 2136.451 & 6.975 & 0.022 & 92.94 & \\
\hline BX01 & $11 / 14 / 90$ & $12: 01$ & 0.26 & 2143.501 & 7.050 & 0.037 & 93.20 & \\
\hline BX01 & $11 / 28 / 90$ & $10: 41$ & 0.49 & 2157.445 & 13.944 & 0.035 & 93.69 & \\
\hline BX01 & $12 / 05 / 90$ & $08: 53$ & 0.21 & 2164.370 & 6.925 & 0.030 & 93.90 & \\
\hline $8 \times 01$ & $12 / 13 / 90$ & $09: 30$ & 0.10 & 2172.396 & 8.026 & 0.012 & 94.00 & \\
\hline
\end{tabular}


TABLE A-2 (Continued)

BRINE ACCUMULATION DATA TABLE

Data through December 31, 1993

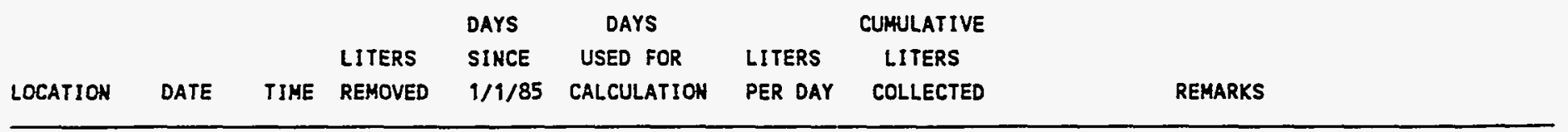

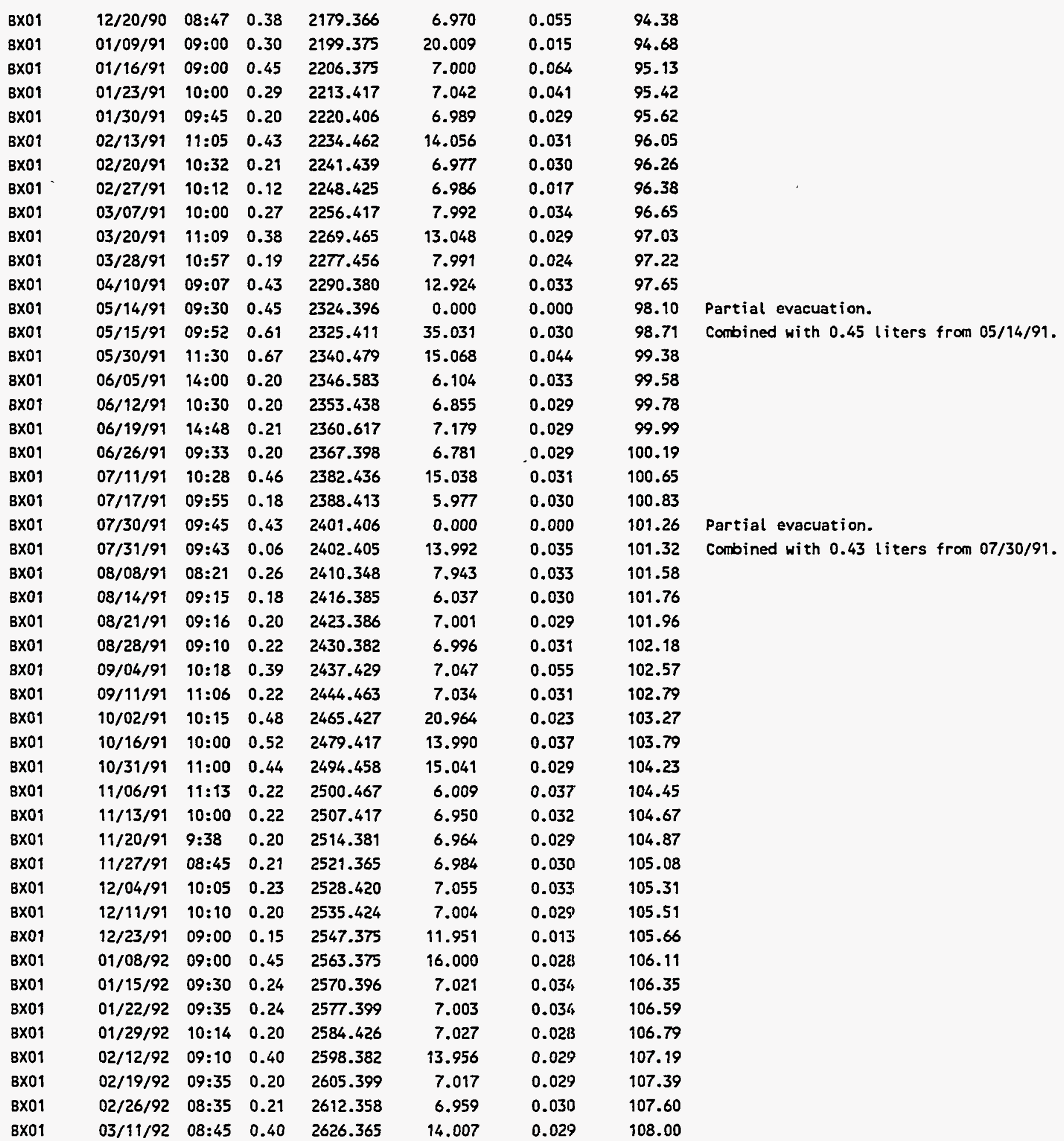


TABLE A-2 (Continued)

BRINE ACCUMULATION DATA TABLE

Data through December 31, 1993

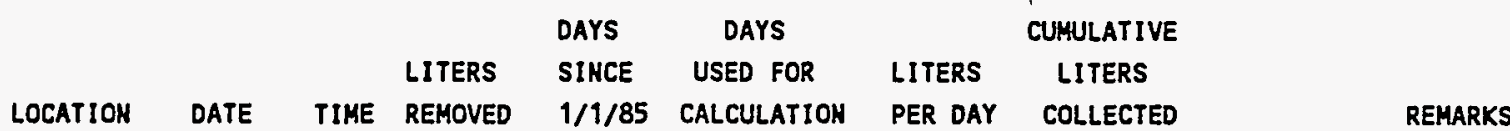

\begin{tabular}{|c|c|c|c|c|c|c|c|c|}
\hline $8 \times 01$ & $03 / 18 / 92$ & 08:35 & 0.22 & 2633.358 & 6.993 & 0.031 & 108.22 & \\
\hline BX01 & $03 / 25 / 92$ & $09: 45$ & 0.20 & 2640.406 & 7.048 & 0.028 & 108.42 & \\
\hline$B \times 01$ & $04 / 01 / 92$ & $09: 45$ & 0.20 & 2647.406 & 7.000 & 0.029 & 108.62 & \\
\hline BX01 & $04 / 07 / 92$ & $09: 50$ & 0.17 & 2653.410 & 6.004 & 0.028 & 108.79 & \\
\hline BX01 & $04 / 15 / 92$ & $08: 30$ & 0.23 & 2661.354 & 7.944 & 0.029 & 109.02 & \\
\hline BX01 & $04 / 22 / 92$ & $09: 53$ & 0.20 & 2668.412 & 7.058 & 0.028 & 109.22 & \\
\hline BX01 & $05 / 06 / 92$ & $10: 43$ & 0.09 & 2682.447 & 0.000 & 0.000 & 109.31 & Partial evacuation. No vacuum. \\
\hline$B \times 01$ & $05 / 07 / 92$ & $09: 45$ & 0.33 & 2683.406 & 14.994 & 0.028 & 109.64 & $\begin{array}{l}\text { Combined with } 0.09 \text { liters removed } 05 / 06 / 92 \\
\text { for total volume. }\end{array}$ \\
\hline BX01 & $05 / 13 / 92$ & $13: 18$ & 0.19 & 2689.554 & 6.148 & 0.031 & 109.83 & \\
\hline $8 \times 01$ & $05 / 21 / 92$ & $10: 52$ & 0.34 & 2697.453 & 7.899 & 0.043 & 110.17 & \\
\hline BX01 & $05 / 27 / 92$ & $09: 32$ & 0.18 & 2703.397 & 5.944 & 0.030 & 110.35 & . \\
\hline BX01 & $06 / 09 / 92$ & $09: 40$ & 0.36 & 2716.403 & 13.006 & 0.028 & 110.71 & \\
\hline $8 \times 01$ & $06 / 18 / 92$ & $09: 10$ & 0.28 & 2725.382 & 8.979 & 0.031 & 110.99 & \\
\hline BX01 & $06 / 25 / 92$ & $10: 07$ & 0.21 & 2732.422 & 7.040 & 0.030 & 111.20 & \\
\hline $8 \times 01$ & $07 / 01 / 92$ & $08: 59$ & 0.16 & 2738.374 & 5.952 & 0.027 & 111.36 & \\
\hline $8 \times 01$ & $07 / 08 / 92$ & $09: 55$ & 0.20 & 2745.413 & 7.039 & 0.028 & 111.56 & \\
\hline BX01 & $07 / 15 / 92$ & $08: 50$ & 0.21 & 2752.368 & 6.955 & 0.030 & 111.77 & \\
\hline BX01 & $07 / 22 / 92$ & $10: 00$ & 0.19 & 2759.417 & 7.049 & .0 .027 & 111.96 & \\
\hline BX01 & $07 / 29 / 92$ & $10: 12$ & 0.21 & 2766.425 & 7.008 & 0.030 & 112.17 & \\
\hline BX01 & $08 / 04 / 92$ & $09: 20$ & 0.17 & 2772.389 & 5.964 & 0.029 & 112.34 & \\
\hline $8 \times 01$ & $08 / 18 / 92$ & $09: 25$ & 0.40 & 2786.392 & 14.003 & 0.029 & 112.74 & . \\
\hline $8 \times 01$ & $09 / 02 / 92$ & $09: 24$ & 0.40 & 2801.392 & 15.000 & 0.027 & 113.14 & \\
\hline BX01 & $09 / 09 / 92$ & $09: 20$ & 0.23 & 2808.389 & 6.997 & 0.033 & 113.37 & \\
\hline BX01 & $09 / 17 / 92$ & $09: 45$ & 0.23 & 2816.406 & 8.017 & 0.029 & 113.60 & \\
\hline$B \times 01$ & $09 / 23 / 92$ & $09: 30$ & 0.17 & 2822.396 & 5.990 & 0.028 & 113.77 & \\
\hline BX01 & $09 / 30 / 92$ & $10: 13$ & 0.21 & 2829.426 & 7.030 & 0.030 & 113.98 & \\
\hline BX01 & $10 / 12 / 92$ & $12: 55$ & 0.36 & 2841.538 & 12.112 & 0.030 & 114.34 & \\
\hline$B \times 01$ & $10 / 21 / 92$ & $12: 30$ & 0.05 & 2850.521 & 8.983 & 0.006 & 114.39 & \\
\hline BX01 & $10 / 28 / 92$ & $08: 55$ & 0.38 & 2857.372 & 6.851 & 0.055 & 114.77 & \\
\hline BX01 & $11 / 11 / 92$ & $10: 35$ & 0.20 & 2871.441 & 14.069 & 0.014 & 114.97 & \\
\hline$B \times 01$ & $11 / 18 / 92$ & $12: 45$ & 0.15 & 2878.531 & 7.090 & 0.021 & 115.12 & \\
\hline BX01 & $11 / 25 / 92$ & $09: 55$ & 0.20 & 2885.413 & 6.882 & 0.029 & 115.32 & \\
\hline BX01 & $12 / 09 / 92$ & $13: 10$ & 0.14 & 2899.549 & 14.136 & 0.010 & 115.46 & \\
\hline BX01 & $12 / 16 / 92$ & $09: 40$ & 0.20 & 2906.403 & 6.854 & 0.029 & 115.66 & \\
\hline BX01 & $01 / 07 / 93$ & $09: 10$ & 0.23 & 2928.382 & 21.979 & 0.010 & 115.89 & - \\
\hline $8 \times 01$ & $01 / 13 / 93$ & $08: 44$ & 0.26 & 2934.364 & 5.982 & 0.043 & 116.15 & \\
\hline BX01 & $01 / 28 / 93$ & $09: 44$ & 0.25 & 2949.406 & 15.042 & 0.017 & 116.40 & \\
\hline BX01 & $02 / 11 / 93$ & $09: 25$ & 0.27 & 2963.392 & 13.986 & 0.019 & 116.67 & \\
\hline BX01 & $02 / 26 / 93$ & $08: 00$ & 0.30 & 2978.333 & 14.941 & 0.020 & 116.97 & \\
\hline$B \times 01$ & $03 / 19 / 93$ & $10: 30$ & 0.52 & 2999.438 & 21.105 & 0.025 & 117.49 & \\
\hline BX01 & $03 / 25 / 93$ & $09: 15$ & 0.57 & 3005.385 & 5.947 & 0.096 & 118.06 & \\
\hline BX01 & $04 / 28 / 93$ & $10: 35$ & 0.24 & 3039.441 & 34.056 & 0.007 & 118.30 & Last time sampled. \\
\hline BX01 & $08 / 19 / 93$ & & & 3152.000 & 0.000 & 0.000 & 118.30 & Collecting hose plugged; \\
\hline
\end{tabular}

OH36 $11 / 21 / 84 \quad 00: 00$ KA $\quad-41.000 \quad 112.559 \quad 0.000 \quad 0.00$ Approximate date this part of Roon G 
TABLE A-2 (Continued)

BRINE ACCUMULATION DATA TABLE

Data through December 31, 1993

\begin{tabular}{|c|c|c|c|c|c|c|c|c|}
\hline & & & & DAYS & DAYS & & CUMULATIVE & \\
\hline & & & LITERS & SIHCE & USED FOR & LITERS & LITERS & \\
\hline LOCATION & DATE & TIME & REMOVED & $1 / 1 / 85$ & CALCULATION & PER DAY & COLLECTED & REMARKS \\
\hline
\end{tabular}

\begin{tabular}{|c|c|c|c|c|c|c|c|c|}
\hline & & & & & & & & excavated. \\
\hline OH36 & $01 / 26 / 85$ & $00: 00$ & NA & 25.000 & 66,000 & 0.000 & 0.00 & Downhole drilled 1/26/85. \\
\hline DH36 & $01 / 28 / 85$ & $09: 00$ & MA & 27.375 & 0.000 & 0.000 & 0.00 & Moist muck at the bottom. \\
\hline DH36 & $02 / 05 / 85$ & $11: 15$ & 02.50 & 35.469 & 10.469 & 0.239 & 2.50 & $\begin{array}{l}\text { About } 1 \text { ft. muck, brine and hydraulic } \\
\text { fluid. First time bailed. }\end{array}$ \\
\hline DH36 & $02 / 11 / 85$ & $11: 00$ & 01.51 & 41.458 & 5.989 & 0.252 & 4.01 & Brine, muck, hydraulic fluid. \\
\hline OH36 & $02 / 19 / 85$ & $12: 10$ & 01.78 & 49.507 & 8.049 & 0.221 & 5.79 & some muck. \\
\hline DH36 & $02 / 26 / 85$ & $10: 45$ & 01.48 & 56.448 & 6.941 & 0.213 & 7.27 & Brine and muck. \\
\hline $\mathrm{DH} 36$ & $03 / 05 / 85$ & $10: 00$ & 01.76 & 63.417 & 6.969 & 0.253 & 9.03 & \\
\hline DH36 & $03 / 12 / 85$ & $10: 00$ & 01.55 & 70.417 & 7.000 & 0.221 & 10.58 & \\
\hline DH36 & $03 / 20 / 85$ & $10: 26$ & 01.59 & 78.435 & 8.018 & 0.198 & 12.17 & \\
\hline DH36 & $03 / 26 / 85$ & $09: 45$ & 01.35 & 84.406 & 5.971 & 0.226 & 13.52 & \\
\hline DH36 & $04 / 02 / 85$ & $10: 15$ & 01.58 & 91.427 & 7.021 & 0.225 & 15.10 & \\
\hline DH36 & $04 / 10 / 85$ & $10: 25$ & 01.71 & 99.434 & 8.007 & 0.214 & 16.81 & \\
\hline DH36 & $04 / 17 / 85$ & $13: 30$ & 01.49 & 106.563 & 7.129 & 0.209 & 18.30 & \\
\hline DH36 & $04 / 23 / 85$ & $11: 46$ & 01.45 & 112.490 & 5.927 & 0.245 & 19.75 & \\
\hline OH36 & $04 / 30 / 85$ & $11: 21$ & 01.49 & 119.473 & 6.983 & 0.213 & 21.24 & \\
\hline DH36 & $05 / 07 / 85$ & $09: 58$ & 01.55 & 126.415 & 6.942 & 0.223 & 22.79 & \\
\hline DH36 & $05 / 14 / 85$ & $10: 54$ & 01.77 & 133.454 & 7.039 & .0 .251 & 24.56 & \\
\hline DH36 & $05 / 21 / 85$ & $10: 45$ & 01.69 & 140.448 & 6.994 & 0.230 & 26.17 & \\
\hline $\mathrm{OH} 36$ & $05 / 29 / 85$ & $10: 00$ & 01.50 & 148.417 & 7.969 & 0.188 & 27.67 & \\
\hline DH36 & $06 / 04 / 85$ & $11: 33$ & 01.40 & 154.481 & 6.064 & 0.231 & 29.07 & \\
\hline DH36 & $06 / 11 / 85$ & $11: 15$ & 01.55 & 161.469 & 6.988 & 0.222 & 30.62 & \\
\hline DH36 & $06 / 18 / 85$ & $10: 17$ & 01.58 & 168.428 & 6.959 & 0.227 & 32.20 & \\
\hline DH36 & $06 / 25 / 85$ & $10: 40$ & 09.43 & 175.444 & 7.016 & 0.204 & 33.63 & \\
\hline DH36 & $07 / 02 / 85$ & $11: 00$ & 01.59 & 182.458 & 7.014 & 0.227 & 35.22 & \\
\hline DH36 & $07 / 09 / 85$ & $11: 15$ & 01.54 & 189.469 & 7.011 & 0.220 & 36.76 & \\
\hline DH36 & $07 / 16 / 85$ & $11: 50$ & 01.58 & 196.493 & 7.024 & 0.225 & 38.34 & Brine effervesces. \\
\hline DH36 & $07 / 24 / 85$ & $10: 46$ & 01.78 & 204.449 & 7.956 & 0.226 & 40.12 & \\
\hline DH36 & $07 / 30 / 85$ & $10: 20$ & 01.39 & 210.431 & 5.982 & $0.23 i$ & 41.51 & \\
\hline DH36 & $08 / 06 / 85$ & $10: 43$ & 01.70 & 217.447 & 7.016 & $0.24 \%$ & 43.21 & \\
\hline DH36 & $08 / 14 / 85$ & $11: 02$ & 01.58 & 225.460 & 8.013 & 0.197 & 44.79 & $\begin{array}{l}\text { Valve leaked, some brine drained back down } \\
\text { hole. }\end{array}$ \\
\hline $\mathrm{DH} 36$ & $08 / 20 / 85$ & $11: 19$ & 01.42 & 231.466 & 6.006 & 0.236 & 46.21 & \\
\hline DH36 & $08 / 28 / 85$ & $10: 00$ & 01.94 & 239.417 & 7.951 & 0.244 & 48.15 & \\
\hline DH36 & $09 / 04 / 85$ & $10: 32$ & 01.69 & 246.439 & 7.022 & 0.241 & 49.84 & \\
\hline DH36 & $09 / 10 / 85$ & $10: 35$ & 01.41 & 252.441 & 6.002 & 0.235 & 51.25 & \\
\hline DH36 & $09 / 17 / 85$ & $09: 42$ & 01.53 & 259.404 & 6.963 & 0.220 & 52.78 & \\
\hline DH36 & $09 / 24 / 85$ & $09: 50$ & 01.53 & 266.410 & 7.006 & 0.218 & 54.31 & \\
\hline DH36 & $10 / 01 / 85$ & $09: 55$ & 01.58 & 273.413 & 7.003 & 0.226 & 55.89 & \\
\hline DH36 & $10 / 08 / 85$ & $10: 52$ & 01.63 & 280.453 & 7.040 & 0.232 & 57.52 & \\
\hline DH36 & $10 / 15 / 85$ & $10: 30$ & 01.58 & 287.438 & 6.985 & 0.226 & 59.10 & \\
\hline $0 H 36$ & $10 / 23 / 85$ & $10: 23$ & 01.82 & 295.433 & 7.995 & 0.228 & 60.92 & \\
\hline DH36 & $10 / 29 / 85$ & $09: 51$ & 01.36 & 301.410 & 5.977 & 0.228 & 62.28 & \\
\hline DH36 & $11 / 05 / 85$ & $09: 27$ & 01.63 & 308.394 & 6.984 & 0.233 & 63.91 & \\
\hline DH36 & $11 / 13 / 85$ & $10: 14$ & 01.79 & 316.426 & 8.032 & 0.223 & 65.70 & \\
\hline DH36 & $11 / 21 / 85$ & $11: 36$ & 01.91 & 324.483 & 8.057 & 0.237 & 67.61 & \\
\hline
\end{tabular}


TABLE A-2 (Continued)

BRINE ACCUMULATION DATA TABLE

Data through December 31, 1993

\begin{tabular}{|c|c|c|c|c|c|c|c|c|}
\hline LOCATIOH & DATE & TIME & $\begin{array}{l}\text { LITERS } \\
\text { REMOVED }\end{array}$ & $\begin{array}{l}\text { DAYS } \\
\text { SIKCE } \\
1 / 1 / 85\end{array}$ & $\begin{array}{l}\text { OAYS } \\
\text { USED FOR } \\
\text { CALCULATIYOH }\end{array}$ & $\begin{array}{l}\text { LITERS } \\
\text { PER DAY }\end{array}$ & $\begin{array}{c}\text { CUMULATIVE } \\
\text { LITERS } \\
\text { COLLECTED }\end{array}$ & REMARKS \\
\hline DH36 & $11 / 26 / 85$ & $19: 30$ & 01.01 & 329.479 & 4.996 & 0.202 & 68.62 & - \\
\hline DH36 & $12 / 03 / 85$ & $13: 35$ & 01.50 & 336.566 & 7.087 & 0.212 & 70.12 & $\begin{array}{l}.50 \text { liters for chemical analysis, sample } \\
\# 4 \text {. }\end{array}$ \\
\hline DH36 & $12 / 10 / 85$ & $12: 15$ & 01.52 & 343.510 & 6.944 & 0.219 & 71.64 & \\
\hline DH36 & $01 / 23 / 86$ & $11: 00$ & 09.30 & 387.458 & 43.948 & 0.212 & 80.94 & $\begin{array}{l}\text { Entry restricted since } 12 / 10 / 85 \text { due to } \\
\text { mining activities. }\end{array}$ \\
\hline DH36 & $01 / 31 / 86$ & $12: 20$ & 01.38 & 395.514 & 8.056 & 0.171 & 82.32 & \\
\hline DH36 & $02 / 12 / 86$ & $11: 00$ & 03.02 & 407.458 & 11.944 & 0.253 & 85.34 & \\
\hline DH36 & $02 / 19 / 86$ & $11: 45$ & 01.55 & 414.490 & 7.032 & 0.220 & 86.89 & \\
\hline $\mathrm{OH} 36$ & $02 / 28 / 86$ & $13: 20$ & 01.85 & 423.556 & 9.066 & 0.204 & 88.74 & \\
\hline DH36 & $03 / 06 / 86$ & $10: 45$ & 01.30 & 429.448 & 5.892 & 0.221 & 90.04 & Volume was estimated. \\
\hline DH36 & $03 / 13 / 86$ & $10: 10$ & 01.50 & 436.424 & 6.976 & 0.215 & 91.54 & \\
\hline DH36 & $03 / 26 / 86$ & $10: 20$ & 02.56 & 449.431 & 13.007 & 0.197 & 94.10 & \\
\hline DH36 & $04 / 02 / 86$ & $09: 40$ & 01.75 & 456.403 & 6.972 & 0.251 & 95.85 & \\
\hline DH36 & $04 / 08 / 86$ & $09: 45$ & 00.97 & 462.406 & 6.003 & 0.162 & 96.82 & \\
\hline DH36 & $04 / 16 / 86$ & $12: 25$ & 01.65 & 470.517 & 8.111 & 0.203 & 98.47 & \\
\hline $\mathrm{OH} 36$ & $04 / 24 / 86$ & $10: 20$ & 02.00 & 478.431 & 7.914 & 0.253 & 100.47 & \\
\hline OH36 & $04 / 30 / 86$ & $10: 55$ & 01.21 & 484.455 & 6.024 & 0.201 & 101.68 & \\
\hline OH36 & $05 / 06 / 86$ & $10: 14$ & 01.20 & 490.426 & 5.971 & .0 .201 & 102.88 & \\
\hline DH36 & $05 / 13 / 86$ & $11: 13$ & 01.42 & 497.467 & 7.041 & 0.202 & 104.30 & \\
\hline DH36 & $05 / 20 / 86$ & $11: 10$ & 01.50 & 504.465 & 6.998 & 0.214 & 105.80 & \\
\hline DH36 & $05 / 27 / 86$ & $15: 45$ & 01.40 & 511.656 & 7.191 & 0.195 & 107.20 & \\
\hline DH36 & $06 / 03 / 86$ & $10: 10$ & 01.38 & 518.424 & 6.768 & 0.204 & 108.58 & \\
\hline DH36 & $06 / 10 / 86$ & $11: 35$ & 01.24 & 525.483 & 7.059 & 0.176 & 109.82 & $\begin{array}{l}\text { Valve leaked, some brine drained back down } \\
\text { hole. }\end{array}$ \\
\hline DH36 & $06 / 17 / 86$ & $11: 00$ & 01.65 & 532.458 & 6.975 & 0.237 & 111.47 & Sample for brine chemistry, \#24. \\
\hline DH36 & $06 / 24 / 86$ & $11: 00$ & 01.45 & 539.458 & 7.000 & 0.207 & 112.92 & \\
\hline OH36 & $07 / 01 / 86$ & $14: 05$ & 01.55 & 546.587 & 7.129 & 0.217 & 114.47 & \\
\hline DH36 & $07 / 08 / 86$ & $10: 45$ & 01.40 & 553.448 & 6.861 & 0.204 & 115.87 & \\
\hline DH36 & $07 / 16 / 86$ & $10: 45$ & 01.76 & 561.448 & 8.000 & 0.220 & 117.63 & \\
\hline DH36 & $07 / 22 / 86$ & $10: 07$ & 01.29 & 567.422 & 5.974 & 0.216 & 118.92 & \\
\hline OH36 & $07 / 29 / 86$ & $10: 40$ & 01.45 & 574.444 & 7.022 & 0.206 & 120.37 & \\
\hline DH36 & $08 / 05 / 86$ & $11: 20$ & 01.46 & 581.472 & 7.028 & 0.208 & 121.83 & \\
\hline DH36 & $08 / 12 / 86$ & $10: 37$ & 01.50 & 588.442 & 6.970 & 0.215 & 123.33 & $\cdot$ \\
\hline DH36 & $08 / 19 / 86$ & $11: 35$ & 01.38 & 595.483 & 7.041 & 0.196 & 124.71 & \\
\hline DH36 & $08 / 26 / 86$ & $10: 38$ & 01.49 & 602.443 & 6.960 & 0.214 & 126.20 & Static level not measured. \\
\hline DH36 & $09 / 04 / 86$ & $10: 41$ & 01.70 & 611.445 & 9.002 & 0.189 & 127.90 & \\
\hline DH36 & $09 / 09 / 86$ & $10: 15$ & 01.20 & 616.427 & 4.982 & 0.241 & 129.10 & Sample \#26. \\
\hline DH36 & $09 / 16 / 86$ & $10: 20$ & 01.37 & 623.431 & 7.004 & 0.196 & 130.47 & \\
\hline DH36 & $09 / 23 / 86$ & $10: 18$ & 01.40 & 630.429 & 6.998 & 0.200 & 131.87 & \\
\hline DH36 & $10 / 01 / 86$ & $12: 18$ & 01.76 & 638.513 & 8.084 & 0.218 & 133.63 & \\
\hline OH36 & $10 / 08 / 86$ & $11: 10$ & 01.44 & 645.465 & 6.952 & 0.207 & 135.07 & $\begin{array}{l}\text { Brine efferveces as it is poured into } \\
\text { beaker. }\end{array}$ \\
\hline DH36 & $10 / 14 / 86$ & $11: 57$ & 01.21 & 651.498 & 6.033 & 0.201 & 136.28 & static level not measured. \\
\hline DH36 & $11 / 05 / 86$ & $11: 38$ & 4.28 & 673.485 & 21.987 & 0.195 & 140.56 & \\
\hline DH36 & $11 / 20 / 86$ & $12: 35$ & 03.12 & 688.524 & 15.039 & 0.207 & 143.68 & \\
\hline DH36 & $12 / 30 / 86$ & $12: 25$ & 01.72 & 728.517 & 0.000 & 0.000 & 145.40 & Partial evacuation. Ho calculation. Do not \\
\hline
\end{tabular}




\section{TABLE A-2 (Continued) \\ BRINE ACCUMULATION DATA TABLE}

Data through December 31, 1993

\begin{tabular}{lllllll} 
& & \multicolumn{2}{c}{ DAYS } & DAYS & CUMULATIVE \\
LOCATION & LITERS & SIMCE & USED FOR & LITERS & LITERS \\
\hline
\end{tabular}

DH36

$06 / 18 / 87 \quad 12: 10 \quad 0.49 \quad 898.507$

42.026

0.184

183.93

plot or use zero value.

\begin{tabular}{|c|c|c|c|c|c|c|c|c|}
\hline 0436 & $12 / 31 / 86$ & $12: 38$ & 6.54 & 729.526 & 41.002 & 0.201 & 151.94 & $\begin{array}{l}\text { Calculated using } 8.26 \text { liters in } 41.002 \text { days } \\
(1.72 \text { liters } 12 / 30 / 86 \text { plus } 6.54 \text { liters } \\
12 / 31 / 86) \text {. }\end{array}$ \\
\hline DH36 & $02 / 03 / 87$ & $13: 35$ & 06.84 & 763.566 & 34.040 & 0.201 & 158.78 & \\
\hline DH36 & $03 / 06 / 87$ & $11: 20$ & 5.84 & 794.472 & 30.906 & 0.189 & 164.62 & \\
\hline DH36 & $03 / 30 / 87$ & $11: 27$ & 4.95 & 818.477 & 24.005 & 0.206 & 169.57 & \\
\hline DH36 & $05 / 07 / 87$ & $11: 33$ & 6.62 & 856.481 & 38.004 & 0.174 & 176.19 & \\
\hline DH36 & $06 / 17 / 87$ & $10: 45$ & 7.25 & 897.448 & 0.000 & 0.000 & 183.44 & $\begin{array}{l}\text { Sample for chen. \#108A, \#108B, \#114A, } \\
\# 114 B, \# 121 A, \# 121 B, \# 127 A, \# 127 B, \# 134 A \text {, } \\
\# 134 B \text {. Some brine left in hole, no } \\
\text { calculation. }\end{array}$ \\
\hline DH36 & $06 / 18 / 87$ & $12: 10$ & 0.49 & 898.507 & 42.026 & 0.184 & 183.93 & $\begin{array}{l}\text { Original L/day calculation too high due to } \\
\text { residual brine left in hole. Recalculated } \\
\text { using } 7.74 \text { \& }(7.25 \mid 6 / 17 / 87 \text { plus } 0.49 \text { । } \\
6 / 18 / 87) \text {. }\end{array}$ \\
\hline DH36 & $07 / 28 / 87$ & $11: 27$ & 7.76 & 938.477 & 39.970 & 0.194 & 191.69 & \\
\hline DH36 & $09 / 01 / 87$ & $10: 50$ & 6.99 & 973.451 & 34.974 & 0.200 & 198.68 & $\begin{array}{l}\text { Collected for chemistry, sample \#153 A\&B, } \\
\# 160 \text { A\&B, \#163 A\&B, \#158 A\&B, \#155 A\&B, } \\
\# 167 \text { A\&8. }\end{array}$ \\
\hline DH36 & $10 / 20 / 87$ & $11: 56$ & 8.58 & 1022.497 & 49.046 & 0.175 & 207.26 & \\
\hline DH36 & $11 / 19 / 87$ & $11: 30$ & 4.19 & 1052.479 & 29.982 & 0.140 & 211.45 & $\begin{array}{l}\text { Collected for chemistry, sample \#199, \#205, } \\
\# 208 \text {, \& \#211. }\end{array}$ \\
\hline DH36 & $01 / 04 / 88$ & $11: 50$ & 6.74 & 1098.493 & 46.014 & 0.146 & 218.19 & \\
\hline 0436 & $02 / 08 / 88$ & $11: 50$ & 4.90 & 1133.493 & 35.000 & 0.140 & 223.09 & $\begin{array}{l}\text { Collected for chemistry, sample \#261, \#262, } \\
\# 263, \# 264, \# 265, \# 266, \# 267, \# 268, \# 269 \& \\
\# 270 .\end{array}$ \\
\hline DH36 & $03 / 29 / 88$ & $11: 35$ & 7.25 & 1183.483 & 49.990 & 0.145 & 230.34 & $\begin{array}{l}\text { Collected for chemistry, sample } \$ 367 \text { - } \\
\$ 378 \text {. }\end{array}$ \\
\hline DH36 & $05 / 05 / 88$ & $09: 45$ & 5.01 & 1220.406 & 36.923 & 0.136 & 235.35 & Sampled for SNL PA. \\
\hline DH36 & $05 / 12 / 88$ & $09: 50$ & 1.30 & 1227.410 & 7.004 & 0.186 & 236.65 & Sampled for SHL PA. \\
\hline DH36 & $07 / 12 / 88$ & $08: 50$ & 7.90 & 1288.368 & 60.958 & 0.130 & 244.55 & $\begin{array}{l}\text { Collected for chemistry, sample \#422 - } \\
\# 436 \text {. }\end{array}$ \\
\hline DH36 & $07 / 28 / 88$ & $10: 25$ & 1.50 & 1304.434 & 16.066 & 0.093 & 246.05 & Sampled for SNL PA. \\
\hline DH36 & $08 / 11 / 88$ & $10: 30$ & 3.66 & 1318.438 & 14.004 & 0.261 & 249.71 & Sampled for SNL PA: \\
\hline OH36 & $08 / 25 / 88$ & $09: 24$ & 2.05 & 1332.392 & 13.954 & 0.147 & 251.76 & Sampled for SNL PA: \\
\hline DH36 & $09 / 08 / 88$ & $14: 50$ & & 1346.618 & 0.000 & 0.000 & 251.76 & Did not sample. \\
\hline OH36 & $09 / 14 / 88$ & $08: 40$ & 2.36 & 1352.361 & 19.969 & 0.118 & 254.12 & slight orange color. \\
\hline DH36 & $09 / 27 / 88$ & $10: 45$ & 1.30 & 1365.448 & 13.087 & 0.099 & 255.42 & $\begin{array}{l}\text { Collected for chemistry, sample \#537 - } \\
\# 539 \text {. }\end{array}$ \\
\hline DH36 & $12 / 13 / 88$ & $10: 00$ & 10.63 & 1442.417 & 76.969 & 0.138 & 266.05 & $\begin{array}{l}\text { Collected for chemistry, sample \#570 - } \\
\# 581 \text {. }\end{array}$ \\
\hline OH36 & $03 / 14 / 89$ & $10: 10$ & 11.16 & 1533.424 & 91.007 & 0.123 & 277.21 & $\begin{array}{l}\text { Sample saved for chemistry, sample \#684 - } \\
695 \text {. }\end{array}$ \\
\hline OH36 & $04 / 06 / 89$ & $09: 31$ & 2.73 & 1556.397 & 22.973 & 0.119 & 279.94 & 2.5 liters saved for SHL brine study. \\
\hline DH36 & $04 / 20 / 89$ & $09: 40$ & 1.79 & 1570.403 & 14.006 & 0.128 & 281.73 & Sample saved for SNL/NM brine study. \\
\hline DH36 & $05 / 17 / 89$ & $10: 20$ & 6.45 & 1597.431 & 27.028 & 0.239 & 288.18 & Sample saved for SNL/NM brine study. \\
\hline
\end{tabular}


TABLE A-2 (Continued)

\section{BRINE ACCUMULATION DATA TABLE}

Data through December 31, 1993

\begin{tabular}{|c|c|c|c|c|c|c|c|c|}
\hline & & & & DAYS & DAYS & & CUHULATIVE & \\
\hline & & & ITF & SIHCE & USED FOR & LITERS & LITERS & \\
\hline OCATION & DATE & TIME & REMOVED & $1 / 1 / 85$ & CALCULATIOH & PER DAY & COLLECTED & \\
\hline
\end{tabular}

\begin{tabular}{|c|c|c|c|c|c|c|c|c|}
\hline DH36 & $06 / 06 / 89$ & $10: 10$ & 2.62 & 1617.424 & 19.993 & 0.131 & 290.80 & Sample saved for chemistry. \\
\hline DH36 & $06 / 29 / 89$ & $10: 35$ & 2.42 & 1640.441 & 23.017 & 0.105 & 293.22 & Sample saved for SHL/NM brine study. \\
\hline DH36 & 07/06/89 & $09: 10$ & 1.08 & 1647.382 & 6.949 & 0.156 & 294.30 & \\
\hline DH36 & $07 / 25 / 89$ & $09: 55$ & 2.35 & 1666.413 & 19.031 & 0.123 & 296.65 & Sample saved for SNL/NM brine study. \\
\hline DH36 & $08 / 16 / 89$ & $09: 27$ & 2.75 & 1688.394 & 21.981 & 0.125 & 299.40 & Sample saved for SHL/NM brine study. \\
\hline DH36 & $09 / 12 / 89$ & $09: 30$ & 3.81 & 1715.396 & 27.002 & 0.141 & 303.21 & Sample saved for chemistry. \\
\hline DH36 & $12 / 13 / 89$ & $11: 10$ & 11.07 & 1807.465 & 92.069 & 0.120 & 314.28 & Sample saved for chemistry, sample $\$ 900$. \\
\hline DH36 & $01 / 10 / 90$ & $10: 18$ & 2.48 & 1835.429 & 27.964 & 0.089 & 316.76 & \\
\hline DH36 & $01 / 24 / 90$ & $09: 37$ & 2.0 & 1849.401 & 13.972 & 0.143 & 318.76 & \\
\hline DH36 & $02 / 07 / 90$ & $10: 17$ & 1.53 & 1863.428 & 14.027 & 0.109 & 320.29 & \\
\hline DH36 & $02 / 21 / 90$ & $09: 50$ & 1.75 & 1877.410 & 13.982 & 0.125 & 322.04 & \\
\hline DH36 & 03/05/90 & $09: 25$ & 1.10 & 1889.392 & 11.982 & 0.092 & 323.14 & \\
\hline DH36 & $03 / 14 / 90$ & $12: 30$ & NA & 1898.521 & 0.000 & 0.000 & 323.14 & Installed sampler. \\
\hline $\mathrm{DH} 36$ & $03 / 19 / 90$ & $10: 36$ & 0.80 & 1903.442 & 0.000 & 0.000 & 323.94 & Brine probably left in hole. \\
\hline DH36 & $03 / 21 / 90$ & $10: 16$ & 0.57 & 1905.428 & 16.036 & 0.085 & 324.51 & $\begin{array}{l}\text { Combined with } 0.80 \text { liters from } 03 / 19 / 90 \text {. } \\
\text { Used } 1.37 \text { liters for calculation. }\end{array}$ \\
\hline DH36 & $04 / 04 / 90$ & 09:09 & 1.08 & 1919.381 & 13.953 & 0.077 & 325.59 & \\
\hline DH36 & $04 / 10 / 90$ & $08: 34$ & 0.97 & 1925.357 & 5.976 & 0.162 & 326.56 & \\
\hline DH36 & $04 / 17 / 90$ & $10: 17$ & 0.85 & 1932.428 & 7.071 & .0 .120 & 327.41 & \\
\hline DH36 & $04 / 24 / 90$ & $09: 14$ & 0.86 & 1939.385 & 0.000 & 0.000 & 328.27 & \\
\hline DH36 & $04 / 25 / 90$ & $08: 45$ & 0.57 & 1940.365 & 7.937 & 0.180 & 328.84 & $\begin{array}{l}\text { Combined with } 0.86 \text { liters from } 04 / 27 / 90 \text {. } \\
\text { Used } 1.43 \text { liters for calculation. }\end{array}$ \\
\hline DH36 & $05 / 02 / 90$ & $10: 24$ & 1.37 & 1947.433 & 7.068 & 0.194 & 330.21 & \\
\hline DH36 & $05 / 09 / 90$ & $08: 35$ & 0.68 & 1954.358 & 6.925 & 0.098 & 330.89 & \\
\hline DH36 & $05 / 16 / 90$ & $08: 45$ & 0.78 & 1961.365 & 7.007 & 0.111 & 331.67 & \\
\hline $\mathrm{DH} 36$ & $05 / 17 / 90$ & $07: 50$ & 0.17 & 1962.326 & 0.961 & 0.177 & 331.84 & \\
\hline DH36 & $05 / 23 / 90$ & $12: 02$ & 0.68 & 1968.501 & 6.175. & 0.110 & 332.52 & \\
\hline DH36 & $05 / 31 / 90$ & $08: 38$ & 0.85 & 1976.360 & 7.859 & 0.108 & 333.37 & \\
\hline DH36 & $06 / 01 / 90$ & $11: 00$ & 0.15 & 1977.458 & 1.098 & 0.137 & 333.52 & Repaired sampler, evacuated hole. \\
\hline DH36 & $06 / 06 / 90$ & $08: 47$ & 0.45 & 1982.366 & 4.908 & 0.092 & 333.97 & \\
\hline DH36 & $06 / 14 / 90$ & $08: 38$ & 0.82 & 1990.360 & 7.994 & 0.103 & 334.79 & \\
\hline DH36 & $06 / 20 / 90$ & $09: 53$ & 0.59 & 1996.412 & 6.052 & 0.097 & 335.38 & \\
\hline $\mathrm{DH} 36$ & $06 / 28 / 90$ & $08: 38$ & 0.88 & 2004.360 & 7.948 & 0.111 & 336.26 & \\
\hline $\mathrm{OH} 36$ & $07 / 17 / 90$ & $10: 52$ & 0.41 & 2023.453 & 0.000 & 0.000 & 336.67 & \\
\hline DH36 & $07 / 18 / 90$ & $10: 20$ & 0.62 & 2024.431 & 20.071 & 0.051 & 337.29 & $\begin{array}{l}\text { Combined with } 0.41 \text { liters from } 07 / 17 / 90 \text {. } \\
\text { Used } 1.03 \text { liters for calculation. }\end{array}$ \\
\hline DH36 & $07 / 25 / 90$ & $09: 45$ & 0.61 & 2031.406 & 6.975 & 0.087 & 337.90 & \\
\hline DH36 & $08 / 01 / 90$ & $10: 38$ & 0.61 & 2038.443 & $7.037^{-}$ & 0.087 & 338.51 & \\
\hline DH36 & $12 / 12 / 90$ & $09: 47$ & 11.54 & 2171.408 & 132.965 & 0.087 & 350.05 & First evacuation since $08 / 07 / 90$ \\
\hline DH36 & $12 / 19 / 90$ & $11: 22$ & 3.61 & 2178.474 & 0.000 & 0.000 & 353.66 & $\begin{array}{l}\text { Combined with } 11.54 \text { liters from } 12 / 12 / 90 \text {. } \\
\text { Brine stored in fractures may have drained } \\
\text { into hole after evacuation of } 11.5 \text { liters } \\
\text { on } 12 / 12 / 90 \text {. Used } 140.03 \text { days, } 15.15 \\
\text { liters. }\end{array}$ \\
\hline DH36 & $01 / 09 / 91$ & $09: 50$ & 2.34 & 2199.410 & 28.002 & 0.212 & 356.00 & \\
\hline DH36 & $01 / 16 / 91$ & $08: 35$ & 0.73 & 2206.358 & 6.948 & 0.105 & 356.73 & \\
\hline DH36 & $01 / 23 / 91$ & $08: 35$ & 0.54 & 2213.358 & 7.000 & 0.077 & 357.27 & \\
\hline
\end{tabular}


TABLE A-2 (Continued)

\section{BRINE ACCUMULATION DATA TABLE}

Data through December 31, 1993

\begin{tabular}{|c|c|c|c|c|c|c|c|c|}
\hline LOCATION & DATE & TIME & $\begin{array}{l}\text { LITERS } \\
\text { REMOVED }\end{array}$ & $\begin{array}{l}\text { DAYS } \\
\text { SIHCE } \\
1 / 1 / 85\end{array}$ & $\begin{array}{l}\text { DAYS } \\
\text { USED FOR } \\
\text { CALCULATION }\end{array}$ & $\begin{array}{l}\text { LITERS } \\
\text { PER DAY }\end{array}$ & $\begin{array}{l}\text { CUMULATIVE } \\
\text { LITERS } \\
\text { COLLECTED }\end{array}$ & REMARKS \\
\hline DH36 & $02 / 13 / 91$ & $10: 30$ & 1.90 & 2234.438 & 21.080 & 0.090 & 359.17 & \\
\hline DH36 & $02 / 20 / 91$ & $10: 30$ & 0.58 & 2241.438 & 7.000 & 0.083 & 359.75 & \\
\hline DH36 & $02 / 27 / 91$ & $09: 58$ & 0.32 & 2248.415 & 6.977 & 0.046 & 360.07 & \\
\hline DH36 & $03 / 07 / 91$ & $09: 45$ & 0.02 & 2256.406 & 0.000 & 0.000 & 360.09 & Partial evacuation. \\
\hline DH36 & $03 / 20 / 91$ & $10: 07$ & 2.72 & 2269.422 & 0.000 & 0.000 & 362.81 & $\begin{array}{l}\text { Partial evacuation. First evacuation with } \\
\text { bailer, second with pump. Sampler } \\
\text { malfunctioning. }\end{array}$ \\
\hline DH36 & $03 / 21 / 91$ & $08: 30$ & 0.38 & 2270.354 & 21.939 & 0.142 & 363.19 & $\begin{array}{l}\text { Combined with } 0.02 \text { liters from } 03 / 07 / 91 \text { and } \\
2.72 \text { liters from } 03 / 20 / 91 \text {. Repaired } \\
\text { sampler. }\end{array}$ \\
\hline DH36 & $03 / 28 / 91$ & $10: 40$ & 0.90 & 2277.444 & 7.090 & 0.127 & 364.09 & \\
\hline DH36 & $04 / 10 / 91$ & $8: 25$ & 0.87 & 2290.337 & 0.000 & 0.000 & 364.96 & Partial evacuation. \\
\hline DH36 & $04 / 11 / 91$ & $08: 55$ & 0.64 & 2291.372 & 13.928 & 0.108 & 365.60 & Combined with 0.87 liters from $04 / 10 / 91$ \\
\hline DH36 & $04 / 17 / 91$ & $10: 34$ & 0.63 & 2297.440 & 6.068 & 0.104 & 366.23 & \\
\hline DH36 & $04 / 24 / 91$ & $09: 15$ & 0.52 & 2304.385 & 6.945 & 0.075 & 366.75 & \\
\hline OH36 & $05 / 01 / 91$ & $09: 26$ & 0.65 & 2311.393 & 7.008 & 0.093 & 367.40 & \\
\hline $\mathrm{DH} 36$ & $05 / 08 / 91$ & $08: 37$ & 0.42 & 2318.359 & 6.966 & 0.060 & 367.82 & \\
\hline DH36 & $05 / 15 / 91$ & $09: 14$ & 0.62 & 2325.385 & 0.000 & 0.000 & 368.44 & Partial evacuation. \\
\hline DH36 & $05 / 29 / 91$ & $09: 30$ & 2.75 & 2339.396 & 21.037 & .0 .160 & 371.19 & Combined with 0.62 liters from $05 / 15 / 91$. \\
\hline DH36 & $06 / 05 / 91$ & $13: 50$ & 0.52 & 2346.576 & 7.180 & 0.072 & 371.71 & \\
\hline DH36 & $06 / 12 / 91$ & $10: 20$ & 0.60 & 2353.431 & 6.855 & 0.088 & 372.31 & \\
\hline DH36 & $06 / 19 / 91$ & $14: 10$ & 0.53 & 2360.590 & 7.159 & 0.074 & 372.84 & \\
\hline DH36 & $06 / 26 / 91$ & $08: 55$ & 0.58 & 2367.372 & 6.782 & 0.086 & 373.42 & \\
\hline DH36 & $07 / 11 / 91$ & $10: 26$ & 0.70 & 2382.435 & 15.063 & 0.046 & 374.12 & \\
\hline DH36 & $07 / 17 / 91$ & $09: 35$ & 0.59 & 2388.399 & 0.000 & 0.000 & .374 .71 & Partial evacuation. \\
\hline OH36 & $07 / 18 / 91$ & $09: 59$ & 0.52 & 2389.416 & 6.981 & 0.159 & 375.23 & Combined with 0.59 liters from $07 / 17 / 91$. \\
\hline DH36 & $07 / 30 / 91$ & $10: 35$ & 0.60 & 2401.441 & 0.000 & 0.000 & 375.83 & Partial evacuation. \\
\hline DH36 & $07 / 31 / 91$ & $09: 35$ & 0.72 & 2402.399 & 0.000 & 0.000 & 376.55 & Partial evacuation. \\
\hline DH36 & $08 / 01 / 91$ & $10: 30$ & 0.83 & 2403.438 & 0.000 & 0.000 & 377.38 & Partial evacuation. \\
\hline DH36 & $08 / 02 / 91$ & $10: 00$ & 0.54 & 2404.417 & 15.001 & 0.179 & 377.92 & $\begin{array}{l}\text { Combined with } 0.60 \text { liters from } 07 / 30 / 91 \text {, } \\
0.72 \text { liters from } 07 / 31 / 91 \text {, and } 0.83 \text { liters } \\
\text { from } 08 / 01 / 91 \text {. }\end{array}$ \\
\hline DH36 & $08 / 08 / 91$ & $09: 23$ & 0.51 & 2410.391 & 0.000 & 0.000 & 378.43 & Some brine may have been left in hole. \\
\hline DH36 & $08 / 14 / 91$ & $10: 04$ & 1.63 & 2416.419 & 12.002 & 0.178 & 380.06 & $\begin{array}{l}\text { Combined with } 0.51 \text { liters from } 08 / 08 / 91 \text {. } \\
\text { Used bailer. }\end{array}$ \\
\hline DH36 & $08 / 21 / 91$ & $11: 00$ & 0.55 & 2423.458 & 0.000 & 0.000 & 380.61 & Partial evacuation. \\
\hline DH36 & $08 / 22 / 91$ & $10: 00$ & 0.53 & 2424.417 & 7.998 & 0.135 & 381.14 & Combined with 0.55 liters from $08 / 21 / 91$ \\
\hline DH36 & $08 / 28 / 91$ & $09: 50$ & 0.98 & 2430.410 & 5.993 & 0.164 & 382.12 & \\
\hline DH36 & $09 / 25 / 91$ & $11: 25$ & 3.12 & 2458.476 & 28.066 & 0.111 & 385.24 & \\
\hline DH36 & $10 / 23 / 91$ & $09: 50$ & 3.25 & 2486.410 & 27.934 & 0.116 & 388.49 & \\
\hline DH36 & $11 / 20 / 91$ & $11: 10$ & 3.00 & 2514.465 & 28.055 & 0.107 & 391.49 & \\
\hline DH36 & $12 / 18 / 91$ & $09: 25$ & 2.55 & 2542.392 & 27.927 & 0.091 & 394.04 & \\
\hline OH36 & $01 / 22 / 92$ & $08: 53$ & 3.11 & 2577.370 & 34.978 & 0.089 & 397.15 & Sampled for SNL project. \\
\hline DH36 & $02 / 19 / 92$ & $08: 45$ & 2.66 & 2605.365 & 27.995 & 0.095 & 399.81 & \\
\hline OH36 & $03 / 25 / 92$ & & 3.53 & 2640.000 & 34.635 & 0.102 & 403.34 & \\
\hline OH36 & $04 / 22 / 92$ & $09: 15$ & 2.81 & 2668.385 & 28.385 & 0.099 & 406.15 & \\
\hline DH36 & $05 / 27 / 92$ & $11: 00$ & 3.78 & 2703.458 & 35.073 & 0.108 & 409.93 & \\
\hline
\end{tabular}




\section{TABLE A-2 (Continued) \\ BRINE ACCUMULATION DATA TABLE}

Data through December 31, 1993

\begin{tabular}{|c|c|c|c|c|c|c|c|}
\hline & & & LIT & $\begin{array}{l}\text { DAYS } \\
\text { SINCE }\end{array}$ & $\begin{array}{l}\text { DAYS } \\
\text { USED FOR }\end{array}$ & & CUMULATIVE \\
\hline CATIOH & DATE & TIME & REMOVED & $1 / 1 / 85$ & CALCULATIOH & PER DAY & COLLECTED \\
\hline
\end{tabular}

\begin{tabular}{|c|c|c|c|c|c|c|c|c|}
\hline DH36 & $06 / 25 / 92$ & $09: 25$ & 4.0 & 2732.392 & 28.934 & 0.138 & 413.93 & \\
\hline DH36 & $07 / 29 / 92$ & $09: 30$ & 4.17 & 2766.396 & 34.004 & 0.123 & 418.10 & Saved for SHL. \\
\hline DH36 & $08 / 20 / 92$ & $08: 50$ & 1.00 & 2788.368 & 0.000 & 0.000 & 419.10 & $\begin{array}{l}\text { Partial evacuation for BSEP analytic } \\
\text { program. }\end{array}$ \\
\hline DH36 & $09 / 09 / 92$ & $10: 25$ & 4.45 & 2808.434 & 42.038 & 0.130 & 423.55 & $\begin{array}{l}\text { Combine with } 1.0 \text { liter evacuated on } \\
08-20-92 \text {. }\end{array}$ \\
\hline DH36 & $11 / 11 / 92$ & $09: 00$ & 8.23 & 2871.375 & 62.941 & 0.131 & 431.78 & \\
\hline DH36 & $02 / 26 / 93$ & $09: 30$ & 13.85 & 2978.396 & 107.021 & 0.129 & 445.63 & \\
\hline DH36 & $04 / 28 / 93$ & $10: 20$ & 8.40 & 3039.431 & 61.035 & 0.138 & 454.03 & \\
\hline DH36 & $06 / 16 / 93$ & $11: 10$ & 7.75 & 3088.465 & 49.034 & 0.158 & 461.78 & Used bailer. \\
\hline DH36 & $08 / 18 / 93$ & $14: 00$ & 8.17 & 3151.583 & 63.118 & 0.129 & 469.95 & Used bailer. \\
\hline DH36 & $11 / 12 / 93$ & $10: 07$ & 11.63 & 3237.422 & 85.839 & 0.135 & 481.58 & Used bailer. \\
\hline DH38 & $12 / 05 / 84$ & $.00: 00$ & NA & 0.000 & 0.000 & 0.000 & 0.00 & $\begin{array}{l}\text { Approximate date this part of Room } G \\
\text { excavated. }\end{array}$ \\
\hline DH3B & $01 / 26 / 85$ & $00: 00$ & NA & 25.000 & 52.000 & 0.000 & 0.00 & Downhole drilled $1 / 25 / 85$ to $1 / 26 / 85$. \\
\hline DH38 & $01 / 28 / 85$ & $09: 00$ & KA & 27.375 & 2.375 & 0.000 & 0.00 & Dry. \\
\hline DH38 & $02 / 05 / 85$ & $19: 15$ & HA & 35.469 & 8.094 & .0 .000 & 0.00 & Wet at bottom. \\
\hline DH38 & $02 / 19 / 85$ & $12: 10$ & 00.80 & 49.507 & 14.038 & 0.057 & 0.80 & Brine and fine muck. \\
\hline DH38 & $02 / 26 / 85$ & $10: 45$ & 01.26 & 56.448 & 6.941 & 0.182 & 2.06 & Brine and fine muck. \\
\hline DH38 & $03 / 05 / 85$ & $10: 00$ & 00.45 & 63.417 & 6.969 & 0.065 & 2.51 & - \\
\hline DH38 & $03 / 12 / 85$ & $10: 00$ & 00.39 & 70.417 & 7.000 & 0.056 & 2.90 & \\
\hline DH38 & $03 / 20 / 85$ & $10: 37$ & 00.45 & 78.442 & 8.025 & 0.056 & 3.35 & \\
\hline DH3B & $03 / 26 / 85$ & $09: 50$ & 00.36 & 84.410 & 5.968 & 0.060 & 3.71 & \\
\hline DH3B & $04 / 02 / 85$ & $10: 25$ & 00.41 & 91.434 & 7.024 & 0.058 & 4.12 & Some muck. \\
\hline DH38 & $04 / 10 / 85$ & $10: 31$ & 00.44 & 99.438 & 8.004 & 0.055 & 4.56 & \\
\hline DH3B & $04 / 17 / 85$ & $13: 30$ & 00.41 & 106.563 & 7.125 & 0.058 & 4.97 & \\
\hline DH38 & $04 / 23 / 85$ & $11: 41$ & 00.34 & 112.487 & 5.924 & 0.057 & 5.31 & \\
\hline DH38 & $04 / 30 / 85$ & $11: 05$ & 00.39 & 119.462 & 6.975 & 0.056 & 5.70 & \\
\hline DH38 & $05 / 07 / 85$ & $09: 50$ & 00.42 & 126.410 & 6.948 & 0.060 & 6.12 & \\
\hline DH38 & $05 / 14 / 85$ & $10: 45$ & 00.41 & 133.448 & 7.038 & 0.058 & 6.53 & \\
\hline DH38 & $05 / 21 / 85$ & $10: 35$ & 00.41 & 140.441 & 6.993 & 0.059 & 6.94 & \\
\hline DH38 & $05 / 29 / 85$ & $11: 35$ & 00.47 & 148.483 & 8.042 & 0.058 & 7.41 & \\
\hline OH3B & $06 / 04 / 85$ & $11: 25$ & 00.35 & 154.476 & 5.993 & 0.058 & 7.76 & - \\
\hline OH38 & $06 / 11 / 85$ & $10: 35$ & 00.40 & 161.441 & 6.965 & 0.057 & $8 .-16$ & $-\cdot-$ \\
\hline DH38 & $06 / 18 / 85$ & 10:09 & 00.39 & 168.423 & 6.982 & 0.056 & 8.55 & \\
\hline DH38 & $06 / 25 / 85$ & $10: 50$ & 00.42 & 175.451 & 7.028 & 0.060 & 8.97 & \\
\hline DH38 & $07 / 02 / 85$ & $11: 00$ & 00.44 & 182.458 & 7.007 & 0.063 & 9.41 & \\
\hline DH38 & $07 / 09 / 85$ & $11: 05$ & 00.43 & 189.462 & 7.004 & 0.061 & 9.84 & \\
\hline DH38 & $07 / 16 / 85$ & $11: 45$ & 00.43 & 196.490 & 7.028 & 0.061 & 10.27 & Brine effervesces. \\
\hline DH38 & $07 / 24 / 85$ & $10: 35$ & 00.49 & 204.441 & 7.951 & 0.062 & 10.76 & \\
\hline DH38 & $07 / 30 / 85$ & $10: 14$ & 00.38 & 210.426 & 5.985 & 0.063 & 11.14 & \\
\hline $\mathrm{OH} 38$ & $08 / 06 / 85$ & $10: 34$ & 00.42 & 217.440 & 7.014 & 0.060 & 11.56 & \\
\hline $\mathrm{OH} 38$ & $08 / 14 / 85$ & $10: 51$ & 00.49 & 225.452 & 8.012 & 0.061 & 12.05 & \\
\hline DH38 & $08 / 20 / 85$ & $11: 02$ & 00.37 & 231.460 & 6.008 & 0.062 & 12.42 & \\
\hline DH38 & $08 / 28 / 85$ & $10: 00$ & 00.51 & 239.417 & 7.957 & 0.064 & 12.93 & \\
\hline
\end{tabular}


TABLE A-2 (Continued)

BRINE ACCUMULATION DATA TABLE

Data through December 31, 1993

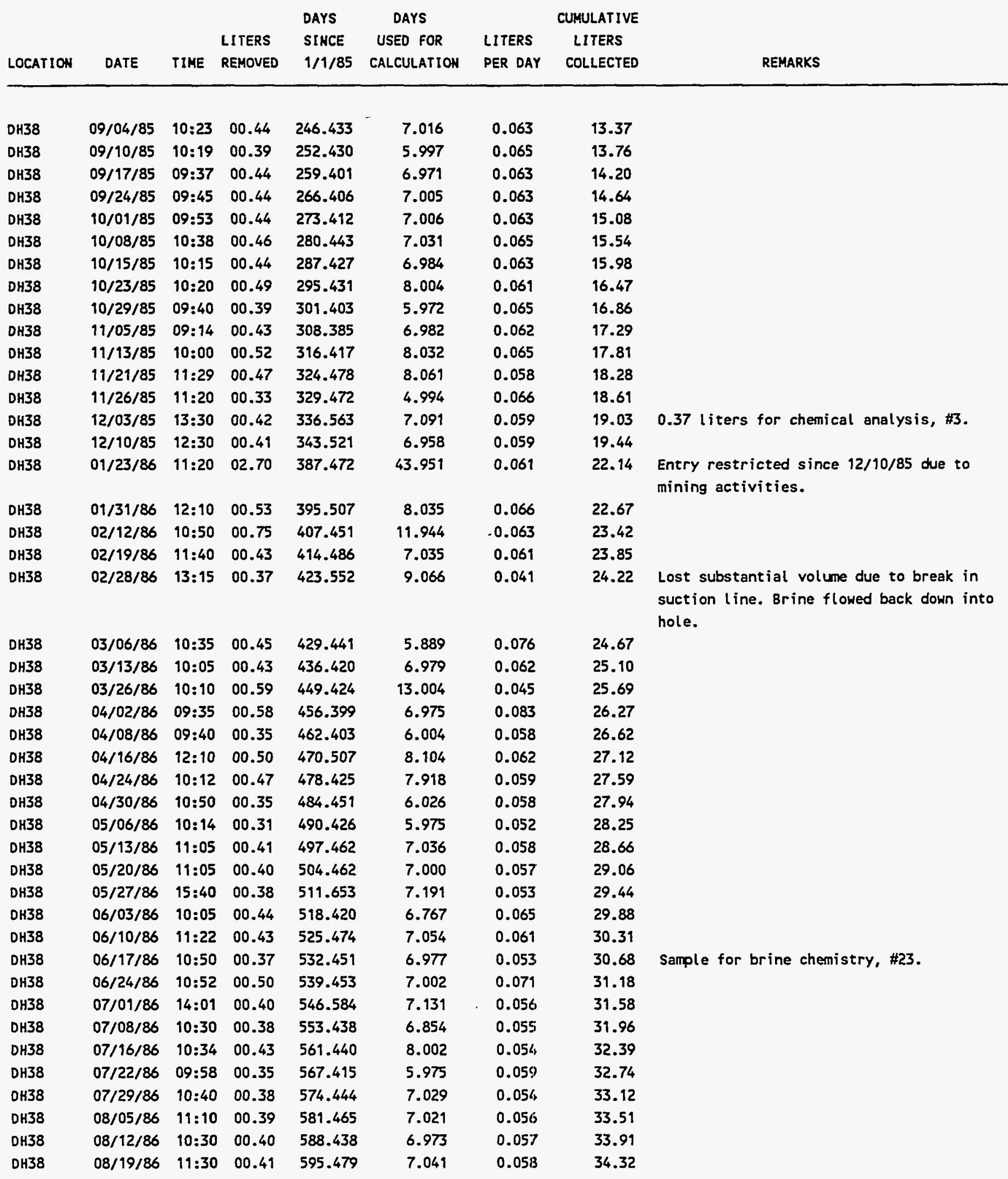


TABLE A-2 (Continued)

\section{BRINE ACCUMULATION DATA TABLE}

Data through December 31, 1993

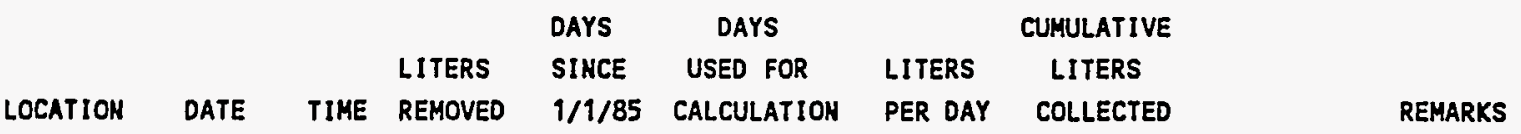

\begin{tabular}{|c|c|c|c|c|c|c|c|c|}
\hline DH38 & $08 / 26 / 86$ & $10: 32$ & 00.36 & 602.439 & 6.960 & 0.052 & 34.68 & Static level not measured. \\
\hline DH38 & $09 / 04 / 86$ & $10: 35$ & 00.49 & 611.441 & 9.002 & 0.054 & 35.17 & \\
\hline DH38 & $09 / 09 / 86$ & $10: 00$ & 00.30 & 616.417 & 4.976 & 0.060 & 35.47 & Sample \#25. \\
\hline DH38 & $09 / 16 / 86$ & $10: 11$ & 00.38 & 623.424 & 7.007 & 0.054 & 35.85 & \\
\hline OH38 & $09 / 23 / 86$ & $10: 10$ & 00.37 & 630.424 & 7.000 & 0.053 & 36.22 & \\
\hline DH38 & $10 / 01 / 86$ & $12: 07$ & 00.43 & 638.505 & 8.081 & 0.053 & 36.65 & \\
\hline OH38 & $10 / 08 / 86$ & $11: 30$ & 00.36 & 645.479 & 6.974 & 0.052 & 37.01 & \\
\hline OH38 & $10 / 14 / 86$ & $11: 45$ & 00.35 & 651.490 & 6.011 & 0.058 & 37.36 & \\
\hline DH38 & $11 / 05 / 86$ & $11: 26$ & 1.10 & 673.476 & 21.986 & 0.050 & 38.46 & \\
\hline OH38 & $11 / 20 / 86$ & $12: 27$ & 00.82 & 688.519 & 15.043 & 0.055 & 39.28 & \\
\hline OH38 & $12 / 30 / 86$ & $12: 15$ & 01.87 & 728.510 & 39.991 & 0.047 & 41.15 & Brôwn color, pH. 5.89 . \\
\hline DH38 & $02 / 03 / 87$ & $13: 15$ & 01.72 & 763.552 & 35.042 & 0.049 & 42.87 & \\
\hline DH38 & $03 / 06 / 87$ & $11: 05$ & 1.58 & 794.462 & 30.910 & 0.051 & 44.45 & \\
\hline DH38 & $03 / 30 / 87$ & $11: 13$ & 1.17 & 818.467 & 24.005 & 0.049 & 45.62 & \\
\hline OH38 & $05 / 07 / 87$ & $11: 20$ & 1.89 & 856.472 & 38.005 & 0.050 & 47.51 & \\
\hline DH38 & $06 / 17 / 87$ & $10: 45$ & 1.91 & 897.448 & 0.000 & 0.000 & 49.42 & $\begin{array}{l}\text { Samples removed for chemistry, \#106A, } \\
\text { \#109A, \#109B. Some brine left in hole, no } \\
\text { calculation. }\end{array}$ \\
\hline DH38 & $06 / 18 / 87$ & $12: 05$ & 0.16 & 898.503 & 42.031 & 0.049 & $49: 58$ & $\begin{array}{l}\text { Calculated using } 2.07 \text { liters }(1.91 \text { liters } \\
6 / 17 / 87 \text { plus } 0.16 \text { liters } 6 / 18 / 87) \text {. }\end{array}$ \\
\hline OH38 & $07 / 28 / 87$ & $10: 53$ & 1.88 & 938.453 & 39.950 & 0.047 & 51.46 & Collected for chemistry, sample \#106. \\
\hline DH38 & $09 / 01 / 87$ & $10: 45$ & 1.70 & 973.448 & 34.995 & 0.049 & 53.16 & Collected for chemistry, sample \#152 A\&B. \\
\hline DH38 & $10 / 20 / 87$ & $11: 40$ & 2.29 & 1022.486 & 49.038 & 0.047 & 55.45 & \\
\hline DH38 & $11 / 19 / 87$ & $11: 05$ & 1.42 & 1052.462 & 29.976 & 0.047 & 56.87 & Collected for chemistry, sample $\# 230$. \\
\hline DH38 & $01 / 04 / 88$ & $11: 35$ & 2.05 & 1098.483 & 46.021 & 0.045 & 58.92 & \\
\hline OH38 & $02 / 08 / 88$ & $11: 40$ & 1.48 & 1133.486 & 35.003 & 0.042 & 60.40 & $\begin{array}{l}\text { Collected for chemistry, sample \#258, \#259 } \\
\& \# 260 \text {. }\end{array}$ \\
\hline DH38 & $03 / 29 / 88$ & $11: 30$ & 2.10 & 1183.479 & 49.993 & 0.042 & 62.50 & $\begin{array}{l}\text { Collected for chemistry, sample } \# 363 \text { - } \\
\# 366 \text {. }\end{array}$ \\
\hline DH38 & $05 / 05 / 88$ & $09: 55$ & 1.70 & 1220.413 & 36.934 & 0.046 & 64.20 & Sampled for SNL/NM PA. \\
\hline OH3B & $05 / 12 / 88$ & $11: 20$ & 0.31 & 1227.472 & 7.059 & 0.044 & 64.51 & Sampled for SHL/HM PA. \\
\hline OH38 & $07 / 12 / 88$ & $08: 45$ & 2.44 & 1288.365 & 60.893 & 0.040 & 66.95 & $\begin{array}{l}\text { Collected for chemistry, sample \#417 - } \\
\# 421 \text {. }\end{array}$ \\
\hline OH38 & $07 / 28 / 88$ & $10: 20$ & .0 .88 & 1304.431 & 16.066 & 0.055 & 67.83 & Sampled for SKL/KH PA. \\
\hline DH38 & $09 / 27 / 88$ & $10: 30$ & 1.92 & 1365.438 & 61.007 & 0.031 & 69.75 & $\begin{array}{l}\text { Collected for chemistry, sample \#533 - } \\
\# 536 \text {. }\end{array}$ \\
\hline OH3B & $12 / 13 / 88$ & $09: 55$ & 3.45 & 1442.413 & 76.975 & 0.045 & 73.20 & $\begin{array}{l}\text { Collected for chemistry, sample } \# 582 \text { - } \\
\# 587 \text {. }\end{array}$ \\
\hline DH38 & $03 / 14 / 89$ & $09: 55$ & 3.25 & 1533.413 & 91.000 & 0.036 & 76.45 & $\begin{array}{l}\text { Sample saved for chemistry, sample } \$ 696 \text { - } \\
701 \text {. }\end{array}$ \\
\hline OH3B & $04 / 06 / 89$ & $09: 45$ & 1.03 & 1556.406 & 22.993 & 0.045 & 77.48 & \\
\hline OH38 & $04 / 20 / 89$ & $09: 35$ & 0.75 & 1570.399 & 13.993 & 0.054 & 78.23 & \\
\hline DH38 & $05 / 17 / 89$ & $10: 05$ & 1.11 & 1597.420 & 27.021 & 0.041 & 79.34 & \\
\hline DH38 & $06 / 06 / 89$ & $10: 00$ & 0.70 & 1617.417 & 19.997 & 0.035 & 80.04 & Sample saved for chemistry. \\
\hline DH38 & $06 / 29 / 89$ & $10: 30$ & 0.64 & 1640.438 & 23.021 & 0.028 & 80.68 & • \\
\hline OH38 & $07 / 25 / 89$ & $10: 27$ & 0.92 & 1666.435 & 25.997 & 0.035 & 81.60 & \\
\hline DH38 & $08 / 16 / 89$ & $09: 57$ & 0.81 & 1688.415 & 21.980 & 0.037 & 82.41 & \\
\hline
\end{tabular}


TABLE A-2 (Continued)

BRINE ACCUMULATION DATA TABLE

Data through December 31, 1993

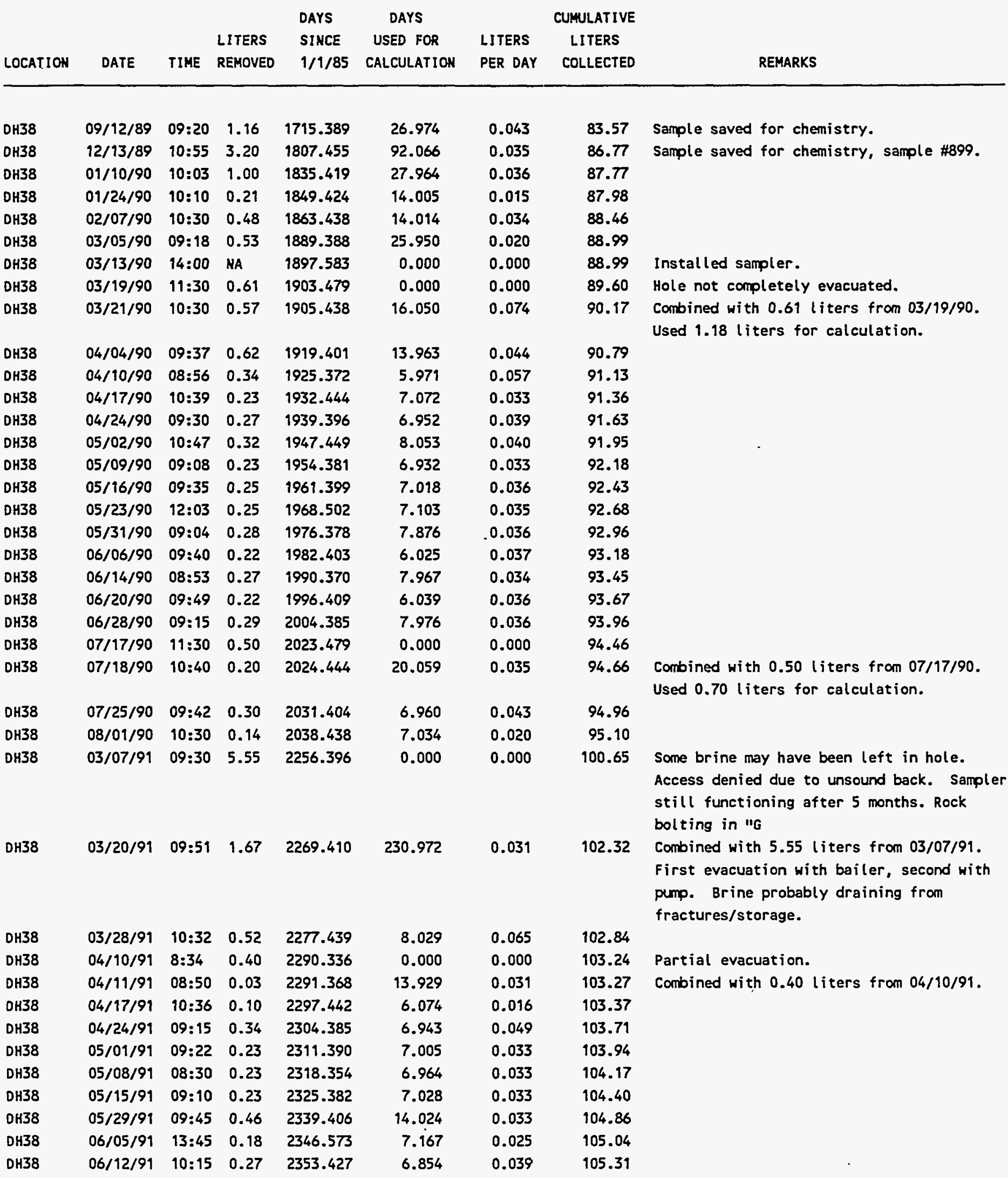


TABLE A-2 (Continued) BRINE ACCUMULATION DATA TABLE

Data through December 31, 1993

\begin{tabular}{|c|c|c|c|c|c|c|c|c|}
\hline & & & & DAYS & DAYS & & UHULATIVE & \\
\hline & & & LI & SIKCE & USED FOR & LITERS & LITERS & \\
\hline & DATE & TIME & REMOVED & $1 / 1 / 85$ & CALCULATIOH & PER DAY & COLLECTED & EMARKS \\
\hline
\end{tabular}

\begin{tabular}{|c|c|c|c|c|c|c|c|c|}
\hline DH38 & $06 / 19 / 99$ & $13: 54$ & 0.25 & 2360.579 & 7.152 & 0.035 & 105.56 & \\
\hline OH38 & $06 / 26 / 91$ & $09: 01$ & 0.00 & 2367.376 & 0.000 & 0.000 & 105.56 & No Vacuum, clamp failed. \\
\hline DH38 & $07 / 11 / 91$ & $10: 35$ & 0.58 & 2382.441 & 21.862 & 0.027 & 106.14 & \\
\hline OH38 & $07 / 17 / 91$ & $09: 30$ & 0.31 & 2388.396 & 5.955 & 0.052 & 106.45 & \\
\hline OH38 & $07 / 30 / 91$ & $10: 20$ & 0.45 & 2401.431 & 13.035 & 0.035 & 106.90 & \\
\hline DH38 & $08 / 08 / 91$ & $09: 18$ & 0.32 & 2410.388 & 8.957 & 0.036 & 107.22 & \\
\hline DH38 & $08 / 14 / 91$ & $10: 35$ & 0.22 & 2416.441 & 6.053 & 0.036 & 107.44 & \\
\hline DH38 & $08 / 21 / 91$ & $10: 55$ & 0.23 & 2423.455 & 7.014 & 0.033 & 107.67 & \\
\hline OH38 & $08 / 28 / 91$ & $10: 20$ & 0.17 & 2430.431 & 6.976 & 0.024 & 107.84 & \\
\hline DH38 & $09 / 04 / 91$ & $11: 18$ & 0.25 & 2437.471 & 7.040 & 0.036 & 108.09 & \\
\hline DH38 & $09 / 11 / 91$ & $11: 50$ & 0.23 & 2444.493 & 7.022 & 0.033 & 108.32 & \\
\hline DH38 & $09 / 18 / 91$ & $09: 20$ & 0.26 & 2451.389 & 6.896 & 0.038 & 108.58 & \\
\hline DH38 & $09 / 25 / 91$ & $12: 00$ & 0.25 & 2458.500 & 7.111 & 0.035 & 108.83 & \\
\hline DH38 & $\begin{array}{l}10 / 02 / 91 \\
10 / 16 / 01\end{array}$ & $\begin{array}{l}11: 09 \\
0.30\end{array}$ & 0.23 & 2465.465 & 6.965 & 0.033 & 109.06 & \\
\hline $\begin{array}{l}\text { DH38 } \\
\text { DH38 }\end{array}$ & $\begin{array}{l}10 / 16 / 91 \\
10 / 23 / 91\end{array}$ & $\begin{array}{l}09: 30 \\
09: 45\end{array}$ & $\begin{array}{l}0.46 \\
0.16\end{array}$ & $\begin{array}{l}2479.396 \\
2486.406\end{array}$ & $\begin{array}{r}13.931 \\
0.000\end{array}$ & 0.033 & 109.52 & Some brine may have been left in hole \\
\hline OHSO & $10 / 23 / 91$ & $08: 45$ & 0.10 & 2480.400 & 0.000 & 0.000 & 109.68 & $\begin{array}{l}\text { Some brine may have been left in hole. } \\
\text { Hose broke, lost vacuum. }\end{array}$ \\
\hline DH38 & $10 / 31 / 91$ & $09: 43$ & 0.45 & 2494.405 & 15.009 & 0.041 & 110.13 & Combined with 0.16 liters from 10/23/91. \\
\hline DH38 & $11 / 06 / 91$ & 10:07 & 0.22 & 2500.422 & 6.017 & .0 .037 & 110.35 & \\
\hline DH38 & $11 / 13 / 91$ & $09: 25$ & 0.24 & 2507.392 & 6.970 & 0.034 & 110.59 & \\
\hline DH38 & $11 / 20 / 91$ & $11: 15$ & 0.24 & 2514.469 & 7.077 & 0.034 & 110.83 & \\
\hline DH38 & $11 / 27 / 91$ & $09: 55$ & 0.24 & 2521.413 & 6.944 & 0.035 & 111.07 & \\
\hline DH38 & $12 / 04 / 91$ & $09: 52$ & 0.24 & 2528.411 & 6.998 & 0.034 & 111.31 & \\
\hline DH38 & $12 / 11 / 91$ & $09: 35$ & 0.22 & 2535.399 & 6.988 & 0.031 & 111.53 & \\
\hline DH38 & $12 / 18 / 91$ & $09: 20$ & 0.00 & 2542.389 & 0.000 & 0.000 & 191.53 & $\begin{array}{l}\text { Some brine may have been left in hole. } \\
\text { Vacuum gone. }\end{array}$ \\
\hline DH38 & $12 / 23 / 91$ & $08: 45$ & 0.37 & 2547.365 & 11.966 & 0.031 & 111.90 & \\
\hline DH38 & $01 / 08 / 92$ & $09: 55$ & 0.51 & 2563.413 & 16.048 & 0.032 & 112.41 & \\
\hline DH38 & $01 / 15 / 92$ & $09: 50$ & 0.25 & 2570.410 & 6.997 & 0.036 & 112.66 & \\
\hline DH38 & $01 / 22 / 92$ & $08: 47$ & 0.23 & 2577.366 & 6.956 & 0.033 & 112.89 & \\
\hline DH38 & $01 / 29 / 92$ & $10: 01$ & 0.23 & 2584.417 & 7.051 & 0.033 & 113.12 & \\
\hline DH38 & $02 / 12 / 92$ & 08:59 & 0.42 & 2598.374 & 13.957 & 0.030 & 113.54 & \\
\hline DH38 & $02 / 19 / 92$ & $09: 00$ & 0.33 & 2605.375 & 7.001 & 0.047 & 113.87 & \\
\hline DH38 & $02 / 26 / 92$ & $09: 50$ & 0.24 & 2612.410 & 7.035 & 0.034 & 114.91 & \\
\hline DH38 & $03 / 11 / 92$ & $09: 45$ & 0.43 & 2626.406 & 13.996 & 0.031 & 114.54 & \\
\hline DH38 & $03 / 18 / 92$ & $09: 55$ & 0.24 & 2633.413 & 7.007 & 0.034 & 114.78 & \\
\hline DH38 & $03 / 25 / 92$ & $10: 15$ & 0.23 & 2640.427 & 7.014 & 0.033 & 115.01 & • \\
\hline DH38 & $04 / 01 / 92$ & $09: 20$ & 0.25 & 2647.389 & 6.962 & 0.036 & 115.26 & \\
\hline DH38 & $04 / 07 / 92$ & $09: 25$ & 0.19 & 2653.392 & 6.003 & 0.032 & 115.45 & \\
\hline DH38 & $04 / 15 / 92$ & $09: 20$ & 0.31 & 2661.389 & 7.997 & 0.039 & 115.76 & \\
\hline DH38 & $04 / 22 / 92$ & $09: 38$ & 0.22 & 2668.401 & 7.012 & 0.031 & 115.98 & 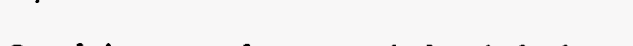 \\
\hline DH38 & $05 / 06 / 92$ & $11: 25$ & 0.45 & 2682.476 & 0.000 & 0.000 & 116.43 & $\begin{array}{l}\text { Partial evacuation, some brine left in } \\
\text { hole. }\end{array}$ \\
\hline DH38 & $05 / 07 / 92$ & $09: 35$ & 0.07 & 2683.399 & 14.998 & 0.035 & 116.50 & $\begin{array}{l}\text { Combine with } 0.45 \text { liters removed } 05 / 06 / 92 \\
\text { for total volume. }\end{array}$ \\
\hline DH38 & $05 / 13 / 92$ & $13: 50$ & 0.21 & 2689.576 & 6.177 & 0.034 & 116.71 & \\
\hline DH38 & $05 / 21 / 92$ & $10: 33$ & 0.27 & 2697.440 & 7.864 & 0.034 & 116.98 & \\
\hline
\end{tabular}


TABLE A-2 (Continued) BRINE ACCUMULATION DATA TABLE

Data through December 31, 1993

\begin{tabular}{|c|c|c|c|c|c|c|c|c|}
\hline & & & & DAYS & DAYS & & CUMULATIVE & \\
\hline & & & LITERS & SIMCE & USED FOR & LITERS & LITERS & \\
\hline LOCATION & DATE & TIME & REMOVED & $1 / 1 / 85$ & CALCULATIOH & PER DAY & COLLECTED & REMARKS \\
\hline
\end{tabular}

\begin{tabular}{|c|c|c|c|c|c|c|c|c|}
\hline DH38 & $05 / 27 / 92$ & $09: 25$ & 0.00 & 2703.392 & 0.000 & 0.000 & 116.98 & Ho vacuum. \\
\hline OH38 & $06 / 09 / 92$ & $09: 25$ & 0.51 & 2716.392 & 18.952 & 0.027 & 117.49 & \\
\hline DH38 & $06 / 18 / 92$ & $10: 05$ & 0.42 & 2725.420 & 9.028 & 0.047 & 117.91 & \\
\hline OH38 & $06 / 25 / 92$ & $09: 55$ & 0.25 & 2732.413 & 6.993 & 0.036 & 118.16 & \\
\hline DH38 & $07 / 01 / 92$ & $09: 36$ & 0.33 & 2738.400 & 5.987 & 0.055 & 118.49 & \\
\hline DH38 & $07 / 08 / 92$ & $09: 35$ & 0.15 & 2745.399 & 6.999 & 0.021 & 118.64 & \\
\hline DH38 & $07 / 15 / 92$ & $09: 30$ & 0.24 & 2752.396 & 6.997 & 0.034 & 118.88 & \\
\hline DH38 & $07 / 22 / 92$ & $09: 20$ & 0.29 & 2759.389 & 6.993 & 0.041 & 119.17 & \\
\hline $\mathrm{OH} 38$ & $07 / 29 / 92$ & $10: 05$ & 0.26 & 2766.420 & 7.031 & 0.037 & 119.43 & \\
\hline DH38 & $08 / 04 / 92$ & $09: 53$ & 0.25 & 2772.412 & 5.992 & 0.042 & 119.68 & \\
\hline OH38 & $08 / 18 / 92$ & $10: 22$ & 0.38 & 2786.432 & 14.020 & 0.027 & 120.06 & \\
\hline DH38 & $09 / 02 / 92$ & $10: 20$ & 0.56 & 2801.431 & 14.999 & 0.037 & 120.62 & \\
\hline DH38 & $09 / 09 / 92$ & $10: 15$ & 0.34 & 2808.427 & 6.996 & 0.049 & 120.96 & \\
\hline DH38 & $09 / 17 / 92$ & $09: 25$ & 0.23 & 2816.392 & 7.965 & 0.029 & 121.19 & \\
\hline DH38 & $09 / 23 / 92$ & $10: 15$ & 0.24 & 2822.427 & 6.035 & 0.040 & 121.43 & \\
\hline DH38 & $09 / 30 / 92$ & $11: 15$ & 0.27 & 2829.469 & 7.042 & 0.038 & 121.70 & \\
\hline DH38 & $10 / 12 / 92$ & $12: 50$ & 0.38 & 2841.535 & 12.066 & 0.031 & 122.08 & \\
\hline DH38 & $10 / 21 / 92$ & & 0.38 & 2850.000 & 8.465 & 0.045 & 122.46 & \\
\hline DH38 & $10 / 28 / 92$ & $09: 25$ & 0.28 & 2857.392 & 7.392 & .0 .038 & 122.74 & \\
\hline DH38 & $11 / 11 / 92$ & $09: 45$ & 0.43 & 2871.406 & 14.014 & 0.031 & 123.17 & \\
\hline DH38 & $11 / 18 / 92$ & $13: 22$ & 0.36 & 2878.557 & 7.151 & 0.050 & 123.53 & \\
\hline DH38 & $11 / 25 / 92$ & $09: 45$ & 0.26 & 2885.406 & 6.849 & 0.038 & 123.79 & \\
\hline DH38 & $12 / 09 / 92$ & $12: 55$ & 0.28 & 2899.538 & 14.132 & 0.020 & 124.07 & \\
\hline OH38 & $12 / 16 / 92$ & $10: 25$ & 0.42 & 2906.434 & 6.896 & 0.061 & 124.49 & \\
\hline DH38 & $01 / 07 / 93$ & $08: 45$ & 0.35 & 2928.365 & 21.931 & 0.016 & 124.84 & \\
\hline DH38 & $01 / 13 / 93$ & $09: 40$ & 0.53 & 2934.403 & 6.038 & 0.088 & 125.37 & \\
\hline DH38 & $01 / 28 / 93$ & $10: 35$ & 0.50 & 2949.441 & 15.038 & 0.033 & 125.87 & \\
\hline DH38 & $02 / 11 / 93$ & $10: 00$ & 0.50 & 2963.417 & 13.976 & 0.036 & 126.37 & \\
\hline DH38 & $02 / 26 / 93$ & $10: 50$ & 0.40 & 2978.451 & 15.034 & 0.027 & 126.77 & \\
\hline DH38 & $03 / 10 / 93$ & $10: 45$ & 0.35 & 2990.448 & 11.997 & 0.029 & 127.12 & \\
\hline DH38 & $03 / 19 / 93$ & $09: 25$ & 0.46 & 2999.392 & 8.944 & 0.051 & 127.58 & \\
\hline DH38 & $03 / 25 / 93$ & $10: 35$ & 0.44 & 3005.441 & 6.049 & 0.073 & 128.02 & \\
\hline DH38 & $03 / 31 / 93$ & $13: 25$ & 0.27 & 3011.559 & 6.118 & 0.044 & 128.29 & \\
\hline DH38 & $04 / 28 / 93$ & $09: 30$ & 0.22 & 3039.396 & 0.000 & 0.000 & 128.51 & Partial evacuation. \\
\hline DH38 & $06 / 16 / 93$ & $11: 20$ & 1.11 & 3088.472 & 76.913 & 0.017 & 129.62 & $\begin{array}{l}\text { Used bailer. Combine with } 0.22 \text { liters from } \\
04-28-93 \text {. }\end{array}$ \\
\hline DH38 & $08 / 18 / 93$ & $14: 19$ & 0.28 & 3151.597 & 0.000 & 0.000 & 129.90 & $\begin{array}{l}\text { Partial evacuation. Unable to sample since } \\
06-16-93 \text {. }\end{array}$ \\
\hline DH38 & $08 / 19 / 93$ & $14: 02$ & 2.10 & 3152.585 & 0.000 & 0.000 & 132.00 & Partial evacuation. \\
\hline DH38 & $08 / 20 / 93$ & $09: 56$ & 0.33 & 3153.414 & 64.942 & 0.042 & 132.33 & $\begin{array}{l}\text { Combine with } 0.28 \text { liters from } 08-18-93 \text { and } \\
2.10 \text { liters from } 08-19-93 \text {. }\end{array}$ \\
\hline 0438 & $11 / 12 / 93$ & $10: 41$ & 3.01 & 3237.445 & 84.031 & 0.036 & 135.34 & Used bailer. \\
\hline $\mathrm{OH} 40$ & $12 / 13 / 84$ & $00: 00$ & NA & 0.000 & 0.000 & 0.000 & 0.00 & $\begin{array}{l}\text { Approximate date this part of Room } G \\
\text { excavated. }\end{array}$ \\
\hline DH4O & $01 / 25 / 85$ & $00: 00$ & MA & 24.000 & 43.000 & 0.000 & 0.00 & Downhole drilled $1 / 24 / 85$ to $1 / 25 / 85$. \\
\hline
\end{tabular}


TABLE A-2 (Continued)

BRINE ACCUMULATION DATA TABLE

Data through December 31, 1993

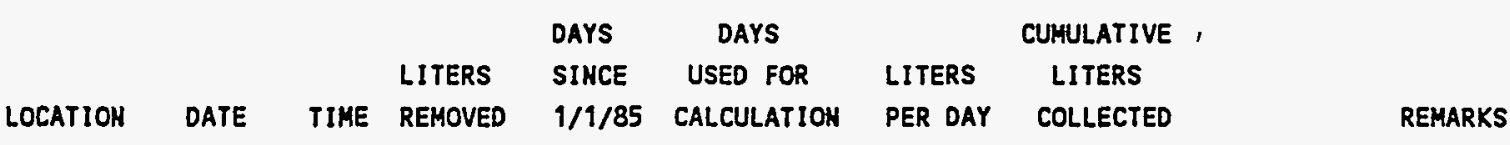

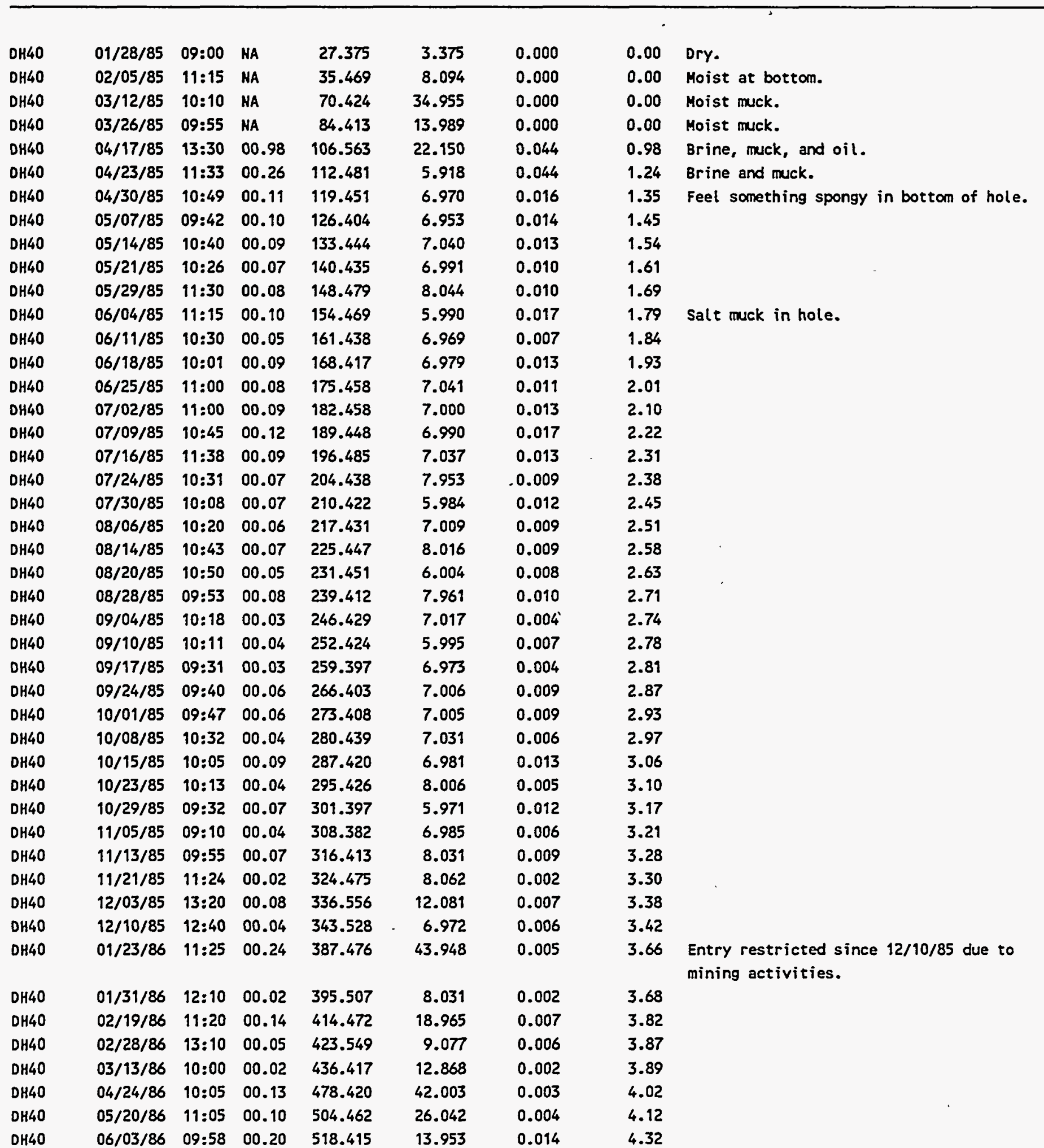


TABLE A-2 (Continued) BRINE ACCUMULATION DATA TABLE

Data through December 31, 1993

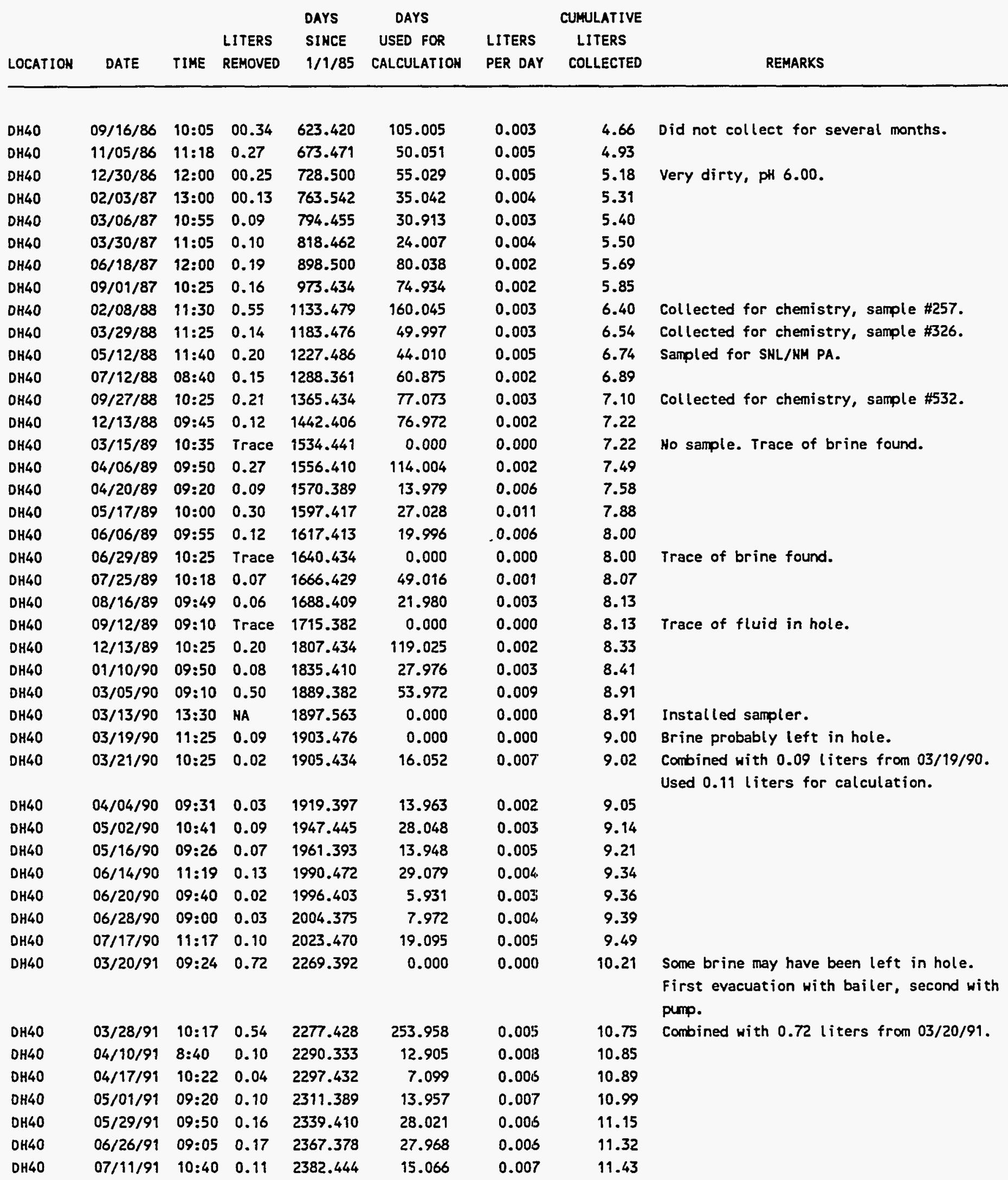


TABLE A-2 (Continued)

BRINE ACCUMULATION DATA TABLE

Data through December 31, 1993

\begin{tabular}{|c|c|c|c|c|c|c|c|}
\hline \multirow{2}{*}{ OCATION } & \multirow{2}{*}{ DATE } & \multirow{2}{*}{ TIME } & \multirow{2}{*}{\multicolumn{2}{|c|}{$\begin{array}{l}\text { DAYS } \\
\text { SIKCE }\end{array}$}} & DAYS & \multicolumn{2}{|r|}{ CUMULATIVE } \\
\hline & & & & & USED FOR & LITERS & LITERS \\
\hline
\end{tabular}

\begin{tabular}{|c|c|c|c|c|c|c|c|}
\hline DH4O & $08 / 08 / 91$ & 09:15 & 0.18 & 2410.385 & 27.941 & 0.006 & 11.61 \\
\hline $\mathrm{OH} 40$ & $08 / 21 / 91$ & $10: 50$ & 0.10 & 2423.451 & 13.066 & 0.008 & 11.71 \\
\hline DH40 & $09 / 18 / 91$ & $09: 15$ & 0.14 & 2451.385 & 27.934 & 0.005 & 11.85 \\
\hline $\mathrm{DH} 40$ & $09 / 25 / 91$ & $11: 55$ & 0.13 & 2458.497 & 7.112 & 0.018 & 11.98 \\
\hline $\mathrm{DH} 40$ & $10 / 16 / 91$ & $09: 25$ & 0.15 & 2479.392 & 20.895 & 0.007 & 12.13 \\
\hline $\mathrm{OH} 40$ & $10 / 23 / 91$ & $09: 40$ & 0.07 & 2486.403 & 7.019 & 0.010 & 12.20 \\
\hline $\mathrm{OH} 4 \mathrm{O}$ & $11 / 13 / 91$ & $09: 30$ & 0.14 & 2507.396 & 20.993 & 0.007 & 12.34 \\
\hline DH4O & $11 / 27 / 91$ & $10: 00$ & 0.10 & 2521.417 & 14.021 & 0.007 & 12.44 \\
\hline DH4O & $12 / 11 / 91$ & $09: 40$ & 0.11 & 2535.403 & 13.986 & 0.008 & 12.55 \\
\hline DH4O & $01 / 08 / 92$ & $09: 52$ & 0.13 & 2563.411 & 28.008 & 0.005 & 12.68 \\
\hline DH4O & $01 / 22 / 92$ & $08: 42$ & 0.10 & 2577.363 & 13.952 & 0.007 & 12.78 \\
\hline DH4O & $01 / 29 / 92$ & $09: 56$ & 0.05 & 2584.414 & 7.051 & 0.007 & 12.83 \\
\hline DH4O & $02 / 12 / 92$ & $08: 53$ & 0.11 & 2598.370 & 13.956 & 0.008 & 12.94 \\
\hline DH4O & $02 / 19 / 92$ & $09: 15$ & 0.07 & 2605.385 & 7.015 & 0.010 & 13.01 \\
\hline DH4O & $02 / 26 / 92$ & $09: 45$ & 0.05 & 2612.406 & 7.021 & 0.007 & 13.06 \\
\hline OH4O & $03 / 11 / 92$ & $09: 40$ & 0.02 & 2626.403 & 13.997 & 0.001 & 13.08 \\
\hline DH4O & $03 / 18 / 92$ & $09: 50$ & 0.18 & 2633.410 & 7.007 & 0.026 & 13.26 \\
\hline DH4O & $03 / 25 / 92$ & $10: 30$ & 0.04 & 2640.438 & 7.028 & 0.006 & 13.30 \\
\hline $\mathrm{OH} 40$ & $04 / 15 / 92$ & $09: 15$ & 0.15 & 2661.385 & 20.947 & .0 .007 & 13.45 \\
\hline $\mathrm{OH} 4 \mathrm{O}$ & $04 / 22 / 92$ & $09: 35$ & 0.07 & 2668.399 & 7.014 & 0.010 & 13.52 \\
\hline OH4O & $05 / 06 / 92$ & $11: 20$ & 0.13 & 2682.472 & 14.073 & 0.009 & 13.65 \\
\hline DH4O & $05 / 27 / 92$ & $09: 15$ & 0.16 & 2703.385 & 20.913 & 0.008 & 13.81 \\
\hline OH4O & $06 / 09 / 92$ & $09: 15$ & 0.11 & 2716.385 & 13.000 & 0.008 & 13.92 \\
\hline DH4O & $06 / 18 / 92$ & $10: 00$ & 0.09 & 2725.417 & 9.032 & 0.010 & 14.01 \\
\hline DH4O & $06 / 25 / 92$ & $09: 50$ & 0.08 & 2732.410 & 6.993 & 0.011 & 14.09 \\
\hline DH40 & 07/01/92 & $09: 30$ & 0.18 & 2738.396 & 5.986 & 0.030 & 14.27 \\
\hline DH40 & $07 / 15 / 92$ & $09: 25$ & 0.19 & 2752.392 & 13.996 & 0.014 & 14.46 \\
\hline DH4O & $07 / 22 / 92$ & $09: 15$ & 0.16 & 2759.385 & 6.993 & 0.023 & 14.62 \\
\hline DH40 & $07 / 29 / 92$ & $09: 57$ & 0.10 & 2766.415 & 7.030 & 0.014 & 14.72 \\
\hline $\mathrm{OH} 40$ & $08 / 04 / 92$ & $09: 45$ & 0.12 & 2772.406 & 5.991 & 0.020 & 14.84 \\
\hline DH40 & $08 / 18 / 92$ & $09: 59$ & 0.28 & 2786.416 & 14.010 & 0.020 & 15.12 \\
\hline DH40 & $09 / 02 / 92$ & $10: 00$ & 0.30 & 2801.417 & 15.001 & 0.020 & 15.42 \\
\hline DH40 & $09 / 09 / 92$ & $10: 05$ & 0.15 & 2808.420 & 7.003 & 0.021 & 15.57 \\
\hline DH40 & $09 / 17 / 92$ & $09: 15$ & 0.16 & 2816.385 & 7.965 & 0.020 & 15.73 \\
\hline DH40 & $09 / 23 / 92$ & $10: 10$ & 0.11 & 2822.424 & 6.039 & 0.018 & 15.84 \\
\hline DH4O & $10 / 12 / 92$ & $12: 45$ & 0.20 & 2841.531 & 19.107 & 0.010 & 16.04 \\
\hline $\mathrm{DH} 40$ & $10 / 21 / 92$ & $13: 10$ & 0.28 & 2850.549 & 9.018 & 0.031 & 16.32 \\
\hline $\mathrm{DH} 40$ & $10 / 28 / 92$ & $09: 20$ & 0.10 & 2857.389 & 6.840 & 0.015 & 16.42 \\
\hline $\mathrm{DH} 40$ & $11 / 11 / 92$ & $09: 40$ & 0.38 & 2871.403 & 14.014 & 0.027 & 16.80 \\
\hline DH4O & $11 / 18 / 92$ & $13: 15$ & 0.17 & 2878.552 & 7.149 & 0.024 & 16.97 \\
\hline DH4O & $11 / 25 / 92$ & $09: 40$ & 0.09 & 2885.403 & 6.851 & 0.013 & 17.06 \\
\hline DH4O & $12 / 09 / 92$ & $12: 45$ & 0.25 & 2899.531 & 14.128 & 0.018 & 17.31 \\
\hline $\mathrm{OH} 4 \mathrm{O}$ & $12 / 16 / 92$ & $10: 20$ & 0.04 & 2906.431 & 6.900 & 0.006 & 17.35 \\
\hline DH4O & $01 / 07 / 93$ & $08: 40$ & 0.27 & 2928.361 & 21.930 & 0.012 & 17.62 \\
\hline $\mathrm{OH} 40$ & $01 / 13 / 93$ & $09: 53$ & 0.53 & 2934.412 & 6.051 & 0.088 & 18.15 \\
\hline DH4O & $01 / 28 / 93$ & $10: 25$ & 0.30 & 2949.434 & 15.022 & 0.020 & 18.45 \\
\hline 40 & $2 / 11 / 93$ & $9: 55$ & 0.30 & 413 & .979 & 0.021 & 18.75 \\
\hline
\end{tabular}


TABLE A-2 (Continued)

\section{BRINE ACCUMULATION DATA TABLE}

Data through December 31, 1993

\begin{tabular}{lccccccc} 
LOCATION & DATE & TIME & $\begin{array}{l}\text { LITERS } \\
\text { REMOVED }\end{array}$ & $\begin{array}{l}\text { DAYS } \\
\text { SIHCE } \\
1 / 1 / 85\end{array}$ & $\begin{array}{c}\text { DAYS } \\
\text { USED FOR } \\
\text { CALCULATION }\end{array}$ & $\begin{array}{l}\text { LITERS } \\
\text { PER DAY }\end{array}$ & $\begin{array}{c}\text { CUMULATIVE } \\
\text { LITERS } \\
\text { COLLECTED }\end{array}$ \\
\hline & & & & & & & \\
DH4O & $02 / 26 / 93$ & $10: 45$ & 0.23 & 2978.448 & 15.035 & 0.015 & 18.98 \\
DH4O & $03 / 10 / 93$ & $10: 40$ & 0.43 & 2990.444 & 11.996 & 0.036 & 19.41 \\
DH40 & $03 / 19 / 93$ & $09: 28$ & 0.22 & 2999.394 & 8.950 & 0.025 & 19.63 \\
DH40 & $03 / 25 / 93$ & $10: 15$ & 0.10 & 3005.427 & 6.033 & 0.017 & 19.73 \\
DH40 & $03 / 31 / 93$ & $13: 05$ & 0.13 & 3011.545 & 6.118 & 0.021 & 19.86 \\
DH40 & $04 / 28 / 93$ & $09: 15$ & 0.48 & 3039.385 & 27.840 & 0.017 & 20.34 \\
OH40 & $06 / 16 / 93$ & $11: 30$ & 0.45 & 3088.479 & 49.094 & 0.009 & 20.79 \\
DH40 & $08 / 19 / 93$ & $14: 12$ & 0.42 & 3152.592 & 64.113 & 0.007 & 21.21 \\
DH40 & $11 / 12 / 93$ & $10: 28$ & 0.78 & 3237.436 & 84.844 & 0.009 & 21.99
\end{tabular}

\begin{tabular}{|c|c|c|c|c|c|c|c|c|}
\hline $\mathrm{OH} 42$ & $12 / 30 / 84$ & $00: 00$ & NA & 0.000 & 0.000 & 0.000 & 0.00 & $\begin{array}{l}\text { Approximate date this part of Room } \mathrm{G} \\
\text { excavated. }\end{array}$ \\
\hline DH42 & $01 / 23 / 85$ & $00: 00$ & NA & 22.000 & 24.000 & 0.000 & 0.00 & Downhole drilled. \\
\hline DH42 & $01 / 28 / 85$ & $09: 00$ & NA & 27.375 & 0.000 & 0.000 & 0.00 & Moist muck at the bottom. \\
\hline $\mathrm{DH} 42$ & $02 / 05 / 85$ & $11: 15$ & 00.27 & 35.469 & 13.469 & 0.020 & 0.27 & First time collected. \\
\hline DH42 & $02 / 11 / 85$ & $11: 00$ & 00.30 & 41.458 & 5.989 & 0.050 & 0.57 & \\
\hline DH42 & $02 / 19 / 85$ & $13: 10$ & 00.33 & 49.549 & 8.091 & 0.041 & 0.90 & \\
\hline OH42 & $02 / 26 / 85$ & $10: 45$ & 00.26 & 56.448 & 6.899 & 0.038 & 1.16 & \\
\hline OH42 & $03 / 05 / 85$ & $10: 00$ & 00.28 & 63.417 & 6.969 & 0.040 & 1.44 & \\
\hline DH42 & $03 / 12 / 85$ & $10: 20$ & 00.25 & 70.431 & 7.014 & 0.036 & 1.69 & \\
\hline OH42 & $03 / 20 / 85$ & $10: 54$ & 00.25 & 78.454 & 8.023 & 0.031 & 1.94 & $\begin{array}{l}\text { Valve leaked, some brine drained back down } \\
\text { hole. }\end{array}$ \\
\hline 0442 & $03 / 26 / 85$ & $10: 06$ & 00.28 & 84.421 & 5.967 & 0.047 & 2.22 & \\
\hline DH42 & $04 / 02 / 85$ & $10: 45$ & 00.26 & 91.448 & 7.027 & 0.037 & 2.48 & \\
\hline DH42 & $04 / 10 / 85$ & $10: 45$ & 00.29 & 99.448 & 8.000 & 0.036 & 2.77 & . \\
\hline DH42 & $04 / 17 / 85$ & $13: 30$ & 00.24 & 106.563 & 7.115 & 0.034 & 3.01 & \\
\hline DH42 & $04 / 23 / 85$ & $13: 23$ & 00.04 & 112.558 & 5.995 & 0.007 & 3.05 & $\begin{array}{l}\text { Significant volume of brine drained back } \\
\text { down hole. }\end{array}$ \\
\hline DH42 & $04 / 30 / 85$ & $10: 31$ & 00.38 & 119.438 & 6.880 & 0.055 & 3.43 & \\
\hline DH42 & $05 / 07 / 85$ & $09: 25$ & 00.33 & 126.392 & 6.954 & 0.047 & 3.76 & \\
\hline DH42 & $05 / 14 / 85$ & $10: 30$ & 00.25 & 133.438 & 7.046 & 0.035 & 4.01 & \\
\hline DH42 & $05 / 21 / 85$ & $10: 17$ & 00.26 & 140.428 & 6.990 & 0.037 & 4.27 & \\
\hline DH42 & $05 / 29 / 85$ & $10: 10$ & 00.30 & 148.424 & 7.996 & 0.038 & 4.57 & \\
\hline DH42 & $06 / 04 / 85$ & $10: 45$ & 00.22 & 154.448 & 6.024 & 0.037 & 4.79 & \\
\hline DH42 & $06 / 11 / 85$ & $10: 10$ & 00.25 & 161.424 & 6.976 & 0.036 & 5.04 & \\
\hline DH42 & $06 / 18 / 85$ & $09: 53$ & 00.25 & 168.412 & 6.988 & 0.036 & 5.29 & \\
\hline DH42 & $06 / 25 / 85$ & $11: 15$ & 00.25 & 175.469 & 7.057 & 0.035 & 5.54 & \\
\hline $\mathrm{OH} 42$ & $07 / 02 / 85$ & $11: 00$ & 00.24 & 182.458 & 6.989 & 0.034 & 5.78 & \\
\hline DH42 & $07 / 09 / 85$ & $10: 30$ & 00.25 & 189.438 & 6.980 & 0.036 & 6.03 & \\
\hline DH42 & $07 / 16 / 85$ & $11: 08$ & 00.25 & 196.464 & 7.026 & 0.036 & 6.28 & Brine effervesces. \\
\hline DH42 & $07 / 24 / 85$ & $10: 19$ & 00.28 & 204.430 & 7.966 & 0.035 & 6.56 & \\
\hline $\mathrm{OH} 42$ & $07 / 30 / 85$ & $09: 57$ & 00.22 & 210.415 & 5.985 & 0.037 & 6.78 & \\
\hline DH42 & $08 / 06 / 85$ & $10: 13$ & 00.26 & 217.426 & 7.019 & 0.037 & 7.04 & \\
\hline DH42 & $08 / 14 / 85$ & $10: 59$ & 00.27 & 225.458 & 8.032 & 0.034 & 7.31 & \\
\hline $\mathrm{OH} 42$ & $08 / 20 / 85$ & $10: 45$ & 00.21 & 231.448 & 5.990 & 0.035 & 7.52 & \\
\hline DH42 & $08 / 28 / 85$ & $09: 45$ & 00.29 & 239.406 & 7.958 & 0.036 & 7.81 & \\
\hline
\end{tabular}


TABLE A-2 (Continued) BRINE ACCUMULATION DATA TABLE

Data through December 31, 1993

\begin{tabular}{|c|c|c|c|c|c|c|c|c|}
\hline LOCATION & DATE & TIME & $\begin{array}{l}\text { LITERS } \\
\text { REMOVED }\end{array}$ & $\begin{array}{l}\text { DAYS } \\
\text { SIHCE } \\
1 / 1 / 85\end{array}$ & $\begin{array}{l}\text { DAYS } \\
\text { USED FOR } \\
\text { CALCULATIOH }\end{array}$ & $\begin{array}{l}\text { LITERS } \\
\text { PER DAY }\end{array}$ & $\begin{array}{c}\text { CUMULATIVE } \\
\text { LITERS } \\
\text { COLLECTED }\end{array}$ & REMARKS \\
\hline DH42 & $09 / 04 / 85$ & $10: 12$ & 00.25 & 246.425 & 7.019 & 0.036 & 8.06 & \\
\hline DH42 & $09 / 10 / 85$ & $09: 56$ & 00.21 & 252.414 & 5.989 & 0.035 & 8.27 & \\
\hline DH42 & $09 / 17 / 85$ & $09: 26$ & 00.28 & 259.393 & 6.979 & 0.040 & 8.55 & \\
\hline DH42 & $09 / 24 / 85$ & $09: 37$ & 00.24 & 266.401 & 7.008 & 0.034 & 8.79 & \\
\hline DH42 & $10 / 01 / 85$ & $09: 44$ & 00.24 & 273.406 & 7.005 & 0.034 & 9.03 & \\
\hline DH42 & $10 / 08 / 85$ & $10: 25$ & 00.23 & 280.434 & 7.028 & 0.033 & 9.26 & \\
\hline DH42 & $10 / 15 / 85$ & $10: 00$ & 00.23 & 287.417 & 6.983 & 0.033 & 9.49 & \\
\hline DH42 & $10 / 23 / 85$ & $10: 07$ & 00.26 & 295.422 & 8.005 & 0.032 & 9.75 & \\
\hline $\mathrm{DH} 42$ & $10 / 29 / 85$ & $09: 16$ & 00.24 & 301.386 & 5.964 & 0.040 & 9.99 & \\
\hline DH42 & $11 / 05 / 85$ & $09: 05$ & 00.22 & 308.378 & 6.992 & 0.031 & 10.21 & \\
\hline DH42 & $11 / 13 / 85$ & $09: 46$ & 00.26 & 316.407 & 8.029 & 0.032 & 10.47 & \\
\hline DH42 & $11 / 21 / 85$ & $10: 53$ & 00.26 & 324.453 & 8.046 & 0.032 & 10.73 & \\
\hline DH42 & $11 / 26 / 85$ & $10: 59$ & 00.16 & 329.458 & 5.005 & 0.032 & 10.89 & \\
\hline DH42 & $12 / 03 / 85$ & $13: 10$ & 00.20 & 336.549 & 7.091 & 0.028 & 11.09 & Sample for chemistry analysis, $\# 2$. \\
\hline OH42 & $12 / 10 / 85$ & $12: 50$ & 00.22 & 343.535 & 6.986 & 0.031 & 11.31 & \\
\hline DH42 & $01 / 23 / 86$ & $11: 30$ & 01.32 & 387.479 & 43.944 & 0.030 & 12.63 & $\begin{array}{l}\text { Entry restricted since } 12 / 10 / 85 \text { due to } \\
\text { mining activities. }\end{array}$ \\
\hline DH42 & $01 / 31 / 86$ & $12: 05$ & 00.30 & 395.503 & 8.024 & 0.037 & 12.93 & \\
\hline DH42 & $02 / 12 / 86$ & $10: 35$ & 00.38 & 407.441 & 11.938 & .0 .032 & 13.31 & \\
\hline DH42 & $02 / 19 / 86$ & $11: 10$ & 00.22 & 414.465 & 7.024 & 0.031 & 13.53 & \\
\hline DH42 & $02 / 28 / 86$ & $13: 00$ & 00.31 & 423.542 & 9.077 & 0.034 & 13.84 & \\
\hline DH42 & $03 / 06 / 86$ & $10: 30$ & 00.17 & 429.438 & 5.896 & 0.029 & 14.01 & \\
\hline DH42 & $03 / 13 / 86$ & $09: 53$ & 00.21 & 436.412 & 6.974 & 0.030 & 14.22 & \\
\hline DH42 & $03 / 26 / 86$ & $10: 00$ & 00.39 & 449.417 & 13.005 & 0.030 & 14.61 & \\
\hline DH42 & $04 / 02 / 86$ & $09: 25$ & 00.20 & 456.392 & 6.975 & 0.029 & 14.81 & \\
\hline DH42 & $04 / 08 / 86$ & $09: 30$ & 00.20 & 462.396 & 6.004 & 0.033 & 15.01 & \\
\hline DH42 & $04 / 16 / 86$ & $11: 55$ & 00.24 & 470.497 & 8.101 & 0.030 & 15.25 & \\
\hline DH42 & $04 / 24 / 86$ & $09: 55$ & 00.21 & 478.413 & 7.916 & 0.027 & 15.46 & \\
\hline DH42 & $04 / 30 / 86$ & $10: 41$ & 00.17 & 484.445 & 6.032 & 0.028 & 15.63 & \\
\hline DH42 & $05 / 06 / 86$ & $10: 10$ & 00.19 & 490.424 & 5.979 & 0.032 & 15.82 & \\
\hline DH42 & $05 / 13 / 86$ & $10: 00$ & 00.20 & 497.417 & 6.993 & 0.029 & .16 .02 & \\
\hline DH42 & $05 / 20 / 86$ & $11: 00$ & 00.20 & $504.45 ̣ 8$ & 7.041 & 0.028 & 16.22 & \\
\hline DH42 & $05 / 27 / 86$ & $15: 35$ & 00.20 & 511.649 & 7.191 & 0.028 & 16.42 & \\
\hline DH42 & $06 / 03 / 86$ & $09: 50$ & 00.20 & 518.410 & 6.761 & 0.030 & 16.62 & \\
\hline DH42 & $06 / 10 / 86$ & $11: 13$ & 00.17 & 525.467 & 7.057 & 0.024 & 16.79 & \\
\hline DH42 & $06 / 17 / 86$ & $10: 40$. & 00.20 & 532.444 & 6.977 & 0.029 & 16.99 & Sample for brine chemistry, \#22. \\
\hline DH42 & $06 / 24 / 86$ & $10: 40$ & 00.18 & 539.444 & 7.000 & 0.026 & 17.17 & \\
\hline DH42 & $07 / 01 / 86$ & $13: 45$ & 00.20 & 546.573 & 7.129 & 0.028 & 17.37 & \\
\hline DH42 & $07 / 08 / 86$ & $10: 22$ & 00.20 & 553.432 & 6.859 & 0.029 & 17.57 & \\
\hline DH42 & $07 / 16 / 86$ & $10: 15$ & 00.30 & 561.427 & 7.995 & 0.038 & 17.87 & \\
\hline DH42 & $07 / 22 / 86$ & $09: 50$ & 00.16 & 567.410 & 5.983 & 0.027 & 18.03 & \\
\hline DH42 & $07 / 29 / 86$ & $10: 25$ & 00.20 & 574.434 & 7.024 & 0.028 & 18.23 & \\
\hline DH42 & $08 / 05 / 86$ & $11: 00$ & 00.22 & 581.458 & 7.024 & 0.031 & 18.45 & \\
\hline DH42 & $08 / 12 / 86$ & $10: 20$ & 00.20 & 588.431 & 6.973 & 0.029 & 18.65 & \\
\hline DH42 & $08 / 19 / 86$ & $11: 20$ & 00.18 & 595.472 & 7.041 & 0.026 & 18.83 & \\
\hline DH42 & $08 / 26 / 86$ & $10: 25$ & 00.20 & 602.434 & 6.962 & 0.029 & 19.03 & Static level not measured. \\
\hline DH42 & $09 / 04 / 86$ & $10: 20$ & 00.25 & 611.431 & 8.997 & 0.028 & 19.28 & \\
\hline
\end{tabular}


TABLE A-2 (Continued) BRINE ACCUMULATION DATA TABLE

Data through December 31, 1993

\begin{tabular}{|c|c|c|c|c|c|c|c|c|}
\hline & & & & DAYS & DAYS & & CUMULATIVE & \\
\hline & & & HITERS & SINCE & USED FOR & LITERS & LITERS & \\
\hline DCATION & DATE & TIME & REMOVED & $1 / 1 / 85$ & CALCULATION & PER DAY & COLLECTED & IARKS \\
\hline
\end{tabular}

\begin{tabular}{|c|c|c|c|c|c|c|c|c|}
\hline DH42 & $09 / 09 / 86$ & $09: 46$ & 00.14 & 616.407 & 4.976 & 0.028 & 19.42 & Sample \#24. \\
\hline DH42 & $09 / 16 / 86$ & $09: 52$ & 00.20 & 623.411 & 7.004 & 0.029 & 19.62 & \\
\hline $\mathrm{OH} 42$ & $09 / 23 / 86$ & $09: 58$ & 00.15 & 630.415 & 7.004 & 0.021 & 19.77 & \\
\hline $\mathrm{OH} 42$ & $10 / 01 / 86$ & $12: 03$ & 00.36 & 638.502 & 8.087 & 0.045 & 20.13 & \\
\hline DH42 & $10 / 08 / 86$ & $10: 55$ & 00.15 & 645.455 & 6.953 & 0.022 & 20.28 & \\
\hline DH42 & $10 / 14 / 86$ & $11: 19$ & 00.15 & 651.472 & 6.017 & 0.025 & 20.43 & \\
\hline DH42 & $11 / 05 / 86$ & $11: 07$ & 0.52 & 673.463 & 21.991 & 0.024 & 20.95 & \\
\hline $\mathrm{OH} 42$ & $11 / 20 / 86$ & $12: 10$ & 00.33 & 688.507 & 15.044 & 0.022 & 21.28 & \\
\hline OH42 & $12 / 30 / 86$ & $11: 45$ & 00.78 & 728.490 & 39.983 & 0.020 & 22.06 & 0.50 liters for sample, pH 5.91 . \\
\hline OH42 & $02 / 03 / 87$ & $12: 50$ & 00.85 & 763.535 & 35.045 & 0.024 & 22.91 & \\
\hline OH42 & $03 / 06 / 87$ & $10: 45$ & 0.68 & 794.448 & 30.913 & 0.022 & 23.59 & \\
\hline DH42 & $03 / 30 / 87$ & $11: 00$ & 0.53 & 818.458 & 24.010 & 0.022 & 24.12 & \\
\hline OH42 & $05 / 07 / 87$ & $11: 15$ & 0.90 & 856.469 & 38.011 & 0.024 & 25.02 & Brine effervesces. \\
\hline DH42 & $06 / 17 / 87$ & $10: 35$ & 0.91 & 897.441 & 0.000 & 0.000 & 25.93 & $\begin{array}{l}\text { Samples removed for chemistry, \#112A, } \\
\# 1128 \text {. Wood fragments in hole. Some brine } \\
\text { left in hole, no calculation. }\end{array}$ \\
\hline DH42 & $06 / 18 / 87$ & $11: 56$ & 0.10 & 898.497 & 42.028 & 0.024 & 26.03 & $\begin{array}{l}\text { Calculated using } 1.01 \text { liters }(0.91 \text { liters } \\
6 / 17 / 87 \text { plus } 0.10 \text { liters } 6 / 18 / 87) \text {. }\end{array}$ \\
\hline DH42 & $07 / 28 / 87$ & $11: 10$ & 0.94 & 938.465 & 39.968 & 0.024 & 26.97 & \\
\hline OH42 & $09 / 01 / 87$ & $10: 15$ & 0.79 & 973.427 & 34.962 & 0.023 & 27.76 & Collected for chemistry, sample \#151 A\&B. \\
\hline OH42 & $10 / 20 / 87$ & $11: 31$ & 1.29 & 1022.480 & 49.053 & 0.026 & 29.05 & \\
\hline DH42 & $11 / 19 / 87$ & $10: 55$ & 0.75 & 1052.455 & 29.975 & 0.025 & 29.80 & Collected for chemistry, sample \#229. \\
\hline DH42 & $01 / 04 / 88$ & $11: 30$ & 1.13 & 1098.479 & 46.024 & 0.025 & 30.93 & \\
\hline DH42 & $02 / 08 / 88$ & $11: 20$ & 0.75 & 1133.472 & 34.993 & 0.021 & 31.68 & $\begin{array}{l}\text { Collected for chemistry, sample \#255 \& } \\
\# 256 \text {. }\end{array}$ \\
\hline DH42 & $03 / 29 / 88$ & $11: 20$ & 1.10 & 1983.472 & 50.000 & 0.022 & 32.78 & $\begin{array}{l}\text { Collected for chemistry, sample } \$ 323 \text { - } \\
\$ 325 \text {. }\end{array}$ \\
\hline DH42 & $05 / 05 / 88$ & $09: 30$ & 0.75 . & 1220.396 & 36.924 & 0.020 & 33.53 & Sampled for SNL/NM PA. \\
\hline OH42 & $05 / 12 / 88$ & $09: 45$ & 0.13 & 1227.406 & 7.010 & 0.019 & 33.66 & Sampled for SHL/NM PA. \\
\hline DH42 & $07 / 12 / 88$ & $08: 35$ & 1.15 & 1288.358 & 60.952 & 0.019 & 34.81 & $\begin{array}{l}\text { Collected for chemistry, sample \#415 \& } \\
\# 416 \text {. }\end{array}$ \\
\hline DH42 & $07 / 28 / 88$ & $10: 10$ & 0.34 & 1304.424 & 16.066 & 0.021 & 35.15 & Sampled for SNL/MM PA. \\
\hline DH42 & $09 / 27 / 88$ & $10: 20$ & 0.66 & 1365.431 & 61.007 & 0.011 & 35.81 & $\begin{array}{l}\text { Collected for chemistry, sample } \# 530 \text { \& } \\
\# 531 \text {. }\end{array}$ \\
\hline DH42 & $12 / 13 / 88$ & $09: 38$ & 1.71 & 1442.401 & 76.970 & 0.022 & 37.52 & $\begin{array}{l}\text { Collected for chemistry. sample } \# 628 \text {. } \\
\# 631 \text {. }\end{array}$ \\
\hline $\mathrm{DH} 42$ & $03 / 15 / 89$ & $10: 30$ & 1.50 & 1534.438 & 92.037 & 0.015 & 39.02 & $\begin{array}{l}\text { Sample saved for chemistry, sample } \# 722 \text { - } \\
724 \text {. }\end{array}$ \\
\hline DH42 & $04 / 06 / 89$ & $10: 10$ & 0.54 & 1556.424 & 21.986 & 0.025 & 39.56 & \\
\hline $\mathrm{DH} 42$ & $04 / 20 / 89$ & $09: 10$ & 0.50 & 1570.382 & 13.958 & 0.036 & 40.06 & \\
\hline DH42 & $05 / 17 / 89$ & $09: 45$ & 0.66 & 1597.406 & 27.024 & 0.024 & 40.72 & \\
\hline DH42 & $06 / 06 / 89$ & $09: 50$ & 0.41 & 1617.410 & 20.004 & 0.020 & 41.13 & Sample saved for chemistry. \\
\hline $\mathrm{DH} 42$ & $06 / 29 / 89$ & $10: 20$ & 0.35 & 1640.431 & 23.021 & 0.015 & 41.48 & \\
\hline DH42 & $07 / 25 / 89$ & $10: 10$ & 0.55 & 1666.424 & 25.993 & 0.021 & 42.03 & Sample saved for SHL brine study. \\
\hline $\mathrm{OH} 42$ & $08 / 16 / 89$ & $09: 40$ & 0.36 & 1688.403 & 21.979 & 0.016 & 42.39 & \\
\hline $\mathrm{DH} 42$ & $09 / 12 / 89$ & 09:00 & 0.35 & 1715.375 & 26.972 & 0.013 & 42.74 & Sample saved for chemistry. \\
\hline DH42 & $12 / 13 / 89$ & $10: 03$ & 1.50 & 1807.419 & 92.044 & 0.016 & 44.24 & Sample saved for chemistry, sample \#898. \\
\hline
\end{tabular}


TABLE A-2 (Continued)

\section{BRINE ACCUMULATION DATA TABLE}

Data through December 31, 1993

\begin{tabular}{|c|c|c|c|c|c|c|c|c|}
\hline LOCATION & DATE & TIME & $\begin{array}{l}\text { LITERS } \\
\text { REMOVED }\end{array}$ & $\begin{array}{l}\text { DAYS } \\
\text { SIKCE } \\
1 / 1 / 85\end{array}$ & $\begin{array}{l}\text { DAYS } \\
\text { USED FOR } \\
\text { CALCULATIOH }\end{array}$ & $\begin{array}{l}\text { LITERS } \\
\text { PER DAY }\end{array}$ & $\begin{array}{l}\text { CUMULATIVE } \\
\text { LITERS } \\
\text { COLLECTED }\end{array}$ & REMARKS \\
\hline DH42 & $01 / 10 / 90$ & $09: 45$ & 0.70 & 1835.406 & 27.987 & 0.025 & 44.94 & \\
\hline $\mathrm{OH} 42$ & $01 / 24 / 90$ & $09: 57$ & 0.27 & 1849.415 & 14.009 & 0.019 & 45.21 & \\
\hline $\mathrm{OH} 42$ & $02 / 07 / 90$ & $10: 23$ & 0.34 & 1863.433 & 14.018 & 0.024 & 45.55 & . \\
\hline $\mathrm{OH} 42$ & $02 / 21 / 90$ & $10: 19$ & 0.32 & 1877.430 & 13.997 & 0.023 & 45.87 & \\
\hline $\mathrm{OH} 42$ & $03 / 05 / 90$ & $09: 00$ & 0.36 & 1889.375 & 11.945 & 0.030 & 46.23 & \\
\hline DH42 & $03 / 13 / 90$ & $12: 00$ & HA & 1897.500 & 0.000 & 0.000 & 46.23 & Installed sampler. \\
\hline DH42 & $03 / 19 / 90$ & $11: 12$ & 0.06 & 1903.467 & 14.092 & 0.004 & 46.29 & Brine probably left in hole. \\
\hline DH42 & $03 / 21 / 90$ & $10: 23$ & 0.08 & 1905.433 & 1.966 & 0.041 & 46.37 & $\begin{array}{l}\text { Combined with } 0.06 \text { liters from } 03 / 19 / 90 \text {. } \\
\text { Used } 0.14 \text { liters for calculation. }\end{array}$ \\
\hline DH42 & $04 / 04 / 90$ & $09: 24$ & 0.24 & 1919.392 & 13.959 & 0.017 & 46.61 & \\
\hline DH42 & $04 / 10 / 90$ & $08: 43$ & 0.14 & 1925.363 & 5.971 & 0.023 & 46.75 & \\
\hline OH42 & $04 / 17 / 90$ & $10: 29$ & 0.14 & 1932.437 & 7.074 & 0.020 & 46.89 & \\
\hline DH42 & $04 / 24 / 90$ & $09: 26$ & 0.13 & 1939.393 & 6.956 & 0.019 & 47.02 & \\
\hline DH42 & $05 / 02 / 90$ & $10: 39$ & 0.15 & 1947.444 & 8.051 & 0.019 & 47.17 & \\
\hline DH42 & $05 / 09 / 90$ & $09: 01$ & 0.13 & 1954.376 & 6.932 & 0.019 & 47.30 & \\
\hline DH42 & $05 / 16 / 90$ & $09: 11$ & 0.13 & 1961.383 & 7.007 & 0.019 & 47.43 & \\
\hline OH42 & $05 / 23 / 90$ & 12:08 & 0.14 & 1968.506 & 7.123 & 0.020 & 47.57 & \\
\hline DH42 & $05 / 31 / 90$ & $08: 59$ & 0.13 & 1976.374 & 7.868 & 0.017 & 47.70 & \\
\hline DH42 & $06 / 06 / 90$ & $09: 37$ & 0.13 & 1982.401 & 6.027 & 0.022 & 47.83 & \\
\hline DH42 & $06 / 14 / 90$ & $08: 46$ & 0.16 & 1990.365 & 7.964 & 0.020 & 47.99 & \\
\hline DH42 & $06 / 20 / 90$ & $09: 35$ & 0.12 & 1996.399 & 6.034 & 0.020 & 48.11 & \\
\hline DH42 & $06 / 28 / 90$ & $08: 55$ & 0.15 & 2004.372 & 7.973 & 0.019 & 48.26 & \\
\hline DH42 & $07 / 17 / 90$ & $11: 08$ & 0.31 & 2023.464 & 19.092 & 0.016 & 48.57 & \\
\hline OH42 & $07 / 25 / 90$ & $09: 40$ & 0.20 & 2031.403 & 7.939 & 0.025 & 48.77 & \\
\hline $\mathrm{OH} 42$ & $08 / 01 / 90$ & $10: 20$ & 0.15 & 2038.431 & 7.028 & 0.021 & 48.92 & \\
\hline DH42 & $03 / 28 / 91$ & $10: 06$ & 3.02 & 2277.421 & 0.000 & 0.000 & 51.94 & $\begin{array}{l}\text { Some brine may have been left in hole. } \\
\text { Sampler still functioning. }\end{array}$ \\
\hline DH42 & $04 / 10 / 91$ & $08: 46$ & 0.90 & 2290.365 & 0.000 & 0.000 & 52.84 & Partial evacuation. \\
\hline DH42 & $04 / 11 / 91$ & $08: 42$ & 0.50 & 2291.363 & 252.932 & 0.017 & 53.34 & $\begin{array}{l}\text { Conbined with } 3.02 \text { liters from } 03 / 28 / 91 \text { and } \\
0.90 \text { liters from } 04 / 10 / 91 \text {. }\end{array}$ \\
\hline DH42 & $04 / 17 / 91$ & $10: 17$ & 0.11 & 2297.428 & 6.065 & 0.018 & 53.45 & \\
\hline $\mathrm{OH} 42$ & $04 / 24 / 91$ & 09:05 & 0.12 & 2304.378 & 6.950 & 0.017 & 53.57 & \\
\hline DH42 & $05 / 01 / 91$ & $09: 11$ & 0.12 & 2311.383 & 7.005 & 0.017 & 53.69 & \\
\hline $\mathrm{DH} 42$ & $05 / 08 / 91$ & $08: 24$ & 0.12 & 2318.350 & 6.967 & 0.017 & 53.81 & \\
\hline $\mathrm{OH} 42$ & $05 / 15 / 91$ & $09: 02$ & 0.12 & 2325.376 & 7.026 & 0.017 & 53.93 & \\
\hline DH42 & $05 / 29 / 91$ & 09:55 & 0.27 & 2339.413 & 14.037 & 0.019 & 54.20 & \\
\hline DH42 & $06 / 05 / 91$ & $13: 40$ & 0.14 & 2346.569 & 7.156 & 0.020 & 54.34 & \\
\hline DH42 & $06 / 12 / 91$ & $10: 07$ & 0.12 & 2353.422 & 6.853 & 0.018 & 54.46 & \\
\hline DH42 & $06 / 19 / 91$ & $13: 35$ & 0.13 & 2360.566 & 7.144 & 0.018 & 54.59 & \\
\hline $\mathrm{DH} 42$ & $06 / 26 / 91$ & 09:10 & 0.12 & 2367.382 & 6.816 & 0.018 & 54.71 & \\
\hline DH42 & $07 / 11 / 91$ & $10: 49$ & 0.27 & 2382.451 & 15.069 & 0.018 & 54.98 & \\
\hline DH42 & $07 / 17 / 91$ & $09: 22$ & 0.11 & 2388.390 & 5.939 & 0.019 & 55.09 & \\
\hline DH42 & $07 / 30 / 91$ & $10: 15$ & 0.30 & 2401.427 & 13.037 & 0.023 & 55.39 & \\
\hline DH42 & $08 / 08 / 91$ & $09: 10$ & 0.24 & 2410.382 & 8.955 & 0.027 & 55.63 & \\
\hline DH42 & $08 / 14 / 91$ & $10: 33$ & 0.16 & 2416.440 & 6.058 & 0.026 & 55.79 & \\
\hline $\mathrm{DH} 42$ & $08 / 21 / 91$ & $10: 45$ & 0.17 & 2423.448 & 7.008 & 0.024 & 55.96 & \\
\hline DH42 & $08 / 28 / 91$ & $10: 10$ & 0.24 & 2430.424 & 6.976 & 0.034 & 56.20 & \\
\hline
\end{tabular}


TABLE A-2 (Continued)

BRINE ACCUMULATION DATA TABLE

Data through December 31, 1993

\begin{tabular}{lllllll} 
& \multicolumn{2}{c}{ DAYS } & DAYS & \multicolumn{2}{c}{ CUMULATIVE } \\
LOCATION & LITERS & SINCE & USED FOR & LITERS & LITERS \\
L TIME REMOVEO & $1 / 1 / 85$ & CALCULATION & PER DAY & COLLECTED & REMARKS
\end{tabular}

\begin{tabular}{|c|c|c|c|c|c|c|c|c|}
\hline DH42 & $09 / 04 / 91$ & $11: 10$ & 0.18 & 2437.465 & 7.041 & 0.026 & 56.38 & \\
\hline OH42 & $09 / 11 / 91$ & $19: 40$ & 0.16 & 2444.486 & 7.021 & 0.023 & 56.54 & \\
\hline DH42 & $09 / 18 / 91$ & $09: 10$ & 0.18 & 2451.382 & 6.896 & 0.026 & 56.72 & \\
\hline OH42 & $09 / 25 / 91$ & $11: 52$ & 0.15 & 2458.494 & 7.112 & 0.021 & 56.87 & \\
\hline DH42 & $10 / 02 / 91$ & $11: 00$ & 0.15 & 2465.458 & 6.964 & 0.022 & 57.02 & \\
\hline OH42 & $10 / 16 / 91$ & $09: 15$ & 0.26 & 2479.385 & 13.927 & 0.019 & 57.28 & \\
\hline DH42 & $10 / 23 / 91$ & $09: 31$ & 0.16 & 2486.397 & 7.012 & 0.023 & 57.44 & \\
\hline DH42 & $11 / 06 / 91$ & $10: 03$ & 0.28 & 2500.419 & 14.022 & 0.020 & 57.72 & \\
\hline DH42 & $11 / 13 / 91$ & $09: 35$ & 0.15 & 2507.399 & 6.980 & 0.021 & 57.87 & \\
\hline DH42 & $11 / 20 / 91$ & $11: 20$ & 0.14 & 2514.472 & 7.073 & 0.020 & 58.01 & \\
\hline DH42 & $11 / 27 / 91$ & $10: 05$ & 0.13 & 2521.420 & 6.948 & 0.019 & 58.14 & \\
\hline DH42 & $12 / 04 / 91$ & $09: 52$ & 0.12 & 2528.411 & $6.991^{\circ}$ & 0.017 & 58.26 & \\
\hline DH42 & $12 / 11 / 91$ & $09: 35$ & 0.10 & 2535.399 & 6.988 & 0.014 & 58.36 & \\
\hline DH42 & $12 / 18 / 91$ & $09: 15$ & 0.13 & 2542.385 & 6.986 & 0.019 & 58.49 & \\
\hline OH42 & $12 / 23 / 91$ & $08: 40$ & 0.10 & 2547.361 & 4.976 & 0.020 & 58.59 & \\
\hline DH42 & $01 / 08 / 92$ & $09: 50$ & 0.31 & 2563.410 & 16.049 & 0.019 & 58.90 & \\
\hline DH42 & $01 / 15 / 92$ & $09: 40$ & 0.11 & 2570.403 & 6.993 & 0.016 & 59.01 & \\
\hline DH42 & $01 / 22 / 92$ & $08: 35$ & 0.16 & 2577.358 & 6.955 & 0.023 & 59.17 & \\
\hline DH42 & $01 / 29 / 92$ & $09: 53$ & 0.17 & 2584.412 & 7.054 & .0 .024 & 59.34 & \\
\hline OH42 & $02 / 12 / 92$ & $08: 49$ & 0.26 & 2598.367 & 13.955 & 0.019 & 59.60 & \\
\hline DH42 & $02 / 19 / 92$ & $09: 18$ & 0.10 & 2605.388 & 7.021 & 0.014 & 59.70 & \\
\hline $\mathrm{OH} 42$ & $02 / 26 / 92$ & $09: 35$ & 0.17 & 2612.399 & 7.011 & 0.024 & 59.87 & \\
\hline DH42 & $03 / 11 / 92$ & $09: 30$ & 0.23 & 2626.396 & 13.997 & 0.016 & 60.10 & \\
\hline DH42 & $03 / 18 / 92$ & $09: 52$ & 0.13 & 2633.411 & 7.015 & 0.019 & 60.23 & \\
\hline DH42 & $03 / 25 / 92$ & $10: 25$ & 0.39 & 2640.434 & 7.023 & 0.056 & 60.62 & \\
\hline DH42 & $04 / 01 / 92$ & $09: 10$ & 0.13 & 2647.382 & 6.948 & 0.019 & 60.75 & \\
\hline DH42 & $04 / 07 / 92$ & $09: 13$ & 0.09 & 2653.384 & 6.002 & 0.015 & 60.84 & \\
\hline DH42 & $04 / 15 / 92$ & $09: 08$ & 0.15 & 2661.381 & 7.997 & 0.019 & 60.99 & \\
\hline OH42 & $04 / 22 / 92$ & $09: 30$ & 0.13 & 2668.396 & 7.015 & 0.019 & 61.12 & \\
\hline DH42 & $05 / 06 / 92$ & $11: 15$ & 0.06 & 2682.469 & 14.073 & 0.004 & 61.18 & Hole examined, no brine left in hole. \\
\hline DH42 & $05 / 13 / 92$ & $13: 47$ & 0.19 & 2689.574 & 7.105 & 0.027 & 61.37 & \\
\hline DH42 & $05 / 21 / 92$ & $10: 29$ & 0.26 & 2697.437 & 7.863 & 0.033 & 61.63 & \\
\hline DH42 & $05 / 27 / 92$ & $09: 10$ & 0.12 & 2703.382 & 5.945 & 0.020 & 61.75 & \\
\hline DH42 & $06 / 09 / 92$ & $09: 10$ & 0.22 & 2716.382 & 13.000 & 0.017 & 61.97 & \\
\hline DH42 & $06 / 18 / 92$ & $09: 55$ & 0.09 & 2725.413 & 9.031 & 0.010 & 62.06 & \\
\hline DH42 & $06 / 25 / 92$ & $09: 45$ & 0.17 & 2732.406 & 6.993 & 0.024 & 62.23 & \\
\hline OH42 & $07 / 01 / 92$ & $09: 15$ & 0.09 & 2738.385 & 5.979 & 0.015 & 62.32 & \\
\hline DH42 & $07 / 08 / 92$ & $09: 25$ & 0.12 & 2745.392 & 7.007 & 0.017 & 62.44 & \\
\hline OH42 & $07 / 15 / 92$ & $09: 18$ & 0.12 & 2752.388 & 6.996 & 0.017 & 62.56 & \\
\hline DH42 & $07 / 22 / 92$ & $09: 10$ & 0.12 & 2759.382 & 6.994 & 0.017 & 62.68 & \\
\hline DH42 & $07 / 29 / 92$ & $09: 51$ & 0.14 & 2766.410 & 7.028 & 0.020 & 62.82 & \\
\hline DH42 & $08 / 04 / 92$ & $09: 37$ & 0.10 & 2772.401 & 5.991 & 0.017 & 62.92 & \\
\hline DH42 & $08 / 18 / 92$ & $09: 54$ & 0.23 & 2786.413 & 14.012 & 0.016 & 63.15 & \\
\hline $\mathrm{DH} 42$ & $09 / 02 / 92$ & $09: 53$ & 0.23 & 2801.412 & 14.999 & 0.015 & 63.38 & \\
\hline DH42 & $09 / 09 / 92$ & $10: 00$ & 0.16 & 2808.417 & 7.005 & 0.023 & 63.54 & \\
\hline OH42 & $09 / 17 / 92$ & $09: 10$ & 0.08 & 2816.382 & 7.965 & 0.010 & 63.62 & \\
\hline OH42 & $09 / 23 / 92$ & $10: 04$ & 0.12 & 2822.419 & 6.037 & 0.020 & 63.74 & \\
\hline
\end{tabular}


TABLE A-2 (Continued)

\section{BRINE ACCUMULATION DATA TABLE}

Data through December 31, 1993

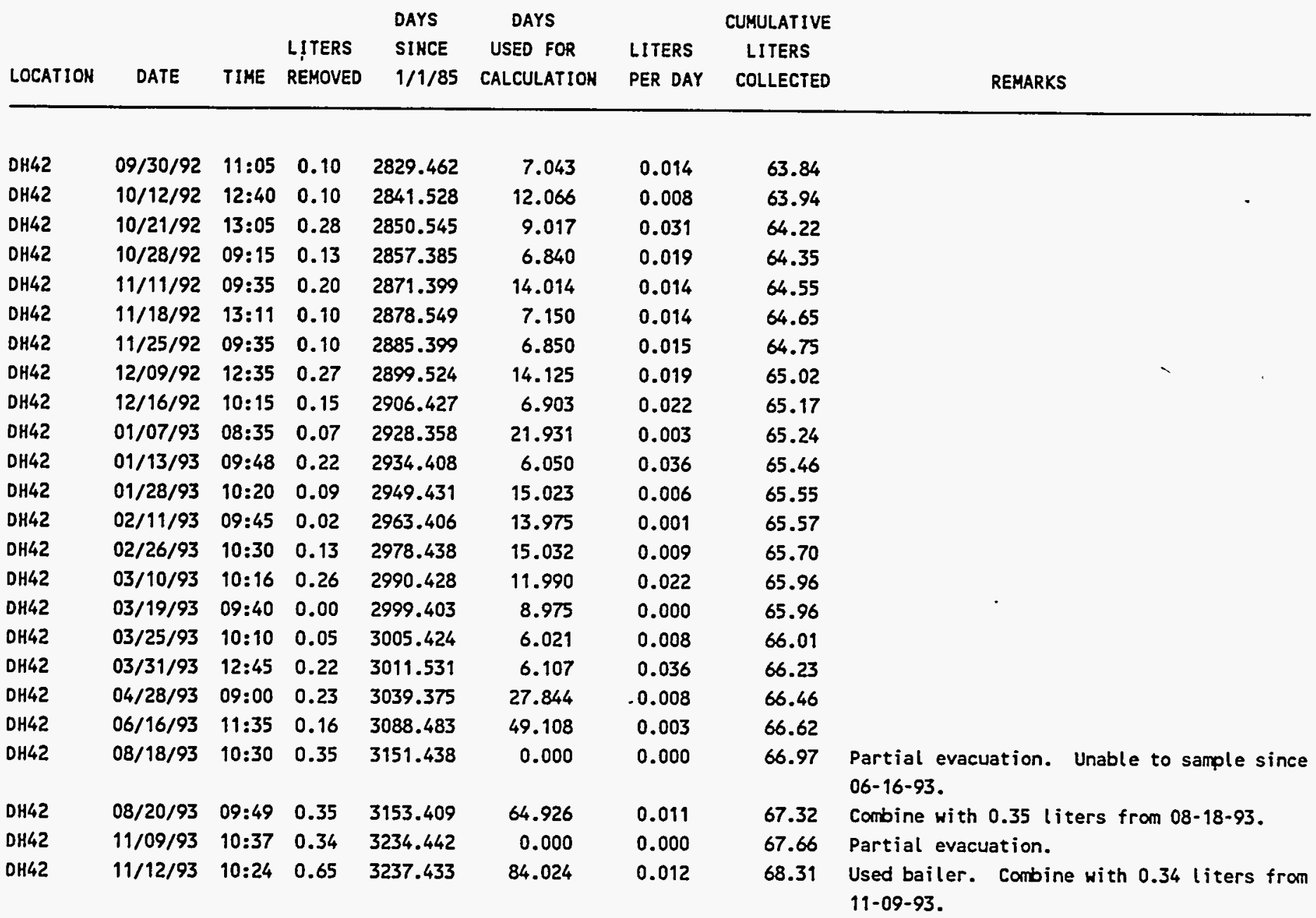

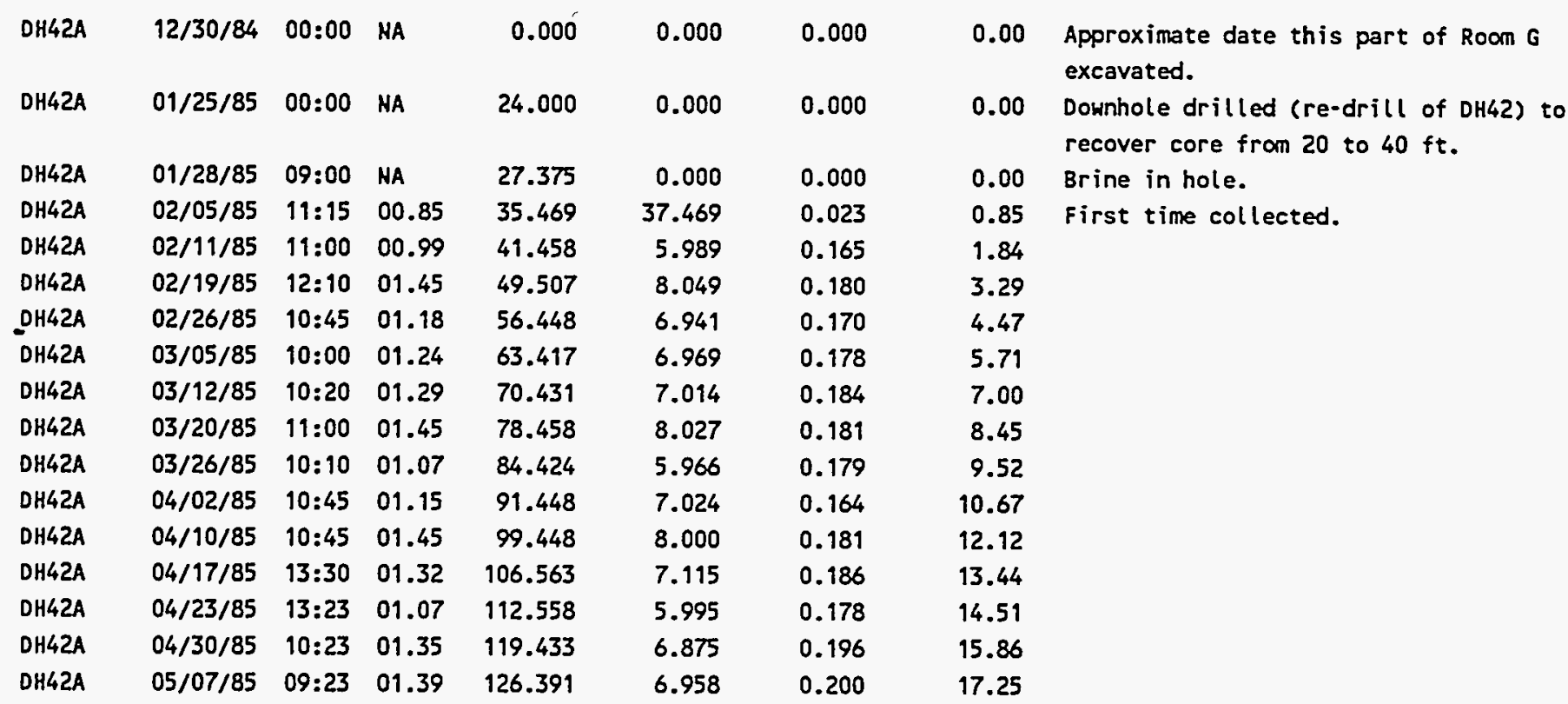


TABLE A-2 (Continued) BRINE ACCUMULATION DATA TABLE

Data through December 31, 1993

\begin{tabular}{|c|c|c|c|c|c|c|c|c|}
\hline \multirow[b]{3}{*}{ LOCATIOH } & \multirow[b]{3}{*}{ DATE } & \multirow[b]{3}{*}{ TIME } & \multirow{3}{*}{$\begin{array}{l}\text { LITERS } \\
\text { REMOVED }\end{array}$} & \multirow{3}{*}{$\begin{array}{l}\text { DAYS } \\
\text { SINCE } \\
1 / 1 / 85\end{array}$} & DAYS & \multicolumn{2}{|r|}{ CUMULATIVE } & \multirow[b]{3}{*}{ REMARKS } \\
\hline & & & & & USED FOR & LITERS & LITERS & \\
\hline & & & & & CALCULATION & PER DAY & COLLECTED & \\
\hline DH42A & $05 / 14 / 85$ & $10: 25$ & 01.34 & 133.434 & 7.043 & 0.190 & 18.59 & \\
\hline DH42A & $05 / 21 / 85$ & $10: 14$ & 01.29 & 140.426 & 6.992 & 0.184 & 19.88 & \\
\hline OH42A & $05 / 29 / 85$ & $10: 30$ & 01.28 & 148.438 & 8.012 & 0.160 & 21.16 & \\
\hline DH42A & $06 / 04 / 85$ & $10: 50$ & 01.03 & 154.451 & 6.013 & 0.171 & 22.19 & \\
\hline DH42A & $06 / 11 / 85$ & $10: 15$ & 01.19 & 161.427 & 6.976 & 0.171 & 23.38 & \\
\hline OH42A & $06 / 18 / 85$ & $09: 51$ & 01.18 & 168.410 & 6.983 & 0.169 & 24.56 & \\
\hline DH42A & $06 / 25 / 85$ & $11: 05$ & 01.16 & 175.462 & 7.052 & 0.164 & 25.72 & \\
\hline DH42A & $07 / 02 / 85$ & $11: 00$ & 01.12 & 182.458 & 6.996 & 0.160 & 26.84 & \\
\hline DH42A & $07 / 09 / 85$ & $10: 25$ & 01.12 & 189.434 & 6.976 & 0.161 & 27.96 & Gas effervescing from sample. \\
\hline DH42A & $07 / 16 / 85$ & $11: 10$ & 01.11 & 196.465 & 7.031 & 0.158 & 29.07 & Brine effervesces. \\
\hline DH42A & $07 / 24 / 85$ & $10: 25$ & 01.23 & 204.434 & 7.969 & 0.154 & 30.30 & \\
\hline DH42A & $07 / 30 / 85$ & $09: 54$ & 00.94 & 210.413 & 5.979 & 0.157 & 31.24 & \\
\hline DH42A & $08 / 06 / 85$ & $10: 10$ & 01.05 & 217.424 & 7.011 & 0.150 & 32.29 & \\
\hline DH42A & $08 / 14 / 85$ & $10: 33$ & 01.11 & 225.440 & 8.016 & 0.138 & 33.40 & \\
\hline DH42A & $08 / 20 / 85$ & $10: 14$ & 00.92 & 231.426 & 5.986 & 0.154 & 34.32 & \\
\hline DH42A & $08 / 28 / 85$ & $09: 40$ & 01.17 & 239.403 & 7.977 & $0.14 i$ & 35.49 & \\
\hline DH42A & $09 / 04 / 85$ & $10: 10$ & 00.99 & 246.424 & 7.021 & 0.149 & 36.48 & \\
\hline DH42A & $09 / 10 / 85$ & $09: 55$ & 00.83 & 252.413 & 5.989 & 0.139 & 37.31 & \\
\hline DH42A & $09 / 17 / 85$ & $09: 25$ & 00.92 & 259.392 & 6.979 & $0.13 ?$ & 38.23 & \\
\hline OH42A & $09 / 24 / 85$ & $09: 25$ & 00.94 & 266.392 & 7.000 & $0.13 \%$ & 39.17 & \\
\hline DH42A & $10 / 01 / 85$ & $09: 40$ & 00.93 & 273.403 & 7.011 & 0.133 & 40.10 & \\
\hline DH42A & $10 / 08 / 85$ & $10: 24$ & 00.96 & 280.433 & 7.030 & 0.137 & 41.06 & \\
\hline DH42A & $10 / 15 / 85$ & $10: 15$ & 00.81 & 287.427 & 6.994 & 0.116 & 41.87 & \\
\hline $\mathrm{DH} 42 \mathrm{~A}$ & $10 / 23 / 85$ & $10: 10$ & 01.02 & 295.424 & 7.997 & 0.128 & 42.89 & \\
\hline DH42A & $10 / 29 / 85$ & $09: 20$ & $00 . \sqrt{5}$ & 301.389 & 5.965 & 0.125 & 43.64 & \\
\hline DH42A & $11 / 05 / 85$ & $09: 00$ & 00.86 & 308.375 & 6.986 & 0.123 & 44.50 & \\
\hline DH42A & $11 / 13 / 85$ & $09: 44$ & 01.03 & 316.406 & 8.031 & 0.128 & 45.53 & \\
\hline DH42A & $11 / 21 / 85$ & $10: 50$ & 00.94 & 324.451 & 8.045 & 0.117 & 46.47 & \\
\hline $\mathrm{OH} 42 \mathrm{~A}$ & $11 / 26 / 85$ & $10: 55$ & 00.61 & 329.455 & 5.004 & 0.122 & 47.08 & \\
\hline $\mathrm{DH} 42 \mathrm{~A}$ & $12 / 03 / 85$ & $13: 05$ & 00.78 & 336.545 & 7.090 & 0.110 & 47.86 & Sample for chemistry analysis, \#1. \\
\hline DH42A & $12 / 10 / 85$ & $12: 50$ & 00.86 & 343.535 & 6.990 & 0.123 & 48.72 & \\
\hline DH42A & $01 / 23 / 86$ & $11: 40$ & 05.13 & 387.486 & 43.951 & 0.117 & 53.85 & $\begin{array}{l}\text { Entry restricted since } 12 / 10 / 85 \text { due to } \\
\text { mining activities. }\end{array}$ \\
\hline DH42A & $01 / 31 / 86$ & $12: 00$ & 00.92 & 395.500 & 8.014 & 0.115 & 54.77 & \\
\hline OH42A & $02 / 12 / 86$ & $10: 40$ & 01.36 & 407.444 & 11.944 & 0.114 & 56.13 & \\
\hline $\mathrm{DH} 42 \mathrm{~A}$ & $02 / 19 / 86$ & $11: 15$ & 00.80 & 414.469 & 7.025 & 0.114 & 56.93 & \\
\hline DH42A & $02 / 28 / 86$ & $12: 55$ & 00.90 & 423.538 & 9.069 & 0.099 & 57.83 & \\
\hline DH42A & $03 / 06 / 86$ & $10: 25$ & 00.70 & 429.434 & 5.896 & 0.119 & 58.53 & \\
\hline DH42A & $03 / 13 / 86$ & $09: 48$ & 00.73 & 436.408 & 6.974 & 0.105 & 59.26 & \\
\hline DH42A & $03 / 26 / 86$ & $09: 40$ & 01.39 & 449.403 & 12.995 & 0.107 & 60.65 & \\
\hline DH42A & $04 / 02 / 86$ & $09: 20$ & 00.80 & 456.389 & 6.986 & 0.115 & 61.45 & \\
\hline DH42A & $04 / 08 / 86$ & $09: 28$ & 00.63 & 462.394 & 6.005 & 0.105 & 62.08 & \\
\hline DH42A & $04 / 16 / 86$ & $11: 50$ & 00.89 & 470.493 & 8.099 & 0.110 & 62.97 & \\
\hline DH42A & $04 / 24 / 86$ & $09: 50$ & 00.67 & 478.410 & 7.917 & 0.085 & 63.64 & · \\
\hline DH42A & $04 / 30 / 86$ & $10: 36$ & 00.76 & 484.442 & 6.032 & 0.126 & 64.40 & \\
\hline DH42A & $05 / 06 / 86$ & $10: 00$ & 00.55 & 490.417 & 5.975 & 0.092 & 64.95 & \\
\hline DH42A & $05 / 13 / 86$ & $10: 00$ & 00.73 & 497.417 & 7.000 & 0.104 & 65.68 & \\
\hline
\end{tabular}


TABLE A-2 (Continued)

\section{BRINE ACCUMULATION DATA TABLE}

Data through December 31, 1993

\begin{tabular}{|c|c|c|c|c|c|c|c|}
\hline & & & & DAYS & DAYS & & CUMULATIVE \\
\hline & & & LITER & SIMCE & USED FOR & LITERS, & LITERS \\
\hline OCATIOH & DATE & TIME & REMOVED & $1 / 1 / 85$ & CALCULATION & PER DAY & COLLECTED \\
\hline
\end{tabular}

\begin{tabular}{|c|c|c|c|c|c|c|c|c|}
\hline DH42A & $05 / 20 / 86$ & $19: 00$ & 00.70 & 504.458 & 7.041 & 0.099 & 66.38 & \\
\hline DH42A & $05 / 27 / 86$ & $15: 35$ & 00.65 & 511.649 & 7.191 & 0.090 & 67.03 & \\
\hline DH42A & $06 / 03 / 86$ & $09: 50$ & 00.66 & 518.410 & 6.761 & 0.098 & 67.69 & \\
\hline DH42A & $06 / 10 / 86$ & $11: 15$ & 00.54 & 525.469 & 7.059 & 0.076 & 68.23 & \\
\hline OH42A & $06 / 17 / 86$ & $10: 31$ & 00.65 & 532.438 & 6.969 & 0.093 & 68.88 & Sample for brine chemisrtry, \#21. \\
\hline OH42A & $06 / 24 / 86$ & $10: 45$ & 00.63 & 539.448 & 7.010 & 0.090 & 69.51 & \\
\hline DH42A & $07 / 01 / 86$ & $13: 50$ & 00.71 & 546.576 & 7.128 & 0.100 & 70.22 & \\
\hline DH42A & $07 / 08 / 86$ & $10: 25$ & 00.63 & 553.434 & 6.858 & 0.092 & 70.85 & \\
\hline DH42A & $07 / 16 / 86$ & $10: 00$ & 00.66 & 561.417 & 7.983 & 0.083 & 71.51 & \\
\hline DH42A & $07 / 22 / 86$ & $09: 48$ & 00.61 & 567.408 & 5.991 & 0.102 & 72.12 & \\
\hline DH42A & $07 / 29 / 86$ & $10: 25$ & 00.71 & 574.434 & 7.026 & 0.101 & 72.83 & \\
\hline DH42A & $08 / 05 / 86$ & $10: 55$ & 00.66 & 581.455 & 7.021 & 0.094 & 73.49 & \\
\hline DH42A & $08 / 12 / 86$ & $10: 23$ & 00.63 & 588.433 & 6.978 & 0.090 & 74.12 & \\
\hline OH42A & $08 / 19 / 86$ & $11: 22$ & 00.68 & 595.474 & 7.041 & 0.097 & 74.80 & \\
\hline $\mathrm{OH} 42 \mathrm{~A}$ & $08 / 26 / 86$ & $10: 28$ & 00.68 & 602.436 & 6.962 & 0.098 & 75.48 & Static level not measured. \\
\hline DH42A & $09 / 04 / 86$ & $10: 25$ & 00.71 & 611.434 & 8.998 & 0.079 & 76.19 & $\begin{array}{l}\text { Valve broke off and left in hole ofter } \\
\text { collecting most of brine. Some brine left } \\
\text { in hole. }\end{array}$ \\
\hline DH42A & $09 / 09 / 86$ & $09: 40$ & 00.07 & 616.403 & 4.969 & .0 .014 & 76.26 & $\begin{array}{l}\text { Bottom obstructed by object in hole. } \\
\text { Sample } \# 23 \text {. }\end{array}$ \\
\hline DH42A & $09 / 16 / 86$ & $09: 59$ & 00.95 & 623.416 & 7.013 & 0.135 & 77.21 & \\
\hline DH42A & $09 / 23 / 86$ & $10: 02$ & 00.60 & 630.418 & 7.002 & 0.086 & 77.81 & \\
\hline DH42A & $10 / 01 / 86$ & $11: 57$ & 00.43 & 638.498 & 8.080 & 0.053 & 78.24 & \\
\hline DH42A & $10 / 08 / 86$ & $10: 55$ & 00.81 & 645.455 & 6.957 & 0.116 & 79.05 & \\
\hline DH42A & $10 / 14 / 86$ & $11: 24$ & 00.56 & 651.475 & 6.020 & 0.093 & 79.61 & \\
\hline DH42A & $11 / 05 / 86$ & $11: 04$ & 1.94 & 673.461 & 21.986 & 0.088 & 81.55 & \\
\hline DH42A & $11 / 20 / 86$ & $12: 08$ & 01.40 & 688.506 & 15.045 & 0.093 & 82.95 & \\
\hline $\mathrm{DH} 42 \mathrm{~A}$ & $12 / 31 / 86$ & $11: 30$ & 02.91 & 729.479 & 40.973 & 0.071 & 85.86 & 0.99 liters for sample, pH 5.86 . \\
\hline DH42A & $02 / 03 / 87$ & $12: 35$ & 03.15 & 763.524 & 34.045 & 0.093 & 89.01 & \\
\hline DH42A & $03 / 06 / 87$ & $10: 45$ & 2.61 & 794.448 & 30.924 & 0.084 & 91.62 & \\
\hline DH42A & $03 / 30 / 87$ & $10: 56$ & 2.52 & 818.456 & 24.008 & 0.105 & 94.14 & \\
\hline DH42A & $05 / 07 / 87$ & $11: 10$ & 3.17 & 856.465 & 38.009 & 0.083 & 97.31 & \\
\hline DH42A & $06 / 17 / 87$ & $10: 30$ & 2.94 & 897.438 & 0.000 & 0.000 & 100.25 & $\begin{array}{l}\text { Samples removed for chemistry. \#113A, } \\
\# 113 B, \# 115 A, \# 115 B \text {, \#118A, \#118B. } \\
\text { Approximately } 0.01 \text { liter spilled. Some } \\
\text { brine left in hole, no calculation. }\end{array}$ \\
\hline DH42A & $06 / 18 / 87$ & $11: 54$ & 0.11 & 898.496 & 42.031 & 0.073 & 100.36 & $\begin{array}{l}\text { Calculated using } 3.05 \text { liters }(2.94 \text { liters } \\
\text { from } 6 / 17 / 87 \text { plus } 0.11 \text { liters from } \\
6 / 18 / 87) \text {. }\end{array}$ \\
\hline DH42A & $07 / 28 / 87$ & $11: 03$ & 3.07 & 938.460 & 39.964 & 0.077 & 103.43 & \\
\hline DH42A & $09 / 01 / 87$ & $10: 08$ & 2.69 & 973.422 & 34.962 & 0.077 & 106.12 & $\begin{array}{l}\text { Collected for chemistry, sample \#154 A\&B } \\
\text { and Sample \#150 A\&B. Samples effervesce. }\end{array}$ \\
\hline $\mathrm{OH} 42 \mathrm{~A}$ & $10 / 20 / 87$ & $11: 28$ & 3.73 & 1022.478 & 49.056 & 0.076 & 109.85 & \\
\hline DH42A & $11 / 19 / 87$ & $10: 55$ & 2.17 & 1052.455 & 29.977 & 0.072 & 112.02 & $\begin{array}{l}\text { Collected for chemistry, sample \#228 \& } \\
\# 233 \text {. }\end{array}$ \\
\hline OH42A & $01 / 04 / 88$ & $11: 25$ & 3.28 & 1098.476 & 46.021 & 0.071 & 115.30 & \\
\hline $\mathrm{OH} 42 \mathrm{~A}$ & $02 / 08 / 88$ & $11: 10$ & 2.47 & 1133.465 & 34.989 & 0.071 & 117.77 & Collected for chemistry, sample $\# 250, \# 251$, \\
\hline
\end{tabular}




\section{TABLE A-2 (Continued) BRINE ACCUMULATION DATA TABLE}

Data through December 31, 1993

\begin{tabular}{|c|c|c|c|c|c|c|c|c|}
\hline & & & & DAYS & DAYS & & CUMULATIVE & \\
\hline & & & LITERS & SINCE & USED FOR & LITERS & LITERS & \\
\hline LOCATION & DATE & TIME & REMOVED & $1 / 1 / 85$ & CALCULATION & PER DAY & COLLECTED & REMARKS \\
\hline
\end{tabular}

\begin{tabular}{|c|c|c|c|c|c|c|c|c|}
\hline DH42A & $03 / 29 / 88$ & $11: 15$ & 3.57 & 1183.469 & 50.004 & 0.071 & 121.34 & $\begin{array}{l}\# 252, \# 253, \& \text { \& } \# 254 \text {. } \\
\text { Collected for chemistry, sample } \# 316 \text { - } \\
\# 322 \text {. }\end{array}$ \\
\hline$D H 42 A$ & $05 / 05 / 88$ & $09: 00$ & 2.38 & 1220.375 & 36.906 & 0.064 & 123.72 & Sampled for SNL/NM PA. \\
\hline $\mathrm{OH} 42 \mathrm{~A}$ & $05 / 12 / 88$ & $09: 40$ & 0.50 & 1227.403 & 7.028 & 0.071 & 124.22 & Sampled for SNL/MM PA. \\
\hline DH42A & $07 / 12 / 88$ & $08: 30$ & 4.06 & 1288.354 & 60.951 & 0.067 & 128.28 & $\begin{array}{l}\text { Collected for chemistry, sample \#407 - } \\
\# 14 \text {. }\end{array}$ \\
\hline DH42A & $07 / 28 / 88$ & $10: 15$ & 1.25 & 1304.427 & 16.073 & 0.078 & 129.53 & Sampled for SNL/NM PA. \\
\hline DH42A & $09 / 14 / 88$ & $08: 45$ & 3.00 & 1352.365 & 47.938 & 0.063 & 132.53 & \\
\hline DH42A & $09 / 27 / 88$ & $10: 10$ & 1.07 & 1365.424 & 13.059 & 0.082 & 133.60 & $\begin{array}{l}\text { Collected for chemistry, sample } \# 528 \text { \& } \\
\# 529 \text {. }\end{array}$ \\
\hline DH42A & $12 / 13 / 88$ & $09: 35$ & 7.95 & 1442.399 & 76.975 & 0.103 & 141.55 & $\begin{array}{l}\text { Collected for chemistry, sample \#618- } \\
\# 627 \text {. }\end{array}$ \\
\hline DH42A & 03/15/89 & $10: 00$ & 5.82 & 1534.417 & 92.018 & 0.063 & 147.37 & $\begin{array}{l}\text { Sample saved for chemistry, sample } \$ 714 \text { - } \\
721 \text {. }\end{array}$ \\
\hline DH42A & 04/06/89 & $10: 15$ & 1.44 & 1556.427 & 22.010 & 0.065 & 148.81 & \\
\hline DH42A & $04 / 20 / 89$ & $09: 00$ & 0.75 & 1570.375 & 13.948 & 0.054 & 149.56 & \\
\hline DH42A & $05 / 17 / 89$ & $09: 45$ & 1.91 & 1597.406 & $27.03 i$ & 0.071 & 151.47 & \\
\hline DH42A & $06 / 06 / 89$ & $09: 45$ & 1.30 & 1617.406 & 20.000 & .0 .065 & 152.77 & Sample saved for chemistry. \\
\hline $\mathrm{DH} 42 \mathrm{~A}$ & $06 / 29 / 89$ & $10: 15$ & 1.35 & 1640.427 & 23.021 & 0.059 & 154.12 & \\
\hline DH42A & $07 / 25 / 89$ & $10: 05$ & 1.51 & 1666.420 & 25.993 & 0.058 & 155.63 & Sample saved for SHL/NM brine study. \\
\hline DH42A & $08 / 16 / 89$ & $09: 31$ & 1.48 & 1688.397 & 21.977 & 0.067 & 157.11 & Sample saved for SHL/NM brine study. \\
\hline DH42A & $09 / 12 / 89$ & $08: 50$ & 1.63 & 1715.368 & 26.971 & 0.060 & 158.74 & Sample saved for chemistry. \\
\hline DH42A & $12 / 13 / 89$ & $09: 20$ & 5.28 & 1807.389 & 92.021 & 0.057 & 164.02 & Sample saved for chemistry, $\# 897$. \\
\hline DH42A & $01 / 10 / 90$ & $09: 36$ & 1.95 & 1835.400 & 28.011 & 0.070 & 165.97 & \\
\hline DH42A & $01 / 24 / 90$ & $09: 52$ & 0.75 & 1849.411 & 14.011 & 0.054 & 166.72 & \\
\hline $\mathrm{DH} 42 \mathrm{~A}$ & $02 / 07 / 90$ & $10: 20$ & 0.95 & 1863.431 & 14.020 & 0.068 & 167.67 & \\
\hline DH42A & $02 / 21 / 90$ & $09: 56$ & 0.81 & 1877.414 & 13.983 & 0.058 & 168.48 & \\
\hline DH42A & $03 / 05 / 90$ & $08: 47$ & 0.68 & 1889.366 & 11.952 & 0.057 & 169.16 & \\
\hline $\mathrm{DH} 42 \mathrm{~A}$ & $03 / 13 / 90$ & $11: 36$ & NA & 1897.483 & 0.000 & 0.000 & 169.16 & Installed sampler. \\
\hline DH42A & $03 / 19 / 90$ & $11: 07$ & 0.51 & 1903.463 & 0.000 & 0.000 & 169.67 & Partial evacuation. \\
\hline DH42A & $03 / 21 / 90$ & $10: 21$ & 0.28 & 1905.431 & 16.065 & 0.049 & 169.95 & $\begin{array}{l}\text { Combined with } 0.51 \text { liters from } 03 / 19 / 90 \text {. } \\
\text { Used } 0.79 \text { liters for calculation. }\end{array}$ \\
\hline DH42A & $04 / 04 / 90$ & $09: 14$ & 0.60 & 1919.385 & 13.954 & 0.043 & 170.55 & \\
\hline $\mathrm{DH} 42 \mathrm{~A}$ & $04 / 10 / 90$ & $08: 40$ & 0.58 & 1925.361 & 5.976 & 0.097 & 171.13 & \\
\hline DH42A & $04 / 17 / 90$ & $10: 19$ & 0.38 & 1932.430 & 7.069 & 0.054 & 171.51 & \\
\hline DH42A & $04 / 24 / 90$ & $09: 18$ & 0.42 & 1939.388 & 6.958 & 0.060 & 171.93 & \\
\hline DH42A & $05 / 02 / 90$ & $10: 32$ & 0.51 & 1947.439 & 8.051 & 0.063 & 172.44 & \\
\hline DH42A & $05 / 09 / 90$ & $08: 48$ & 0.39 & 1954.367 & 6.928 & 0.056 & 172.83 & \\
\hline DH42A & $05 / 16 / 90$ & $09: 07$ & 0.43 & 1961.380 & 7.013 & 0.061 & 173.26 & \\
\hline DH42A & $05 / 23 / 90$ & $12: 08$ & 0.40 & 1968.506 & 7.126 & 0.056 & 173.66 & \\
\hline DH42A & $05 / 31 / 90$ & $08: 47$ & 0.46 & 1976.366 & 7.860 & 0.059 & 174.12 & \\
\hline DH42A & $06 / 06 / 90$ & $09: 30$ & 0.34 & 1982.396 & 6.030 & 0.056 & 174.46 & \\
\hline DH42A & $06 / 14 / 90$ & $08: 38$ & 0.39 & 1990.360 & 7.964 & 0.049 & 174.85 & \\
\hline DH42A & $06 / 20 / 90$ & $09: 33$ & 0.45 & 1996.398 & 6.038 & 0.075 & 175.30 & \\
\hline DH42A & $06 / 28 / 90$ & $08: 50$ & 0.45 & 2004.368 & 7.970 & 0.056 & 175.75 & \\
\hline DH42A & $07 / 17 / 90$ & $11: 04$ & 0.56 & 2023.461 & 0.000 & 0.000 & 176.31 & ion. \\
\hline
\end{tabular}


TABLE A-2 (Continued)

BRINE ACCUMULATION DATA TABLE

Data through December 31, 1993

$\begin{array}{llclc} & \text { OAYS } & \text { OAYS } & & \text { CUHULATIVE } \\ \text { LITERS } & \text { SINCE } & \text { USED FOR } & \text { LITERS } & \text { LITERS } \\ \text { REMOVED } & 1 / 1 / 85 & \text { CALCULATION } & \text { PER DAY } & \text { COLLECTED }\end{array}$

REMARKS

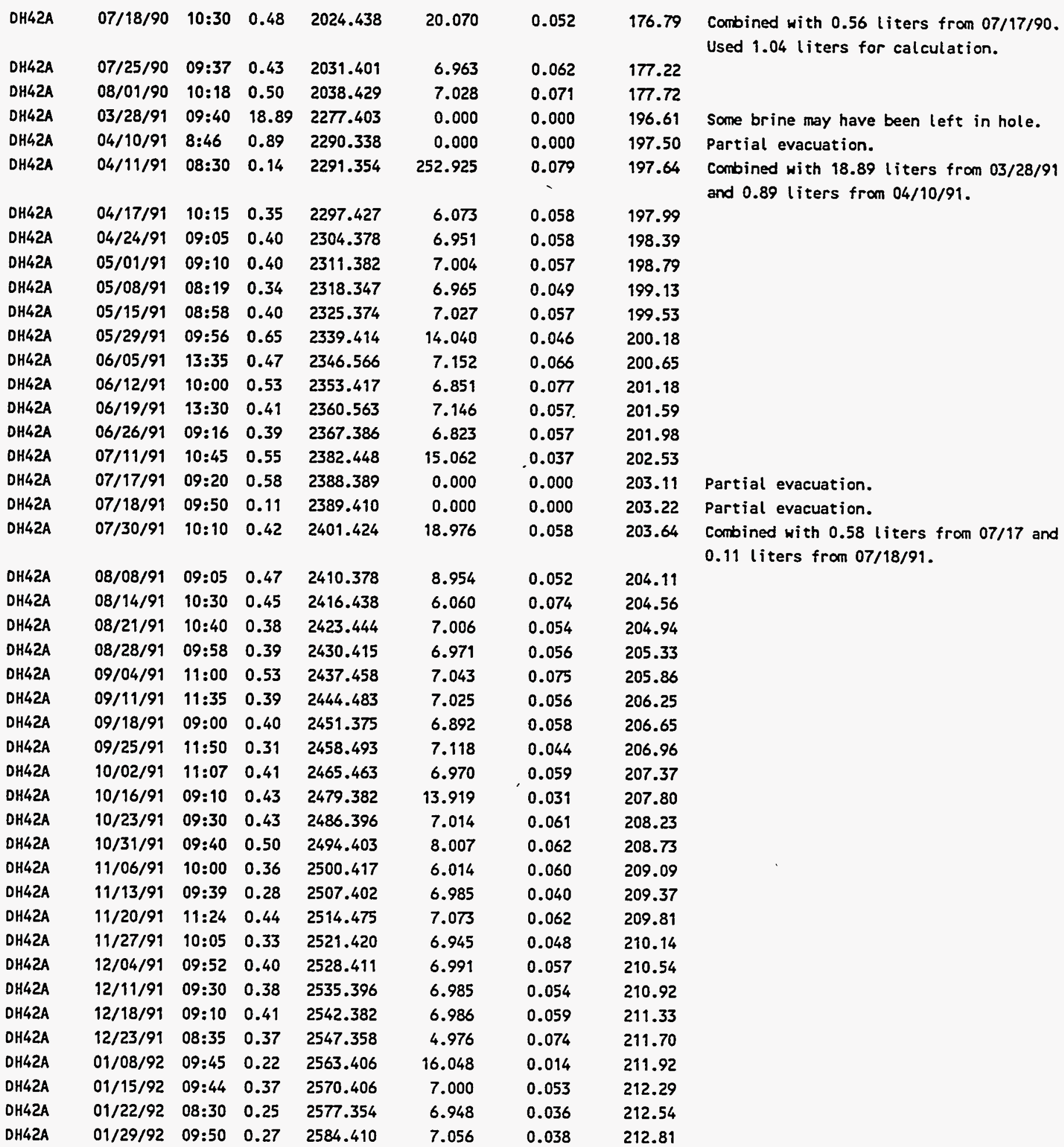


TABLE A-2 (Continued)

BRINE ACCUMULATION DATA TABLE

Data through December 31, 1993

\begin{tabular}{|c|c|c|c|c|c|c|c|c|}
\hline LOCATION & DATE & TIME & $\begin{array}{l}\text { LITERS } \\
\text { REMOVED }\end{array}$ & $\begin{array}{l}\text { DAYS } \\
\text { SIHCE } \\
1 / 1 / 85\end{array}$ & $\begin{array}{l}\text { DAYS } \\
\text { USED FOR } \\
\text { CALCULATIOH }\end{array}$ & $\begin{array}{l}\text { LITERS } \\
\text { PER DAY }\end{array}$ & $\begin{array}{l}\text { CUMULATIVE } \\
\text { LITERS } \\
\text { COLLECTED }\end{array}$ & REMARKS \\
\hline OH42A & $02 / 12 / 92$ & $08: 40$ & 0.40 & 2598.361 & 13.951 & 0.029 & 213.21 & \\
\hline DH42A & $02 / 19 / 92$ & 09:21 & 0.37 & 2605.390 & 7.029 & 0.053 & 213.58 & \\
\hline $\mathrm{DH} 42 \mathrm{~A}$ & $02 / 26 / 92$ & $09: 35$ & 0.40 & 2612.399 & 7.009 & 0.057 & 213.98 & \\
\hline DH42A & $03 / 11 / 92$ & 09:30 & 0.48 & 2626.396 & 13.997 & 0.034 & 214.46 & \\
\hline DH42A & $03 / 18 / 92$ & $09: 51$ & 0.47 & 2633.410 & 7.014 & 0.067 & 214.93 & \\
\hline DH42A & $03 / 25 / 92$ & $10: 23$ & 0.13 & 2640.433 & 7.023 & 0.019 & 215.06 & \\
\hline DH42A & $04 / 01 / 92$ & $09: 00$ & 0.45 & 2647.375 & 6.942 & 0.065 & 215.51 & \\
\hline DH42A & $04 / 07 / 92$ & $09: 10$ & 0.40 & 2653.382 & 6.007 & 0.067 & 215.91 & \\
\hline DH42A & $04 / 15 / 92$ & 09:00 & 0.48 & 2661.375 & 7.993 & 0.060 & 216.39 & \\
\hline DH42A & $04 / 22 / 92$ & $09: 30$ & 0.44 & 2668.396 & 7.021 & 0.063 & 216.83 & \\
\hline DH42A & $05 / 06 / 92$ & $11: 10$ & 0.45 & 2682.465 & 0.000 & 0.000 & 217.28 & $\begin{array}{l}\text { Partial evacuation, some brine left in } \\
\text { hole. }\end{array}$ \\
\hline OH42A & $05 / 07 / 92$ & $09: 30$ & 0.07 & 2683.396 & 15.000 & 0.035 & 217.35 & $\begin{array}{l}\text { Combine with } 0.45 \text { liters removed } 05 / 06 / 92 \\
\text { for total volume. }\end{array}$ \\
\hline DH42A & $05 / 13 / 92$ & $13: 45$ & 0.40 & 2689.573 & 6.177 & 0.065 & 217.75 & \\
\hline DH42A & $05 / 21 / 92$ & $10: 22$ & 0.43 & 2697.432 & 7.859 & 0.055 & 218.18 & \\
\hline DH42A & $05 / 27 / 92$ & 09:05 & 0.35 & 2703.378 & 5.946 & 0.059 & 218.53 & \\
\hline DH42A & $06 / 09 / 92$ & $09: 00$ & 0.44 & 2716.375 & 12.997 & 0.034 & 218.97 & \\
\hline DH42A & $06 / 18 / 92$ & $09: 45$ & 0.29 & 2725.406 & 9.031 & 0.032 & 219.26 & \\
\hline DH42A & $06 / 25 / 92$ & $09: 40$ & 0.46 & 2732.403 & 6.997 & 0.066 & 219.72 & \\
\hline $\mathrm{DH} 42 \mathrm{~A}$ & $07 / 01 / 92$ & $09: 10$ & 0.58 & 2738.382 & 5.979 & 0.097 & 220.30 & \\
\hline DH42A & $07 / 08 / 92$ & $09: 22$ & 0.44 & 2745.390 & 7.008 & 0.063 & 220.74 & \\
\hline $\mathrm{DH} 42 \mathrm{~A}$ & $07 / 15 / 92$ & $09: 14$ & 0.40 & 2752.385 & 6.995 & 0.057 & 221.14 & \\
\hline DH42A & $07 / 22 / 92$ & $09: 00$ & 0.46 & 2759.375 & 6.990 & 0.066 & 221.60 & \\
\hline DH42A & $07 / 29 / 92$ & $09: 48$ & 0.48 & 2766.408 & 7.033 & 0.068 & 222.08 & \\
\hline $\mathrm{DH} 42 \mathrm{~A}$ & $08 / 04 / 92$ & $09: 34$ & 0.41 & 2772.399 & 5.991 & 0.068 & 222.49 & \\
\hline DH42A & $08 / 18 / 92$ & $09: 56$ & 0.67 & 2786.414 & 14.015 & 0.048 & 223.16 & \\
\hline DH42A & $09 / 02 / 92$ & $09: 50$ & 0.59 & 2801.410 & 14.996 & 0.039 & 223.75 & \\
\hline $\mathrm{DH} 42 \mathrm{~A}$ & $09 / 09 / 92$ & $10: 03$ & 2.38 & 2808.419 & 7.009 & 0.340 & 226.13 & \\
\hline $\mathrm{OH} 42 \mathrm{~A}$ & $09 / 17 / 92$ & $09: 00$ & 0.28 & 2816.375 & 7.956 & 0.035 & 226.41 & \\
\hline $\mathrm{DH} 42 \mathrm{~A}$ & $09 / 23 / 92$ & $10: 03$ & 0.43 & 2822.419 & 6.044 & 0.071 & 226.84 & \\
\hline DH42A & $09 / 30 / 92$ & $11: 00$ & 0.49 & 2829.458 & 7.039 & 0.070 & 227.33 & \\
\hline DH42A & $10 / 12 / 92$ & $12: 35$ & 0.34 & 2841.524 & 12.066 & 0.028 & 227.67 & \\
\hline DH42A & $10 / 21 / 92$ & $13: 03$ & 0.69 & 2850.544 & 9.020 & 0.076 & 228.36 & \\
\hline $\mathrm{DH} 42 \mathrm{~A}$ & $10 / 28 / 92$ & $09: 10$ & 0.66 & 2857.382 & 6.838 & 0.097 & 229.02 & \\
\hline DH42A & $11 / 11 / 92$ & $09: 30$ & 0.90 & 2871.396 & 14.014 & 0.064 & 229.92 & \\
\hline DH42A & $11 / 18 / 92$ & $13: 09$ & 0.30 & 2878.548 & 7.152 & 0.042 & 230.22 & \\
\hline DH42A & $11 / 25 / 92$ & $09: 30$ & 0.39 & 2885.396 & 6.848 & 0.057 & 230.61 & \\
\hline DH42A & $12 / 09 / 92$ & $12: 30$ & 0.65 & 2899.521 & 14.125 & 0.046 & 231.26 & \\
\hline DH42A & $12 / 16 / 92$ & $10: 15$ & 0.46 & 2906.427 & 6.906 & 0.067 & 231.72 & \\
\hline DH42A & $01 / 07 / 93$ & $08: 30$ & 0.55 & 2928.354 & 21.927 & 0.025 & 232.27 & \\
\hline DH42A & $01 / 13 / 93$ & $09: 45$ & 0.69 & 2934.406 & 6.052 & 0.114 & 232.96 & \\
\hline DH42A & $01 / 28 / 93$ & $10: 10$ & 0.75 & 2949.424 & 15.018 & 0.050 & 233.71 & \\
\hline DH42A & $02 / 11 / 93$ & $09: 40$ & 0.63 & 2963.403 & 13.979 & 0.045 & 234.34 & \\
\hline DH42A & $02 / 26 / 93$ & $10: 15$ & 1.25 & 2978.427 & 15.024 & 0.083 & 235.59 & \\
\hline DH42A & $03 / 10 / 93$ & $10: 10$ & 0.67 & 2990.424 & 11.997 & 0.056 & 236.26 & \\
\hline $\mathrm{DH} 42 \mathrm{~A}$ & $03 / 19 / 93$ & $09: 37$ & 0.43 & 2999.401 & 8.977 & 0.048 & 236.69 & \\
\hline
\end{tabular}


TABLE A-2 (Continued)

BRINE ACCUMULATION DATA TABLE

Data through December 31, 1993

\begin{tabular}{|c|c|c|c|c|c|c|c|c|}
\hline & & & & DAYS & DAYS & & CUMULATIVE & \\
\hline & & & LITERS & SINCE & USED FOR & LITERS & LITERS & \\
\hline LOCATION & DATE & TIME & REMOVED & $1 / 1 / 85$ & CALCULATIOH & PER DAY & COLLECTED & REMARKS \\
\hline
\end{tabular}

\begin{tabular}{|c|c|c|c|c|c|c|c|c|}
\hline $\mathrm{DH} 42 \mathrm{~A}$ & $03 / 25 / 93$ & $09: 50$ & 0.28 & 3005.410 & 6.009 & 0.047 & 236.97 & \\
\hline DH42A & $03 / 31 / 93$ & $12: 35$ & 0.40 & 3011.524 & 6.114 & 0.065 & 237.37 & \\
\hline DH42A & $04 / 28 / 93$ & $08: 45$ & 1.26 & 3039.365 & 27.841 & 0.045 & 238.63 & Used bailer, hole left dry. \\
\hline DH42A & $06 / 16 / 93$ & $11: 50$ & 1.85 & 3088.493 & 49.128 & 0.038 & 240.48 & Used bailer. \\
\hline DH42A & $08 / 18 / 93$ & $10: 48$ & 3.25 & 3151.450 & 0.000 & 0.000 & 243.73 & $\begin{array}{l}\text { Partial evacuation. Unable to sample since } \\
06-16-93 \text {. }\end{array}$ \\
\hline OH42A & $08 / 20 / 93$ & $09: 36$ & 0.50 & 3153.400 & 64.907 & 0.058 & 244.23 & Combine with 3.25 liters from 08-18-93. \\
\hline DH42A & $11 / 09 / 93$ & $10: 33$ & 0.38 & 3234.440 & 0.000 & 0.000 & 244.61 & Partial evacuation. \\
\hline DH42A & $11 / 12 / 93$ & $10: 21$ & 0.58 & 3237.431 & 84.031 & 0.011 & 245.19 & $\begin{array}{l}\text { Used bailer. Combine with } 0.38 \text { liter from } \\
11-09-93 \text {. }\end{array}$ \\
\hline DHP402A & $10 / 29 / 86$ & $00: 00$ & NA & 0.000 & 0.000 & 0.000 & 0.00 & Drift excavated at S1950/E1320. \\
\hline DHP402A & $12 / 05 / 86$ & $00: 00$ & HA & 703.000 & 37.000 & 0.000 & 0.00 & Downhole completed. \\
\hline DHP402A & $03 / 06 / 87$ & $09: 40$ & 0.14 & 794.403 & 0.000 & 0.000 & 0.14 & First time sampled. \\
\hline DHP402A & $03 / 30 / 87$ & $09: 15$ & 0.00 & 818.385 & 0.000 & 0.000 & 0.14 & \\
\hline DHP402A & $04 / 22 / 87$ & $11: 24$ & 0.03 & 841.475 & 138.475 & 0.001 & 0.17 & $\begin{array}{l}\text { Bailer stuck in hole. Hole appears offset } \\
\text { or blocked at } 45 \text { feet. There may be a rock } \\
\text { bolt or piece of rod in the hole. }\end{array}$ \\
\hline DHP402A & $07 / 08 / 87$ & $00: 00$ & HA & 918.000 & 0.000 & 0.000 & 0.17 & $\begin{array}{l}\text { Horizontal pilot hole for Room } 7 \text { of the } \\
\text { first Waste Storage Panal started just } \\
\text { north of this location, drilled with brine. }\end{array}$ \\
\hline DHP402A & $07 / 16 / 87$ & $09: 20$ & 0.00 & 926.389 & 0.000 & 0.000 & 0.17 & $\begin{array}{l}\text { Hole entirely filled with brine from } \\
\text { drilling the pilot /gas release hole for } \\
\text { the last room of the first panel. }\end{array}$ \\
\hline DHP402A & $07 / 28 / 87$ & $10: 20$ & 17.50 & 938.431 & 0.000 & 0.000 & 17.67 & $\begin{array}{l}\text { Removed } 17.5 \text { liters of brine from hole, } \\
\text { mostly drilling fluid. }\end{array}$ \\
\hline DHP402A & $07 / 29 / 87$ & $09: 10$ & 15.00 & 939.382 & 0.000 & 0.000 & 32.67 & $\begin{array}{l}\text { Drilling brine removed from hole. Partial } \\
\text { evacuation, brine left in hole. }\end{array}$ \\
\hline DHP402A & $08 / 16 / 87$ & $00: 00$ & HA & 957.000 & 0.000 & 0.000 & 32.67 & $\begin{array}{l}\text { Brine from the AIS sump spread in Panel } 1 \\
\text { to assist in the reconstitution of loose } \\
\text { muck on the floor. }\end{array}$ \\
\hline DHP402A & $08 / 20 / 87$ & $00: 00$ & NA & 961.000 & 0.000 & 0.000 & 32.67 & $\begin{array}{l}\text { Brine from the AIS sump spread in Panel } 1 \\
\text { to assist in the reconstitution of loose } \\
\text { muck on the floor. }\end{array}$ \\
\hline DHP402A & $10 / 01 / 87$ & $00: 00$ & HA & 1003.000 & 0.000 & 0.000 & 32.67 & $\begin{array}{l}\text { Approximate date the salt muck stockpile } \\
\text { was placed at the east end of s1950, } \\
\text { covering the collar of this hole. }\end{array}$ \\
\hline DHP402A & $07 / 12 / 88$ & $13: 50$ & & 1288.576 & 0.000 & 0.000 & 32.67 & Muck piled over hole, could not collect. \\
\hline DHP402A & $08 / 19 / 88$ & $10: 00$ & 57.25 & 1326.417 & 484.942 & 0.185 & 89.92 & $\begin{array}{l}\text { Collected for chemistry, sample \#492 - } \\
\# 497 \text {. Used } 72.25 \text { liters for calculation } \\
(15.0 \text { on } 7 / 29+57.25 \text { on } 8 / 19) \text {. }\end{array}$ \\
\hline DHP402A & $08 / 30 / 88$ & $11: 00$ & 42.75 & 1337.458 & 11.041 & 3.872 & 132.67 & $\begin{array}{l}\text { Depth of water } 28.8 \text { feet below floor. } \\
\text { Bottom of hole at } 44.3 \text { feet. } 5.7 \text { feet of } \\
\text { salt on botton of hole. }\end{array}$ \\
\hline DHP402A & $09 / 15 / 88$ & $10: 00$ & 0.24 & 1353.417 & 0.000 & 0.000 & 132.91 & $\begin{array}{l}\text { Not fully evacuated. Don't use for } \\
\text { calculation. Sampled for bacteriology. }\end{array}$ \\
\hline
\end{tabular}


TABLE A-2 (Continued) BRINE ACCUMULATION DATA TABLE

Data through December 31, 1993

\begin{tabular}{|c|c|c|c|c|c|c|c|c|}
\hline LOCATION & DATE & TIME & $\begin{array}{l}\text { LITERS } \\
\text { REMOVED }\end{array}$ & $\begin{array}{l}\text { DAYS } \\
\text { SINCE } \\
1 / 1 / 85\end{array}$ & $\begin{array}{l}\text { DAYS } \\
\text { USED FOR } \\
\text { CALCULATION }\end{array}$ & $\begin{array}{l}\text { LITERS } \\
\text { PER DAY }\end{array}$ & $\begin{array}{l}\text { CUHULATIVE } \\
\text { LITERS } \\
\text { COLLECTED }\end{array}$ & REMARKS \\
\hline DHP402A & $09 / 22 / 88$ & $09: 00$ & 63.75 & 1360.375 & 22.917 & 2.792 & 196.66 & $\begin{array}{l}\text { Hole evacuated to } 44.21 \text { level. Chemistry } \\
\text { samples } \# 498 \text { - } \# 503 \text {. }\end{array}$ \\
\hline DHP402A & $10 / 18 / 88$ & $13: 45$ & 45 & 1386.573 & 26.198 & 1.718 & 241.66 & $\begin{array}{l}\text { Some moisture could have entered hole due } \\
\text { to water spread for dust control }\end{array}$ \\
\hline DHP402A & $11 / 15 / 88$ & $10: 30$ & 40.65 & 1414.438 & 27.865 & 1.459 & 282.31 & $\begin{array}{l}\text { Evacuated to } 43.75 \text { foot level. Obstruction } \\
\text { near botton of hole prevents additional } \\
\text { evacuation. }\end{array}$ \\
\hline DHP402A & $12 / 13 / 88$ & $10: 50$ & 6.0 & 1442.451 & 0.000 & 0.000 & 288.31 & $\begin{array}{l}\text { Collected for chemistry, sample } \$ 606 \text { - } \\
\# 617 \text {. Not fully evacuated, some brine left } \\
\text { in hole. }\end{array}$ \\
\hline DHP402A & $12 / 29 / 88$ & $12: 00$ & 43.60 & 1458.500 & 44.062 & 1.126 & 331.91 & $\begin{array}{l}\text { Used } 49.6 \text { liters for calculation ( } 6.0 \text { on } \\
12 / 13+43.6 \text { on } 12 / 29) \text {. }\end{array}$ \\
\hline DHP402A & $01 / 04 / 89$ & $13: 30$ & 13.5 & 1464.563 & 6.063 & 2.227 & 345.41 & $\begin{array}{l}\text { Complete evacuation to } 43.3 \mathrm{ft} \text {. level. } \\
\text { strong ador of diesel from hole and bailer. }\end{array}$ \\
\hline DHP402A & $01 / 20 / 89$ & $10: 30$ & 19 & 1480.438 & 15.875 & 1.197 & 364.41 & $\begin{array}{l}\text { Volume removed includes } 2.5 \text { gallons of } \\
\text { brine introduced to hole by Intera. }\end{array}$ \\
\hline DHP402A & $02 / 28 / 89$ & $11: 50$ & 12.1 & 1519.493 & 39.055 & 0.310 & 376.51 & Hole open to 44.2 feet. \\
\hline DHP402A & $04 / 06 / 89$ & $13: 30$ & 1.19 & 1556.563 & 37.070 & 0.032 & 377.70 & Sample removed from above packer. \\
\hline DHP402A & $04 / 20 / 89$ & $13: 05$ & NA & 1570.545 & 0.000 & 0.000 & 377.70 & Level measured at 33.1 feet. \\
\hline DHP402A & $04 / 26 / 89$ & $10: 30$ & HA & 1576.438 & 0.000 & 0.000 & 377.70 & Level of brine at 27.2 feet. \\
\hline DHP402A & $04 / 27 / 89$ & $10: 00$ & 49.00 & 1577.417 & 20.854 & 2.350 & 426.70 & Hole bottom measured at 44.3 feet. \\
\hline DHP402A & $05 / 17 / 89$ & $09: 00$ & 33 & 1597.375 & 19.958 & 1.653 & 459.70 & Fluid level at 44.6 feet. \\
\hline DHP402A & $06 / 20 / 89$ & $10: 00$ & NA & 1631.417 & 0.000 & 0.000 & 459.70 & $\begin{array}{l}\text { Fluid measured at } 39.8 \text { feet. Hole not } \\
\text { evacuated. }\end{array}$ \\
\hline DHP402A & $06 / 29 / 89$ & $09: 00$ & HA & 1640.375 & 0.000 & 0.000 & 459.70 & Measured hole fluid level at 37.6 feet. \\
\hline DHP402A & $07 / 24 / 89$ & $09: 50$ & 24 & 1665.410 & 68.035 & 0.353 & 483.70 & $\begin{array}{l}\text { Sample saved for Intera brine study. Hole } \\
\text { pumped to fluid level of } 41.1 \text { feet. }\end{array}$ \\
\hline DHP402A & $08 / 16 / 89$ & $09: 00$ & NA & 1688.375 & 0.000 & 0.000 & 483.70 & $\begin{array}{l}\text { Sample not obtained. Fluid level at } 36.5 \\
\text { feet. }\end{array}$ \\
\hline DHP402A & $08 / 23 / 89$ & $11: 45$ & NA & 1695.490 & 0.000 & 0.000 & 483.70 & $\begin{array}{l}\text { Observed fluid level at } 35.4 \text { feet. Not } \\
\text { sampled. }\end{array}$ \\
\hline OHP402A & $09 / 12 / 89$ & $12: 30$ & 6.30 & 1715.521 & 50.111 & 0.126 & 490.00 & Partial collection for chemistry. \\
\hline DHP402A & $10 / 02 / 89$ & $11: 00$ & 25.5 & 1735.458 & 19.937 & 1.279 & 515.50 & Sample saved for Intera brine study. \\
\hline DHP402A & $11 / 15 / 89$ & $10: 30$ & 16 & 1779.438 & 43.980 & 0.364 & 531.50 & Sample saved for Intera brine study. \\
\hline DHP402A & $12 / 13 / 89$ & $12: 12$ & 15.62 & 1807.508 & 28.070 & 0.556 & 547.12 & $\begin{array}{l}\text { Sample saved for chemistry and for Intera } \\
\text { brine study, sample } \# 901 \text {. }\end{array}$ \\
\hline DHP402A & $03 / 22 / 90$ & $08: 53$ & 4.0 & 1906.370 & 0.000 & 0.000 & 551.12 & Hole not completely evacuated. \\
\hline DHP402A & $03 / 26 / 90$ & $09: 25$ & 7.0 & 1910.392 & 0.000 & 0.000 & 558.12 & Hole not completely evacuated. \\
\hline DHP402A & $05 / 31 / 90$ & $10: 03$ & 0.0 & 1976.419 & 0.000 & 0.000 & 558.12 & Hole not sampled, water level at 36.0 feet. \\
\hline DHP402A & $06 / 20 / 90$ & $10: 31$ & 15.0 & 1996.438 & 188.930 & 0.138 & 573.12 & $\begin{array}{l}2 \text { liters for BSEP, . } 25 \text { liters for SNL/NM. } \\
\text { Partial evacuation. Combined with } 4.0 \\
\text { liters }(3 / 22) \text { and } 7.0 \text { liters }(3 / 26) \text {. }\end{array}$ \\
\hline DHP402A & $10 / 05 / 90$ & $09: 30$ & 2.250 & 2103.396 & 0.000 & 0.000 & 575.37 & Partial evacuation. \\
\hline DHP402A & $11 / 14 / 90$ & $10: 20$ & 0.0 & 2143.431 & 0.000 & 0.000 & 575.37 & Hole not sampled, water level at 34.2 feet. \\
\hline DHP402A & $12 / 20 / 90$ & $10: 05$ & 40.7 & 2179.420 & 182.982 & 0.235 & 616.07 & $\begin{array}{l}\text { Combined with } 2.25 \text { liters from } 10 / 05 / 90 \text {. } \\
\text { Used } 42.95 \text { liters for calculation. }\end{array}$ \\
\hline OHP402A & $02 / 20 / 91$ & $13: 00$ & 2.0 & 2241.542 & 0.000 & 0.000 & 618.07 & Partial evacuation. \\
\hline
\end{tabular}


TABLE A-2 (Continued)

\section{BRINE ACCUMULATION DATA TABLE}

Data through December 31, 1993

\begin{tabular}{|c|c|c|c|c|c|c|c|c|}
\hline & & & & DAYS & DAYS & & CUAULATIVE & \\
\hline & & & LITERS & SIHCE & USED FOR & LITERS & LITERS & \\
\hline LOCATION & DATE & TIME & REMOVED & $1 / 1 / 85$ & CALCULATIOH & PER DAY & COLLECTED & EHARKS \\
\hline
\end{tabular}

\begin{tabular}{|c|c|c|c|c|c|c|c|c|}
\hline DHP402A & $03 / 11 / 91$ & $10: 45$ & 12.72 & 2260.448 & 0.000 & 0.000 & 630.79 & $\begin{array}{l}\text { Partial evacuation. Removed for SHL/HM } \\
\text { study. }\end{array}$ \\
\hline DHP402A & $03 / 27 / 91$ & $10: 27$ & 5.20 & 2276.435 & 97.015 & 0.205 & 635.99 & $\begin{array}{l}\text { Combined with } 2.0 \text { liters from } 02 / 20 / 91 \text { and } \\
12.72 \text { liters from } 03 / 11 / 91 \text {. Sample given } \\
\text { to INTERA. }\end{array}$ \\
\hline DHP402A & $07 / 11 / 91$ & $10: 00$ & 2.00 & 2382.417 & 0.000 & 0.000 & 637.99 & Partial evacuation. \\
\hline DHP402A & $09 / 18 / 91$ & $10: 15$ & 0.06 & 2451.427 & 0.000 & 0.000 & 638.05 & Collected over two week period. \\
\hline DHP402A & $09 / 25 / 91$ & $12: 43$ & 2.0 & 2458.530 & 182.095 & 0.022 & 640.05 & $\begin{array}{l}\text { Combined with } 2.0 \text { liters from } 07 / 11 / 91 \text { and } \\
0.06 \text { liters from } 09 / 18 / 91 \text {. }\end{array}$ \\
\hline DHP402A & $04 / 16 / 92$ & $12: 15$ & 3.00 & 2662.510 & 203.980 & 0.015 & 643.05 & Saved for BSEP. \\
\hline DHP402A & $08 / 20 / 92$ & $09: 20$ & 1.00 & 2788.389 & 125.879 & 0.008 & 644.05 & $\begin{array}{l}\text { Partial evacuation for BSEP analytical } \\
\text { program. }\end{array}$ \\
\hline
\end{tabular}

\begin{tabular}{|c|c|c|c|c|c|}
\hline GSEEP & $11 / 21 / 84$ & 0.000 & 0.000 & 0.000 & 0.00 \\
\hline GSEEP & $08 / 28 / 85$ & 239.000 & 0.000 & 0.000 & 0.00 \\
\hline & $11 / 12 / 85$ & 315.001 & 0.000 & 0.000 & .00 \\
\hline
\end{tabular}

Approximate date this part of Room G excavated.

Noticed damp area on floor at this location.

0.00 Damp area on floor near $s$. rib approx. . E1140 (45 ft. E. of DH35) and at E1149. Crusted moist area is about $41 \times 4$ ', has increased noticeably in size over the last two months.

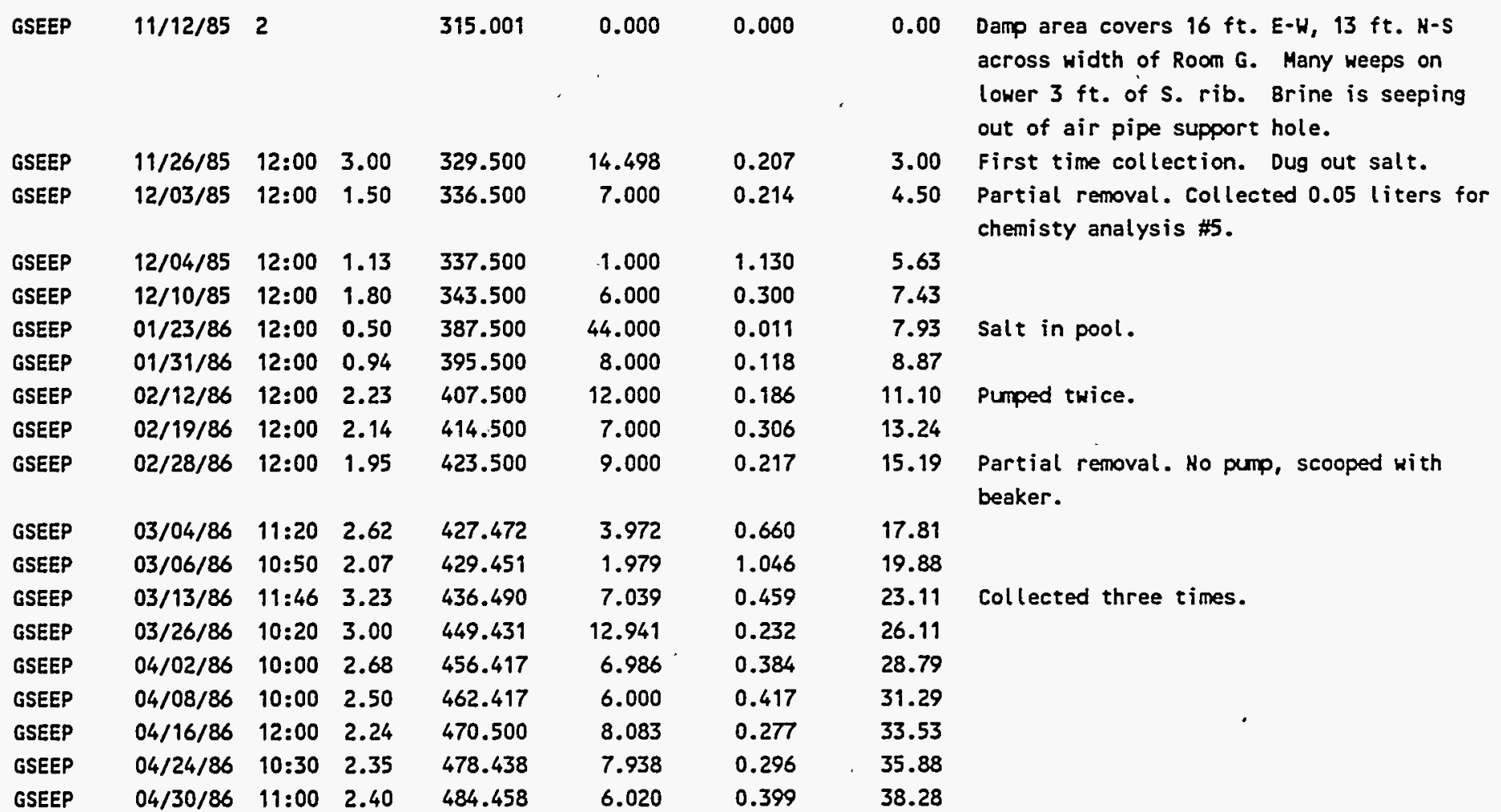


TABLE A-2 (Continued) BRINE ACCUMULATION DATA TABLE

Data through December 31, 1993

\begin{tabular}{|c|c|c|c|c|c|c|c|c|}
\hline \multirow[b]{3}{*}{ LOCATIOA } & \multirow[b]{3}{*}{ DATE } & \multirow[b]{3}{*}{ TIME } & \multirow{3}{*}{$\begin{array}{l}\text { LITERS } \\
\text { REMOVED }\end{array}$} & \multirow{3}{*}{$\begin{array}{l}\text { DAYS } \\
\text { SINCE } \\
1 / 1 / 85\end{array}$} & DAYS & \multicolumn{2}{|r|}{ CUHULATIVE } & \multirow[b]{3}{*}{ REMARKS } \\
\hline & & & & & USED FOR & LITERS & LITERS & \\
\hline & & & & & CALCULATION & PER DAY & COLLECTED & \\
\hline GSEEP & $05 / 06 / 86$ & $10: 30$ & 2.49 & 490.438 & 5.980 & 0.416 & 40.77 & \\
\hline GSEEP & $05 / 13 / 86$ & $11: 20$ & 2.66 & 497.472 & 7.034 & 0.378 & 43.43 & \\
\hline GSEEP & $05 / 20 / 86$ & $11: 20$ & 2.44 & 504.472 & 7.000 & 0.349 & 45.87 & \\
\hline GSEEP & $05 / 27 / 86$ & $15: 30$ & 3.11 & 511.646 & 7.174 & 0.434 & 48.98 & \\
\hline GSEEP & $06 / 03 / 86$ & $10: 40$ & 3.31 & 518.444 & 6.798 & 0.487 & 52.29 & \\
\hline GSEEP & $06 / 10 / 86$ & $11: 38$ & 3.21 & 525.485 & 7.041 & 0.456 & 55.50 & \\
\hline GSEEP & $06 / 17 / 86$ & $11: 15$ & 3.11 & 532.469 & 6.984 & 0.445 & 58.61 & Sample for brine chemistry, \#20. \\
\hline GSEEP & $06 / 24 / 86$ & $19: 00$ & 4.60 & 539.458 & 6.989 & 0.658 & 63.21 & Very humid air in workings. \\
\hline GSEEP & $07 / 01 / 86$ & $14: 00$ & 5.43 & 546.583 & 7.125 & 0.762 & 68.64 & Very humid last week, rain on surface. \\
\hline GSEEP & $07 / 08 / 86$ & $10: 50$ & 4.14 & 553.451 & 6.868 & 0.603 & 72.78 & \\
\hline GSEEP & $07 / 16 / 86$ & $10: 50$ & 3.32 & 561.451 & 8.000 & 0.415 & 76.10 & \\
\hline GSEEP & $07 / 22 / 86$ & $10: 15$ & 2.29 & 567.427 & 5.976 & 0.383 & 78.39 & \\
\hline GSEEP & $07 / 29 / 86$ & $10: 45$ & 2.68 & 574.448 & 7.021 & 0.382 & 81.07 & \\
\hline GSEEP & $08 / 05 / 86$ & $11: 20$ & 2.60 & 581.472 & 7.024 & 0.370 & 83.67 & \\
\hline GSEEP & $08 / 12 / 86$ & $10: 45$ & 3.67 & 588.448 & 6.976 & 0.526 & 87.34 & \\
\hline GSEEP & $08 / 19 / 86$ & $11: 40$ & 3.90 & 595.486 & 7.038 & 0.554 & 91.24 & \\
\hline GSEEP & $08 / 26 / 86$ & $11: 00$ & 3.73 & 602.458 & 6.972 & 0.535 & 94.97 & \\
\hline GSEEP & $09 / 04 / 86$ & $10: 55$ & 5.15 & 611.455 & 8.997 & 0.572 & 100.12 & Last week has been humid and rainy. \\
\hline GSEEP & $09 / 09 / 86$ & $10: 00$ & 3.70 & 616.417 & 4.962 & .0 .746 & 103.82 & \\
\hline GSEEP & $09 / 16 / 86$ & $10: 25$ & 3.82 & 623.434 & 7.017 & 0.544 & 107.64 & \\
\hline GSEEP & $09 / 23 / 86$ & $10: 20$ & 4.29 & 630.431 & 6.997 & 0.613 & 111.93 & \\
\hline GSEEP & $10 / 01 / 86$ & $12: 24$ & 3.70 & 638.517 & 8.086 & 0.458 & 115.63 & \\
\hline GSEEP & $10 / 08 / 86$ & $10: 45$ & 3.80 & 645.448 & 0.000 & 0.000 & 119.43 & Partial collection. \\
\hline GSEEP & $10 / 08 / 86$ & $14: 57$ & 1.87 & 645.623 & 7.106 & 0.798 & 121.30 & $\begin{array}{l}\text { Second collection for this day. Use }(3.80 \\
+1.87) /(6.931+0.175)=0.798 \text { l/day. }\end{array}$ \\
\hline GSEEP & $10 / 10 / 86$ & $09: 16$ & 1.24 & 647.386 & 1.763 & 0.703 & 122.54 & \\
\hline GSEEP & $10 / 14 / 86$ & $11: 10$ & 2.19 & 651.465 & 4.079 & 0.537 & 124.73 & \\
\hline GSEEP & $11 / 05 / 86$ & $10: 45$ & 4.44 & 673.448 & 21.983 & 0.202 & 129.17 & $\begin{array}{l}\text { First time } 3.74 \text { liters, second time } 0.70 \\
\text { liters. }\end{array}$ \\
\hline GSEEP & $11 / 20 / 86$ & $12: 02$ & 3.84 & 688.501 & 15.053 & 0.255 & 133.01 & \\
\hline GSEEP & $12 / 30 / 86$ & $12: 50$ & 4.44 & 728.535 & 40.034 & 0.111 & 137.45 & \\
\hline GSEEP & $02 / 03 / 87$ & $13: 45$ & 3.45 & 763.573 & 35.038 & 0.098 & 140.90 & \\
\hline GSEEP & $03 / 06 / 87$ & $11: 30$ & 3.0 & 794.479 & 30.906 & 0.097 & 143.90 & \\
\hline GSEEP & $03 / 30 / 87$ & $11: 34$ & 2.51 & 818.482 & 24.003 & 0.105 & 146.41 & \\
\hline GSEEP & $05 / 07 / 87$ & $11: 48$ & 3.31 & 856.492 & 38.010 & 0.087 & 149.72 & \\
\hline GSEEP & $06 / 30 / 87$ & $10: 00$ & 12.24 & 910.417 & 53.925 & 0.227 & 161.96 & \\
\hline GSEEP & $07 / 16 / 87$ & $10: 30$ & 11.66 & 926.438 & 16.021 & 0.728 & 173.62 & \\
\hline GSEEP & $07 / 23 / 87$ & $09: 20$ & 3.87 & 933.389 & 6.951 & 0.557 & 177.49 & \\
\hline GSEEP & $07 / 28 / 87$ & $11: 35$ & 2.36 & 938.483 & 5.094 & 0.463 & 179.85 & \\
\hline GSEEP & $08 / 07 / 87$ & $09: 15$ & 5.33 & 948.385 & 9.902 & 0.538 & 185.18 & \\
\hline GSEEP & $08 / 12 / 87$ & $10: 12$ & 2.80 & 953.425 & 5.040 & 0.556 & 187.98 & \\
\hline GSEEP & $08 / 24 / 87$ & $08: 46$ & 6.53 & 965.365 & 11.940 & 0.547 & 194.51 & \\
\hline GSEEP & $09 / 01 / 87$ & $11: 00$ & 5.26 & 973.458 & 8.093 & 0.650 & 199.77 & $\begin{array}{l}\text { Collected for chemistry, sample \#164 A\&B, } \\
\# 166 \text { A\&B, \#169 A\&B, \#165 A\&B, \#168 A\&B. }\end{array}$ \\
\hline GSEEP & $09 / 11 / 87$ & $09: 00$ & 5.03 & 983.375 & 9.917 & 0.507 & 204.80 & \\
\hline GSEEP & $09 / 16 / 87$ & $09: 33$ & 2.42 & 988.398 & 5.023 & 0.482 & 207.22 & \\
\hline GSEEP & $09 / 25 / 87$ & $08: 55$ & 4.12 & 997.372 & 8.974 & 0.459 & 211.34 & Sump drilled to facilitate accumulation of \\
\hline
\end{tabular}


TABLE A-2 (Continued)

BRINE ACCUMULATION DATA TABLE

Data through December 31, 1993

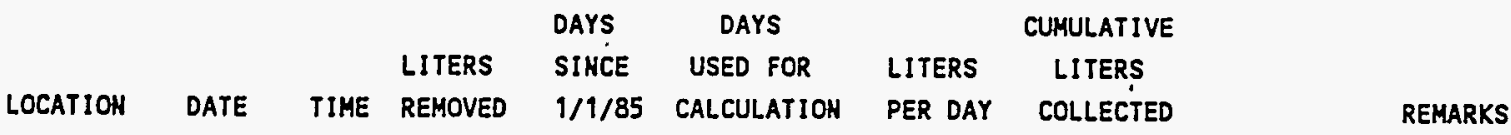

\begin{tabular}{|c|c|c|c|c|c|c|c|c|}
\hline GSEEP & $10 / 01 / 87$ & $12: 15$ & 2.81 & 1003.510 & 6.138 & 0.458 & 214.15 & ?. \\
\hline GSEEP & $10 / 08 / 87$ & $10: 25$ & 2.97 & 1010.434 & 6.924 & 0.429 & 217.12 & \\
\hline GSEEP & $10 / 16 / 87$ & $10: 41$ & 3.37 & 1018.445 & 8.011 & 0.421 & 220.49 & \\
\hline GSEEP & $10 / 20 / 87$ & $11: 59$ & 2.06 & 1022.499 & 4.054 & 0.508 & 222.55 & \\
\hline GSEEP & $11 / 12 / 87$ & $10: 41$ & 10.21 & 1045.445 & 22.946 & 0.445 & 232.76 & \\
\hline GSEEP & $11 / 19 / 87$ & $11: 35$ & 2.90 & 1052.483 & 7.038 & 0.412 & 235.66 & $\begin{array}{l}\text { Collected for chemistry, sample } \# 202, \# 219 \\
\& \# 231 \text {. }\end{array}$ \\
\hline GSEEP & $12 / 07 / 87$ & $12: 50$ & 7.02 & 1070.535 & 18.052 & 0.389 & 242.68 & Collected for chemistry, sample \#239. \\
\hline GSEEP & $01 / 04 / 88$ & $12: 10$ & 16.11 & 1098.507 & 27.972 & 0.576 & 258.79 & \\
\hline GSEEP & $01 / 20 / 88$ & $11: 25$ & 8.68 & 1114.476 & 15.969 & 0.544 & 267.47 & \\
\hline GSEEP & $02 / 08 / 88$ & $12: 15$ & 9.58 & 1133.510 & 19.034 & 0.503 & 277.05 & $\begin{array}{l}\text { Collected for chemistry, sample } \# 271, \# 272 \text {, } \\
\# 273, \# 274, \# 275, \# 276, \# 277, \# 278, \# 279, \\
\# 280, \# 281, \# 282, \# 283, \# 284, \# 285, \& \# 286 .\end{array}$ \\
\hline GSEEP & $02 / 25 / 88$ & $10: 40$ & 11.87 & 1150.444 & 16.934 & 0.701 & 288.92 & \\
\hline GSEEP & $03 / 09 / 88$ & $10: 18$ & 7.35 & 1163.429 & 12.985 & 0.566 & 296.27 & \\
\hline GSEEP & $03 / 17 / 88$ & $11: 20$ & 4.45 & 1171.472 & 8.043 & 0.553 & 300.72 & \\
\hline GSEEP & $03 / 29 / 88$ & $11: 45$ & 5.42 & 1183.490 & 12.018 & 0.451 & 306.14 & $\begin{array}{l}\text { Collected for chemistry, sample } \$ 327 \text { - } \\
\$ 337 \text {. }\end{array}$ \\
\hline GSEEP & $04 / 15 / 88$ & $11: 01$ & 7.43 & 1200.459 & 16.969 & 0.438 & 313.57 & \\
\hline GSEEP & $05 / 05 / 88$ & $10: 10$ & 9.34 & 1220.424 & 19.965 & 0.468 & 322.91 & Sampled for SNL/NM PA. \\
\hline GSEEP & $05 / 12 / 88$ & $09: 30$ & 3.55 & 1227.396 & 6.972 & 0.509 & 326.46 & Sampled for SHL/HM PA. \\
\hline GSEEP & $06 / 09 / 88$ & $08: 45$ & 12.00 & 1255.365 & 27.969 & 0.429 & 338.46 & Removed for SNL/KM PA. \\
\hline GSEEP & $06 / 16 / 88$ & $09: 43$ & 4.13 & 1262.405 & 7.040 & 0.587 & 342.59 & Sampled for SNL/NM PA. \\
\hline GSEEP & $06 / 30 / 88$ & $08: 30$ & 6.00 & 1276.354 & 13.949 & 0.430 & 348.59 & Sampled for SNL/NM PA. \\
\hline GSEEP & $07 / 12 / 88$ & 09:00 & 6.40 & 1288.375 & 12.021 & 0.532 & 354.99 & $\begin{array}{l}\text { Collected for chemistry, sample } \# 437 \text { - } \\
\# 448 \text {. }\end{array}$ \\
\hline GSEEP & $07 / 28 / 88$ & $10: 30$ & 11.35 & 1304.438 & 16.063 & 0.707 & 366.34 & Sampled for SNL/MM PA. \\
\hline GSEEP & $08 / 11 / 88$ & $10: 00$ & 12.02 & 1318.417 & 13.979 & 0.860 & 378.36 & Sampled for SHL/NM PA. \\
\hline GSEEP & $08 / 25 / 88$ & $09: 07$ & 6.72 & 1332.380 & 13.963 & 0.481 & 385.08 & $\begin{array}{l}\text { Hole covered with tight fitting brattice } \\
\text { cloth. Sampled for SHL/NM PA. }\end{array}$ \\
\hline GSEEP & $09 / 08 / 88$ & $14: 48$ & 7.31 & 1346.617 & 14.237 & 0.513 & 392.39 & Sampled for SNL/NM PA. \\
\hline GSEEP & $09 / 14 / 88$ & $08: 30$ & 3.00 & 1352.354 & 5.737 & 0.523 & 395.39 & \\
\hline GSEEP & $09 / 27 / 88$ & $10: 50$ & 6.45 & 1365.451 & 13.097 & 0.492 & 401.84 & $\begin{array}{l}\text { Collected for chemistry, sample } \# 545 \text { - } \\
\# 556 \text {. }\end{array}$ \\
\hline GSEEP & $10 / 18 / 88$ & $10: 22$ & 10.20 & 1386.432 & 20.981 & 0.486 & 412.04 & . \\
\hline GSEEP & $11 / 10 / 88$ & $09: 08$ & 12.62 & 1409.381 & 22.949 & 0.550 & 424.66 & $\begin{array}{l}\text { Smell of urine in sample and coming from } \\
\text { hole. }\end{array}$ \\
\hline GSEEP & $12 / 13 / 88$ & $10: 20$ & 17.81 & 1442.431 & 33.050 & 0.539 & 442.47 & $\begin{array}{l}\text { Collected for chemistry, sample \#564 - } \\
\text { \#69. Sample effervesces and brine feels } \\
\text { warmer than usual. }\end{array}$ \\
\hline GSEEP & $01 / 10 / 89$ & $13: 30$ & 17.38 & 1470.563 & 28.132 & 0.618 & 459.85 & Sample saved for SNL/NM brine study. \\
\hline GSEEP & 02/09/89 & $10: 22$ & 19.5 & 1500.432 & 29.869 & 0.653 & 479.35 & Sample saved for SHL/HM brine study. \\
\hline GSEEP & 03/01/89 & $10: 00$ & 3.90 & 1520.417 & 19.985 & 0.195 & 483.25 & Partial collection for Westinghouse. \\
\hline GSEEP & $03 / 14 / 89$ & $12: 45$ & 19.57 & 1533.531 & 13.114 & 1.492 & 502.82 & $\begin{array}{l}\text { Sample saved for chemistry, sample } \# 672 \text { - } \\
683 \text {. Add } 3.9 \text { liters collected } 3 / 01 / 90 \text { to } \\
19.57 \text { liters Use } 23.47 \text { liters for }\end{array}$ \\
\hline
\end{tabular}




\section{TABLE A-2 (Continued) BRINE ACCUMULATION DATA TABLE}

Data through December 31, 1993

\begin{tabular}{|c|c|c|c|c|c|c|c|c|}
\hline & & & & DAYS & DAYS & & CUMULATIVE & \\
\hline & & & ITEDS & SIHCE & USED FOR & LITERS & LITERS & \\
\hline CATION & DATE & TIME & REMOVED & $9 / 9 / 85$ & CALCULATIOH & PER DAY & COLLECTED & RKS \\
\hline
\end{tabular}

\begin{tabular}{|c|c|c|c|c|c|c|c|c|}
\hline GSEEP & $04 / 06 / 89$ & $08: 56$ & 16.35 & 1556.372 & 22.841 & 0.716 & 519.17 & $\begin{array}{l}16 \text { liters of sample saved for SNL/NM brine } \\
\text { study. }\end{array}$ \\
\hline GSEEP & $04 / 20 / 89$ & $08: 45$ & 10.43 & 1570.365 & 13.993 & 0.745 & 529.60 & Sample saved for SHL/NM brine study. \\
\hline GSEEP & $05 / 17 / 89$ & $09: 40$ & 19.72 & 1597.403 & 27.038 & 0.729 & 549.32 & Sample saved for SNL/MM brine study. \\
\hline GSEEP & $06 / 06 / 89$ & $09: 40$ & 14.52 & 1617.403 & 20.000 & 0.726 & 563.84 & $\begin{array}{l}\text { Sample saved for chemistry. Extra saved for } \\
\text { SWL/NM brine study. }\end{array}$ \\
\hline GSEEP & $06 / 29 / 89$ & $10: 01$ & 15.95 & 1640.417 & 23.014 & 0.693 & 579.79 & Sample saved for SNL/NM brine study. \\
\hline GSEEP & $07 / 06 / 89$ & $09: 00$ & 4.67 & 1647.375 & 6.958 & 0.671 & 584.46 & Sample saved for SHL/HM brine study. \\
\hline GSEEP & $07 / 25 / 89$ & $09: 30$ & 12.60 & 1666.396 & 19.021 & 0.662 & 597.06 & Sample saved for SNL/HM brine study. \\
\hline GSEEP & $08 / 16 / 89$ & $09: 15$ & 14.73 & 1688.385 & 21.989 & 0.670 & 611.79 & Sample saved for SNL/NM brine study. \\
\hline GSEEP & $09 / 12 / 89$ & $08: 30$ & 18.68 & 1715.354 & 26.969 & 0.693 & 630.47 & Sample saved for chemistry. \\
\hline GSEEP & $10 / 11 / 89$ & $09: 47$ & 17.70 & 1744.408 & 29.054 & 0.609 & 648.17 & Sample saved for SHL/HM brine study. \\
\hline GSEEP & $11 / 15 / 89$ & $09: 30$ & 21.44 & 1779.396 & 34.988 & 0.613 & 669.61 & Sample saved for SNL/HM brine study. \\
\hline GSEEP & $12 / 13 / 89$ & $09: 13$ & 16.30 & 1807.384 & 27.988 & 0.582 & 685.91 & $\begin{array}{l}\text { Sample saved for SHL/NM brine study, sample } \\
\text { \#96. }\end{array}$ \\
\hline GSEEP & $01 / 10 / 90$ & $09: 21$ & 16.40 & 1835.390 & 28.006 & 0.586 & 702.31 & \\
\hline GSEEP & $01 / 24 / 90$ & $09: 19$ & 9.0 & 1849.388 & 13.998 & 0.643 & 711.31 & \\
\hline GSEEP & $02 / 07 / 90$ & $10: 07$ & 9.0 & 1863.422 & 14.034 & 0.641 & 720.31 & \\
\hline GSEEP & $02 / 21 / 90$ & $09: 40$ & 8.32 & 1877.403 & 13.981 & 0.595 & 728.63 & \\
\hline GSEEP & $03 / 21 / 90$ & $09: 49$ & 16.55 & 1905.409 & 28.006 & 0.591 & 745.18 & \\
\hline GSEEP & $04 / 24 / 90$ & $11: 16$ & 20.33 & 1939.469 & 34.060 & 0.597 & 765.51 & \\
\hline GSEEP & $05 / 23 / 90$ & $11: 51$ & 16.66 & 1968.494 & 29.025 & 0.574 & 782.17 & \\
\hline GSEEP & $06 / 06 / 90$ & $12: 30$ & 10.50 & 1982.521 & 0.000 & 0.000 & 792.67 & \\
\hline GSEEP & $06 / 20 / 90$ & $08: 56$ & 15.72 & 1996.372 & 27.878 & 0.941 & 808.39 & \\
\hline GSEEP & $07 / 25 / 90$ & $08: 50$ & 15.0 & 2031.368 & 34.996 & 0.429 & 823.39 & \\
\hline GSEEP & $12 / 11 / 90$ & $10: 30$ & 2.0 & 2170.438 & 0.000 & 0.000 & 825.39 & $\begin{array}{l}\text { Partial removal. First time sampled since } \\
07 / 25 / 90 \text {. }\end{array}$ \\
\hline GSEEP & $12 / 13 / 90$ & $08: 56$ & 49.89 & 2172.372 & 141.004 & 0.368 & 875.28 & $\begin{array}{l}\text { Combined with } 2.0 \text { liters from } 12 / 11 / 90 \text {. } \\
\text { Used } 51.89 \text { liters for calculation. }\end{array}$ \\
\hline GSEEP & $12 / 20 / 90$ & $08: 23$ & 0.0 & 2179.349 & 0.000 & 0.000 & 875.28 & Could not sample. \\
\hline GSEEP & $01 / 23 / 91$ & $09: 30$ & 26.14 & 2213.396 & 41.024 & 0.637 & 901.42 & $\begin{array}{l}\text { Combined with } 2.0 \text { liters from } 12 / 11 / 90 \text { and } \\
49.89 \text { liters from } 12 / 13 / 90 \text {. }\end{array}$ \\
\hline GSEEP & $02 / 27 / 91$ & $09: 52$ & 17.6 & 2248.411 & 35.015 & 0.503 & 919.02 & \\
\hline GSEEP & $03 / 11 / 91$ & $08: 20$ & 6.9 & 2260.347 & 11.936 & 0.578 & 925.92 & $\begin{array}{l}\text { Removed out of cycle for SHL/NM biology } \\
\text { study. }\end{array}$ \\
\hline GSEEP & $03 / 20 / 91$ & $10: 10$ & 2.02 & 2269.424 & 0.000 & 0.000 & 927.94 & $\begin{array}{l}\text { Partial evacuation. First evacuation with } \\
\text { bailer, second with pump. }\end{array}$ \\
\hline GSEEP & $03 / 21 / 91$ & $08: 45$ & 3.17 & 2270.365 & 10.018 & 0.518 & 931.11 & Combined with 2.02 liters from $03 / 20 / 91$. \\
\hline GSEEP & $04 / 24 / 91$ & $09: 02$ & 15.85 & 2304.376 & 34.019 & 0.466 & 946.96 & \\
\hline GSEEP & $05 / 29 / 91$ & 09:06 & 15.72 & 2339.379 & 35.003 & 0.449 & 962.68 & \\
\hline GSEEP & $06 / 26 / 91$ & $08: 50$ & 12.0 & 2367.368 & 27.989 & 0.429 & 974.68 & \\
\hline GSEEP & $07 / 11 / 91$ & $10: 20$ & 2.25 & 2382.431 & 0.000 & 0.000 & 976.93 & Partial evacuation. \\
\hline GSEEP & $07 / 31 / 91$ & $09: 30$ & 11.72 & 2402.396 & 35.028 & 0.399 & 988.65 & Combined with 2.25 liters from $07 / 11 / 91$. \\
\hline GSEEP & $08 / 28 / 91$ & $09: 15$ & 11.40 & 2430.385 & 27.989 & 0.407 & 1000.05 & \\
\hline GSEEP & $09 / 25 / 91$ & $11: 20$ & 2.0 & 2458.472 & 0.000 & 0.000 & 1002.05 & Some brine may have been left in hole. \\
\hline GSEEP & $10 / 23 / 91$ & $09: 55$ & 15.0 & 2486.413 & 56.028 & 0.303 & 1017.05 & ined with 2 liters from $10 / 23 / 91$. \\
\hline
\end{tabular}


TABLE A-2 (Continued)

\section{BRINE ACCUMULATION DATA TABLE}

Data through December 31, 1993

\begin{tabular}{|c|c|c|c|c|c|c|c|c|}
\hline LOCATION & DATE & TIME & $\begin{array}{l}\text { LITERS } \\
\text { REMOVED }\end{array}$ & $\begin{array}{l}\text { OAYS } \\
\text { SIHCE } \\
1 / 1 / 85\end{array}$ & $\begin{array}{l}\text { DAYS } \\
\text { USED FOR } \\
\text { CALCULATION }\end{array}$ & $\begin{array}{l}\text { LITERS } \\
\text { PER DAY }\end{array}$ & $\begin{array}{l}\text { CUMULATIVE } \\
\text { LITERS } \\
\text { COLLECTED }\end{array}$ & REMARKS \\
\hline GSEEP & $11 / 27 / 91$ & $09: 40$ & 10.0 & 2521.403 & 34.990 & 0.286 & 1027.05 & - \\
\hline GSEEP & $12 / 10 / 91$ & $10: 30$ & 1.7 & 2534.438 & 0.000 & 0.000 & 1028.75 & Partial removal for SNL/NM. \\
\hline GSEEP & $01 / 29 / 92$ & $09: 42$ & 13.10 & 2584.404 & 63.001 & 0.235 & 1041.85 & SHL/NM sampling. \\
\hline GSEEP & $02 / 26 / 92$ & $09: 30$ & 7.23 & 2612.396 & 27.992 & 0.258 & 1049.08 & Saved for SNL/MM. \\
\hline GSEEP & $04 / 16 / 92$ & $10: 30$ & 3.00 & 2662.438 & 50.042 & 0.060 & 1052.08 & Saved for BSEP. \\
\hline GSEEP & $05 / 27 / 92$ & 09:01 & 13.23 & 2703.376 & 40.938 & 0.323 & 1065.31 & Saved for SNL/NM Chemistry. \\
\hline GSEEP & $07 / 29 / 92$ & $09: 35$ & 11.75 & 2766.399 & 63.023 & 0.186 & 1077.06 & Saved for SHL/MM. \\
\hline GSEEP & $0 B / 20 / 92$ & $08: 58$ & 1.00 & 2788.374 & 0.000 & 0.000 & 1078.06 & $\begin{array}{l}\text { Partial evacuation for BSEP analytical } \\
\text { program. }\end{array}$ \\
\hline GSEEP & $02 / 26 / 93$ & $08: 45$ & 7.40 & 2978.365 & 211.966 & 0.040 & 1085.46 & \\
\hline GSEEP & $05 / 19 / 93$ & $10: 15$ & 6.0 & 3060.427 & 82.062 & 0.073 & 1091.46 & $\begin{array}{l}\text { Saved for SHL/HM Brine Study. Cleaned hole } \\
\text { of accumulated salt buildup: removed all } \\
\text { loose material. Pumped hole dry. } \\
\text { Saved for SNL/NM Chemistry. }\end{array}$ \\
\hline EEPP & $08 / 19 / 93$ & $13: 44$ & 4.10 & 3152.572 & 64.122 & 0.064 & 1098.31 & \\
\hline ISEEP & $11 / 12 / 93$ & $09: 44$ & 2.46 & 3237.406 & 84.834 & 0.029 & 1100.77 & \\
\hline
\end{tabular}

\begin{tabular}{|c|c|c|c|c|c|c|c|c|}
\hline он20 & $09 / 03 / 85$ & $14: 00$ & NA & 245.583 & 0.000 & $.0: 000$ & 0.00 & $\begin{array}{l}\text { Approximated date this part of drift } \\
\text { excavated. }\end{array}$ \\
\hline он20 & $03 / 29 / 89$ & $14: 00$ & HA & 1548.583 & 0.000 & 0.000 & 0.00 & $\begin{array}{l}\text { Horizontal hole drilled } 3 / 28 / 89 \text { to } 3 / 29 / 89 \text {. } \\
\text { Hole drilled with brine. Fluorescien added } \\
\text { to drilling fluid. }\end{array}$ \\
\hline ОН2O & $03 / 30 / 89$ & 11:00 & NA & 1549.458 & 0.000 & 0.000 & 0.00 & $\begin{array}{l}\text { Hew hole. Installed collection device. } \\
\text { Hole dry. }\end{array}$ \\
\hline ОH2O & $04 / 18 / 89$ & $09: 45$ & 0 & 1568.406 & 1322.830 & 0.000 & 0.00 & Device left with 50 centibars suction. \\
\hline ОН20 & $04 / 26 / 89$ & $09: 50$ & 0 & 1576.410 & 8.004 & 0.000 & 0.00 & Device left with 50 centibars suction. \\
\hline OH2O & $06 / 05 / 89$ & $09: 00$ & 0.31 & 1616.375 & 39.965 & 0.008 & 0.31 & $\begin{array}{l}\text { First time sample recovered from this hole. } \\
\text { Sample colored with Fluorescien dye. } \\
\text { Replaced collection device. Sample saved } \\
\text { for chemistry. }\end{array}$ \\
\hline ОН 20 & $06 / 20 / 89$ & $08: 30$ & 0.03 & 1631.354 & 14.979 & 0.002 & 0.34 & \\
\hline OH2O & $07 / 06 / 89$ & $11: 00$ & 0.02 & 1647.458 & 16.104 & 0.001 & 0.36 & $\begin{array}{l}\text { Collection device retained vacuum. Sample } \\
\text { collected for chemistry. }\end{array}$ \\
\hline OH2O & $08 / 09 / 89$ & $10: 00$ & 0.29 & 1681.417 & 33.959 & 0.009 & 0.65 & $\begin{array}{l}\text { Sample collected for chemistry. Pumped } \\
\text { collection device, repaired hose end. }\end{array}$ \\
\hline $\mathrm{OH} 2 \mathrm{O}$ & $08 / 23 / 89$ & $11: 22$ & 0.16 & 1695.474 & 14.057 & 0.011 & 0.81 & $\begin{array}{l}\text { Sample collected for chemistry. Still } \\
\text { yellowish green in color. }\end{array}$ \\
\hline ОН20 & $09 / 14 / 89$ & $11: 05$ & 0.21 & 1717.462 & 21.988 & 0.010 & 1.02 & Sample saved for chemistry. \\
\hline OH2O & $10 / 02 / 89$ & $11: 20$ & 0.27 & 1735.472 & 18.010 & 0.015 & 1.29 & Sample saved for chemistry. \\
\hline OH2O & $10 / 20 / 89$ & $11: 25$ & 0.26 & 1753.476 & 18.004 & 0.014 & 1.55 & Sample saved for chemistry, sample $\# 855$. \\
\hline OH2O & $11 / 10 / 89$ & $10: 18$ & 0.29 & 1774.429 & 20.953 & 0.014 & 1.84 & Sample saved for chemistry, sample $\# 868$. \\
\hline OH2O & $11 / 29 / 89$ & $13: 00$ & 0.37 & 1793.542 & 19.113 & 0.019 & 2.21 & Sample saved for chemistry, sample $\# 876$. \\
\hline OH2O & $12 / 12 / 89$ & $10: 06$ & 0.20 & 1806.421 & 12.879 & 0.016 & 2.41 & Sample saved for chemistry, sample \#888. \\
\hline OH2O & $01 / 04 / 90$ & $11: 52$ & 0.27 & 1829.494 & 23.073 & 0.012 & 2.68 & \\
\hline ОН 20 & $01 / 17 / 90$ & 09:59 & 0.21 & 1842.416 & 12.922 & 0.016 & 2.89 & \\
\hline OH2O & $01 / 31 / 90$ & $10: 38$ & 0.21 & 1856.443 & 14.027 & 0.015 & 3.10 & \\
\hline
\end{tabular}


TABLE A-2 (Continued)

\section{BRINE ACCUMULATION DATA TABLE}

Data through December 31, 1993

\begin{tabular}{|c|c|c|c|c|c|c|c|c|}
\hline & & & & DAYS & DAYS & & CUHULAT IVE & \\
\hline & & & LITERS & SINCE & USED FOR & LITERS & LITERS & . \\
\hline LOCATION & DATE & TIME & REMOVED & $1 / 1 / 85$ & CALCULATION & PER DA' & COLLECTED & REMARKS \\
\hline
\end{tabular}

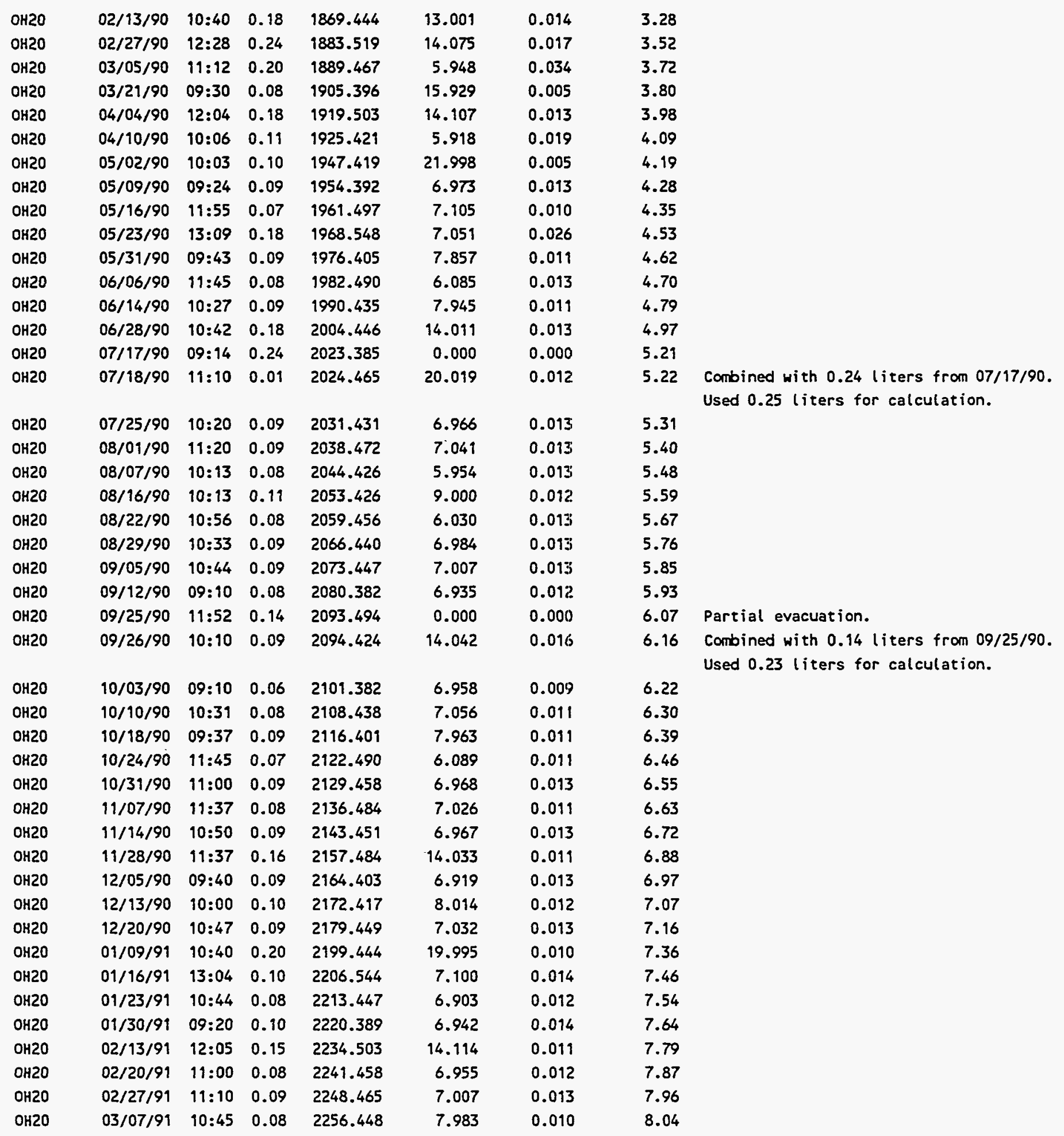


TABLE A-2 (Continued)

\section{BRINE ACCUMULATION DATA TABLE}

Data through December 31, 1993

\begin{tabular}{|c|c|c|c|c|c|c|c|c|}
\hline & & & & DAYS & DAYS & & CURULATIVE & \\
\hline & & & LITERS & SIHCE & USED FOR & LITERS & LITERS & \\
\hline LOCATION & DATE & TIME & REMOVED & $1 / 1 / 85$ & CALCULATIOH & PER DAY & COLLECTED & REMARKS \\
\hline
\end{tabular}

\begin{tabular}{|c|c|c|c|c|c|c|c|}
\hline OH2O & $03 / 20 / 91$ & $12: 51$ & 0.15 & 2269.535 & 13.087 & 0.011 & 8.19 \\
\hline OH2O & $03 / 28 / 91$ & $12: 34$ & 0.10 & 2277.524 & 7.989 & 0.013 & 8.29 \\
\hline OH2O & $04 / 10 / 91$ & $09: 44$ & 0.14 & 2290.406 & 12.882 & 0.011 & 8.43 \\
\hline ОН2О & $04 / 17 / 91$ & $11: 10$ & 0.09 & 2297.465 & 7.059 & 0.013 & 8.52 \\
\hline ОН2O & $04 / 24 / 91$ & $10: 05$ & 0.09 & 2304.420 & 6.955 & 0.013 & 8.61 \\
\hline OH2O & $05 / 01 / 91$ & $10: 10$ & 0.09 & 2311.424 & 7.004 & 0.013 & 8.70 \\
\hline $\mathrm{OH} 20$ & $05 / 08 / 91$ & $09: 10$ & 0.09 & 2318.382 & 6.958 & 0.013 & 8.79 \\
\hline OH2O & $05 / 15 / 91$ & $10: 45$ & 0.08 & 2325.448 & 7.066 & 0.011 & 8.87 \\
\hline OH2O & $05 / 29 / 91$ & $10: 33$ & 0.15 & 2339.440 & 13.992 & 0.011 & 9.02 \\
\hline OH2O & $06 / 05 / 91$ & $13: 13$ & 0.09 & 2346.551 & 7.111 & 0.013 & 9.11 \\
\hline OH2O & $06 / 12 / 91$ & $09: 15$ & 0.08 & 2353.385 & 6.834 & 0.012 & 9.19 \\
\hline ОН2О & $06 / 19 / 91$ & $15: 45$ & 0.09 & 2360.656 & 7.271 & 0.012 & 9.28 \\
\hline OH2O & $06 / 26 / 91$ & $08: 20$ & 0.08 & 2367.347 & 6.691 & 0.012 & 9.36 \\
\hline $\mathrm{OH} 2 \mathrm{O}$ & $07 / 11 / 91$ & $11: 54$ & 0.16 & 2382.496 & 15.149 & 0.011 & 9.52 \\
\hline OH2O & $07 / 17 / 91$ & $10: 36$ & 0.06 & 2388.442 & 5.946 & 0.010 & 9.58 \\
\hline $\mathrm{OH} 2 \mathrm{O}$ & $07 / 30 / 91$ & $10: 50$ & 0.14 & 2401.451 & 13.009 & 0.011 & 9.72 \\
\hline ОН2О & $08 / 08 / 91$ & $09: 45$ & 0.10 & 2410.406 & 8.955 & 0.011 & 9.82 \\
\hline OH2O & $08 / 14 / 91$ & $11: 00$ & 0.07 & 2416.458 & 6.052 & 0.012 & 9.89 \\
\hline OH2O & $08 / 21 / 91$ & $11: 25$ & 0.15 & 2423.476 & 7.018 & 0.021 & 10.04 \\
\hline ОН2O & $08 / 28 / 91$ & $10: 55$ & 0.07 & 2430.455 & 6.979 & 0.010 & 10.11 \\
\hline $\mathrm{OH} 2 \mathrm{O}$ & $09 / 04 / 91$ & $11: 30$ & 0.08 & 2437.479 & 7.024 & 0.011 & 10.19 \\
\hline ОН 20 & $09 / 11 / 91$ & $12: 15$ & 0.09 & 2444.510 & 7.031 & 0.013 & 10.28 \\
\hline OH2O & $09 / 18 / 91$ & $09: 35$ & 0.08 & 2451.399 & 6.889 & 0.012 & 10.36 \\
\hline $\mathrm{OH} 2 \mathrm{O}$ & $09 / 25 / 91$ & $10: 37$ & 0.02 & 2458.442 & 7.043 & 0.003 & 10.38 \\
\hline $\mathrm{OH} 2 \mathrm{O}$ & $10 / 02 / 91$ & $11: 48$ & 0.10 & 2465.492 & 7.050 & 0.014 & 10.48 \\
\hline ОН2О & $10 / 16 / 91$ & $10: 50$ & 0.11 & 2479.451 & 13.959 & 0.008 & 10.59 \\
\hline ОH 20 & $10 / 23 / 91$ & $12: 41$ & 0.09 & 2486.528 & 7.077 & 0.013 & 10.68 \\
\hline $\mathrm{OH} 2 \mathrm{O}$ & $10 / 31 / 91$ & $11: 55$ & 0.08 & 2494.497 & 7.969 & 0.010 & 10.76 \\
\hline OH2O & $11 / 06 / 91$ & $11: 50$ & 0.11 & 2500.493 & 5.996 & 0.018 & 10.87 \\
\hline $\mathrm{OH} 2 \mathrm{O}$ & $11 / 13 / 91$ & $11: 14$ & 0.14 & 2507.468 & 6.975 & 0.020 & 11.01 \\
\hline OH2O & $11 / 20 / 91$ & $11: 55$ & 0.15 & 2514.497 & 7.029 & 0.021 & 11.16 \\
\hline ОH2O & $11 / 27 / 91$ & $10: 15$ & 0.04 & 2521.427 & 6.930 & 0.006 & 11.20 \\
\hline OH2O & $12 / 04 / 91$ & $12: 05$ & 0.09 & 2528.503 & 7.076 & 0.013 & 11.29 \\
\hline $\mathrm{OH} 2 \mathrm{O}$ & $12 / 11 / 91$ & $11: 15$ & 0.10 & 2535.469 & 6.966 & 0.014 & 11.39 \\
\hline $\mathrm{OH} 2 \mathrm{O}$ & $12 / 18 / 91$ & $10: 20$ & 0.04 & 2542.431 & 6.962 & 0.006 & 11.43 \\
\hline $\mathrm{OH} 2 \mathrm{O}$ & $01 / 08 / 92$ & $11: 07$ & 0.16 & 2563.463 & .21 .032 & 0.008 & 11.59 \\
\hline $\mathrm{OH} 2 \mathrm{O}$ & $01 / 15 / 92$ & $10: 15$ & 0.08 & 2570.427 & 6.964 & 0.011 & 11.67 \\
\hline $\mathrm{OH} 2 \mathrm{O}$ & $01 / 22 / 92$ & $09: 55$ & 0.14 & 2577.413 & 6.986 & 0.020 & 11.81 \\
\hline ОН2О & $01 / 29 / 92$ & & 0.11 & 2584.000 & 6.587 & 0.017 & 11.92 \\
\hline OH2O & $02 / 12 / 92$ & $10: 00$ & 0.15 & 2598.417 & 14.417 & 0.010 & 12.07 \\
\hline OH2O & $02 / 19 / 92$ & $10: 25$ & 0.14 & 2605.434 & 7.017 & 0.020 & 12.21 \\
\hline OH2O & $02 / 26 / 92$ & $10: 06$ & 0.06 & 2612.421 & 6.987 & 0.009 & 12.27 \\
\hline $\mathrm{OH} 2 \mathrm{O}$ & $03 / 11 / 92$ & $10: 15$ & 0.08 & 2626.427 & 14.006 & 0.006 & 12.35 \\
\hline OH2O & $03 / 18 / 92$ & $10: 15$ & 0.15 & 2633.427 & 7.000 & 0.021 & 12.50 \\
\hline $\mathrm{OH} 2 \mathrm{O}$ & $03 / 25 / 92$ & $12: 30$ & 0.08 & 2640.521 & 7.094 & 0.011 & 12.58 \\
\hline ОН2O & $04 / 01 / 92$ & $10: 10$ & 0.18 & 2647.424 & 6.903 & 0.026 & 12.76 \\
\hline OH2O & $04 / 07 / 92$ & $10: 25$ & 0.16 & 2653.434 & 6.010 & 0.027 & 12.92 \\
\hline
\end{tabular}


TABLE A-2 (Continued) BRINE ACCUMULATION DATA TABLE

Data through December 31, 1993

\begin{tabular}{|c|c|c|c|c|c|c|c|c|}
\hline & & & & DAYS & DAYS & & CUMULAT IVE & \\
\hline & & & LITERS & SINCE & USED FOR & LITERS & LITERS & \\
\hline LOCATIOH & DATE & TIME & REMOVED & $1 / 1 / 85$ & CALCULATION & PER DAY & COLLECTED & REMARKS \\
\hline
\end{tabular}

\begin{tabular}{|c|c|c|c|c|c|c|c|c|}
\hline $\mathrm{OH} 20$ & $04 / 15 / 92$ & $09: 45$ & 0.09 & 2661.406 & 7.972 & 0.011 & 13.01 & \\
\hline ОН20 & $04 / 22 / 92$ & $11: 03$ & 0.07 & 2668.460 & 7.054 & 0.010 & 13.08 & \\
\hline ОН2О & $05 / 06 / 92$ & $11: 40$ & 0.17 & 2682.486 & 14.026 & 0.012 & 13.25 & \\
\hline ОН 20 & $05 / 13 / 92$ & $14: 10$ & 0.15 & 2689.590 & 7.104 & 0.021 & 13.40 & \\
\hline он20 & $05 / 21 / 92$ & $11: 30$ & 0.06 & 2697.479 & 7.889 & 0.008 & 13.46 & \\
\hline $\mathrm{OH} 2 \mathrm{O}$ & $05 / 27 / 92$ & $10: 08$ & 0.03 & 2703.422 & 5.943 & 0.005 & 13.49 & \\
\hline $\mathrm{OH} 20$ & $06 / 09 / 92$ & $10: 00$ & 0.14 & 2716.417 & 12.995 & 0.011 & 13.63 & \\
\hline ОН20 & $06 / 18 / 92$ & $10: 20$ & 0.10 & 2725.431 & 9.014 & 0.011 & 13.73 & . \\
\hline ОН2O & $06 / 25 / 92$ & $10: 55$ & 0.05 & 2732.455 & 7.024 & 0.007 & 13.78 & \\
\hline $\mathrm{OH} 20$ & $07 / 01 / 92$ & $10: 10$ & 0.03 & 2738.424 & 5.969 & 0.005 & 13.81 & \\
\hline ОН 20 & $07 / 08 / 92$ & & 0.06 & 2745.000 & 6.576 & 0.009 & 13.87 & \\
\hline ОН 20 & $07 / 15 / 92$ & $10: 00$ & 0.05 & 2752.417 & 7.417 & 0.007 & 13.92 & \\
\hline OH2O & $07 / 22 / 92$ & $11: 30$ & 0.06 & 2759.479 & 7.062 & 0.008 & 13.98 & \\
\hline OH2O & $07 / 29 / 92$ & $10: 45$ & 0.05 & 2766.448 & 6.969 & 0.007 & 14.03 & \\
\hline OH2O & $08 / 04 / 92$ & $10: 20$ & 0.07 & 2772.431 & 5.983 & 0.012 & 14.10 & \\
\hline $\mathrm{OH} 2 \mathrm{O}$ & $08 / 18 / 92$ & $10: 35$ & 0.13 & 2786.441 & 14.010 & 0.009 & 14.23 & \\
\hline ОН20 & $09 / 02 / 92$ & $10: 37$ & 0.15 & 2801.442 & 15.001 & 0.010 & 14.38 & \\
\hline $\mathrm{OH} 2 \mathrm{O}$ & $09 / 09 / 92$ & $10: 30$ & 0.08 & 2808.438 & 6.996 & 0.011 & 14.46 & \\
\hline $\mathrm{OH} 2 \mathrm{O}$ & $09 / 17 / 92$ & $10: 25$ & 0.05 & 2816.434 & 7.996 & 0.006 & 14.51 & \\
\hline ОН2O & $09 / 23 / 92$ & $10: 20$ & 0.04 & 2822.431 & 5.997 & 0.007 & 14.55 & \\
\hline OH2O & $09 / 30 / 92$ & $11: 40$ & 0.04 & 2829.486 & 7.055 & 0.006 & 14.59 & \\
\hline OH2O & $10 / 12 / 92$ & $13: 20$ & 0.10 & 2841.556 & 12.070 & 0.008 & 14.69 & \\
\hline ОН20 & $10 / 21 / 92$ & $13: 25$ & 0.04 & 2850.559 & 9.003 & 0.004 & 14.73 & \\
\hline ОН 20 & $10 / 28 / 92$ & $09: 45$ & 0.04 & 2857.406 & 6.847 & 0.006 & 14.77 & \\
\hline ОН2O & $11 / 11 / 92$ & $13: 15$ & 0.03 & 2871.552 & 14.146 & 0.002 & 14.80 & \\
\hline OH2O & $11 / 18 / 92$ & $13: 30$ & 0.03 & 2878.563 & 7.011 & 0.004 & 14.83 & \\
\hline OH2O & $11 / 25 / 92$ & $10: 20$ & 0.00 & 2885.431 & 0.000 & 0.000 & 14.83 & Lost vacuum. \\
\hline $\mathrm{OH} 20$ & $12 / 09 / 92$ & $13: 40$ & 0.04 & 2899.569 & 21.006 & 0.002 & 14.87 & \\
\hline ОН 20 & $01 / 07 / 93$ & $09: 45$ & 0.03 & 2928.406 & 28.837 & 0.001 & 14.90 & \\
\hline ОН20 & $01 / 13 / 93$ & $10: 16$ & 0.02 & 2934.428 & 6.022 & 0.003 & 14.92 & \\
\hline ОН20 & $01 / 28 / 93$ & $10: 45$ & 0.03 & 2949.448 & 15.020 & 0.002 & 14.95 & \\
\hline OH2O & $02 / 11 / 93$ & $10: 20$ & 0.02 & 2963.431 & 13.983 & 0.001 & 14.97 & \\
\hline $\mathrm{OH} 20$ & $02 / 26 / 93$ & $11: 45$ & 0.01 & 2978.490 & 15.059 & 0.001 & 14.98 & \\
\hline $\mathrm{OH} 2 \mathrm{O}$ & $03 / 10 / 93$ & $10: 58$ & 0.00 & 2990.457 & 11.967 & 0.000 & 14.98 & \\
\hline $\mathrm{OH} 20$ & $03 / 25 / 93$ & $10: 55$ & 0.01 & 3005.455 & 14.998 & 0.001 & 14.99 & \\
\hline ОН 20 & $04 / 28 / 93$ & $10: 50$ & 0.00 & 3039.451 & 33.996 & 0.000 & 14.99 & \\
\hline $\mathrm{OH} 20$ & $06 / 16 / 93$ & $09: 15$ & 0.20 & 3088.385 & 48.934 & 0.004 & 15.99 & \\
\hline OH2O & $08 / 19 / 93$ & $10: 03$ & 0.19 & 3152.419 & 0.000 & 0.000 & 15.38 & Partial evacuation. \\
\hline ОH2O & $08 / 20 / 93$ & $09: 16$ & 0.04 & 3153.386 & 65.001 & 0.004 & 15.42 & Combine with 0.19 liter from 08-19-93. \\
\hline OH2O & $11 / 09 / 93$ & $09: 54$ & 0.35 & 3234.413 & 0.000 & 0.000 & 15.77 & Partial evacuation. \\
\hline ОН20 & $11 / 12 / 93$ & $11: 15$ & 0.03 & 3237.469 & 84.083 & 0.005 & 15.80 & Combine with 0.35 liter from 11-09-93. \\
\hline OH21 & $09 / 03 / 85$ & $14: 00$ & NA & 245.583 & 0.000 & 0.000 & 0.00 & $\begin{array}{l}\text { Approximate date this part of drift } \\
\text { excavated. }\end{array}$ \\
\hline OH21 & $12 / 12 / 88$ & $14: 00$ & HA & 1441.583 & 0.000 & 0.000 & 0.00 & $\begin{array}{l}\text { Horizontal hole drilled } 12 / 12 / 88 \text { to } \\
12 / 19 / 88 \text {. Hole drilled with brine. }\end{array}$ \\
\hline
\end{tabular}


TABLE A-2 (Continued) BRINE ACCUMULATION DATA TABLE

Data through December 31, 1993

\begin{tabular}{|c|c|c|c|c|c|c|c|}
\hline & & & & DAYS & DAYS & & CUMULATIVE \\
\hline & & & LITERS & SIHCE & USED FOR & LITERS & LITERS \\
\hline LOCATIOH & DATE & TIME & REMOVED & $1 / 1 / 85$ & CALCULATION & PER DAY & COLLECTED \\
\hline
\end{tabular}

REMARKS

\begin{tabular}{|c|c|c|c|c|c|c|c|c|}
\hline \multirow[b]{2}{*}{$\mathrm{OH} 21$} & \multirow[b]{2}{*}{$02 / 06 / 89$} & \multirow[b]{2}{*}{$10: 00$} & \multirow[b]{2}{*}{ NA } & \multirow[b]{2}{*}{1497.417} & \multicolumn{2}{|l|}{ - } & \multirow[b]{2}{*}{0.00} & \multirow{2}{*}{$\begin{array}{l}\text { Fluorescien added to drilling fluid. } \\
\text { New hole. Installed collection device a } \\
53 \text { ' in hole. Hole dry. }\end{array}$} \\
\hline & & & & & 0.000 & 0.000 & & \\
\hline OH21 & $02 / 14 / 89$ & $09: 25$ & 0 & 1505.392 & 1259.810 & 0.000 & 0.00 & $\begin{array}{l}\text { Hole plugged with foam. Hole holding vacuum } \\
\text { at approx. } 50 \text { centibars. }\end{array}$ \\
\hline OH21 & $02 / 21 / 89$ & $10: 30$ & 0 & 1512.438 & 7.046 & 0.000 & 0.00 & Holding vacuun. \\
\hline OH21 & $02 / 28 / 89$ & $10: 50$ & 0 & 1519.451 & 7.013 & 0.000 & 0.00 & Holding vacuum. \\
\hline OH21 & $03 / 01 / 89$ & $11: 45$ & NA & 1520.490 & 0.000 & 0.000 & 0.00 & $\begin{array}{l}\text { Device left with approximately } 70 \text { centibars } \\
\text { suction. }\end{array}$ \\
\hline OH21 & 03/0B/89 & $09: 45$ & 0 & 1527.406 & 7.955 & 0.000 & 0.00 & $\begin{array}{l}\text { Device left with approximately } 50 \text { centibars } \\
\text { suction. }\end{array}$ \\
\hline OH21 & $03 / 15 / 89$ & $11: 35$ & 0 & 1534.483 & 7.077 & 0.000 & 0.00 & Hole dry. \\
\hline OH21 & $03 / 30 / 89$ & $10: 20$ & 0 & 1549.431 & 14.948 & 0.000 & 0.00 & Hole dry. \\
\hline OH21 & $04 / 18 / 89$ & $09: 50$ & 0 & 1568.410 & 18.979 & 0.000 & 0.00 & $\begin{array}{l}\text { Device left with approximately } 50 \text { centibars } \\
\text { suction. }\end{array}$ \\
\hline $\mathrm{OH} 29$ & $04 / 26 / 89$ & $09: 55$ & 0 & 1576.413 & 8.003 & 0.000 & 0.00 & $\begin{array}{l}\text { Device left with approximately } 50 \text { centibars } \\
\text { suction. }\end{array}$ \\
\hline OH29 & $06 / 05 / 89$ & $09: 10$ & 0 & 1616.382 & 39.969 & 0.000 & 0.00 & $\begin{array}{l}\text { Hole dry, no vacuum in collection device. } \\
\text { Removed and replaced collection device. }\end{array}$ \\
\hline ОН21 & $06 / 20 / 89$ & $08: 40$ & 0 & 1631.361 & 14.979 & 0.000 & 0.00 & Hole dry. \\
\hline OH21 & $07 / 06 / 89$ & $11: 10$ & 0 & 1647.465 & 16.104 & 0.000 & 0.00 & $\begin{array}{l}\text { Hole dry. Collection device retained } \\
\text { vacuum. }\end{array}$ \\
\hline OH21 & $08 / 09 / 89$ & $10: 05$ & 0 & 1681.420 & 33.955 & 0.000 & 0.00 & $\begin{array}{l}\text { Hole dry. Pumped collection device, } \\
\text { repaired hose ends. }\end{array}$ \\
\hline ОН21 & $08 / 23 / 89$ & $11: 20$ & 0 & 1695.472 & 14.052 & 0.000 & 0.00 & Hole dry. \\
\hline OH21 & $10 / 02 / 89$ & $11: 25$ & 0 & 1735.476 & 40.004 & 0.000 & 0.00 & Hole dry. \\
\hline OH29 & $10 / 20 / 89$ & $11: 25$ & 0 & 1753.476 & 18.000 & 0.000 & 0.00 & Hole dry. \\
\hline OH21 & $11 / 10 / 89$ & $10: 20$ & 0 & 1774.431 & 20.955 & 0.000 & 0.00 & Hole dry. \\
\hline OH21 & $11 / 29 / 89$ & $12: 52$ & 0 & 1793.536 & 19.105 & 0.000 & 0.00 & Hole dry. \\
\hline ОН21 & $12 / 12 / 89$ & $10: 10$ & 0 & 1806.424 & 12.888 & 0.000 & 0.00 & $\begin{array}{l}\text { Hole dry. Reseat collection device } \\
\text { (leaking). }\end{array}$ \\
\hline OH21 & $03 / 28 / 91$ & $12: 45$ & 0.00 & 2277.531 & 479.107 & 0.000 & 0.00 & Air blowing through tube. \\
\hline OH21 & $04 / 24 / 91$ & $10: 07$ & 0.00 & 2304.422 & 26.891 & 0.000 & 0.00 & Air blowing through tube. \\
\hline OH21 & $07 / 17 / 91$ & $10: 36$ & 0.00 & 2388.442 & 84.020 & 0.000 & 0.00 & Air blowing through tube. \\
\hline OH21 & $09 / 25 / 91$ & $10: 35$ & 0.00 & 2458.441 & 69.999 & 0.000 & 0.00 & $\begin{array}{l}\text { Dry. Air blowing through tube. Sampler } \\
\text { under vacuum. }\end{array}$ \\
\hline OH21 & $10 / 31 / 91$ & $11: 48$ & 0.00 & 2494.492 & 36.051 & 0.000 & 0.00 & Dry. \\
\hline $\mathrm{OH} 21$ & $03 / 2$ & $11: 15$ & .08 & 3005.469 & 510.977 & 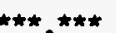 & 0.08 & Liter \\
\hline
\end{tabular}

OH21 03/25/93 11.15 $0.08 \quad 3005.469 \quad 510.977 \quad$ *t* ***

0.08 Liters/day value $>0.000$ and $<0.001$.

\begin{tabular}{|c|c|c|c|c|c|c|c|c|}
\hline OH22 & $09 / 03 / 85$ & $14: 00$ & HA & 245.583 & 0.000 & 0.000 & 0.00 & $\begin{array}{l}\text { Approximate date this part of drift } \\
\text { excavated. }\end{array}$ \\
\hline OH22 & $12 / 19 / 88$ & $14: 00$ & HA & 1448.583 & 1203.000 & 0.000 & 0.00 & $\begin{array}{l}\text { Horizontal hole drilled } 12 / 12 / 88 \text { to } \\
12 / 19 / 88 \text {. Hole drilled with brine. } \\
\text { Fluorescien added to drilling fluid. }\end{array}$ \\
\hline OH22 & $02 / 06 / 89$ & $11: 00$ & HA & 1497.458 & 0.000 & 0.000 & 0.00 & $\begin{array}{l}\text { New hole. Installed collection device a } \\
52.4^{\prime} \text { in hole. Hole dry. }\end{array}$ \\
\hline
\end{tabular}


TABLE A-2 (Continued)

BRINE ACCUMULATION DATA TABLE

Data through December 31, 1993

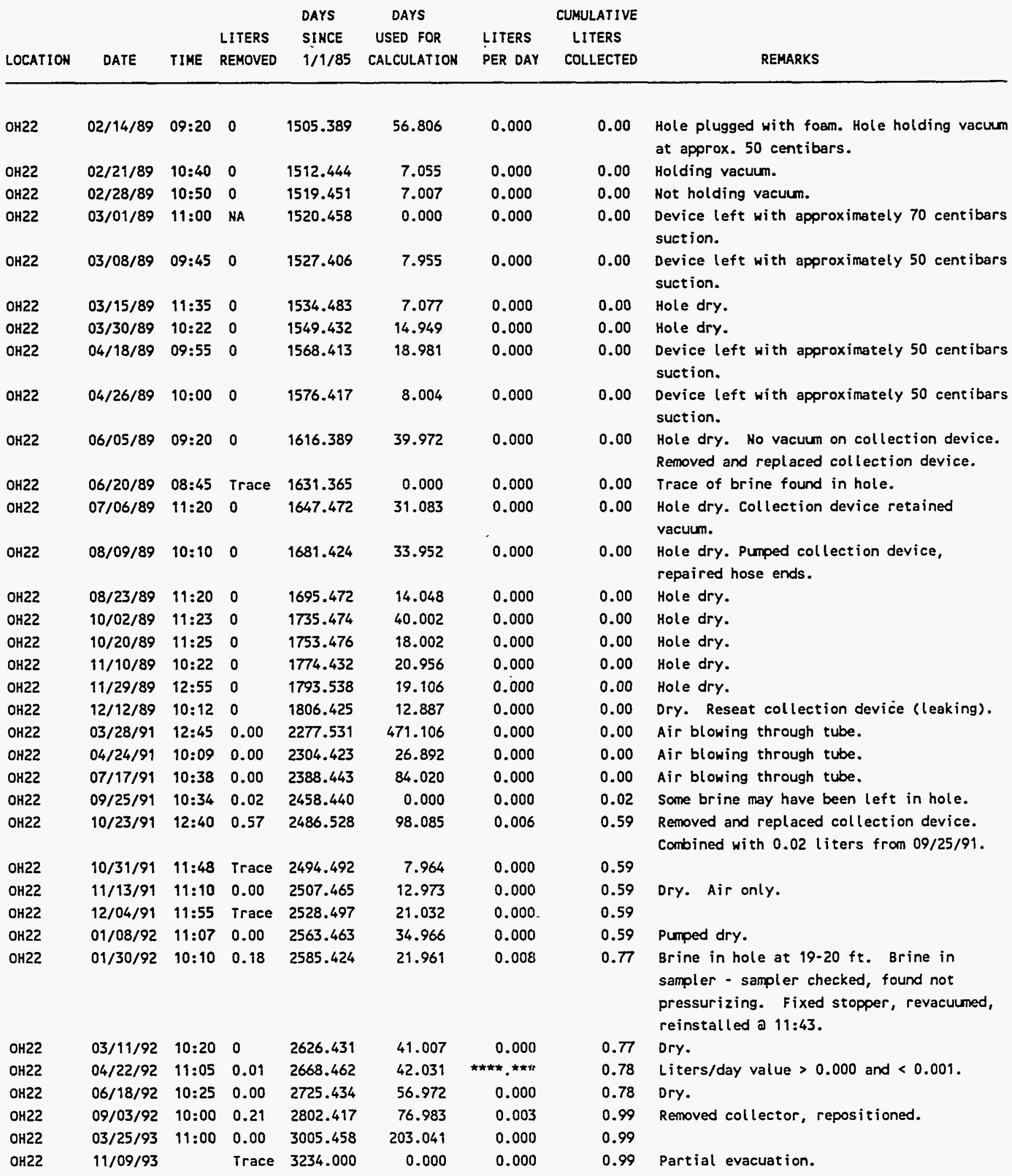


TABLE A-2 (Continued)

BRINE ACCUMULATION DATA TABLE

Data through December 31, 1993

\begin{tabular}{|c|c|c|c|c|c|c|c|c|c|}
\hline & & & & DAYS & DAYS & & CUMULATIVE & & \\
\hline & & & LITERS & SINCE & USED FOR & LITERS & LITERS & & \\
\hline LOCATION & DATE & TIHE & REMOVED & $1 / 1 / 85$ & CALCULATION & PER DAY & COLLECTED & REMARKS & \\
\hline
\end{tabular}

\begin{tabular}{|c|c|c|c|c|c|c|c|c|}
\hline OH23 & $12 / 08 / 85$ & $14: 00$ & NA & 341.583 & 0.000 & 0.000 & 0.00 & $\begin{array}{l}\text { Approximate date this part of drift } \\
\text { excavated. }\end{array}$ \\
\hline OH23 & $02 / 06 / 89$ & $14: 00$ & HA & 1497.583 & 1384.540 & 0.000 & 0.00 & $\begin{array}{l}\text { Horizontal hole drilled } 2 / 6 / 89 \text {. Hole } \\
\text { drilled with brine. Fluorescien added to } \\
\text { drilling fluid. }\end{array}$ \\
\hline OH23 & 02/07/89 & $14: 00$ & NA & 1498.583 & 1.000 & 0.000 & 0.00 & $\begin{array}{l}\text { Hew hole. Installed collection device a } \\
153^{\prime} \text { in hole. Hole dry. }\end{array}$ \\
\hline OH23 & $02 / 14 / 89$ & $09: 08$ & 0 & 1505.381 & 6.798 & 0.000 & 0.00 & $\begin{array}{l}\text { Hole plugged with foam. Hole holding vacuum } \\
\text { at approx. } 50 \text { centibars. }\end{array}$ \\
\hline OH23 & $02 / 21 / 89$ & $10: 00$ & 0.00 & 1512.417 & 7.036 & 0.000 & 0.00 & Holding vacuum. \\
\hline 애23 & 02/28/89 & $10: 00$ & 0.43 & 1519.417 & 7.000 & 0.061 & 0.43 & Sample clear, warm and effervescent. \\
\hline OH23 & 03/08/89 & $09: 30$ & 0.30 & 1527.396 & 7.979 & 0.038 & 0.73 & $\begin{array}{l}\text { Device left with approximately } 50 \text { centibars } \\
\text { suction. }\end{array}$ \\
\hline OH23 & 03/15/89 & $11: 45$ & 0.21 & 1534.490 & 7.094 & 0.030 & 0.94 & Sample saved for chemistry, sample $\# 671$. \\
\hline OH23 & 03/30/89 & $10: 15$ & 0.52 & 1549.427 & 14.937 & 0.035 & 1.46 & Sample saved for chemistry. \\
\hline OH23 & $04 / 04 / 89$ & $09: 30$ & 0.10 & 1554.396 & 4.969 & 0.020 & 1.56 & $\begin{array}{l}\text { Sample saved for chemistry. Device left } \\
\text { with approximately } 50 \text { centibars suction. } \\
\text { Outer } 75 \text { feet (approx.) of hole dry. }\end{array}$ \\
\hline OH23 & $04 / 18 / 89$ & $09: 55$ & 0.10 & 1568.413 & 14.017 & 0.007 & 1.66 & $\begin{array}{l}\text { No sample. Device left with approximately } \\
50 \text { centibars suction. }\end{array}$ \\
\hline OH23 & $04 / 26 / 89$ & $09: 35$ & 0.15 & 1576.399 & 7.986 & 0.019 & 1.81 & $\begin{array}{l}\text { Device left with approximately } 50 \text { centibars } \\
\text { suction. Combined sample saved for } \\
\text { chemistry. }\end{array}$ \\
\hline OH23 & 06/05/89 & 09:30 & 0.35 & 1616.396 & 39.997 & 0.009 & 2.16 & Sample saved for chemistry. \\
\hline OH23 & $06 / 20 / 89$ & $08: 50$ & 0.62 & 1631.368 & 14.972 & 0.041 & 2.78 & \\
\hline OH23 & $07 / 06 / 89$ & $11: 30$ & 0.37 & 1647.479 & 16.111 & 0.023 & 3.15 & $\begin{array}{l}\text { Collection device retained vacuum. Sample } \\
\text { saved for chemistry. }\end{array}$ \\
\hline OH23 & $08 / 09 / 89$ & $10: 15$ & 0.76 & 1681.427 & 33.948 & 0.022 & 3.91 & $\begin{array}{l}\text { Sample saved for chemistry. Pumped } \\
\text { collection device. }\end{array}$ \\
\hline OH23 & $08 / 23 / 89$ & $11: 13$ & 0.35 & 1695.467 & 14.040 & 0.025 & 4.26 & Sample saved for chemistry. \\
\hline OH23 & $09 / 14 / 89$ & $11: 14$ & 0.51 & 1717.468 & 22.001 & 0.023 & 4.77 & Sample saved for chemistry. \\
\hline OH23 & $10 / 02 / 89$ & $11: 30$ & 0.36 & 1735.479 & 18.011 & 0.020 & 5.13 & Sample saved for chemistry. \\
\hline OH23 & $10 / 20 / 89$ & $11: 35$ & 0.46 & 1753.483 & 18.004 & 0.026 & 5.59 & Sample saved for chemistry, sample $\# 856$. \\
\hline OH23 & $11 / 10 / 89$ & $10: 24$ & NA & 1774.433 & 0.000 & 0.000 & 5.59 & $\begin{array}{l}\text { Collection device exploded-in-hole due to } \\
\text { overpressuring during sampling. }\end{array}$ \\
\hline ОH23 & $11 / 15 / 89$ & 09:00 & HA & 1779.375 & 0.000 & 0.000 & 5.59 & Reinstalled collection device. \\
\hline OH23 & $11 / 29 / 89$ & $12: 51$ & 0.26 & 1793.535 & 40.052 & 0.006 & 5.85 & Sample saved for chemistry, sample $\# 875$. \\
\hline OH23 & $12 / 12 / 89$ & $09: 52$ & 0.13 & 1806.411 & 12.876 & 0.010 & 5.98 & $\begin{array}{l}\text { Sample saved for chemistry, sample } \# 887 \text {. } \\
\text { Reseat collection device (leaking). }\end{array}$ \\
\hline OH23 & $01 / 04 / 90$ & $11: 57$ & 0.11 & 1829.498 & 23.087 & 0.005 & 6.09 & \\
\hline OH23 & $01 / 17 / 90$ & $09: 20$ & 0.23 & 1842.389 & 12.891 & 0.018 & 6.32 & \\
\hline OH23 & $03 / 26 / 90$ & $09: 15$ & 0.60 & 1910.385 & 0.000 & 0.000 & 6.92 & Brine probably left in hole. \\
\hline OH23 & $04 / 04 / 90$ & $11: 53$ & 0.58 & 1919.495 & 0.000 & 0.000 & 7.50 & 8rine probably left in hole. \\
\hline OH23 & $04 / 10 / 90$ & $09: 39$ & 0.33 & 1925.402 & 83.013 & 0.018 & 7.83 & $\begin{array}{l}\text { Combined with } 0.60 \text { liters from } 03 / 26 / 90 \text { and } \\
0.58 \text { liters from } 04 / 04 / 90 \text {. Used } 1.51 \text { liters }\end{array}$ \\
\hline
\end{tabular}


TABLE A-2 (Continued) BRINE ACCUMULATION DATA TABLE

Data through December 31, 1993

\begin{tabular}{lllllll} 
& & \multicolumn{2}{c}{ DAYS } & DAYS & & CUMULATIVE \\
LOCATION & LITERS & SINCE & USED FOR & LITERS & LITERS \\
\hline
\end{tabular}

\begin{tabular}{|c|c|c|c|c|c|c|c|}
\hline Он23 & $04 / 24 / 90$ & $08: 46$ & 0.29 & 1939.365 & 13.963 & 0.021 & 8.12 \\
\hline $\mathrm{OH} 23$ & $05 / 02 / 90$ & $09: 52$ & 0.17 & 1947.411 & 8.046 & 0.021 & 8.29 \\
\hline OH23 & $05 / 09 / 90$ & $09: 32$ & 0.15 & 1954.397 & 6.986 & 0.021 & 8.44 \\
\hline $\mathrm{OH} 23$ & $05 / 16 / 90$ & $11: 45$ & 0.17 & 1961.490 & 7.093 & 0.024 & 8.61 \\
\hline ОH23 & $05 / 23 / 90$ & $13: 07$ & 0.13 & 1968.547 & 7.057 & 0.018 & 8.74 \\
\hline $\mathrm{OH} 23$ & $05 / 31 / 90$ & $09: 35$ & 0.16 & 1976.399 & 7.852 & 0.020 & 8.90 \\
\hline ОН23 & $06 / 06 / 90$ & $11: 40$ & 0.12 & 1982.486 & 6.087 & 0.020 & 9.02 \\
\hline ОН23 & $06 / 14 / 90$ & $10: 35$ & 0.17 & 1990.441 & 7.955 & 0.021 & 9.19 \\
\hline $\mathrm{OH} 23$ & $06 / 28 / 90$ & $10: 36$ & 0.38 & 2004.442 & 14.001 & 0.027 & 9.57 \\
\hline $\mathrm{OH} 23$ & $07 / 17 / 90$ & $09: 04$ & 0.33 & 2023.378 & 0.000 & 0.000 & 9.90 \\
\hline $\mathrm{OH} 23$ & $07 / 18 / 90$ & $11: 05$ & 0.10 & 2024.462 & 20.020 & 0.021 & 10.00 \\
\hline $\mathrm{OH} 23$ & $07 / 25 / 90$ & $10: 15$ & 0.10 & 2031.427 & 6.965 & 0.014 & 10.10 \\
\hline OH23 & $08 / 01 / 90$ & $11: 15$ & 0.14 & 2038.469 & 7.042 & 0.020 & 10.24 \\
\hline OH23 & $08 / 07 / 90$ & $09: 58$ & 0.14 & 2044.415 & 5.946 & 0.024 & 10.38 \\
\hline ОН 23 & $08 / 16 / 90$ & $09: 42$ & 0.15 & 2053.404 & 8.989 & 0.017 & 10.53 \\
\hline OH 23 & $08 / 22 / 90$ & $10: 51$ & 0.10 & 2059.452 & 6.048 & 0.017 & 10.63 \\
\hline OH23 & $08 / 29 / 90$ & $10: 30$ & 0.15 & 2066.438 & 6.986 & 0.021 & 10.78 \\
\hline OH23 & $09 / 05 / 90$ & $10: 40$ & 0.17 & 2073.444 & 7.006 & 0.024 & 10.95 \\
\hline $\mathrm{OH} 23$ & $09 / 12 / 90$ & $09: 00$ & 0.10 & 2080.375 & 6.931 & 0.014 & 11.05 \\
\hline OH23 & $09 / 25 / 90$ & $11: 42$ & 0.21 & 2093.488 & 0.000 & 0.000 & 11.26 \\
\hline $\mathrm{OH} 23$ & $09 / 26 / 90$ & $09: 53$ & 0.06 & 2094.412 & 14.037 & 0.019 & 11.32 \\
\hline $\mathrm{OH} 23$ & $10 / 03 / 90$ & $09: 05$ & 0.11 & 2101.378 & 6.966 & 0.016 & 11.43 \\
\hline $\mathrm{OH} 23$ & $10 / 10 / 90$ & $10: 22$ & 0.13 & 2108.432 & 7.054 & 0.018 & 11.56 \\
\hline $\mathrm{OH} 23$ & $10 / 18 / 90$ & $09: 30$ & 0.15 & 2116.396 & 7.964 & 0.019 & 11.71 \\
\hline $\mathrm{OH} 23$ & $10 / 24 / 90$ & $11: 30$ & 0.10 & 2122.479 & 6.083 & 0.016 & 11.81 \\
\hline $\mathrm{OH} 23$ & $10 / 31 / 90$ & $10: 53$ & 0.11 & 2129.453 & 6.974 & 0.016 & 11.92 \\
\hline OH23 & $11 / 07 / 90$ & $11: 40$ & 0.10 & 2136.486 & 7.033 & 0.014 & 12.02 \\
\hline $\mathrm{OH} 23$ & $11 / 14 / 90$ & $10: 45$ & 0.13 & 2143.448 & 6.962 & 0.015 & 12.15 \\
\hline OH23 & $11 / 28 / 90$ & $11: 32$ & 0.22 & 2157.481 & 14.033 & 0.016 & 12.37 \\
\hline ОН23 & $12 / 05 / 90$ & $09: 35$ & 0.10 & 2164.399 & 6.918 & 0.016 & 12.47 \\
\hline $\mathrm{OH} 23$ & $12 / 13 / 90$ & $10: 15$ & 0.14 & 2172.427 & 8.028 & 0.017 & 12.61 \\
\hline он23 & $12 / 20 / 90$ & $10: 30$ & 0.10 & 2179.438 & 7.011 & 0.014 & 12.71 \\
\hline $\mathrm{OH} 23$ & $01 / 09 / 91$ & $10: 48$ & 0.24 & 2199.450 & 0.000 & 0.000 & 12.95 \\
\hline $\mathrm{OH} 23$ & $01 / 16 / 91$ & $13: 15$ & 0.43 & 2206.552 & 27.114 & 0.025 & 13.38 \\
\hline ОН23 & $01 / 23 / 91$ & $10: 50$ & 0.08 & 2213.451 & 6.899 & 0.012 & 13.46 \\
\hline $\mathrm{OH} 23$ & $01 / 30 / 91$ & 09:01 & 0.12 & 2220.376 & 6.925 & 0.017 & 13.58 \\
\hline $\mathrm{OH} 23$ & $02 / 13 / 91$ & $12: 15$ & 0.20 & 2234.510 & 14.134 & 0.014 & 13.78 \\
\hline OH23 & $02 / 20 / 91$ & $11: 20$ & 0.12 & 2241.472 & 6.962 & 0.017 & 13.90 \\
\hline $\mathrm{OH} 23$ & $02 / 27 / 91$ & $11: 15$ & 0.11 & 2248.469 & 6.997 & 0.016 & 14.01 \\
\hline OH23 & $03 / 07 / 91$ & $10: 50$ & 0.11 & 2256.451 & 7.982 & 0.014 & 14.12 \\
\hline OH23 & $03 / 20 / 91$ & $12: 50$ & 0.21 & 2269.535 & 13.084 & 0.016 & 14.33 \\
\hline $\mathrm{OH} 23$ & $03 / 28 / 91$ & $12: 15$ & 0.12 & 2277.510 & 7.975 & 0.015 & 14.45 \\
\hline OH23 & $04 / 10 / 91$ & $09: 55$ & 0.20 & 2290.413 & 12.903 & 0.016 & 14.65 \\
\hline
\end{tabular}

for calculation.

Combined with 0.33 liters from $07 / 17 / 90$. Used 0.43 liters for calculation.

Combined with 0.21 liters from $09 / 25 / 90$. Used 0.27 liters for calculation.

Some brine may have been left in hole. Combined with 0.24 liters from 01/09/91. Collection device replaced on $01 / 10 / 91$. 
TABLE A-2 (Continued) BRINE ACCUMULATION DATA TABLE

Data through December 31, 1993

\begin{tabular}{|c|c|c|c|c|c|c|c|c|}
\hline & & & & DAYS & DAYS & & CUMULATIVE & \\
\hline & & & LITERS & SIKCE & USEO FOR & LITERS & LITERS & \\
\hline DCATION & DATE & TIME & REMOVED & $1 / 1 / 85$ & CALCULATION & PER DAY & COLLECTED & RKS \\
\hline
\end{tabular}

\begin{tabular}{|c|c|c|c|c|c|c|c|}
\hline ОН23 & $04 / 17 / 91$ & $10: 59$ & 0.11 & 2297.458 & 7.045 & 0.016 & 14.76 \\
\hline ОН23 & $04 / 24 / 91$ & $10: 10$ & 0.07 & 2304.424 & 6.966 & 0.010 & 14.83 \\
\hline OH23 & $05 / 01 / 91$ & $10: 05$ & 0.12 & 2311.420 & 6.996 & 0.017 & 14.95 \\
\hline ОН23 & $05 / 08 / 91$ & $09: 15$ & 0.06 & 2318.385 & 6.965 & 0.009 & 15.01 \\
\hline $\mathrm{OH} 23$ & $05 / 15 / 91$ & $11: 00$ & 0.13 & 2325.458 & 7.073 & $0: 018$ & 15.14 \\
\hline ОН23 & $05 / 29 / 91$ & $10: 28$ & 0.04 & 2339.436 & 13.978 & 0.003 & 15.18 \\
\hline он23 & $06 / 05 / 91$ & $13: 10$ & 0.04 & 2346.549 & 7.113 & 0.006 & 15.22 \\
\hline $\mathrm{OH} 23$ & $06 / 12 / 91$ & 09:00 & 0.02 & 2353.375 & 6.826 & 0.003 & 15.24 \\
\hline ОН23 & $06 / 19 / 91$ & $15: 35$ & 0.43 & 2360.649 & 7.274 & 0.059 & 15.67 \\
\hline ОН23 & $06 / 26 / 91$ & $08: 15$ & 0.10 & 2367.344 & 6.695 & 0.015 & 15.77 \\
\hline OH23 & $07 / 11 / 91$ & $12: 05$ & 0.23 & 2382.503 & 15.159 & 0.015 & 16.00 \\
\hline ОН23 & $07 / 17 / 91$ & $10: 40$ & 0.09 & 2388.444 & 5.941 & 0.015 & 16.09 \\
\hline $\mathrm{OH} 23$ & $07 / 30 / 91$ & $10: 45$ & 0.18 & 2401.448 & 13.004 & 0.014 & 16.27 \\
\hline ОН23 & $08 / 08 / 91$ & $09: 42$ & 0.14 & 2410.404 & 8.956 & 0.016 & 16.41 \\
\hline ОH23 & $08 / 14 / 91$ & $11: 30$ & 0.13 & 2416.479 & 6.075 & 0.021 & 16.54 \\
\hline OH23 & $08 / 21 / 91$ & $11: 20$ & 0.12 & 2423.472 & 6.993 & 0.017 & 16.66 \\
\hline ОН23 & $08 / 28 / 91$ & $10: 50$ & 0.07 & 2430.451 & 6.979 & 0.010 & 16.73 \\
\hline ОН23 & $09 / 04 / 91$ & $11: 35$ & 0.14 & 2437.483 & 7.032 & 0.020 & 16.87 \\
\hline он23 & $09 / 11 / 91$ & $12: 10$ & 0.04 & 2444.507 & 7.024 & .0 .006 & 16.91 \\
\hline OH23 & $09 / 18 / 91$ & $09: 30$ & 0.16 & 2451.396 & 6.889 & 0.023 & 17.07 \\
\hline ОН 23 & $09 / 25 / 91$ & $10: 30$ & 0.09 & 2458.438 & 7.042 & 0.013 & 17.16 \\
\hline $\mathrm{OH} 23$ & $10 / 02 / 91$ & $11: 44$ & 0.10 & 2465.489 & 7.051. & 0.014 & 17.26 \\
\hline $\mathrm{OH} 23$ & $10 / 16 / 91$ & $10: 57$ & 0.16 & 2479.456 & 13.967 & 0.011 & 17.42 \\
\hline ОН 23 & $10 / 23 / 91$ & $12: 35$ & 0.11 & 2486.524 & 7.068 & 0.016 & 17.53 \\
\hline OH23 & $10 / 31 / 91$ & $11: 40$ & 0.08 & 2494.486 & 7.962 & 0.010 & 17.61 \\
\hline он23 & $11 / 06 / 91$ & $12: 03$ & 0.09 & 2500.502 & 6.016 & 0.015 & 17.70 \\
\hline OH23 & $11 / 13 / 91$ & $11: 00$ & 0.05 & 2507.458 & 6.956 & 0.007 & 17.75 \\
\hline ОН23 & $11 / 20 / 91$ & $11: 50$ & 0.16 & 2514.493 & 7.035 & 0.023 & 17.91 \\
\hline $\mathrm{OH} 23$ & $11 / 27 / 91$ & $10: 30$ & 0.10 & 2521.438 & 6.945 & 0.014 & 18.01 \\
\hline OH23 & $12 / 04 / 91$ & $11: 50$ & 0.10 & 2528.493 & 7.055 & 0.014 & 18.11 \\
\hline $\mathrm{OH} 23$ & $12 / 11 / 91$ & $11: 30$ & 0.09 & 2535.479 & 6.986 & 0.013 & 18.20 \\
\hline OH23 & $12 / 18 / 91$ & $11: 00$ & 0.07 & 2542.458 & 6.979 & 0.010 & 18.27 \\
\hline OH23 & $01 / 08 / 92$ & $11: 03$ & 0.26 & 2563.460 & 21.002 & 0.012 & 18.53 \\
\hline OH 23 & $01 / 15 / 92$ & $10: 10$ & 0.08 & 2570.424 & 6.964 & 0.011 & 18.61 \\
\hline OH23 & $01 / 22 / 92$ & $09: 58$ & 0.11 & 2577.415 & 6.991 & 0.016 & 18.72 \\
\hline ОН 23 & $01 / 29 / 92$ & & 0.13 & 2584.000 & -6.585 & 0.020 & -18.85 \\
\hline $\mathrm{OH} 23$ & $02 / 12 / 92$ & $10: 15$ & 0.13 & $2598.427^{\circ}$ & 14.427 & 0.009 & 18.98 \\
\hline $\mathrm{OH} 23$ & $02 / 19 / 92$ & $10: 28$ & 0.14 & 2605.436 & 7.009 & 0.020 & 19.12 \\
\hline 애23 & $02 / 26 / 92$ & $10: 12$ & 0.05 & 2612.425 & 6.989 & 0.007 & 19.17 \\
\hline $\mathrm{OH} 23$ & $03 / 11 / 92$ & $10: 25$ & 0.21 & 2626.434 & 14.009 & 0.015 & 19.38 \\
\hline $\mathrm{OH} 23$ & $03 / 18 / 92$ & $10: 25$ & 0.10 & 2633.434 & 7.000 & 0.014 & 19.48 \\
\hline OH23 & $03 / 25 / 92$ & $12: 35$ & 0.08 & 2640.524 & 7.090 & 0.011 & 19.56 \\
\hline OH 23 & $04 / 01 / 92$ & $10: 20$ & 0.10 & 2647.431 & 6.907 & 0.014 & 19.66 \\
\hline OH23 & $04 / 07 / 92$ & $10: 35$ & 0.07 & 2653.441 & 6.010 & 0.012 & 19.73 \\
\hline $\mathrm{OH} 23$ & $04 / 15 / 92$ & $09: 55$ & 0.14 & 2661.413 & 7.972 & 0.018 & 19.87 \\
\hline OH23 & $04 / 22 / 92$ & & 0.10 & 2668.000 & 6.587 & 0.015 & 19.97 \\
\hline $\mathrm{OH} 23$ & $05 / 06 / 92$ & $11: 45$ & 0.20 & 2682.490 & 14.490 & 0.014 & 20.17 \\
\hline
\end{tabular}


TABLE A-2 (Continued) BRINE ACCUMULATION DATA TABLE

Data through December 31, 1993

\begin{tabular}{|c|c|c|c|c|c|c|c|c|}
\hline LOCATION & DATE & TIME & $\begin{array}{l}\text { LITERS } \\
\text { REHOVED }\end{array}$ & $\begin{array}{l}\text { DAYS } \\
\text { SINCE } \\
1 / 1 / 85\end{array}$ & $\begin{array}{c}\text { DAYS } \\
\text { USED FOR } \\
\text { CALCULATION }\end{array}$ & $\begin{array}{l}\text { LITERS } \\
\text { PER DAY }\end{array}$ & $\begin{array}{c}\text { CUMULATIVE } \\
\text { LITERS } \\
\text { COLLECTED }\end{array}$ & REMARKS \\
\hline $\mathrm{OH} 23$ & $05 / 13 / 92$ & $14: 25$ & 0.17 & 2689.601 & 7.111 & 0.024 & 20.34 & \\
\hline ОН 23 & $05 / 21 / 92$ & $11: 23$ & 0.10 & 2697.474 & 7.873 & 0.013 & 20.44 & \\
\hline $\mathrm{OH} 23$ & $05 / 27 / 92$ & $10: 02$ & 0.08 & 2703.418 & 5.944 & 0.013 & 20.52 & \\
\hline ОН 23 & $06 / 09 / 92$ & $10: 10$ & 0.16 & 2716.424 & 13.006 & 0.012 & 20.68 & \\
\hline $\mathrm{OH} 23$ & $06 / 18 / 92$ & $10: 30$ & 0.12 & 2725.438 & 9.014 & 0.013 & 20.80 & \\
\hline он23 & $06 / 25 / 92$ & $10: 50$ & 0.08 & 2732.451 & 7.013 & 0.011 & 20.88 & \\
\hline он 23 & $07 / 01 / 92$ & $10: 15$ & 0.08 & 2738.427 & 5.976 & 0.013 & 20.96 & \\
\hline OH23 & $07 / 08 / 92$ & & 0.09 & 2745.000 & 6.573 & 0.014 & 21.05 & \\
\hline ОН23 & $07 / 15 / 92$ & $10: 20$ & 0.09 & 2752.431 & 7.431 & 0.012 & 21.14 & \\
\hline ОН23 & $07 / 22 / 92$ & $11: 35$ & 0.09 & 2759.483 & 7.052 & 0.013 & 21.23 & \\
\hline $\mathrm{OH} 23$ & $07 / 29 / 92$ & $10: 42$ & 0.085 & 2766.446 & 6.963 & 0.012 & 21.32 & \\
\hline OH23 & $08 / 04 / 92$ & $10: 27$ & 0.09 & 2772.435 & 5.989 & 0.015 & 21.41 & \\
\hline OH23 & $08 / 18 / 92$ & $10: 40$ & 0.17 & 2786.444 & 14.009 & 0.012 & 21.58 & \\
\hline $\mathrm{OH} 23$ & $09 / 02 / 92$ & $10: 20$ & 0.16 & 2801.431 & 14.987 & 0.011 & 21.74 & \\
\hline $\mathrm{OH} 23$ & $09 / 09 / 92$ & $10: 35$ & 0.11 & 2808.441 & 7.010 & 0.016 & 21.85 & \\
\hline OH23 & $09 / 17 / 92$ & $10: 30$ & 0.09 & 2816.438 & 7.997 & 0.011 & 21.94 & \\
\hline OH 23 & $09 / 23 / 92$ & $10: 25$ & 0.08 & 2822.434 & 5.996 & 0.013 & 22.02 & \\
\hline Он23 & $09 / 30 / 92$ & $11: 45$ & 0.10 & 2829.490 & 7.056 & 0.014 & 22.12 & \\
\hline ОН23 & $10 / 12 / 92$ & $13: 25$ & 0.14 & 2841.559 & 12.069 & 0.012 & 22.26 & \\
\hline ОН 23 & $10 / 21 / 92$ & $13: 30$ & 0.10 & 2850.563 & 9.004 & 0.011 & 22.36 & \\
\hline $\mathrm{OH} 23$ & $10 / 28 / 92$ & $09: 55$ & 0.08 & 2857.413 & 6.850 & 0.012 & 22.44 & \\
\hline $\mathrm{OH} 23$ & $11 / 11 / 92$ & $13: 35$ & 0.15 & 2871.566 & 14.153 & 0.011 & 22.59 & \\
\hline ОН23 & $11 / 18 / 92$ & $13: 35$ & 0.09 & 2878.566 & 7.000 & 0.013 & 22.68 & \\
\hline ОН23 & $11 / 25 / 92$ & $10: 25$ & 0.10 & 2885.434 & 6.868 & 0.015 & 22.78 & \\
\hline OH23 & $12 / 09 / 92$ & $13: 50$ & 0.15 & 2899.576 & 14.142 & 0.011 & 22.93 & \\
\hline он 23 & $01 / 07 / 93$ & $09: 55$ & 0.34 & 2928.413 & 28.837 & 0.012 & 23.27 & \\
\hline $\mathrm{OH} 23$ & $01 / 13 / 93$ & $10: 20$ & 0.07 & 2934.431 & 6.018 & 0.012 & 23.34 & \\
\hline OH23 & $01 / 28 / 93$ & $11: 00$ & 0.18 & 2949.458 & 15.027 & 0.012 & 23.52 & \\
\hline OH23 & $02 / 11 / 93$ & $10: 40$ & 0.17 & 2963.444 & 13.986 & 0.012 & 23.69 & \\
\hline $\mathrm{OH} 23$ & $02 / 26 / 93$ & $19: 55$ & 0.14 & 2978.497 & 15.053 & 0.009 & 23.83 & \\
\hline ОН23 & $03 / 10 / 93$ & $11: 05$ & 0.14 & 2990.462 & 11.965 & 0.012 & 23.97 & \\
\hline OH23 & $03 / 25 / 93$ & $11: 20$ & 0.20 & 3005.472 & 15.010 & 0.013 & 24.17 & \\
\hline ОН 23 & $04 / 28 / 93$ & $10: 55$ & 0.41 & 3039.455 & 33.983 & 0.012 & 24.58 & \\
\hline ОН23 & $06 / 16 / 93$ & $09: 10$ & 0.55 & 3088.382 & 48.927 & 0.011 & 25.13 & \\
\hline ОН23 & $08 / 19 / 93$ & $09: 38$ & 0.65 & 3152.401 & 0.000 & 0.000 & 25.78 & Partial evacuation. \\
\hline OH23 & $08 / 20 / 93$ & $09: 12$ & 0.08 & 3153.383 & 65.001 & 0.011 & 25.86 & Combine with 0.65 liters from 08-19-93. \\
\hline он23 & $11 / 09 / 93$ & $09: 51$ & 0.62 & 3234.410 & 0.000 & 0.000 & 26.48 & Partial evacuation. \\
\hline $\mathrm{OH} 23$ & $11 / 12 / 93$ & $11: 20$ & 0.27 & $\stackrel{3237.472}{\longrightarrow}$ & 84.089 & 0.011 & 26.75 & Combine with 0.62 liters from 11-09-93. \\
\hline OH24 & $12 / 08 / 85$ & $14: 00$ & MA & 341.583 & 0.000 & 0.000 & 0.00 & $\begin{array}{l}\text { Approximate date this part of drift } \\
\text { excavated. }\end{array}$ \\
\hline $\mathrm{OH} 24$ & $03 / 06 / 89$ & $14: 00$ & NA & 1525.583 & 1184.000 & 0.000 & 0.00 & Horizontal hole drilled $3 / 2 / 89$ to $3 / 6 / 89$. \\
\hline $\mathrm{OH} 24$ & 03/08/89 & $09: 50$ & NA & 1527.410 & 0.000 & 0.000 & 0.00 & $\begin{array}{l}\text { Ney hole. Installed collection device. } \\
\text { Hole ory. }\end{array}$ \\
\hline $\mathrm{OH} 24$ & $03 / 15 / 89$ & $11: 45$ & 0 & 1534.490 & 8.907 & 0.000 & 0.00 & Hole dry. \\
\hline OH24 & $03 / 30 / 89$ & $10: 25$ & 0 & 1549.434 & 14.944 & 0.000 & 0.00 & Hole dry. \\
\hline
\end{tabular}


TABLE A-2 (Continued)

BRINE ACCUMULATION DATA TABLE

Data through December 31, 1993

\begin{tabular}{|c|c|c|c|c|c|c|c|c|}
\hline & & & & DAYS & DAYS & & CUMULATIVE & \\
\hline & & & LITERS & SIMCE & USED FOR & LITERS & LITERS & \\
\hline LOCATIOH & DATE & TIME & REMOVED & $1 / 1 / 85$ & CALCULATION & PER DAY & COLLECTED & REMARKS \\
\hline
\end{tabular}

\begin{tabular}{|c|c|c|c|c|c|c|c|c|}
\hline ОН24 & $04 / 18 / 89$ & $10: 00$ & 0 & 1568.417 & 18.983 & 0.000 & 0.00 & $\begin{array}{l}\text { Device left with approximately } 50 \text { centibars } \\
\text { suction. }\end{array}$ \\
\hline ОН24 & $04 / 26 / 89$ & $09: 40$ & 0 & 1576.403 & 7.986 & 0.000 & 0.00 & $\begin{array}{l}\text { Device left with approximately } 50 \text { centibars } \\
\text { suction. }\end{array}$ \\
\hline $\mathrm{OH} 24$ & $06 / 05 / 89$ & $09: 40$ & 0.05 & 1616.403 & 40.000 & 0.001 & 0.05 & $\begin{array}{l}\text { First time sample recovered. No vacuum in } \\
\text { collection device. Collection device } \\
\text { removed and replaced. }\end{array}$ \\
\hline ОН24 & $06 / 20 / 89$ & $09: 00$ & 0.03 & 1631.375 & 14.972 & 0.002 & 0.08 & \\
\hline ОН24 & $07 / 06 / 89$ & $11: 40$ & 0.01 & 1647.486 & 16.111 & 0.001 & 0.09 & $\begin{array}{l}\text { Collection device retained vacuum. Sample } \\
\text { saved for chemistry. }\end{array}$ \\
\hline ОН24 & $08 / 09 / 89$ & $10: 20$ & 0 & 1681.431 & 33.945 & 0.000 & 0.09 & Hole dry. Pumped collection device. \\
\hline OH24 & $08 / 23 / 89$ & $11: 18$ & 0 & 1695.471 & 14.040 & 0.000 & 0.09 & Hole dry. \\
\hline $\mathrm{OH} 24$ & $10 / 02 / 89$ & $11: 35$ & 0 & 1735.483 & 40.012 & 0.000 & 0.09 & Hole dry. \\
\hline OH24 & $10 / 20 / 89$ & $11: 35$ & 0 & 1753.483 & 18.000 & 0.000 & 0.09 & Hole dry. \\
\hline OH24 & $11 / 10 / 89$ & $10: 26$ & 0 & 1774.435 & 20.952 & 0.000 & 0.09 & Hole dry. \\
\hline ОН24 & $11 / 29 / 89$ & $12: 58$ & 0 & 1793.540 & 19.105 & 0.000 & 0.09 & Hole dry. \\
\hline ОH24 & $12 / 12 / 89$ & $09: 54$ & 0 & 1806.413 & 12.873 & 0.000 & 0.09 & $\begin{array}{l}\text { Hole dry. Reseat collection device } \\
\text { (leaking). }\end{array}$ \\
\hline $\mathrm{OH} 24$ & $04 / 10 / 90$ & $09: 46$ & 0.09 & 1925.407 & 118.994 & .0 .001 & 0.18 & \\
\hline $\mathrm{OH} 24$ & $04 / 24 / 90$ & $08: 46$ & 0.03 & 1939.365 & 13.958 & 0.002 & 0.21 & \\
\hline ОН24 & $05 / 02 / 90$ & $09: 55$ & NA & 1947.413 & 0.000 & 0.000 & 0.21 & Trace. \\
\hline OH24 & $08 / 10 / 90$ & $09: 40$ & NA & 2047.403 & 0.000 & 0.000 & 0.21 & $\begin{array}{l}\text { Cleaned, checked, and reinstalled vacuum up } \\
\text { to } 50 \text { centibars. Checked in one hour. } \\
\text { Sampler holding vacuum. }\end{array}$ \\
\hline $\mathrm{OH} 24$ & $04 / 24 / 91$ & $10: 12$ & 0.00 & 2304.425 & 0.000 & 0.000 & 0.21 & Air blowing through tube. \\
\hline OH24 & $07 / 17 / 91$ & $10: 45$ & 0.00 & 2388.448 & 0.000 & 0.000 & 0.21 & Air blowing through tube. \\
\hline OH24 & $09 / 25 / 91$ & $10: 18$ & 0.00 & 2458.429 & 0.000 & 0.000 & 0.21 & Air blowing through tube. \\
\hline OH24 & $10 / 23 / 91$ & $12: 30$ & 0.53 & 2486.521 & 547.156 & 0.001 & 0.74 & $\begin{array}{l}\text { Replaced broken collection device. Used } \\
547.156 \text { days. }\end{array}$ \\
\hline OH24 & $10 / 31 / 91$ & $11: 42$ & Trace & 2494.488 & 7.967 & 0.000 & 0.74 & Hole wet at 25 feet. \\
\hline OH24 & $11 / 13 / 91$ & $11: 05$ & 0.02 & 2507.462 & 12.974 & 0.002 & 0.76 & \\
\hline OH24 & $12 / 04 / 91$ & $11: 45$ & Trace & 2528.490 & 21.028 & 0.000 & 0.76 & \\
\hline OH24 & $01 / 08 / 92$ & $11: 03$ & 0.00 & 2563.460 & 34.970 & 0.000 & 0.76 & Pumped dry. \\
\hline ОН24 & $01 / 30 / 92$ & $10: 30$ & 0.03 & 2585.438 & 21.978 & 0.001 & 0.79 & $\begin{array}{l}\text { Hole shows brine on plastic hose a } 19.5 \mathrm{ft} \text {. } \\
\text { (into hole) Sampler had minimal brine. } \\
\text { Revacuumed and reinstalled a } 11: 18 \text {. }\end{array}$ \\
\hline OH24 & $03 / 11 / 92$ & $10: 30$ & 0 & 2626.438 & 41.000 & 0.000 & 0.79 & ory. \\
\hline OH24 & $04 / 22 / 92$ & & 0.00 & 2668.000 & 41.562 & 0.000 & 0.79 & \\
\hline OH24 & $06 / 18 / 92$ & $10: 35$ & 0.0 & 2725.441 & 57.441 & 0.000 & 0.79 & Dry. \\
\hline OH24 & $09 / 03 / 92$ & $10: 30$ & Trace & 2802.438 & 76.997 & 0.000 & 0.79 & Removed collector, repositioned. \\
\hline OH24 & $03 / 25 / 93$ & $11: 25$ & 0.26 & 3005.476 & 203.038 & 0.001 & 1.05 & \\
\hline ОH24 & $11 / 09 / 93$ & & Trace & 3234.000 & 0.000 & 0.000 & 1.05 & artial evacuation. \\
\hline
\end{tabular}

\begin{tabular}{|c|c|c|c|c|c|c|c|c|}
\hline OH25 & $12 / 08 / 85$ & $14: 00$ & HA & 341.583 & 228.524 & 0.000 & 0.00 & $\begin{array}{l}\text { Approximate date this part of drift } \\
\text { excavated. }\end{array}$ \\
\hline ОН25 & $03 / 27 / 89$ & $14: 00$ & NA & 1546.583 & 1205.000 & 0.000 & 0.00 & Horizontal hole drilled on $3 / 27 / 89$. \\
\hline
\end{tabular}


TABLE A-2 (Continued)

\section{BRINE ACCUMULATION DATA TABLE}

Data through December 31, 1993

\begin{tabular}{|c|c|c|c|c|c|c|c|c|}
\hline LOCATION & DATE & TIME & $\begin{array}{l}\text { LITERS } \\
\text { REMOVED }\end{array}$ & $\begin{array}{l}\text { DAYS } \\
\text { SIHCE } \\
1 / 1 / 85\end{array}$ & $\begin{array}{l}\text { DAYS } \\
\text { USED FOR } \\
\text { CALCULATION }\end{array}$ & $\begin{array}{l}\text { LITERS } \\
\text { PER DAY }\end{array}$ & $\begin{array}{l}\text { CUMULATIVE } \\
\text { LITERS } \\
\text { COLLECTED }\end{array}$ & REMARKS \\
\hline OH25 & $03 / 30 / 89$ & $10: 27$ & 0 & 1549.435 & 0.000 & 0.000 & 0.00 & Hole dry. \\
\hline $\mathrm{OH} 25$ & $04 / 18 / 89$ & $10: 05$ & 0 & 1568.420 & 21.837 & 0.000 & 0.00 & $\begin{array}{l}\text { Device left with approximately } 50 \text { centibars } \\
\text { suction. }\end{array}$ \\
\hline OH25 & $04 / 26 / 89$ & $09: 45$ & 0 & 1576.406 & 7.986 & 0.000 & 0.00 & $\begin{array}{l}\text { Device left with approximately } 50 \text { centibars } \\
\text { suction. }\end{array}$ \\
\hline он25 & $06 / 05 / 89$ & $09: 50$ & 0 & 1616.410 & 40.004 & 0.000 & 0.00 & $\begin{array}{l}\text { Hole dry. Ho vacum on collection device. } \\
\text { Collection device removed and replaced. }\end{array}$ \\
\hline $\mathrm{OH} 25$ & $06 / 20 / 89$ & $09: 10$ & 0 & 1631.382 & 14.972 & 0.000 & 0.00 & Hole dry. \\
\hline $\mathrm{OH} 25$ & $07 / 06 / 89$ & $11: 40$ & 0.01 & 1647.486 & 16.104 & 0.001 & 0.01 & $\begin{array}{l}\text { Collection device retained vacuum. Sample } \\
\text { saved for chemistry. }\end{array}$ \\
\hline OH25 & 08/09/89 & $10: 25$ & 0 & 1689.434 & 33.948 & 0.000 & 0.01 & Hole dry. \\
\hline OH 25 & $08 / 23 / 89$ & $11: 18$ & 0 & 1695.471 & 14.037 & 0.000 & 0.01 & Hole dry. \\
\hline OH25 & $10 / 02 / 89$ & $11: 35$ & 0 & 1735.483 & 40.012 & 0.000 & 0.01 & Hole dry. \\
\hline ОН25 & $10 / 20 / 89$ & $11: 35$ & 0 & 1753.483 & 18.000 & 0.000 & 0.01 & Hole dry. \\
\hline OH25 & $11 / 10 / 89$ & $10: 30$ & 0 & 1774.438 & 20.955 & 0.000 & 0.01 & Hole dry. \\
\hline ОН25 & $11 / 29 / 89$ & 13:02 & 0 & 1793.543 & 19.105 & 0.000 & 0.01 & Hole dry. \\
\hline OH25 & $12 / 12 / 89$ & $09: 58$ & 0 & 1806.415 & 12.872 & 0.000 & 0.01 & $\begin{array}{l}\text { Hole dry. Reseat collection device } \\
\text { (leaking). }\end{array}$ \\
\hline OH25 & $08 / 10 / 90$ & $09: 50$ & NA & 2047.410 & 0.000 & 0.000 & 0.01 & $\begin{array}{l}\text { Cleaned, checked, and reinstalled vacuum up } \\
\text { to } 50 \text { centibars. Checked in one hour. } \\
\text { Sampler holding vacuum. }\end{array}$ \\
\hline OH25 & $04 / 24 / 91$ & $10: 14$ & 0.00 & 2304.426 & 498.011 & 0.000 & 0.01 & Air blowing through tube. \\
\hline $\mathrm{OH} 25$ & $07 / 17 / 91$ & $10: 47$ & 0.00 & 2388.449 & 84.023 & 0.000 & 0.01 & Air blowing through tube. \\
\hline OH25 & $09 / 25 / 91$ & $10: 15$ & 0.06 & 2458.427 & 69.978 & 0.001 & 0.07 & \\
\hline $\mathrm{OH} 25$ & $10 / 31 / 91$ & $11: 42$ & 0.00 & 2494.488 & 36.061 & 0.000 & 0.07 & Dry. Hole wet at 30 feet. \\
\hline $\mathrm{OH} 25$ & $03 / 25 / 93$ & $11: 27$ & 0.07 & 3005.477 & 510.989 & 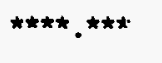 & 0.14 & Liter/day value is $>0.000$ and $<0.001$ \\
\hline OH26 & $08 / 05 / 86$ & $14: 00$ & NA & 581.583 & 0.000 & 0.000 & 0.00 & $\begin{array}{l}\text { Approximate date this part of drift } \\
\text { excavated. }\end{array}$ \\
\hline ОН 26 & $03 / 27 / 89$ & $14: 00$ & NA & 1546.583 & 965.000 . & 0.000 & 0.00 & $\begin{array}{l}\text { Horizontal hole drilled on } 3 / 27 / 89 \text {. Hole } \\
\text { drilled with brine. Fluorescien added to } \\
\text { drilling fluid. }\end{array}$ \\
\hline OH26 & $03 / 30 / 89$ & $10: 00$ & NA & 1549.417 & 0.000 & 0.000 & 0.00 & $\begin{array}{l}\text { New hole. Installed collection device. } \\
\text { Hole dry. }\end{array}$ \\
\hline OH26 & $04 / 18 / 89$ & $10: 10$ & 0 & 1568.424 & 21.841 & 0.000 & 0.00 & $\begin{array}{l}\text { Device left with approximately } 50 \text { centibars } \\
\text { suction. }\end{array}$ \\
\hline $\mathrm{OH} 26$ & $04 / 26 / 89$ & $09: 15$ & 0 & 1576.385 & 7.961 & 0.000 & 0.00 & $\begin{array}{l}\text { Device left with approximately } 50 \text { centibars } \\
\text { suction. }\end{array}$ \\
\hline $\mathrm{OH} 26$ & $06 / 05 / 89$ & $10: 00$ & 0.20 & 1616.417 & 40.032 & 0.005 & 0.20 & $\begin{array}{l}\text { First time sample recovered. Collection } \\
\text { device removed and replaced. Sample saved } \\
\text { for chemistry. }\end{array}$ \\
\hline OH26 & $06 / 20 / 89$ & $09: 15$ & 0.05 & 1631.385 & 14.968 & 0.003 & 0.25 & \\
\hline $\mathrm{OH} 26$ & $07 / 06 / 89$ & $11: 50$ & 0.49 & 1647.493 & 16.108 & 0.030 & 0.74 & $\begin{array}{l}\text { Collection device retained vacuum. Sample } \\
\text { saved for chemistry. }\end{array}$ \\
\hline OH26 & $08 / 09 / 89$ & $10: 30$ & 0.67 & 1681.438 & 33.945 & 0.020 & 1.41 & Sample saved for chemistry. \\
\hline $\mathrm{OH} 26$ & $08 / 23 / 89$ & $10: 30$ & 0.55 & 1695.438 & 14.000 & 0.039 & 1.96 & Sample saved for chemistry. \\
\hline
\end{tabular}


TABLE A-2 (Continued)

BRINE ACCUMULATION DATA TABLE

Data through December 31, 1993

\begin{tabular}{|c|c|c|c|c|c|c|c|}
\hline & & & & DAYS & DAYS & & CUMULATIVE \\
\hline & & & LITERS & SIHCE & USED FOR & LITERS & LITERS \\
\hline LOCATION & DATE & TIME & REMOVED & $1 / 1 / 85$ & CALCULATION & PER DAY & COLLECTED \\
\hline
\end{tabular}

\begin{tabular}{|c|c|c|c|c|c|c|c|c|}
\hline OH26 & $09 / 14 / 89$ & $11: 21$ & 0.51 & 1717.473 & 22.035 & 0.023 & 2.47 & Sample saved for chemistry. \\
\hline Он26 & $10 / 02 / 89$ & $11: 40$ & 0.56 & 1735.486 & 18.013 & 0.031 & 3.03 & Sample saved for chemistry. \\
\hline OH26 & $10 / 20 / 89$ & $11: 45$ & 0.45 & 1753.490 & 18.004 & 0.025 & 3.48 & Sample saved for chemistry, sample $\$ 857$. \\
\hline OH26 & $11 / 10 / 89$ & $11: 04$ & 0.48 & 1774.461 & 20.971 & 0.023 & 3.96 & Sample saved for chemistry, sample $\# 866$. \\
\hline OH26 & $11 / 29 / 89$ & $12: 40$ & 0.32 & 1793.528 & 19.067 & 0.017 & 4.28 & Sample saved for chemistry, sample $\# 874$. \\
\hline OH26 & $12 / 12 / 89$ & $09: 38$ & 0.32 & 1806.401 & 12.873 & 0.025 & 4.60 & Sample saved for chemistry, sample $\# 885$. \\
\hline OH26 & $01 / 04 / 90$ & $12: 05$ & 0.23 & 1829.503 & 23.102 & 0.010 & 4.83 & \\
\hline OH26 & $01 / 17 / 90$ & $08: 58$ & 0.36 & 1842.374 & 12.871 & 0.028 & 5.19 & \\
\hline OH26 & $01 / 31 / 90$ & $10: 54$ & 0.26 & 1856.454 & $14.080^{\circ}$ & 0.018 & 5.45 & \\
\hline OH26 & $02 / 13 / 90$ & $11: 30$ & 0.26 & 1869.479 & 13.025 & 0.020 & 5.71 & \\
\hline OH26 & $02 / 27 / 90$ & $12: 46$ & 0.21 & 1883.532 & 14.053 & 0.015 & 5.92 & Brine probably left in hole. \\
\hline OH26 & $03 / 05 / 90$ & $11: 27$ & 0.26 & 1889.477 . & 5.945 & 0.044 & 6.18 & \\
\hline OH26 & $03 / 21 / 90$ & $09: 26$ & 0.18 & 1905.393 & 15.916 & 0.011 & 6.36 & \\
\hline OH26 & $04 / 04 / 90$ & $11: 49$ & 0.28 & 1919.492 & 14.099 & 0.020 & 6.64 & Brine probably left in hole. \\
\hline OH26 & $04 / 10 / 90$ & $09: 17$ & 0.22 & 1925.387 & 5.895 & 0.037 & 6.86 & \\
\hline OH26 & $04 / 24 / 90$ & $08: 33$ & 0.19 & 1939.356 & 13.969 & 0.014 & 7.05 & \\
\hline OH26 & $05 / 02 / 90$ & $09: 45$ & 0.24 & 1947.406 & 8.050 & 0.030 & 7.29 & \\
\hline OH26 & $05 / 09 / 90$ & $09: 46$ & 0.21 & 1954.407 & 7.001 & 0.030 & 7.50 & \\
\hline OH26 & $05 / 16 / 90$ & $11: 30$ & 0.15 & 1961.479 & 7.072 & .0 .021 & 7.65 & \\
\hline $\mathrm{OH} 26$ & $05 / 23 / 90$ & $13: 03$ & 0.12 & 1968.544 & 7.065 & 0.017 & 7.77 & \\
\hline ОН26 & $05 / 31 / 90$ & $09: 29$ & 0.14 & 1976.395 & 7.851 & 0.018 & 7.91 & \\
\hline OH26 & $06 / 06 / 90$ & $11: 35$ & 0.14 & 1982.483 & 6.088 & 0.023 & 8.05 & \\
\hline $\mathrm{OH} 26$ & $06 / 14 / 90$ & $10: 42$ & 0.14 & 1990.446 & 7.963 & 0.018 & 8.19 & \\
\hline OH26 & $06 / 28 / 90$ & $10: 27$ & 0.16 & 2004.435 & 13.989 & 0.091 & 8.35 & \\
\hline ОН26 & $07 / 17 / 90$ & $08: 56$ & 0.18 & 2023.372 & 0.000 & 0.000 & 8.53 & \\
\hline OH26 & $07 / 18 / 90$ & $11: 00$ & 0.28 & 2024.458 & 20.023 & 0.023 & 8.81 & $\begin{array}{l}\text { Combined with } 0.18 \text { liters } 07 / 17 / 90 \text {. Used } \\
0.46 \text { liters for calculation. }\end{array}$ \\
\hline OH26 & $07 / 25 / 90$ & $10: 07$ & 0.05 & 2031.422 & 6.964 & 0.007 & 8.86 & Brine probably left in hole. \\
\hline OH26 & $08 / 01 / 90$ & $11: 05$ & 0.25 & 2038.462 & 7.040 & 0.036 & 9.11 & - \\
\hline OH26 & $08 / 07 / 90$ & $09: 40$ & 0.11 & 2044.403 & 5.941 & 0.019 & 9.22 & \\
\hline OH26 & $08 / 16 / 90$ & $09: 18$ & 0.12 & 2053.388 & 8.985 & 0.013 & 9.34 & \\
\hline OH26 & $08 / 22 / 90$ & $10: 44$ & 0.10 & 2059.447 & 6.059 & 0.017 & 9.44 & \\
\hline OH26 & $08 / 29 / 90$ & $10: 23$ & 0.11 & 2066.433 & 6.986 & 0.016 & 9.55 & \\
\hline $\mathrm{OH} 26$ & $09 / 05 / 90$ & $10: 34$ & 0.11 & 2073.440 & 7.007 & 0.016 & 9.66 & \\
\hline $\mathrm{OH} 26$ & $09 / 12 / 90$ & $08: 45$ & 0.10 & 2080.365 & 6.925 & 0.014 & 9.76 & \\
\hline OH26 & $09 / 25 / 90$ & $11: 26$ & 0.19 & 2093.476 & 0.000 & 0.000 & 9.95 & \\
\hline OH26 & $09 / 26 / 90$ & $09: 48$ & 0.10 & 2094.408 & 14.043 & 0.021 & 10.05 & $\begin{array}{l}\text { Combined with } 0.19 \text { liters from } 09 / 25 / 90 \text {. } \\
\text { Used } 0.29 \text { liters for calculation. }\end{array}$ \\
\hline OH26 & $10 / 03 / 90$ & $08: 55$ & 0.10 & 2101.372 & 6.964 & 0.014 & 10.15 & \\
\hline ОН26 & $10 / 10 / 90$ & $10: 14$ & 0.11 & 2108.426 & 7.054 & 0.016 & 10.26 & \\
\hline OH26 & $10 / 18 / 90$ & $09: 25$ & 0.13 & 2116.392 & 7.966 & 0.016 & 10.39 & \\
\hline $\mathrm{OH} 26$ & $10 / 24 / 90$ & $11: 16$ & 0.11 & 2122.469 & 6.077 & 0.018 & 10.50 & \\
\hline OH26 & $10 / 31 / 90$ & $10: 43$ & 0.12 & 2129.447 & 6.978 & 0.017 & 10.62 & \\
\hline ОН 26 & $11 / 07 / 90$ & $11: 43$ & 0.13 & 2136.488 & 7.041 & 0.018 & 10.75 & \\
\hline OH26 & $11 / 14 / 90$ & $10: 40$ & 0.10 & 2143.444 & 6.956 & 0.014 & 10.85 & \\
\hline ОН26 & $11 / 28 / 90$ & $11: 20$ & 0.21 & 2157.472 & 14.028 & 0.015 & 11.06 & \\
\hline он26 & $12 / 05 / 90$ & $09: 30$ & 0.14 & 2164.396 & 6.924 & 0.020 & 11.20 & \\
\hline
\end{tabular}


TABLE A-2 (Continued) BRINE ACCUMULATION DATA TABLE

Data through December 31, 1993

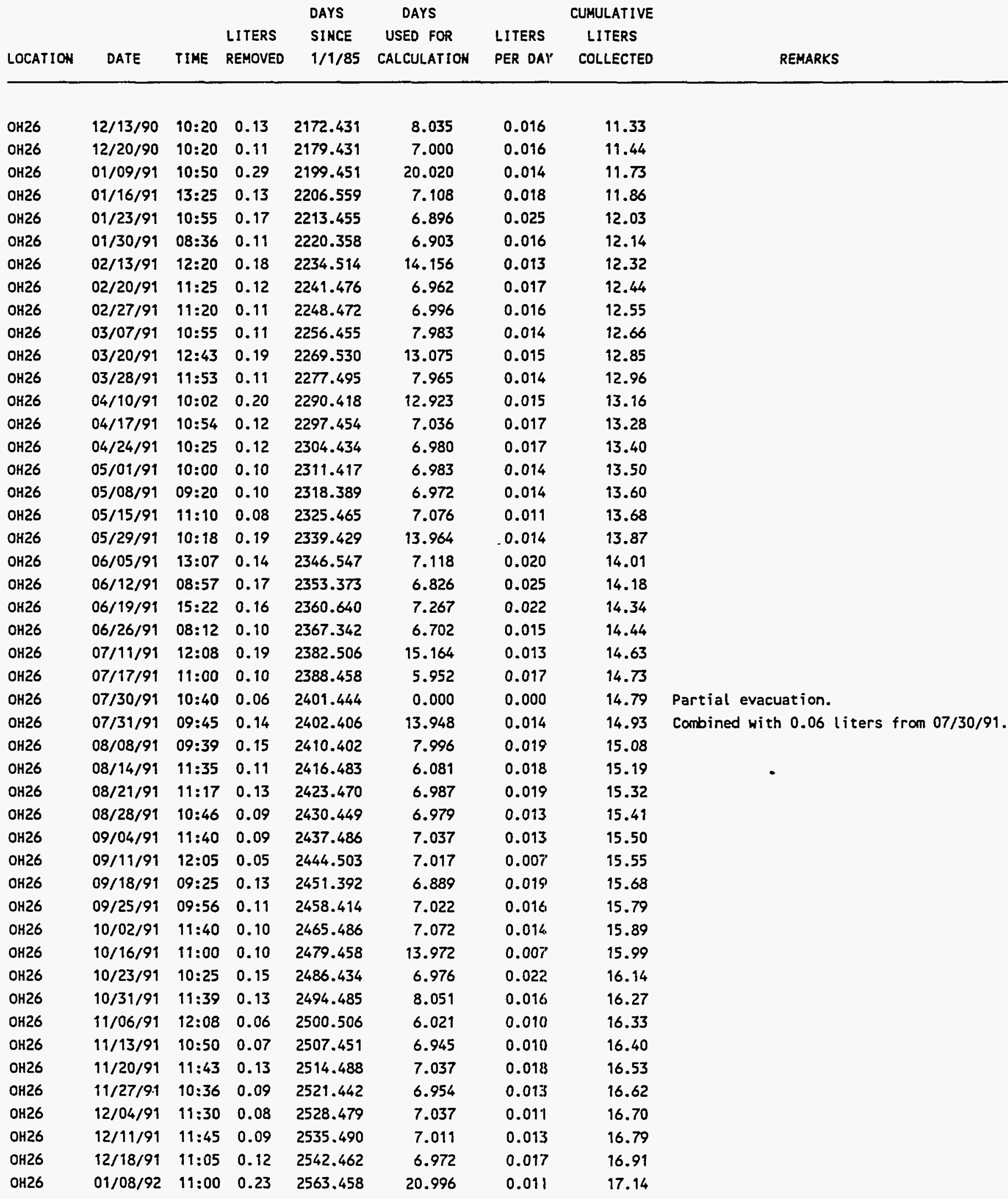


TABLE A-2 (Continued)

BRINE ACCUMULATION DATA TABLE

Data through December 31, 1993

\begin{tabular}{|c|c|c|c|c|c|c|c|c|}
\hline & & & ; & DAYS & DAYS & & CUMULATIVE & \\
\hline & & & LITERS & SIHCE & USED FOR & LITERS & LITERS & - \\
\hline LOCATIOH & DATE & TIME & REMOVED & $1 / 1 / 85$ & CALCULATIOH & PER DAY & COLLECTED & REMARKS \\
\hline
\end{tabular}

\begin{tabular}{|c|c|c|c|c|c|c|c|}
\hline OH26 & $01 / 15 / 92$ & $10: 08$ & 0.10 & 2570.422 & 6.964 & 0.014 & 17.24 \\
\hline OH26 & $01 / 22 / 92$ & $10: 10$ & 0.08 & 2577.424 & 7.002 & 0.011 & 17.32 \\
\hline OH26 & $01 / 29 / 92$ & & 0.14 & 2584.000 & 6.576 & 0.021 & 17.46 \\
\hline OH26 & $02 / 12 / 92$ & $10: 20$ & 0.15 & 2598.431 & 14.431 & 0.010 & 17.61 \\
\hline OH26 & $02 / 19 / 92$ & $10: 32$ & 0.11 & 2605.439 & 7.008 & 0.016 & 17.72 \\
\hline OH26 & $02 / 26 / 92$ & $10: 15$ & 0.06 & 2612.427 & 6.988 & 0.009 & 17.78 \\
\hline OH26 & $03 / 11 / 92$ & $10: 35$ & 0.16 & 2626.441 & 14.014 & 0.011 & 17.94 \\
\hline OH26 & $03 / 18 / 92$ & $10: 35$ & 0.11 & 2633.441 & 7.000 & 0.016 & 18.05 \\
\hline OH26 & $03 / 25 / 92$ & $12: 40$ & 0.10 & 2640.528 & 7.087 & 0.014 & 18.15 \\
\hline OH26 & $04 / 01 / 92$ & $10: 35$ & 0.08 & 2647.441 & 6.913 & 0.012 & 18.23 \\
\hline OH26 & $04 / 07 / 92$ & $10: 45$ & 0.05 & 2653.448 & 6.007 & 0.008 & 18.28 \\
\hline OH26 & $04 / 15 / 92$ & $10: 05$ & 0.13 & 2661.420 & 7.972 & 0.016 & 18.41 \\
\hline OH26 & $04 / 22 / 92$ & & 0.09 & 2668.000 & 6.580 & 0.014 & 18.50 \\
\hline $0 \mathrm{H} 26$ & $05 / 06 / 92$ & $11: 50$ & 0.18 & 2682.493 & 14.493 & 0.012 & 18.68 \\
\hline $\mathrm{OH} 26$ & $05 / 13 / 92$ & $14: 40$ & 0.16 & 2689.611 & 7.118 & 0.022 & 18.84 \\
\hline OH26 & $05 / 21 / 92$ & $11: 18$ & 0.08 & 2697.471 & 7.860 & 0.010 & 18.92 \\
\hline $\mathrm{OH} 26$ & $05 / 27 / 92$ & $10: 14$ & 0.06 & 2703.426 & 5.955 & 0.010 & 18.98 \\
\hline OH26 & $06 / 09 / 92$ & $10: 20$ & 0.15 & 2716.431 & 13.005 & 0.012 & 19.13 \\
\hline ОH26 & $06 / 18 / 92$ & $10: 40$ & 0.15 & 2725.444 & 9.013 & .0 .017 & 19.28 \\
\hline OH26 & $06 / 25 / 92$ & $10: 45$ & 0.07 & 2732.448 & 7.004 & 0.010 & 19.35 \\
\hline OH26 & $07 / 01 / 92$ & $10: 20$ & 0.08 & 2738.431 & 5.983 & 0.013 & 19.43 \\
\hline OH26 & $07 / 08 / 92$ & & 0.11 & 2745.000 & 6.569 & 0.017 & 19.54 \\
\hline $\mathrm{OH} 26$ & $07 / 15 / 92$ & $10: 40$ & 0.07 & 2752.444 & 7.444 & 0.009 & 19.61 \\
\hline OH26 & $07 / 22 / 92$ & $11: 40$ & 0.10 & 2759.486 & 7.042 & 0.014 & 19.71 \\
\hline ОН26 & $07 / 29 / 92$ & $10: 38$ & 0.09 & 2766.443 & 6.957 & 0.013 & 19.80 \\
\hline OH26 & $08 / 04 / 92$ & $10: 33$ & 0.07 & 2772.440 & 5.997 & 0.012 & 19.87 \\
\hline OH26 & $08 / 18 / 92$ & $10: 47$ & 0.15 & 2786.449 & 14.009 & 0.011 & 20.02 \\
\hline OH26 & $09 / 02 / 92$ & $10: 15$ & 0.18 & 2801.427 & 14.978 & 0.012 & 20.20 \\
\hline ОН26 & $09 / 09 / 92$ & $10: 40$ & 0.09 & 2808.444 & 7.017 & 0.013 & 20.29 \\
\hline OH26 & $09 / 17 / 92$ & $10: 35$ & 0.14 & 2816.441 & 7.997 & 0.018 & 20.43 \\
\hline OH26 & $09 / 23 / 92$ & $10: 30$ & 0.05 & 2822.438 & 5.997 & 0.008 & 20.48 \\
\hline OH26 & $09 / 30 / 92$ & $11: 50$ & 0.06 & 2829.493 & 7.055 & 0.009 & 20.54 \\
\hline OH26 & $10 / 12 / 92$ & $13: 30$ & 0.15 & 2841.563 & 12.070 & 0.012 & 20.69 \\
\hline OH26 & $10 / 21 / 92$ & $13: 45$ & 0.09 & 2850.573 & 9.010 & 0.010 & 20.78 \\
\hline $\mathrm{OH} 26$ & $10 / 28 / 92$ & $10: 05$ & 0.08 & 2857.420 & 6.847 & 0.012 & 20.86 \\
\hline OH26 & $11 / 11 / 92$ & $13: 35$ & 0.15 & 2871.566 & 14.146 & 0.011 & 21.01 \\
\hline ОН26 & $11 / 18 / 92$ & $13: 40$ & 0.10 & 2878.569 & 7.003 & 0.014 & 21.11 \\
\hline OH26 & $11 / 25 / 92$ & $10: 30$ & 0.07 & 2885.438 & 6.869 & 0.010 & 21.18 \\
\hline OH26 & $12 / 09 / 92$ & $14: 00$ & 0.16 & 2899.583 & 14.145 & 0.011 & 21.34 \\
\hline $\mathrm{OH} 26$ & $01 / 07 / 93$ & $10: 05$ & 0.32 & 2928.420 & 28.837 & 0.011 & 21.66 \\
\hline OH26 & $01 / 13 / 93$ & $10: 23$ & 0.06 & 2934.433 & 6.013 & 0.010 & 21.72 \\
\hline $\mathrm{OH} 26$ & $01 / 28 / 93$ & $11: 15$ & 0.18 & 2949.469 & 15.036 & 0.012 & 21.90 \\
\hline OH26 & $02 / 11 / 93$ & $10: 58$ & 0.11 & 2963.457 & 13.988 & 0.008 & 22.01 \\
\hline $\mathrm{OH} 26$ & $02 / 26 / 93$ & $12: 10$ & 0.23 & 2978.507 & 15.050 & 0.015 & 22.24 \\
\hline OH26 & $03 / 10 / 93$ & $11: 10$ & 0.13 & 2990.465 & 11.958 & 0.011 & 22.37 \\
\hline $0 \mathrm{H} 26$ & $03 / 25 / 93$ & 11:35 & 0.18 & 3005.483 & 15.018 & 0.012 & 22.55 \\
\hline $\mathrm{OH} 26$ & $04 / 28 / 93$ & $11: 20$ & 0.38 & 3039.472 & 33.989 & 0.011 & 22.93 \\
\hline
\end{tabular}


TABLE A-2 (Continued) BRINE ACCUMULATION DATA TABLE

Data through December 31, 1993

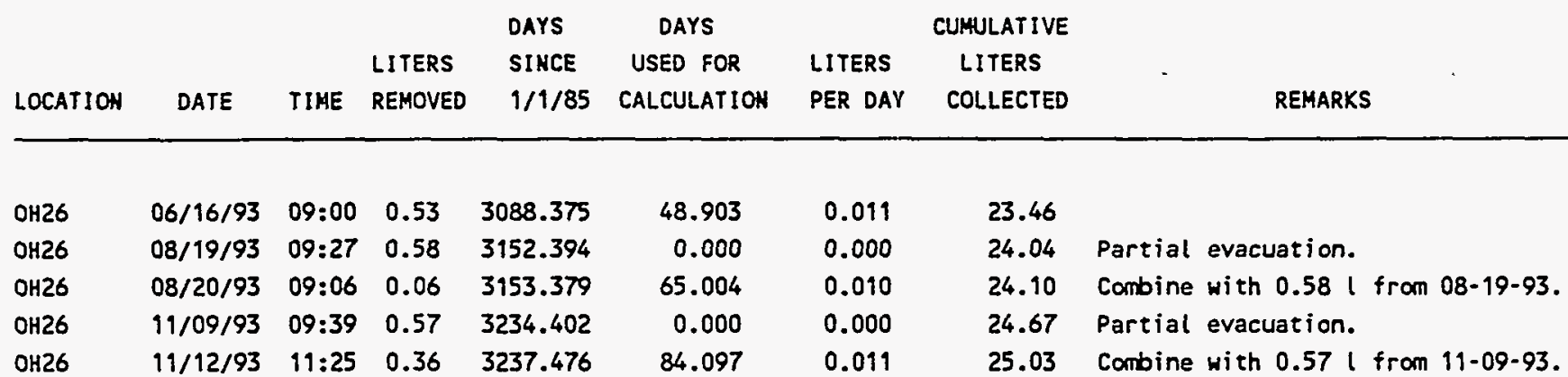

\begin{tabular}{|c|c|c|c|c|c|c|c|c|}
\hline ОН28 & $08 / 05 / 86$ & $14: 00$ & NA & 581.583 & 0.000 & 0.000 & 0.00 & $\begin{array}{l}\text { Approximate date this part of drift } \\
\text { excavated. }\end{array}$ \\
\hline OH28 & $04 / 12 / 89$ & $14: 00$ & NA & 1562.583 & 981.000 & 0.000 & 0.00 & Horizontal hole drilled $4 / 11 / 89$ to $4 / 12 / 89$. \\
\hline он28 & $04 / 18 / 89$ & $10: 25$ & 0 & 1568.434 & 0.000 & 0.000 & 0.00 & $\begin{array}{l}\text { Device left with approximetely } 50 \text { centibars } \\
\text { suction. }\end{array}$ \\
\hline OH28 & $04 / 26 / 89$ & $09: 30$ & 0 & 1576.396 & 13.813 & 0.000 & 0.00 & $\begin{array}{l}\text { Device left with approximately } 50 \text { centibars } \\
\text { suction. }\end{array}$ \\
\hline OH28 & $06 / 05 / 89$ & $10: 30$ & 0.08 & 1616.438 & 40.042 & 0.002 & 0.08 & $\begin{array}{l}\text { First time sample recovered. Collection } \\
\text { device removed and replaced. Sample saved } \\
\text { for chemistry. }\end{array}$ \\
\hline OH28 & $06 / 20 / 89$ & $09: 30$ & 0.03 & 1631.396 & 14.958 & 0.002 & 0.11 & \\
\hline OH28 & $07 / 06 / 89$ & $12: 00$ & 0 & 1647.500 & $16: 104$ & .0 .000 & 0.11 & $\begin{array}{l}\text { Hole dry. Collection device retained } \\
\text { vacuum. }\end{array}$ \\
\hline OH28 & $08 / 09 / 89$ & $10: 45$ & 0 & 1681.448 & 33.948 & 0.000 & 0.11 & Hole dry. \\
\hline OH28 & $08 / 23 / 89$ & $10: 46$ & 0 & 1695.449 & 14.001 & 0.000 & 0.11 & Hole dry. \\
\hline OH28 & $10 / 02 / 89$ & $11: 50$ & 0.05 & 1735.493 & 40.044 & 0.001 & 0.16 & Sample saved for chemistry. \\
\hline OH28 & $10 / 20 / 89$ & $11: 45$ & 0 & 1753.490 & 17.997 & 0.000 & 0.16 & Hole dry. \\
\hline OH28 & $11 / 10 / 89$ & $11: 10$ & 0.07 & 1774.465 & 20.975 & 0.003 & 0.23 & Sample saved for chemistry, sample $\# 867$. \\
\hline OH28 & $11 / 29 / 89$ & $12: 48$ & 0 & 1793.533 & 19.068 & 0.000 & 0.23 & Hole dry. \\
\hline $\mathrm{OH} 28$ & $12 / 12 / 89$ & $09: 48$ & 0.10 & 1806.408 & 12.873 & 0.008 & 0.33 & Sample saved for chemistry, sample $\# 886$. \\
\hline ОН28 & $04 / 10 / 90$ & $09: 36$ & 0.14 & 1925.400 & 118.992 & 0.001 & 0.47 & \\
\hline OH28 & $04 / 24 / 90$ & $08: 36$ & 0.18 & 1939.358 & 13.958 & 0.013 & 0.65 & \\
\hline OH28 & $05 / 02 / 90$ & $09: 35$ & 0.01 & 1947.399 & 8.041 & 0.001 & 0.66 & \\
\hline ОН28 & $05 / 09 / 90$ & $09: 40$ & Trace & 1954.403 & 0.000 & 0.000 & 0.66 & Trace. \\
\hline OH28 & $05 / 16 / 90$ & $11: 38$ & 0.02 & 1961.485 & 14.086 & 0.001 & 0.68 & \\
\hline $\mathrm{OH} 28$ & $05 / 31 / 90$ & $09: 33$ & 0.01 & 1976.398 & 14.913 & 0.001 & 0.69 & \\
\hline OH28 & $08 / 07 / 90$ & $09: 42$ & 0.10 & 2044.404 & 68.006 & 0.001 & 0.79 & \\
\hline OH28 & $08 / 10 / 90$ & $09: 10$ & NA & 2047.382 & 0.000 & 0.000 & 0.79 & $\begin{array}{l}\text { Cleaned, checked, and reinstalled vacuum up } \\
\text { to } 50 \text { centibars. Checked in one hour. } \\
\text { Sampler holding vacurm. }\end{array}$ \\
\hline OH28 & $09 / 12 / 90$ & $08: 40$ & 0.04 & 2080.361 & 35.957 & 0.001 & 0.83 & \\
\hline OH28 & $09 / 26 / 90$ & $09: 50$ & 0.05 & 2094.410 & 14.049 & 0.004 & 0.88 & \\
\hline OH28 & $11 / 28 / 90$ & $11: 28$ & 0.08 & 2157.478 & 63.068 & 0.001 & 0.96 & \\
\hline OH28 & $12 / 20 / 90$ & $10: 27$ & 0.07 & 2179.435 & 21.957 & 0.003 & 1.03 & \\
\hline ОН28 & $01 / 30 / 91$ & $08: 51$ & 0.07 & 2220.369 & 40.934 & 0.002 & 1.10 & \\
\hline OH28 & $03 / 20 / 91$ & $11: 55$ & 0.18 & 2269.497 & 49.128 & 0.004 & 1.28 & \\
\hline OH28 & $03 / 28 / 91$ & $11: 45$ & 0.01 & 2277.490 & 7.993 & 0.001 & 1.29 & \\
\hline ОН28 & $04 / 24 / 91$ & $10: 29$ & 0.00 & 2304.437 & 26.947 & 0.000 & 1.29 & Air blowing through tube. \\
\hline OH28 & $07 / 11 / 91$ & $12: 08$ & 0.00 & 2382.506 & 78.069 & 0.000 & 1.29 & Dry. \\
\hline OH28 & $07 / 17 / 91$ & $11: 07$ & 0,00 & 2388.463 & 5.957 & 0.000 & 1.29 & Air blowing through \\
\hline
\end{tabular}


TABLE A-2 (Continued)

BRINE-ACCUMULATION DATA TABLE

Data through December 31, 1993

\begin{tabular}{|c|c|c|c|c|c|c|c|}
\hline & & & & DAYS & DAYS & & CUHULATIVE \\
\hline & & & LITERS & SINCE & USED FOR & LITERS & LITERS \\
\hline LOCATIOH & DATE & TIME & REMOVED & $1 / 1 / 85$ & CALCULATION & PER DAY & COLLECTED \\
\hline
\end{tabular}

$\begin{array}{lrlllllll}\text { OH28 } & 09 / 25 / 91 & 09: 56 & 0.00 & 2458.414 & 69.951 & 0.000 & 1.29 \\ \text { OH28 } & 10 / 23 / 91 & 10: 27 & 0.27 & 2486.435 & 28.021 & 0.010 & 1.56 \\ & & & & & & & & \\ \text { OH28 } & 10 / 31 / 91 & 11: 36 & 0.00 & 2494.483 & 8.048 & 0.000 & 1.56 \\ \text { OH28 } & 11 / 13 / 91 & 10: 55 & 0.02 & 2507.455 & 12.972 & 0.002 & 1.58 \\ \text { OH28 } & 12 / 04 / 91 & 11: 35 & \text { Trace } & 2528.483 & 21.028 & 0.000 & 1.58 \\ \text { OH28 } & 01 / 08 / 92 & 11: 00 & 0.00 & 2563.458 & 34.975 & 0.000 & 1.58 \\ \text { OH28 } & 01 / 30 / 92 & 11: 45 & 0.09 & 2585.490 & 22.032 & 0.004 & 1.67\end{array}$

29 Air blowing through tube.

.56 First time successfull collection since 03/20/91. Used 216.94 days and 0.28 liters to calculate flow rate.

1.56

Dry. No Vacuum. Hole wet at 25 feet.

1.58

1.58 Pumped dry.

1.67 Brine on push rods a 25-28 $\mathrm{ft}$. Water pushed out of hole by sampler when removed (lots of brine) sampler checked, found in working condit

\begin{tabular}{lllll} 
OH28 & $03 / 11 / 92$ & $10: 40$ & 0 & 2626.444 \\
OH28 & $04 / 22 / 92$ & & 0.00 & 2668.000 \\
OH28 & $06 / 18 / 92$ & $10: 45$ & 0.18 & 2725.448 \\
OH28 & $09 / 03 / 92$ & $11: 00$ & 0.05 & 2802.458 \\
OH28 & $03 / 25 / 93$ & $11: 25$ & 0.04 & 3005.476 \\
OH28 & $11 / 09 / 93$ & $09: 45$ & Trace & 3234.406 \\
\hline
\end{tabular}

$\begin{array}{rrr}40.954 & 0.000 & 1.67 \\ 41.556 & 0.000 & 1.67 \\ 57.448 & 0.003 & 1.85 \\ 77.010 & 0.001 & 1.90 \\ 203.018 & \star \star \star \star . \star \star \star & 1.94 \\ 0.000 & 0.000 & 1.94\end{array}$

1.67

1.67

1.90

moved collector, repositioned.

Liter/day value is $>0.000$ and $<0.001$. Partial evacuation.

\begin{tabular}{|c|c|c|c|c|c|c|c|c|}
\hline $0 H 45$ & 05/08/89 & $14: 00$ & HA & 1588.583 & 0.000 & 0.000 & 0.00 & $\begin{array}{l}\text { Approximate date this part of underground } \\
\text { core storage room excavated. }\end{array}$ \\
\hline OH45 & $06 / 15 / 89$ & $14: 00$ & NA & 1626.583 & 0.000 & 0.000 & 0.00 & Horizontal hole drilled $6 / 9 / 89$ to $6 / 15 / 89$ \\
\hline OH45 & $06 / 23 / 89$ & $11: 00$ & HA & 1634.458 & 0.000 & 0.000 & 0.00 & New hole. Installed collection device. \\
\hline OH45 & $08 / 09 / 89$ & $14: 00$ & 0 & 1681.583 & 321.930 & 0.000 & 0.00 & $\begin{array}{l}\text { No vacuum. Reinstalled collection device. } \\
\text { Hole dry. }\end{array}$ \\
\hline OH45 & $08 / 23 / 89$ & $11: 30$ & 0.45 & 1695.479 & 13.896 & 0.032 & 0.45 & $\begin{array}{l}\text { First time hole sampled. Sample saved for } \\
\text { chemistry. }\end{array}$ \\
\hline OH45 & $09 / 12 / 89$ & $12: 35$ & 0.15 & 1715.524 & 20.045 & 0.007 & 0.60 & Sample saved for chemistry. \\
\hline OH45 & $10 / 02 / 89$ & $12: 15$ & 0.13 & 1735.510 & 19.986 & 0.007 & 0.73 & Sample saved for chemistry. \\
\hline OH45 & $10 / 20 / 89$ & $11: 10$ & 0.11 & 1733.465 & 17.955 & 0.006 & 0.84 & Sample saved for chemistry, sample $\# 852$. \\
\hline OH45 & $11 / 10 / 89$ & $10: 20$ & 0.13 & 1774.431 & 20.966 & 0.006 & 0.97 & Sample saved for chemistry, sample $\# 863$. \\
\hline OH45 & $11 / 29 / 89$ & $13: 11$ & 0.11 & 1793.549 & 19.118 & 0.006 & 1.08 & Sample saved for chemistry, sample $\# 878$. \\
\hline OH45 & $12 / 12 / 89$ & $10: 19$ & 0.08 & 1806.430 & 12.881 & 0.006 & 1.16 & $\begin{array}{l}\text { Sample saved for chemistry, sample } \# 889 \text {. } \\
\text { Sample bubbling. }\end{array}$ \\
\hline OH45 & $01 / 04 / 90$ & $11: 41$ & 0.14 & 1829.487 & 23.057 & 0.006 & 1.30 & \\
\hline $\mathrm{OH} 45$ & $01 / 17 / 90$ & $11: 54$ & 0.08 & 1842.496 & 13.009 & 0.006 & 1.38 & \\
\hline OH45 & $01 / 31 / 90$ & $11: 08$ & 0.01 & 1856.464 & 13.968 & 0.001 & 1.39 & \\
\hline OH45 & $02 / 13 / 90$ & $10: 54$ & 0.01 & 1869.454 & 12.990 & 0.001 & 1.40 & \\
\hline OH45 & $02 / 27 / 90$ & $12: 56$ & 0.11 & 1883.539 & 14.085 & 0.008 & 1.51 & Removed and replaced sampler. \\
\hline $\mathrm{OH} 45$ & $03 / 05 / 90$ & $11: 45$ & 0.08 & 1889.490 & 5.951 & 0.013 & 1.59 & \\
\hline OH45 & $03 / 21 / 90$ & $11: 34$ & Trace & 1905.482 & 0.000 & 0.000 & 1.59 & Trace. \\
\hline OH45 & $04 / 10 / 90$ & $10: 28$ & Trace & 1925.436 & 0.000 & 0.000 & 1.59 & Trace. \\
\hline OH45 & $05 / 02 / 90$ & $09: 12$ & 0.06 & 1947.383 & 57.893 & 0.001 & 1.65 & \\
\hline OH45 & $05 / 09 / 90$ & $10: 03$ & Trace & 1954.419 & 0.000 & 0.000 & 1.65 & Trace. \\
\hline OH45 & $05 / 17 / 90$ & $09: 20$ & 0.05 & 1962.389 & 0.000 & 0.000 & 1.70 & \\
\hline OH45 & $05 / 23 / 90$ & $13: 10$ & 0.01 & 1968.549 & 21.166 & 0.003 & 1.71 & \\
\hline
\end{tabular}


TABLE A-2 (Continued)

BRINE ACCUMULATION DATA TABLE

Data through December 31, 1993

\begin{tabular}{|c|c|c|c|c|c|c|c|c|}
\hline LOCATION & DATE & TIME & $\begin{array}{l}\text { LITERS } \\
\text { REMOVED }\end{array}$ & $\begin{array}{l}\text { DAYS } \\
\text { SINCE } \\
1 / 1 / 85\end{array}$ & $\begin{array}{l}\text { DAYS } \\
\text { USED FOR } \\
\text { CALCULATION }\end{array}$ & $\begin{array}{l}\text { LITERS } \\
\text { PER DAY }\end{array}$ & $\begin{array}{l}\text { CUMULATIVE } \\
\text { LITERS } \\
\text { COLLECTED }\end{array}$ & REMARKS \\
\hline OH45 & $06 / 14 / 90$ & $10: 15$ & 0.01 & 1990.427 & 0.000 & 0.000 & 1.72 & Brine probably left in hole. \\
\hline OH45 & $07 / 17 / 90$ & $11: 58$ & 0.46 & 2023.499 & 54.950 & 0.009 & 2.18 & \\
\hline $\mathrm{OH} 45$ & $08 / 07 / 90$ & $08: 50$ & Trace & 2044.368 & 0.000 & 0.000 & 2.18 & $\begin{array}{l}\text { Trace. Could not sample. Brine probably } \\
\text { left in hole. }\end{array}$ \\
\hline $\mathrm{OH} 45$ & $08 / 29 / 90$ & $12: 01$ & 0.27 & 2066.501 & 43.002 & 0.006 & 2.45 & \\
\hline OH45 & $09 / 13 / 90$ & $10: 40$ & 0.02 & 208.1 .444 & 14.943 & 0.001 & 2.47 & \\
\hline $\mathrm{OH} 45$ & $10 / 18 / 90$ & $10: 14$ & 0.05 & 2116.426 & 0.000 & 0.000 & 2.52 & \\
\hline $\mathrm{OH} 45$ & $02 / 13 / 91$ & $12: 40$ & Trace & 2234.528 & 0.000 & 0.000 & 2.52 & Did not save. \\
\hline $\mathrm{OH} 45$ & $03 / 21 / 91$ & $10: 30$ & 0.80 & 2270.438 & 188.994 & 0.004 & 3.32 & $\begin{array}{l}\text { Repaired and reinstalled sampler. Used } 189 \\
\text { days and } 0.85 \text { liters. }\end{array}$ \\
\hline OH45 & $03 / 28 / 91$ & $11: 21$ & 0.00 & 2277.473 & 7.035 & 0.000 & 3.32 & Air blowing through tube. \\
\hline OH45 & $04 / 10 / 91$ & $12: 15$ & 0.00 & 2290.510 & 13.037 & 0.000 & 3.32 & Dry. \\
\hline OH45 & $05 / 01 / 91$ & $10: 30$ & 0.11 & 2311.438 & 20.928 & 0.005 & 3.43 & \\
\hline OH45 & $05 / 08 / 91$ & $09: 35$ & 0.00 & 2318.399 & 6.961 & 0.000 & 3.43 & Air blowing through tube. \\
\hline $\mathrm{OH} 45$ & $07 / 18 / 91$ & $10: 20$ & 0.14 & 2389.431 & 71.032 & 0.002 & 3.57 & \\
\hline OH45 & $08 / 14 / 91$ & $11: 15$ & 0.05 & 2416.469 & 27.038 & 0.002 & 3.62 & \\
\hline $\mathrm{OH} 45$ & $09 / 25 / 91$ & $12: 10$ & 0.19 & 2458.507 & 42.038 & 0.005 & 3.81 & \\
\hline OH45 & $10 / 23 / 91$ & $10: 09$ & 0.11 & 2486.423 & 27.916 & 0.004 & 3.92 & \\
\hline $\mathrm{OH} 45$ & $10 / 31 / 91$ & $11: 36$ & 0.06 & 2494.483 & 8.060 & 0.007 & 3.98 & \\
\hline $\mathrm{OH} 45$ & $11 / 13 / 91$ & $10: 36$ & 0.05 & 2507.442 & 12.959 & 0.004 & 4.03 & \\
\hline OH45 & $12 / 04 / 91$ & $11: 40$ & 0.08 & 2528.486 & 21.044 & 0.004 & 4.11 & \\
\hline OH45 & $12 / 18 / 91$ & $11: 07$ & 0.02 & 2542.463 & 13.977 & 0.001 & 4.13 & \\
\hline OH45 & $01 / 08 / 92$ & $10: 35$ & 0.10 & 2563.441 & 20.978 & 0.005 & 4.23 & \\
\hline $\mathrm{OH} 45$ & $01 / 29 / 92$ & $10: 52$ & 0.09 & 2584.453 & 21.012 & 0.004 & 4.32 & \\
\hline OH45 & $02 / 19 / 92$ & $10: 15$ & 0.08 & 2605.427 & 20.974 & 0.004 & 4.40 & \\
\hline $\mathrm{OH} 45$ & $02 / 26 / 92$ & $10: 02$ & Trace & 2612.418 & 6.991 & 0.000 & 4.40 & \\
\hline OH45 & $03 / 11 / 92$ & $10: 00$ & 0.15 & 2626.417 & 13.999 & 0.011 & 4.55 & \\
\hline $\mathrm{OH} 45$ & $03 / 18 / 92$ & $10: 00$ & 0.05 & 2633.417 & 7.000 & 0.007 & 4.60 & \\
\hline OH45 & $03 / 25 / 92$ & $12: 45$ & 0.09 & 2640.531 & 7.114 & 0.001 & 4.61 & \\
\hline OH45 & $04 / 07 / 92$ & $11: 20$ & 0.06 & 2653.472 & 12.941 & 0.005 & 4.67 & \\
\hline $\mathrm{OH} 45$ & $04 / 95 / 92$ & $09: 35$ & Trace & 2661.399 & 7.927 & 0.000 & 4.67 & \\
\hline OH45 & $04 / 22 / 92$ & $10: 10$ & Trace & 2668.424 & 7.025 & 0.000 & 4.67 & \\
\hline $\mathrm{OH} 45$ & $05 / 07 / 92$ & $09: 00$ & Trace & 2683.375 & 14.951 & 0.000 & 4.67 & \\
\hline OH45 & $06 / 18 / 92$ & $10: 50$ & 0.14 & 2725.451 & 42.076 & 0.003 & 4.81 & \\
\hline $\mathrm{OH} 45$ & $07 / 01 / 92$ & $10: 00$ & 0.05 & 2738.417 & 12.966 & 0.004 & 4.86 & \\
\hline OH45 & $07 / 15 / 92$ & $10: 50$ & 0.05 & 2752.451 & 14.034 & 0.004 & 4.91 & \\
\hline $\mathrm{OH} 45$ & $08 / 18 / 92$ & $10: 28$ & 0.10 & 2786.436 & 33.985 & 0.003 & 5.01 & \\
\hline $\mathrm{OH} 45$ & $09 / 02 / 92$ & $10: 30$ & 0.15 & 2801.438 & 15.002 & 0.010 & 5.16 & \\
\hline $\mathrm{OH} 45$ & $09 / 24 / 92$ & $09: 30$ & 0.07 & 2823.396 & 21.958 & 0.003 & 5.23 & \\
\hline OH45 & $10 / 21 / 92$ & $14: 00$ & 0.15 & 2850.583 & 27.187 & 0.006 & 5.38 & \\
\hline OH45 & $01 / 07 / 93$ & $09: 35$ & 0.25 & 2928.399 & 77.816 & 0.003 & 5.63 & \\
\hline $\mathrm{OH} 45$ & $01 / 28 / 93$ & $11: 20$ & 0.13 & 2949.472 & 21.073 & 0.006 & 5.76 & \\
\hline $\mathrm{OH} 45$ & $03 / 25 / 93$ & $10: 45$ & 0.05 & 3005.448 & 55.976 & 0.001 & 5.81 & \\
\hline OH45 & $04 / 28 / 93$ & $11: 30$ & 0.19 & 3039.479 & 34.031 & 0.006 & 6.00 & \\
\hline OH45 & $06 / 16 / 93$ & $08: 43$ & 0.15 & 3088.363 & 48.884 & 0.003 & 6.15 & \\
\hline OH45 & $08 / 19 / 93$ & & 0.20 & 3152.000 & 63.637 & 0.003 & 6.35 & \\
\hline $\mathrm{OH} 45$ & $11 / 12 / 93$ & $11: 09$ & 0.24 & 3237.465 & 85.465 & 0.003 & 6.59 & \\
\hline
\end{tabular}


TABLE A-2 (Continued) BRINE ACCUMULATION DATA TABLE

Data through December 31, 1993

\begin{tabular}{|c|c|c|c|c|c|c|c|c|}
\hline \multirow{3}{*}{ LOCATIOH } & \multirow[b]{3}{*}{ DATE } & \multirow[b]{3}{*}{ TIME } & \multirow{3}{*}{$\begin{array}{l}\text { LITERS } \\
\text { REMOVED }\end{array}$} & \multirow{3}{*}{$\begin{array}{l}\text { DAYS } \\
\text { SIHCE } \\
1 / 1 / 85\end{array}$} & \multirow{3}{*}{$\begin{array}{l}\text { DAYS } \\
\text { USED FOR } \\
\text { CALCULATION }\end{array}$} & \multicolumn{2}{|r|}{ CUMULATIVE } & \\
\hline & & & & & & LITERS & LITERS & \\
\hline & & & & & & PER DAY & COLLECTED & REMARKS \\
\hline
\end{tabular}

\begin{tabular}{|c|c|c|c|c|c|c|c|c|}
\hline $\mathrm{OH} 46$ & 05/08/89 & $14: 00$ & NA & 1588.583 & 0.000 & 0.000 & 0.00 & $\begin{array}{l}\text { Approximate date this part of underground } \\
\text { core storage room excavated. }\end{array}$ \\
\hline OH46 & $06 / 20 / 89$ & $14: 00$ & MA & 1631.583 & 0.000 & 0.000 & 0.00 & Downhole drilled $6 / 16 / 89$ to $6 / 20 / 89$ \\
\hline OH46 & $07 / 06 / 89$ & $11: 30$ & NA & 1647.479 & 0.000 & 0.000 & 0.00 & $\begin{array}{l}\text { First day of observation for hole, blown } \\
\text { dry. }\end{array}$ \\
\hline $\mathrm{OH} 46$ & $07 / 25 / 89$ & $10: 48$ & 0.28 & 1666.450 & 77.867 & 0.004 & 0.28 & $\begin{array}{l}\text { First time hole sampled. Sample yellow- } \\
\text { with wood chips and other debris. } \\
\text { Hydrocarbon odor (diesel lubricant?). }\end{array}$ \\
\hline $\mathrm{OH} 46$ & $08 / 16 / 89$ & 10:05 & 0.68 & 1688.420 & 21.970 & 0.031 & 0.96 & Sample saved for chenistry. \\
\hline $\mathrm{OH} 46$ & $09 / 12 / 89$ & $12: 35$ & 0.47 & 1715.524 & 27.104 & 0.017 & 1.43 & Sample saved for chemistry. \\
\hline OH46 & $10 / 02 / 89$ & $12: 30$ & 0.05 & 1735.521 & 0.000 & 0.000 & 1.48 & $\begin{array}{l}\text { Sample saved for chemistry. Some brine } \\
\text { probably left in hole. }\end{array}$ \\
\hline OH46 & $10 / 20 / 89$ & $11: 10$ & 0.57 & 1753.465 & 37.941 & 0.016 & 2.05 & $\begin{array}{l}\text { Sample saved for chemistry, sample } \# 853 \text {. } \\
\text { Combined with } 0.05 \text { liters from } 10-02-89 \text {. } \\
\text { Used } 0.62 \text { liters for calculation. }\end{array}$ \\
\hline OH46 & $11 / 10 / 89$ & $10: 30$ & 0.68 & 1774.438 & 20.973 & 0.032 & 2.73 & Sample saved for chemistry, sample $\# 865$. \\
\hline $\mathrm{OH} 46$ & $11 / 29 / 89$ & $13: 15$ & 0.53 & 1793.552 & 19.114 & 0.028 & 3.26 & Sample saved for chemistry, sample $\# 879$. \\
\hline OH46 & $12 / 12 / 89$ & $10: 20$ & 0.46 & 1806.431 & 12.879 & 0.036 & 3.72 & Sample saved for chemistry, sample $\# 890$. \\
\hline OH46 & $01 / 04 / 90$ & $11: 44$ & 0.45 & 1829.489 & 23.058 & 0.020 & 4.17 & \\
\hline OH46 & $01 / 17 / 90$ & $11: 58$ & 0.25 & 1842.499 & 13.010 & 0.019 & 4.42 & \\
\hline $\mathrm{OH} 46$ & $01 / 31 / 90$ & $11: 12$ & 0.25 & 1856.467 & 13.968 & 0.018 & 4.67 & \\
\hline $\mathrm{OH} 46$ & $02 / 13 / 90$ & $11: 16$ & 0.22 & 1869.469 & 13.002 & 0.017 & 4.89 & \\
\hline $\mathrm{OH} 46$ & $02 / 27 / 90$ & $13: 10$ & 0.27 & 1883.549 & 14.080 & 0.019 & 5.16 & Brine probably left in hole. \\
\hline OH46 & $03 / 05 / 90$ & $11: 54$ & 0.27 & 1889.496 & 5.947 & 0.045 & 5.43 & - \\
\hline $\mathrm{OH} 46$ & $03 / 21 / 90$ & $11: 34$ & 0.13 & 1905.482 & 15.986 & 0.008 & 5.56 & Brine probably left in hole. \\
\hline $\mathrm{OH} 46$ & $04 / 11 / 90$ & $10: 33$ & 0.32 & 1926.440 & 20.958 & 0.015 & 5.88 & \\
\hline OH46 & $05 / 02 / 90$ & $09: 10$ & 0.25 & 1947.382 & 20.942 & 0.012 & 6.13 & Brine probably left in hole. \\
\hline $\mathrm{OH} 46$ & $05 / 08 / 90$ & $10: 05$ & 0.15 & 1953.420 & 6.038 & 0.025 & 6.28 & \\
\hline $\mathrm{OH} 46$ & $05 / 17 / 90$ & $09: 30$ & 0.14 & 1962.396 & 8.976 & 0.016 & 6.42 & \\
\hline OH46 & $05 / 23 / 90$ & $13: 30$ & 0.10 & 1968.563 & 6.167 & 0.016 & 6.52 & \\
\hline $\mathrm{OH} 46$ & $06 / 14 / 90$ & $10: 01$ & 0.32 & 1990.417 & 21.854 & 0.015 & 6.84 & \\
\hline $\mathrm{OH} 46$ & $06 / 28 / 90$ & $11: 06$ & 0.20 & 2004.463 & 14.046 & 0.014 & 7.04 & \\
\hline OH46 & $07 / 17 / 90$ & $11: 50$ & 0.30 & 2023.493 & 19.030 & 0.016 & 7.34 & \\
\hline $\mathrm{OH} 46$ & $07 / 25 / 90$ & $10: 50$ & 0.15 & 2031.451 & $\cdot 7.958$ & 0.019 & 7.49 & . \\
\hline $\mathrm{OH} 46$ & $08 / 07 / 90$ & $08: 50$ & 0.19 & 2044.368 & 12.917 & 0.015 & 7.68 & - \\
\hline OH46 & $08 / 16 / 90$ & $10: 30$ & 0.17 & 2053.438 & 9.070 & 0.019 & 7.85 & \\
\hline $\mathrm{OH} 46$ & $08 / 22 / 90$ & $11: 05$ & 0.11 & 2059.462 & 6.024 & 0.018 & 7.96 & \\
\hline OH46 & $08 / 29 / 90$ & $11: 45$ & 0.11 & 2066.490 & 7.028 & 0.016 & 8.07 & \\
\hline OH46 & $09 / 05 / 90$ & $11: 04$ & 0.12 & 2073.461 & 6.971 & 0.017 & 8.19 & \\
\hline $\mathrm{OH} 46$ & $09 / 13 / 90$ & $10: 42$ & 0.12 & 2081.446 & 7.985 & 0.015 & 8.31 & \\
\hline OH46 & $09 / 28 / 90$ & $10: 10$ & 0.22 & 2096.424 & 14.978 & 0.015 & 8.53 & \\
\hline OH46 & $10 / 18 / 90$ & $09: 52$ & 0.26 & 2116.411 & 19.987 & 0.013 & 8.79 & \\
\hline OH46 & $02 / 13 / 91$ & $12: 50$ & 0.74 & 2234.535 & 118.124 & 0.006 & 9.53 & \\
\hline OH46 & $02 / 27 / 91$ & $11: 05$ & 0.55 & 2248.462 & 13.927 & 0.039 & 10.08 & \\
\hline OH46 & $03 / 20 / 91$ & $13: 37$ & 0.58 & 2269.567 & 21.105 & 0.027 & 10.66 & \\
\hline
\end{tabular}




\section{TABLE A-2 (Continued) BRINE ACCUMULATION DATA TABLE \\ Data through December 31, 1993}

\begin{tabular}{|c|c|c|c|c|c|c|c|}
\hline & & & & DAYS & DAYS & & CUMULATIVE \\
\hline & & & LITERS & SIHCE & USED FOR & LITERS & LITERS \\
\hline CATION & DATE & TIME & REMOVED & $1 / 1 / 85$ & CALCULATIOH & PER DAY & COLLECTED \\
\hline
\end{tabular}

\begin{tabular}{|c|c|c|c|c|c|c|c|}
\hline $\mathrm{OH} 46$ & $03 / 28 / 91$ & $11: 21$ & 0.19 & 2277.473 & 7.906 & 0.024 & 10.85 \\
\hline OH46 & $04 / 10 / 91$ & $12: 15$ & 0.15 & 2290.510 & 13.037 & 0.012 & 11.00 \\
\hline OH 46 & $04 / 17 / 91$ & $11: 21$ & 0.28 & 2297.473 & 6.963 & 0.040 & 11.28 \\
\hline $\mathrm{OH} 46$ & $05 / 01 / 91$ & $10: 30$ & 0.18 & 2311.438 & 13.965 & 0.013 & 19.46 \\
\hline $\mathrm{OH} 46$ & $05 / 08 / 91$ & $08: 59$ & 0.09 & 2318.374 & 6.936 & 0.013 & 19.55 \\
\hline OH46 & $05 / 15 / 91$ & $10: 48$ & 0.09 & 2325.450 & 7.076 & 0.013 & 11.64 \\
\hline $\mathrm{OH} 46$ & $06 / 12 / 91$ & $09: 45$ & 0.29 & 2353.406 & 27.956 & 0.010 & 11.93 \\
\hline $\mathrm{OH} 46$ & $06 / 19 / 91$ & $15: 57$ & 0.10 & 2360.665 & 7.259 & 0.014 & 12.03 \\
\hline $\mathrm{OH} 46$ & $06 / 26 / 91$ & $10: 00$ & 0.10 & 2367.417 & 6.752 & 0.015 & 12.13 \\
\hline OH46 & $07 / 11 / 91$ & $10: 20$ & 0.20 & 2382.431 & 15.014 & 0.013 & 12.33 \\
\hline $\mathrm{OH} 46$ & $07 / 17 / 91$ & $11: 04$ & 0.08 & 2388.461 & 6.030 & 0.013 & 12.41 \\
\hline $\mathrm{OH} 46$ & $07 / 30 / 91$ & $11: 00$ & 0.16 & 2401.458 & 12.997 & 0.012 & 12.57 \\
\hline OH46 & $08 / 08 / 91$ & $09: 50$ & 0.13 & 2410.410 & 8.952 & 0.015 & 12.70 \\
\hline $\mathrm{OH} 46$ & $08 / 14 / 91$ & $10: 45$ & 0.08 & 2416.448 & 6.038 & 0.013 & 12.78 \\
\hline $\mathrm{OH} 46$ & $08 / 28 / 91$ & $11: 11$ & 0.17 & 2430.466 & 14.018 & 0.012 & 12.95 \\
\hline OH 46 & $09 / 18 / 91$ & $09: 40$ & 0.26 & 2451.403 & 20.937 & 0.012 & 13.21 \\
\hline $\mathrm{OH} 46$ & $09 / 25 / 91$ & $12: 35$ & 0.15 & 2458.524 & 7.121 & 0.021 & 13.36 \\
\hline $\mathrm{OH} 46$ & $10 / 16 / 91$ & $10: 45$ & 0.22 & 2479.448 & 20.924 & 0.011 & 13.58 \\
\hline $\mathrm{OH} 46$ & $10 / 23 / 91$ & $10: 10$ & 0.12 & 2486.424 & 6.976 & 0.017 & 13.70 \\
\hline $\mathrm{OH} 46$ & $10 / 31 / 91$ & $11: 30$ & 0.10 & 2494.479 & 8.055 & 0.012 & 13.80 \\
\hline $\mathrm{OH} 46$ & $11 / 06 / 91$ & $12: 10$ & 0.09 & 2500.507 & 6.028 & 0.015 & 13.89 \\
\hline $\mathrm{OH} 46$ & $11 / 13 / 91$ & $10: 36$ & 0.08 & 2507.442 & 6.935 & 0.012 & 13.97 \\
\hline OH46 & $11 / 27 / 91$ & $10: 11$ & 0.14 & 2521.424 & 13.982 & 0.010 & 14.11 \\
\hline $\mathrm{OH} 46$ & $12 / 04 / 91$ & $11: 45$ & 0.11 & 2528.490 & 7.066 & 0.016 & 14.22 \\
\hline $\mathrm{OH} 46$ & $12 / 18 / 91$ & $10: 25$ & 0.15 & 2542.434 & 13.944 & 0.011 & 14.37 \\
\hline $\mathrm{OH} 46$ & $01 / 08 / 92$ & $10: 35$ & 0.23 & 2563.441 & 21.007 & 0.011 & 14.60 \\
\hline OH46 & $01 / 29 / 92$ & $10: 53$ & 0.22 & 2584.453 & 21.012 & 0.010 & 14.82 \\
\hline $\mathrm{OH} 46$ & $02 / 12 / 92$ & $09: 35$ & 0.18 & 2598.399 & 13.946 & 0.013 & 15.00 \\
\hline $\mathrm{OH} 46$ & $02 / 19 / 92$ & $10: 15$ & 0.05 & 2605.427 & 7.028 & 0.007 & 15.05 \\
\hline $\mathrm{OH} 46$ & $02 / 26 / 92$ & $10: 04$ & 0.08 & 2612.419 & 6.992 & 0.019 & 15.13 \\
\hline $\mathrm{OH} 46$ & $03 / 11 / 92$ & $10: 00$ & 0.05 & 2626.417 & 13.998 & 0.004 & 15.18 \\
\hline $\mathrm{OH} 46$ & $03 / 18 / 92$ & $10: 05$ & 0.08 & 2633.420 & 7.003 & 0.011 & 15.26 \\
\hline OH46 & $03 / 25 / 92$ & $12: 45$ & 0.16 & 2640.531 & 7.111 & 0.023 & 15.42 \\
\hline $\mathrm{OH} 46$ & $04 / 01 / 92$ & $10: 50$ & 0.08 & 2647.451 & 6.920 & 0.012 & 15.50 \\
\hline $\mathrm{OH} 46$ & $04 / 07 / 92$ & $11: 25$ & 0.08 & 2653.476 & 6.025 & 0.013 & 15.58 \\
\hline $\mathrm{OH} 46$ & $04 / 15 / 92$ & $09: 35$ & 0.10 & 2661.399 & 7.923 & 0.013 & 15.68 \\
\hline $\mathrm{OH} 46$ & $04 / 22 / 92$ & $10: 20$ & 0.08 & 2668.431 & 7.032 & 0.011 & 15.76 \\
\hline $\mathrm{OH} 46$ & $05 / 07 / 92$ & $09: 00$ & 0.15 & 2683.375 & 14.944 & 0.010 & 15.91 \\
\hline $\mathrm{OH} 46$ & $05 / 27 / 92$ & $10: 00$ & 0.22 & 2703.417 & 20.042 & 0.011 & 16.13 \\
\hline $\mathrm{OH} 46$ & $06 / 18 / 92$ & $10: 55$ & 0.24 & 2725.455 & 22.038 & 0.011 & 16.37 \\
\hline $\mathrm{OH} 46$ & $06 / 25 / 92$ & $10: 39$ & 0.10 & 2732.444 & 6.989 & 0.014 & 16.47 \\
\hline $\mathrm{OH} 46$ & $07 / 01 / 92$ & $10: 00$ & 0.07 & 2738.417 & 5.973 & 0.012 & 16.54 \\
\hline $\mathrm{OH} 46$ & $07 / 08 / 92$ & & 0.08 & 2745.000 & 6.583 & 0.012 & 16.62 \\
\hline $\mathrm{OH} 46$ & $07 / 15 / 92$ & $10: 50$ & 0.09 & 2752.451 & 7.451 & 0.012 & 16.71 \\
\hline $\mathrm{OH} 46$ & $07 / 22 / 92$ & $11: 45$ & 0.08 & 2759.490 & 7.039 & 0.019 & 16.79 \\
\hline OH46 & $08 / 04 / 92$ & $10: 05$ & 0.15 & 2772.420 & 12.930 & 0.012 & 16.94 \\
\hline $\mathrm{OH} 46$ & $08 / 18 / 92$ & $10: 28$ & 0.15 & 2786.436 & 14.016 & 0.011 & 17.09 \\
\hline
\end{tabular}


TABLE A-2 (Continued)

\section{BRINE ACCUMULATION DATA TABLE}

Data through December 31, 1993

\begin{tabular}{|c|c|c|c|c|c|c|c|c|}
\hline LOCATION & DATE & TIME & $\begin{array}{l}\text { LITERS } \\
\text { REMOVED }\end{array}$ & $\begin{array}{l}\text { DAYS } \\
\text { SINCE } \\
1 / 1 / 85\end{array}$ & $\begin{array}{c}\text { DAYS } \\
\text { USED FOR } \\
\text { CALCULATION }\end{array}$ & $\begin{array}{l}\text { LITERS } \\
\text { PER DAY }\end{array}$ & $\begin{array}{l}\text { CUHULATIVE } \\
\text { LITERS } \\
\text { COLLECTED }\end{array}$ & REMARKS \\
\hline OH46 & $09 / 02 / 92$ & $10: 35$ & 0.18 & 2801.449 & 15.005 & 0.012 & 17.27 & \\
\hline OH46 & $09 / 24 / 92$ & $09: 32$ & 0.01 & 2823.397 & 21.956 & 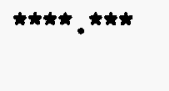 & 17.28 & $\begin{array}{l}\text { Liters/day value is }>0.000 \text { and }<0.001 \\
(0.4 E-03) \text {. }\end{array}$ \\
\hline $\mathrm{OH} 46$ & $10 / 21 / 92$ & $14: 05$ & 0.50 & 2850.587 & 27.190 & 0.018 & 17.78 & \\
\hline $\mathrm{OH} 46$ & $10 / 28 / 92$ & $09: 35$ & 0.06 & 2857.399 & 6.812 & 0.009 & 17.84 & \\
\hline OH46 & $11 / 11 / 92$ & $12: 50$ & 0.15 & 2871.535 & 14.136 & 0.011 & 17.99 & \\
\hline OH46 & $12 / 09 / 92$ & $13: 30$ & 0.28 & 2899.563 & 28.028 & 0.010 & 18.27 & \\
\hline $\mathrm{OH} 46$ & $12 / 16 / 92$ & $10: 40$ & 0.04 & 2906.444 & 6.881 & 0.006 & 18.31 & \\
\hline OH46 & $01 / 07 / 93$ & $09: 40$ & 0.23 & 2928.403 & 21.959 & 0.010 & 18.54 & \\
\hline OH46 & $01 / 13 / 93$ & $10: 10$ & 0.06 & 2934.424 & 6.021 & 0.010 & 18.60 & \\
\hline OH 46 & $01 / 28 / 93$ & $11: 25$ & 0.13 & 2949.476 & 15.052 & 0.009 & 18.73 & \\
\hline OH46 & $03 / 25 / 93$ & $10: 45$ & 0.49 & 3005.448 & 55.972 & 0.009 & 19.22 & \\
\hline $\mathrm{OH} 46$ & $04 / 28 / 93$ & $11: 30$ & 0.33 & 3039.479 & 34.031 & 0.010 & 19.55 & \\
\hline $\mathrm{OH} 46$ & $06 / 16 / 93$ & $08: 43$ & 0.33 & 3088.363 & 48.884 & 0.007 & 19.88 & \\
\hline OH46 & $08 / 19 / 93$ & & 0.45 & 3152.000 & 63.637 & 0.007 & 20.33 & \\
\hline $\mathrm{OH} 46$ & $11 / 12 / 93$ & $11: 09$ & 0.42 & 3237.465 & 85.465 & 0.005 & 20.75 & \\
\hline
\end{tabular}




\section{THIS PAGE INTENTIONALLY LEFT BLANK}




\section{APPENDIX B \\ GRAPHS OF BRINE ACCUMULATION DATA}


THIS PAGE INTENTIONALLY LEFT BLANK 


\section{APPENDIX B GRAPHS OF BRINE ACCUMULATION DATA}

This appendix contains graphs of data presented in Appendix A for selected locations. As described in Deal and Case (1987), much of the variability in the quantity of brine collected resulted from limitations of the collection techniques, rather than variations in the actual inflow of brine from bedrock at the collecting locations. As a result, plotting of the inflow data from the data tables (Appendix A) results in an irregular plot that implies variations in inflow which, in fact, do not exist. An 11-point moving average was used to smooth the line. The smoothed data reflect trends in the body of the curve that are representative of the brine seepage rates, while still showing variations that are probably the result of collection techniques.

At the beginning and end of each curve, moving average smoothing projects the calculated trend. As a result, initial and ending values tend to be distorted by the 11-point moving average smoothing program. A "step-down" moving average was used for the data points at the beginning and end of the curve to correct the distortion. The "step-down" moving average involves stepping down from an 11-point average to a 9-point, 7-point, 5-point and a 3-point average at both ends of the line, utilizing the actual data for the last point. Additional discussion of the collection and data handling is provided in Deal and Case (1987). 


\section{THIS PAGE INTENTIONALLY LEFT BLANK}



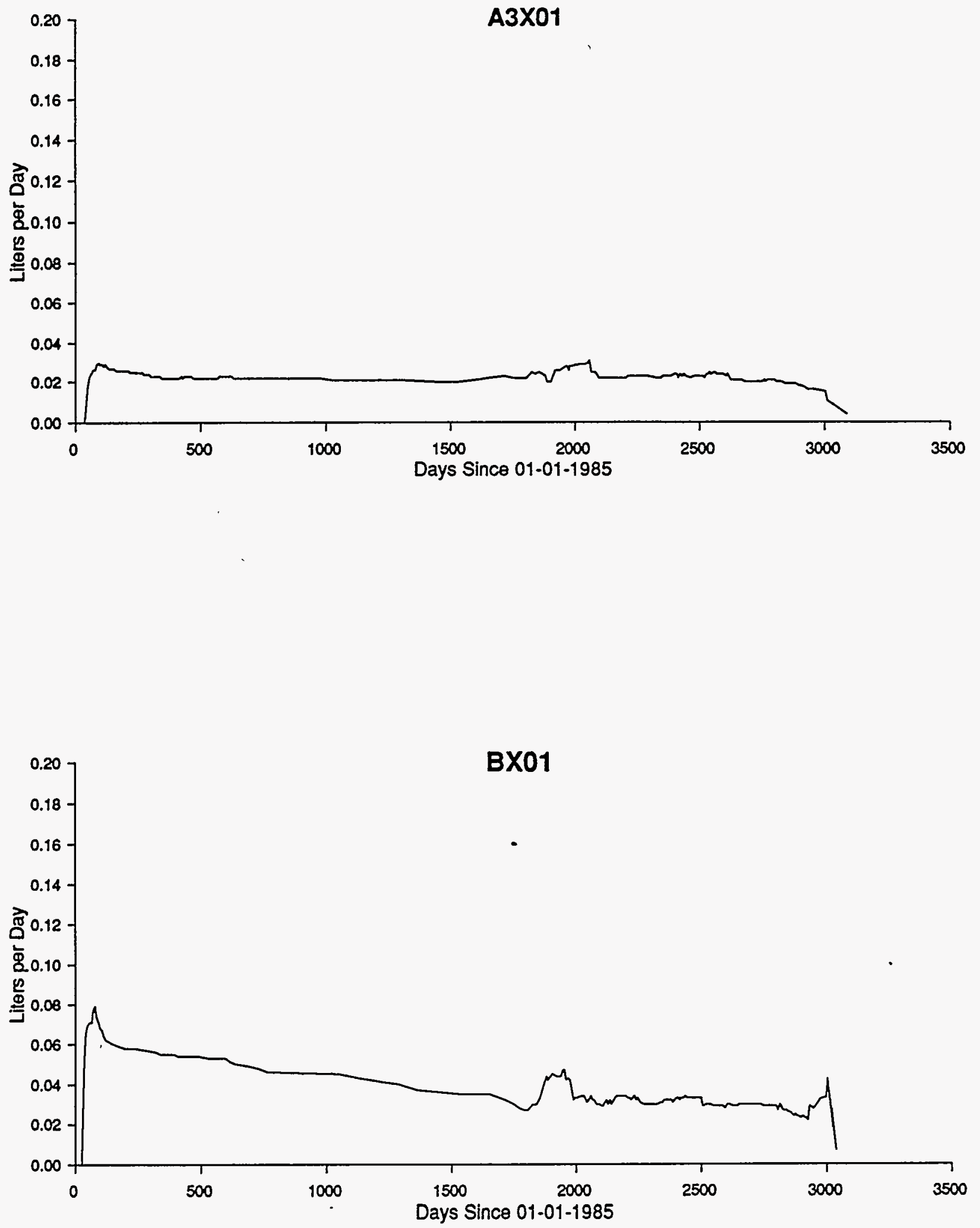

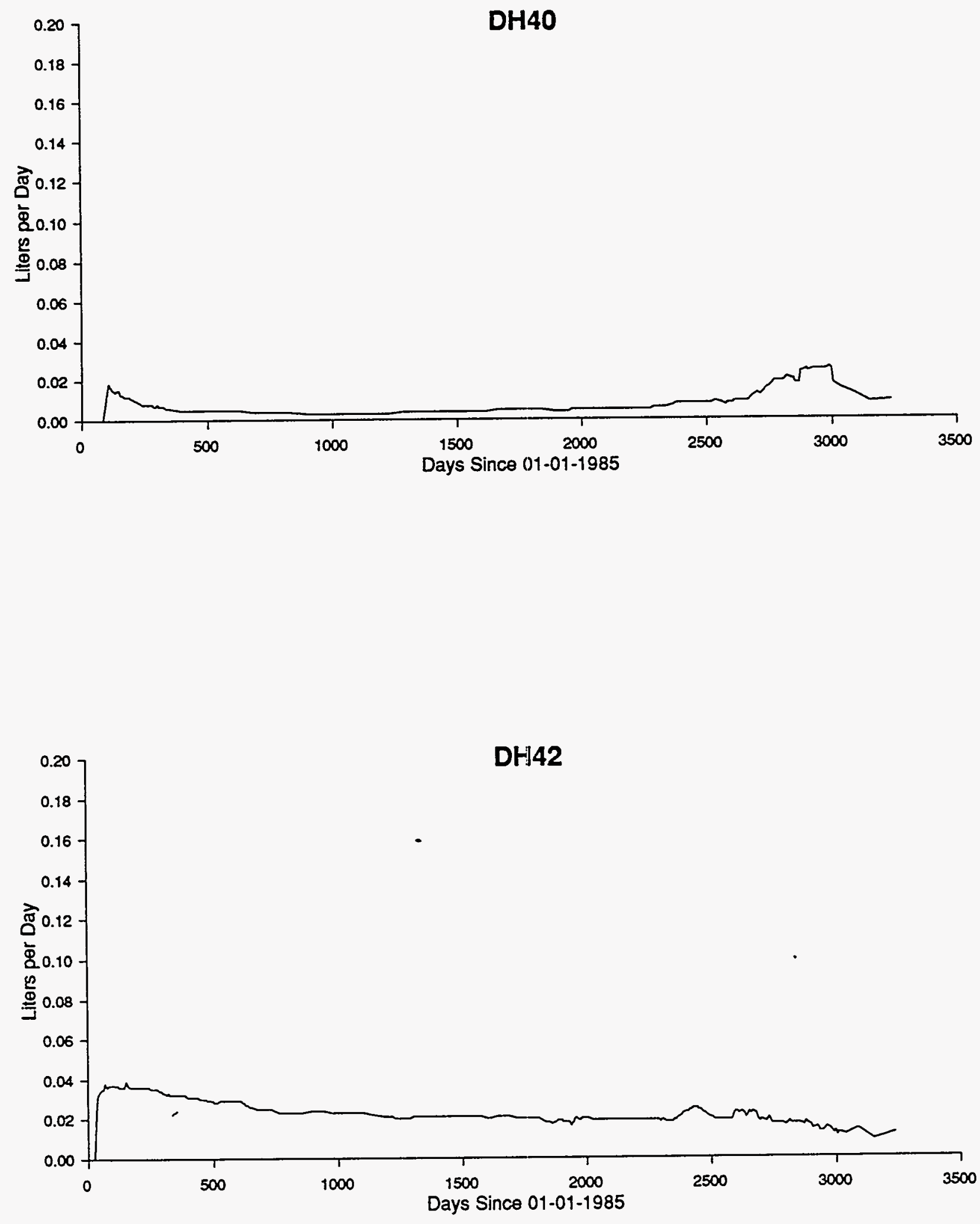

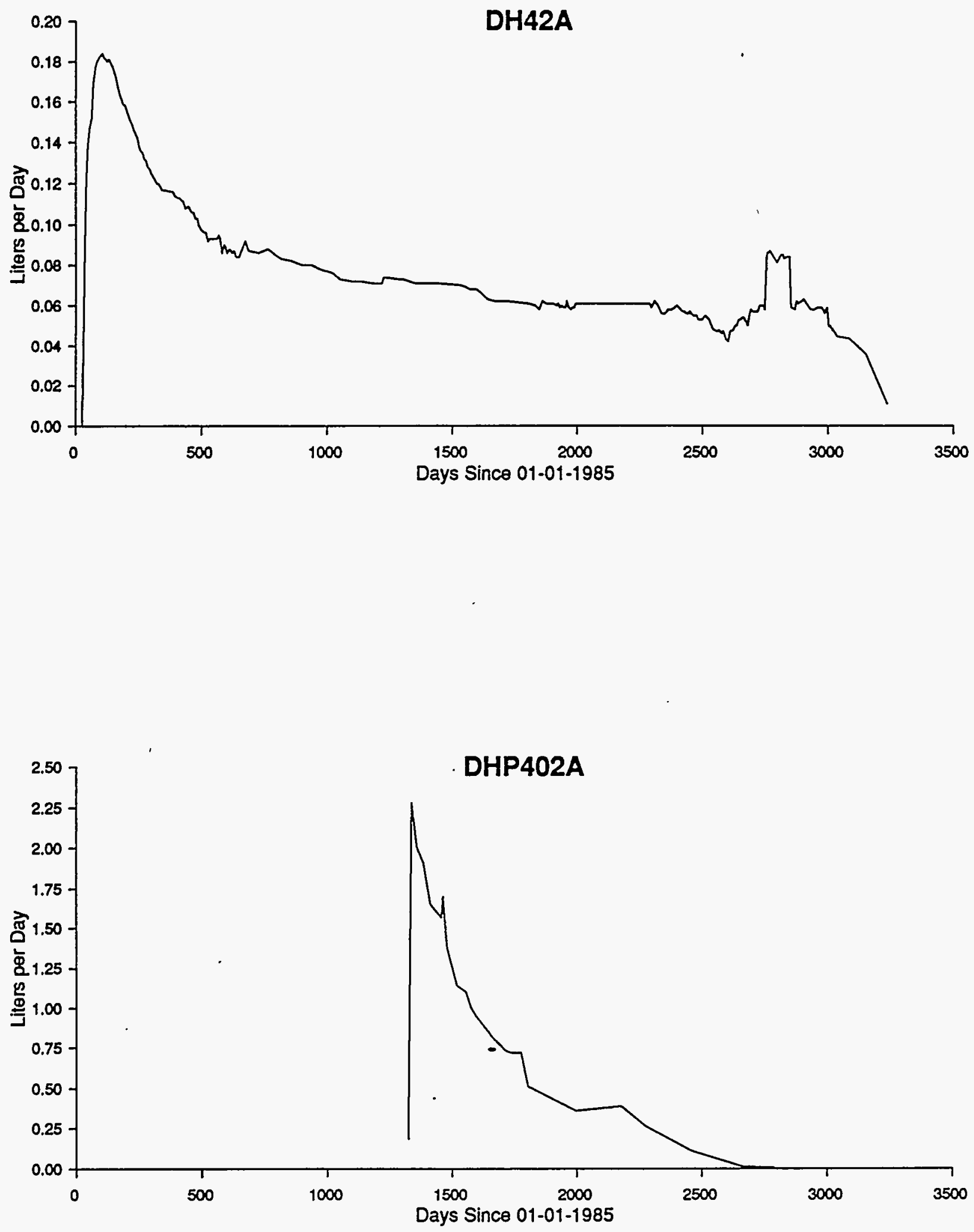

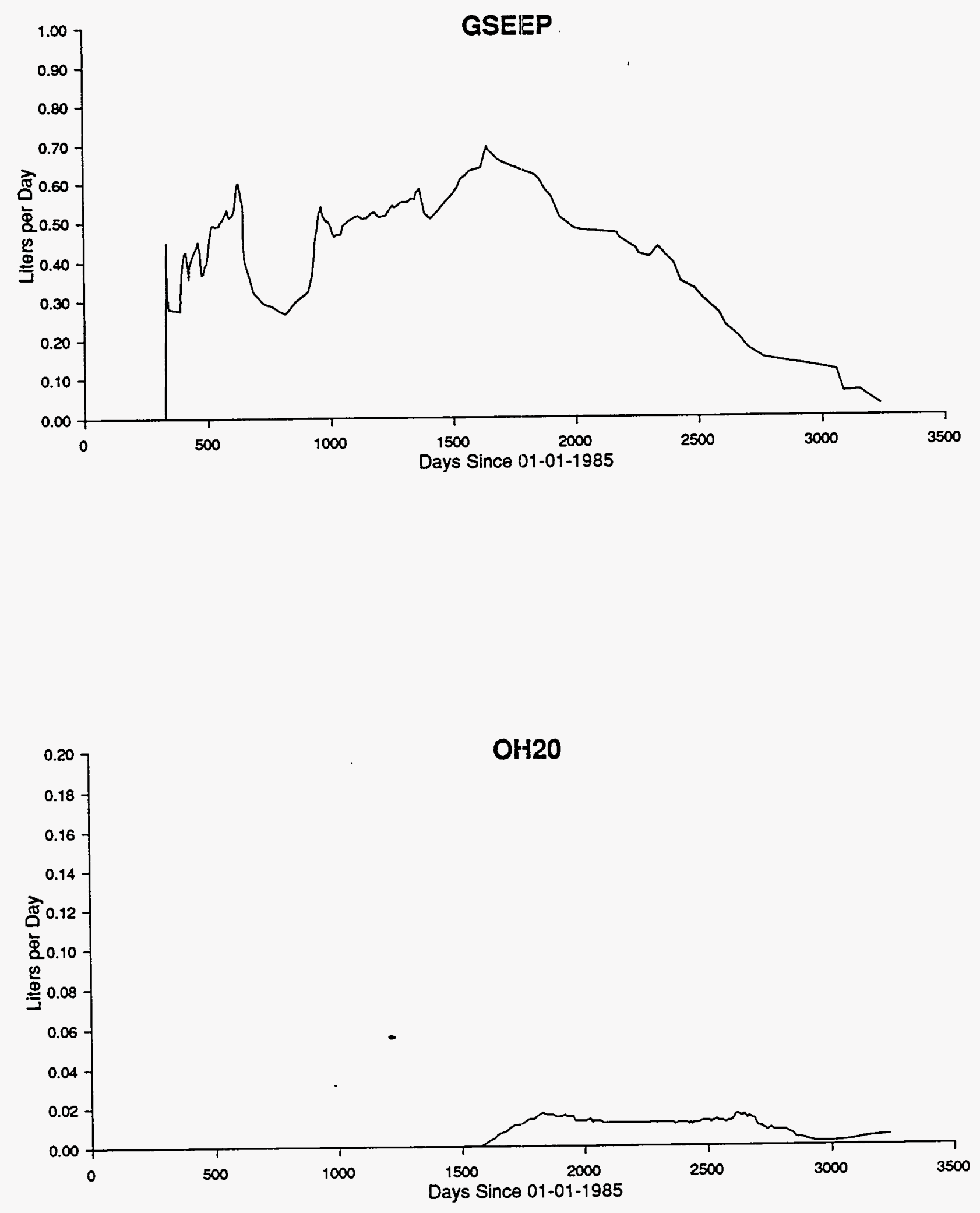

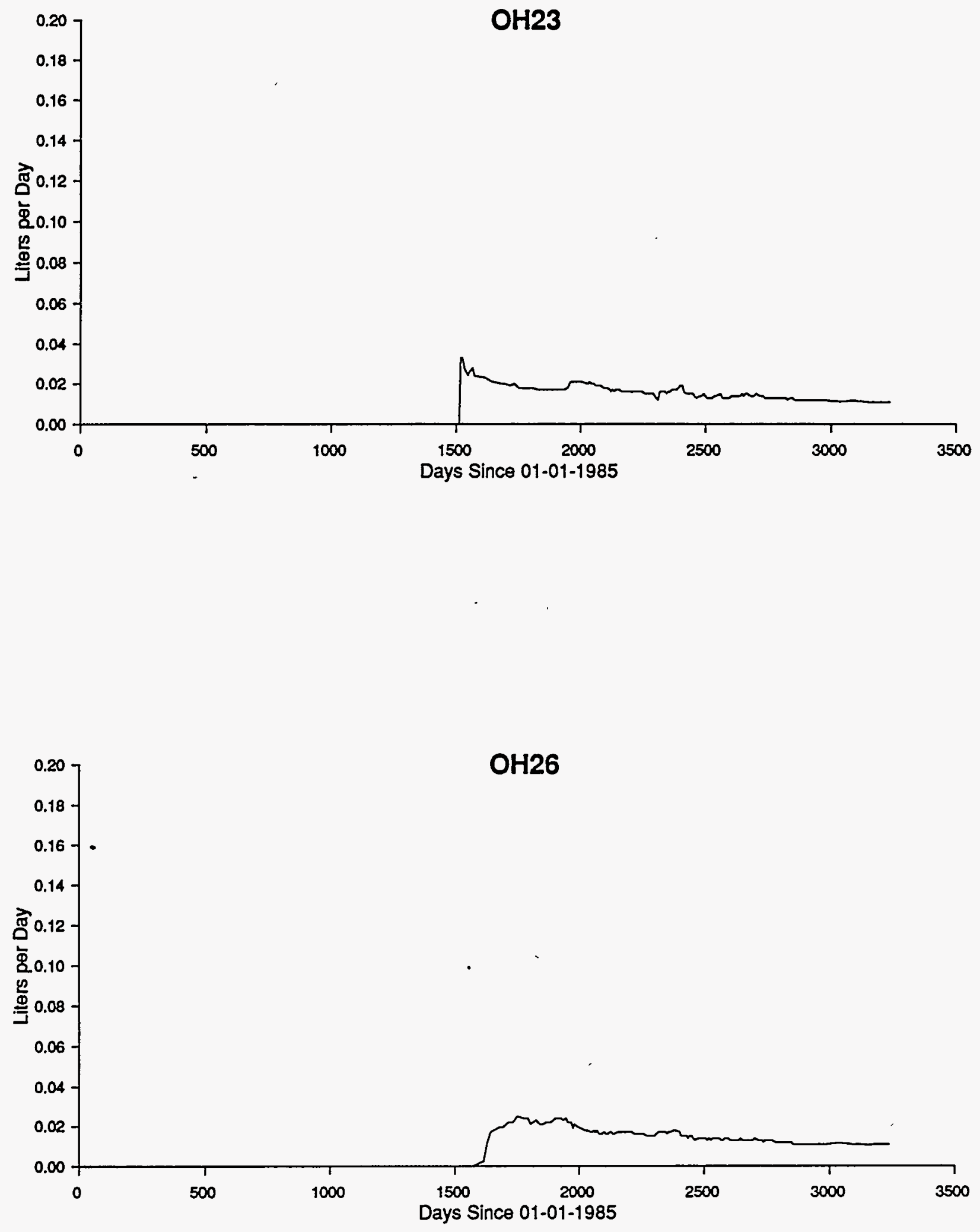

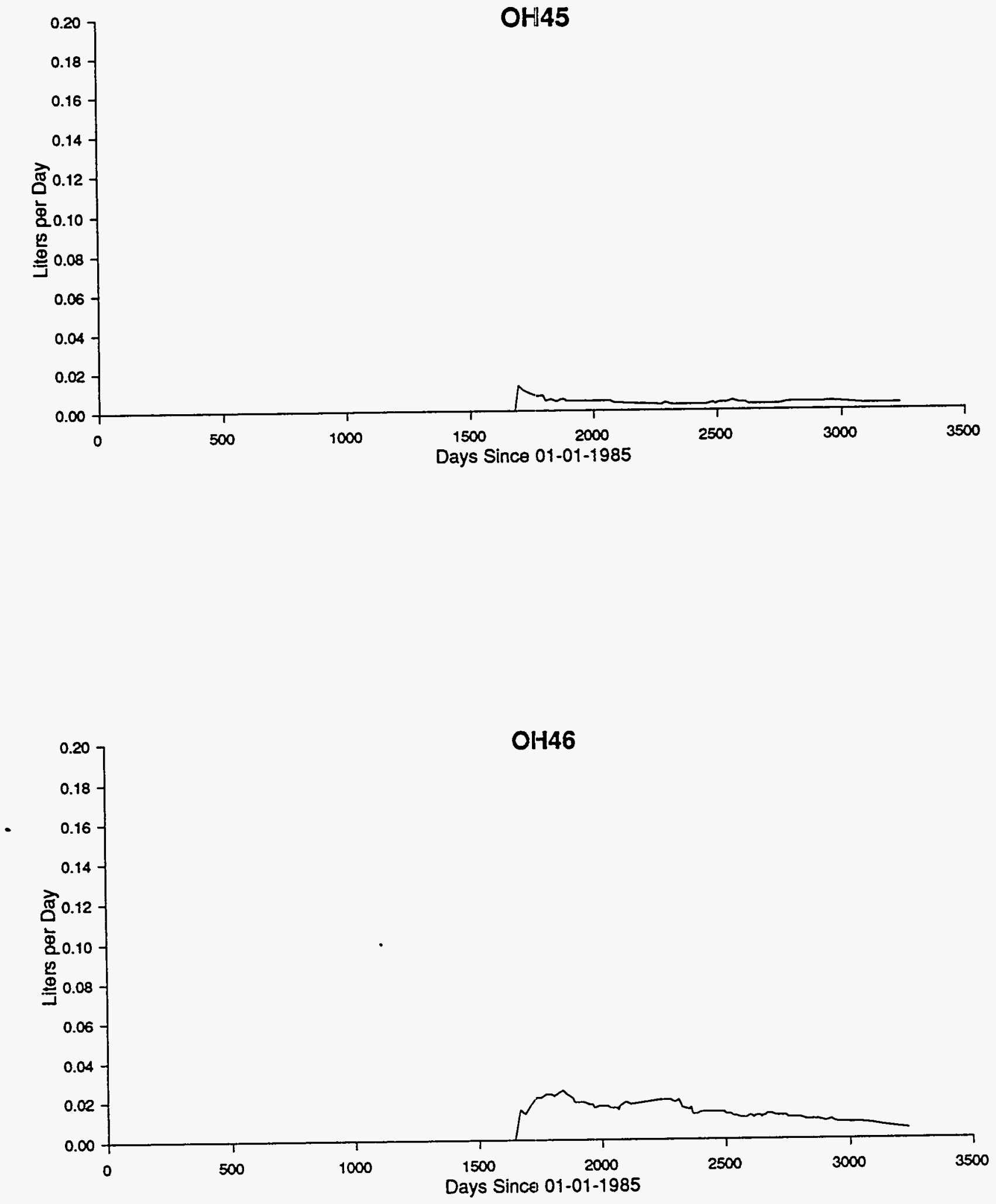


\section{APPENDIX C AIR INTAKE SHAFT INSPECTION}




\section{THIS PAGE INTENTIONALLY LEFT BLANK}




\section{Table of Contents}

C.1.0 General Observations $\ldots \ldots \ldots \ldots \ldots \ldots \ldots \ldots \ldots \ldots \ldots \ldots \ldots \ldots \ldots \ldots \ldots \ldots \ldots \ldots .2$

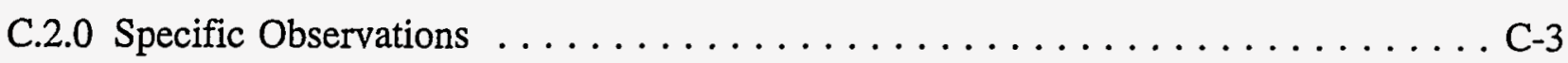

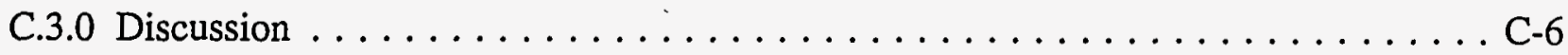

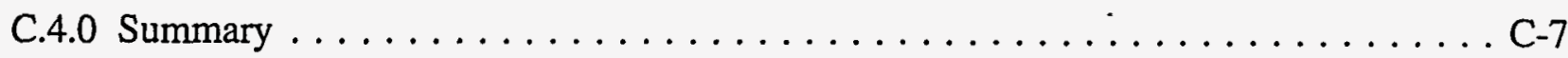

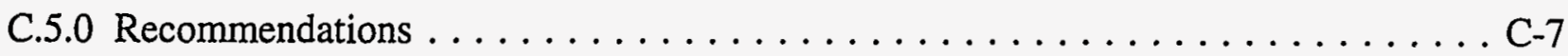

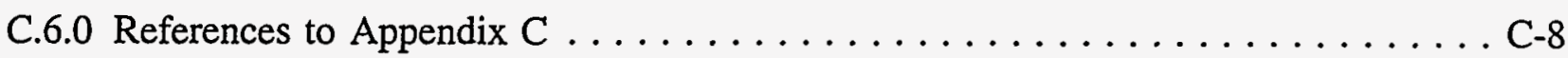




\section{THIS PAGE INTENTIONALLY LEFT BLANK}




\section{AIR INTAKE SHAFT INSPECTION}

Surfaces of excavated areas for the Waste Isolation Pilot Plant (WIPP) have been observed to develop salt encrustations from weeps in some locations (TSC-D'Appolonia, 1983; Deal and others, 1989). These weeps develop salt encrustations over a period of time, if flow is sufficient, and then dry up and cease to flow within periods ranging from hours to a few years (Deal and others, 1989). Similar phenomena are observed within some potash mine areas that are also in the Salado Formation in the vicinity of WIPP (Deal and Case, 1987, Section 3.2).

The source of the brine, the process of fluid flow, and the rate and volume of fluid flow to repository panels are significant factors in assessing the volume of water that will accumulate within the panels. The water volume is an important parameter for calculating gas generation during breakdown of waste; gas pressure might be sufficient to fracture the surrounding rock and breach containment (SNL/NM, 1992). Deal and Bills (1994) and Deal and others (1994) have concluded that empirical data from 11 years of observations, as well as some simplified modeling, indicate that fluid flow will be limited in duration and volume. They conclude that the brine will be a small fraction of that required to corrode metal and to generate gas pressures high enough to fracture the surrounding rock. Because of this, it is appropriate to examine the evidence developed empirically about the weeps, determine limits to inferences based on this data, and seek any further evidence bearing on interpretations already provided.

The Air Intake Shaft (AIS) at the WIPP provides a different perspective on the distribution and development of weeps within the Salado Formation. The underground workings reveal a significant horizontal area within a fairly narrow stratigraphic range and at relatively constant depth. The underground areas open to visual observation are predominantly halite beds. Sulfate interbeds are exposed in only a few locations and have been altered by deformation in the disturbed rock zone (DRZ). It is difficult to accurately determine the undisturbed hydrologic properties of the sulfate beds from those exposures. The AIS is a vertical, circular shaft with reasonable access to the beds from the repository level to near the top of the Salado Formation. Sulfate marker beds, clay-rich units, and halitic beds with differing minor components are observable at different depths. Although a DRZ has formed around the shaft, the extensive fracturing that characterizes the DRZ around the rectangular, horizontal WIPP excavations (Appendix E, Figure E-2-1) does not exist. 
A portion of the shaft wall was cleaned with high-pressure water during 1989 and was then allowed to dry. The AIS was mapped in considerable detail based on the cleaned section (Holt and Powers, 1990). Some sections of the shaft were noted to be yielding some fluid during mapping. The cleaned and mapped portion of the shaft was observed during recent inspections to have remained relatively clean and well-exposed, providing the opportunity to compare the present surface with that mapped during 1989. (For consistency with that report, English units are used in this appendix.)

On July 19, 1994, an initial assessment of the development of the weeps in the AIS was made from the man cage. The units were viewed at inspection speed on the down trip to the repository level, confirming that the south shaft wall was still well-exposed. Significant marker beds were identified and weeps were found to be present. Salt encrustations were noted on the stratigraphic log of the shaft mapping (Holt and Powers, 1990). A more detailed examination was performed during the ascent.

All observations were made from the confines of the man cage, approximately centered in the shaft and about $9 \mathrm{ft}$ from the shaft wall. Lighting was provided by battery powered miner's lamps and a flashlight.

\section{C.1.0 General Observations.}

A strip ranging from approximately 5 to $20 \mathrm{ft}$ wide along the south side of the AIS was cleaned with high-pressure water and allowed to dry before the AIS was mapped during 1989 (Holt and Powers, 1990). This strip remains in good condition for observing geologic features nearly five years later, although the surface has altered somewhat under the intake of air varying in temperature and humidity.

Salt encrustations are much more common below the depth of $1,500 \mathrm{ft}$ in the shaft, approximately the midpoint of the exposed Salado in the AIS. Only a few units above 1,500 ft show that weeps have existed, though Marker Bed (MB) 103 (near the top of the Salado) was the only bed observed to have a wet surface at the time of this survey. The observations do not differentiate between zones where weeps have ceased and zones where there might still be seepage under the salt encrustation.

As noted in some of the specific comments below, many of the encrustations appear to emanate from or are closely associated with contacts between units where Holt and Powers (1990) inferred exposure surfaces of Permian age, based on the compositions, sedimentary 
features, and textures of the units underlying the contact. The bulk of the encrustations are also closely associated with argillaceous units.

\section{C.2.0 Specific Observations}

The specific observations of salt encrustations in the AIS have been summarized (see Table C-2-1) for reference relative to the original mapping (Holt and Powers, 1990). There is a minor difference in depth below approximately $2,000 \mathrm{ft}$ between the present depth markers and the original depth reference for mapping. Except as noted, the depths in this report correspond to the original mapping depth to aid in relating them to the stratigraphy presented from the mapping. Specific examples of features are described briefly; they are ordered here by increasing depth.

MB 103 (1,029 ft) displays the most significant salt encrustations for any sulfate marker bed within the AIS (Figure C-2-1). The weeps were restricted to a zone about $1 \mathrm{ft}$ thick that is dolomitic, overlies a claystone, and was observed to be wet during inspection. The same zone was yielding fluid during the 1989 mapping. Rock bolts from the argillaceous halite, below the marker bed and claystone, at a depth of approximately 1,030 ft were also wet.

The lower 5-6 ft of the Vaca Triste Sandstone Member (1,342-1,348 ft) displays significant encrustations, many around the heads of rock bolts used to hold screen in place (Figures C-2-2 and C-2-3). The upper $2 \mathrm{ft}$ (see Figure C-2-2) reveals sedimentary features, dish-shaped structures, and soft-sediment deformation consistent with synsedimentary remobilization of halite in a mud pan. This zone is also halitic and shows no evidence of weeps (see Figure C-2-2). Encrustations are large in the lower part (see Figure C-3).

Many of the marker beds show no encrustations associated with weeps, as illustrated by the Union Anhydrite (1,529-1,535 ft) (Figure C-2-4). A distinctive argillaceous unit from about 1,673 to $1,676 \mathrm{ft}$ has produced a number of small encrustations within the unit as well as at and slightly above the upper surface (see Figure C-2-5). Subaqueous primary halite overlies the unit. The halite immediately above the argillaceous halite should be examined at close range to more precisely determine the relationship of weeps to halite. In most areas, highpurity halite shows no encrustations.

A series of stratigraphically controlled encrustations (see Figure C-2-6) from a zone at a depth of approximately 1,767 to $1,776 \mathrm{ft}$ is related to several exposure surfaces over that interval. 
During original mapping, some of these zones, as well as others nearby, were observed to have some brine seeps.

Within Figure C-6, two zones (one at approximately $1,770 \mathrm{ft}$ and one from 1,775 ft to approximately $1,777 \mathrm{ft}$ ) do not seem to have yielded any fluid or to have developed any encrustations. From the descriptions (Holt and Powers, 1990), these two zones, or beds, differ from adjacent zones in two ways: a higher content of clay and a higher proportion of "podular" textures. These areas may also warrant closer examination from the work platform or galloway.

Clay minerals distributed throughout the uppermost deposits of an exposure surface seem to be the significant control for a number of the intervals with encrustations. The exposure surface at the 1,825-ft depth (Figure C-2-7) is overlain by halite still exhibiting some primary fluid inclusion zones. The surface itself developed some of the larger and deeper (some to 10 ft) synsedimentary dissolution pipes within the Salado Formation as the penecontemporaneous water table dropped below the exposed surface. The clays do not occur in large proportions under this surface, but they are greater than in underlying or overlying units.

There is very little association of encrustations with marker beds composed mostly of polyhalite. A thin (less than $1 \mathrm{ft}$ ) unit of mixed polyhalite and halite (see Figure C-2-8) at a depth of approximately $1,845 \mathrm{ft}$ ) overlying an exposure surface developed some significant encrustations. Minor encrustations also developed in the slightly polyhalitic halite overlying this unit. Because the polyhalite-halite bed appeared slumped and contorted, it may be that clays are significant in the unit, though not noted during mapping. Solution pipes with collapse textures underlie this bed and developed small encrustations from collapse material.

Claystones and argillaceous halite underlying several of the marker beds have developed encrustations. The gray claystone (see Figure C-2-9) underlying MB 131 shows this stratigraphic control. The exposure surface at approximately $1,867 \mathrm{ft}$, shown near the bottom of Figure C-2-9, also developed a line of encrustations similar to other exposure surfaces. As in other parts of the shaft, it is notable that rock bolts within approximately a foot of the upper line of encrustations did not develop observable evidence of weeps.

An unnamed anhydrite at approximately 1,940 ft (see Figure C-2-10) shows encrustations developed at, and possibly slightly above, the underlying claystone. Rock bolts within the upper part of the anhydrite indicate that some weeps have developed. This unit should be 
examined more closely for cracking as well as for lithologic control of the weeps. The basal part of Figure C-2-10 shows possible minor weeps originating from the argillaceous halite underlying the claystone.

MB 134, at a depth from 1,956 to $1,967 \mathrm{ft}$, appears to have developed 2 or 3 isolated weeps within the upper few feet of the unit (see Figure C-2-11). These are unusual among the observed encrustations in the AIS for two reasons: they are within a thick anhydrite, and they are isolated. This entire unit should be carefully examined for evidence of fractures and controls on the weeps.

The claystone at the base of MB 135 appears to control weep locations, as do some other marker beds (Figure C-2-12 at a depth of approximately 1,985 ft). Some encrustations developed above MB 135 from clay-filled synsedimentary pits or caves (Figure C-2-12) (Holt and Powers, 1990). The anhydrite in MB 135 should be more carefully examined for evidence of fractures.

The major exposure surface at approximately 1,995 $\mathrm{ft}$ (Figure C-2-13), as well as secondary surfaces below that, controls the location of many of the encrustations in that zone. In addition to discrete surfaces, the clay content increases in the upper $5 \mathrm{ft}$ of this zone of weeps. The overlying, relatively pure halite did not develop weeps, as in many similar sequences throughout the AIS.

From a depth of about 2,075 to nearly $2,100 \mathrm{ft}$, a series of stratigraphically controlled encrustations developed along mapped exposure surfaces (Figure C-2-14). The clay content generally increases upward toward the exposure surface, and the overlying halite is relatively pure with remains of primary fluid inclusion zones in some halite.

The base of MB 138 (Figure C-2-15) shows weeps similar to some other sulfate beds. It is the closest such claystone-sulfate unit above the repository horizon to develop encrustations. "Anhydrite a," at an original mapping depth of approximately 2,123 to $2,124(2,121$ to $2,122 \mathrm{ft}$ according to new depth-marker signs) shows a visible fracture but no evidence of seepage.

Discontinuous small encrustations developed along and above the exposure surface (referred to as clay $I$ in reference stratigraphy for rock mechanics) originally mapped at approximately $2,113 \mathrm{ft}$ (Figure C-2-16). Above the more significant exposure surface, the halite is thinly 
bedded with anhydrite stringers indicating minor exposure. These encrustations are the closest observed to the repository horizon.

\section{C.3.0 Discussion}

The most common denominator for the salt encrustations observed in the AIS appears to be stratigraphic discontinuities interpreted by Holt and Powers (1990) as produced by a period of exposure during the Permian Age before deposition of the overlying sediment. It could also be reasonably argued that clay content is the common denominator. Exposure surfaces and increasing clay content upward in a depositional cycle are associated. Nonetheless, the mere presence of either an exposure surface or a surface of clay does not mean that a weep will form; most do not develop weeps.

At this time, there is insufficient evidence to draw conclusions from the AIS as to whether the units that developed salt encrustations might do so if intercepted at another location. Various locations underground at WIPP do produce weeps within the same stratigraphic units; therefore, it is suspected that a unit producing weeps in the AIS will be prone to produce weeps if intercepted elsewhere. This has not been demonstrated by observation. Nor can it be demonstrated that a shaft some distance from the AIS would only produce weeps from the exact same stratigraphic intervals. It is expected that any intercept through these units would produce weeps that preferentially, but not uniquely, are associated with exposure surfaces and attendant argillaceous halite.

Claystones under some sulfate beds do produce weeps. These are more common at greater depth. Some might also be interpreted to overlie exposure surfaces. By inspection, it appears that claystones underlying sulfate beds less than $2 \mathrm{ft}$ thick more frequently produce weeps than those underlying thicker sulfate beds.

MB 103 has the most significant weeps of any sulfate unit in the AIS, and it also has a wet surface. In contrast, "anhydrite a" is visibly fractured parallel to bedding but appears not to have developed any weeps. This observation limits the amount of fluid available from the unit to that which might have flowed before cleaning and mapping.

In contrast, $\mathrm{MB} 103$ persisted with flow after this period of cleaning and mapping. As the uppermost unit yielding weeps, unloading may be more of a factor, but it must also be noted that other marker beds above and below MB 103 did not similarly yield brine. 


\section{C.4.0 Summary}

The AIS intercepts a variety of evaporite lithologies and bedding relationships. A number of these units have developed salt encrustations over the five years since a strip was cleaned and mapped.

Encrustations are more abundant below a depth of approximately 1,500 ft, about the midpoint of the exposed Salado Formation in the AIS. The salt encrustations are frequently, but not uniquely, associated with Permian age exposure surfaces and related argillaceous halite. Claystones under a few sulfate beds in the lower half also yielded brine. MB 103, near the top of the exposed Salado, has much more salt encrustation than any other marker bed, and it shows a small surface that is observably wet now. Most of the sulfate beds, especially polyhalitic units, show no weeps or encrustations.

Observations were made from approximately $9 \mathrm{ft}$ away. The encrustations all appeared to be dry.

\section{C.5.0 Recommendations}

It is recommended that some additional, closer, observations of the AIS weep occurrences be performed to supplement the preliminary data reported here. Selected stratigraphic intervals should be examined in detail from the galloway to confirm the stratigraphic and textural relationships between encrustations and previous mapping.

A limited, two-part program in the AIS to confirm the preliminary observations is recommended. This should be within the capabilities of on-site geotechnical personnel, especially if trained as indicated above; outside observers can confirm information, if necessary. The first phase is to document the encrustations in considerably more detail by demonstrating very specific textural and stratigraphic relationships to the encrustations. Scaled photographic records should also be included.

During the first phase, some areas of encrustations should be removed within carefully documented and marked areas. The samples should be preserved for possible analysis pending further observations. Within selected stratigraphic intervals, part of the encrustations may be removed to compare any future precipitation with current encrustations. The main purpose of scraping the areas is to determine if some weeps might still be active and would develop again. 
The second phase is a modest program of reinspections to determine if weeping is continuing where the encrustations had been removed, as well as a general survey of the shaft for any other evidence of renewed weeps. The initial inspections should be within a few weeks, at most, to not miss small amounts of weeping. Within a year, a few inspections should reveal any renewed activity. If there are additional weeps, the program of observation can be reevaluated.

\section{C.6.0 References to Appendix C}

Deal, D. E., and R. A. Bills, 1994, "Conclusions After Eleven Years of Studying Brine at the Waste Isolation Pilot Plant," Waste Management '94, Tucson, Arizona, March 2, 1994, IT Corporation, Albuquerque, New Mexico, and U.S. Department of Energy, Carlsbad, New Mexico.

Deal, D. E., and J. B. Case, 1987, "Brine Sampling and Evaluation Program, Phase I Report," DOE-WIPP 87-008, prepared for the U.S. Department of Energy by IT Corporation and Westinghouse Electric Corporation, Carlsbad, New Mexico, 163 pp.

Deal, D. E., R. M. Holt, J. M. Melvin, and S. M. Djordjevic, 1994, "Calculation of Brine Seepage from Anhydrite Marker Bed 139 into a Waste Storage Room at the Waste Isolation Pilot Plant," DOE-WIPP 94-007, Westinghouse Electric Corp., Carlsbad, New Mexico.

Deal, D. E., R. J. Abitz, D. S. Belski, J. B. Case, M. E. Crawley, R. M. Deshler, P. E. Drez, C. A. Givens, R. B King, B. A. Lauctes, J. Myers, S. Niou, J. M. Pietz, W. M. Roggenthen, J. R. Tyburski, and M. G. Wallace, 1989, "Brine Sampling and Evaluation Program Report, 1988," DOE-WIPP 89-015, prepared for the U.S. Department of Energy by IT Corporation and Westinghouse Electric Corporation, Carlsbad, NNew Mexico.

Holt, R. M., and D. W. Powers, 1990, "Geologic Mapping of the Air Intake Shaft at the Waste Isolation Pilot Plant," DOE/WIPP 90-051, prepared for the U.S. Department of Energy by IT Corporation, Carlsbad, New Mexico.

Sandia National Laboratories/New Mexico (SNL/NM), 1992, "Preliminary Performance Assessment for the Waste Isolation Pilot Plant, December 1992, Volume 3: Model Parameters," SAND92-0700/3, WIPP Performance Assessment Division, Sandia National Laboratories, New Mexico.

TSC-D’Appolonia, 1983, "Geologic Mapping of Access Drifts, 'Double Box' Area, Geotechnical Field Data Report No. 5," Carlsbad, New Mexico. 


\section{Table C-2-1}

\section{Air Intake Shaft Observations of Significant Salt Encrustations}

\begin{tabular}{|c|c|}
\hline $\begin{array}{l}\text { Depth } \\
(\mathrm{ft})\end{array}$ & Geological Relationships or Observations \\
\hline 998 & Minor exposure surface; in SMPH ${ }^{1}$. \\
\hline $1026-28 P^{2}$ & Lower MB 103 , in dolomitic anhydrite above claystone. \\
\hline 1030 & $\begin{array}{l}\text { Slightly argillaceous halite, upper SMPH below MB } 103 \text { claystone; mainly from } \\
\text { around rock bolts. }\end{array}$ \\
\hline 1161 & Argillaceous nodular anhydrite in lower third of MB 109; from around rock bolts. \\
\hline 1175 & $\begin{array}{l}\text { Argillaceous halite }(\mathrm{DMRH}) \text { below halitic claystone }(\mathrm{HM}) \text { at top of desiccating } \\
\text { sequence. }\end{array}$ \\
\hline 1200 & Argillaceous halite (DMRH), at and below contact with overlying SMPH. \\
\hline 1253 & Halite with trace clay and polyhalite, underlying thin polyhalite. \\
\hline 1277 & $\begin{array}{l}\text { Halite, trace clay, from exposure surface at top SMPH, underlying DMRH with } \\
\text { halite growth textures. }\end{array}$ \\
\hline $1302-03$ & Halite, trace clay, from exposure surface overlying argillaceous halite. \\
\hline 1320 & Single weep, near center MB 115, from zone of wavy bedding. \\
\hline 1342-48 PP & $\begin{array}{l}\text { Siltstone, Vaca Triste Sandstone Member, from below upper } 2 \mathrm{ft} \text { of halite- } \\
\text { cemented mudstone; many weeps. }\end{array}$ \\
\hline 1495.5 & Base polyhalite (MB 121), above claystone; zone of small salt encrustations. \\
\hline 1510 & Argillaceous halite (DMRH), just below contact with overlying SMPH. \\
\hline 1515 & Halite (SMPH), just above bedded zone of primary halite ${ }^{3}$ and polyhalite laminae. \\
\hline $1549-52$ & Halite, slightly polyhalitic or slightly argillaceous, lower SMPH; small encrustations. \\
\hline 1613-14 & Polyhalite in lower third of MB 123 anhydrite. \\
\hline 1627.5 & Anhydrite, top of zone of contorted bedding in lower $1 \mathrm{ft}$. \\
\hline $1673-75.5 P$ & $\begin{array}{l}\text { Argillaceous halite, at contact with overlying SMPH as well as within unit } \\
\text { underlying exposure surface. }\end{array}$ \\
\hline $1687-88$ & Argillaceous halite (HM and DMRH), below exposure surface; overlain by SMPH. \\
\hline 1701 & $\begin{array}{l}\text { Halite, trace of clay, from inches above major exposure surface; maybe at minor } \\
\text { bedding plane or exposure surface. }\end{array}$ \\
\hline 1708 & $\begin{array}{l}\text { Halite, trace of clay, primary halite and podular texture; from level equivalent to } \\
\text { base of pits and pipes. }\end{array}$ \\
\hline 1710.5 & Argillaceous halite, at top of DMRH zone; SMPH overlies exposure surface. \\
\hline 1721 & Halite, trace polyhalite; at or from clay at base of polyhalite (MB 126). \\
\hline 1726 & Halite (SMPH), at transition from trace of polyhalite to slightly argillaceous halite. \\
\hline 1739.5 & $\begin{array}{l}\text { Argillaceous halite, podular zone just above exposure surface; underlain by SMPH } \\
\text { with primary halite. }\end{array}$ \\
\hline 1768 & $\begin{array}{l}\text { Halite, within SMPH, and secondary exposure surface where argillaceous halite } \\
\text { overlies polyhalitic halite. }\end{array}$ \\
\hline
\end{tabular}

Refer to footnotes at end of table. 
Table C-2-1 (Continued)

Air Intake Shaft Observations of Significant Salt Encrustations

\begin{tabular}{|c|c|}
\hline $\begin{array}{l}\text { Depth } \\
\text { (ft) }\end{array}$ & Geological Relationships or Observations \\
\hline $1770 P$ & Podular halite, at or across exposure surface of argillaceous halite. \\
\hline $1774 \mathrm{P}$ & $\begin{array}{l}\text { Top of podular halite, at same level as base of pits; just above zone of primary } \\
\text { halite; encrustations drape about } 1 \mathrm{ft} \text { of primary halite zone. }\end{array}$ \\
\hline 1776 & $\begin{array}{l}\text { At exposure surface, with argillaceous halite overlying polyhalitic and argillaceous } \\
\text { halite. }\end{array}$ \\
\hline 1783 & Top of MB 129, polyhalite overlain by SMPH primary halite. \\
\hline 1784.5 & Base of MB 129, polyhalite overlying argillaceous halite. \\
\hline 1788 & Argillaceous halite overlying secondary exposure surface. \\
\hline 1793 & Argillaceous halite under exposure surface overlain by primary SMPH. \\
\hline 1799 & $\begin{array}{l}\text { Halite, trace polyhalite, at exposure surface overlain by primary halite, slightly } \\
\text { argillaceous. }\end{array}$ \\
\hline 1807.5 & Halite, trace clay, at or above exposure surface overlain by podular halite. \\
\hline 1815 & Halite, trace of clay, at and below exposure surface in podular halite. \\
\hline $1825 \mathrm{P}$ & Same as 1815. \\
\hline 1833 & Halite, trace of clay, at and below exposure surface; overlain by primary halite. \\
\hline 1837 & Same as 1833. \\
\hline $1844.5 \mathrm{P}$ & At top and possible from base of thin polyhalite overlain by primary halite. \\
\hline 1847 & From within synsedimentary dissolution pipe with collapse material. \\
\hline 1862 & Top of MB 131, polyhalite overlain by primary halite. \\
\hline $1863 P$ & Base MB 131, at contact of polyhalite with argillaceous halite. \\
\hline 1867 & At exposure surface, with primary halite both above and below. \\
\hline $1873-75$ & $\begin{array}{l}\text { At and below exposure surface in argillaceous halite overlain by primary halite; } \\
\text { also within argillaceous halite with displacive halite. }\end{array}$ \\
\hline 1881 & Argillaceous halite, at exposure surface; within bed with polyhalitic halite. \\
\hline 1883 & Argillaceous halite, podular halite; within bed with polynalitic halite. \\
\hline 1887 & $\begin{array}{l}\text { Halite, with trace of polyhalite, at exposure surface overlain by primary halite with } \\
\text { trace of clay. }\end{array}$ \\
\hline 1895 & At exposure surface, probably from clay underlying polyhalite (MB 132). \\
\hline 1914.5 & $\begin{array}{l}\text { Extensive encrustations mainly associated with rock bolts at claystone under } \\
\text { polyhalite (MB 133). }\end{array}$ \\
\hline 1933 & $\begin{array}{l}\text { Halite with trace of clay and some polyhalite, under exposure surface overlain by } \\
\text { primary halite. }\end{array}$ \\
\hline $1940 P$ & $\begin{array}{l}\text { Claystone, polyhalitic at base of anhydrite (about } 2 \mathrm{ft} \text { thick); at major exposure } \\
\text { surface. }\end{array}$ \\
\hline 1947.5 & Argillaceous halite at exposure surface, overlain by primary halite. \\
\hline $1959.5 \mathrm{P}$ & Single encrustation in upper third of MB 134 (about 11-ft-thick anhydrite). \\
\hline
\end{tabular}

Refer to footnotes at end of table. 


\section{Table C-2-1 (Continued)}

\section{Air Intake Shaft Observations of Significant Salt Encrustations}

\begin{tabular}{|c|c|}
\hline $\begin{array}{l}\text { Depth } \\
\text { (ft) }\end{array}$ & Geological Relationships or Observations \\
\hline 1973 & $\begin{array}{l}\text { Small encrustations at exposure surface; argillaceous halite above and below } \\
\text { surface. }\end{array}$ \\
\hline $1985 \mathrm{P}$ & Podular argillaceous halite at exposure surface under anhydrite (MB 135). \\
\hline 1989 & Minor encrustations in halite at level of base of secondary pits and pipes. \\
\hline $1995.5 P$ & At exposure surface in argillaceous halite, overlain by halite. \\
\hline $2000,2001 P$ & At secondary exposure surface in argillaceous halite overlying halite. \\
\hline 2012.5 & At exposure surface, may come from primary halite just above contact. \\
\hline 2016 & Argillaceous halite below exposure surface; overlain by primary halite. \\
\hline 2022 & $\begin{array}{l}\text { Podular halite, at change from polyhalite and polyhalitic halite to increasing clay } \\
\text { upward. }\end{array}$ \\
\hline 2027 & $\begin{array}{l}\text { Encrustations around rock bolts at polyhalite-anhydrite transition, upper third of MB } \\
136 \text {. }\end{array}$ \\
\hline 2040 & $\begin{array}{l}\text { At thin anhydrite overlying exposure surface; may also be at contact with overlying } \\
\text { primary halite. }\end{array}$ \\
\hline 2076.5 & Claystone at exposure surface, overlain by thin anhydrite. \\
\hline $2079.5 P$ & $\begin{array}{l}\text { Halitic claystone (also called } M-1 \text { in reference stratigraphy for rock mechanics } \\
\text { calculations) at exposure surface. }\end{array}$ \\
\hline $2084.5 P$ & $\begin{array}{l}\text { Halitic claystone ( } L \text { in reference stratigraphy for rock mechanics calculations) at } \\
\text { exposure surface. }\end{array}$ \\
\hline 2090 & $\begin{array}{l}\text { Argillaceous halite in podular zone at top secondary exposure surface; overlain by } \\
\text { primary halite. }\end{array}$ \\
\hline 2094 & $\begin{array}{l}\text { At secondary exposure surface where podular argillaceous halite overlies } \\
\text { polyhalitic halite. }\end{array}$ \\
\hline $2099 \mathrm{P}$ & Top of anhydrite (MB 138), overlain by primary halite. \\
\hline $2099.5 P$ & $\begin{array}{l}\text { Argillaceous halite and claystone ( } \mathrm{K} \text { in reference stratigraphy for rock mechanics } \\
\text { calculations) at exposure surface at base of } \mathrm{MB} 138 .\end{array}$ \\
\hline 2108 & Argillaceous halite in podular zone underlying exposure surface. \\
\hline $2110-11 P$ & At minor exposure surfaces within halite with trace polyhalite. \\
\hline
\end{tabular}

${ }^{1}$ Facies designations (e.g., SMPH) are taken from Holt and Powers (1990) "Geologic Mapping.of the Air Intake Shaft at the Waste Isolation Pilot Plant," DOEMIPP 90-051, U.S. Department of Energy, Carlsbad, New Mexico.

${ }^{2} \mathrm{P}$ signifies a photograph is included as a figure in this report.

3"Primary halite" refers to halite with observable textures as evidence of subaqueous growth. All Salado Formation halite is believed to have been deposited during the Permian Age. 


\section{THIS PAGE INTENTIONALLY LEFT BLANK}




\section{Figure C-2-1}

Composite photograph of MB 103 and encrustations in lower part.

Taken toward south, near $1029 \mathrm{ft}$ depth. Note gray claystone in lower third of photograph.

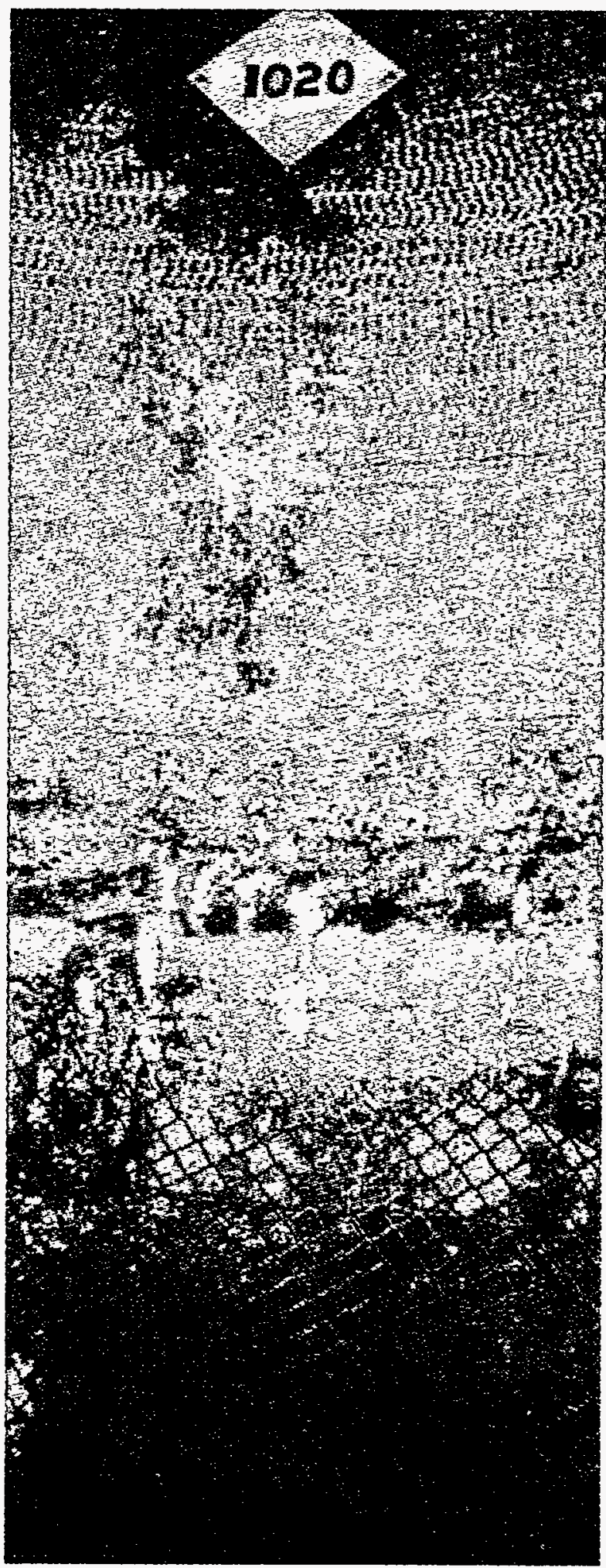




\section{Figure $\mathrm{C}-2-2$}

Photograph of uppermost Vaca Triste SS Mbr (1340 - 1342) with weeps below halite cemented zone.

Taken toward south.
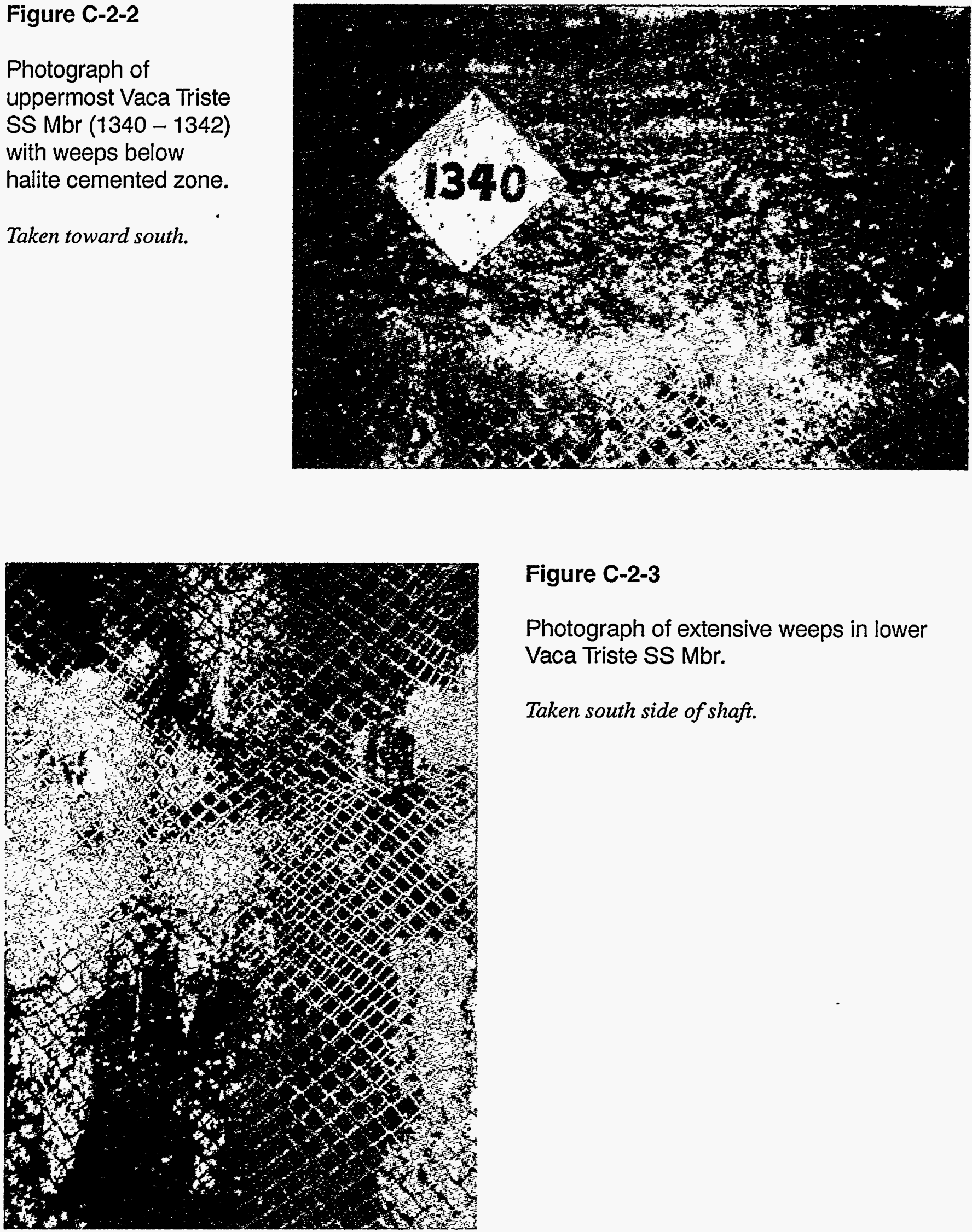

Figure C-2-3

Photograph of extensive weeps in lower Vaca Triste SS Mbr.

Taken south side of shaft. 
Figure C-2-4

Photograph of upper Union Anhydrite $\mathrm{Mbr}$ (about $1530 \mathrm{ft}$ )

Taken toward south, note lack of weeps.

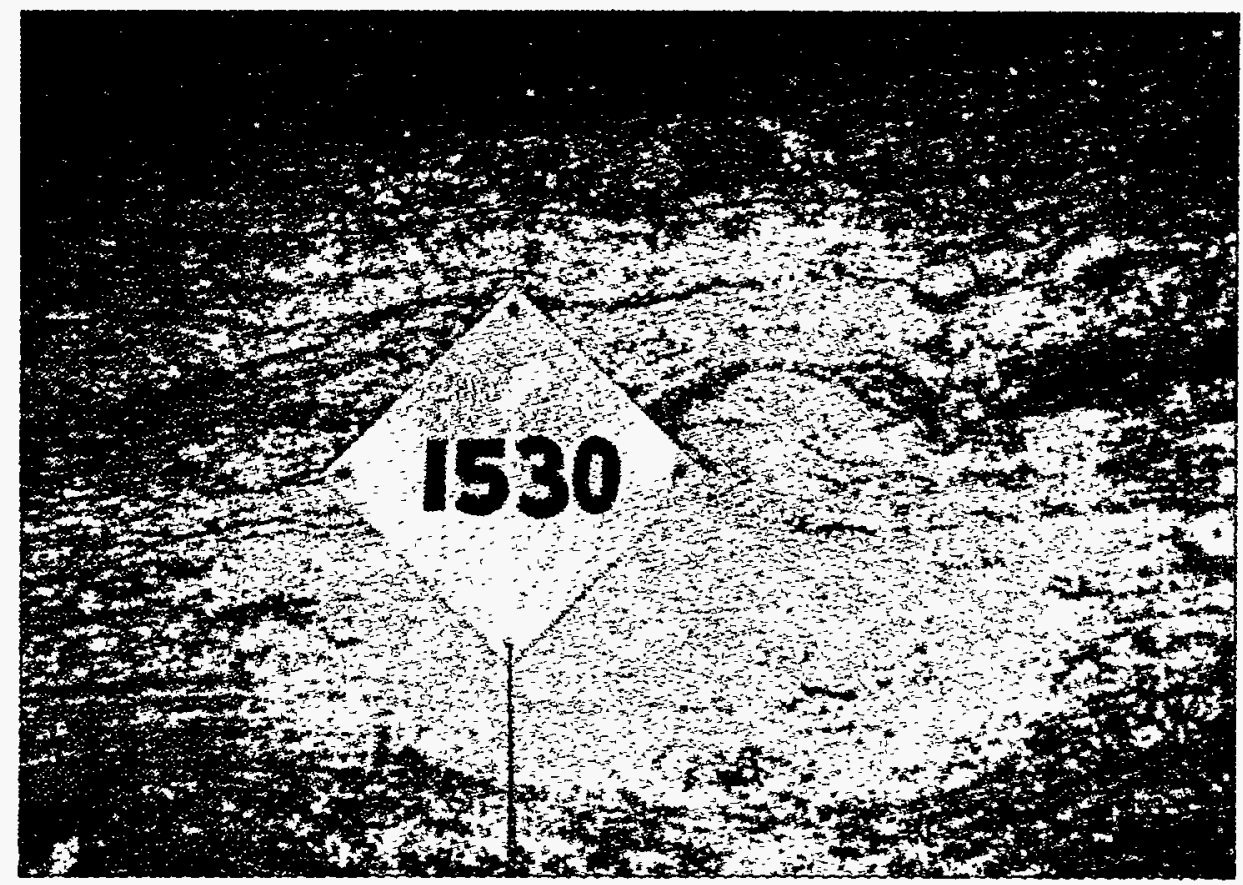

Figure C-2-4

Small encrustations at the exposure surface over argillaceous halite.

Taken south side of shaft, about $1672 \mathrm{ft}$. 


\section{Figure C-2-6}

Photograph of encrustations at exposure surfaces in argillaceous halite.

Taken toward south, about $1765-1775 \mathrm{ft}$.

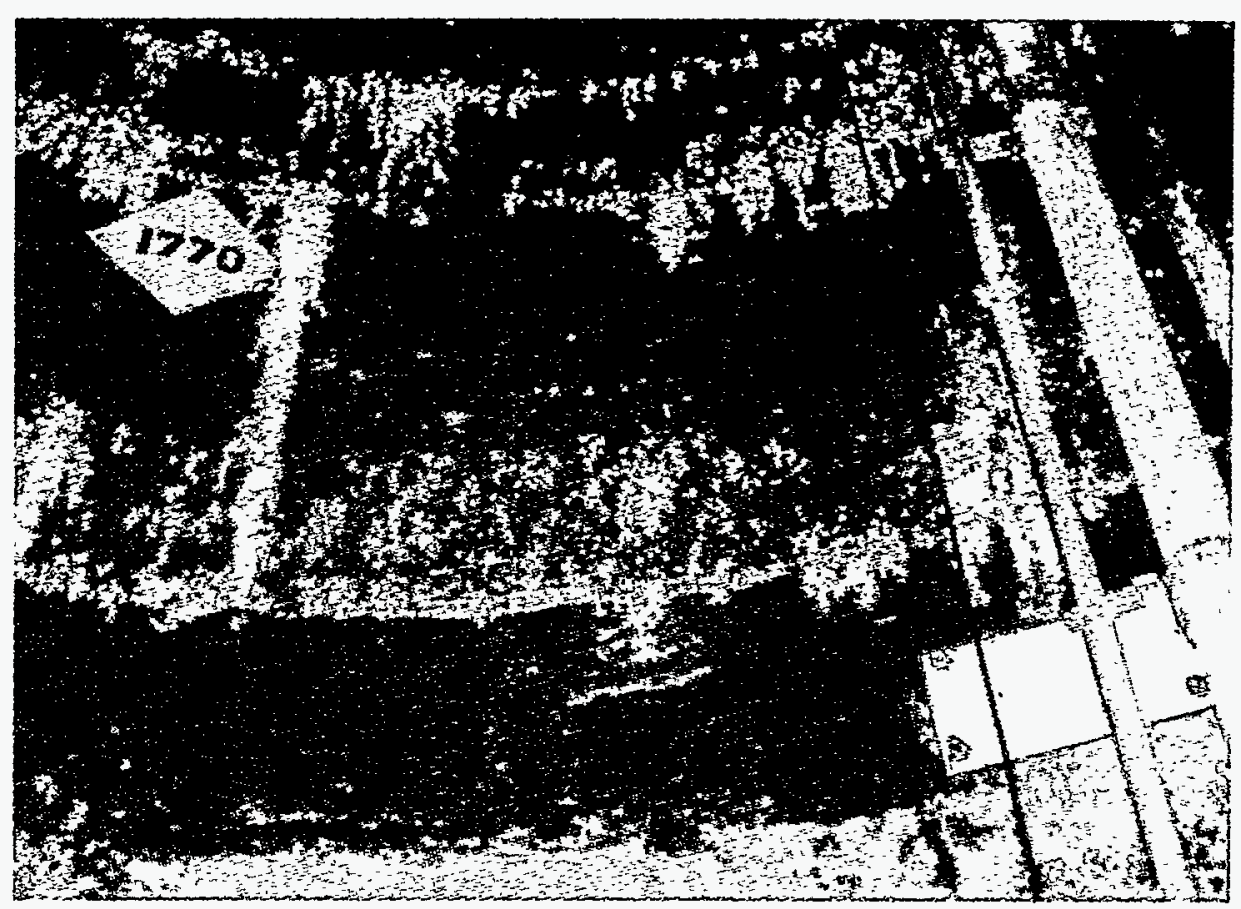

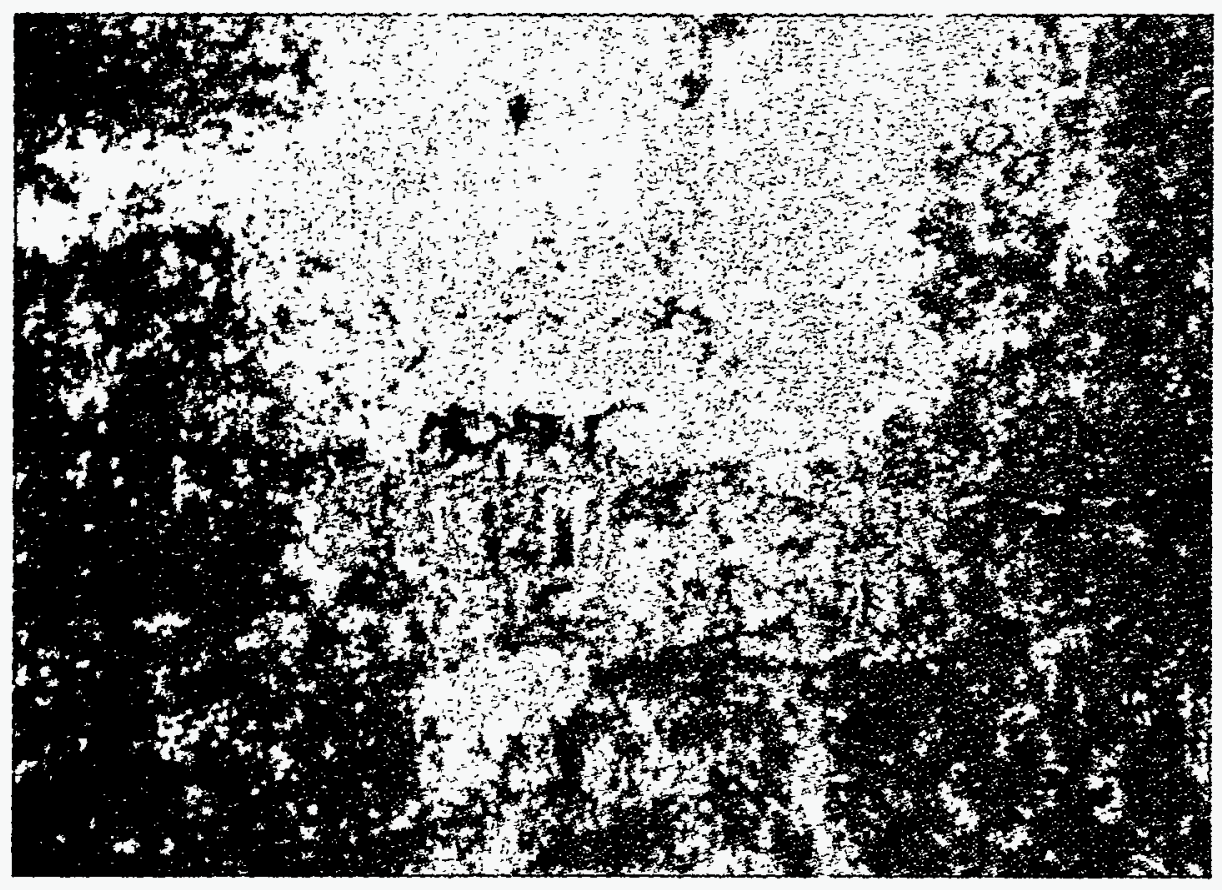

Figure $\mathrm{C}-2-7$

Photograph of small encrustations in argillaceous halite at and below exposure surface at $1825 \mathrm{ft}$.

Taken south side of shaft, about $1825 \mathrm{ft}$.

Purer halite with primary textures above exposure surface shows no encrustations. 


\section{Figure C-2-8}

Photograph of encrustations at polyhalite at $1844.5 \mathrm{ft}$.

Taken toward south, about $1844.5 \mathrm{ft}$.

Note small encrustations from primary halite with polyhalite from about 1843 $1844 \mathrm{ft}$.

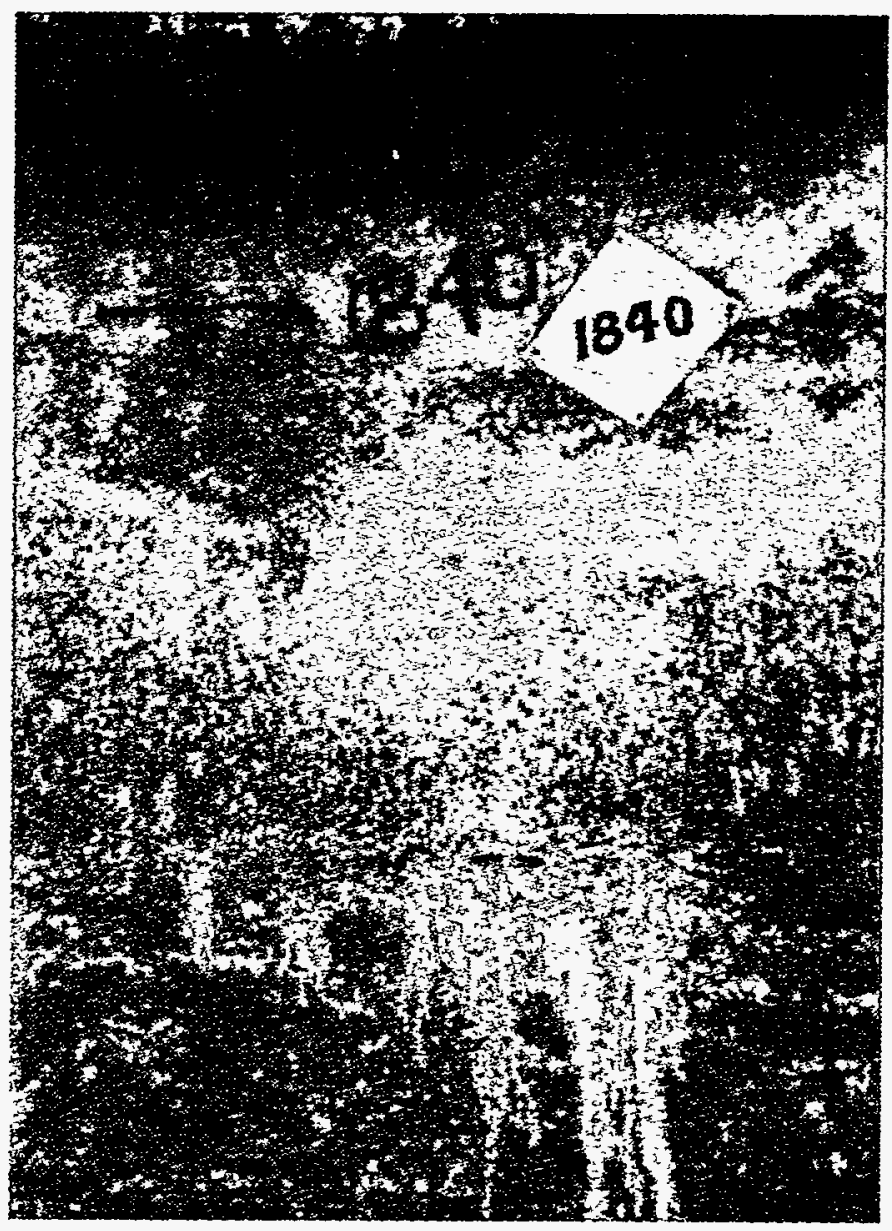

Figure C-2-9

Encrustations in claystone under MB 131 at $1863 \mathrm{ft}$. Additional encrustations from argillaceous halite below exposure surface.

Taken south side of shaft, about $1863 \mathrm{ft}$.

"Podular" halite textures are well shown in lower third of photograph. 
'H 096I moqo 'ffoys fo ap!s yłnos uวभp L

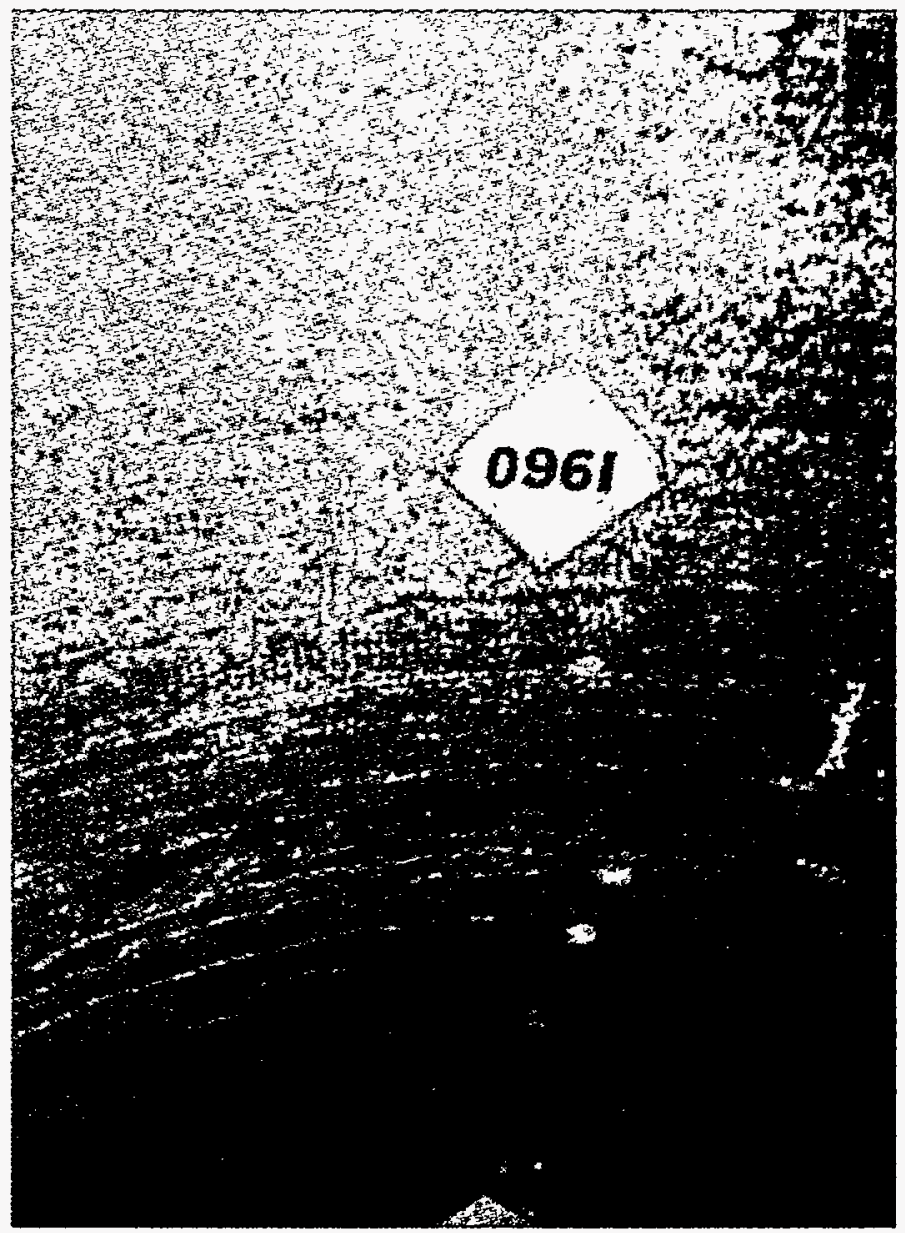

\section{เト-て-つ วגก6!」}

-aspfins adnsodxa dapun an!า y snoaวpl1!8.10 uoy suonpisndour Ilpus $270 \mathrm{~N}$

- f0t6I apau

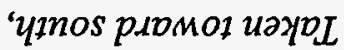

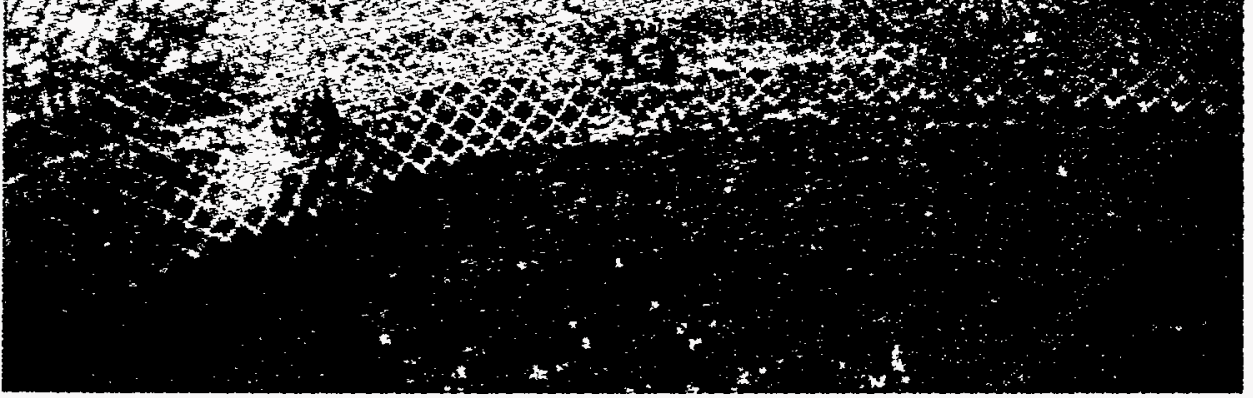

-әt!..pरिчue jo әseq fe suo!̣eisndouə 10 ydesboloud 0เ-て-つ әגก6!」 


\section{Figure $\mathbf{C - 2 - 1 2}$}

Photograph of encrustations at base anhydrite of MB 135

Taken toward south, near $1985 \mathrm{ft}$ depth.

Note small encrustations from gray argillaceous halite in synsedimentary pit or "cave" above $M B$.

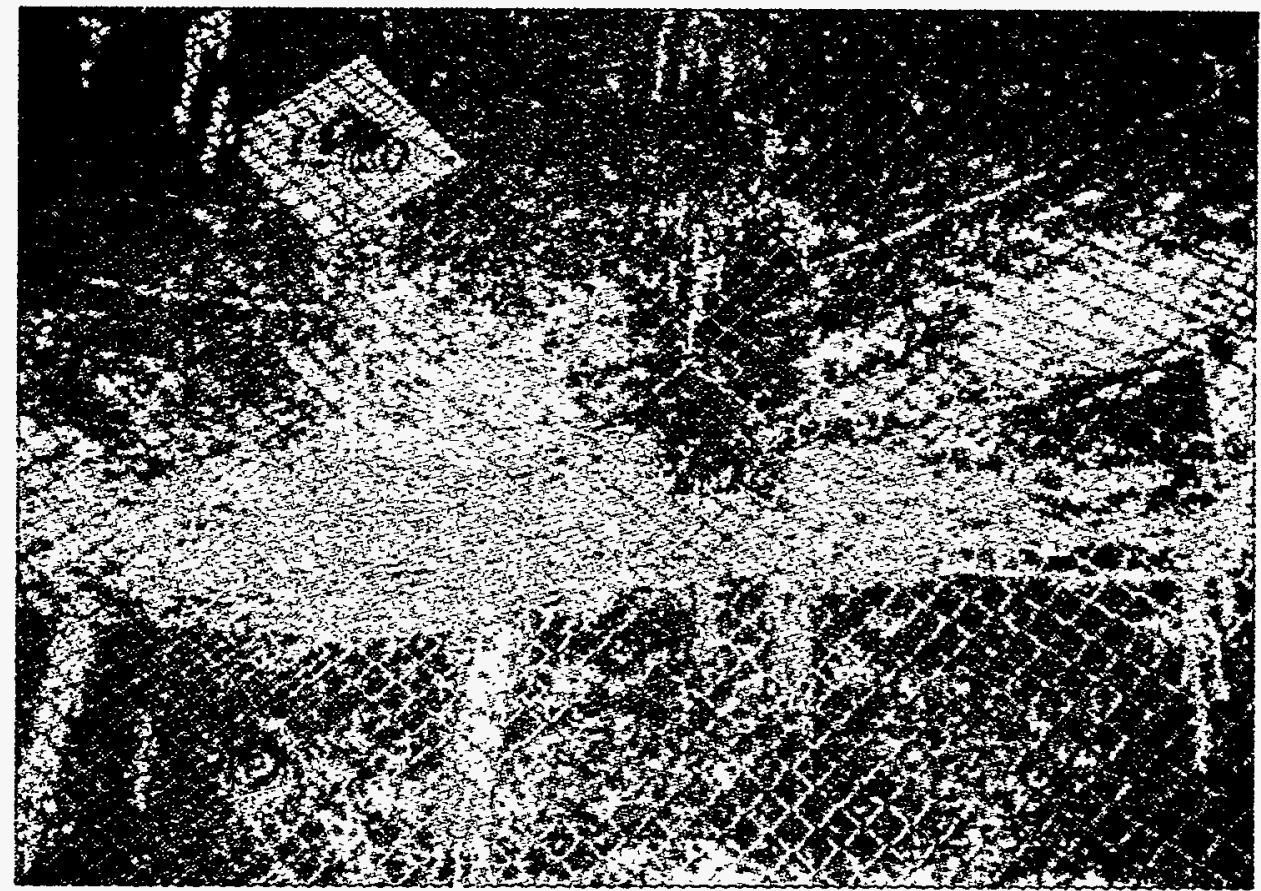

Figure C-2-13

Encrustations in argillaceous halite at top of exposure surface overlain by halite at about $1995 \mathrm{ft}$.

Taken south side of shaft, note dish-shaped structures below exposure surface, isolated encrustations. 


\section{Figure $\mathrm{C}-2-14$}

Photograph of encrustations at exposure surfaces with claystones $\mathrm{M}-1$ (above sign) and $L$ (below sign).

Taken toward south, about $2080 \mathrm{ft}$.

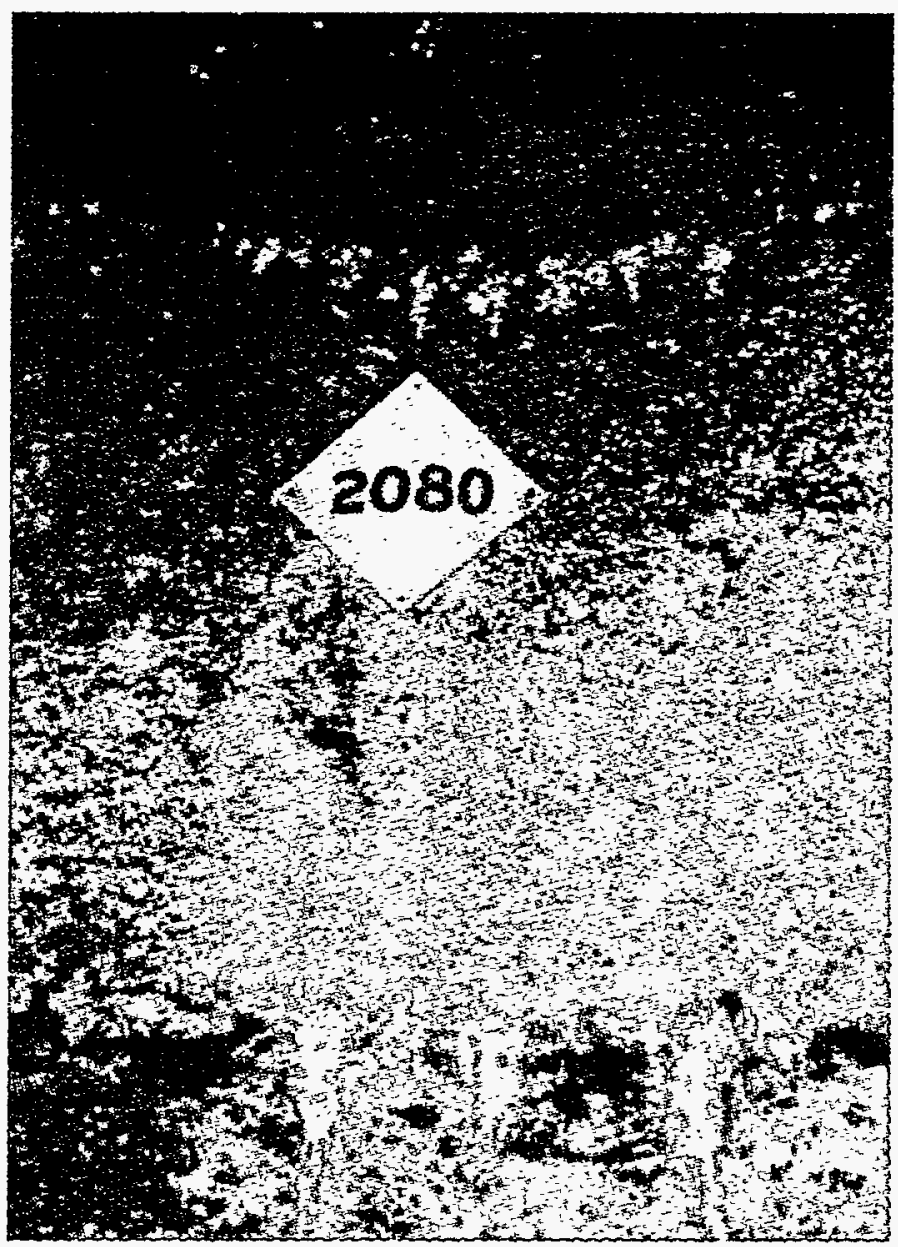

Figure C-2-15

Encrustations at claystone $K$ at exposure surface under MB 138.

Taken south side of shaft, about $2100 \mathrm{ft}$.

Clay-MB contact about middle of photograph. 


\section{Figure C-2-16}

Small encrustations at exposure surface overlying argillaceous halite and along polyhalitic laminae in halite.

Taken about $2110 \mathrm{ft}$ depth, south wall.

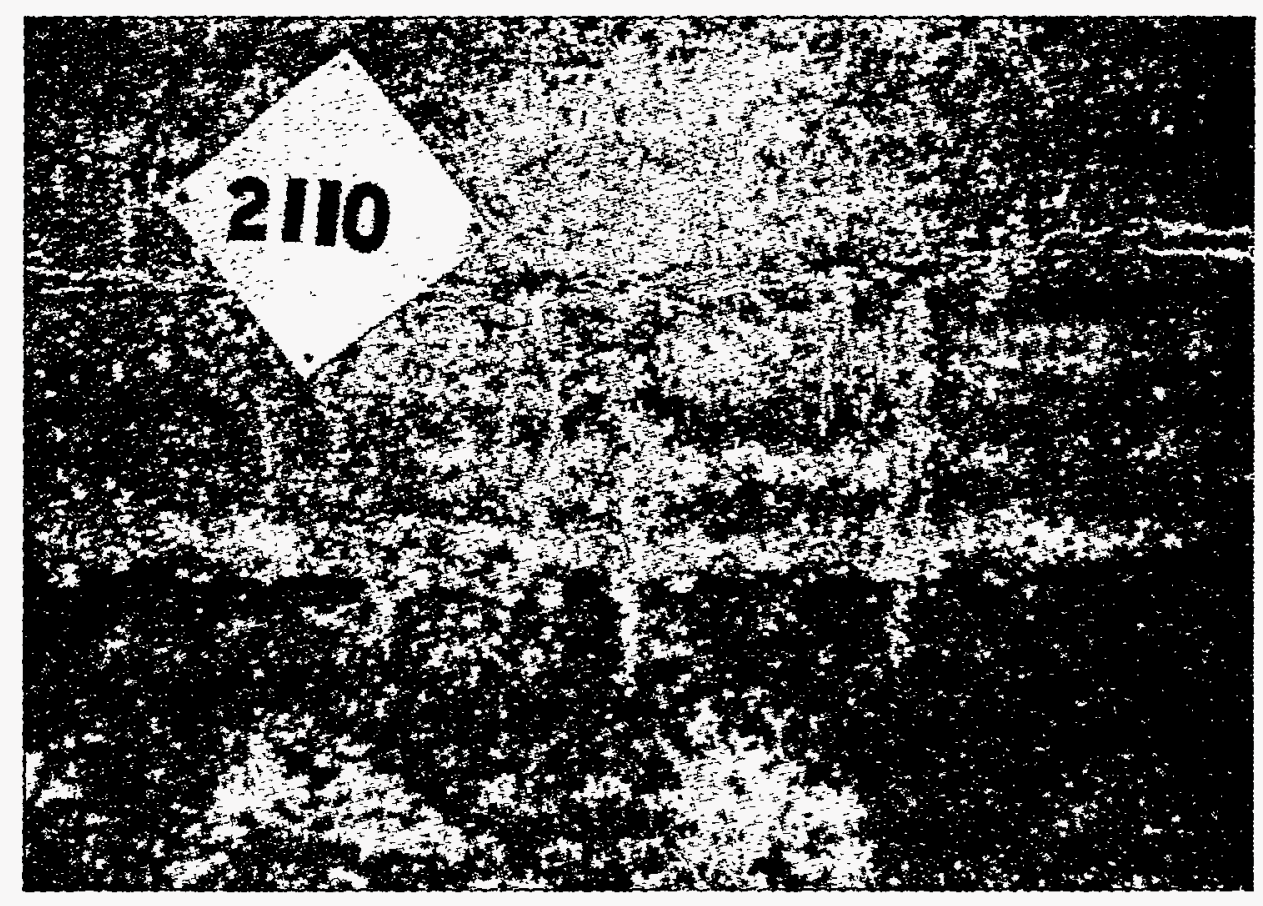




\section{APPENDIX D \\ ANALYTICAL RESULTS}

PART I-1992-1993 ANALYTICAL RESULTS FOR SALADO BRINE

PART II-ANALYTICAL RESULTS FOR CULEBRA AND CONSTRUCTION BRINES 


\section{THIS PAGE INTENTIONALLY LEFT BLANK}




\section{APPENDIX D}

\section{ANALYTICAL RESULTS}

PART I-1992-1993 ANALYTICAL RESULTS FOR SALADO BRINE 
THIS PAGE INTENTIONALLY LEFT BLANK 
TABLE D-1

ANALYTICAL RESULTS FOR SALADO BRINES

SAMPLE HOLE NUMBER

NUMBER \& DIRECTION

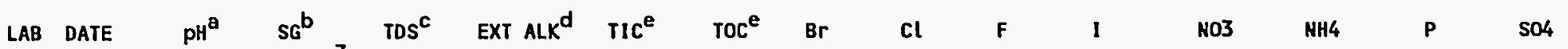
(s.u.) $\left(\mathrm{g} / \mathrm{cm}^{3}\right)(\mathrm{mg} / \mathrm{L})(\mathrm{mg} / \mathrm{L}) \quad(\mathrm{mg} / \mathrm{L}) \quad(\mathrm{mg} / \mathrm{L})(\mathrm{mg} / \mathrm{L})(\mathrm{mg} / \mathrm{L})(\mathrm{mg} / \mathrm{L})(\mathrm{mg} / \mathrm{L})(\mathrm{mg} / \mathrm{L}) \quad(\mathrm{mg} / \mathrm{L}) \quad(\mathrm{mg} / \mathrm{L})(\mathrm{mg} / \mathrm{L})$

\begin{tabular}{|c|c|c|c|c|c|c|c|c|c|c|c|c|c|c|c|c|c|c|}
\hline 4037 & A1X02 & UP & UNC & $04 / 16 / 92$ & 5.8 & 1.24 & 394000 & 897 & $<2.5$ & 15 & 2310 & 195000 & 6.8 & 15.6 & 0.3 & 166 & $<0.1$ & 21000 \\
\hline 4050 & A1X02 & UP & UNC & $08 / 20 / 92$ & 3.5 & 1.23 & 415000 & NA & $<2.5$ & 41 & 6330 & 259000 & $<1$ & 40.7 & $<1$ & 356 & 0.2 & 36600 \\
\hline 4038 & $A 3 \times 01$ & DN & UNC & $04 / 16 / 92$ & 6.1 & 1.23 & 374000 & 1019 & 4.1 & 25 & 1660 & 188000 & 6.7 & 16.2 & 0.2 & 175 & $<0.1$ & 16100 \\
\hline 4049 & $A 3 \times 01$ & DN & UNC & $08 / 20 / 92$ & 6.0 & 1.23 & 338000 & 937 & 3.0 & 15 & 1480 & 192000 & 6 & 13.6 & $<1$ & 177 & $<0.1$ & 16500 \\
\hline 4036 & $8 \times 01$ & DH & UNC & $04 / 16 / 92$ & 6.2 & 1.23 & 373000 & 897 & 2.5 & 15 & 1580 & 190000 & 5.8 & 15.5 & $<0.2$ & 148 & $<0.1$ & 16000 \\
\hline 4052 & BX01 & DN & UNC & $08 / 20 / 92$ & 6.0 & 1.22 & 344000 & 876 & 2.5 & 5 & 1460 & 189000 & 6 & 13.3 & $<1$ & 175 & $<0.1$ & 16600 \\
\hline 4024 & DH36 & DN & UNC & $04 / 16 / 92$ & 6.2 & 1.24 & 375000 & 848 & 5.1 & 36 & 1560 & 192000 & 4.6 & 24.5 & $<0.2$ & 189 & $<0.1$ & 16000 \\
\hline 4025 & DH36 & DN & UNC & $04 / 16 / 92$ & 6.2 & 1.24 & 372000 & 848 & 5.6 & 30 & 1590 & 193000 & 4.7 & 24.2 & $<0.2$ & 188 & $<0.1$ & 16000 \\
\hline 4026 & DH36 & DN & UNC & $04 / 16 / 92$ & 6.2 & 1.24 & 377000 & 848 & 5.1 & 36 & 1590 & 192000 & 4.8 & 20.2 & $<0.2$ & 164 & $<0.1$ & 16000 \\
\hline 4048 & DH36 & DN & UNC & $08 / 20 / 92$ & 6.2 & 1.23 & 348000 & 821 & 5.6 & 15 & 1450 & 192000 & 4 & 15.6 & $<1$ & 186 & $<0.1$ & 16500 \\
\hline 4034 & DH38 & $\mathrm{DN}$ & UNC & $04 / 16 / 92$ & 6.3 & 1.24 & 372000 & 958 & 4.1 & 61 & 1540 & 190000 & 4.2 & 21.2 & 0.2 & 193 & $<0.1$ & 14900 \\
\hline 4051 & DH38 & DN & UNC & $08 / 20 / 92$ & 6.2 & 1.22 & 357000 & 931 & 4.1 & 15 & 1420 & 194000 & 4 & 15.3 & $<1$ & 190 & $<0.1$ & 15600 \\
\hline 4035 & DH4O & DN & UNC & $04 / 16 / 92$ & 6.4 & 1.22 & 365000 & 1140 & 6.1 & 71 & 1580 & 186000 & 4.3 & 21.8 & 0.5 & 184 & 0.1 & 15200 \\
\hline 4055 & DH4O & DN & UNC & $08 / 20 / 92$ & 6.4 & 1.23 & 345000 & 1199 & 5.1 & 56 & 1460 & 188000 & 5 & 15.2 & $<1$ & 196 & $<0.1$ & 15700 \\
\hline 4033 & DH42 & DN & UNC & $04 / 16 / 92$ & 6.3 & 1.25 & 372000 & 915 & 3.6 & 41 & 1540 & 187000 & 4.4 & 19.7 & 0.4 & 195 & $<0.1$ & 14800 \\
\hline 4053 & DH42 & DN & UNC & $08 / 20 / 92$ & 6.2 & 1.23 & 344000 & 906 & 3.0 & 46 & 1410 & 189000 & 4 & 15.0 & $<1$ & 197 & 0.1 & 15300 \\
\hline 4021 & DH42A & DN & UNC & $04 / 16 / 92$ & 6.2 & 1.24 & 374000 & 848 & 4.1 & 15 & 1490 & 193000 & 4.4 & 23.2 & $<0.2$ & 191 & $<0.1$ & 15100 \\
\hline 4022 & DH42A & $D N$ & UNC & $04 / 16 / 92$ & 6.2 & 1.24 & 376000 & 848 & 4.6 & 10 & 1500 & 192000 & 4.2 & 25.8 & $<0.2$ & 214 & $<0.1$ & 15100 \\
\hline 4023 & $\mathrm{OH} 42 \mathrm{~A}$ & DN & UNC & $04 / 16 / 92$ & 6.2 & 1.24 & 373000 & 842 & 5.6 & 15 & 1510 & 194000 & 4.5 & 25.8 & $<0.2$ & 185 & $<0.1$ & 15100 \\
\hline 4054 & DH42A & DN & UNC & $08 / 20 / 92$ & 6.1 & 1.23 & 349000 & 833 & 3.0 & $<5$ & 1400 & 190000 & 4 & 15.2 & $<1$ & 215 & $<0.1$ & 15300 \\
\hline 4030 & DHP402A & DN & UNC & $04 / 16 / 92$ & .6 .1 & 1.24 & 378000 & 763 & 3.6 & 10 & 1690 & 192000 & 6.4 & 14.5 & 0.3 & 150 & $<0.1$ & 16700 \\
\hline 4031 & DHP402A & $D N$ & UNC & $04 / 16 / 92$ & 6.1 & 1.24 & 381000 & 787 & 3.6 & 15 & 1670 & 193000 & 6.3 & 14.3 & $<0.2$ & 142 & $<0.1$ & 16600 \\
\hline 4032 & DHP402A & DN & UNC & $04 / 16 / 92$ & 6.1 & 1.25 & 380000 & 787 & 2.5 & 10 & 1690 & 192000 & 6.2 & 14.5 & 0.2 & 138 & $<0.1$ & 16600 \\
\hline 4047 & DHP402A & DH & UNC & $08 / 20 / 92$ & 6.0 & 1.23 & 339000 & 730 & 7.1 & 15 & 1540 & 192000 & 7 & 12.0 & $<1$ & 175 & $<0.1$ & 17400 \\
\hline 4027 & GSEEP & DN & UNC & $04 / 16 / 92$ & 6.3 & 1.24 & 374000 & 946 & 3.0 & $<5$ & 1520 & 186000 & 3.2 & 21.9 & $<0.2$ & 205 & $<0.1$ & 26700 \\
\hline 4028 & GSEEP & DH & UNC & $04 / 16 / 92$ & 6.3 & 1.24 & 373000 & 958 & 2.5 & $<5$ & 1520 & 186000 & 3.0 & 20.7 & 0.3 & 197 & $<0.1$ & 26700 \\
\hline
\end{tabular}

a s.u. = standard units

b Specific Gravity

C Total Disolved Solids

${ }^{d}$ Extended Alkal inity measured to an endpoint $\mathrm{pH}$ of 2.5 and reported as equivalent bicarbonate $\left(\mathrm{HCO}_{3}\right)$.

e TIC (Total Inorganic Carbon) and TOC (Total Organic Carbon) are reported as equivalent bicarbonate.

HA Not available.

DN DOWn

HZ Horizontal 
TABLE $D-1$

ANALYTICAL RESULTS FOR SALADO BRINES

SAMPLE MOLE NUMBER

NUMBER \& DIRECTION

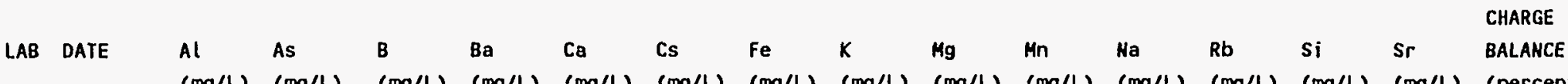
$(m g / L)(m g / L) \quad(m g / L)(m g / L)(m g / L) \quad(m g / L)(m g / L)(m g / L)(m g / L)(m g / L)(m g / L)(m g / L)(m g / L)(m g / L)(p e r c e n t)$

\begin{tabular}{|c|c|c|c|c|c|c|c|c|c|c|c|c|c|c|c|c|c|c|}
\hline 4037 & $\mathrm{~A} 1 \times 02$ & up & UNC & $04 / 16 / 92<0.05$ & 0.01 & 1700 & 0.053 & 252 & 0.379 & $<0.50$ & 15700 & 35000 & 4.69 & 63000 & 18.5 & 1.29 & 5.77 & MA \\
\hline 4050 & A1X02 & UP & UNC & $08 / 20 / 92$ & 0.02 & 4590 & 0.026 & 27 & 0.566 & 0.56 & 13800 & 86000 & 5.27 & 8300 & 14.9 & 3.37 & $<0.1$ & NA \\
\hline 4038 & $A 3 \times 01$ & DN & UNC & $04 / 16 / 92<0.05$ & $<0.001$ & 1590 & 0.081 & 249 & 0.368 & $<0.50$ & 16200 & 24000 & 1.51 & 78000 & 16.2 & 1.60 & 1.75 & NA \\
\hline 4049 & $A 3 \times 01$ & DN & UNC & $08 / 20 / 92<0.05$ & 0.002 & 1600 & 0.061 & 245 & 0.368 & $<0.50$ & 16100 & 23200 & 1.45 & 78000 & 15.5 & 1.07 & 1.76 & NA \\
\hline 4036 & $8 \times 01$ & DN & UNC & $04 / 16 / 92<0.05$ & 0.001 & 1560 & 0.074 & 256 & 0.336 & $<0.50$ & 16200 & 22700 & 1.25 & 80000 & 15.2 & 1.22 & 1.80 & NA \\
\hline 4052 & BX01 & DN & UNC & $08 / 20 / 92 \quad 0.06$ & 0.002 & 1570 & 0.067 & 241 & 0.356 & $<0.50$ & 16100 & 22200 & 1.31 & 78000 & 15.3 & 0.76 & 1.79 & NA \\
\hline 4024 & DH36 & DH & UNC & $04 / 16 / 92<0.05$ & 0.003 & 1680 & 0.14 & 296 & 0.285 & $<0.50$ & 18300 & 18400 & 1.12 & 83000 & 14.6 & 1.91 & 1.22 & NA \\
\hline 4025 & DH36 & $D N$ & UNC & $04 / 16 / 92<0.05$ & 0.003 & 1670 & 0.123 & .301 & 0.29 & $<0.50$ & 18800 & 18900 & 1.12 & 84000 & 14.3 & 1.83 & 1.28 & NA \\
\hline 4026 & DH36 & DN & UNC & $04 / 16 / 92<0.05$ & 0.003 & 1660 & 0.064 & 294 & 0.281 & $<0.50$ & 18200 & 19300 & 1.09 & 85000 & 14.2 & 1.88 & 1.20 & NA \\
\hline 4048 & DH36 & DN & UNC & $08 / 20 / 92 \quad 0.06$ & 0.009 & 1650 & 0.095 & 286 & 0.295 & 1.67 & 17700 & 18700 & 1.09 & 86000 & 14.1 & 1.70 & 1.21 & NA \\
\hline 4034 & DH38 & DN & UNC & $04 / 16 / 92<0.05$ & $<0.001$ & 1610 & 0.039 & 305 & 0.267 & $<0.50$ & 18000 & 18300 & 0.94 & 86000 & 14.0 & 2.45 & 0.77 & NA \\
\hline 4051 & DH38 & $D H$ & UNC & $08 / 20 / 92<0.05$ & $<0.001$ & 1630 & 0.06 & 277 & .0 .283 & $<0.50$ & 17700 & 18300 & 0.97 & 85000 & 13.9 & 1.48 & 0.74 & NA \\
\hline 4035 & $\mathrm{DH} 40$ & $\mathrm{DN}$ & UNC & $04 / 16 / 92<0.05$ & 0.002 & 1600 & 0.087 & 302 & 0.259 & $<0.50$ & 18900 & 18200 & 1.72 & 84000 & 13.8 & 2.34 & 0.96 & NA \\
\hline 4055 & DH4O & DN & UNC & $08 / 20 / 92<0.05$ & 0.001 & 1640 & 0.056 & 270 & 0.270 & $<0.50$ & 18200 & 18300 & 3.14 & 84000 & 14.0 & 1.69 & 0.84 & NA \\
\hline 4033 & DH42 & DN & UNC & $04 / 16 / 92<0.05$ & 0.004 & 1600 & 0.055 & 302 & 0.256 & $<0.50$ & 18300 & 17800 & 1.07 & 85000 & 13.5 & 1.82 & 0.84 & NA \\
\hline 4053 & DH42 & DN & UNC & $08 / 20 / 92 \quad 0.08$ & 0.006 & 1630 & 0.056 & 294 & 0.259 & $<0.50$ & 18100 & 17500 & 1.12 & 86000 & 13.5 & 1.42 & 0.80 & NA \\
\hline 4021 & DH42A & DN & UNC & $04 / 16 / 92<0.05$ & 0.004 & 1620 & 0.058 & 294 & 0.253 & $<0.50$ & 18700 & 17700 & 1.00 & 88000 & 13.7 & 2.21 & 0.75 & NA \\
\hline 4022 & DH42A & DN & UNC & $04 / 16 / 92<0.05$ & 0.004 & 1630 & 0.046 & 291 & 0.253 & $<0.50$ & 19100 & 17900 & 0.96 & 88000 & 13.2 & 2.21 & 0.75 & NA \\
\hline 4023 & DH42A & DN & UNC & $04 / 16 / 92<0.05$ & 0.004 & 1620 & 0.057 & 296 & 0.253 & $<0.50$ & 19100 & 17800 & 0.97 & 87000 & 13.7 & 2.12 & 0.75 & NA \\
\hline 4054 & DH42A & DN & UNC & $08 / 20 / 92 \quad 0.06$ & 0.004 & 1540 & 0.056 & 273 & 0.251 & $<0.50$ & 17000 & 17000 & 0.98 & 84000 & 12.9 & 1.57 & 0.75 & NA \\
\hline 4030 & DHP402A & $\mathrm{DN}$ & UNC & $04 / 16 / 92<0.05$ & $<0.001$ & 1370 & 0.065 & 294 & 0.368 & 67.5 & 14900 & 24600 & 2.56 & 75000 & 15.5 & 0.50 & 2.70 & NA \\
\hline 4031 & DHP402A & DN & UNC & $04 / 16 / 92<0.05$ & $<0.001$ & 1380 & 0.056 & 280 & 0.363 & 21.4 & 15000 & 25400 & 2.18 & 77000 & 15.8 & $<0.5$ & 2.44 & NA \\
\hline 4032 & DHP402A & DH & UNC & $04 / 16 / 92<0.05$ & $<0.001$ & 1390 & 0.08 & 283 & 0.352 & 14.0 & 15000 & 25200 & 2.10 & 77000 & 15.3 & $<0.5$ & 2.49 & NA \\
\hline 4047 & DHP402A & $\mathrm{DN}$ & UNC & $08 / 20 / 92<0.05$ & 0.002 & 1540 & 0.155 & 270 & 0.371 & $>99$ & 14800 & 26900 & 3.10 & 79000 & 15.1 & $<0.5$ & 2.47 & NA \\
\hline 4027 & GSEEP & DN & UNC & $04 / 16 / 92<0.05$ & 0.006 & 1720 & 0.061 & 249 & 0.215 & $<0.50$ & 14300 & 15100 & 0.64 & 96000 & 12.2 & 1.25 & 1.96 & NA \\
\hline 4028 & GSEEP & $O N$ & UNC & $04 / 16 / 92<0.05$ & 0.007 & 1750 & 0.074 & 260 & 0.217 & $<0.50$ & 14500 & 15000 & 0.64 & 94000 & 12.2 & 1.22 & 2.24 & NA \\
\hline 4029 & GSEEP & DN & UNC & $04 / 16 / 92<0.05$ & 0.006 & 1710 & 0.031 & 251 & 0.223 & $<0.50$ & 14800 & 15200 & 0.63 & 95000 & 12.3 & 1.35 & 1.96 & NA \\
\hline
\end{tabular}


TABLE D-1

ANALYTICAL RESULTS FOR BALADO BRINES

\begin{tabular}{|c|c|c|c|c|c|c|c|c|c|c|c|c|c|c|c|c|c|c|}
\hline $\begin{array}{l}4029 \\
\text { SAMPLE } \\
\text { NUMBER }\end{array}$ & $\begin{array}{l}\text { GSEEP } \\
\text { HOLE NUMB } \\
\text { \& DIRECTI }\end{array}$ & $\begin{array}{l}D H \\
E R \\
O N\end{array}$ & UNC & $04 / 16 / 92$ & $\begin{array}{l}\mathrm{PH}^{\mathrm{a}} \\
\left(s . u_{.}\right)\end{array}$ & $\begin{array}{l}S G^{b} \\
\left(g / \mathrm{cm}^{3}\right)\end{array}$ & $\begin{array}{l}\operatorname{TDS}^{c} \\
(\mathrm{mg} / \mathrm{L})\end{array}$ & $\begin{array}{l}\text { EXT ALK } \\
(m g / L)\end{array}$ & $\begin{array}{l}\text { TIC }^{\mathrm{e}} \\
(\mathrm{mg} / \mathrm{L})\end{array}$ & $\begin{array}{l}\operatorname{TOC}^{\mathrm{e}} \\
(\mathrm{mg} / \mathrm{L})\end{array}$ & $\begin{array}{l}\mathrm{Br} \\
(\mathrm{mg} / \mathrm{L})\end{array}$ & $\begin{array}{l}\mathrm{cl} \\
(\mathrm{mg} / \mathrm{L})\end{array}$ & $\begin{array}{l}F \\
(m g / L)\end{array}$ & $\begin{array}{l}1 \\
(m g / L)\end{array}$ & $\begin{array}{l}\text { N03 } \\
\text { (mg/L) }\end{array}$ & $\begin{array}{l}\text { NH4 } \\
\text { (mg/L) }\end{array}$ & $\begin{array}{l}P \\
(m g / L)\end{array}$ & $\begin{array}{l}\mathrm{SO}_{4} \\
(\mathrm{mg} / \mathrm{L})\end{array}$ \\
\hline 4046 & GSEEP & $\mathrm{DN}$ & UMC & $08 / 20 / 92$ & 6.3 & 1.24 & 359000 & 943 & $<2.5$ & $<5$ & 1410 & 184000 & 4 & 17.0 & $<1$ & 199 & $<0.1$ & 26900 \\
\hline 4039 & $\mathrm{OH} 2 \mathrm{O}$ & $\mathrm{HZ}$ & UNC & $04 / 16 / 92$ & 6.0 & 1.23 & 385000 & 656 & 3.6 & 56 & 1500 & 193000 & 4.8 & 15.1 & 1.2 & 131 & $<0.1$ & 17100 \\
\hline 4058 & ОH2O & $H Z$ & UNC & $08 / 20 / 92$ & 5.9 & 1.22 & 354000 & 675 & 3.0 & 56 & 1480 & 188000 & $4^{\prime}$ & 13.3 & $<1$ & 175 & $<0.1$ & 16500 \\
\hline 4044 & OH22 & $\mathbf{H Z}$ & UNC & $04 / 16 / 92$ & 6.3 & 1.23 & 371000 & 946 & 5.1 & 81 & 1570 & 188000 & 4.1 & 15.7 & 0.3 & 150 & 0.2 & 15400 \\
\hline 4040 & OH23 & $H Z$ & UMC & $04 / 16 / 92$ & 6.0 & 1.22 & 371000 & 614 & 3.0 & 41 & 1530 & 190000 & 5.2 & 15.2 & 1.1 & 135 & $<0.1$ & 16400 \\
\hline 4056 & $\mathrm{OH} 23$ & $H Z$ & UNC & $08 / 20 / 92$ & 5.9 & 1.22 & 335000 & 597 & $<2.5$ & 36 & 1460 & 185000 & 4 & 13.0 & $<1$ & 170 & $<0.1$ & 15800 \\
\hline 4045 & $\mathrm{OH} 24$ & $\mathrm{HZ}$ & UNC & $04 / 16 / 92$ & 6.3 & 1.23 & 376000 & 909 & 4.6 & 86 & 1610 & 190000 & 4.7 & 16.3 & 0.5 & 175 & 0.4 & 16000 \\
\hline 4041 & OH26 & $\mathrm{HZ}$ & UNC & $04 / 16 / 92$ & 6.1 & 1.23 & 379000 & 681 & 2.5 & 66 & 1530 & 189000 & 4.8 & 15.2 & 0.9 & 136 & 0.1 & 16100 \\
\hline 4057 & $\mathrm{OH} 26$ & $\mathrm{HZ}$ & UNC & $08 / 20 / 92$ & 6.0 & 1.22 & 340000 & 687 & $<2.5$ & 61 & 1480 & 186000 & 4 & 13.5 & $<1$ & 180 & 0.1 & 15800 \\
\hline 4059 & OH28 & DN & UNC & $08 / 20 / 92$ & 6.0 & 1.22 & 335000 & 760 & 10.2 & 97 & 1470 & 188000 & 5 & 14.3 & $<1$ & 168 & 0.7 & 16300 \\
\hline 4042 & OH45 & $\mathrm{HZ}$ & UNC & $04 / 16 / 92$ & 6.3 & 1.23 & 372000 & 946 & 6.6 & 91 & 1650 & 188000 & 4.8 & 15.8 & 0.6 & 150 & $<0.1$ & 16000 \\
\hline 4043 & $0 H 46$ & DN & UNC & $04 / 16 / 92$ & 6.3 & 1.23 & 364000 & 946 & 6.1 & 36 & 1640 & 188000 & 4.7 & 15.8 & $<0.2$ & 149 & $<0.1$ & 15000 \\
\hline 4060 & $\mathrm{OH} 46$ & DN & UNC & $08 / 20 / 92$ & 6.1 & 1.22 & 339000 & 1016 & 4.6 & 30 & 1600 & 188000 & 5 & 14.4 & $<1$ & 182 & $<0.1$ & 16000 \\
\hline
\end{tabular}

\footnotetext{
a.u. = standard units

b Specific Gravity

c Total Disolved Solids

d Extended Alkalinity measured to an endpoint $\mathrm{pH}$ of 2.5 and reported as equivalent bicarbonate $\left(\mathrm{HCO}_{3}\right)$.

e TIC (Total Inorganic Carbon) and TOC (Total Organic Carbon) are reported as equivalent bicarbonate.

NA Not available.

DN Down

HZ Horizontal
} 
TABLE D-1

ANALYTICAL RESULTS FOR SALADO BRINES

SAMPLE HOLE NUMBER

NUMBER \& DIRECTIOH

LAB DATE

AI

Al As B

$\operatorname{res}$

.

$(m g / L)(m g / L) \quad(m g / L)(m g / L)(m g / L)(m g / L)(m g / L)(m g / L)(m g / L)(m g / L)(m g / L)(m g / L)(m g / L)(m g / L)(p e r c e n t)$

\begin{tabular}{|c|c|c|c|c|c|c|c|c|c|c|c|c|c|c|c|c|c|c|c|}
\hline 4046 & GSEEP & DN & UNC & $08 / 20 / 92$ & $<0.05$ & 0.006 & 1800 & 0.045 & 245 & 0.235 & $<0.50$ & 14500 & 15700 & 0.71 & 97000 & 12.3 & 0.93 & 2.01 & NA \\
\hline 4039 & ОН20 & $\mathrm{HZ}$ & UNC & $04 / 16 / 92$ & $<0.05$ & 0.002 & 1480 & 0.03 & 336 & 0.281 & $<0.50$ & 15600 & 21600 & 1.87 & 82000 & 15.1 & 1.43 & 1.54 & NA \\
\hline 4058 & $\mathrm{OH} 20$ & $\mathrm{HZ}$ & UNC & $08 / 20 / 92$ & 0.07 & 0.002 & 1420 & 0.095 & 267 & 0.281 & $<0.50$ & 14800 & 21400 & 2.11 & 78000 & 14.3 & 1.88 & 0.98 & NA \\
\hline 4044 & ОН22 & $\mathrm{HZ}$ & UNC & $04 / 16 / 92$ & $<0.05$ & 0.002 & 1510 & 0.1 & 291 & 0.298 & 1.16 & 16200 & 21400 & 1.58 & 78000 & 15.3 & 2.96 & 0.95 & NA \\
\hline 4040 & ОН23 & $\mathrm{HZ}$ & UNC & $04 / 16 / 92$ & 0.06 & 0.002 & 1470 & 0.045 & 279 & 0.285 & $<0.50$ & 15400 & 21900 & 1.73 & 80000 & 15.3 & 1.54 & 0.89 & NA \\
\hline 4056 & ОН 23 & $\mathrm{HZ}$ & UNC & $08 / 20 / 92$ & 0.13 & 0.002 & 1490 & 0.06 & 270 & 0.288 & $<0.50$ & 15400 & 21300 & 1.70 & 80000 & 14.8 & 0.94 & 0.87 & NA \\
\hline 4045 & ОН24 & $\mathrm{HZ}$ & UNC & $04 / 16 / 92$ & $<0.05$ & 0.003 & 1510 & 0.062 & 294 & 0.291 & $<0.50$ & 15900 & 22300 & 1.85 & 80000 & 15.0 & 2.96 & 0.97 & NA \\
\hline 4041 & $\mathrm{OH} 26$ & $\mathrm{HZ}$ & UNC & $04 / 16 / 92$ & $<0.05$ & $<0.001$ & 1460 & 0.061 & 276 & 0.290 & $<0.50$ & 15500 & 22200 & 1.70 & 80000 & 14.9 & 1.32 & 0.83 & NA \\
\hline 4057 & $\mathrm{OH} 26$ & $\mathrm{HZ}$ & UNC & $08 / 20 / 92$ & 0.18 & 0.001 & 1470 & 0.051 & 261 & 0.294 & $<0.50$ & 15000 & 21600 & 1.67 & 79000 & 14.8 & 0.75 & 0.79 & NA \\
\hline 4059 & $\mathrm{OH} 28$ & DN & UNC & $08 / 20 / 92$ & 0.12 & 0.002 & 1520 & 0.061 & 314 & 0.291 & 1.22 & 15700 & 21500 & 1.79 & 80000 & 14.8 & 1.01 & 1.36 & NA \\
\hline 4042 & OH45 & $\mathrm{HZ}$ & UNC & $04 / 16 / 92$ & 0.2 & 0.002 & 1460 & 0.116 & 286 & 0.272 & $<0.50$ & 16400 & 21000 & 1.54 & 77000 & 15.0 & 1.40 & 2.12 & NA \\
\hline 4043 & $\mathrm{OH} 46$ & DN & UNC & $04 / 16 / 92$ & $<0.05$ & $<0.001$ & 1520 & 0.037 & 277 & 0.286 & $<0.50$ & 16100 & 20800 & 1.31 & 79000 & 14.9 & 1.09 & 1.69 & NA \\
\hline 4060 & 0446 & DN & UNC & $08 / 20 / 92$ & 0.09 & 0.001 & 1590 & 0.088 & 264 & 0.308 & $<0.50$ & 20000 & 21500 & 1.47 & 79000 & 15.3 & 0.73 & 0.98 & NA \\
\hline
\end{tabular}

NA Not available. 


\section{APPENDIX D ANALYTICAL RESULTS}

PART II- ANALYTICAL RESULTS FOR CULEBRA AND CONSTRUCTION BRINES 
THIS PAGE INTENTIONALLY LEFT BLANK 
TABLE D-2

SAMPLE HOLE NUMBER

NUMBER \& DIRECTION

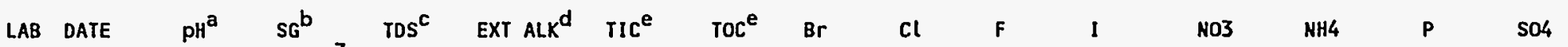
(s.u.) $\left(\mathrm{g} / \mathrm{cm}^{3}\right)(\mathrm{mg} / \mathrm{L})(\mathrm{mg} / \mathrm{L}) \quad(\mathrm{mg} / \mathrm{L}) \quad(\mathrm{mg} / \mathrm{L})(\mathrm{mg} / \mathrm{L})(\mathrm{mg} / \mathrm{L})(\mathrm{mg} / \mathrm{L})(\mathrm{mg} / \mathrm{L})(\mathrm{mg} / \mathrm{L})(\mathrm{mg} / \mathrm{L})(\mathrm{mg} / \mathrm{L})(\mathrm{mg} / \mathrm{L})$

\begin{tabular}{|c|c|c|c|c|c|c|c|c|c|c|c|c|c|c|c|c|c|c|}
\hline 1062 & Als & CLBRA & UNC & $03 / 03 / 90$ & 8.1 & 1.04 & 48000 & 113 & 72.6 & 10 & 28 & 20600 & 0.7 & 0.2 & 0.1 & 0.34 & $<0.1$ & 8230 \\
\hline 0482 & AIs & SUMP & UNC & $07 / 29 / 88$ & 7.1 & 1.21 & 330000 & 122 & 94.0 & 10 & 37 & 190000 & 4 & $<0.1$ & 5 & 4.3 & $<1$ & 6150 \\
\hline 0484 & AIS & SUMP & UNC & $07 / 29 / 88$ & 7.1 & 1.21 & 333000 & 116 & 94.5 & 5 & 44 & 188000 & $<1$ & $<0.1$ & 6 & 4.0 & $<1$ & 6180 \\
\hline 0486 & Als & SUMP & UNC & $07 / 29 / 88$ & 7.1 & 1.21 & 333000 & 128 & 91.9 & 30 & 44 & 187000 & $<1$ & $<0.1$ & 6 & 4.5 & $<1$ & 6170 \\
\hline 0747 & AIs & SUMP & UNC & $04 / 06 / 89$ & 8.2 & 1.06 & 96000 & 122 & 77.7 & 20 & 35 & 50700 & 1.4 & 0.2 & $<1$ & 0.33 & $<0.1$ & 7750 \\
\hline 0748 & AIS & SUMP & UNC & $04 / 26 / 89$ & 7.4 & 1.20 & 331000 & 177 & 112.8 & 91 & 90 & 187000 & $<1$ & 0.2 & .10 & 3.23 & $<0.1$ & 10700 \\
\hline 0738 & B\&E & BRINE & UNC & $03 / 14 / 89$ & 6.6 & 1.21 & 324000 & 201 & 201 & 5 & 26 & 187000 & 6 & 1.6 & 5 & 0.39 & $<0.3$ & 3610 \\
\hline 0740 & B\&E & BRINE & UNC & $03 / 14 / 89$ & 6.5 & 1.22 & 324000 & 188 & 191 & 51 & 19 & 187000 & 2 & 1.4 & 4 & 0.26 & $<0.3$ & 3590 \\
\hline 0742 & B\&E & BRINE & UNC & $03 / 14 / 89$ & 6.8 & 1.22 & 324000 & 183 & 179 & 36 & 23 & 187000 & $<1$ & 1.4 & 4 & 0.28 & $<0.3$ & 3590 \\
\hline
\end{tabular}

Al As $\quad$ B

Ba

\section{.}

CHARGE

$\begin{array}{lllllllllllllll}(m g / L) & (m g / L) & (m g / L) & (m g / L) & (m g / L) & (m g / L) & (m g / L) & (m g / L) & (m g / L) & (m g / L) & (m g / L) & (m g / L) & (m g / L) & (m g / L) & (p e r c e n t)\end{array}$

\begin{tabular}{|c|c|c|c|c|c|c|c|c|c|c|c|c|c|c|c|c|c|c|}
\hline 1062 & AIS CLBRA & UNC & $03 / 03 / 90$ & $<0.05$ & $<0.001$ & 35 & 0.027 & 822 & $N A$ & 0.51 & 376 & 568 & $<0.5$ & 15800 & NA & 3.84 & 10.72 & 1.95 \\
\hline 0482 & AIS SUMP & UNC & $07 / 29 / 88$ & 0.167 & 0.002 & 13 & 0.236 & 950 & 0 NA & 0.13 & 1720 & 1040 & 0.39 & 118000 & NA & 3.32 & 30.7 & -1.67 \\
\hline 0484 & AIS SUMP & UNC & $07 / 29 / 88$ & 0.163 & 0.002 & 12 & 0.239 & 950 & NA & 0.12 & 1720 & 1040 & 0.39 & 118000 & NA & 3.33 & 30.5 & -1.16 \\
\hline 0486 & AIS SUMP & UNC & $07 / 29 / 88$ & 0.169 & 0.002 & 13 & 0.215 & 960 & $N A$ & 0.12 & 1720 & 1040 & 0.4 & 118000 & NA & 3.26 & 30.5 & -0.89 \\
\hline 0747 & AIS SUMP & UNC & $04 / 06 / 89$ & 1.35 & 0.002 & 30 & 0.069 & 989 & $<0.01$ & 0.98 & 496 & 629 & 0.18 & 32600 & NA & 8.19 & 13.9 & -1.99 \\
\hline 0748 & AIS SUMP & UNC & $04 / 26 / 89$ & 2.41 & 0.002 & 31 & 0.196 & 669 & 0.021 & 1.42 & 3210 & 1630 & $<0.1$ & 121000 & $\mathrm{NA}$ & 8.56 & 33.4 & 0.10 \\
\hline 0738 & B\&E BRINE & UNC & $03 / 14 / 89$ & $<0.05$ & 0.004 & 2 & 0.106 & 1550 & 0.054 & $<0.5$ & 11 & 43 & $<0.1$ & 120000 & NA & 49.3 & 23.8 & -0.50 \\
\hline 0740 & B\&E BRINE & UNC & $03 / 14 / 89$ & $<0.05$ & 0.003 & 2 & 0.104 & 1500 & 0.01 & $<0.5$ & 11 & 44 & $<0.1$ & 119000 & NA & 50.3 & 23.6 & -0.92 \\
\hline 0742 & B\&E BRINE & UNC & $03 / 14 / 89$ & $<0.05$ & 0.003 & 1 & 0.112 & 1520 & 0.005 & $<0.5$ & 11 & 42 & $<0.1$ & 121000 & NA & 49.9 & 23.9 & -0.09 \\
\hline
\end{tabular}

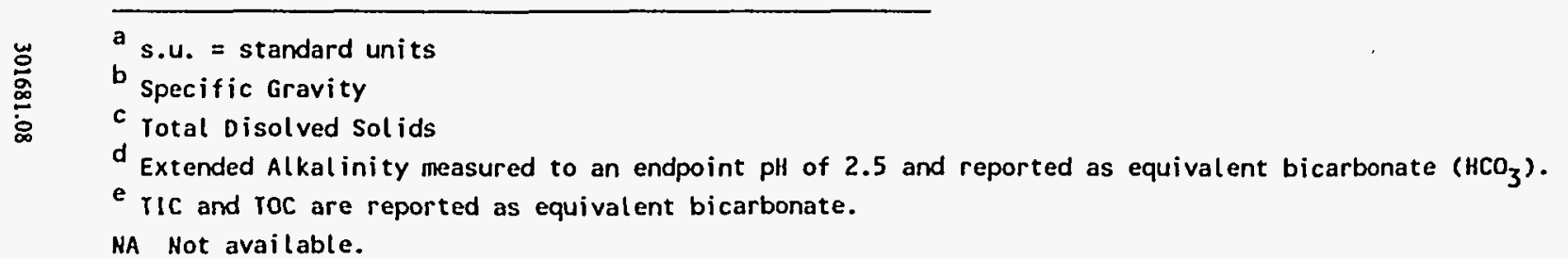


THIS PAGE INTENTIONALLY LEFT BLANK 


\section{APPENDIX E \\ HYDROLOGIC TESTING OF THE FRACTURED PART OF THE DISTURBED ROCK ZONE BENEATH THE WIPP EXCAVATIONS}




\section{Table of Contents}

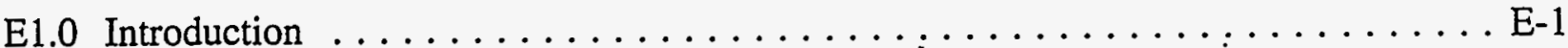

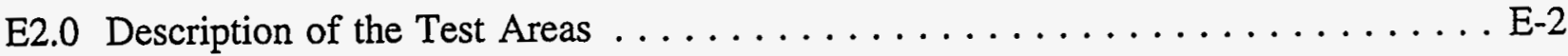

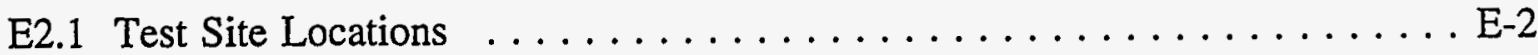

E2.1.1 S90 Near the AIS Test Site $\ldots \ldots \ldots \ldots \ldots \ldots \ldots \ldots \ldots$ E-4

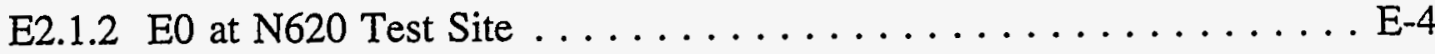

E2.1.3 W170 at the Underground Core Storage Library Test Site . . . . . E-8

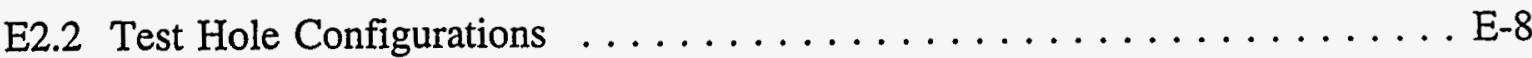

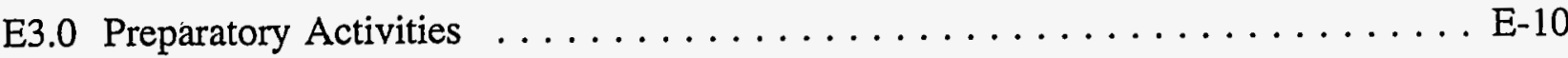

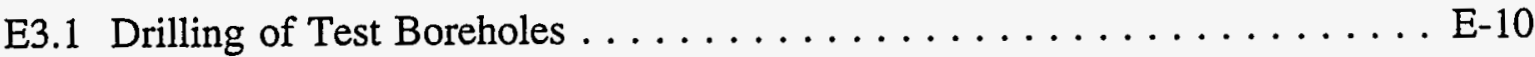

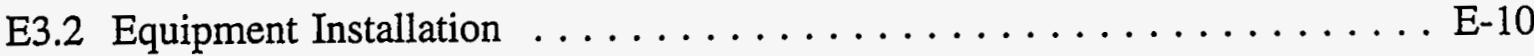

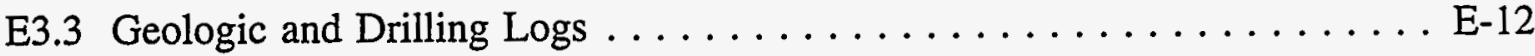

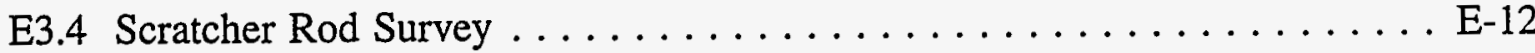

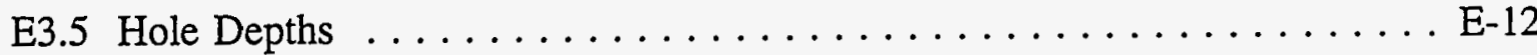

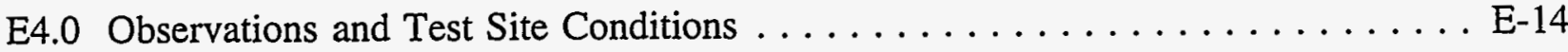

E4.1 E0 Test Site Prior to the Initial Pumping Test $\ldots \ldots \ldots \ldots \ldots \ldots \ldots$ E-15

E4.2 W170/Core Library Test Site . . . . . . . . . . . . . . . E E-19

E4.3 E0 Test Site Prior to the Retest at This Site $\ldots \ldots \ldots \ldots \ldots \ldots \ldots$ E-23

E5.0 Hydrologic Testing and Data Collection ................... E-24

E5.1 Initial Pumping Drawdown Test at the E0 Drift Site $\ldots \ldots \ldots \ldots \ldots$ E-29

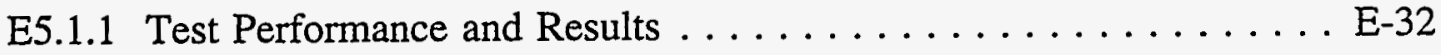

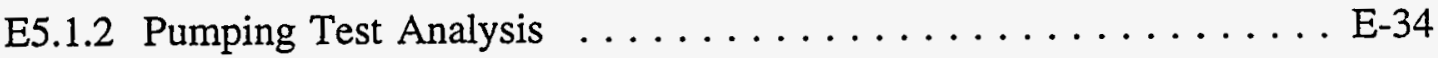

E5.2 Initial Pump Test at the Core Storage Library (W170) Site . . . . . . . E-41

E5.3.1 Test Performance and Results ................ E-50

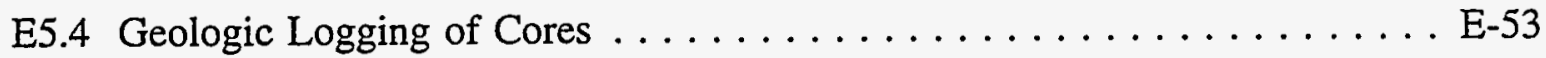

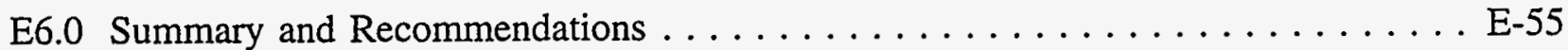

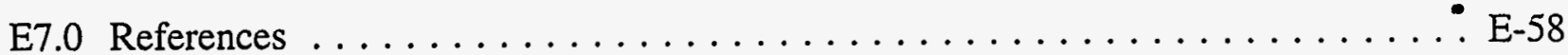




\section{THIS PAGE INTENTIONALLY LEFT BLANK}




\section{APPENDIX E}

\section{E1.0 Introduction}

The main objective of the hydrologic testing of the fractured part of the disturbed rock zone (DRZ) beneath the Waste Isolation Pilot Plant (WIPP) Excavations Program is to characterize the fracture system beneath the floor of the repository. The data resulting from this program will be used by Waste Isolation Division personnel to develop operational plans for predicting brine and gas movement through the fracture system. Additionally, the data obtained may be useful in refining the design of seals to be used within the repository and in assessing the long-term behavior of flow through the fractured zone.

As salt creeps into the WIPP underground excavations, macrofractures develop in the DRZ beneath the excavations (Bechtel, 1986; see also review by Deal and Roggenthen, 1991). The fractures tend to concentrate in but are not limited to Marker Bed (MB) 139, which is about 3 feet (ft) (1 meter [m]) thick and lies 3 to $6 \mathrm{ft}$ ( 1 to $2 \mathrm{~m}$ ) below the floor of most of the WIPP excavations. The developing fracture systems may provide pathways for rapid movement of brine and gas (Deal and Case 1987; Deal and others, 1989; Deal and others, 1991) and are considered to be one of the most likely pathways for migration of constituents of concern (COC) away from the waste storage panels. The hydrologic characteristics of the fractured zone must be understood to predict and, if necessary, modify the movement of fluids and COCs within MB 139 if a release occurred during operation of the facility.

In 1989, a hydraulic test of short duration was conducted in the DRZ beneath the floor of the intersection of the S90 and W620 drifts (Deal and others, 1991). The results indicated that drawdown-type pump testing in the underlying fracture system could be performed successfully and could yield useful hydrologic data about the DRZ. After evaluating the results from the preliminary testing effort, a more comprehensive field testing program was developed, and hydraulic testing was implemented at two additional underground test sites.

This report presents the results of short-duration hydraulic tests conducted at the two additional sites and provides recommendations for further field data collection. 


\section{E2.0 Description of the Test Areas}

The Salado Formation is predominantly halite, consisting of alternating sequences of halite, argillaceous halite, polyhalitic halite, clay partings 0.40 - to 1.2 -inches (in.)- (1- to 3 -centimeters [cm]-) thick clay layers, and thin anhydrite beds as numerous horizontal discontinuities. Anhydrite beds ranging from a few millimeters to about $3.3 \mathrm{ft}(1 \mathrm{~m})$ in thickness are brittle at repository depths. As the salt deforms, the contrast in ductility between the salt and the anhydrite causes preferential fracturing in the brittle anhydrite. $M B$ 139, approximately $3.3 \mathrm{ft}(1 \mathrm{~m})$ thick, is located $6.6 \mathrm{ft}(2 \mathrm{~m})$ beneath the floor of the excavations and shows how local variation in stratigraphy influences macrofracture development. The dish-shaped fractures that normally develop beneath the floor of excavated rooms are distorted and tend to flatten near the room center (Figure E-2-1). Although the fractures concentrate within the anhydrite, especially beneath the center of drifts or rooms, they also cut the halite and other units (Bechtel, 1986). Air-filled fractures up to 5.9 in. (15 $\mathrm{cm}$ ) wide have developed two to five years after excavation (Bechtel, 1986). Five years after excavation, the largest observed separation is about 9 in. $(23 \mathrm{~cm})$ wide.

Some subhorizontal fracturing has been noted just above clay $E$, at the base of MB 139 , approximately $6.6 \mathrm{ft}(2 \mathrm{~m})$ below the floor of the excavations, but no separations at clay $E$ were noted (Bechtel, 1986). This may be the result of creeping salt that deformed upward and pushed against the anhydrite to keep the clay confined.

Near the edges of rooms and drifts, fracturing tends to concentrate in the walls or salt above MB 139 (Figure E-2-1). Eventually fracturing will extend into the salt below MB 139. The zone of macrofractures was expected to extend about $6.6 \mathrm{ft}(2 \mathrm{~m})$ below the floor of the excavation at this location and to extend laterally a lesser distance into the bedrock beyond the edges of the rooms. In map view, the fractured zone under investigation is expected to follow the plan of the underground excavations closely.

\section{E2.1 Test Site Locations}

The hydrologic testing areas were selected to evaluate various room and drift dimensions, excavation ages, areas where water was introduced for construction purposes, and areas isolated from construction fluids. Three sites were selected for drilling and testing as part of this program. 

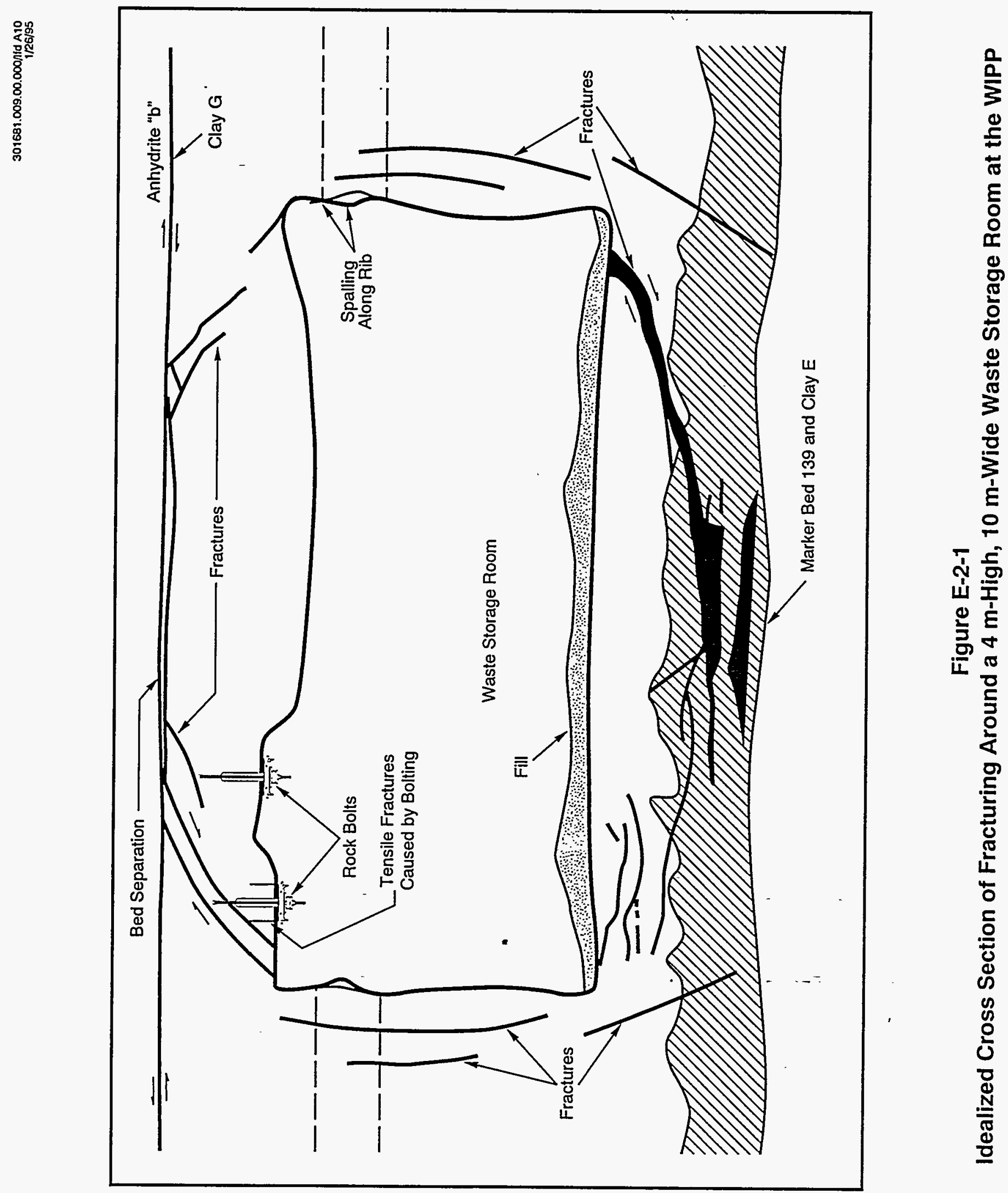
- Test Site No. 1 is at the intersection of the S90 and W620 drifts near the Air Intake Shaft (AIS). This site consists of 10 test holes drilled at the intersection and along the length of the S90 drift (Figure E-2-2). This test site was not accessible during the field investigation period and is shown as the first hydrologic test site on the map in Figure E-2-2.

- Test Site No. 2 is located in the E0 drift in the general area of N620. The site includes nine test holes drilled along the E0 drift (Figure E-2-2).

- Test Site No. 3 is located in the W170 drift immediately in front of the underground core storage room. This site consists of 11 test holes drilled along the W170 drift and into the core library (Figure E-2-2).

\section{E2.1.1 S90 Near the AIS Test Site}

Test Site No. 1 in the S90 drift near the AIS was scheduled to be the third and final location tested for this program. However, hydrologic testing at this site was impossible because of the presence of an electrical substation located in the center of the intersection and electrical transformers located in an adjacent alcove. The position of the electrical equipment covered several of the test holes at this site, making testing impossible. The scope of the field program was revised when it was determined that the electrical equipment could not be moved within the time frame required to meet field-testing program objectives. The final field test was moved from the $\mathrm{S} 90$ site to the initial site in the E0 drift to retest this location. Therefore, the $S 90$ test site will not be discussed in detail. Table E-2-1 lists the test hole number, the location, and the date drilling was completed for the test holes drilled as part of this field program.

\section{E2.1.2 EO at N620 Test Site}

This hydrologic test site is located in the E0 drift, directly in front of the N620 alcove. The site was selected because the EO drift is a very old, wide drift where open brine-filled fractures have been observed beneath the drift floor. Fracturing beneath the drifts develop over time, and they develop fastest beneath the widest drifts.

This site also was easily accessed and offered an existing electrical supply and an area within the N620 alcove to set up instrumentation and store equipment. Figure E-2-3 shows the layout of the EO test site. 


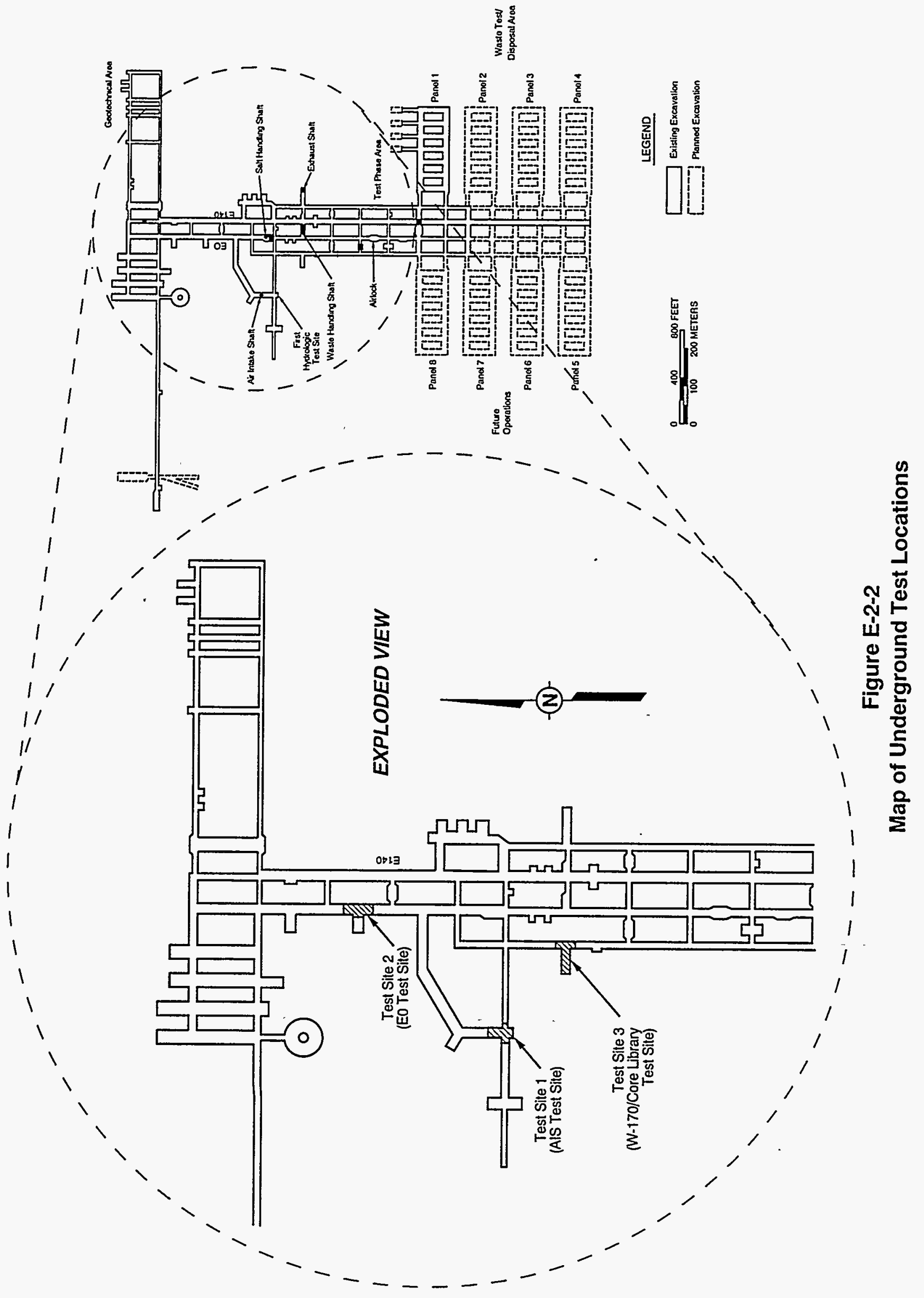


Table E-2-1

Test Holes Drilled as Part of the Hydrologic Testing of the Fractured Zone

\begin{tabular}{|c|c|c|}
\hline Hole Number & Date Drilled & Hole Location \\
\hline $\mathrm{OH} 49$ & $1-5-90$ & W620/S90 \\
\hline $\mathrm{OH} 50$ & $1-9-90$ & W620/S90 \\
\hline $\mathrm{OH} 51$ & $1-5-90$ & W620/S90 \\
\hline $\mathrm{OH} 52$ & $1-9-90$ & W620/S90 \\
\hline $\mathrm{OH} 53$ & $1-11-90$ & W620/S90 \\
\hline $\mathrm{OH} 54$ & $1-11-90$ & W620/S90 \\
\hline $\mathrm{OH} 55$ & $1-11-90$ & W620/S90 \\
\hline $\mathrm{OH} 56$ & $1-16-90$ & W620/S90 \\
\hline $\mathrm{OH} 57$ & $1-10-90$ & W620/S90 \\
\hline OH58 & $1-16-90$ & W620/S90 \\
\hline OH59 & $10-19-92$ & W170/Core Library \\
\hline $\mathrm{OH} 60$ & $10-19-92$ & W170/Core Library \\
\hline $\mathrm{OH} 61$ & $10 / 19 / 92$ & W170/Core Library \\
\hline $\mathrm{OH} 62$ & $1-24-90$ & W170/Core Library \\
\hline $\mathrm{OH} 63$ & $1-19-90$ & W170/Core Library \\
\hline $\mathrm{OH} 64$ & $1-23-90$ & W170/Core Library \\
\hline $\mathrm{OH} 65$ & $1-23-90$ & W170/Core Library \\
\hline $\mathrm{OH} 66$ & $1-18-90$ & W170/Core Library \\
\hline OH67 & $1-22-90$ & W170/Core Library \\
\hline OH68 & $1-19-90$ & W170/Core Library \\
\hline $\mathrm{OH} 69$ & $1-17-90$ & W170/Core Library \\
\hline $\mathrm{OH} 70$ & $1-29-90$ & E0/N620 \\
\hline $\mathrm{OH} 71$ & $1-31-90$ & E0/N620 \\
\hline $\mathrm{OH} 72$ & $1-31-90$ & E0/N620 \\
\hline $\mathrm{OH} 73$ & $1-31-90$ & E0/N620 \\
\hline $\mathrm{OH} 74$ & $1-29-90$ & E0/N620 \\
\hline $\mathrm{OH} 75$ & $1-29-90$ & E0/N620 \\
\hline $\mathrm{OH} 76$ & $1-31-90$ & E0/N620 \\
\hline $\mathrm{OH} 77$ & $1-31-90$ & E0/N620 \\
\hline $\mathrm{OH} 78$ & $1-29-90$ & E0/N620 \\
\hline
\end{tabular}




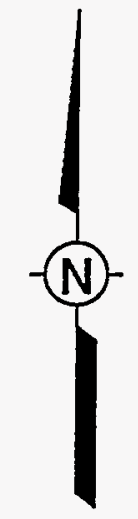

Test Hole Location

Figure not to Scale

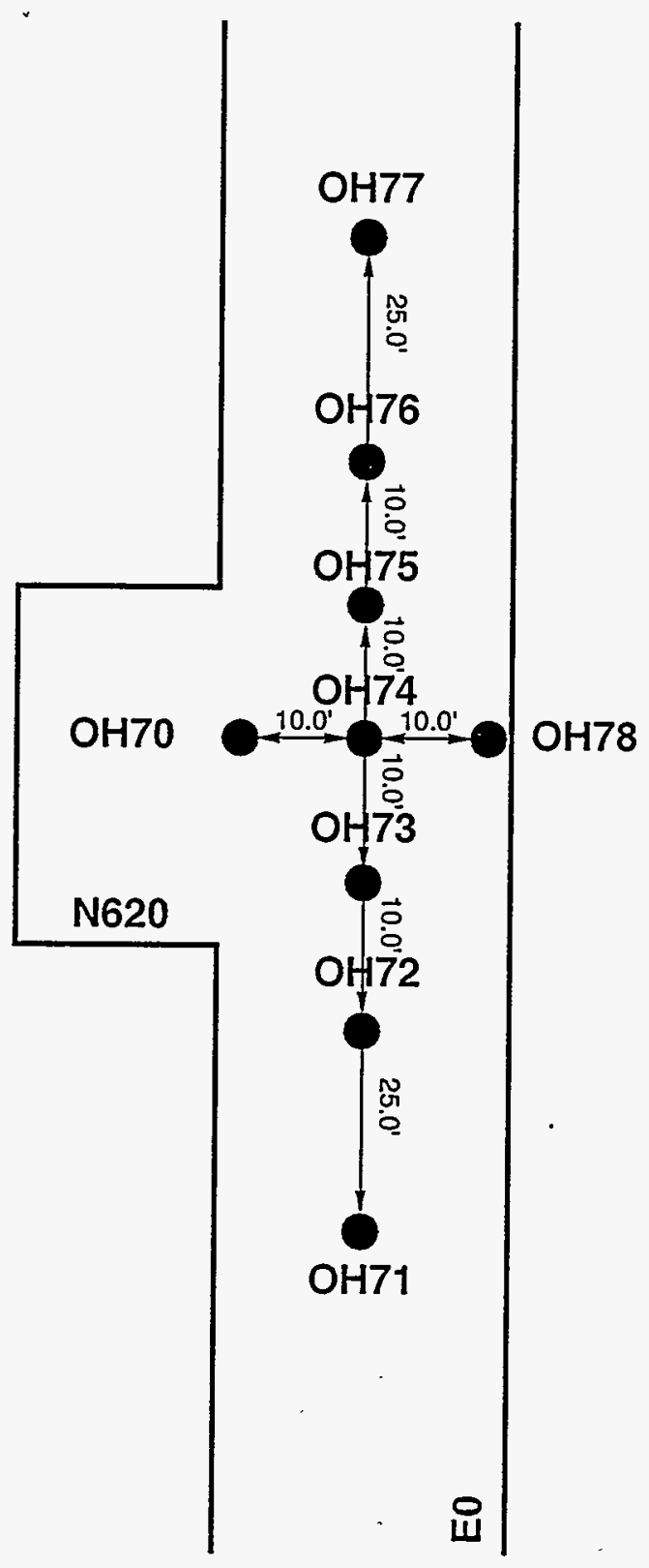

Figure E-2-3

Layout of Test Site No. 2 the E0 Drift in Front of N620, Holes $\mathrm{OH} 70$ through $\mathrm{OH} 78$ 


\section{E2.1.3 W170 at the Underground Core Storage Library Test Site}

The third test location is in the W170 drift in front of the underground core library. To best determine the development of the fracture-related transmissivity with time, this site was selected to initiate an investigation of a wide, new drift in the floor before significant fracturing develops. The underground core storage area is a 25 -ft-wide, relatively new excavation mined in May 1989 and is in a low-traffic area. The W170 drift adjacent to the core library is approximately five years older than the storage room and was expected to exhibit a well-developed fracture system beneath it. In addition, the W170 drift has been exposed to a long period of water spreading to control dust and to assist in roadbed consolidation. The objectives for this site are to test the fracture systems in the W170 drift and to monitor the development of fracturing beneath the core storage area. A series of boreholes were drilled in the core storage area to evaluate the potential hydraulic connection of the fracture systems within W170 and the core library. A future objective will be to monitor the development of fractured-zone hydraulic characteristics beneath the core library area by periodic retesting.

The site also offered electrical power, equipment storage areas, and convenient and safe access to the test holes. Figure E-2-4 shows the configuration of the W170 test site.

\section{E2.2 Test Hole Configurations}

The test locations represent areas with fracture zones that appear to be locally saturated with brine and exhibit some degree of interconnected fracturing. Test holes were installed at each site in a pattern designed to intercept separate fracture systems. At each site, holes were drilled along the length of the drift, as well as perpendicularly to the drift center line. Test holes were also emplaced as close to the drift wall (rib) as possible at both test sites (Figure E-2-3 and E-2-4). Placement of holes in this manner allowed the drawdown response produced by pumping one hole to be observed in multiple directions, potentially identifying individual fracture systems. 


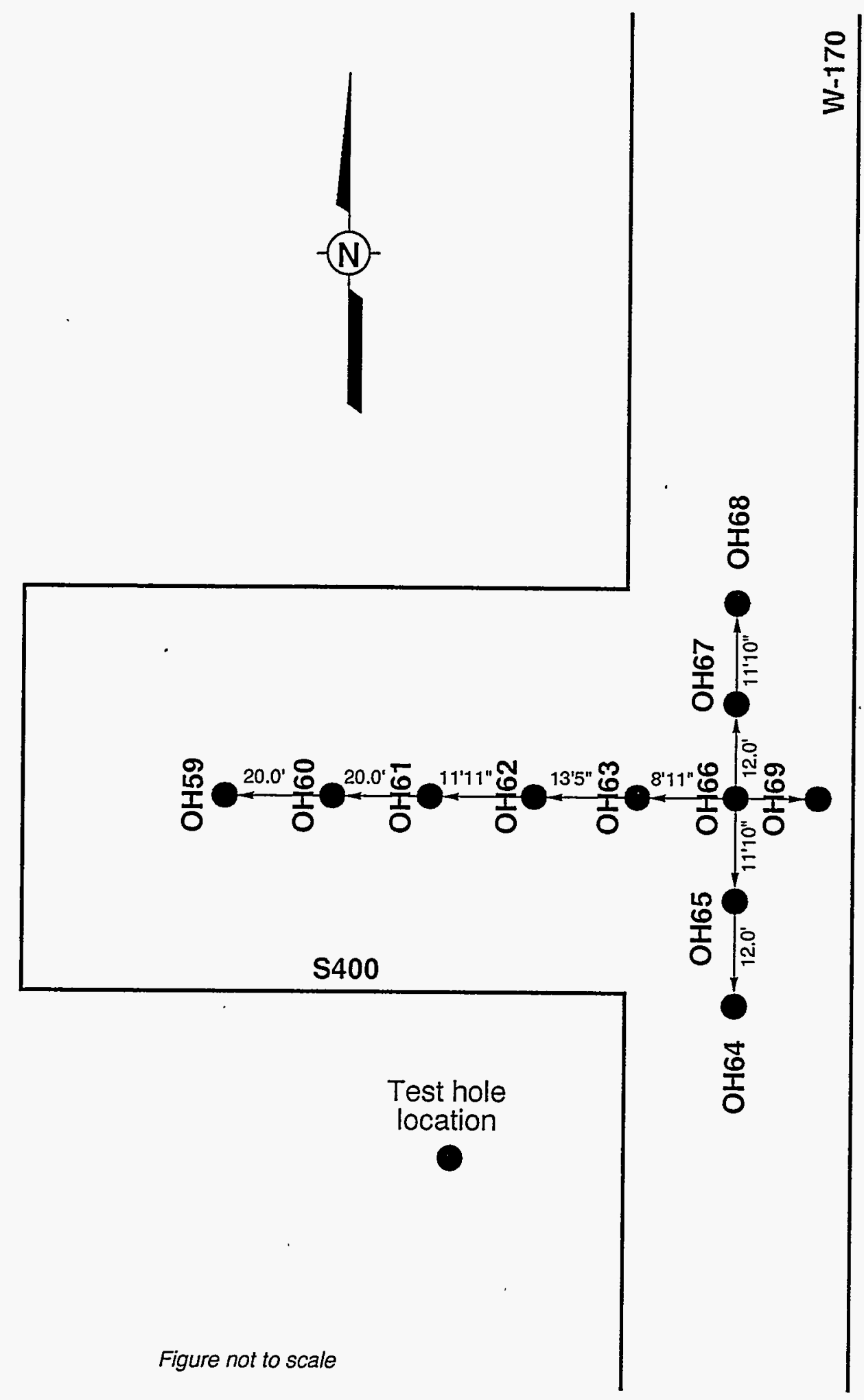

Figure E-2-4

Layout of Test Site No. 3 at the Intersection of S400 and W170 Drifts (Underground Core Library) Holes $\mathrm{OH} 59$ through $\mathrm{OH} 69$ 


\section{E3.0 Preparatory Activities}

The following preparatory activities were performed prior to the pumping drawdown tests:

- Drilling boreholes

- Blowing out boreholes to clear out muck

- Installing equipment

- Surveying hole locations

- Core logging

- Driller logging

- Scratcher rod surveying

- Measuring fluid levels

- Measuring hole depths

- Observing the condition of the holes.

\section{E3.1 Drilling of Test Boreholes}

Boreholes were drilled at three separate test sites in the WIPP underground workings

(Figure E-2-2). All holes were cored vertically downward to a depth of approximately $1 \mathrm{ft}$ $(0.30 \mathrm{~m})$ beneath the base of $\mathrm{MB} 139$ and are $4 \mathrm{in.}(10.2 \mathrm{~cm})$ in diameter. The holes were all drilled with air, and great care was taken to not introduce foreign fluids into the test holes. As mentioned previously, Test Site No. 1 was not used because of the presence of temporary electrical equipment in the drift.

\section{E3.2 Equipment Installation}

Test holes were instrumented with pressure transducers (Geokon Model 4500-H) before the pumping began, during the pumping test, and after the pumping was completed. In addition, a transducer was placed in open air to detect underground air pressure changes that may influence measured pressure in the holes. Before data were collected, the transducers were zeroed for atmospheric pressure using a Geokon 401. All transducers were connected to a junction box, with a single line connecting the junction box to a Geokon CR-10 remote datalogger that stores data obtained from the transducers (Figure E-3-1). A computer cable connected the datalogger to a laptop computer (Toshiba Model 1200) for downloading information and for storage of data on magnetic disk.

A Bennett model air-driven piston pump was used for all tests. This type of pump allows a user-controlled discharge rate from 1.0 to 50 gallons (gal) per hour (gph) (3.8 to 189 liters per hour [Lph]). This pump is capable of very low discharge rates and uses compressed air supplied by a portable air compressor. The pump was lowered into a designated test hole, 


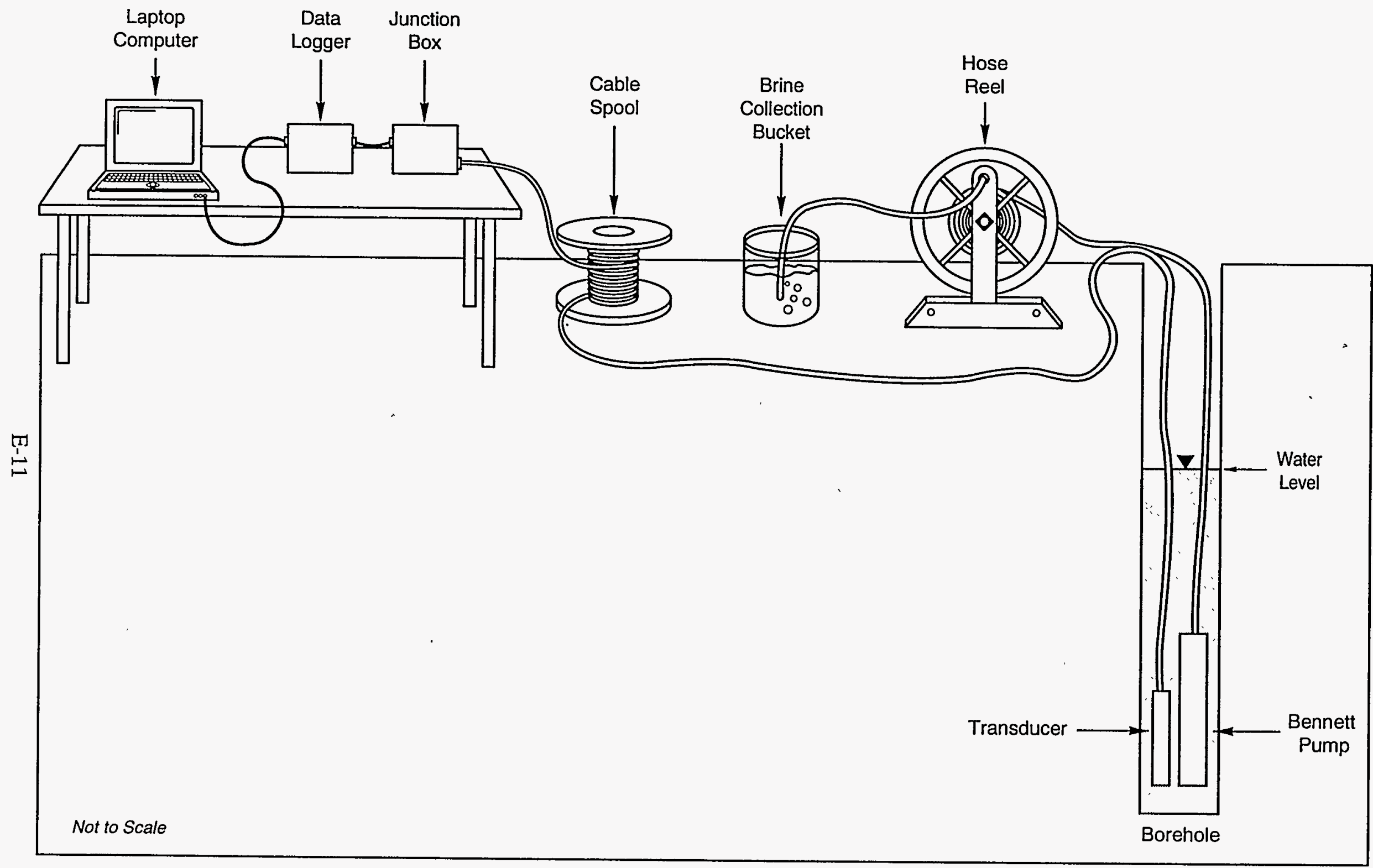

Figure E-3-1

Typical Pump Test Equipment Setup 
and the water level was allowed to reach equilibrium. The flow rate was measured by pumping the brine into a 1 gal $(3.78 \mathrm{~L})$ bucket while measuring the time required to fill the bucket. The bucket of brine was then dumped into a 250-gal (945 L) storage tank.

\section{E3.3 Geologic and Drilling Logs}

Geological logs were prepared for each core. Cores were logged in accordance with WIPP Procedure WP 07-502, "Geologic Rock Coring Logging," (Westinghouse, 1987). In addition to the geological logs, core drilling depth logs were prepared for correlation between the holes.

\section{E3.4 Scratcher Rod Survey}

As part of ongoing investigations, Geotechnical Engineering is observing and evaluating fracturing beneath the WIPP excavation. Fracturing was characterized in the holes using standard procedures for borehole fracture investigation. A scratcher rod was used to determine the fracture location, the orientation, and the approximate size of the fractures. The results of the scratcher rod survey were recorded as part of the Excavation Effects Program.

\section{E3.5 Hole Depths}

The general condition and total depth were observed at each test hole. Table E-3-1 shows the drilled depth, the measured depth, and the date of measurement. Depths were measured using a standard metal measuring tape.

EO at N620 Test Site. With the exception of OH71 (Figure E-2-3), which had no surface plug and was partially filled with debris, each hole was reasonably intact. Westinghouse Experimental Operations staff chipped and vacuumed out much of the debris and fluid and fluid-level equilibrium had not yet been achieved by the start of the test. All holes showed signs that the clay layers surrounding MB 139 were deforming and offsetting the test holes. The bottoms of the test holes appeared either to have filled with muck or to have started to close in at a depth of approximately $9 \mathrm{ft}(2.75 \mathrm{~m})$ and were offset. The muck in the partially filled holes may have changed the observed hydrologic response to pumping.

For some holes, the depths that were measured were somewhat different from the original depths drilled as indicated Table E-3-1. In December 1992, the Westinghouse Experimental Operations staff vacuumed all test holes to remove debris and muck. The test hole depths 
Table E-3-1

\section{Drilled and Measured Depths of Test Holes at the E0 and W170 Test Sites}

\begin{tabular}{|c|c|c|c|}
\hline Hole Number & Test Date & $\begin{array}{l}\text { Measured Hole } \\
\text { Depth (in feet) }\end{array}$ & $\begin{array}{c}\text { Drilled Depths } \\
\text { (in feet) }^{\mathrm{a}}\end{array}$ \\
\hline $\mathrm{OH} 59$ & $11 / 9 / 92$ & 10.29 & 10.17 \\
\hline $\mathrm{OH} 60$ & $11 / 9 / 92$ & 11.25 & 11.33 \\
\hline $\mathrm{OH} 61$ & $11 / 9 / 92$ & 10.00 & 10.00 \\
\hline $\mathrm{OH} 62$ & $11 / 9 / 92$ & 11.25 & 11.32 \\
\hline $\mathrm{OH} 63$ & $11 / 9 / 92$ & 9.50 & 9.39 \\
\hline $\mathrm{OH} 64$ & $11 / 9 / 92$ & 10.20 & 10.70 \\
\hline $\mathrm{OH} 65$ & $11 / 9 / 92$ & 9.25 & 9.98 \\
\hline $\mathrm{OH} 66$ & $11 / 9 / 92$ & 9.00 & 9.15 \\
\hline $\mathrm{OH} 67$ & $11 / 9 / 92$ & 9.67 & 9.40 \\
\hline $\mathrm{OH} 68$ & $11 / 9 / 92$ & 10.42 & 10.68 \\
\hline $\mathrm{OH} 69$ & 11/9/92 & 6.17 & 10.00 \\
\hline $\mathrm{OH} 70$ & $\begin{array}{l}10 / 12 / 92 \\
12 / 17 / 92 \\
\end{array}$ & $\begin{array}{l}8.85 \\
8.92 \\
\end{array}$ & 8.75 \\
\hline $\mathrm{OH} 71$ & $\begin{array}{l}10 / 12 / 92 \\
12 / 17 / 92 \\
\end{array}$ & $\begin{array}{l}9.15 \\
9.08 \\
\end{array}$ & 9.00 \\
\hline $\mathrm{OH} 72$ & $\begin{array}{l}10 / 12 / 92 \\
12 / 17 / 92 \\
\end{array}$ & $\begin{array}{l}9.50 \\
9.42 \\
\end{array}$ & 8.96 \\
\hline $\mathrm{OH} 73$ & $\begin{array}{l}10 / 12 / 92 \\
12 / 17 / 92 \\
\end{array}$ & $\begin{array}{l}9.00 \\
9.00 \\
\end{array}$ & 8.90 \\
\hline $\mathrm{OH} 74$ & $\begin{array}{l}10 / 12 / 92 \\
12 / 17 / 92 \\
\end{array}$ & $\begin{array}{l}9.33 \\
9.33 \\
\end{array}$ & 9.00 \\
\hline $\mathrm{OH} 75$ & $\begin{array}{l}10 / 12 / 92 \\
12 / 17 / 92 \\
\end{array}$ & $\begin{array}{l}7.75 \\
8.00 \\
\end{array}$ & 7.70 \\
\hline $\mathrm{OH} 76$ & $\begin{array}{l}10 / 12 / 92 \\
12 / 17 / 92 \\
\end{array}$ & $\begin{array}{l}9.25 \\
9.08 \\
\end{array}$ & 9.10 \\
\hline $\mathrm{OH} 77$ & $\begin{array}{l}10 / 12 / 92 \\
12 / 17 / 92 \\
\end{array}$ & $\begin{array}{l}8.00 \\
8.15\end{array}$ & 7.90 \\
\hline $\mathrm{OH} 78$ & $\begin{array}{l}10 / 12 / 92 \\
12 / 17 / 92 \\
\end{array}$ & $\begin{array}{l}9.00 \\
8.42 \\
\end{array}$ & 9.10 \\
\hline
\end{tabular}

${ }^{a}$ Depths measured below drift floor surface. 
were remeasured (Table E-3-1). The total hole depths measured in December 1992 are very near those recorded on the original drilling logs from January 1990.

W170 Test Site. With the exception of OH69, all test holes appeared to be fairly clean and free of muck and debris. Hole $\mathrm{OH} 69$ was filled with at least $3.5 \mathrm{ft}(1.1 \mathrm{~m})$ of muck. All holes appeared to be straight and did not show obvious signs of the clay layers surrounding MB 139 squeezing into the holes.

Table E-3-1 shows the measured depths of the boreholes, the as-drilled boreholes depths, and the date drilled. There were some notable differences in water levels across the test site area. Water levels were somewhat higher in the center of the W170 drift, directly in front of the core library (OH65, OH66, and OH67). The depths to water increased by almost $2 \mathrm{ft}(0.61$ $\mathrm{m})$ in the interior of the core library and to an even greater extent elsewhere.

\section{E4.0 Observations and Test Site Conditions}

Thorough characterization of the hydrologic conditions present in the test areas was required before proceeding with actual pumping drawdown tests. These conditions include the number and locations of fractures, the levels of saturation, the occurrence of saturated muck on the drift floors, the structure and thickness of MB 139, and the water pressure and fluid levels in the holes prior to the tests.

Test hole drilling and installation confirmed that fractures saturated with brine occurred beneath much of the areas for both test sites. Fracture observations were made visually and with a nail probe rod (scratcher rod), as described in Section 3.4 of this report. Based on these observations, it appears that fractures and structural separations of individual layers were restricted to the anhydrite (MB 139) and the clay seams associated with $M B 139$. Therefore, the hydrogeologic unit within the DRZ being tested and yielding brine to the test holes is generally fractured MB 139.

Both hydrologic test sites showed variability in fluid levels in the test holes across each respective site. Such variability suggests that each test site contained separate and independent fracture systems acting as isolated brine reservoirs. Geologic logging of the drill hole cores indicate that MB 139 is not uniform in thickness nor in depth to the top or bottom 
of the bed. Logging also revealed that MB 139 appears to be fractured at numerous depths, particularly in the lower one-half of the unit (Crawley et al., 1992).

The following sections describe specific observations and conditions of the E0 and W170 test site areas in more detail.

\section{E4.1 EO Test Site Prior to the Initial Pumping Test}

The initial hydrologic test of this field program was conducted at the EO drift test site during the period of October 8 through 14, 1992. The nine test holes at this site were preexisting, having been drilled more than three years prior to the scheduled pumping test.

Considering the history of brine use for dust control in the EO drift, fluid levels in the boreholes were initially lower than expected, providing only about 3 to $4 \mathrm{ft}(1 \mathrm{~m})$ of saturated hole to be used in pumping. Table E-4-1 shows the measured depths to water in the test holes prior to the pumping tests. The deeper fluid levels suggested that the pumping test at this site would likely be very short in duration, if even possible.

Figures E-4-1 and E-4-2, cross-sectional views of the E0 test site, show the depths to the top and bottom of MB 139 and the depths to the standing-water column in each test hole. Fluid levels in holes $\mathrm{OH} 72, \mathrm{OH} 73, \mathrm{OH} 74, \mathrm{OH} 75$, and $\mathrm{OH} 76$ are similar and are at equilibrium near the top of MB 139 (Figure E-4-1). The depth to the top of the fluid column increases significantly to the north in hole $\mathrm{OH77}$, suggesting that fractures in $\mathrm{MB} 139$ may not be saturated north of the test site area. The fluid level in OH71 on the south end of the test site is somewhat lower than that in the center of the test area. However, the lower fluid level in OH71 may be the result of previously cleaning out and evacuating this test hole and does not reflect the equilibrium fluid level. Figure E-4-2 shows the cross-sectional view across the E0 drift, depicting fluid levels within or slightly above those of MB 139 . Hole OH78 exhibits a lower fluid level than do $\mathrm{OH} 70$ and $\mathrm{OH} 74$, perhaps indicating that the fracture system near the east edge of the drift may not be hydraulically connected to fractures in the center of the drift.

Drilled depths and measured depths of holes shown in Figures E-4-1 and E-4-2 are often different. Muck in the holes may cause the measured depth to be less than the original 
Table E-4-1

Measured Water Levels in Test Holes at the EO Site During the Initial Pumping Test

\begin{tabular}{|c|c|c|}
\hline Hole Number & Date Measured & Depths to Water (in feet) $^{\mathrm{a}}$ \\
\hline \hline $\mathrm{OH} 70$ & $10 / 12 / 92$ & 4.62 \\
\hline $\mathrm{OH} 71$ & $10 / 12 / 92$ & 6.83 \\
\hline $\mathrm{OH} 72$ & $10 / 12 / 92$ & 4.70 \\
\hline $\mathrm{OH} 73$ & $10 / 12 / 92$ & 4.95 \\
\hline $\mathrm{OH} 74$ & $10 / 12 / 92$ & 4.98 \\
\hline $\mathrm{OH} 75$ & $10 / 12 / 92$ & 4.94 \\
\hline $\mathrm{OH} 76$ & $10 / 12 / 92$ & 5.08 \\
\hline $\mathrm{OH} 77$ & $10 / 12 / 92$ & 5.90 \\
\hline $\mathrm{OH} 78$ & $10 / 12 / 92$ & 6.23 \\
\hline
\end{tabular}

${ }^{a}$ Depths to water measured below drift floor surface. 


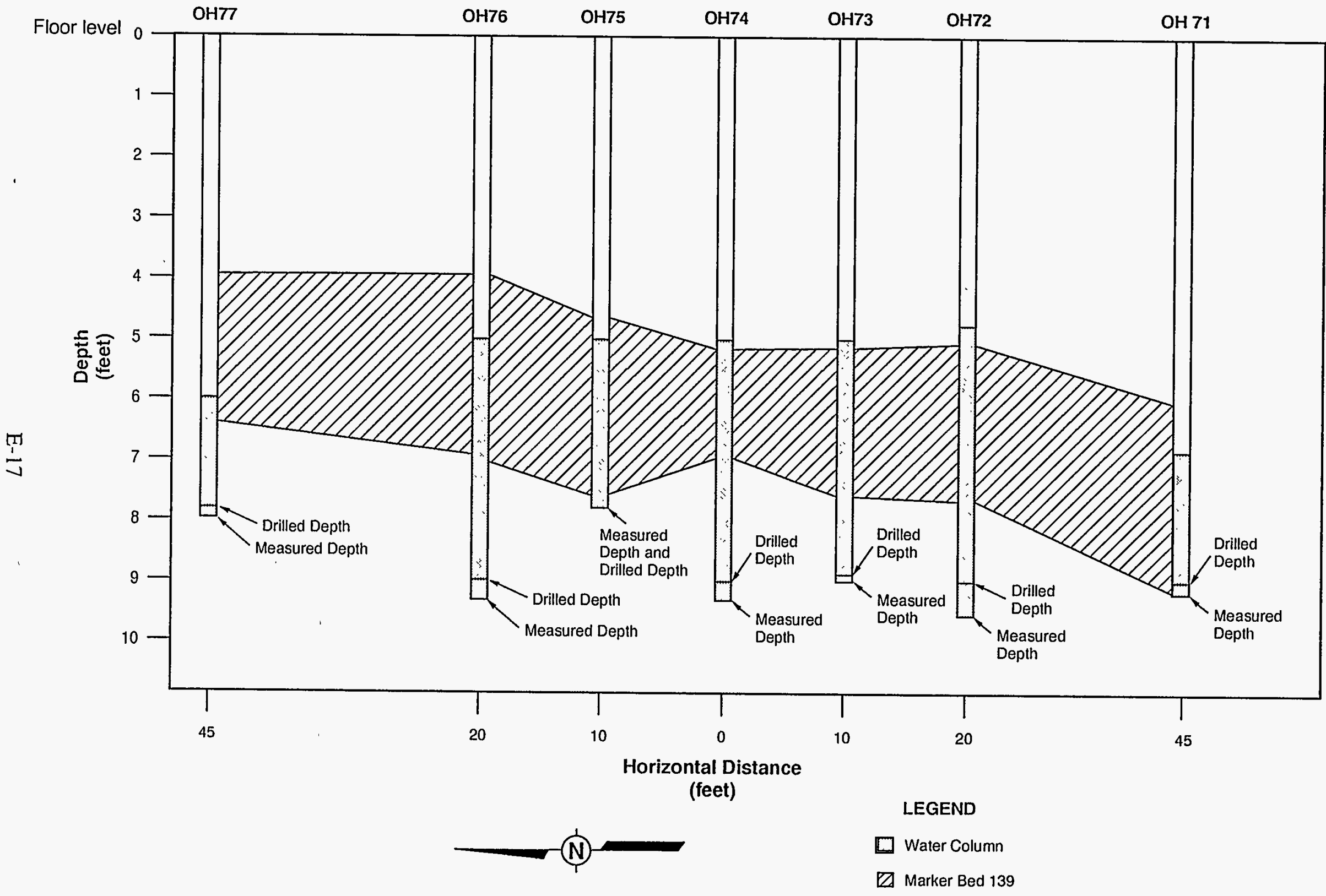

Figure E-4-1

Cross Section of Boreholes Along the E0 Drift Test Site, October 1992 


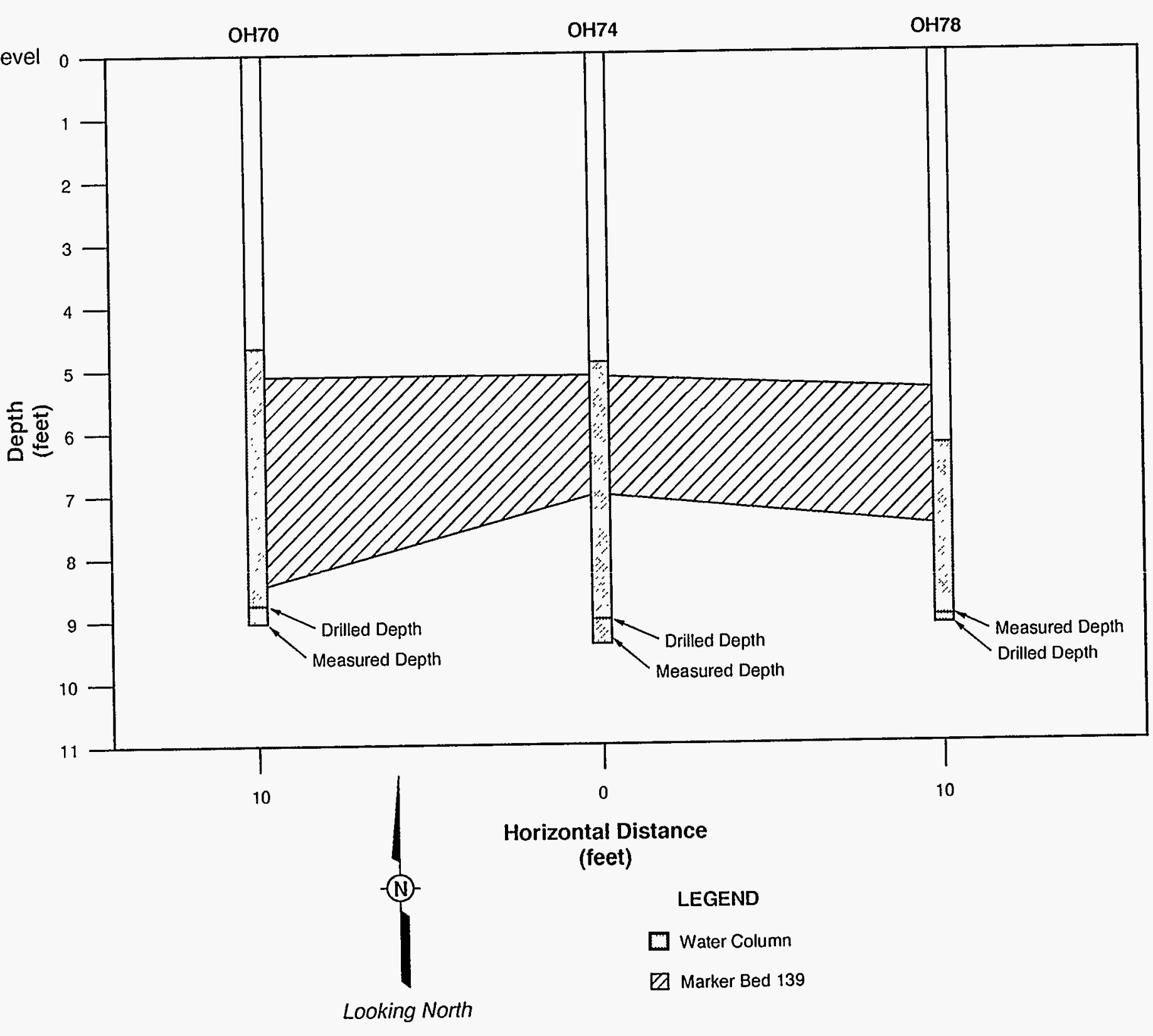


drilled depth. Conversely, muck buildup on the floor of the drift (the reference point for measurements) may cause the measured depth to be greater than the original drilled depth.

Figure E-4-1 shows that MB 139 has an apparent dip to the south in this are and that the depths to the top and bottom of the bed is variable. These structural characteristics may influence fracture orientation and fluid levels.

Based on the initial observations of test hole fluid levels, it was anticipated that the planned pumping test may be short in duration, yielding limited data for hydrologic analysis. Hole OH74 was selected as the primary pumping hole for the test. Data from the preliminary step drawdown test conducted on October 12, 1992 indicated that a pumping drawdown test could be conducted at a very low flow rate (Chapter 5.0).

\section{E4.2 W170/Core Library Test Site}

The second hydrologic test of this field program was conducted at the intersection of the W170 drift and the underground core library from November 9 to 13, 1992. Eight of the eleven test holes at this site were preexisting, having been drilled more than three years prior to the scheduled test at this site. Three of the holes (OH59, OH60, and $\mathrm{OH} 61$ ) were drilled on October 19 and 21, 1992, in preparation for testing at this site.

Water levels were measured in all holes prior to any pumping activity. Water levels in holes in the W170 drift were generally 3 to $5 \mathrm{ft}(0.91$ to $1.5 \mathrm{~m})$ below the drift surface, with a few exceptions (Table E-4-2). Figures E-4-3 and E-4-4 show the depths to the top and bottom of MB 139 and the depths of the standing water columns in each test hole at this site.

As shown in these two cross-sectional figures, there were some notable differences in water levels across the test site area. Water levels were somewhat higher in the center of the W170 drift, directly in front of the core storage library (OH65, OH66, and OH67). The depths to water increased by almost $2 \mathrm{ft}(0.61 \mathrm{~m})$ in the interior of the core storage library and to an even greater extent elsewhere. Two holes, $\mathrm{OH} 60$ and $\mathrm{OH} 68$, exhibited markedly different water levels. OH60, located interior to the core storage library, is a recently drilled hole, the water level may not yet have reached equilibrium, or the borehole does not intersect any brine-filled fractures. 
Table E-4-2

Measured Water Levels in Test Holes at the W170 Site During the Initial Pumping Test

\begin{tabular}{|c|c|c||}
\hline Hole Number & Date Measured & Depths to Water (in feet) $^{\text {a }}$ \\
\hline \hline OH59 & $11 / 9 / 92$ & 6.15 \\
\hline OH60 & $11 / 9 / 92$ & 10.86 \\
\hline OH61 & $11 / 9 / 92$ & 5.43 \\
\hline OH62 & $11 / 9 / 92$ & 5.20 \\
\hline OH63 & $11 / 9 / 92$ & 4.98 \\
\hline OH64 & $11 / 9 / 92$ & 4.65 \\
\hline OH65 & $11 / 9 / 92$ & 3.30 \\
\hline OH66 & $11 / 9 / 92$ & 3.35 \\
\hline OH67 & $11 / 9 / 92$ & 3.00 \\
\hline OH68 & $11 / 9 / 92$ & 7.30 \\
\hline OH69 & $11 / 9 / 92$ & 4.10 \\
\hline
\end{tabular}

${ }^{a}$ Depths to water measured below drift floor surface. 


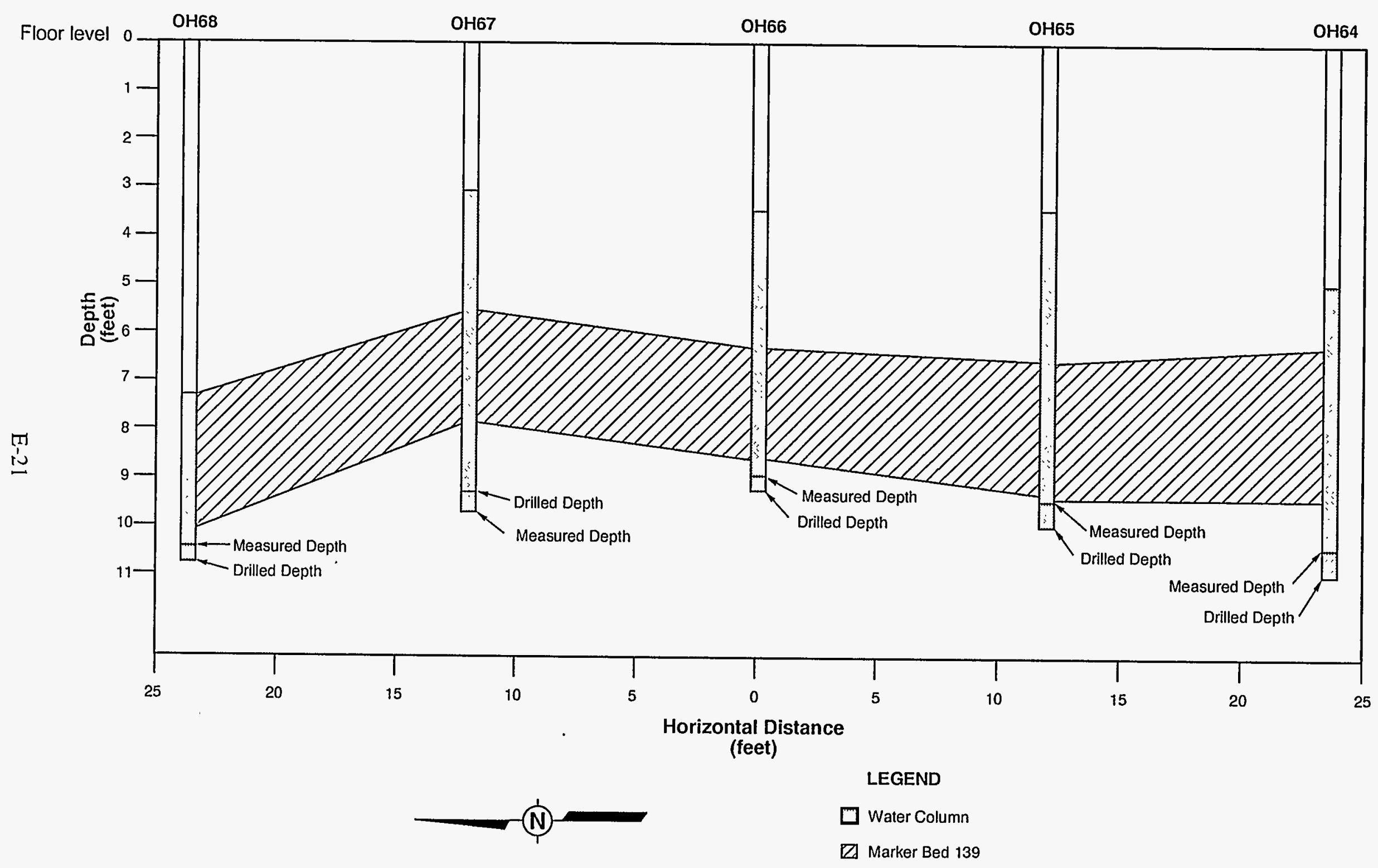




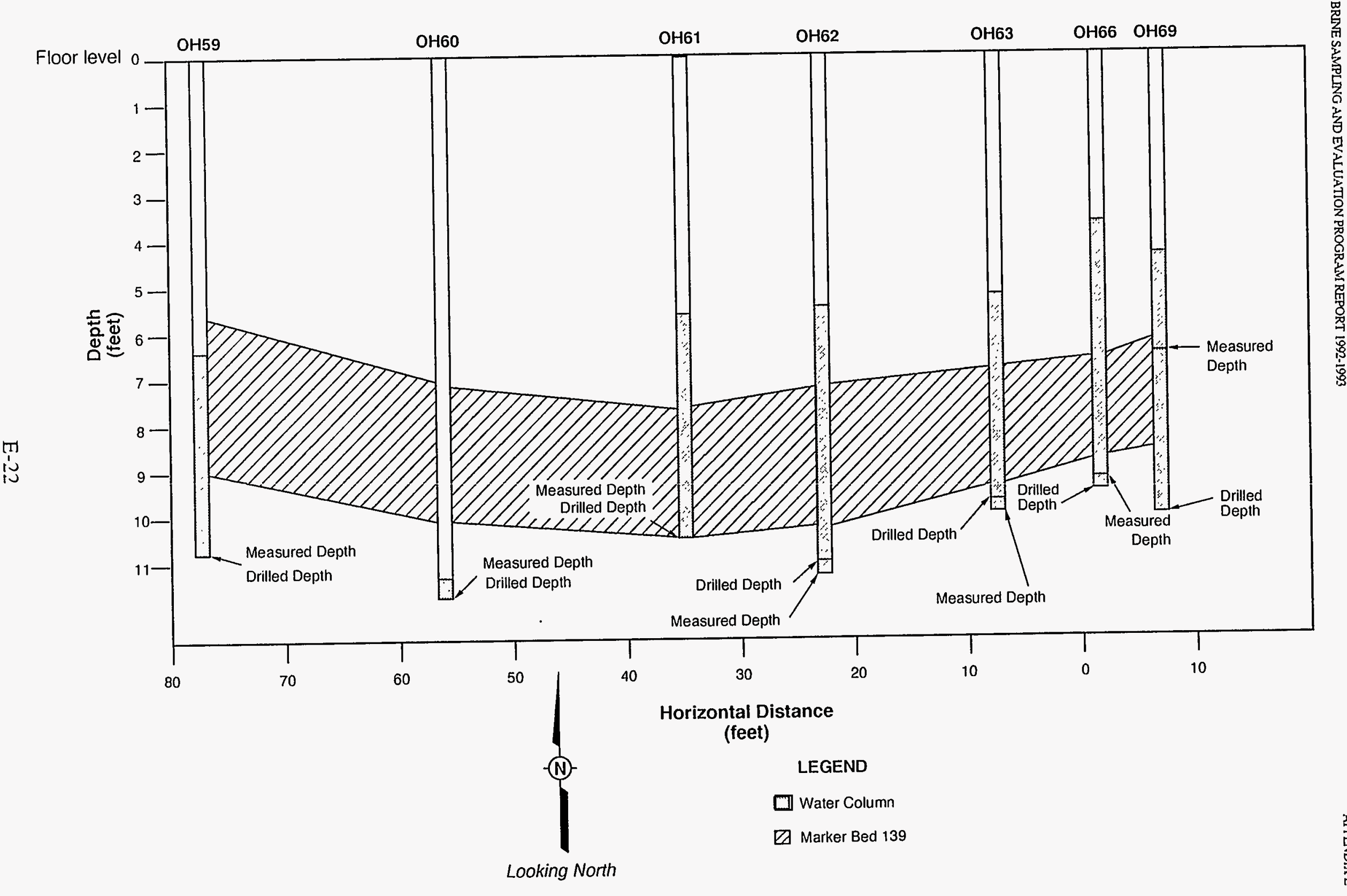

Figure E-4-4

Cross Section of Boreholes Across the W170 Drift and into the Core Library, November 1992 
Hole OH66 was selected as the pumping hole for the initial test based on its location in the middle of the drift (directly in front of the entrance to the core library) and a pretest standing fluid column of approximately $5.65 \mathrm{ft}(1.7 \mathrm{~m})$. The pretest fluid columns in holes OH62, $\mathrm{OH} 63, \mathrm{OH} 64, \mathrm{OH} 65, \mathrm{OH} 66$, and $\mathrm{OH} 67$ were all greater than $5 \mathrm{ft}(1.5 \mathrm{~m})$ in length, suggesting that there would be sufficient water to conduct a pumping test of adequate duration at this site. Because of concerns about potential dewatering of the proposed pumping hole (OH66), no preliminary drawdown step test was performed for this site.

\section{E4.3 EO Test Site Prior to the Retest at This Site}

The third and final field tests for the hydrologic testing of the fractured part of the DRZ project were conducted from December 14 through 17, 1992, at WIPP. These tests were repeats of the initial test conducted at the site located in the E0 drift in front of the N620 alcove from October 9 through 14, 1992.

As described in Section E4.1, the test holes at this site appeared to contain some semiconsolidated muck, which may have had an impact on the results from the initial test. Prior to the second test attempt at this site, the holes were reconditioned by removing some of the muck with a vacuum system. The reconditioning effort also removed all of the brine standing in the holes at that time. The fluid levels in the test holes partially recovered prior to the December 1992 field test period.

The total depth of each hole was measured, and each hole bottom felt solid and free of muck. With the exception of OH76, which has a major offset at approximately 6 to $7 \mathrm{ft}$ (1.8 to $2.1 \mathrm{~m}$ ) beneath the floor of the drift, all holes appeared to be generally straight. This offset closes about one-half of the hole and made instrument installation difficult.

Figures E-4-5 and E-4-6 show the depths of the top and bottom of MB 139 and the depths of the standing water columns in each test hole at this site in December 1992. The depths measured in December 1992 are very near the depths recorded on the original drilling logs in January 1990 (Table E-3-1). Water levels, measured in all holes prior to any pumping activity, ranged from 6.10 to $8.55 \mathrm{ft}(1.86$ to $2.61 \mathrm{~m})$ below the drift floor surface (Table E-4-3). There were some notable differences in water levels between holes OH76 and $\mathrm{OH} 77$ and the other holes at the test site. All of the holes, except these two, had very similar depth-to-water measurements. Holes $\mathrm{OH} 76$ and $\mathrm{OH} 77$ had water levels approximately $2 \mathrm{ft}$ 
$(0.61 \mathrm{~m})$ deeper than the other test holes. The standing fluid column in all holes was less than $3 \mathrm{ft}(0.91 \mathrm{~m})$.

Figures E-4-5 and E-4-6 show that during the December 1992 retest period, the fluid levels in MB 139 were much lower than those measured in October 1992 (Figure E-4-1 and E-4-2). The large decrease in fluid levels may be in response to the removal of fluid from the holes during cleaning in December 1992. The lower fluid levels measured in October suggest that the area may not have fully recovered from the hole reconditioning. However, it is also possible that the continued removal of brine from test holes at the EO drift site may be locally depleting the available fluid reservoir.

Based on the shorter standing fluid column in the test holes, it was anticipated that the pumping test might be shorter in duration than the initial test at this site. Hole OH74, which was selected for the initial test here in October 1992, was also selected in December 1992 as the primary pumping hole for the test.

\section{E5.0 Hydrologic Testing and Data Collection}

The main objective of the field testing program was to determine local hydrologic parameters for the fractured part of the DRZ at the EO and W170 drift test sites using standard pumping drawdown-type testing techniques. Additional goals include developing and refining testing techniques and collecting baseline hydrologic data from comparable old and new areas of the repository. To achieve these objectives, the two test sites were instrumented with downhole pressure transducers to monitor local fluid pressures in the fractured zone beneath the drifts. For the pumping drawdown tests, a Bennett model air-driven piston pump was installed in the selected test hole to provide user-controlled discharge rates from approximately 1 to $50 \mathrm{gph}$ ( 3.8 to $189 \mathrm{Lph}$ ). This type of pump is capable of very low discharge rates and uses compressed air from a portable compressor (Figure E-5-1).

The pressure transducer data were stored in random access memory using Geokon CR-10 remote dataloggers. These data were transferred to magnetic disk for analysis and evaluation using the appropriate computer software. The procedure for conducting pumping drawdowntype hydrologic testing included collecting background water pressure and water-level data prior to the test, evaluating local fluid-level trends, selecting the pumping hole, pumping equipment installation, performing a preliminary step drawdown test, estimating the 


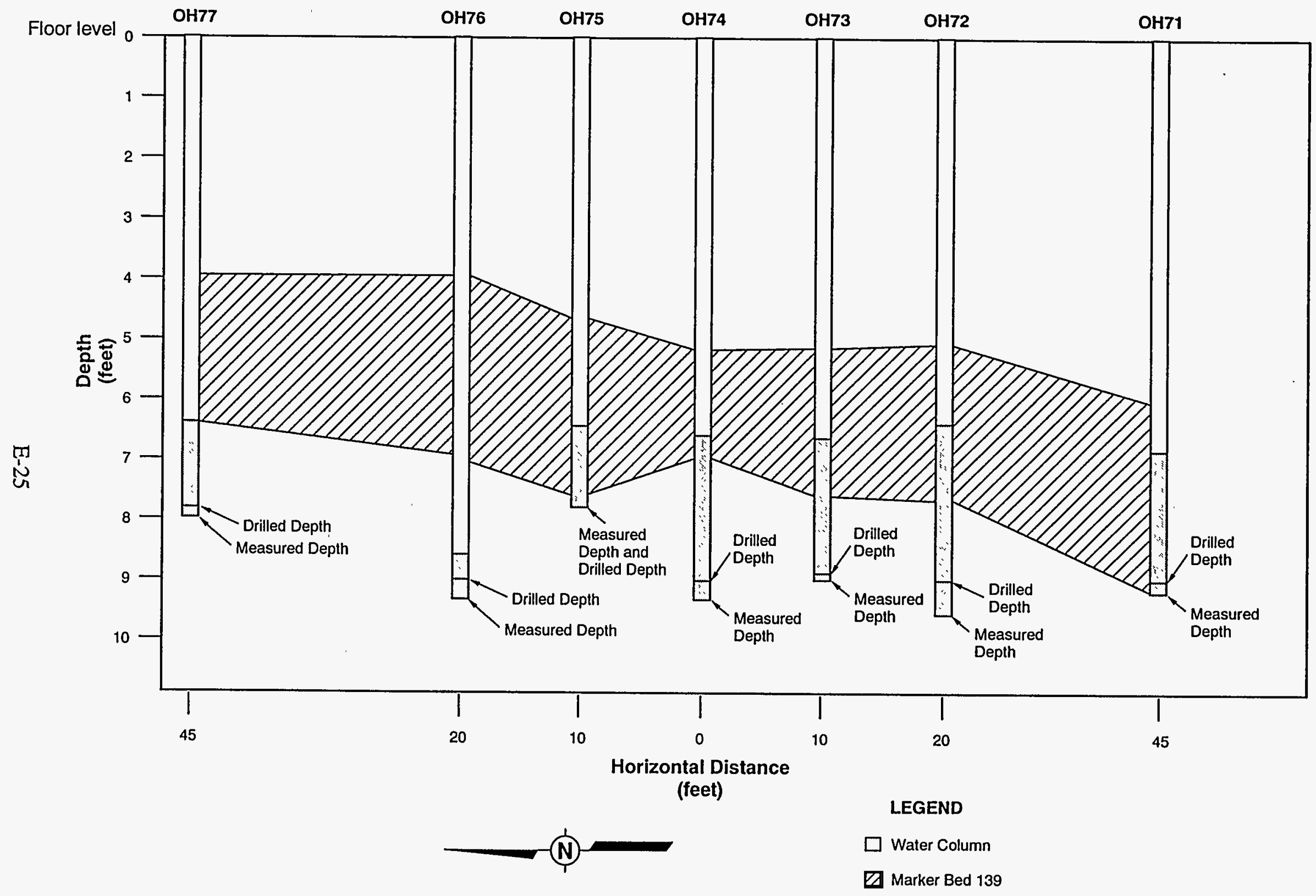

Figure E-4-5

Cross Section of Boreholes Along the E0 Drift Test Site, December 1992 


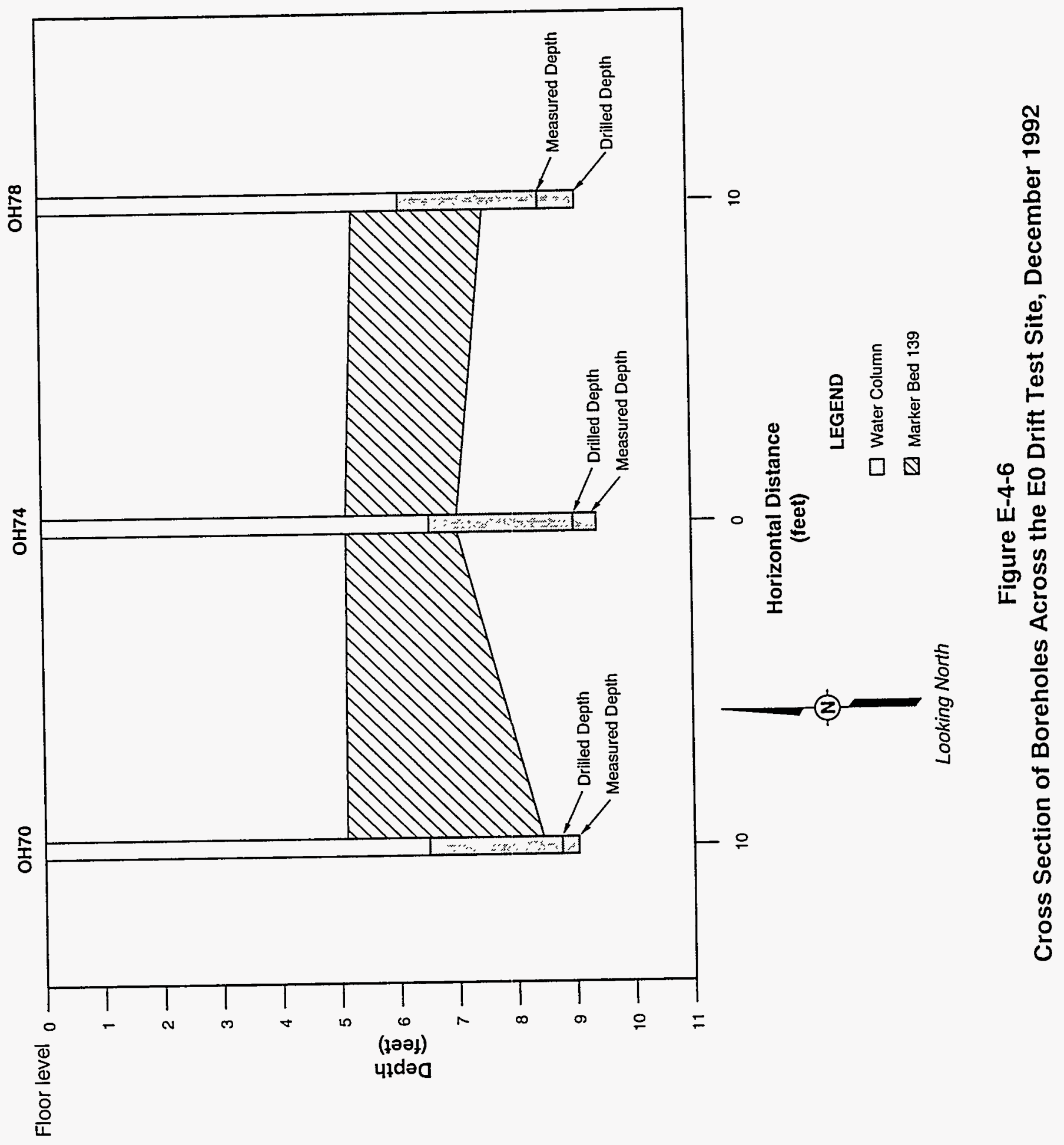




\section{Table E-4-3}

Measured Water Levels in Test Holes at the EO Site During the Second Pumping Test

\begin{tabular}{|c|c|c|}
\hline Hole Number & Date Measured & Depths to Water (in feet) $^{\mathrm{a}}$ \\
\hline \hline $\mathrm{OH70}$ & $12 / 14 / 92$ & 6.50 \\
\hline $\mathrm{OH71}$ & $12 / 14 / 92$ & 6.85 \\
\hline $\mathrm{OH72}$ & $12 / 14 / 92$ & 6.35 \\
\hline $\mathrm{OH73}$ & $12 / 14 / 92$ & 6.75 \\
\hline $\mathrm{OH74}$ & $12 / 14 / 92$ & 6.70 \\
\hline $\mathrm{OH75}$ & $12 / 14 / 92$ & 6.70 \\
\hline $\mathrm{OH76}$ & $12 / 14 / 92$ & 8.55 \\
\hline $\mathrm{OH77}$ & $12 / 14 / 92$ & 8.40 \\
\hline $\mathrm{OH78}$ & $12 / 14 / 92$ & 6.10 \\
\hline
\end{tabular}

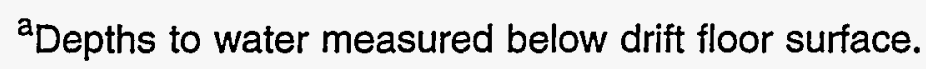




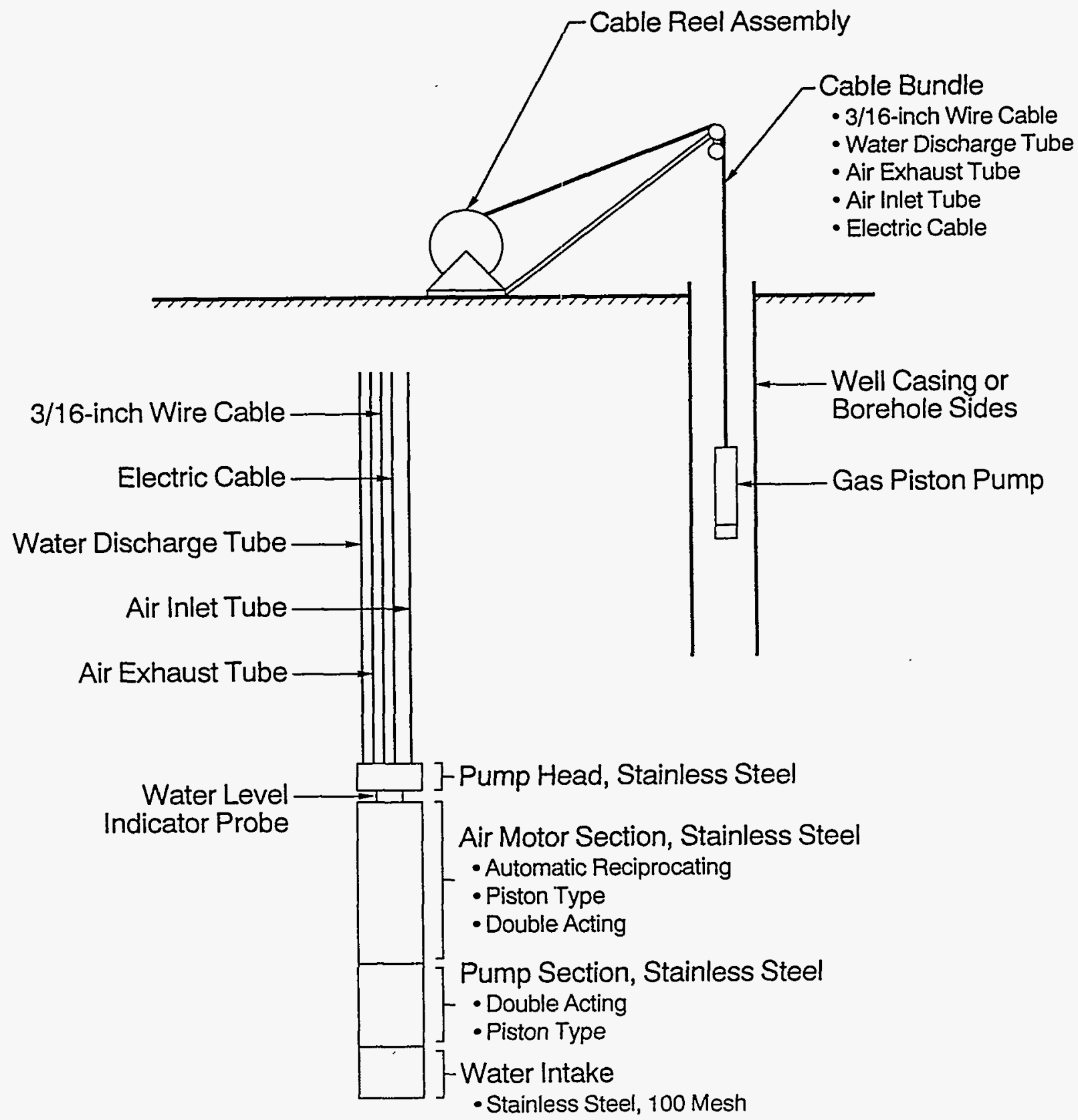

Figure E:-5-1

Schematic of Gas-Driven Piston Pump (Bennett Model 1800) with Exploded View of Cable Bundle 
appropriate flow rate, conducting the pumping test for as long as possible, and monitoring recovery of fluid levels. The sections below present the results of the pumping drawdown tests at the E0 and W170 drift test sites.

\section{E5.1 Initial Pumping Drawdown Test at the EO Drift Site}

Background water pressure and water-level data were collected at the E0 test site to provide some definition of long-term trends, and changes regarding test parameters and for comparison with test results. Background data collection began October 12, 1992, in all holes at the test site.

Figure E-5-2 shows hydrographs of fluid pressures in holes $\mathrm{OH70,OH73,} \mathrm{OH74,} \mathrm{and} \mathrm{OH} 75$, from the initial instrumentation through completion of the pumping drawdown test. These hydrographs show the response to pumping OH74. Pumping commenced at 10:32 a.m., October 13, 1992. The hydrograph also shows that the fluid levels in the holes that responded to pumping did not recover to their pretest levels by the time data collection was terminated. This observation suggests that the fluid reservoir being pumped through $\mathrm{OH} 74$ may be limited and that pumping from the test area may be dewatering the available fluid locally from the fracture systems.

Figure E-5-3 shows hydrographs of fluid pressures in holes $\mathrm{OH} 71, \mathrm{OH} 72, \mathrm{OH} 76, \mathrm{OH} 77$, and $\mathrm{OH} 78$ from the beginning to the end of the pumping test in OH74. These holes show very limited, if any, drawdown response to pumping, indicating that the test area may have separate, unconnected fracture systems that are at least partially saturated with brine.

On October 12, 1992, a preliminary step drawdown test was conducted at the E0 Test Site. $\mathrm{OH} 74$ was pumped at several flow rates to establish a discharge rate that could be maintained for at least several hours. The final flow rate was adjusted to approximately $8.7 \mathrm{gph}$ (32.9 Lph), producing a drawdown that would appear to allow pumping for the required duration. The test was terminated after less than 1 hour of pumping, and the area was allowed to recover overnight. The hole that was pumped and the surrounding holes had only partially recovered by the beginning of the full-scale pumping drawdown test the following morning. 


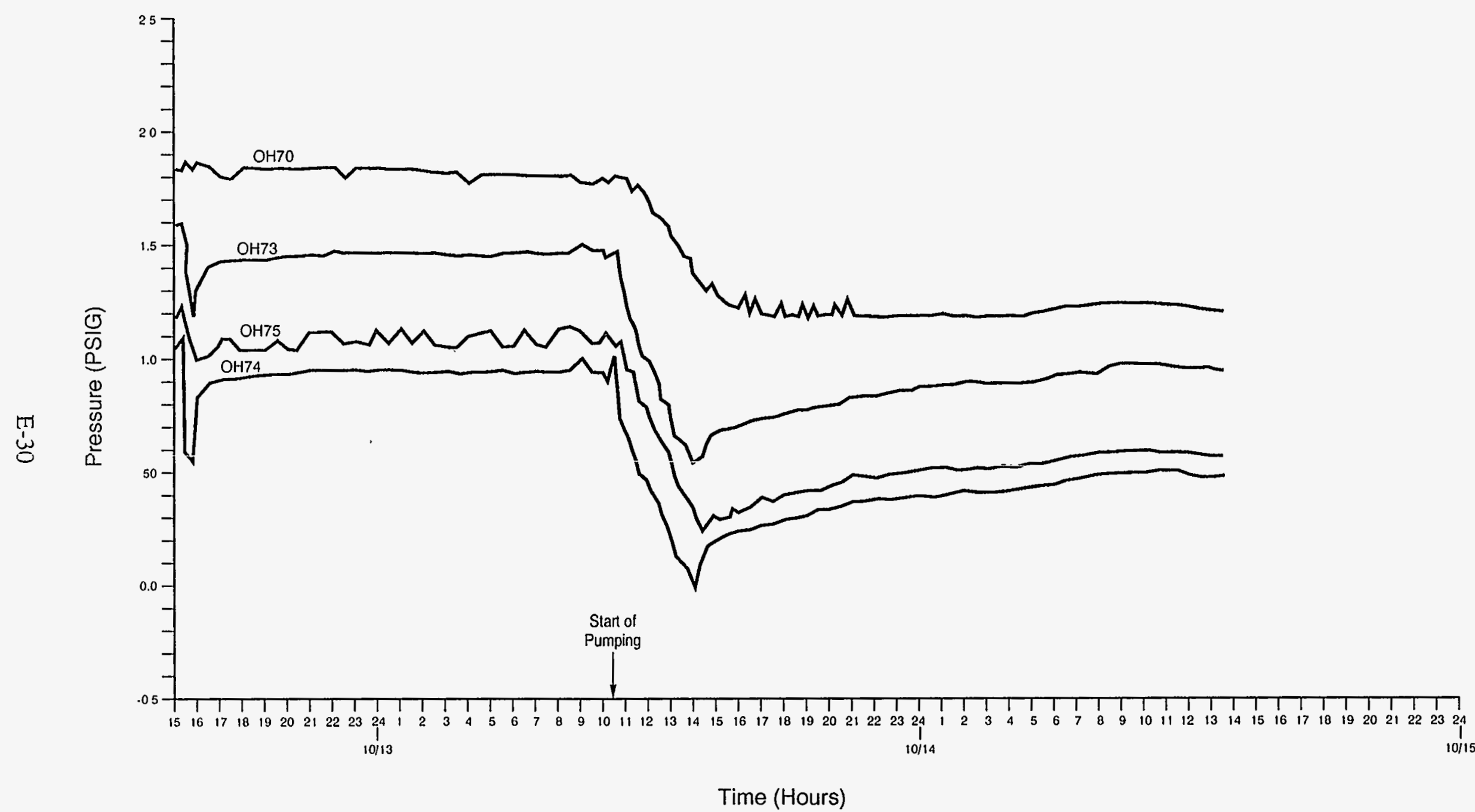

Figure E-5-2

Hydrographs of the EO Site Test Holes $\mathrm{OH} 70, \mathrm{OH} 73, \mathrm{OH} 74$, and OH75 Through the End of the Pumping Test, October 1992 


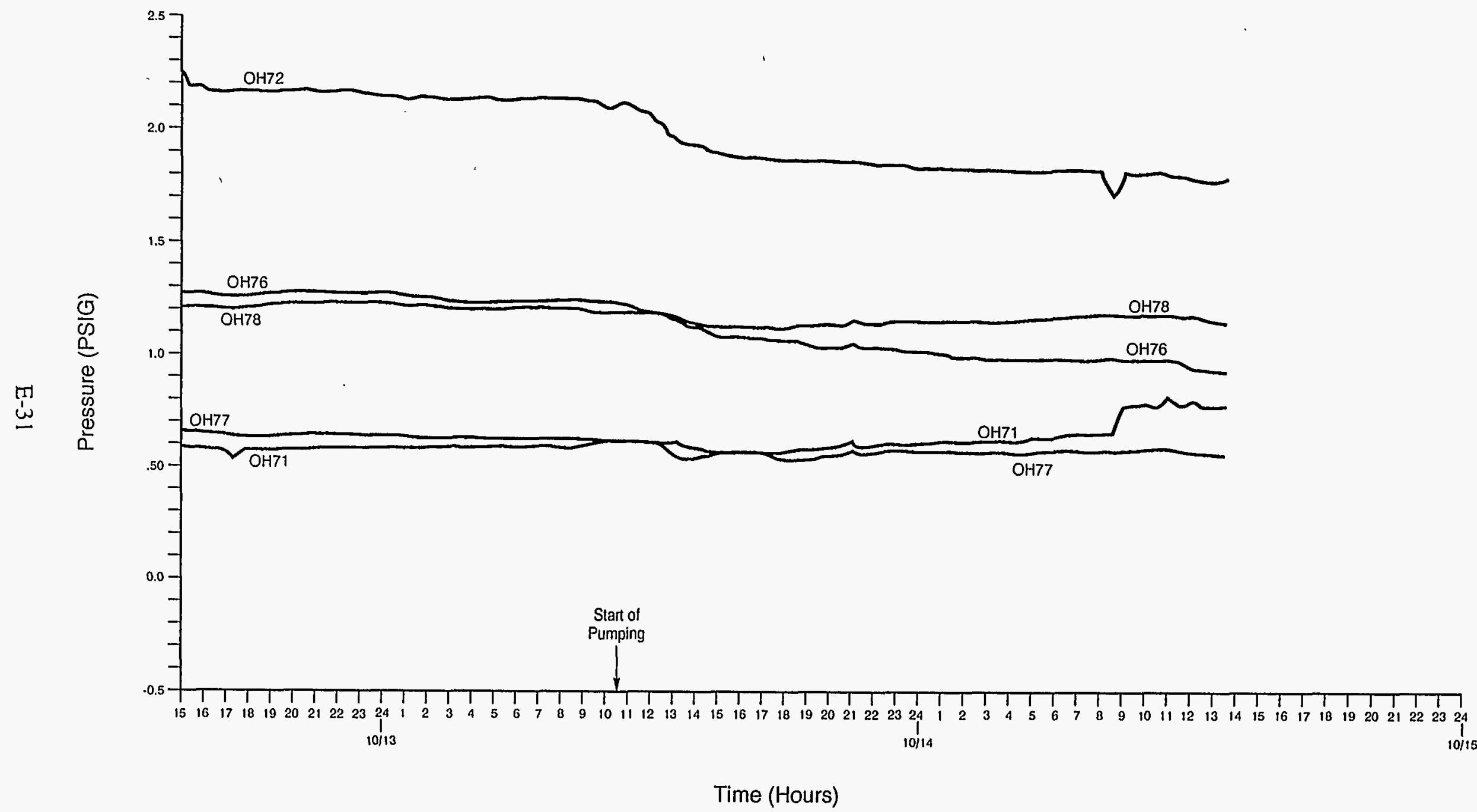

Figure E-5-3

Hydrographs of the E0 Site Test Holes $\mathrm{OH} 71, \mathrm{OH} 72, \mathrm{OH} 76, \mathrm{OH} 77$, and

OH78 Through the End of the Pumping Test, October 1992 
A pumping drawdown-type test was conducted on October 13, 1992, again using hole OH74 as the pumping hole. The test started at 10:00 a.m., but the pump intake soon became clogged with mud. The pump was removed from the hole, and the intake was cleaned. The pump was reinstalled, and the test hole was allowed to recover for 30 minutes (min). During the second pumping attempt at 10:32 a.m., all equipment functioned properly.

The pumping discharge rate was continuously monitored and adjusted until a flow rate of approximately $6 \mathrm{gph}(22.5 \mathrm{Lph})$ was attained. The actual measured pumping rate ranged from 7.57 to $5.14 \mathrm{gph}(28.6$ to $19.5 \mathrm{Lph})$, with an average of $6.06 \mathrm{gph}(22.9 \mathrm{Lph})$. The test lasted for approximately 3 hours and $37 \mathrm{~min}$. During the test, fluid levels in, all holes were continuously monitored using transducers and a datalogger.

\section{E5.1.1 Test Performance and Results}

The pumping discharge rate became stable after approximately $20 \mathrm{~min}$. The discharge rate was slightly variable throughout the test but did not significantly influence the steady drawdown achieved in the pumped hole and surrounding holes. Test hole fluid pressures were monitored at 1-min intervals throughout the test.

The pumping test was terminated at 2:10 p.m., when the fluid level in the pumped hole dropped to a level below the pump intake. The pump was then turned off, and the recovery phase was started. All pumping and monitoring equipment performed well throughout the test.

Drawdown of fluid levels was observed in most of the test holes surrounding OH74 during the pumping period. Only holes $\mathrm{OH} 71$ and $\mathrm{OH} 77$ showed no measurable response. These two holes, located at either end of the line of holes along the axis of the E0 drift, are the most distant holes from the pumping center. Figure E-5-4 shows the distribution of drawdown response at the site during the initial test in October 1992.

The hydrographs in Figures E-5-2 and E-5-3 show that OH70, OH73, and OH75 are the only holes to respond significantly to pumping OH74. Holes OH72 and OH76 showed only a limited response to pumping, and OH78 shows only a very slight long-term lowering of downhole fluid pressure. Holes $\mathrm{OH} 71$ and $\mathrm{OH} 77$ showed no response. 


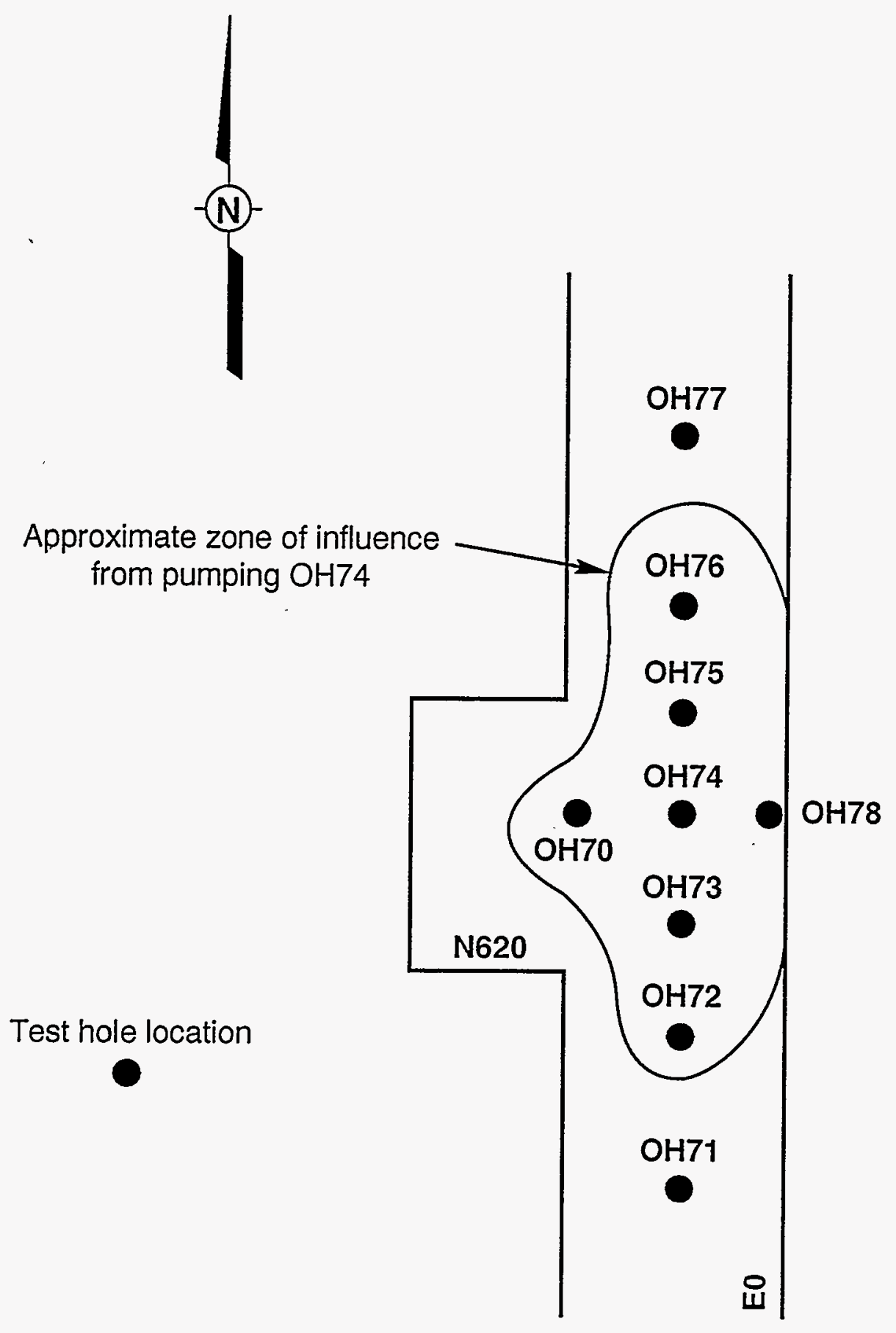

Figure not to scale

Figure E-5-4

Test Holes at the E0 Site Showing Response to Pumping OH74 During the Initial Test at This Site, October 1992 
All holes showing some response to pumping exhibited limited fluid-level recovery upon termination of pumping, including the pumping hole OH74. The limited recovery, combined with the distribution and degree of drawdown response, suggests that the local fractured reservoir is limited in radial extent and that fracture systems may not be well interconnected. Both $\mathrm{OH} 70$ and $\mathrm{OH} 78$ are located only $10 \mathrm{ft}$ from the pumped hole, but their drawdown responses are significantly different. Hole OH70 responded well to pumping, while OH78 did not. The drastic difference in response may indicate that the fracture system intercepted by OH78 (near the EO drift wall) is not connected to the system intercepted by the other test holes in the middle of the EO drift. The fracture system in the middle of the E0 drift is also possibly prominent beneath the N620 alcove because of the effect of the alcove excavation itself. The testing results also suggest that the fractures in MB 139 at the intersection of the EO and N620 are fairly well connected and that the connection dissipates as one moves away from the intersection down the E0 drift or toward the drift wall opposite the alcove.

Long-term fluid-level monitoring was not conducted to determine whether the test hole fluid levels had completely recovered with time. Therefore, no firm conclusion can be made as to the degree of potential dewatering of the fracture systems that occurred during the pumping test.

\section{E5.1.2 Pumping Test Analysis}

The aquifer test design and analysis computer software package AQTESOLV (Geraghty and Miller, 1989) was used to determine the hydrologic properties of the fractured zone at the E0 test site. The drawdown data from observation test holes $\mathrm{OH} 70, \mathrm{OH} 73, \mathrm{OH} 75$, and $\mathrm{OH} 72$ and pumping hole $\mathrm{OH} 74$ were evaluated using the computer software package. The Jacob and the Theis methods (Lohman, 1972) were used to determine transmissivity and storage coefficients (observation holes only) in the holes that responded to pumping.

Figures E-5-5 through E-5-9 show semilog and log-log plots of drawdown-versus-time data for the five wells listed above. The drawdown curves allowed analysis by Jacob and the Theis methods and generally showed linear plots of drawdown versus time and a good fit to Theis-type curves. The calculated transmissivity for the four observation holes, analyzed using both the Theis and the Jacob methods, ranged from 0.7 to 9.9 square feet per day ( $\mathrm{ft}^{2} /$ day), with an average of $3.7 \mathrm{ft}^{2} /$ day. The storage coefficients for the observation holes 
ranged from 0.0004 to a maximum of 0.0034 , indicating that the fracture system is at least partially confined. Transmissivity, calculated from the data for pumping hole OH74, was 3.8 $\mathrm{ft}^{2} /$ day for both Theis and Jacob methods (Figure E-5-9). No storage coefficient can be calculated for the pumping hole.

Both the transmissivity and storage coefficient values, as calculated by the program, agree to within an order of magnitude for all test holes analyzed. The results of the pumping test analysis indicate that the transmissivity of the fractured zone beneath the EO drift test site appears to be uniform (for those holes responding to pumping OH74). The calculated transmissivity for hole $\mathrm{OH} 72$ (Figure E-5-6) is somewhat greater (but by less than a factor of $2 \mathrm{X}$ ) than those calculated for $\mathrm{OH} 74, \mathrm{OH} 73$, and $\mathrm{OH} 75$. The value is well within the error range of the testing and analysis methods, indicating that the transmissivity at $\mathrm{OH} 72$ is consistent with transmissivities calculated for the rest of this test site.

The analysis suggests the fracture system beneath the intersection of the drift and alcove at the test site is hydraulically connected and has similar hydraulic characteristics. The data also show that the fracture system acts as a porous medium. It is noted that these methods require the assumption that the aquifer is infinite. The fracture system tested at this site probably does not meet this requirement. Also, the testing generally did not provide enough data to curve-fit for more than one log cycle; therefore, the reported analytical results may be in violation of this requirement. However, the test results do provide some semiquantitative estimates of the local hydrologic characteristics of the fracture systems.

Figures E-5-5 through E-5-9 show prominent changes in slope of the drawdown-versus-log time plot for these test holes. This change in slope may indicate that the cone of depression produced by pumping encountered a low-permeability boundary or an area void of brine-filled

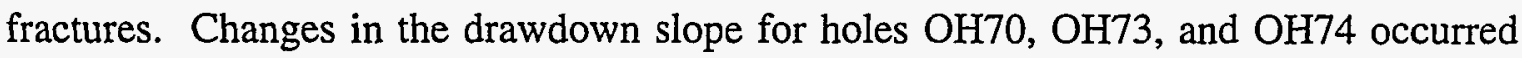
between 80 and $150 \mathrm{~min}$ into the test. The apparent no-flow or low-permeability boundary conditions observed in these plots are consistent with the other hydrologic data resulting from this program and support the conclusion that, at the EO test site, there are separate and hydraulically unconnected fracture systems saturated with brine. 

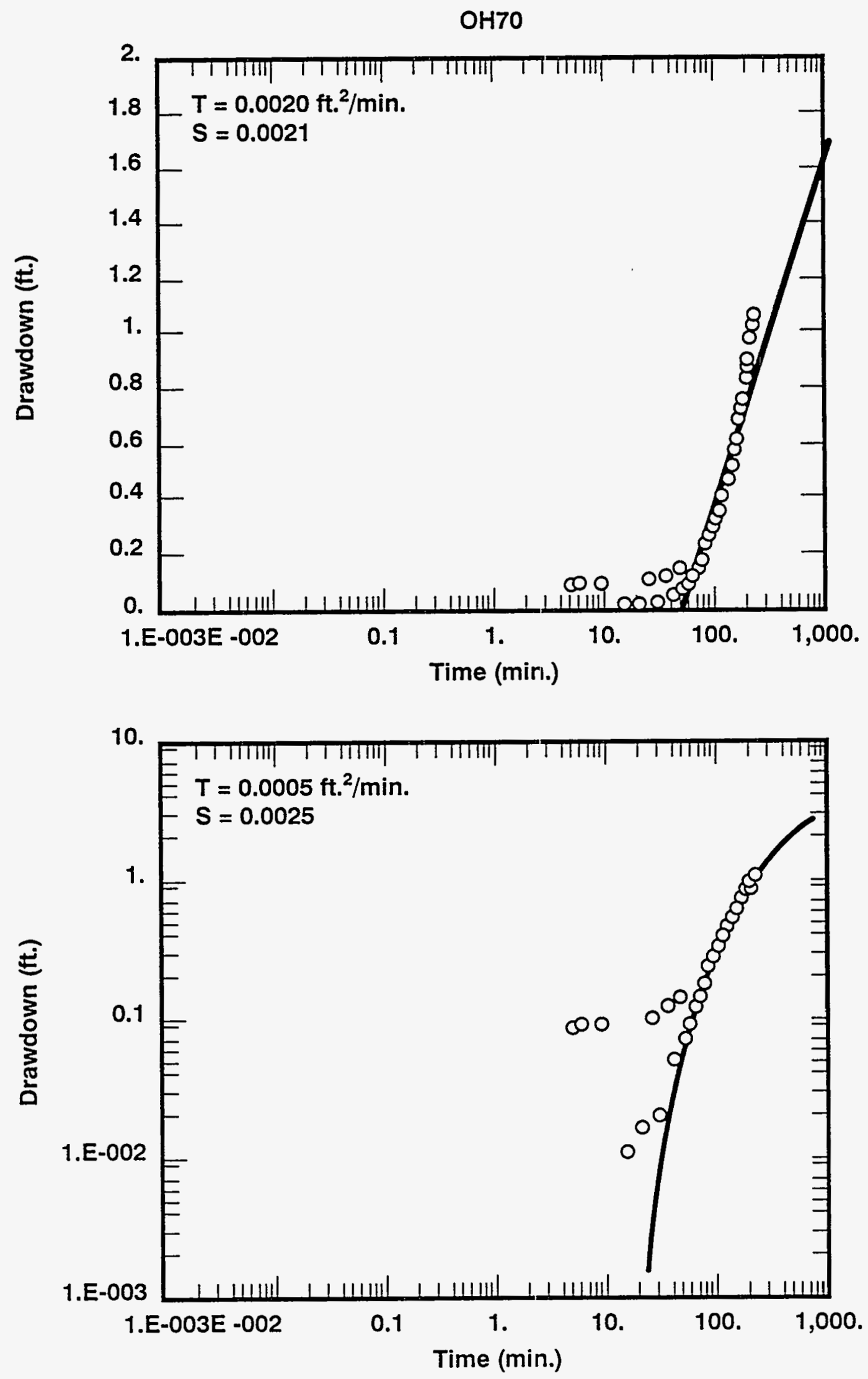

Figure E-5-5

Semilog and Log-log Plots of Drawdown

Versus Time for Observation Hole $\mathrm{OH} 7 \mathrm{O}$ 

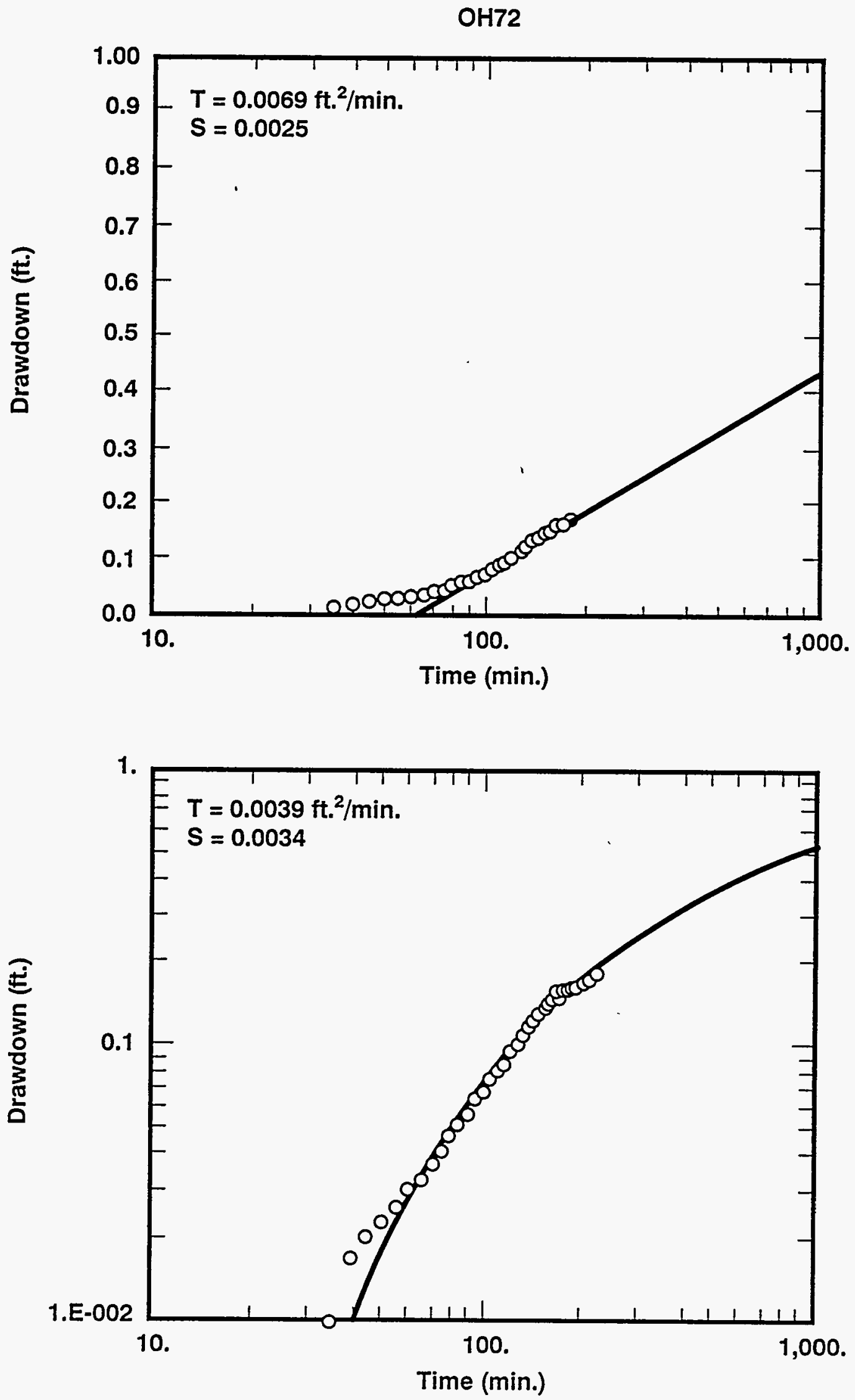

Figure E-5-6

Semi-log and Log-log Plots of Drawdown Versus Time for Observation Hole OH72 

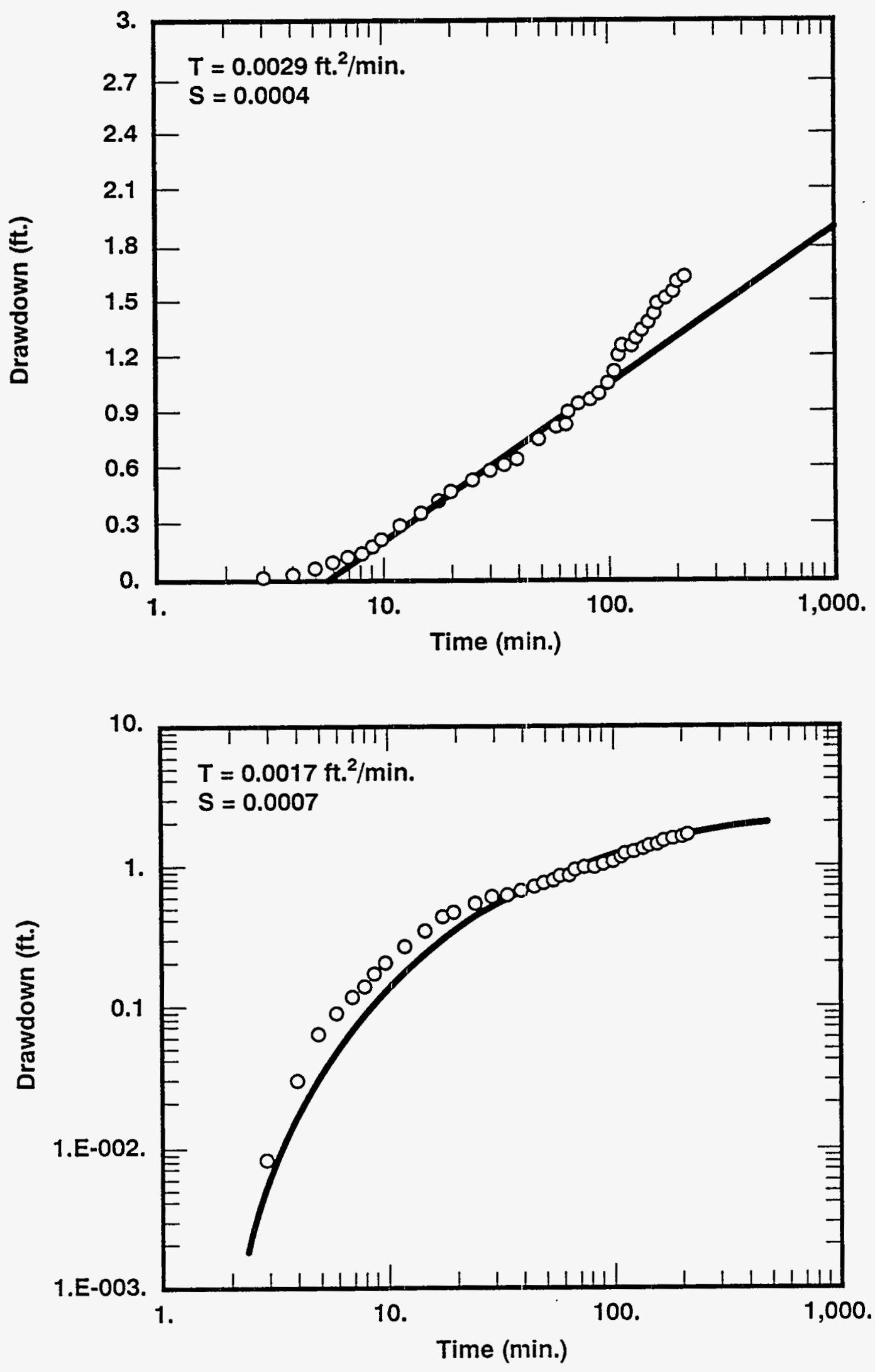

Figure E-5-7

Semilog and Log-log Plots by Drawdown

Versus Time for Observation Hole $\mathrm{OH} 73$ 
OH75
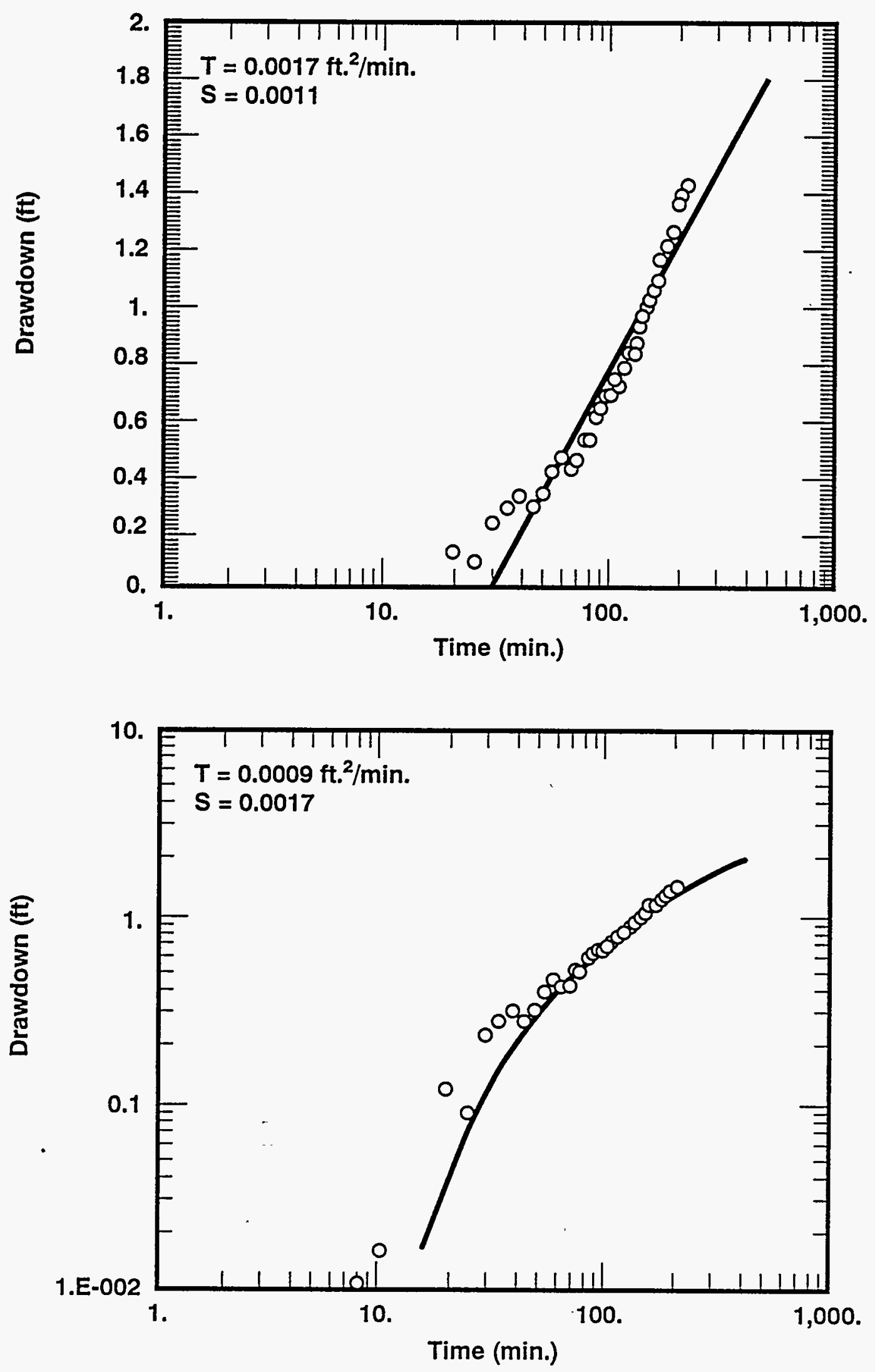

Figure E-5-8

Semilog and Log-log Plots of Drawdown

Versus Time for Observation Hole OH75 

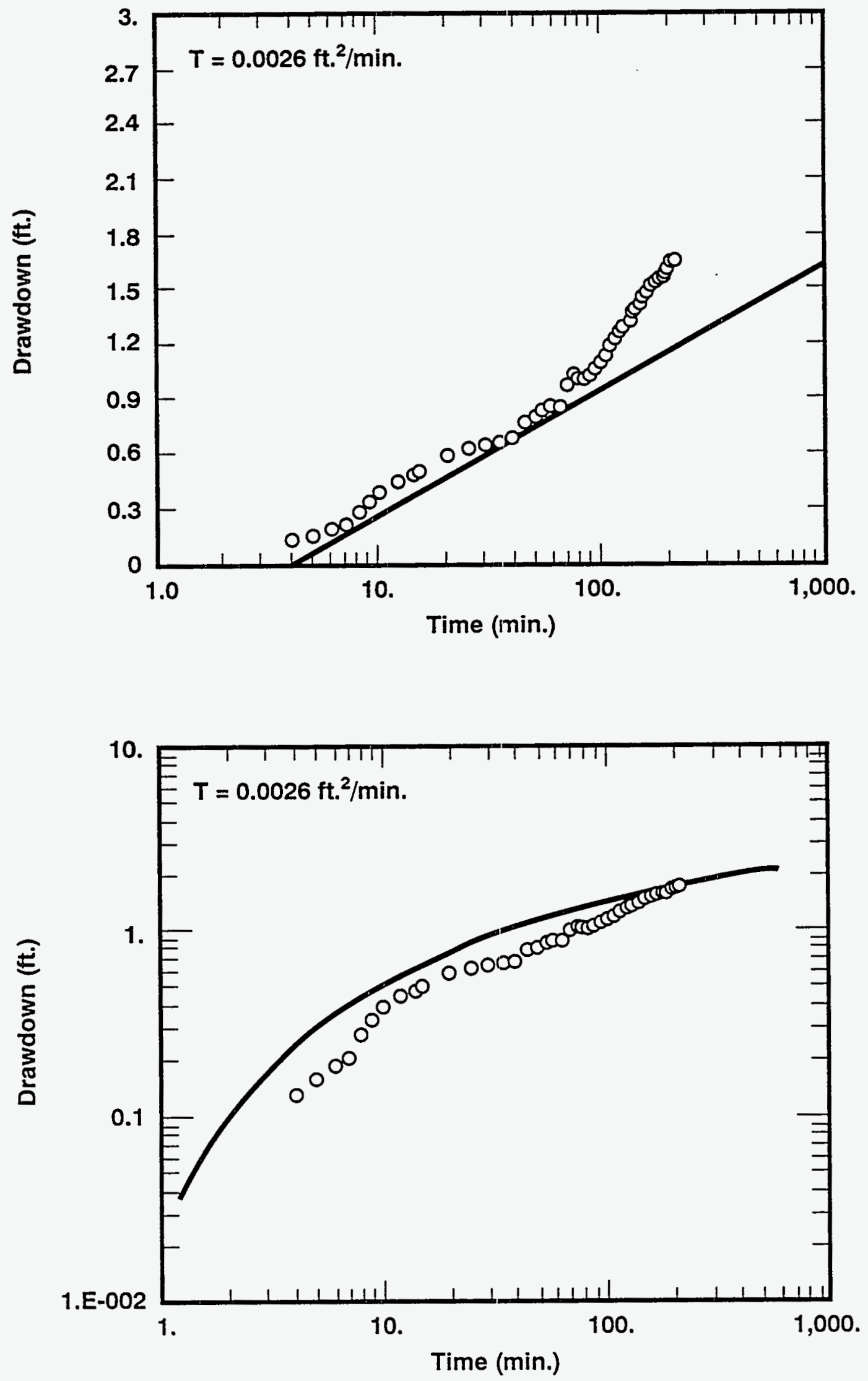

Figure E-5-9

Semilog and Log-log Plots of Drawdown

Versus Time for OH74 (Pumphole) 


\section{E5.2 Initial Pump Test at the Core Storage Library (W170) Site}

The second in a series of three fractured zone pumping tests was conducted during the week of November 9 through 13,1992. This test was performed at the intersection of the W170 drift and the underground core library.

Background water pressure and water-level data were collected to provide some definition of long-term trends and changes regarding test parameters and for comparison with test results. Background data collection began on November 9, 1992, in all holes at the test site, with the exception of $\mathrm{OH} 60$, which was not instrumented.

Figures E-5-10 and E-5-11 show hydrographs of fluid pressures in all holes (with the exception of OH60) from the initial instrumentation through completion of the pump test. These hydrographs show limited response to two different pumping episodes. Figure E-5-10 shows that the first pumped hole, OH66, did not recover from pumping and the limited response of the surrounding holes. Figure E-5-10 also shows that the second pumping hole, OH62, was slowly recovering before the transducer was pulled. These observations suggest that the fractured fluid reservoirs are limited and probably isolated from each other.

Figure E-5-11 shows hydrographs of fluid pressures in holes OH59, OH61, OH64, and OH68 through the end of the second pumping test. These holes, with the exception of $\mathrm{OH} 61$, which is adjacent to the second pump hole, show very limited (if any) response. This indicates some fracture connection between the two holes but limited connection elsewhere.

The pumping drawdown test, using $\mathrm{OH} 66$ as the initial pump hole, began at 11:00 a.m. on November 10,1992. The pump was turned on at 11:00 a.m., and brine was routed to a bucket with a 1-gal graduate scale. The initial flow rate was calculated at $13.3 \mathrm{gph}$ (50.3 $\mathrm{Lph}$ ). The flow rate was adjusted, and two additional readings were taken for an average flow rate of $9 \mathrm{gph}(34 \mathrm{Lph})$. Less than 3.5 gal (13.2 L) were pumped before the hole became dry. The water level reached the pump intake level at approximately 11:28 a.m., and the pump was left on until 11:45 a.m. to clear the pump tubing. Scan rates were changed back to 30-min intervals for overnight recovery data collection.

On November 11,1992, a second pump test was attempted using OH62 as the pumped hole. The pump was installed at approximately 8:35 a.m. The water level in OH62 was allowed to 


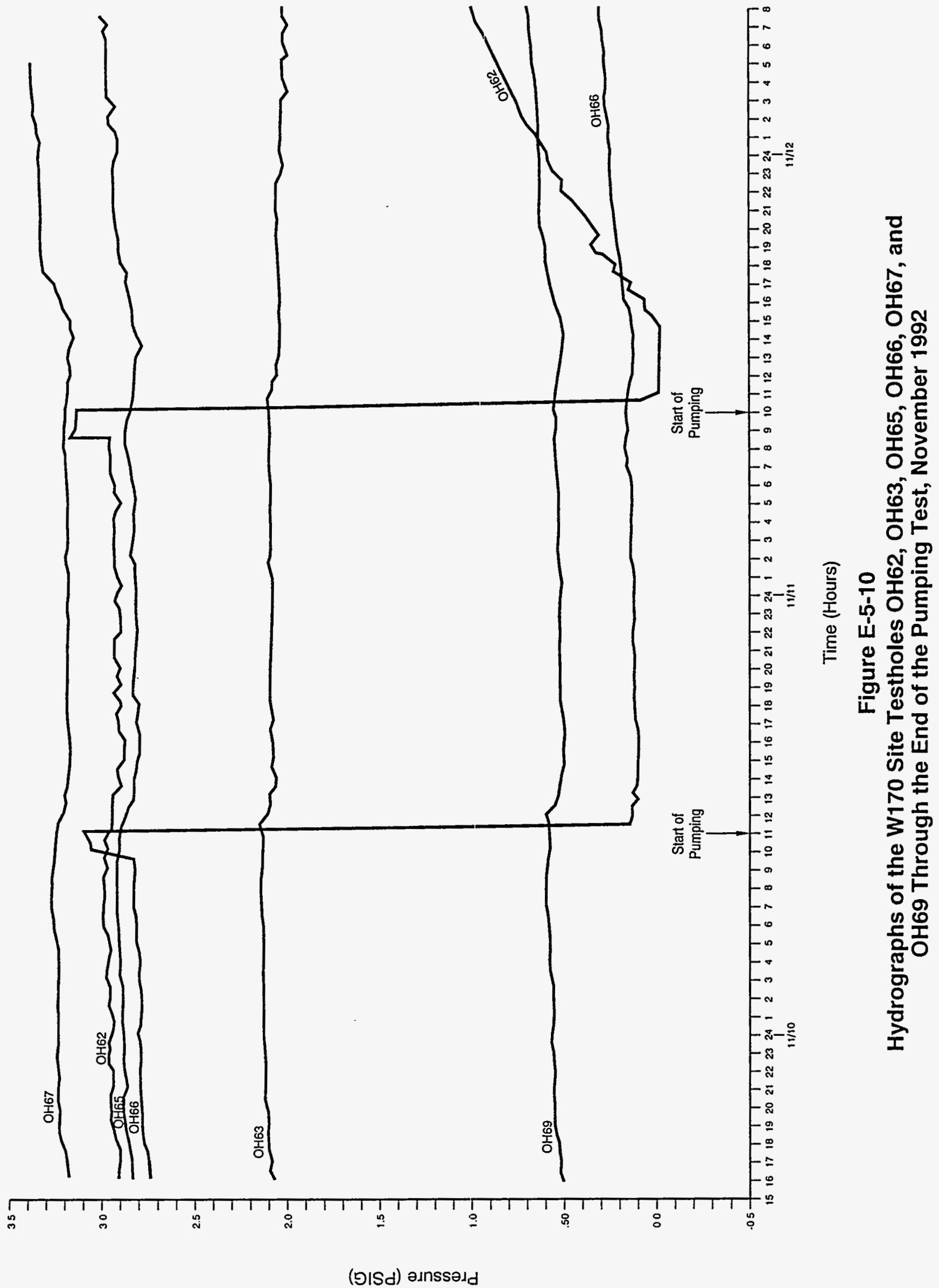

E-42 


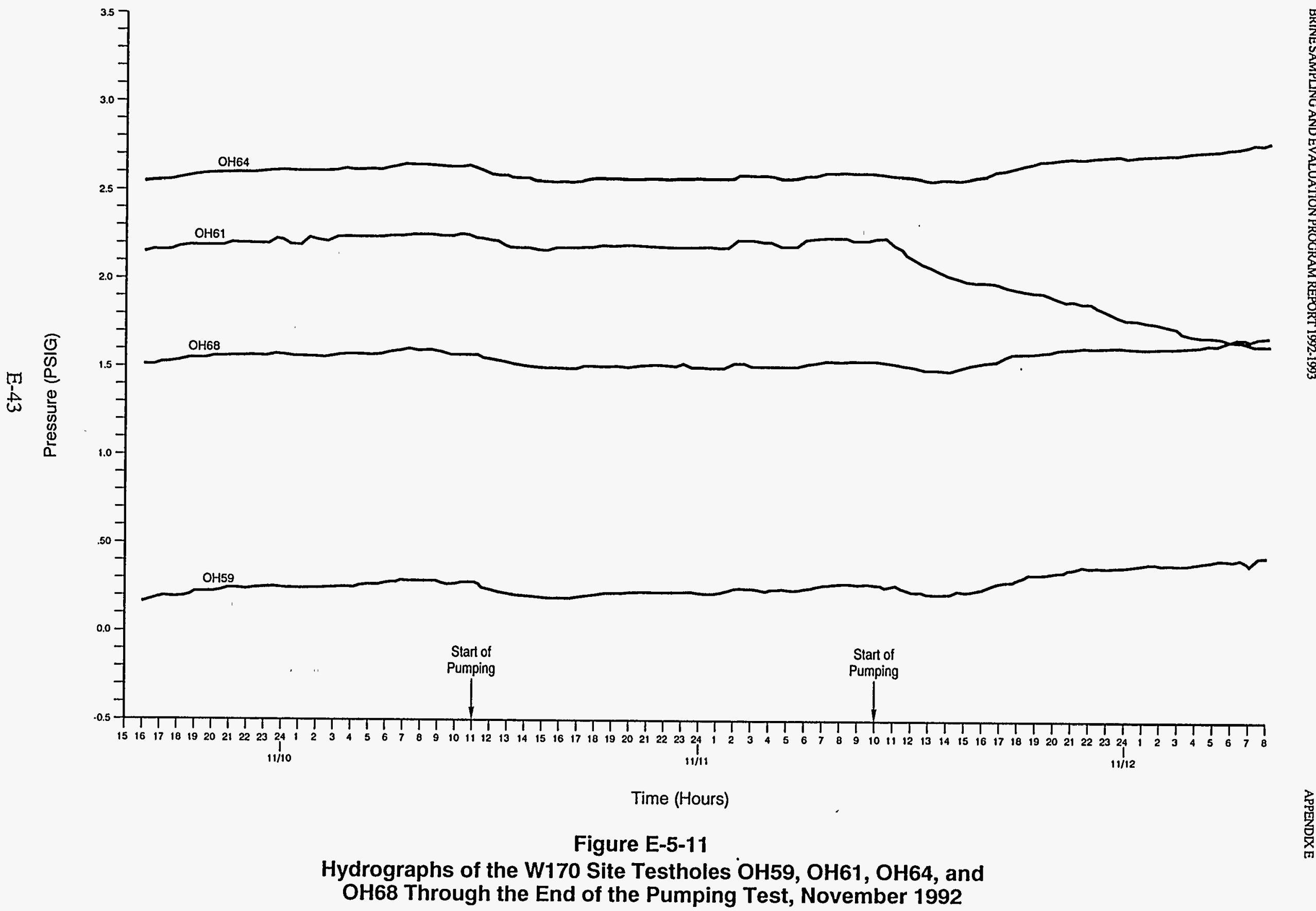


reach equilibrium for approximately $90 \mathrm{~min}$ after pump installation. The scan rate was changed from 30-min to 1-min intervals to accommodate rapid water-level changes that might occur during pumping. Pumping was initiated at 10:00 a.m., with a starting flow rate of 4.8 gph $(18.2 \mathrm{Lph})$. The flow rate was increased to $6 \mathrm{gph}(22 \mathrm{Lph})$. Two additional flow rates were taken before $\mathrm{OH} 62$ became dry at 11:00 a.m. Average flow rate during the approximately 1-hour test was $4.8 \mathrm{gph}(18.2 \mathrm{Lph})$. Scan rates were changed from 1-min intervals during the pump test to 30 -min intervals for overnight recovery data collection. The hydrographs in Figures E-5-10 and E-5-11 show that the pump test holes (OH62 and OH66) showed the only significant response to pumping. The other holes showed very limited or no response at all. OH62 showed fairly rapid recovery after the hole was pumped dry, while OH66 showed no recovery response after being pumped. OH59, OH63, OH64, OH65, OH67, OH68, and OH69 showed very little response (Figures E-5-10 and E-5-11).

Long-term fluid-level monitoring was not conducted to determine whether the test hole fluid levels completely recovered with time. Therefore, no firm conclusion can be made as to the degree of the potential dewatering of the fracture systems that occurred during the pumping tests. Hole OH66 exhibited limited fluid-level recovery upon termination of pumping. The limited recovery, combined with the distribution and degree drawdown, suggests that the local. fractured reservoir is limited in volume and that fracture systems ard marginally interconnected.

Response to OH62 pumping was limited to the holes toward the interior of the core library (Figure E-5-12). The hole adjacent to $\mathrm{OH} 62$ (OH61) showed significant response, as demonstrated in Figure E-5-11. OH63, located only $5 \mathrm{ft}(1.5 \mathrm{~m})$ away, showed no response to OH62 pumping, indicating a very limited fracture system confined to the alcove and not connected to the older W170 drift.

The hydrologic data generated from pumping $\mathrm{OH} 66$ and $\mathrm{OH} 62$ were inadequate for performing aquifer test analysis. Therefore, no analysis other than the evaluation of fracture relationships and drawdown was attempted.

The third pumping drawdown test was conducted at the E0 drift test site (Figure E-2-2) during the period of December 14 through 17, 1992. This field effort was performed as a retest of the initial test conducted here in October 1992. The purpose of the retest was to 


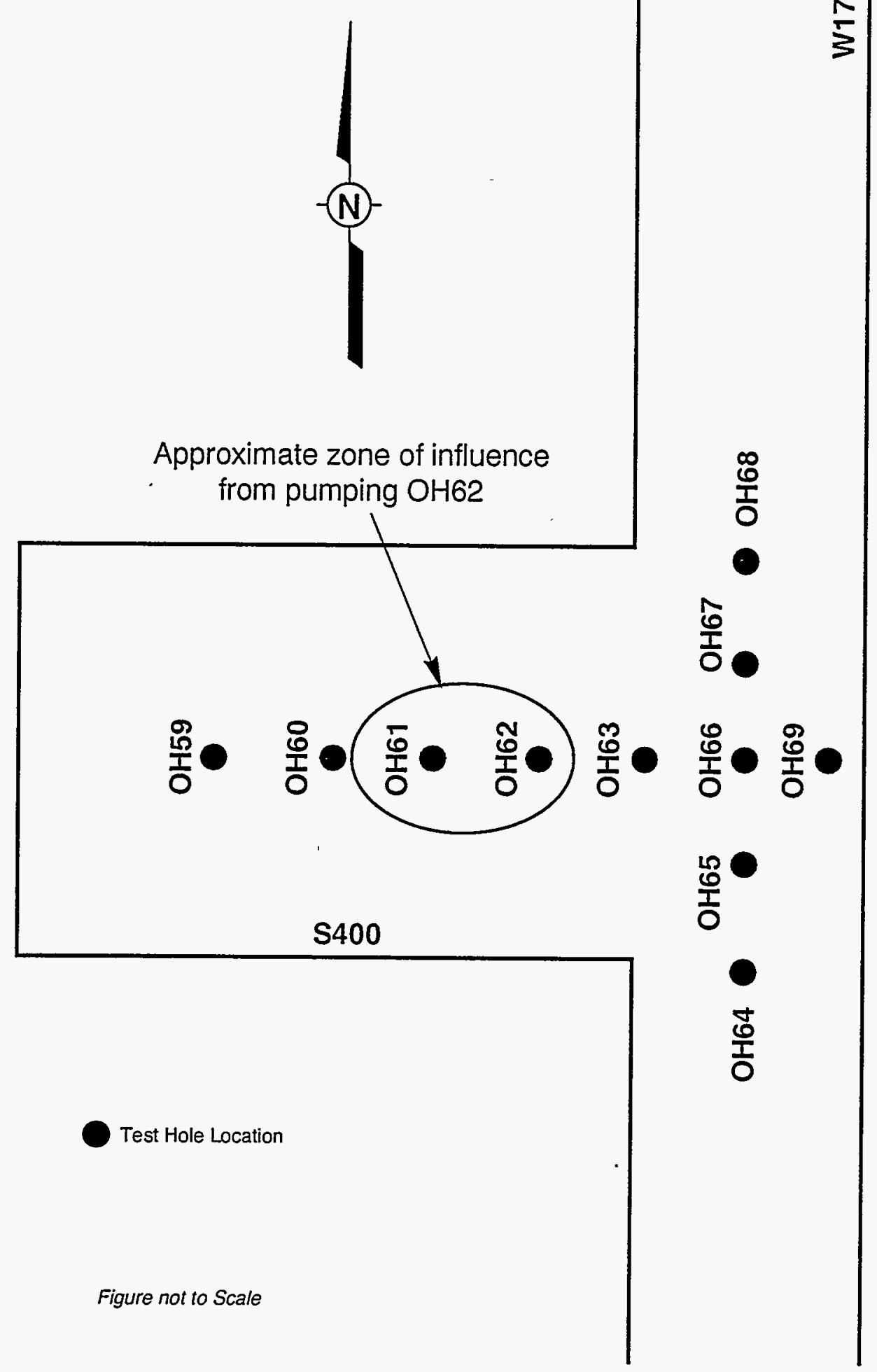

Figure E-5-12

Test Holes at the W170 Site Showing Response to Pumping OH62, November 1992 
determine whether reconditioning of the test holes would enhance the water-yielding characteristics of the holes, as well as the surrounding fractured zone. However, the reconditioning effort removed most of the brine from the test holes, and their fluid levels had not fully recovered by the time this test was initiated. Test hole reconditioning including chipping and vacuuming muck and fill material from the sides and bottoms of the test holes.

For this final test, three separate holes were pumped during individual pumping episodes. Initially the centrally located hole, $\mathrm{OH74,} \mathrm{was} \mathrm{used} \mathrm{as} \mathrm{the} \mathrm{pumping} \mathrm{hole,} \mathrm{as} \mathrm{it} \mathrm{was} \mathrm{during} \mathrm{the}$ first test at this site. On the following day, holes $\mathrm{OH} 72$ and $\mathrm{OH} 71$ were used as pumping holes. In each effort, the selected hole was pumped dry within 2 hours or less, indicating that the fractured zone was not yielding brine to the pumped hole at an adequate rate to keep up with the pumping.

As with the initial test at this site, all holes were instrumented with pressure transducers, and background pretest water-level data were recorded. These fluid-level data are continuous through the end of the pumping tests. Background fluid-level data collection began on December 14, 1992, in preparation for the pumping test scheduled for the following day.

Figures E-5-13 and 5-14 show hydrographs of test holes $\mathrm{OH} 71, \mathrm{OH} 72, \mathrm{OH} 73, \mathrm{OH} 74, \mathrm{OH} 70$, $\mathrm{OH} 75$, and $\mathrm{OH} 78$ through the end of all three pumping tests. These hydrographs show that any significant response to pumping $\mathrm{OH} 74$ (the first of the three holes pumped during this test period) is limited to $\mathrm{OH} 70, \mathrm{OH} 73$, and $\mathrm{OH} 75$. These three test holes are the closest to the pumped hole. However, review of the hydrographs shows that there was at least a minor response to pumping in most of the holes where a response was observed during the initial test in October 1992. The hydrographs also show that fluid levels had not completely recovered by the time that data collection was terminated.

The pumping drawdown-type test was conducted on December 15, 1992, using OH74 as the pumping hole. The test began at 12:00 p.m., attempting to achieve a flow rate of approximately $4.5 \mathrm{gph}(17 \mathrm{Lph})$. The pumping discharge rate was continuously monitored and adjusted until the desired flow rate was attained. The 4.5-gph (17-Lph) flow rate was selected because it was lower than that used during the October test and could possibly prolong pumping and, therefore, generate more data for analysis. The actual pumping rate varied from 4.19 to $5.84 \mathrm{gph}(15.9$ to $22.1 \mathrm{Lph})$, with an average of $4.70 \mathrm{gph}$ (17.8 Lph). 


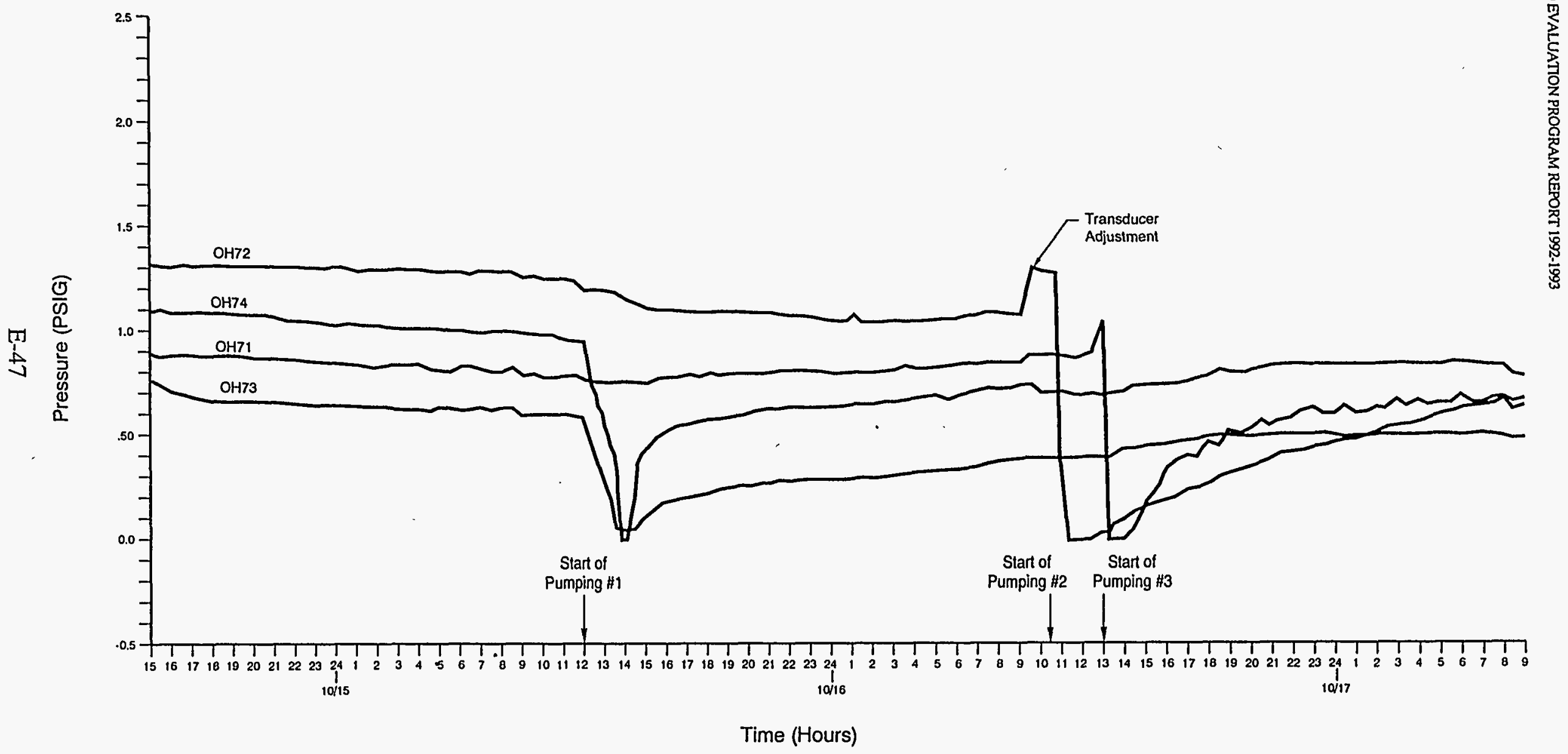

Figure E-5-13

Hydrographs of the EO Site Testholes $\mathrm{OH} 71, \mathrm{OH} 72, \mathrm{OH} 73$, and

OH74 Through the End of the Pumping Test, December 1992 

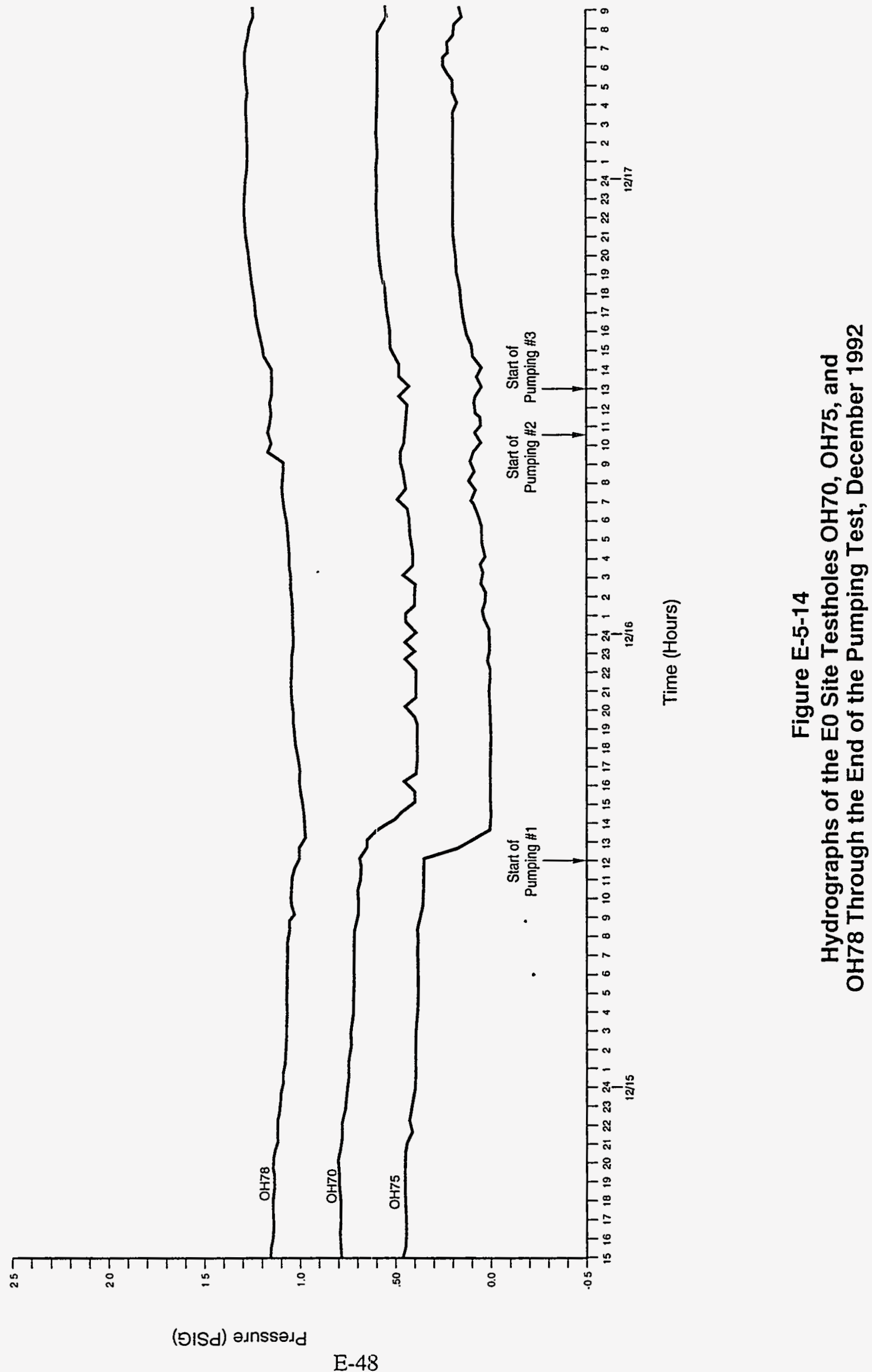
This test lasted until 1:50 p.m., when the fluid level in the pumped hole OH74 drew down to a level below the pump intake for a total test duration of approximately 1 hour and $50 \mathrm{~min}$. This retest was approximately 1.5 hours shorter in duration than the original test at this site. The shorter pumping duration is the result of having a shorter fluid column in the pumping hole, giving less available drawdown. The short duration of the test and rapid drawdown suggest that reconditioning the holes did little to enhance results from the pumped hole. During this test, fluid levels in all holes were continuously monitored using transducers and the datalogger. In addition, one transducer was set up as a barometer to monitor changes in atmospheric pressure.

After allowing the test area to recover overnight, a second pump test, using hole $\mathrm{OH} 72$ as the pumped hole, was attempted on December 16, 1992, at 10:46 a.m. Hole OH74, used for the test the previous day, had not fully recovered by the time the second test started. At the start of the second test, the pumping discharge rate was quickly established at approximately 4.0 gph $(15.1 \mathrm{Lph})$. The fluid level dropped below the pump intake before the next flow rate could be determined. The second pumping event was terminated at 11:14 a.m., after only 28 min of pumping. These results indicate that the surrounding fracture system yielded very little, if any, fluid to the pumped hole. The volume discharged by pumping appears to equal the fluid volume stored in the test hole.

Considering the rapid dewatering of the two previous test holes, the decision was made to perform a third pumping test in a separate test hole. It was anticipated that the staged pumping episodes might yield some additional information about the geometry and interconnection of fracturing at the test site by potentially creating overlapping drawdown responses or different responses in individual test holes.

Prior to initiating the third pumping test, the EO test site was allowed to recover for approximately 2 hours. Fluid levels in all holes were monitored continuously during this recovery period. The third pumping event, using hole $\mathrm{OH} 71$ as the pumped hole, began at 1:00 p.m. on December 16, 1992. Initially, the pumping discharge rate was measured at approximately $6.64 \mathrm{gph}(25.1 \mathrm{Lph})$. The discharge rate was subsequently lowered to less than $5.0 \mathrm{gph}(18.9 \mathrm{Lph})$. However, the hole pumped down to a level below the pump intake before the second flow rate could be accurately determined. The test was stopped 1:16 p.m. Review of the pressure readings in OH71 showed that negative pressure values were 
registered after only 9 to 10 min of pumping, indicating that the water level had already dropped below the transducer port by that time.

Recovery of the test area fluid levels was monitored until the following morning, when all data collection was terminated.

\section{E5.3.1 Test Performance and Results}

Figure E-5-15 shows the holes that exhibited at least some drawdown response to pumping OH74. The hydrographs in Figures E-5-13 and E-5-14 show that OH70, OH73, and OH75 are the only holes to respond significantly to pumping OH74. In contrast to the initial test performed at this site in October 1992, drawdown response during the December 1992 test was not as widespread and was less pronounced. The decreased response to pumping could be the result of the test area now having fewer interconnected saturated fractures, resulting in a decrease in reservoir size. The smaller saturated area may be the result of fluid removal from the test holes during previous pumping and hole reconditioning. The apparent decreased drawdown may also be the result of the holes not having recovered from previous fluid withdrawal and now having much lower fluid levels prior to this pumping test.

The test holes that showed measurable drawdown did exhibit recovery after pumping ceased in hole OH74. The apparent recovery trends as seen in Figures E-5-13 and E-5-14 suggest that with time, fluid levels may return to their pretest levels. Aquifer test analysis of the drawdown data from pumping OH74 was not performed. The data generated from this pump test are inadequate for performing such analysis with confidence.

Figure E-5-16 shows the test holes that showed some response to the pumping of the second test hole (OH72). Only holes $\mathrm{OH73}$ and $\mathrm{OH} 74$ appear to respond to pumping. The apparent response is limited to a slight short-term decrease in fluid pressure or a reduction in the rate of recovery from the OH74 pumping test.

The limited response seen in other holes at the site indicates that $\mathrm{OH} 72$ is not hydraulically well connected by fractures to the central part of the test site. This observation is supported by the drawdown responses seen from the previous tests performed at this site. The holes that did show a slight response to pumping are located along the EO drift centerline and back toward the intersection with the N620 alcove. No response was measured at the holes to the 


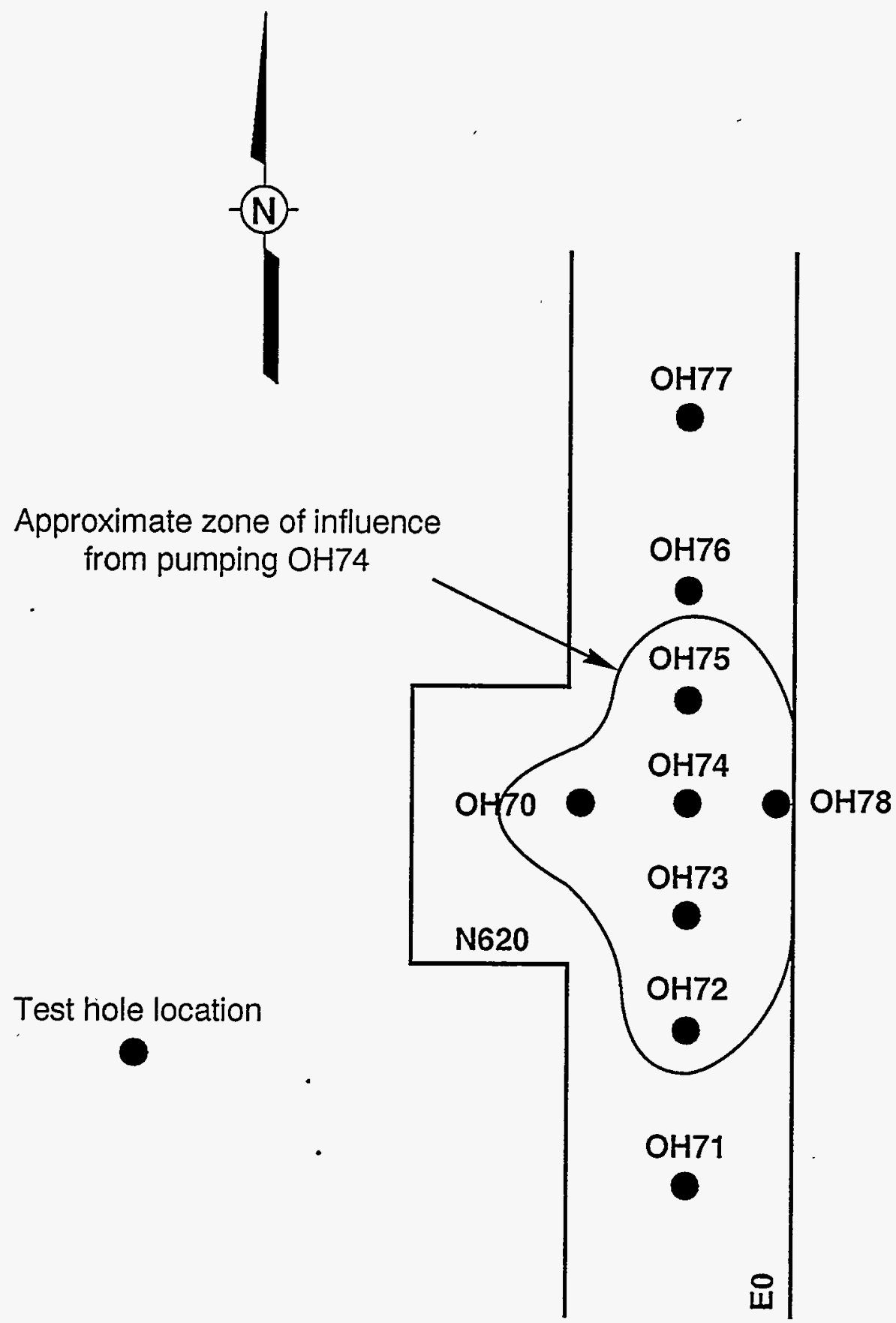

Figure not to scale

Figure E-5-15

Test Holes at the E0 Site Showing Response to Pumping OH74 During the Retest at This Site, December 1992 


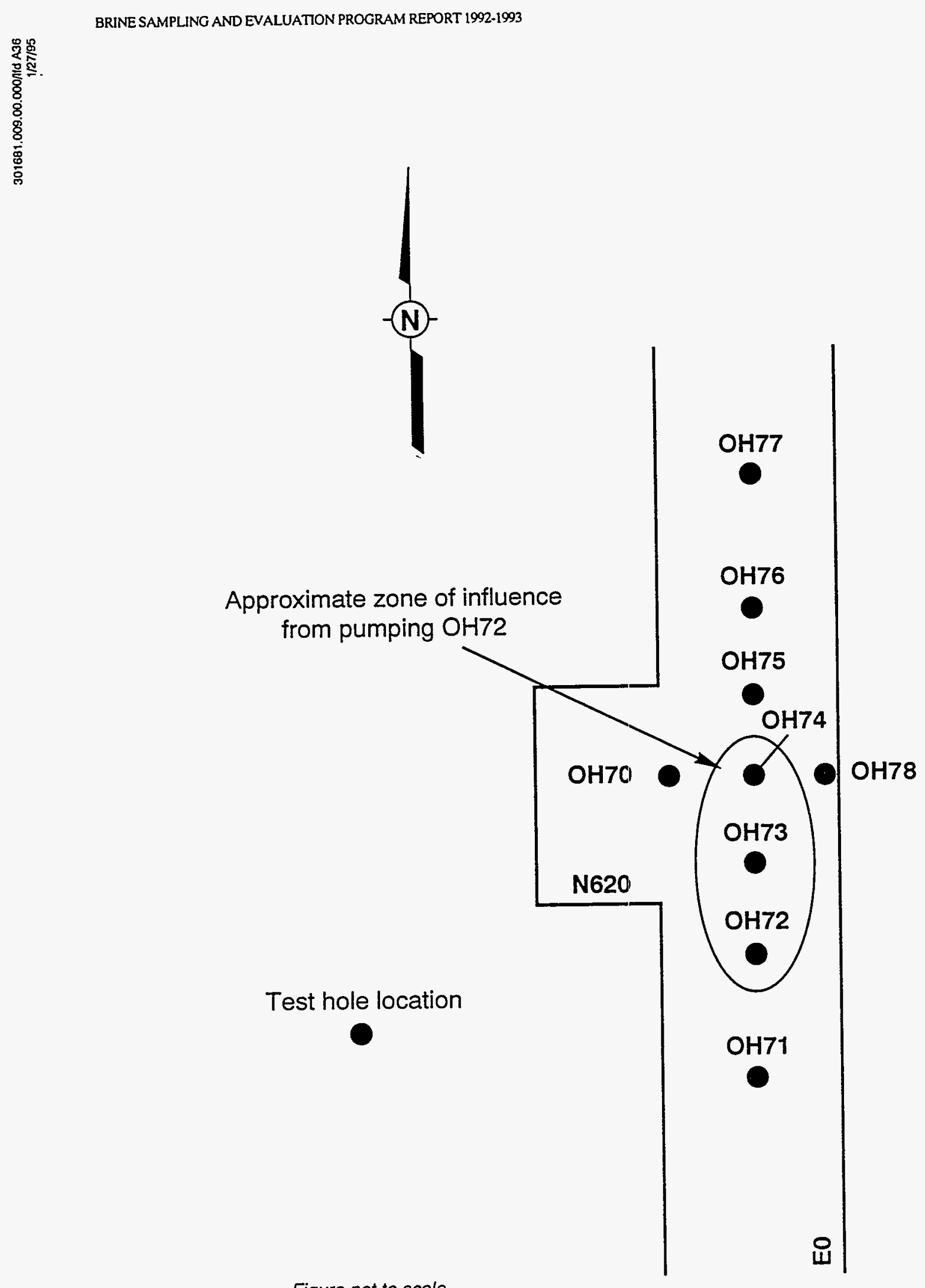

Figure not to scale

Figure E-5-16

Test Holes at the EO Site Showing Response to Pumping OH72 During the Retest at This Site, December 1992 
south of OH72, down the drift, supporting the conclusion that fracturing at this site is somewhat limited to the general area of the intersection. The rapid dewatering of $\mathrm{OH} 72$ indicates that the fracture system around this test hole has a very low permeability and that the available fluid reservoir may be very limited in volume.

The hydrologic data generated from pumping $\mathrm{OH} 72$ are insufficient for performing an analysis to define hydrologic parameters. Therefore, no analysis other than the evaluation of drawdown and fracture relationships was attempted.

The third and final pumping test at the EO drift test site was conducted using hole OH71 as the pumping hole. Figure E-5-17 shows the holes at the test site that responded to pumping. No drawdown response was seen in any of the test holes, except OH71, which was the pumped hole. The test results support the conclusion that interconnected fracture systems are absent or at least not significant away from the intersection of the E0 drift and the N620 alcove. Evaluation of the volume of fluid pumped from $\mathrm{OH} 71$ suggests that no fluid was donated to the pump hole by the surrounding fractured formation. Therefore, the fracture system in the EO drift around $\mathrm{OH} 71$ is of very low permeability, and the available fluid reservoir is small.

The hydrographs in Figures E-5-13 and E-5-14 show that all other test holes continued to recover from previous pumping activity and were not affected by pumping $\mathrm{OH} 71$. Hole $\mathrm{OH} 71$ recovered approximately 50 percent of the drawdown achieved during the test by the time that data collection was terminated. Posttest fluid-level monitoring was not long enough to determine whether any of the test holes recovered to their pretest fluid levels.

No drawdown response to pumping OH71 was observed in any of the test holes at the site. Therefore, no pumping test analysis can be performed for this test.

\section{E5.4 Geologic Logging of Cores}

As part of the program for hydrologic testing of the fractured part of the DRZ, continuous cores were collected from each of the test holes drilled at the EO and W170 test sites. These cores were drilled using air to a depth of at least $1 \mathrm{ft}$ beneath the base of MB 139. The cores were logged according to standard procedures established by the WIPP Geotechnical Engineering Section. Of particular importance to this study are the descriptions of fractures 


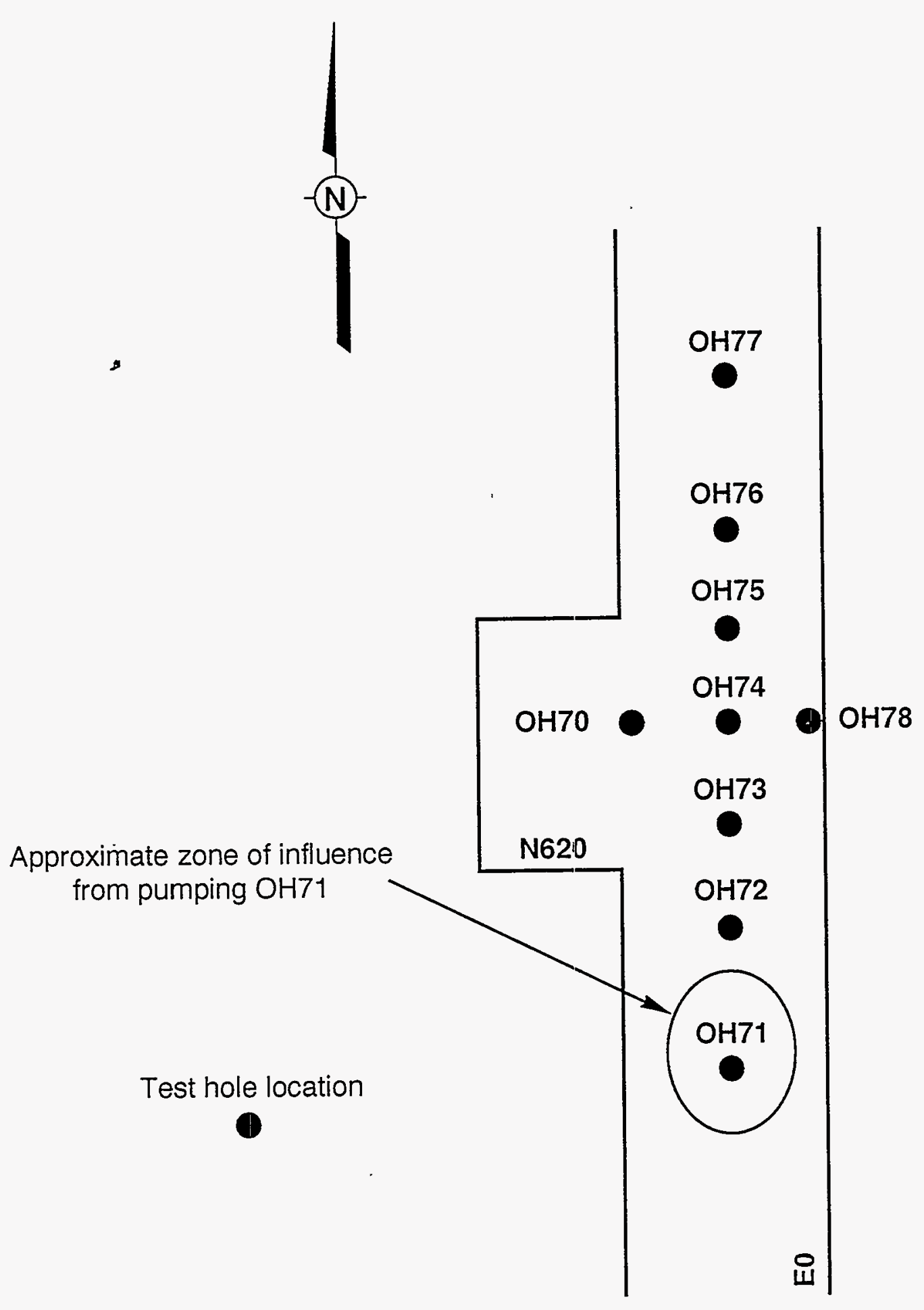

Figure not to scale

Figure E-5-17

Test Holes at the E0 Site Showing Response to Pumping $\mathrm{OH} 71$

During the Retest at This Site, December 1992 
and separations in the core samples. These core descriptions reveal where the fractured part of the hydrologic system being tested occurs. The data presented in the core logs indicate that, in general, the anhydrite of MB 139 often contains numerous fractures and core breaks, especially in the lower one-half of the bed. The breaks noted in the lower part of the core from $M B 139$ may signify the presence of fractures in those locations in the subsurface.

Figure E-5-18 presents the core descriptions of holes $\mathrm{OH} 66$ and $\mathrm{OH} 74$, which were used as pumping holes (and are representative of the conditions) at each of the two test sites. Figure E-5-18 shows that fractures within the lower portion of MB 139 may be the most prominent fluid transmitting part of the fractured zone beneath the WIPP underground excavations. However, the existence of breaks in the core samples does not necessarily represent open brine-filled fractures in the subsurface.

Hole $\mathrm{OH} 66$ was used as the pumping hole during the second pumping test conducted at the W170 test site (Section E5.2). This hole pumped dry quickly, indicating that the local permeability of the fracture system was very low and that the fractures yielded little fluid to the hole during pumping. Therefore, the fractures and breaks observed in the core of $\mathrm{OH} 66$ either may not be open in the subsurface or may not be well connected to the overall test area fracture system.

Hole $\mathrm{OH} 74$, used as a pumping hole at the EO test site, also exhibits fractures and breaks in the core sample examined. In this case, the performance of the hole during pumping suggests that the fractures may be better connected to the surrounding area and are possibly more open to transmit fluid during pumping.

\section{E6.0 Summary and Recommendations}

The main objective of the Hydrogeologic Testing of the Fractured Part of the Disturbed Rock Zone Beneath the WIPP Excavations Program is to characterize the hydrologic conditions of fracture systems beneath the floor of the repository. Short-duration hydraulic tests were conducted at two underground test sites. These tests were conducted at the intersection of the E0 drift and N620 alcove and in the W170 drift, in front of the underground core library. 


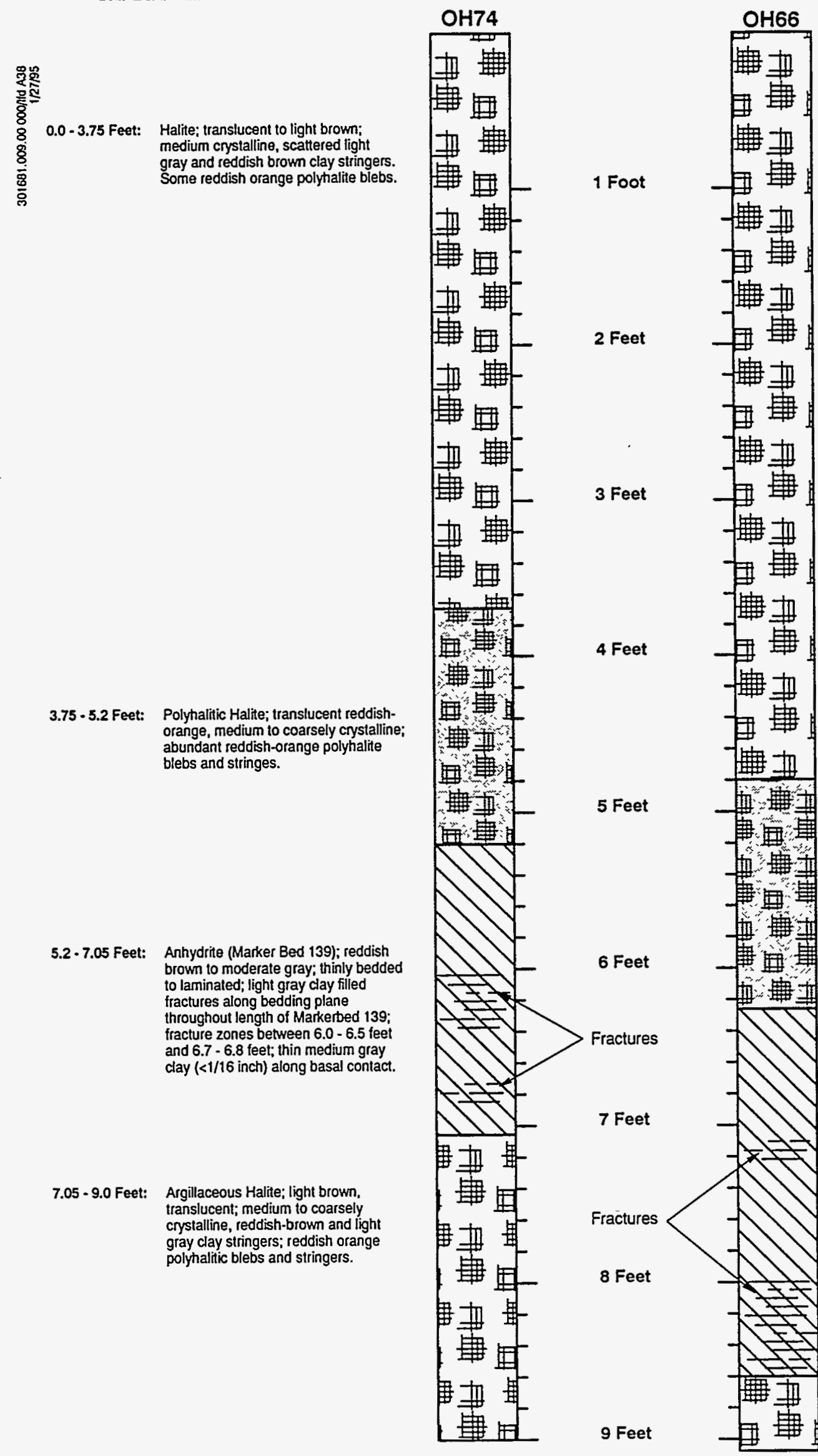

0.0 - 4.8 Feet: Halite; Iranstucent, light brown to reddish brown; medium crystalline, scattered moderate gray clay stringers decreasing downward; some reddish orange polyhalite blebs and stringers: some core washout along clay seams (<1/16 inch) thick between 0.15 and 1.0 feet.

\section{8 - 6.35 Feet: Polyhalitic Halite; translucent moderate reddish orange; coarsely crystalline: scattered reddish orange polynalitic blebs and stringers; zone of clear to translucent halite $\mathbf{5 . 7 5} \cdot \mathbf{5 . 8 5}$ leet; sharp basal contact (-25 degrees).}

6.35 - 8.6 Feet: Anhydrite (Marker Bed 139); moderate reddish.brown to medium gray: banded to thick laminar; thin ( $<1 / 4$ inch) moderate gray clay along basal contact; core breaks at 7.2 feet. 8.0 feet, 8.05 feet, 8.3 feet, 8.55 leet: breaks along bedding planes from 8.0-8.6 feet; sharp horizontal basal contact.

Figure E.5-18

Core Log Description Diagram for Pump Test Holes 
These two sites were selected because of their age, their physical characteristics, their relationships to other excavations, the existence of fractures, and their exposures to long periods of water-spreading to control dust and to assist in roadbed consolidation.

Test results indicate that the significant fracture systems that yield water to test holes are restricted to $M B$ 139. For the two sites tested, there appears to be separate, saturated unconnected fracture systems of fairly low transmissivity. At the E0 test site, fracture systems that are connected are confined to the immediate intersection of the drift and alcove. For the W170 site, the intersection did not contain significant connected fractures. Based on the observed drawdown response to pumping, the area within the core library appeared to be underlain by a somewhat more connected fracture system. This condition could be influenced by the width of the individual excavations. The W170 drift, although much older, has a relatively narrow opening in comparison to the core library. These data suggest that excavation dimensions may play a more important role than age in fracture development.

The posttest fluid-level recovery observed at the test sites suggests that the fracture systems beneath these areas are limited, and the available fluid reservoirs are small. Although longterm fluid-level monitoring was not conducted as part of this field program, the data gathered indicate that pumping at these sites was dewatering the fracture systems.

The results of the pumping tests support the concept of limited, bounded fractured fluid reservoirs. Data analysis from the E0 test site showed clear changes in the slope of the plotted drawdown curves for some test holes, indicating the presence of nearby no-flow or low-permeability boundaries. Testing at the W170 site did not produce adequate data for aquifer test analysis.

The Jacob and Theis methods were used to determine transmissivity and storage coefficients for the first test at the E0 site. The calculated transmissivities for all holes were 0.7 to $9.9 \mathrm{ft}^{2} /$ day. Storage coefficients ranged from 0.00038 to 0.0034 , indicating that the fracture system at the E0 site is partially confined.

Additional test sites should be developed to define better the nature of fracturing in areas other than the intersections of drifts and rooms. The EO test site could be expanded to both the north and south of the current site to allow comparative testing. If the test site was 
expanded, the results of pump testing away from the drift and alcove intersection could be compared to the results produced by this study, and the effects of excavation geometry could be quantified. Additional testing should be conducted at the lowest possible flow rates for the longest time achievable, and fluid-level recovery should be monitored long term.

The test site located near the AIS in the S90 drift should be prepared for hydrologic testing by providing access to all test holes. This will require moving the electrical substation and other equipment away from the site. This is an important test site, because it was the site of the original fractured zone test in 1988. Fracturing may have become better developed since the initial test was conducted. This site may provide the best location to observe timedependent development of fracture systems.

\section{E7.0 References}

Bechtel National, Inc. (Bechtel), 1986, "Interim Geotechnical Field Data Report," WIPP-DOE 86-012, prepared for the U.S. Department of Energy by Bechtel National, Inc., San Francisco, California.

Crawley, M. E., T. W. Cooper, and R. G. Richardson, 1992, "Hydrologic Testing of the Fractured Part of the Disturbed Rock Zone Beneath the WIPP Excavations," report filed by IT Corporation for Westinghouse Electric Corporation, Carlsbad, New Mexico.

Deal, D. E., and J. B. Case, 1987, "Brine Sampling and Evaluation Program, Phase I Report, June, 1987," DOE-WIPP-87-008, prepared for the U.S. Department of Energy by IT Corporation and Westinghouse Electric Corporation, Albuquerque, New Mexico.

Deal, D. E., and W. M. Roggenthen, 1991, "Evolution of Hydrologic Systems and Brine Geochemistry in a Deforming Salt Medium: Data from WIPP Brine Seeps," Proceedings of Waste Management '91, Tucson, Arizona, Vol. 2, pp. 507-516.

Deal, D. E., R. J. Abitz, D. S. Belski, J. B. Case, M. E. Crawley, R. M. Deshler, P. E. Drez, C. A. Givens, R. B. King, B. A. Lauctes, J. Myers, S. Niou, J. M. Pietz, W. M. Roggenthen, J. R. Tyburski, and M. C. Wallace, 1989, "Brine Sampling and Evaluation Program 1988 Report," DOE-WIPP-89-015, Waste Isolation Pilot Plant, U.S. Department of Energy, Carlsbad, New Mexico.

Deal, D. E., R. J. Abitz, D. S. Belski, J. B. Clark, M. E. Crawley, and M. L. Martin, 1991, "Brine Sampling and Evaluation Program, 1989 Report," DOE-WIPP 91-009, Waste Isolation Pilot Plant, U.S. Department of Energy, Carlsbad, New Mexico. 
Geraghty and Miller, 1989, "AQTESOLV," Aquifer Test Design and Analysis Computer Software, Geraghty and Miller Modeling Group, Reston, Virginia.

Lohman, S. W., 1972, "Ground-Water Hydraulics," U.S. Geological Survey Professional . Paper 708, U.S. Government Printing Office, Washington, D.C., 70 pp.

Westinghouse Electric Corporation, 1987, "Geologic Rock Coring Logging," Waste Isolation Pilot Plant Procedure WP 07-502, Carlsbad, New Mexico. 


\section{APPENDIX F}

\section{NUMERICAL MODELING OF BRINE SEEPAGE FROM CLAY COMPACTION}




\section{THIS PAGE INTENTIONALLY LEFT BLANK}




\section{APPENDIX F}

\section{F1.0 Introduction}

These calculations are order-of-magnitude calculations to approximate how much brine might be released to Waste Isolation Pilot Plant (WIPP) excavations assuming that the only source of brine are saturated clays within thin clay seams in the Salado Formation (Deal and others, 1993; Deal and Bills, 1994). The three analyses consider consolidation of thin compressible clay layers due to the redistribution of stress and generation of excess pore pressure within the clay layers after entry or room excavation. The modeling assumptions are as follows:

- Stress redistribution results in a localized increase in stress that is far more significant in generating excess pore pressure than in near-ground surface consolidation. The stress redistribution deforms the clay, plastically generating an excess pore pressure of several megapascals ( $\mathrm{MPa}$ ) within the disturbed rock zone (DRZ).

- Transient flow to the excavation or boundary dissipates the excess pore pressure within the clay layer.

- The rate of flow depends on the consolidation properties of the clay (hydraulic conductivity, compressibility, and porosity), the cross-sectional area of the clay seams intercepting the excavation, and the extent of the DRZ.

- The tributary method predicts the resulting increasing in the total stress of $3 \mathrm{MPa}$. Consider that after 1,000 days (Deal and others, 1989, Figure 5-4), the stress abutment zone extends out about 5 excavation diameters. The average diameter for the room is about 3 meters (m).

- The compressibility of the clay is $10^{-7} \mathrm{~Pa}^{-1}$ corresponding to a clay of medium compressibility. The hydraulic conductivity of the clay is $10^{-8}$ centimeters per second $(\mathrm{cm} / \mathrm{s})$. Under a change in effective stress of $3 \mathrm{MPa}$ after consolidation is complete, the change in porosity is 30 percent.

\section{F2.0 Room Q}

\section{F2.1 Problem Statement}

Calculate the amount of brine released to Room Q for two clay seams 3.5 millimeters $(\mathrm{mm})$ thick above and below the orange band (Map Unit 1). The model for consolidation assumes that the increase in stress deforms the clay seams plastically, resulting in $3 \mathrm{MPa}$ of excess pore pressure. Brine flow is induced to the room excavation because of the excess pore pressure. The consolidation of the clay layer is substantial, with a change in porosity of 30 percent. 


\section{F2.2 Solution}

From Deal and others (1989, p. 5-19), the stress abutment zone extends out about 5 diameters after about 1,000 days. Calculate the size of the abutment for Room Q. The diameter of Room $Q$ is $1.5 \mathrm{~m}$. The length $(L)$ over which flow occurs is:

$$
\mathrm{L}:=5 \cdot 1.5 \cdot \mathrm{m} \quad \mathrm{L}=750^{\circ} \mathrm{cm}
$$

From Freeze and Cherry $(1979$, p. 55$)$, the clay compressibility $\left(\alpha_{\text {clay }}\right)$ and porosity $(\phi)$ are:

$$
\alpha_{\text {clay }}:=10^{-7} \cdot \mathrm{Pa}^{-1} \quad \phi:=0.50
$$

Calculate the change in porosity that results from compression of the clay in the DRZ.

$$
\alpha_{\text {clay }} \cdot 3 \cdot 10^{6} \cdot \mathrm{Pa}=0.3
$$

The change is substantial and agrees with observation of "squeezing ground."

Calculate the void ratio (e), the conductivity $(k)$, and the coefficient of consolidation $\left(c_{v}\right)$ for the clay. From Freeze and Cherry (1979) and Scott (1963):

$$
\begin{array}{cc}
\mathrm{e}:=\frac{\phi}{1-\phi} & \mathrm{e}=1 \\
\mathrm{k}:=10^{-8} \cdot \frac{\mathrm{cm}}{\mathrm{sec}} & \\
\rho_{\text {wo }}:=1 \cdot 0 \cdot \frac{\mathrm{gm}}{\mathrm{cm}^{3}} & \mathrm{c}_{\mathrm{v}}=0.002 \cdot \frac{\mathrm{cm}^{2}}{\mathrm{sec}}
\end{array}
$$

where:

$$
\begin{aligned}
& \rho_{\mathrm{wo}}=\text { unit weight of water } \\
& \mathrm{g} \quad=\text { acceleration constant }
\end{aligned}
$$


Calculate the initial excess pore pressure head $\left(\mathrm{V}_{0}\right)$

$$
\mathrm{V}_{0}:=\frac{3 \cdot 10^{6} \cdot \mathrm{Pa}}{\rho_{\mathrm{wo}} \cdot \mathrm{g}} \quad \mathrm{V}_{0}=3.059 \cdot 10^{4} \cdot \mathrm{cm}
$$

We consider the lateral flow to the room over the length of the abutment zone $L$ with zero pressure at the room boundary and a "no flow" boundary condition at the edge of the DRZ. See Scott (1963, p. 184) for explanation of similarities between the thermal case and the consolidation case. We initally define the complementary error function.

From Carslaw and Jaeger (1959, p. 309), the solution for excess pore pressure dissipation is as follows:

$$
\begin{aligned}
& \operatorname{erfc}(x):=1-\operatorname{erf}(x) \quad \kappa:=c_{v} \\
& v(x, t):=v_{0}-v_{0} \cdot\left[\sum_{n=0}^{20}(-1)^{n} \cdot \operatorname{erfc}\left[\frac{(2 \cdot n+1) \cdot L-x}{2 \cdot \sqrt{\kappa \cdot t}}\right]+\sum_{n=0}^{20}(-1)^{n} \cdot \operatorname{erfc}\left[\frac{(2 \cdot n+1) \cdot L+x}{2 \cdot \sqrt{\kappa \cdot t}}\right]\right]
\end{aligned}
$$

where:

$$
\begin{aligned}
& \mathrm{V}_{0}=\text { initial temperature (analogous to initial excess pore pressure) } \\
& \mathrm{v}(\mathrm{x}, \mathrm{t})=\text { temperature as function of space and time (analogous to excess pore pressure } \\
& \text { over a steady state pore pressure as a function of space and time) } \\
& \kappa=\text { thermal diffusivity (analogous to coefficient of consolidation) } \\
& \mathrm{t}=\text { time } \\
& \mathrm{x}=\text { distance }(\mathrm{x}=750 \mathrm{~cm} \text { at the Room } \mathrm{Q} \text { boundary, and } \mathrm{x}=0 \mathrm{~cm} \text { at the far field) } \\
& \operatorname{erfc}(x)=\text { complimentary error function } \\
& \operatorname{erf}(\mathrm{x})=\text { error function, and } \\
& \mathrm{n}=\text { series index. }
\end{aligned}
$$

With the above properties, plot the distribution of excess pore pressure at several times.

$$
\mathrm{x} .=0 \cdot \mathrm{L}, .01 \cdot \mathrm{L} . . \mathrm{L}
$$




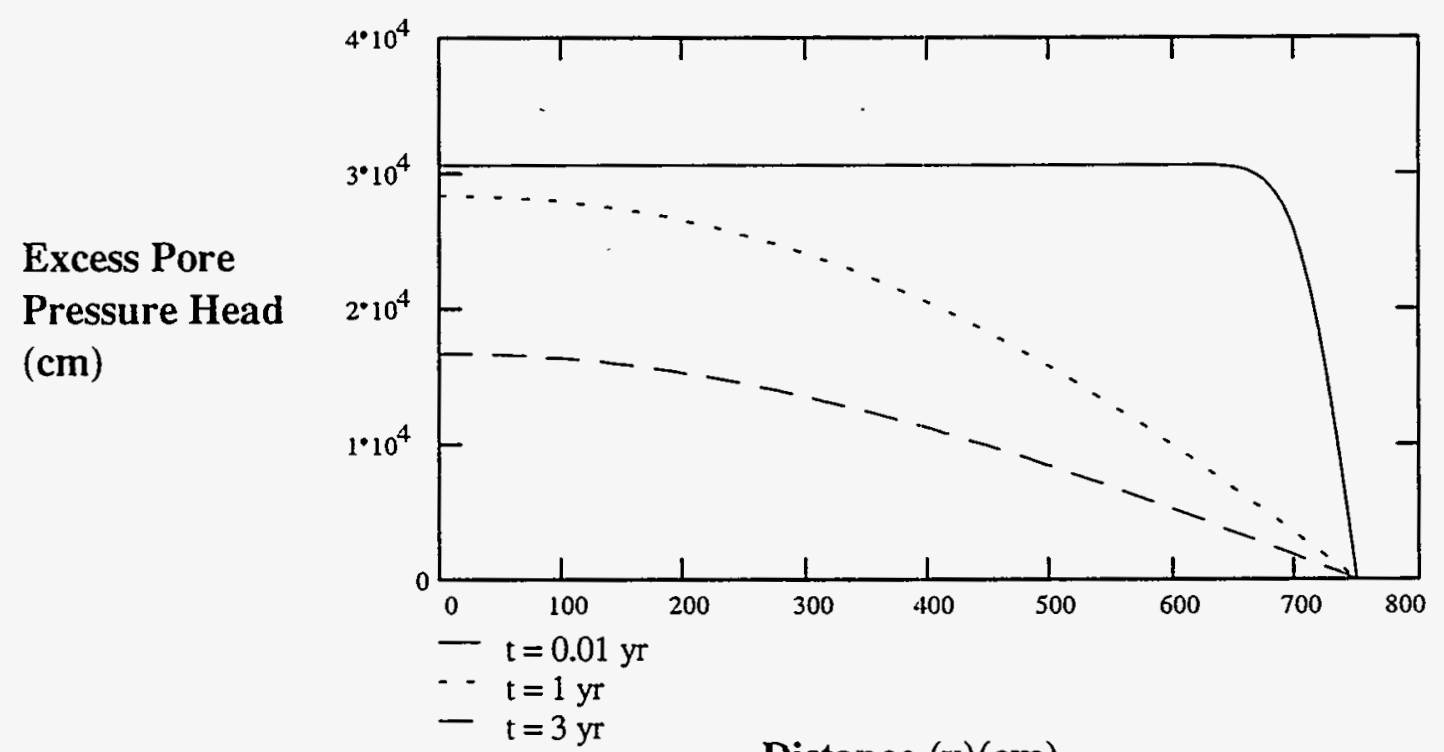

Distance $(\mathbf{x})(\mathrm{cm})$

Figure F-2-1

Pore Pressure Distribution Around Room Q

The analysis shows agreement with boundary conditions of the problem. Differentiate the subexpressions with respect to $\mathrm{x}$ in the brackets to develop the flux rate.

$$
v_{0}-v_{0} \cdot\left[\sum_{n=0}^{20}(-1)^{n} \cdot \operatorname{erfc}\left[\frac{(2 \cdot n+1) \cdot L-x}{2 \cdot \sqrt{k \cdot t}}\right]+\sum_{n=0}^{20}(-1)^{n} \cdot \operatorname{erfc}\left[\frac{(2 \cdot n+1) \cdot L+x}{2 \cdot \sqrt{k \cdot t}}\right]\right]
$$

Differentiate the following subexpressions:

$$
\operatorname{erfc}\left[\frac{(2 \cdot n+1) \cdot L-x}{2 \cdot \sqrt{k \cdot t}}\right] \quad \text { and } \quad \operatorname{erfc}\left[\frac{(2 \cdot n+1) \cdot L+x}{2 \cdot \sqrt{x \cdot t}}\right]
$$

by differentiation with respect to $\mathrm{x}$ yields

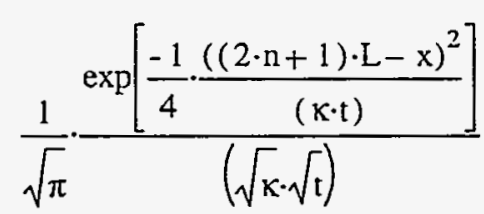

and

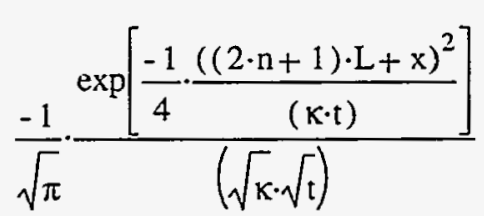


Check the dimensions of the result for correctness.

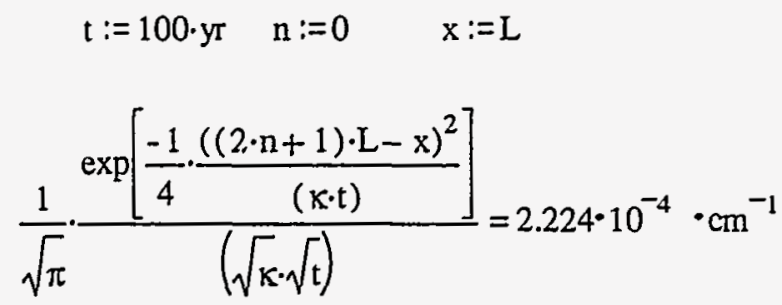

The dimensions of the subexpression are correct.

Substitute the subexpressions into the sums to calculate the hydraulic gradient.

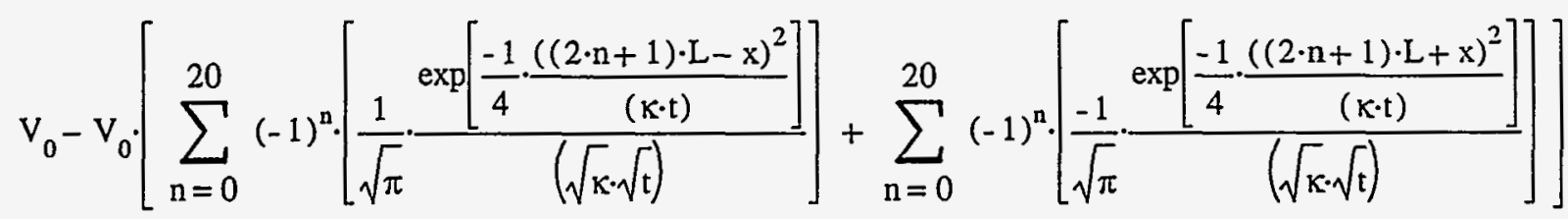

Consider the flux through the cross-sectional area of flow. The flux equals the hydraulic conductivity times the thickness times the length of the clay seam times the number of clay seams. The clay seam sizes are from Table 4-3 of Deal and others (1993). Evaluate the area through which the brine is flowing. There are two clays seams each $3.5 \mathrm{~mm}$ thick and $100 \mathrm{~m}$ long on each side of the room. Area $=2 * 3.5 \mathrm{~mm} * 2 * 100 \mathrm{~m}=1.4 \mathrm{~m}^{2}$. Evaluate the the flux $\mathrm{q}(\mathrm{t})$ at the boundary $\mathrm{x}=\mathrm{L}$.

$\mathrm{x}:=\mathrm{L}$

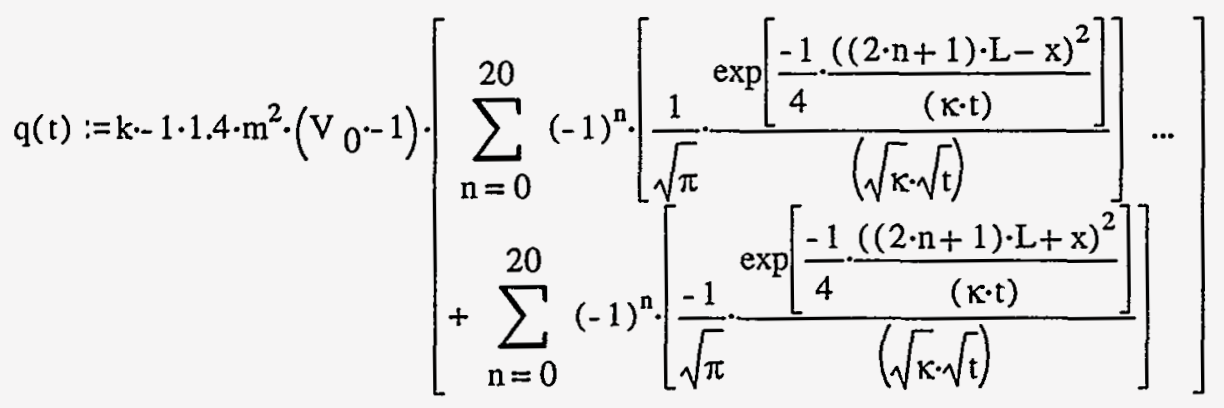

Check the result with an approximate relation for the derivative after one year.

$$
\mathrm{q}(1 \cdot \mathrm{yr})=823 \cdot \frac{\mathrm{cm}^{3}}{\text { day }}
$$

$$
\mathrm{k} \cdot 1 \cdot 1.4 \cdot \mathrm{m}^{2} \cdot \frac{\mathrm{v}(\mathrm{L}, 1 \cdot \mathrm{yr})-\mathrm{v}(.999 \cdot \mathrm{L}, 1 \cdot \mathrm{yr})}{.001 \cdot \mathrm{L}}=823 \cdot \frac{\mathrm{cm}^{3}}{\mathrm{day}}
$$


The results are in agreement. The inflow rate after 10 years and after 20 years will be as follows:

$$
\mathrm{q}(10 \cdot \mathrm{yr})=59 \cdot \frac{\mathrm{cm}^{3}}{\text { day }} \quad \text { and } \quad \mathrm{q}(20 \cdot \mathrm{yr})=3.5 \cdot \frac{\mathrm{cm}^{3}}{\text { day }}
$$

Plot the inflow rate as a function of time over the period from 800 days to 25 years. See Howarth and others (1994) for measurement time period.

$:=800 \cdot$ day, $1000 \cdot$ day. $.10000 \cdot$ day

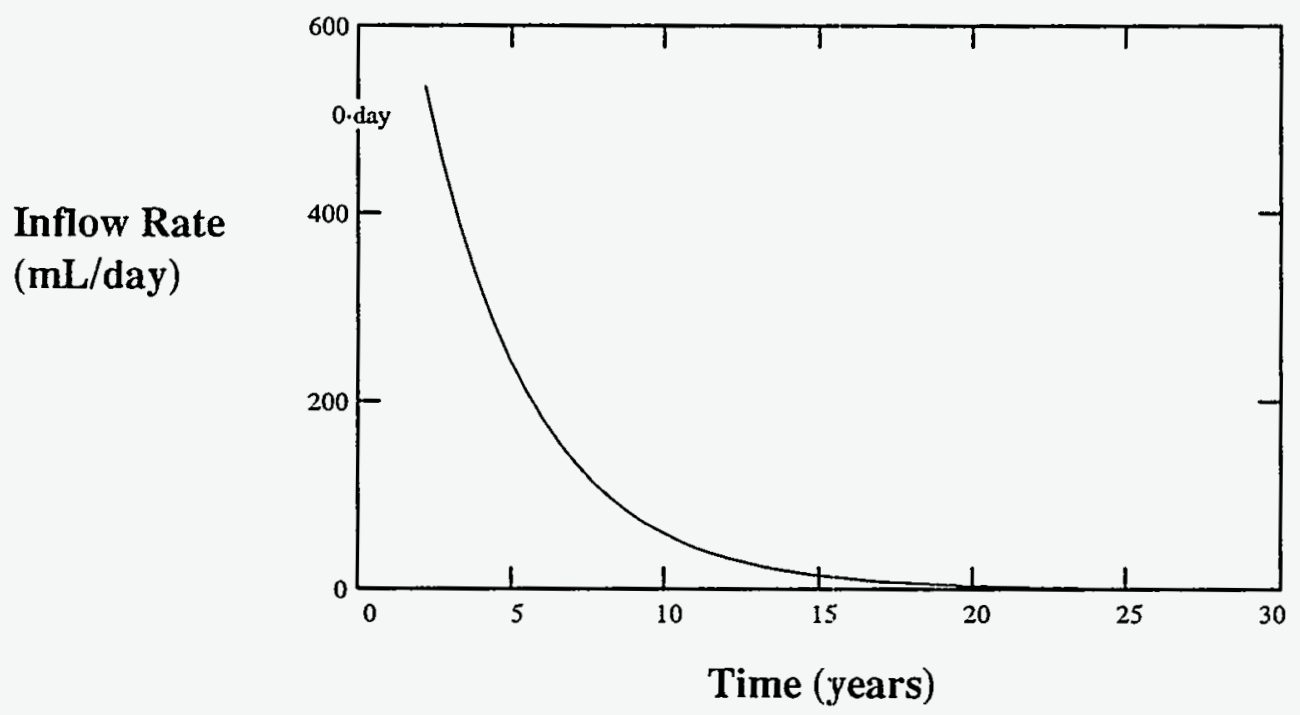

Figure F-2-2

\section{Brine Inflow into Room Q}

Determine the cumulative flow over time by integration of the flow rate relation with time. Integrate each subexpression separately, and combine in the sums.

$$
\left[\frac{1}{\sqrt{\pi}} \cdot \frac{\exp \left[\frac{-1}{4} \cdot \frac{((2 \cdot n+1) \cdot L-x)^{2}}{(\kappa \cdot t)}\right]}{(\sqrt{\kappa} \cdot \sqrt{t})}\right]
$$

by integration, yields

$$
\frac{\left[2 \cdot \sqrt{t} \cdot \exp \left[\frac{-1}{4} \cdot \frac{(-2 \cdot L \cdot n-L+x)^{2}}{(t \cdot K)}\right]+\frac{(-2 \cdot L \cdot n-L+x)}{\sqrt{\kappa}} \cdot \sqrt{\pi} \cdot \operatorname{erf}\left[\frac{1}{2} \cdot \frac{(-2 \cdot L \cdot n-L+x)}{(\sqrt{\kappa} \cdot \sqrt{t})}\right]\right]}{(\sqrt{\pi} \cdot \sqrt{\kappa})}
$$


and the following term:

$$
\left[\frac{-1}{\sqrt{\pi}} \cdot \frac{\exp \left[\frac{-1}{4} \cdot \frac{((2 \cdot n+1) \cdot L+x)^{2}}{(\kappa \cdot t)}\right]}{(\sqrt{\kappa} \cdot \sqrt{t})}\right]
$$

by integration, yields

$$
\frac{-\left[2 \cdot \sqrt{t} \cdot \exp \left[\frac{-1}{4} \cdot \frac{(2 \cdot L \cdot n+L+x)^{2}}{(t \cdot k)}\right]+\frac{(2 \cdot L \cdot n+L+x)}{\sqrt{\kappa}} \cdot \sqrt{\pi} \cdot \operatorname{erf}\left[\frac{1}{2} \cdot \frac{(2 \cdot L \cdot n+L+x)}{(\sqrt{k} \cdot \sqrt{t})}\right]\right]}{(\sqrt{\pi} \cdot \sqrt{k})}
$$

Substituting the subexpressions into a subexpression $(\Theta(t))$ and calculating the cummulative flow rate $(\mathrm{Q}(\mathrm{t}))$ :

$$
\Theta(t):=\left[\begin{array}{l}
\sum_{n=0}^{20}(-1)^{n} \cdot\left[\frac{\left[2 \cdot \sqrt{t} \cdot \exp \left[\frac{-1}{4} \cdot \frac{(-2 \cdot L \cdot n-L+x)^{2}}{(t \cdot K)}\right]+\frac{(-2 \cdot L \cdot n-L+x)}{\sqrt{K}} \cdot \sqrt{\pi} \cdot \operatorname{erf}\left[\frac{1}{2} \cdot \frac{(-2 \cdot L \cdot n-L+x)}{(\sqrt{K} \cdot \sqrt{t})}\right]\right]}{(\sqrt{\pi} \cdot \sqrt{k})}\right] \cdots \\
+\sum_{n=0}^{20}\left(-1^{n}\right)^{n} \cdot\left[\frac{-\left[2 \cdot \sqrt{t} \cdot \exp \left[\frac{-1}{4} \cdot \frac{(2 \cdot L \cdot n+L+x)^{2}}{(t \cdot k)}\right]+\frac{(2 \cdot L \cdot n+L+x)}{\sqrt{k}} \cdot \sqrt{\pi} \cdot \operatorname{erf}\left[\frac{1}{2} \cdot \frac{(2 \cdot L \cdot n+L+x)}{(\sqrt{k} \cdot \sqrt{t})}\right]\right]}{(\sqrt{\pi} \cdot \sqrt{k})}\right]
\end{array}\right] \cdot 1
$$

and

$$
Q(t):=\Theta(t) \cdot\left(k \cdot 1.4 \cdot m^{2} \cdot \frac{V_{0}}{c m} \cdot \sec \right)
$$

Check the analysis against direct numerical integration:

$$
\begin{aligned}
& Q(1600 \cdot \text { day })-Q(800 \cdot \text { day })=317.777 \cdot \mathrm{cm}^{-1} \cdot \mathrm{sec} \quad \cdot \text { liter } \\
& \text { TOL :=1.0 (TOL is calculation tolerance) } \\
& \int_{800 \cdot \text { day }}^{1600 \cdot \text { day }} \mathrm{q}(\mathrm{t}) \mathrm{dt}=317.777 \cdot \mathrm{liter}
\end{aligned}
$$


The results of the closed-form solution agree with the numerical analysis.

Plot the cumulative flow with time.

$\mathrm{t}:=800 \cdot$ day, $900 \cdot$ day. $.1600 \cdot$ day

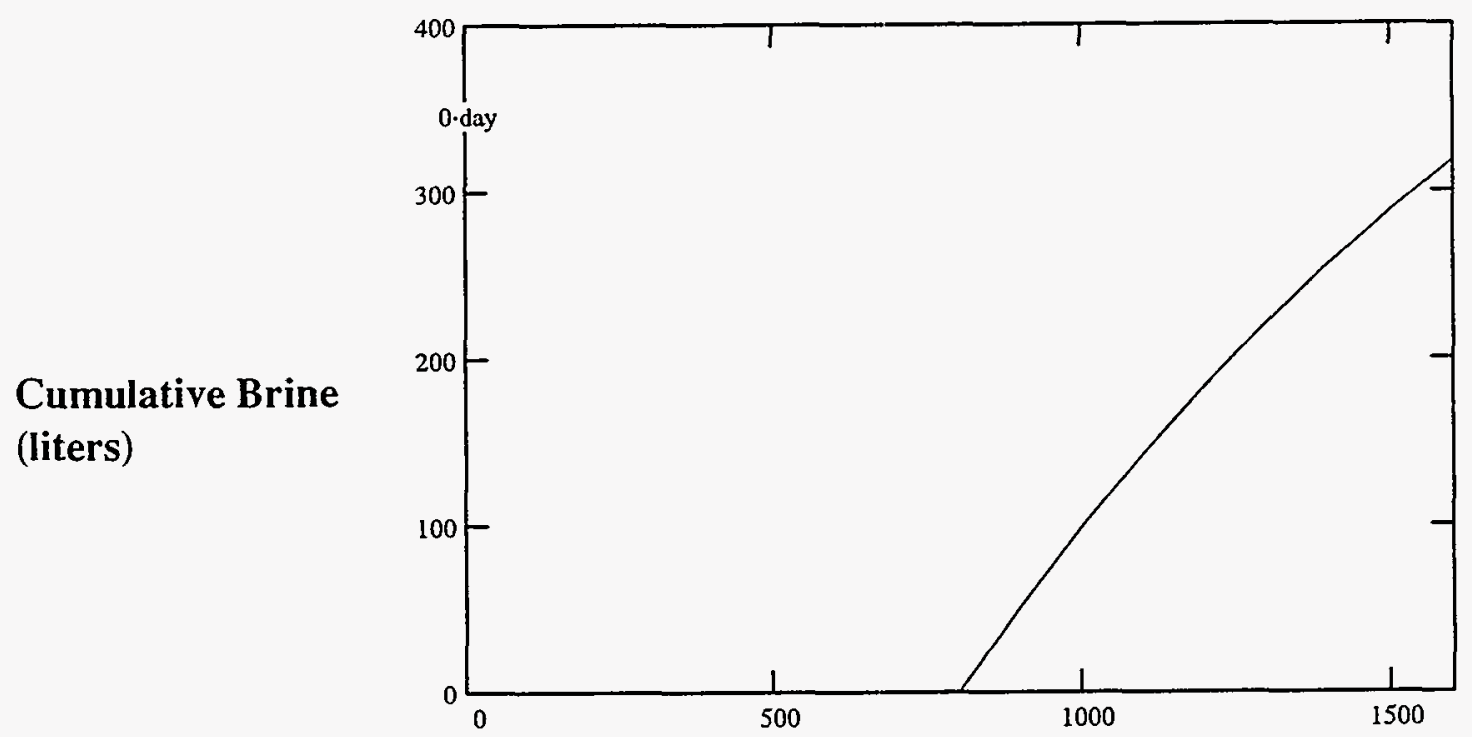

Time (days)

Figure F-2-3

Cumulative Flow into Room Q

\section{F2.3 Conclusions}

The results agree approximately with the measured flow into Room Q (see Howarth and others, 1994). 


\section{F3.0 Standard WIPP Waste Storage Room}

\section{F3.1 Problem Statement}

Calculate the amount of brine released to a Waste Disposal Room, from two clay seams totaling $17.1 \mathrm{~mm}$ thick above and below the orange band (Map Unit 1). The model for consolidation assumes that the increase in stress deforms the clay seams plastically resulting in $3 \mathrm{MPa}$ of excess pore pressure. Brine flow is induced to the room excavation due to the excess pore pressure. The consolidation of the clay layer is substantial with a change in porosity of 30 percent.

\section{F3.2 Solution}

From Deal and others (1989, p. 5-19), the stress abutment zone extends out about 5 diameters after about 1,000 days. Calculate the size of the abutment for the Waste Storage Room. The diameter of a Waste Disposal Room is $3.56 \mathrm{~m}$ (see Deal and others, 1993, p. 2-2). The length (L) over which flow occurs is:

$$
\mathrm{L}:=5 \cdot 3.56 \cdot \mathrm{m} \quad \mathrm{L}=17.8 \cdot \mathrm{m}
$$

From Freeze and Cherry (1979, p. 55), the clay compressibility $\left(\alpha_{\text {clay }}\right)$ and porosity $(\phi)$ are:

$$
\alpha_{\text {clay }}:=10^{-7} \cdot \mathrm{Pa}^{-1} \quad \phi:=0.50
$$

Calculate the change in porosity due to compression of the clay in the DRZ.

$$
\alpha_{\text {clay }} \cdot 3 \cdot 10^{6} \cdot \mathrm{Pa}=0.3
$$

The change is substantial and agrees with observation of "squeezing ground."

Calculate the void ratio (e), the conductivity (k), and the coefficient of consolidation $\left(c_{v}\right)$ for the clay (from Scott, 1963 and Freeze and Cherry, 1979):

$$
\begin{array}{cc}
\mathrm{e}:=\frac{\phi}{1-\phi} & \mathrm{e}=1 \\
\mathrm{k}:=10^{-8} \cdot \frac{\mathrm{cm}}{\mathrm{sec}} & \\
\rho_{\text {wo }}:=1.0 \cdot \frac{\mathrm{gm}}{\mathrm{cm}^{3}} & \mathrm{c}_{\mathrm{v}}=0.002 \cdot \frac{\mathrm{cm}^{2}}{\mathrm{sec}}
\end{array}
$$


Calculate the initial pore pressure head $\left(\mathrm{V}_{0}\right)$

$$
\mathrm{V}_{0}:=\frac{3 \cdot 10^{6} \cdot \mathrm{Pa}}{\rho_{w_{0}} \cdot g} \quad \mathrm{~V}_{0}=3.059 \cdot 10^{4} \cdot \mathrm{cm}
$$

We consider the lateral flow to the room over the length of the abutment zone $L$ with zero pressure at the room boundary and a "no flow" boundary condition at the edge of the DRZ. See Scott (1963, p.184) for more details in the similarities between the thermal case and the consolidation case.

From Carlsaw and Jaeger (1959, p. 309), the solution for excess pore pressure dissipation is as follows:

$$
\begin{aligned}
& \operatorname{erfc}(x):=1-\operatorname{erf}(x) \quad k:=c_{v} \\
& v(x, t):=V_{0}-V_{0} \cdot\left[\sum_{n=0}^{20}(-1)^{n} \cdot \operatorname{erfc}\left[\frac{(2 \cdot n+1) \cdot L-x}{2 \cdot \sqrt{\kappa \cdot t}}\right]+\sum_{n=0}^{20}(-1)^{n} \cdot \operatorname{erfc}\left[\frac{(2 \cdot n+1) \cdot L+x}{2 \cdot \sqrt{\kappa \cdot t}}\right]\right]
\end{aligned}
$$

where:

$$
\begin{aligned}
& \mathrm{V}_{0}=\text { initial temperature (analogous to initial excess pore pressure) } \\
& \mathrm{v}(\mathrm{x}, \mathrm{t})=\text { temperature as function of space and time (analogous to excess pore } \\
& \kappa=\text { thermal diffusivity (analogous to coefficient of consolidation) } \\
& \mathrm{t}=\text { time } \\
& \mathrm{x}=\text { distance } \\
& \text { erfc }(x)=\text { complimentary error function } \\
& \text { erf }(x)=\text { error function, and } \\
& \mathrm{n}=\text { series index }
\end{aligned}
$$


With the above properties, plot the distribution of pore pressure at several times.

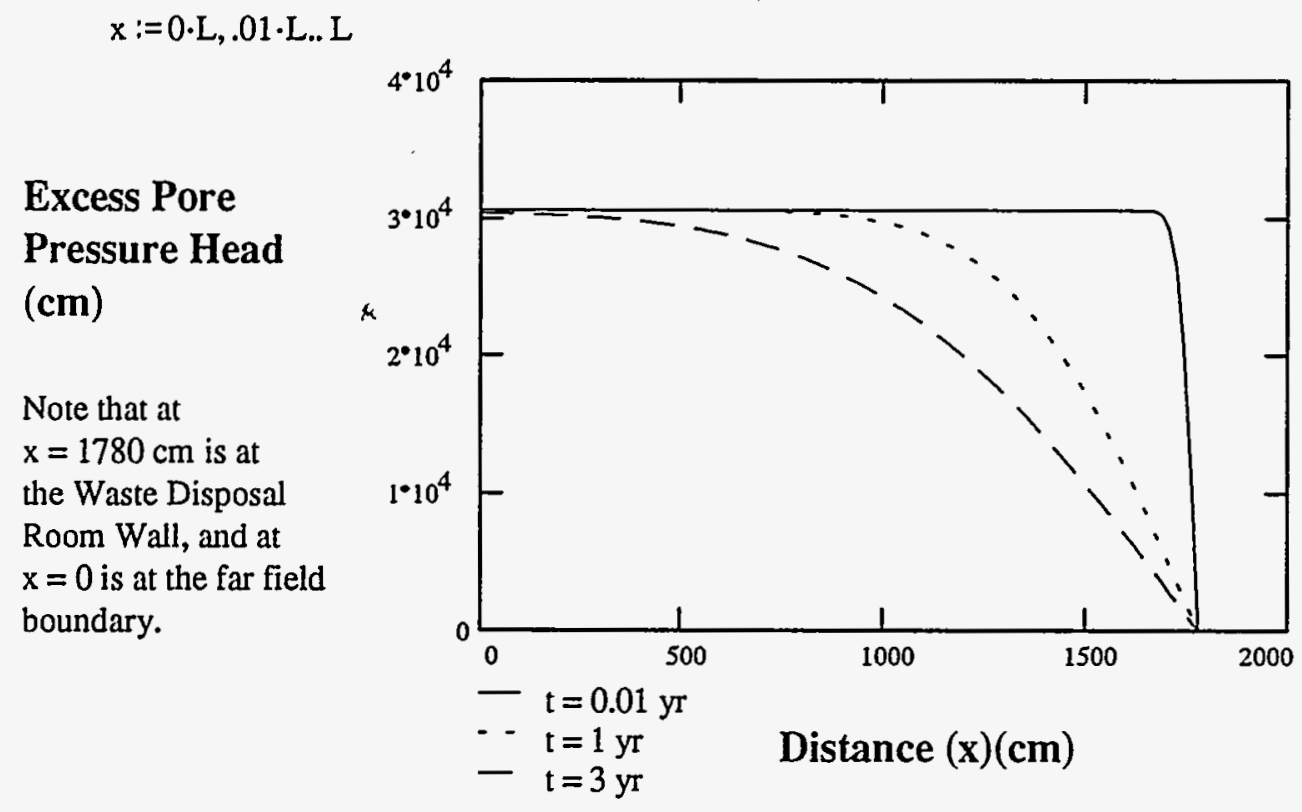

Figure F-3-1

Pore Pressure Distribution Around Standard WIPP Waste Disposal Room

The analysis shows agreement with boundary conditions of the problem. From the former equation:

$$
v_{0}-v_{0}\left[\sum_{n=0}^{20}(-1)^{n} \cdot \operatorname{erfc}\left[\frac{(2 \cdot n+1) \cdot L-x}{2 \cdot \sqrt{x \cdot t}}\right]+\sum_{n=0}^{20}(-1)^{n} \cdot \operatorname{erfc}\left[\frac{(2 \cdot n+1) \cdot L+x}{2 \cdot \sqrt{k \cdot t}}\right]\right]
$$

Differentiate the following subexpressions from the former equation to develop the flux.

$$
\operatorname{erfc}\left[\frac{(2 \cdot n+1) \cdot L-x}{2 \cdot \sqrt{k \cdot t}}\right] \quad \operatorname{erfc}\left[\frac{(2 \cdot n+1) \cdot L+x}{2 \cdot \sqrt{K \cdot t}}\right]
$$

by differentiation with respect to $\mathrm{x}$ yields

$$
\frac{1}{\sqrt{\pi}} \cdot \frac{\exp \left[\frac{-1}{4} \cdot \frac{((2 \cdot n+1) \cdot L-x)^{2}}{(\mathrm{k} \cdot \mathrm{t})}\right]}{(\sqrt{\mathrm{K} \cdot} \cdot \sqrt{\mathrm{t}})}
$$$$
\frac{-1}{\sqrt{\pi}} \cdot \frac{\exp \left[\frac{-1}{4} \cdot \frac{((2 \cdot n+1) \cdot L+x)^{2}}{(\kappa \cdot t)}\right]}{(\sqrt{\kappa} \cdot \sqrt{t})}
$$ 
Check the dimensions of the result for correctness.

$$
\begin{aligned}
& \mathrm{t}:=100 \cdot \mathrm{yr} \quad \mathrm{n}:=0 \quad \mathrm{x}:=\mathrm{L} \quad \mathrm{x}=17.8 \cdot \mathrm{m} \\
& \frac{1}{\sqrt{\pi}} \cdot \frac{\exp \left[\frac{-1}{4} \cdot \frac{((2 \cdot \mathrm{n}+1) \cdot \mathrm{L}-\mathrm{x})^{2}}{(\mathrm{k} \cdot \mathrm{t})}\right]}{(\sqrt{\mathrm{K} \cdot \sqrt{\mathrm{t}})}}=2.224 \cdot 10^{-4} \cdot \mathrm{cm}^{-1} \quad \begin{array}{l}
\text { The dimensions of the } \\
\text { subexpression are correct. }
\end{array}
\end{aligned}
$$

Substitute the subexpressions into the sums to calculate the hydraulic gradient.

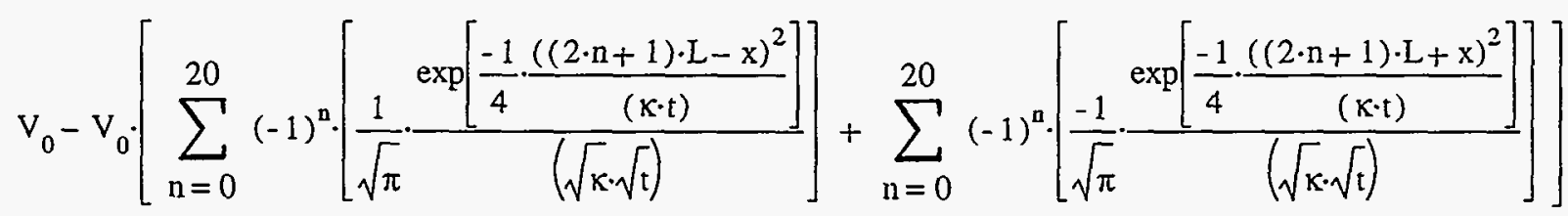

Consider the flux through the cross-sectional area of flow. The flux $q(t)$ equals the hydraulic conductivity times the thickness times the length of the clay seam times the number of clay seams. The clay seam sizes of $(3.5+3.5+10.1)=17.1 \mathrm{~mm}$ for the clay seams are from Table 4-3 of Deal and others (1993). The length of the room is $91.4 \mathrm{~m}$ from Case et al., (1991, p. 2-4). Note that we multiply by 2 to account for both sides of the room. Evaluate the the flux at the boundary $\mathrm{x}=\mathrm{L}$ :

$$
\begin{aligned}
& \mathrm{x}:=\mathrm{L}
\end{aligned}
$$

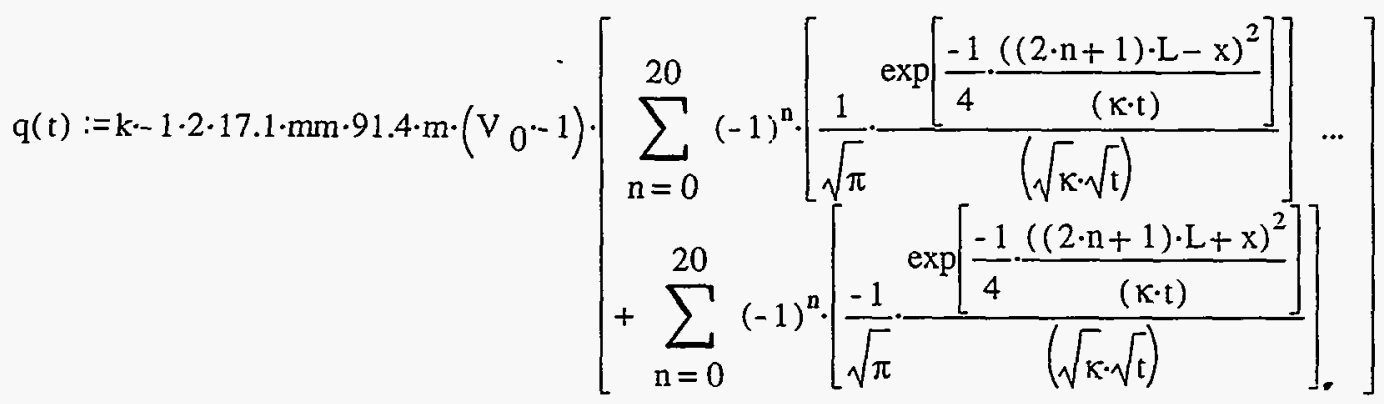

Check the result with an approximate relation for the derivative after one year.

$$
\begin{aligned}
& \mathrm{q}(1 \cdot \mathrm{yr})=1.837 \cdot 10^{3} \cdot \frac{\mathrm{cm}^{3}}{\text { day }} \\
& \mathrm{k} \cdot 1 \cdot 2 \cdot 17.1 \cdot \mathrm{mm} \cdot 91.4 \cdot \mathrm{m} \cdot \frac{\mathrm{v}(\mathrm{L}, 1 \cdot \mathrm{yr})-\mathrm{v}(.999 \cdot \mathrm{L}, \mathrm{l} \cdot \mathrm{yr})}{.001 \cdot \mathrm{L}}=1.837 \cdot 10^{3} \cdot \frac{\mathrm{cm}^{3}}{\mathrm{day}}
\end{aligned}
$$


The results are in agreement. The inflow rates after 10 and 20 years will be:

$$
\mathrm{q}(10 \cdot \mathrm{yr})=573 \cdot \frac{\mathrm{cm}^{3}}{\text { day }} \quad \mathrm{q}(20 \cdot \mathrm{yr})=341 \cdot \frac{\mathrm{cm}^{3}}{\text { day }}
$$

Plot the inflow rate as a function of time over the period from 0 to 200 years.

$\mathrm{t}:=1 \cdot \mathrm{yr}, 2 \cdot \mathrm{yr} . .200 \cdot \mathrm{yr}$

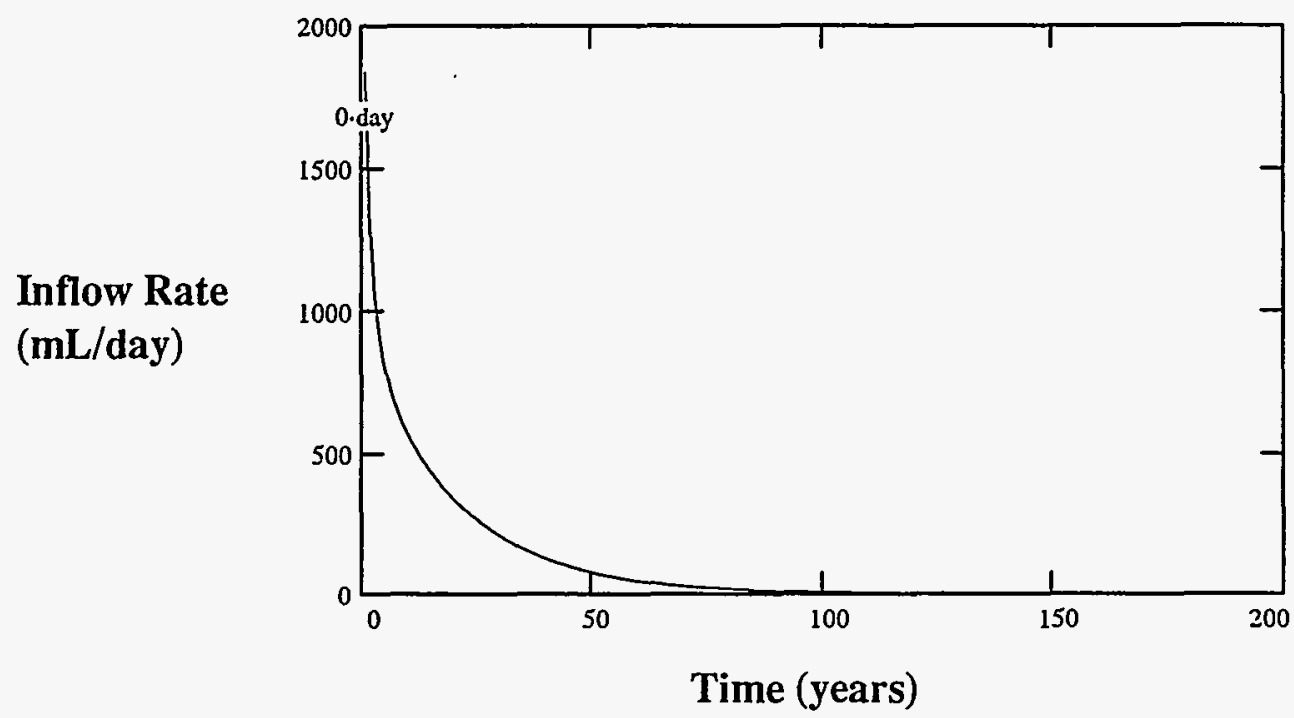

Figure F-3-2

Brine Inflow into a Waste Disposal Room

Determine the cumulative flow over time by integration of the flow rate relation with time. Integrate each subexpression separately and combine in the sums.

$$
\left[\frac{1}{\sqrt{\pi}} \cdot \frac{\exp \left[\frac{-1}{4} \cdot \frac{((2 \cdot n+1) \cdot L-x)^{2}}{(k \cdot t)}\right]}{(\sqrt{k} \cdot \sqrt{t})}\right]
$$

by integration, yields

$$
\frac{\left[2 \cdot \sqrt{t} \cdot \exp \left[\frac{-1}{4} \cdot \frac{(-2 \cdot L \cdot n-L+x)^{2}}{(t \cdot K)}\right]+\frac{(-2 \cdot L \cdot n-L+x)}{\sqrt{\kappa}} \cdot \sqrt{\pi} \cdot \operatorname{erf}\left[\frac{1}{2} \cdot \frac{(-2 \cdot L \cdot n-L+x)}{(\sqrt{K} \cdot \sqrt{t})}\right]\right]}{(\sqrt{\pi} \cdot \sqrt{\kappa})}
$$


similarly,

$$
\left[\frac{-1}{\left.\sqrt{\pi} \cdot \frac{\exp \left[\frac{-1}{4} \cdot \frac{((2 \cdot n+1) \cdot L+x)^{2}}{(\kappa \cdot t)}\right]}{(\sqrt{k} \cdot \sqrt{t})}\right]}\right.
$$

by integration, yields

$$
\frac{-\left[2 \cdot \sqrt{t} \cdot \exp \left[\frac{-1}{4} \cdot \frac{(2 \cdot L \cdot n+L+x)^{2}}{(t \cdot k)}\right]+\frac{(2 \cdot L \cdot n+L+x)}{\sqrt{K}} \cdot \sqrt{\pi} \cdot \operatorname{erf}\left[\frac{1}{2} \cdot \frac{(2 \cdot L \cdot n+L+x)}{(\sqrt{k} \cdot \sqrt{t})}\right]\right]}{(\sqrt{\pi} \cdot \sqrt{k})}
$$

Substituting the subexpressions into to subexpressions $\Theta(t)$, and calculating the cumulative flow rate $\mathrm{Q}(\mathrm{t})$ :

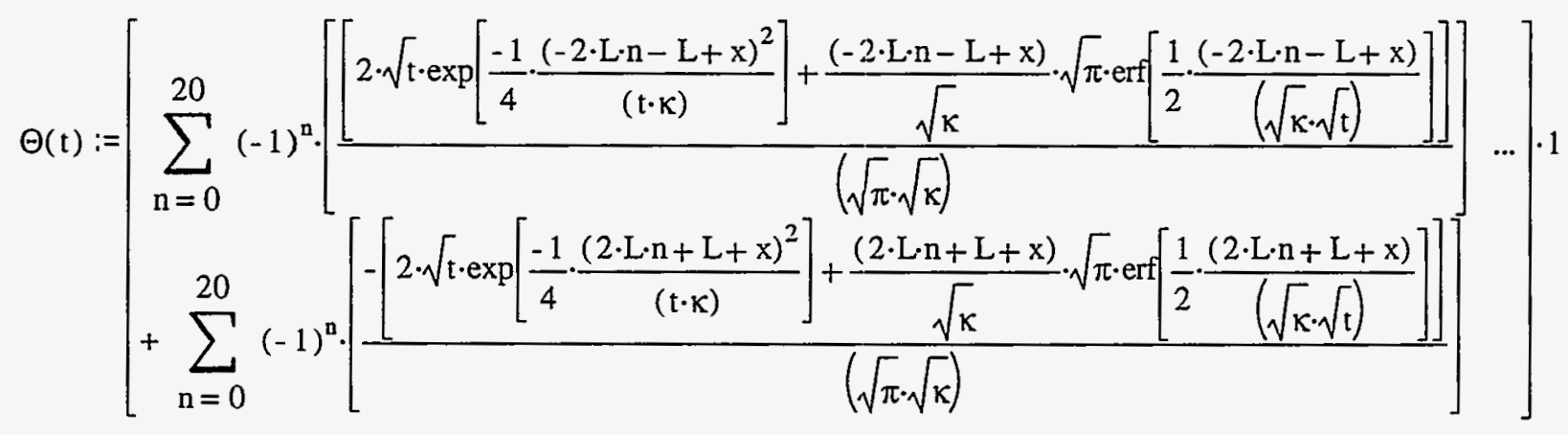

and

$$
Q(t):=\Theta(t) \cdot\left(k \cdot 1 \cdot 2 \cdot 17.1 \cdot \mathrm{mm} \cdot 91.43 \cdot \mathrm{m} \cdot \frac{\mathrm{V}_{0}}{\mathrm{~cm}} \cdot \mathrm{sec}\right)
$$

Check the analysis against direct numerical integration:

TOL $:=0.05 \quad$ TOL is calculation tolerance

$$
\mathrm{Q}(200 \cdot \mathrm{yr})-\mathrm{Q}(0.1 \cdot \mathrm{yr})=7.924 \cdot 10^{3} \cdot \mathrm{cm}^{-1} \cdot \mathrm{sec} \cdot \text { liter }
$$$$
\int_{.1 \cdot \mathrm{yr}}^{200 \cdot \mathrm{yr}} \mathrm{q}(\mathrm{t}) \mathrm{dt}=7.946 \cdot 10^{3} \cdot \text { liter }
$$

The results of the closed form solution agree with the numerical analysis 
Plot the cumulative brine with time.

$\mathrm{t}:=.1 \cdot \mathrm{yr}, 2.1 \cdot \mathrm{yr} . .200 \cdot \mathrm{yr}$

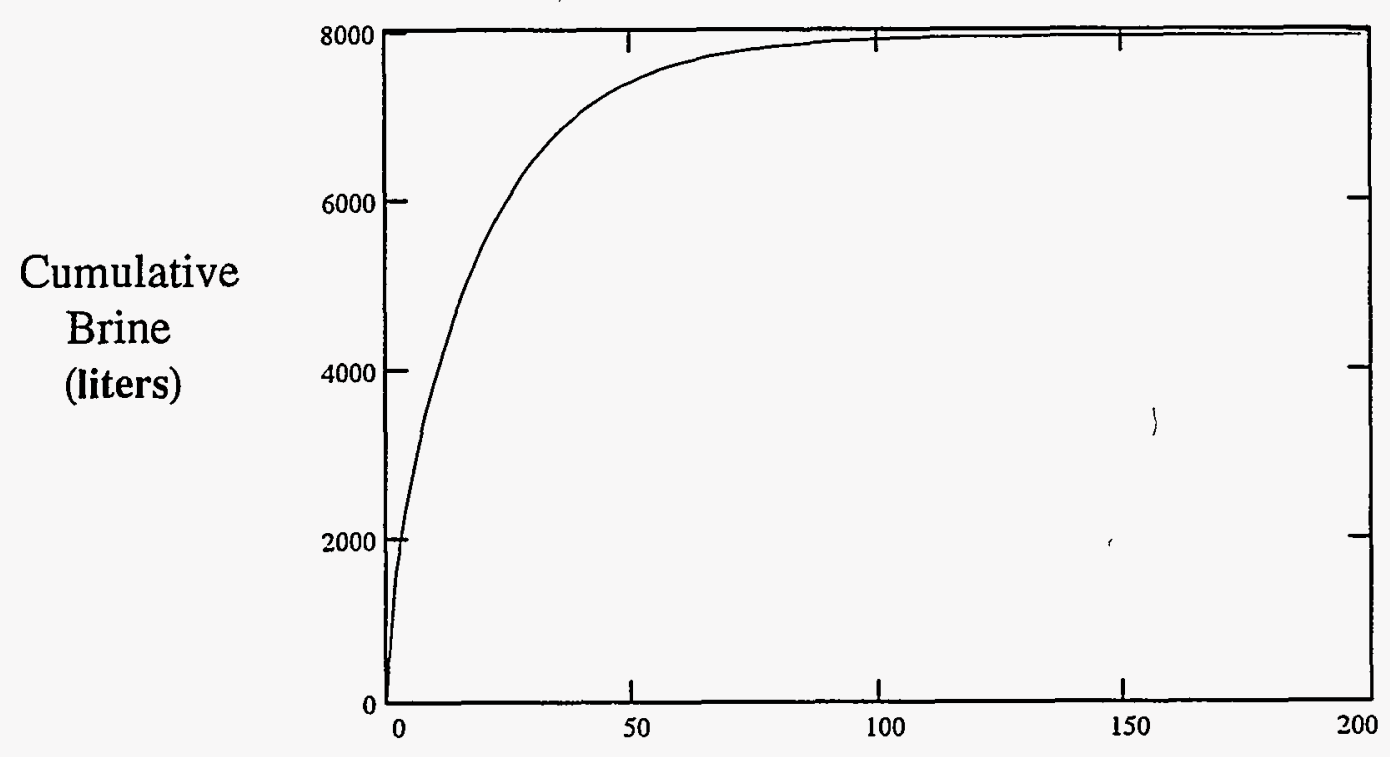

Time (years)

Figure F-3-3

Cumulative Flow into a Waste Disposal Room

\section{F3.3 Conclusion}

The amount of brine entering a waste disposal room is $8,000 \mathrm{~L}$ after about 100 years. 


\section{F4.0 Axial Consolidation Around a Borehole}

\section{F4.1 Problem Statement}

Calculate the amount of brine released to a vertical borehole intercepting a clay layer that is undergoing consolidation. Consider that the borehole is in the floor of Room $G$ and intercepts a 1-cm-thick clay layer $10 \mathrm{~m}$ below the floor.

\section{F4.2 Solution}

From Scott (1963, p. 203), the average degree of consolidation, $U\left(T_{r}, m\right)$ is given by:

$$
\begin{aligned}
& \mathrm{U}\left(\mathrm{T}_{\mathrm{r}}, \mathrm{m}\right):=1-\mathrm{e}^{-2 \cdot \frac{\mathrm{T}_{\mathrm{r}}}{\mathrm{m}}} \\
& \mathrm{m}=\left(\frac{\mathrm{n}^{2}}{\mathrm{n}^{2}-1} \cdot \ln (\mathrm{n})-\frac{3 \cdot \mathrm{n}^{2}-1}{4 \cdot \mathrm{n}^{2}}+\frac{\mathrm{k}_{\mathrm{r}}}{\mathrm{r}_{\mathrm{w}} \cdot \mathrm{K}} \cdot \frac{\mathrm{n}^{2}-1}{\mathrm{n}^{2}}\right) \\
& \mathrm{T}_{\mathrm{r}}=\mathrm{c}_{\mathrm{r}} \cdot \frac{\mathrm{t}}{\mathrm{r}_{\mathrm{e}}{ }^{2}} \\
& \mathrm{n}=\frac{\mathrm{r}_{\mathrm{e}}}{\mathrm{r}_{\mathrm{w}}}
\end{aligned}
$$

where:

$$
\begin{aligned}
& \mathrm{t}=\text { time, } \\
& \mathrm{T}_{\mathrm{r}}=\text { dimensionless time, } \\
& \mathrm{K}=\text { constant related to the surface resistance, } \\
& \mathrm{k}_{\mathrm{r}}=\text { hydraulic conductivity, } \\
& \mathrm{r}_{\mathrm{e}}=\text { effective radius of drainage, } \\
& \mathrm{r}_{\mathrm{W}}=\text { radius of the borehole } \\
& \mathrm{c}_{\mathrm{r}}=\text { coefficient of consolidation } \\
& \mathrm{m}=\text { dimensionless coefficient, and } \\
& \mathrm{n}=\text { dimensionless coeffícient for radius. }
\end{aligned}
$$

Construct a plot of the degree of consolidation with the dimensionless time. 


$$
\mathrm{T}_{\mathrm{r}}:=0.01,0.02 . .5
$$

\section{Degree \\ of \\ Consolidation (U)}

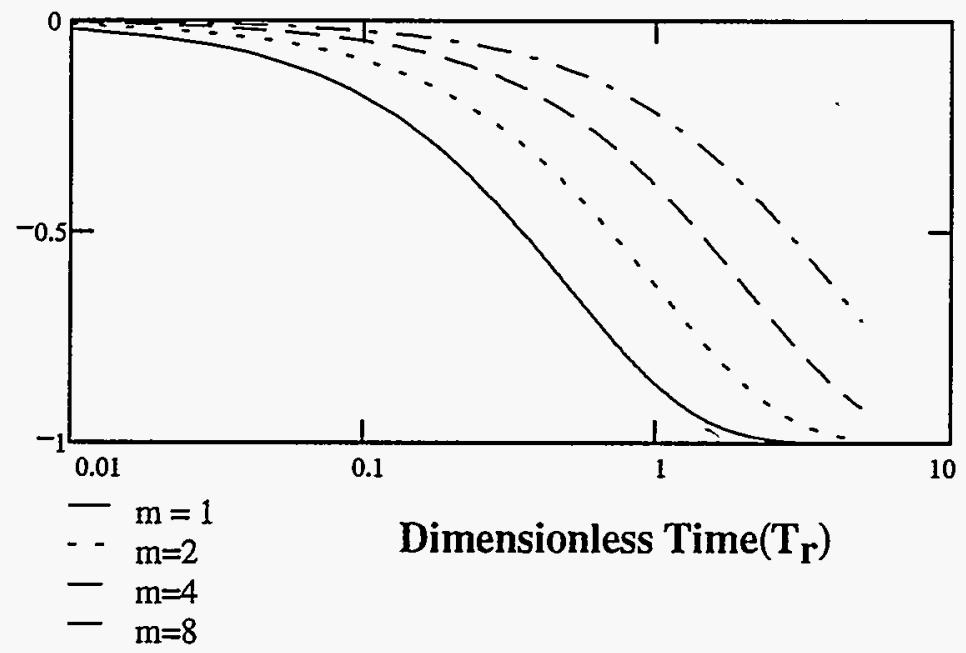

Figure F-4-1

Degree of Consolidation with the Dimensionless Time

The results agree with the results in Scott (1963, p. 202).

From Freeze and Cherry (1979, p. 55), the clay compressibility $\alpha_{\text {clay }}$ is as follows:

$$
\alpha_{\text {clay }}:=10^{-7} \cdot \mathrm{Pa}^{-1}
$$

Note that initially the excavation compresses the sidewalls, with a resulting increase in stress that could be predicted by the tributary method. Consider that after 1,000 days from Deal and others (1989, p. 5-19), the stress abutment zone extends out about 5 excavation diameters. The average diameter for the room is:

$$
\sqrt{\frac{33 \cdot \mathrm{ft} \cdot 13}{\pi} \cdot \mathrm{ft}}=3.562 \cdot \mathrm{m}
$$

At this time the stress abutment zone extends out $17.8 \mathrm{~m}$ as given in the VISCOT analysis in Deal and others (1989), the increase in stress in MPa is

$$
\frac{3.562}{17.8} \cdot 15=3.002
$$


These calculations suggest that the increase in stress is of the order of 3 to $5 \mathrm{MPa}$. Calculate the cumulative amount of brine that would flow to the borehole due to an average increase in stress of 3 $\mathrm{MPa}$.

$$
\begin{aligned}
& \alpha_{\text {clay }} \cdot 3 \cdot 10^{6} \cdot \mathrm{Pa}=0.3 \\
& \Delta \phi:=0.3 \\
& \mathrm{t}:=1 \cdot \mathrm{cm} \\
& \mathrm{A}_{\text {clay }}:=\pi \cdot(17.8 \cdot \mathrm{m})^{2} \quad \mathrm{~A}_{\text {clay }}=9.954 \cdot 10^{6} \cdot\left(\mathrm{cm}^{2}\right) \quad \\
& \text { Volume }:=\frac{\mathrm{t} \cdot \mathrm{A}_{\text {clay }} \cdot \Delta \phi}{1} \quad \text { Volume }=2.986 \cdot 10^{3} \cdot \text { liter } \quad \text { Volume }:=340.234
\end{aligned}
$$

Where:

$\mathrm{t}=$ thickness of the clay seam

A $\quad=$ area affected by a chnage in stress

Volume $=$ volume of brine stored in the clay

$\Delta \phi \quad=$ change in porosity of seam

Consider the properties of the borehole, and the clay. From Deal and others (1989, Table A-1), the diameter of the hole (a) is

$$
\mathrm{a}:=3.5 \cdot \text { in } \quad \mathrm{a}=8.89 \cdot \mathrm{cm}
$$

From Freeze and Cherry (1979, p. 37), the porosity ( $\phi)$ of clays range from 40 to 70 percent. Use

$$
\phi:=0.5
$$

The void ratio (e) is:

$$
\mathrm{e}:=\frac{\phi}{1-\phi} \quad \mathrm{e}=1
$$

From Freeze and Cherry (1979, p. 29), the hydraulic conductivity of clays ( $\mathrm{k}_{\mathrm{r}}$ ) ranges from $10^{-6}$ to $10^{-8} \mathrm{~cm} / \mathrm{sec}$. Use

$$
\mathrm{k}_{\mathrm{r}}:=10^{-8} \cdot \frac{\mathrm{cm}}{\mathrm{sec}} \quad \gamma_{\mathrm{wo}}:=62.4 \cdot \frac{\mathrm{lbf}}{\mathrm{ft}^{3}} \quad \mathrm{~g}=980.665 \cdot \frac{\mathrm{cm}}{\mathrm{sec}^{2}} \quad \frac{\gamma_{\mathrm{wo}}}{\mathrm{g}}=1 \cdot \frac{\mathrm{gm}}{\mathrm{cm}^{3}}
$$


Calculate the coefficient of consolidation. From Scott (1963, p. 184), the coefficient of consolidation is

$$
c_{r}:=k_{r} \cdot \frac{1+e}{\gamma_{w 0} \cdot \alpha_{\text {clay }}} \quad c_{r}=0.002 \cdot \frac{\mathrm{cm}^{2}}{\mathrm{sec}}
$$

Consider the effective radius of drainage $\left(\mathrm{r}_{\mathrm{e}}\right)$ equal to the far-field distance from the center of the borehole (Deal and others, 1989, p. 5-19).

$$
\mathrm{r}_{\mathrm{e}}:=20 \cdot \mathrm{m}
$$

and the diameter of the borehole equal to:

$$
\mathrm{r}_{\mathrm{w}}:=\mathrm{a} \quad \mathrm{r}_{\mathrm{w}}=0.089 \cdot \mathrm{m}
$$

Calculate the dimensionless coefficients. Consider a range for the ratio

$$
\begin{aligned}
& \mathrm{n}:=\frac{\mathrm{r}_{\mathrm{e}}}{\mathrm{r}_{\mathrm{w}}} \quad \mathrm{n}=224.972 \\
& \mathrm{~T}_{\mathrm{r}}=\mathrm{c}_{\mathrm{r}} \cdot \frac{\mathrm{t}_{\mathrm{r}}}{\mathrm{r}_{\mathrm{e}}{ }^{2}} \quad \frac{\mathrm{r}_{\mathrm{e}}{ }^{2}}{\mathrm{c}_{\mathrm{r}}}=62.124 \cdot \mathrm{yr} . \\
& \mu(\text { Ratio }):=\frac{n^{2}}{n^{2}-1} \cdot \ln (n)-\frac{3 \cdot n^{2}-1}{4 \cdot n^{2}}+(\text { Ratio }) \cdot \frac{n^{2}-1}{n^{2}} \\
& \mu \text { (Ratio) = function of the ratio } \frac{k_{r}}{r_{w} \cdot K} \\
& \mathrm{k}_{\mathrm{r}} \quad \cdot \quad=\text { the permeability of the undisturbed clay } \\
& 1 / \mathrm{K}=\text { surface resistance } \\
& \mathrm{T}_{\mathrm{r}} \quad \cdot \quad=\text { dimensionless time factor } \\
& \mathrm{t}_{\mathrm{r}} \quad=\text { time }
\end{aligned}
$$

$\mathrm{m}$ is not sensitive to the ratio. Use 0.2 for a middle range $\mathrm{m}$ value

$$
\text { Ratio := .2 }
$$$$
\mathrm{m}:=\mu \text { (Ratio) } \quad \mathrm{m}=4.866
$$ 
Determine the cumulative amount of brine flowing to the borehole.

$$
T_{r}:=0.01,0.02 . .1 .3
$$

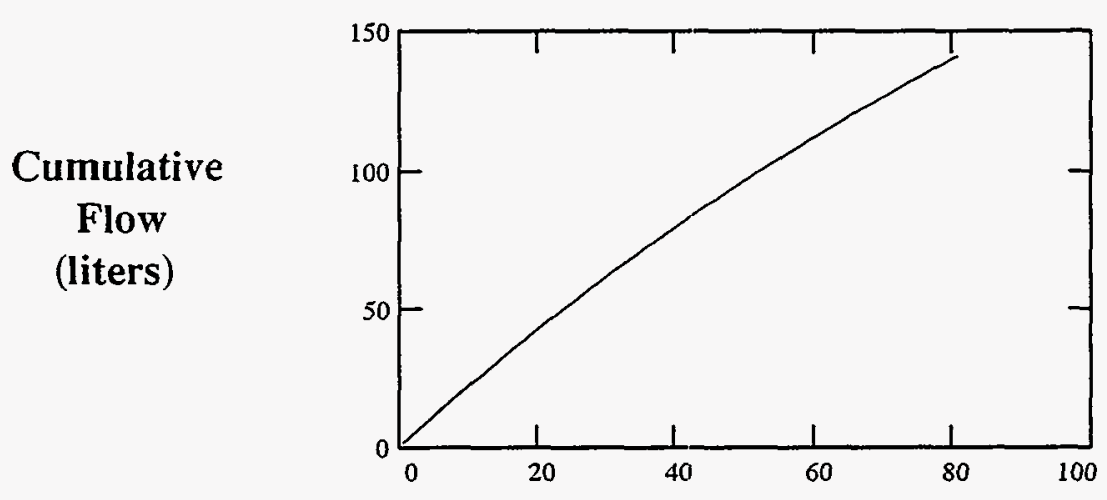

Time (years)

Figure F-4-2

Cumulative Inflow to a Borehole

Consider the flow rate as a function of time.

$$
\begin{aligned}
\mathrm{U}_{\mathrm{d}}(\mathrm{t}, \mathrm{m}):=\frac{2}{\mathrm{~m}} \cdot \exp \left[-2 \cdot \frac{\mathrm{c}_{\mathrm{r}} \cdot \frac{\mathrm{t}}{\mathrm{r}_{\mathrm{e}}^{2}}}{\mathrm{~m}}\right] \cdot \frac{\mathrm{c}_{\mathrm{r}}}{\mathrm{r}_{\mathrm{e}}{ }^{2}} \quad \mathrm{U}_{\mathrm{d}}(1 \cdot \mathrm{yr}, \mathrm{m})=1.799 \cdot 10^{-5} \cdot \mathrm{day}^{-1} \\
\mathrm{t}:=0 \cdot \mathrm{yr}, .1 \cdot \mathrm{yr} . .20 \cdot \mathrm{yr}
\end{aligned}
$$




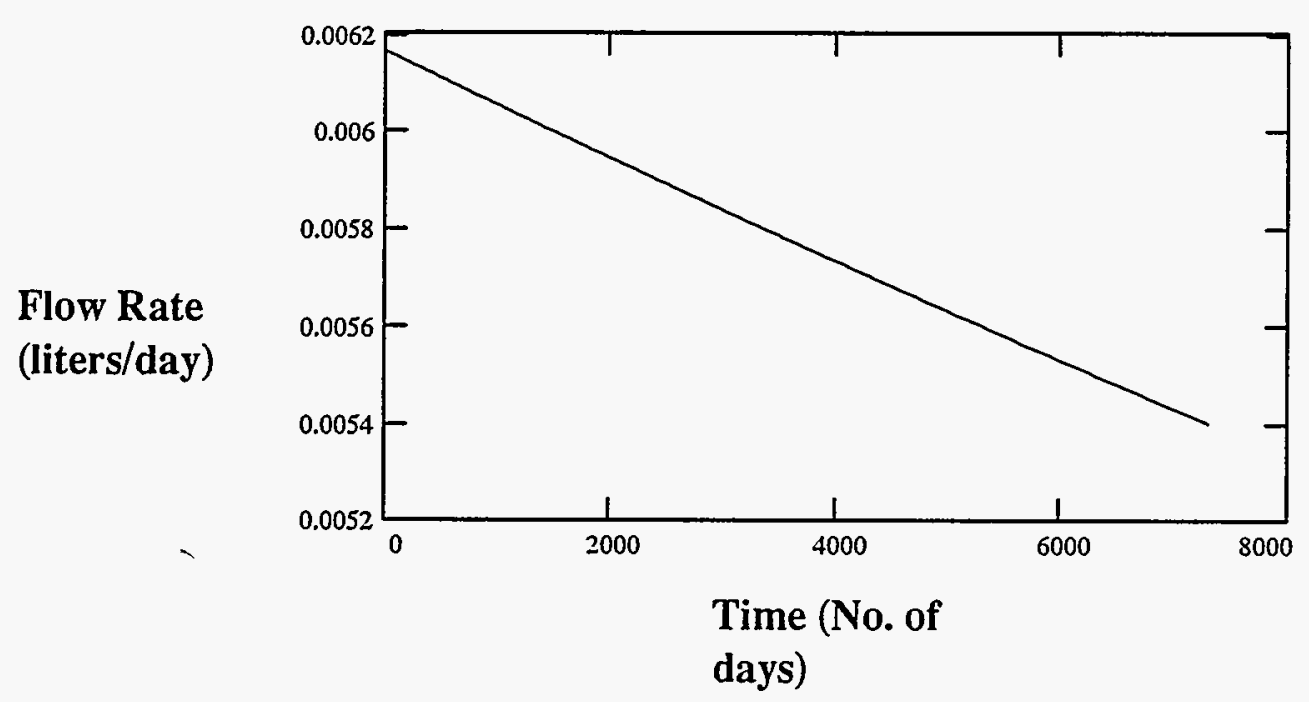

Figure F-4-3

Brine Inflow to a Borehole

\section{F4.3 Conclusion}

It will take a very long time for flow to dissipate pore pressure for a radial hole penetrating a $1 \mathrm{~cm}$ thick clay seam. DH36, DH38, DH40, DH42, and DH42A all intersect the clay seam B and show the following flow rates:

$\begin{array}{lll}\text { DH36 } & 0.1 & \text { L/Day } \\ \text { DH38 } & 0.03 & \text { L/Day } \\ \text { DH40 } & 0.008 & \text { L/Day } \\ \text { DH42 } & 0.01 & \text { L/Day } \\ \text { DH42A } & 0.02 & \text { L/Day }\end{array}$

\section{F5.0 Summary}

These order-of-magnitude seepage calculations compare well with the observed seepages into the WIPP excavations. In the case of Room $Q$, calculated seepage rates are on the order of $0.3 \mathrm{~L} /$ day after 1,600 days, where the observable rate is $0.17 \mathrm{~L} /$ day (Howarth et al.,1994, Figure 3). In this case, the numerical model is for flow toward the room along a thin clay seam. Extending this model to a waste storage room predicts that total seepage into the room will be on the order of $9,000 \mathrm{~L}$, far short of the 220,000 L necessary to completely corrode the susceptible metals that will be emplaced in it (Deal et al., 1991, Section 4.6). Furthermore, seepage into the room will cease after about 100 years. 
The case for seepage into a downhole drilled into strata below a WIPP excavation behaves differently, because flow is radially toward the drillhole. In this case, some seepage continues for a long time, perhaps a thousand years or more. It is clear that seepage into drillholes is strikingly different from seepage into a repository excavation. Deal et al (1993, Section 2.7.2) pointed out that seepage into drillholes probably should not be used to predict long-term seepage into a WIPP Waste Storage Room after sealing and closure. This calculation provides additional support for this caution. 


\section{F6.0 References}

Carslaw, H. S., and J. C. Jaeger, 1959. "Conduction of Heat in Solids," Oxford at the Clarendon Press, Oxford, England.

Case, J. B., C. A. Givens, and J. R. Tyburski, 1991. "The Geotechnical Effects of Alcove Excavation on Panel 1," DOE/WIPP 91-017, U. S. Department of Energy, Carlsbad, NM.

Deal, D. E., and R. A. Bills, 1994, "Conclusions After Eleven Years of Studying Brine at the Waste Isolation Pilot Plant", Waste Management '94, Tucson, Arizona, March 2, 1994, IT Corporation, Albuquerque, New Mexico, and Carlsbad Area Office, U. S. Department of Energy, Carlsbad, New Mexico.

Deal, D. E., R. J. Abitz, J. Myers, D. S. Belski, M. L. Martin, D. J. Milligan, R. W. Sobocinski, P. P. James Lipponer, 1993. "Brine Sampling and Evaluation Program: 1991 Report," DOE-WIPP 93-026, U. S. Department of Energy, Carlsbad, New Mexico.

Deal, D. E., R. J. Abitz, D. S. Belski, J. B. Case, M. E. Crawley, R. M. Deshler, P. E. Drez, C. A. Givens, R. B. King, B. A. Lauctes, J. Myers, S. Niou, J. M. Pietz, W. M. Roggenthen, J. R. Tyburski, and M. G. Wallace, 1989. "Brine Sampling and Evaluation Program: 1988 Report," DOE-WIPP-89-015, U. S. Department of Energy, Carlsbad, New Mexico.

Freeze, R. A. and J. Cherry, 1979. "Groundwater," Prentice Hall, Englewood Cliffs, NJ.

Howarth, S., K. Larson, T. Christian-Frear, R. Beauheim, D. Borns, D. Deal, A.L. Jensen, K. Pickens, R. Roberts, M. Tierney, P. Vaughn, and S. Webb, 1994. "Salado Formation Fluid Flow and Transport Containment Group - White Paper for Systems Prioritization and Technical Baseline, Rev. 1," Prepared by Sandia National Laboratories/New Mexico for the U.S. Department of Energy, Carlsbad, New Mexico.

Scott, R. F., 1963. Principles of Soil Mechanics, Addison Wesley, Reading, Massachusetts. 


\section{THIS PAGE INTENTIONALLLY LEFT BLANK}

